\title{
Federal Buildings Supplemental Survey 1993
}

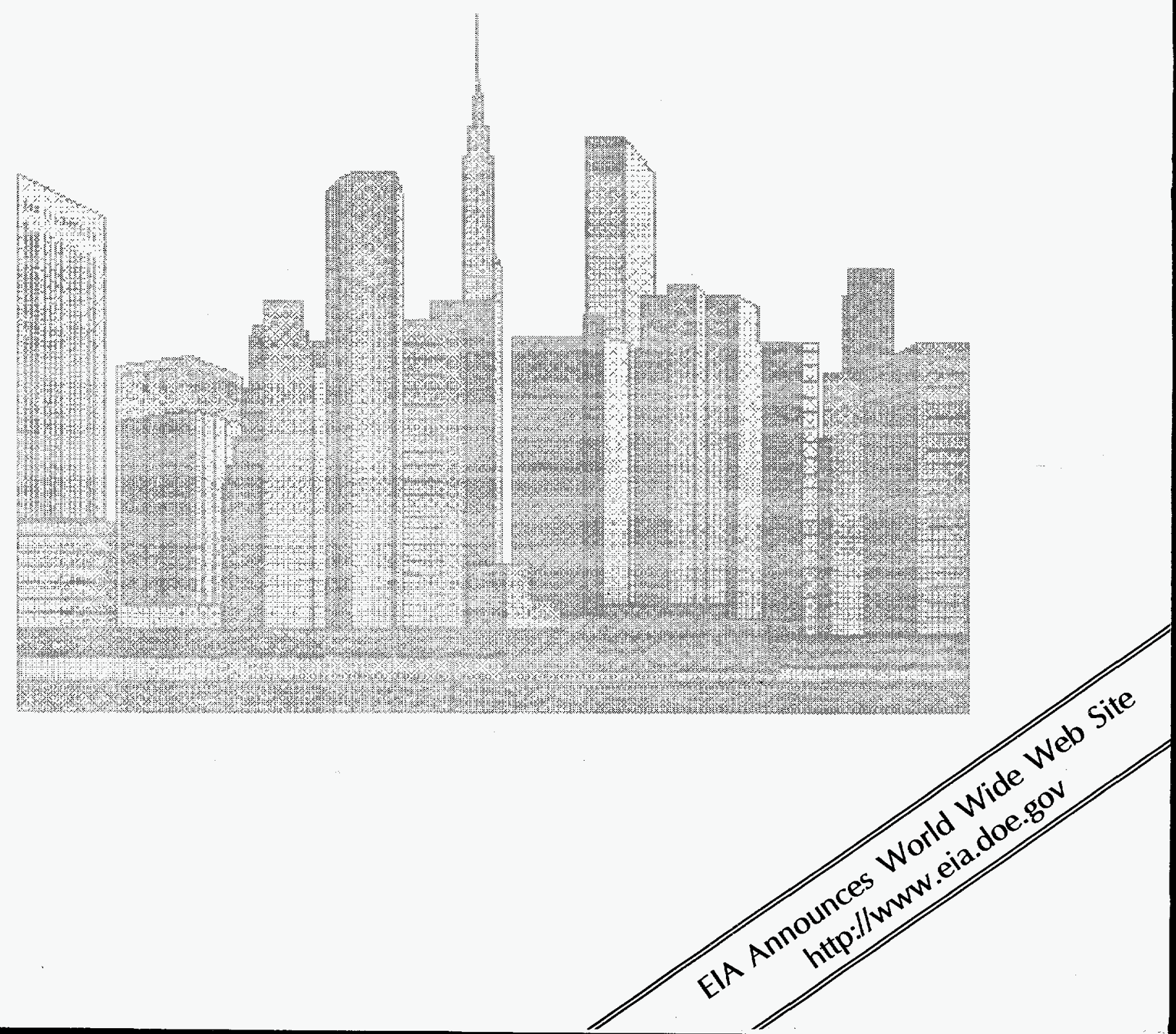


This publication is available free of charge from the National Energy Information. Center at the following address:

National Energy Information Center, EI-231

Energy Information Administration

Forrestal Building, Room 1F-048

Washington, DC 20585

(202) 586-8800

(202) 586-0727 (FAX)

TTY: For people who are deaf or hard

of hearing: (202) 586-1181

9 a.m. to 5 p.m., eastern time, M-F

Internet Addresses:

E-mail: infoctr@eia.doe.gov

World Wide Web Site: http://www.eia.doe.gov

Gopher Site: gopher://gopher.eia.doe.gov

FTP Site: ftp://ftp.eia.doe.gov 


\section{Federal Buildings Supplemental Survey 1993}

November 1995

Energy Information Administration

Office of Energy Markets and End Use

U.S. Department of Energy

Washington, DC 20585

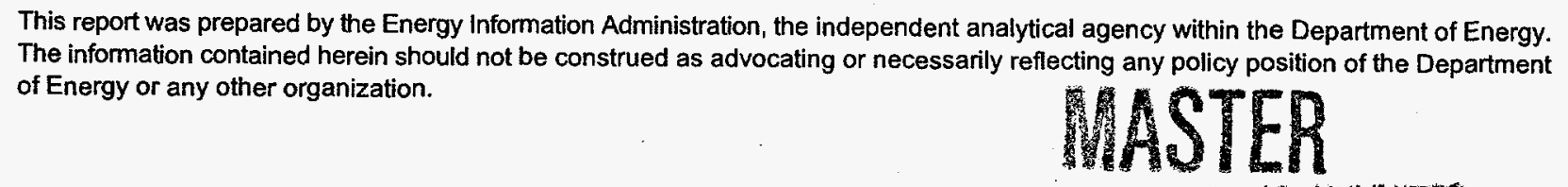




\section{Contacts}

The Energy Information Administration (EIA) prepared this publication under the general direction of W. Calvin Kilgore, Director of the Office of Energy Markets and End Use (202-586-1617). The project was directed by Lynda T. Carlson, Director of the Energy End Use and Integrated Statistics Division (EEUISD) (202-586-1112) and Nancy L. Leach, Chief of the Residential and Commercial Branch (202-586-1114). Specific technical information may be obtained from the Federal Buildings Supplemental Survey (FBSS) Manager, Emilda Rivers (202-586-5744). The Fax number for all EEUISD personnel is 202-586-0018.

For detailed technical questions on the topics indicated, refer to the following members of the EEUISD:

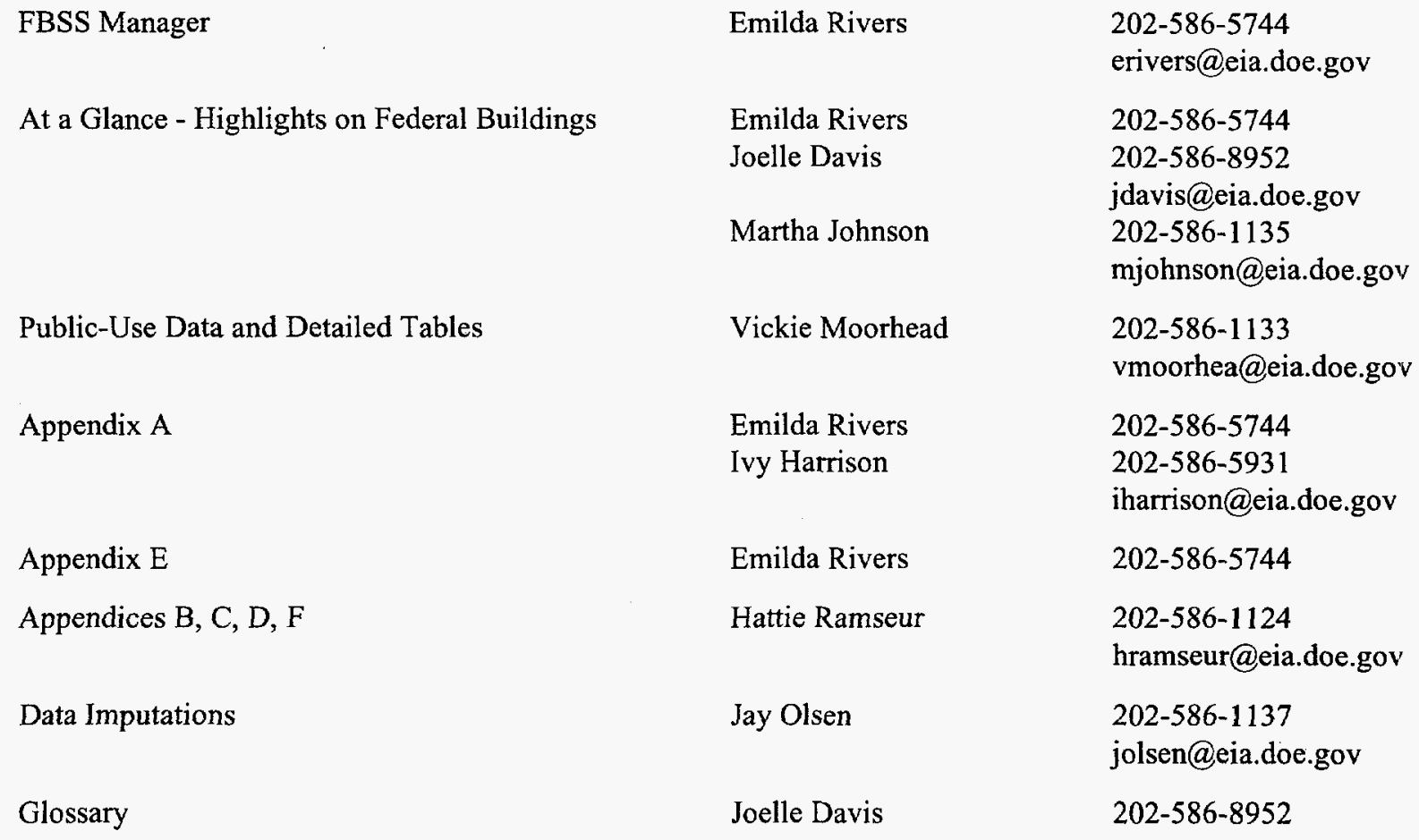

The EEUISD would also like to acknowledge LaVerne Gilchrist for her desktop publishing expertise in this report.

Support for the FBSS was received from the Office of Federal Energy Management Programs (OFEMP), U.S. Department of Energy.

Data in this report are in the public domain and, with appropriate credit, may be reproduced without permission. A suggested citation is "U.S. Department of Energy, Energy Information Administration, Federal Buildings Supplemental Survey 1993" or "U.S. Department of Energy, Energy Information Administration, 1993 Federal Buildings Supplemental Survey."

Public Use Data Diskettes containing FBSS data are available through the Office of Scientific and Technical Information (OSTI) and the National Technical Information Service (NTIS). (See Appendix F, "Related EIA Publications on Energy Consumption," for ordering information.) For questions about the contents of EPUB reports and data availability of this information on CD-ROM, call (202) 586-8800. EIA also maintains a Home Page(http://www.eia.doe.gov) on the Internet. 


\section{DISCLAIMER}

This report was prepared as an account of work sponsored by an agency of the United States Government. Neither the United States Government nor any agency thereof, nor any of their employees, make any warranty, express or implied, or assumes any legal liability or responsibility for the accuracy, completeness, or usefulness of any information, apparatus, product, or process disclosed, or represents that its use would not infringe privately owned rights. Reference herein to any specific commercial product, process, or service by trade name, trademark, manufacturer, or otherwise does not necessarily constitute or imply its endorsement, recommendation, or favoring by the United States Government or any agency thereof. The views and opinions of authors expressed herein do not necessarily state or reflect those of the United States Government or any agency thereof. 


\section{DISCLAIMER}

Portions of this document may be illegible in electronic image products. Images are produced from the best available original document. 


\section{Preface}

The Energy Information Administration (EIA) of the U.S. Department of Energy (DOE) is mandated by Congress to be the agency that collects, analyzes, and disseminates impartial, comprehensive data about energy including the volume consumed, its customers, and the purposes for which it is used. To comply with this Congressional mandate, the EIA conducts a number of energy consumption surveys to provide meaningful, objective, and accurate energy information for a wide audience that includes Congress, Federal and State agencies, industry, and the general public.

The Federal Buildings Supplemental Survey (FBSS) was conducted by EIA in conjunction with DOE's Office of Federal Energy Management Programs (OFEMP) to gain a better understanding of how Federal buildings use energy. This report presents the data from 881 completed telephone interviews with Federal buildings in three Federal regions. These buildings were systematically selected using OFEMP's specifications; therefore, these data do not statistically represent all Federal buildings in the country .

OFEMP requested that the FBSS provide building-level energy-related characteristics for a special sample of commercial buildings owned by the Federal Government. This special sample met the following OFEMP-specified criteria:

- Federal buildings from different areas of the country -- Federal Regions 3,6 , and 9

- Fewer sampled buildings from Department of Defense (DOD) -- Sample selection ratio of 1:10 for DOD buildings in each Federal Region

- Commercial Building Eligibility based on (1) size -- 10,000 square feet or over and (2) building use -exclude buildings with the majority of the floorspace used for warehouse/storage purposes.

The FBSS was conducted by telephone from July to December 1994 with Federal buildings in the following regions:

Federal Region 3 -- Delaware, District of Columbia, Maryland, Pennsylvania, Virginia, and West Virginia

Federal Region 6 -- Arkansas, Louisiana, New Mexico, Oklahoma, and Texas

Federal Region 9 -- Arizona, California, Hawaii, and Nevada.

Information for Federal commercial buildings is also collected in the Commercial Buildings Energy Consumption Survey (CBECS), which is one of the consumption surveys conducted by EIA. CBECS is conducted on a triennial basis, and is a nationwide survey of approximately 6,500 commercial buildings and their energy suppliers. Governmentowned commercial buildings are one type of building that is included in the CBECS sample. In the 1992 CBECS, approximately 15.1 billion square feet (22 percent) of commercial floorspace was government-owned.' Of this government-owned floorspace, 8 percent was in buildings owned by the Federal government. The number of Federal buildings that are selected for the relatively small CBECS sample does not allow for in-depth examination of energy use and characteristics of these buildings, which is necessary to meet the Energy Policy Act of 1992 (EPACT). Therefore, the FBSS was conducted to provide more detailed information about Federal buildings.

The purpose of the FBSS was threefold: (1) to understand the characteristics of Federal buildings and their energy use; (2) to provide a baseline in these three Federal regions to measure future energy use in Federal buildings as required in EPACT; and (3) to compare building characteristics and energy use with the data collected in the CBECS.

${ }^{\text {I} C o m m e r c i a l ~ B u i l d i n g s ~ C h a r a c t e r i s t i c s ~ 1992, ~ D O E / E I A-0246(92) ~(W a s h i n g t o n, ~ D C, ~ A p r i l ~ 1994) . ~}$ 



\section{Contents}

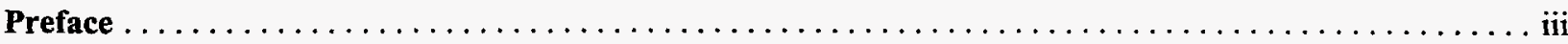

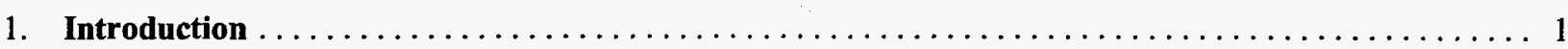

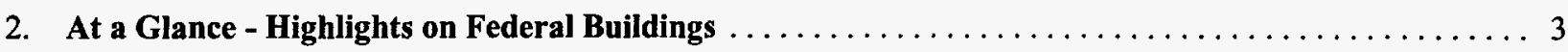

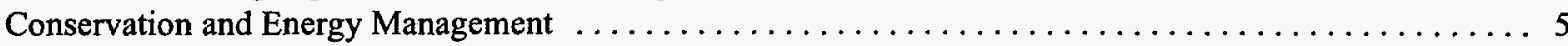

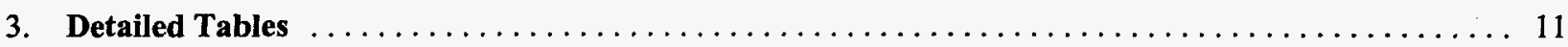

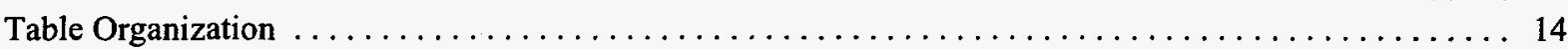

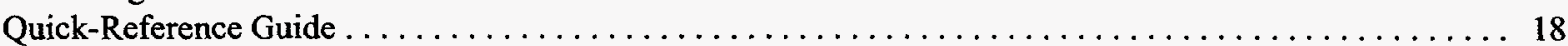

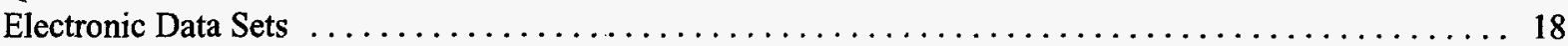

\section{Appendices}

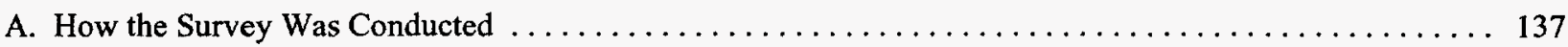

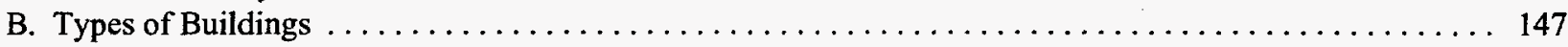

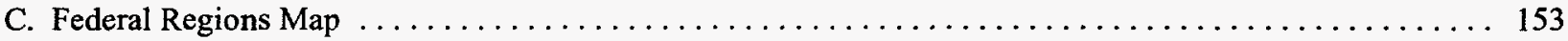

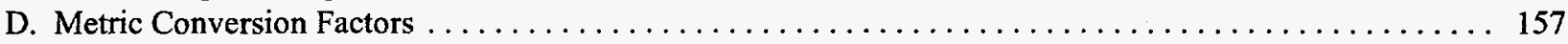

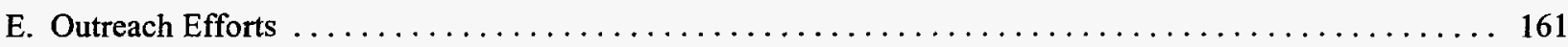

F. Related ElA Publications on Energy Consumption $\ldots \ldots \ldots \ldots \ldots \ldots \ldots \ldots \ldots \ldots \ldots \ldots \ldots \ldots$

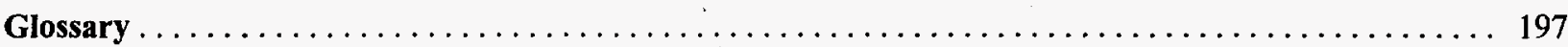

Tables

2.1. Selected Energy-Related Statistics by Federal Agency, $1993 \ldots \ldots \ldots \ldots \ldots \ldots \ldots \ldots \ldots \ldots \ldots$

2.2. Age of Equipment and Whether Retrofitted or Purchased in Past Ten Years,

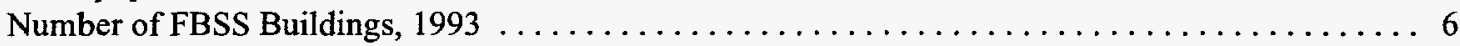

2.3. Sponsor of Retrofit or Purchase of Equipment, Number of FBSS Buildings, $1993 \ldots \ldots \ldots \ldots \ldots .7$

2.4. Type of Assistance Received for Retrofit or Purchase of Equipment, Number of FBSS

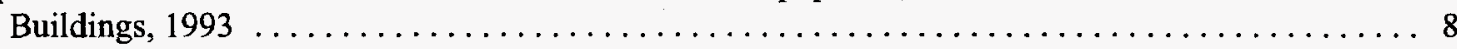

2.5. Motor Characteristics by Equipment Type, Number of FBSS Buildings, $1993 \ldots \ldots \ldots \ldots \ldots \ldots$. 9

3.1. Sample and Weighted Totals in FBSS Buildings in Regions 3, 6, and 9, $1993 \ldots \ldots \ldots \ldots \ldots \ldots 11$

3.2. Regional-level Estimates and Confidence Intervals for Selected Building Characteristics, Number of

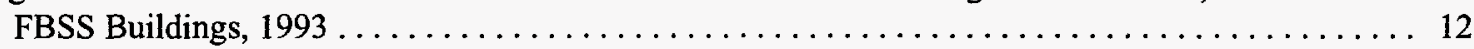

3.3. Regional-level Estimates and Confidence Intervals for Selected Buildings Characteristics, FBSS

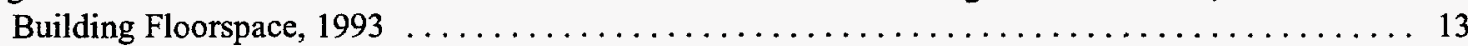

3.4. Regional-level Estimates and Confidence Intervals for Major Fuels, FBSS Consumption and

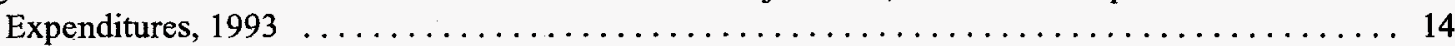

3.5. Number of FBSS Buildings with "Don't Know" Responses, $1993 \ldots \ldots \ldots \ldots \ldots \ldots \ldots \ldots \ldots \ldots$

3.6. Floorspace of FBSS Buildings with "Don't Know" Responses, $1993 \ldots \ldots \ldots \ldots \ldots \ldots \ldots \ldots \ldots 17$

3.7. Summary of FBSS Buildings and Floorspace in Federal Regions 3,6 , and $9,1993 \ldots \ldots \ldots \ldots \ldots 19$

3.8. Energy Sources in FBSS Buildings in Federal Regions 3, 6, and 9, Number Buildings, $1993 \ldots \ldots \ldots 24$

3.9. Energy Sources in FBSS Buildings in Federal Regions 3, 6, and 9, Floorspace, $1993 \ldots \ldots \ldots \ldots 25$

3.10 Heating Equipment in FBSS Buildings in Federal Region 3, Number of Buildings, $1993 \ldots \ldots \ldots \ldots 26$

3.11. Heating Equipment in FBSS Buildings in Federal Region 6, Number of Buildings, $1993 \ldots \ldots \ldots .28$

3.12. Heating Equipment in FBSS Buildings in Federal Region 9, Number of Buildings, $1993 \ldots \ldots \ldots \ldots 30$

3.13. Heating Equipment in FBSS Buildings in Federal Region 3, Floorspace, $1993 \ldots \ldots \ldots \ldots \ldots \ldots 32$

3.14. Heating Equipment in FBSS Buildings in Federal Region 6, Floorspace, $1993 \ldots \ldots \ldots \ldots \ldots \ldots \ldots 34$

3.15. Heating Equipment in FBSS Buildings in Federal Region 9, Floorspace, $1993 \ldots \ldots \ldots \ldots \ldots \ldots$

3.16. Cooling Equipment in FBSS Buildings in Federal Region 3, Number of Buildings, $1993 \ldots \ldots \ldots . .38$ 
3.17. Cooling Equipment in FBSS Buildings in Federal Region 6, Number of Buildings, $1993 \ldots \ldots \ldots .40$

3.18. Cooling Equipment in FBSS Buildings in Federal Region 9, Number of Buildings, $1993 \ldots \ldots \ldots \ldots 42$

3.19. Cooling Equipment in FBSS Buildings in Federal Region 3, Floorspace, 1993 . . . . . . . . . . . 44

3.20. Cooling Equipment in FBSS Buildings in Federal Region 6, Floorspace, $1993 \ldots \ldots \ldots \ldots \ldots \ldots 46$

3.21. Cooling Equipment in FBSS Buildings in Federal Region 9, Floorspace, $1993 \ldots \ldots \ldots \ldots \ldots \ldots 48$

3.22. Refrigeration Equipment in FBSS Buildings in Federal Region 3, Number of Buildings and

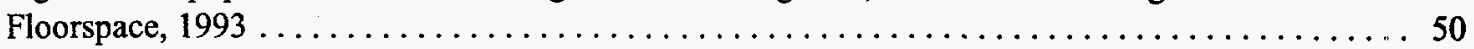

3.23. Refrigeration Equipment in FBSS Buildings in Federal Region 6, Number of Buildings and

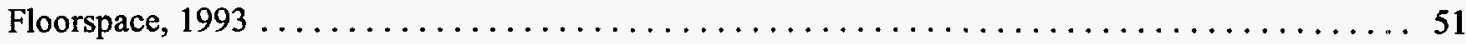

3.24. Refrigeration Equipment in FBSS Buildings in Federal Region 9, Number of Buildings and

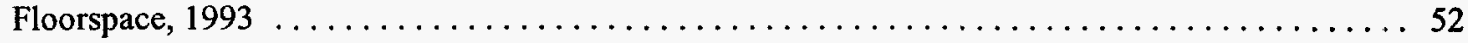

3.25. Water-Heating Equipment in FBSS Buildings in Federal Region 3, Number of Buildings and

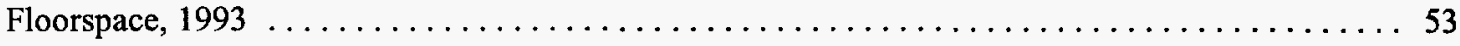

3.26. Water-Heating Equipment in FBSS Buildings in Federal Region 6, Number of Buildings and

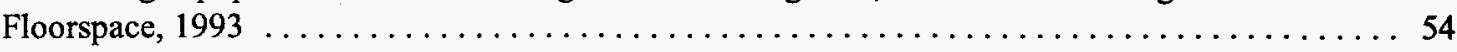

3.27. Water-Heating Equipment in FBSS Buildings in Federal Region 9, Number of Buildings and

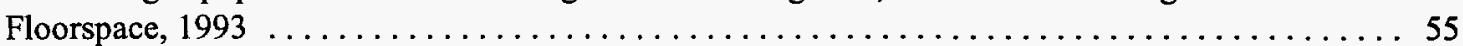

3.28. Lighting Equipment in FBSS Buildings in Federal Region 3, Number of Buildings, 1993 . . . . ... 56

3.29. Lighting Equipment in FBSS Buildings in Federal Region 6, Number of Buildings, $1993 \ldots \ldots \ldots .58$

3.30. Lighting Equipment in FBSS Buildings in Federal Region 9, Number of Buildings, $1993 \ldots \ldots \ldots 60$

3.31. Lighting Equipment in FBSS Buildings in Federal Region 3, Floorspace, $1993 \ldots \ldots \ldots \ldots \ldots \ldots 62$

3.32. Lighting Equipment in FBSS Buildings in Federal Region 6, Floorspace, $1993 \ldots \ldots \ldots \ldots \ldots \ldots 6$

3.33. Lighting Equipment in FBSS Buildings in Federal Region 9, Floorspace, $1993 \ldots \ldots \ldots \ldots \ldots \ldots 6$

3.34. Energy Conservation Features in FBSS Buildings in Federal Region 3, Number of Buildings and

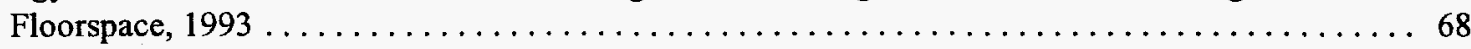

3.35. Energy Conservation Features in FBSS Buildings in Federal Region 6, Number of Buildings and

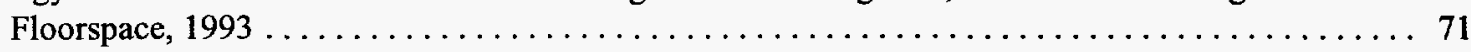

3.36. Energy Conservation Features in FBSS Buildings in Federal Region 9, Number of Buildings and

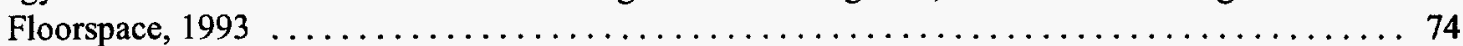

3.37. Energy Management Practices in FBSS Buildings in Federal Region 3, Number of Buildings, 1993 . . 77

3.38. Energy Management Practices in FBSS Buildings in Federal Region 6, Number of Buildings, $1993 \quad \ldots 80$

3.39. Energy Management Practices in FBSS Buildings in Federal Region 9, Number of Buildings, 1993 . . 83

3.40. Energy Management Practices in FBSS Buildings in Federal Region 3, Floorspace, 1993 . . . . . . 86

3.41. Energy Management Practices in FBSS Buildings in Federal Region 6, Floorspace, 1993 . . . . ... 89

3.42. Energy Management Practices in FBSS Buildings in Federal Region 9, Floorspace, 1993 . . . . ... 92

3.43. Consumption and Expenditures for Sum of Major Fuels, Electricity, and Natural Gas in FBSS Buildings

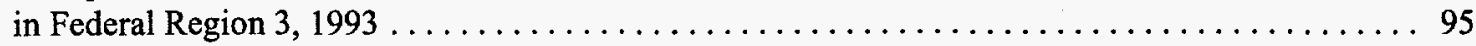

3.44. Consumption and Expenditures for Sum of Major Fuels, Electricity, and Natural Gas in FBSS Buildings

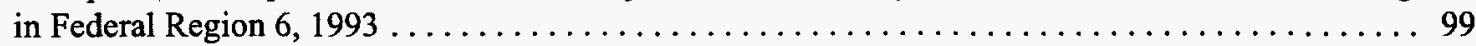

3.45. Consumption and Expenditures for Sum of Major Fuels, Electricity, and Natural Gas in FBSS Buildings

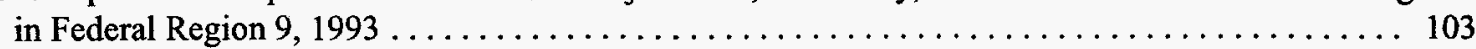

3.46. Electricity Consumption and Expenditure Intensities in FBSS Buildings in Federal Region 3, 1993 . . 107

3.47. Electricity Consumption and Expenditure Intensities in FBSS Buildings in Federal Region 6, 1993 . . 111

3.48. Electricity Consumption and Expenditure Intensities in FBSS Buildings in Federal Region 9, 1993 . . 115

3.49. Natural Gas Consumption and Expenditure Intensities in FBSS Buildings in Federal

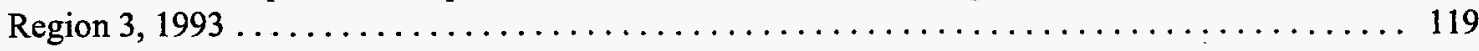

3.50. Natural Gas Consumption and Expenditure Intensities in FBSS Buildings in Federal

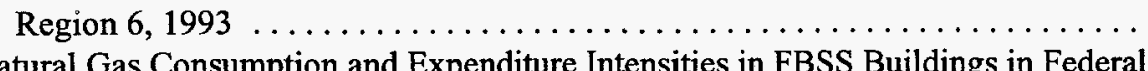

3.51. Natural Gas Consumption and Expenditure Intensities in FBSS Buildings in Federal

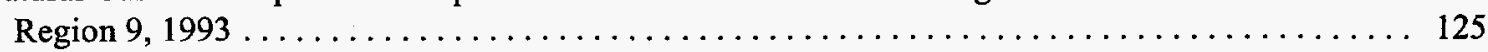

3.52. District Heat Consumption and Expenditure Intensities in FBSS Buildings in Federal

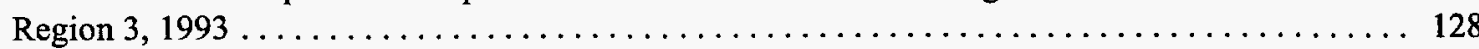

3.53. District Heat Consumption and Expenditure Intensities in FBSS Buildings in Federal

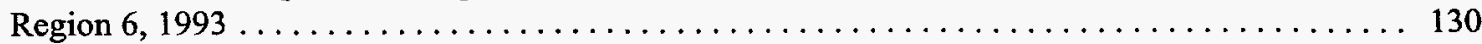


3.54. District Heat Consumption and Expenditure Intensities in FBSS Buildings in Federal

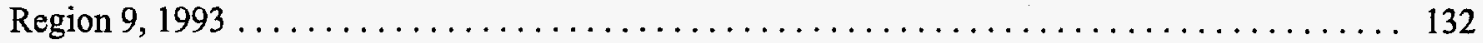

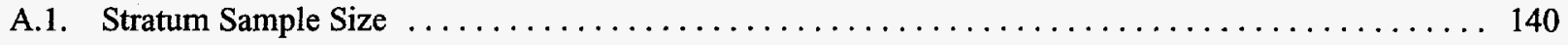

A.2. Regional Response Rates for Panel I and Panel II $\ldots \ldots \ldots \ldots \ldots \ldots \ldots \ldots \ldots \ldots \ldots \ldots \ldots \ldots \ldots \ldots \ldots$

Figure

2.1. Selected Characteristics of Motors, 1993 


\section{Introduction}

The EIA conducts two types of surveys: (1) supply surveys and (2) consumption surveys:

- Supply surveys gather information from energy suppliers and marketers on the quantities and prices of specific energy sources produced or supplied to the market. The results of these surveys are published in fuelspecific EIA publications and in the Monthly Energy Review.

- Consumption surveys gather information directly from energy end users on the types of energy they consume, along with information on the energy-related characteristics of commercial buildings, households, vehicles, and manufacturing establishments. ${ }^{2}$ The results of these surveys are published in energy consumption reports, such as this report, and in special analytical reports.

Respondents to the Federal Buildings Supplemental Survey (FBSS) 1993 were interviewed to collect information about the building, such as the principal building activity, structural characteristics, building use, energy sources, energyusing equipment, and conservation features and programs; as well as to collect billing data on energy consumption and expenditures. These billing data were collected from the Federal building manager because they were expected to have immediate access to their consumption and expenditure account data.

Following are examples of some of the data that were collected about the sampled Federal buildings:

- Structural characteristics: size (square feet of floorspace), year constructed, and roof and wall materials

- Building use: primary building activity, regular and additional operating hours, number of workers, and personal computers/terminals

- Energy sources used: four major sources; (1) electricity, (2) natural gas, (3) fuel oil (including kerosene), and (4) district sources (steam, hot water, or chilled water from a central plant or utility). The use of other energy sources in the building, such as propane, wood, coal, and solar energy, was also collected; however, no consumption statistics were gathered on these energy sources

- Energy end use: heating, air-conditioning, hot water heating, commercial cooking and serving, manufacturing, and electricity generation

- Consumption: consumption and expenditure estimates for electricity, natural gas, and district sources for Fiscal Year 1993

- Energy-using equipment: type of heating and cooling equipment and distribution systems, type of refrigeration, water-heating equipment and lighting

- Motors: number of motors with 10 or more horsepower; age, number of energy-efficient motors, and maintenance practices

- Conservation features and programs: energy audits, conservation features and programs related to building shell; heating, ventilation and air-conditioning (HVAC) systems (regular HVAC maintenance, variable-air volume, economizer cycles); and lighting (daylighting controls, manual dimmer switches, occupancy sensors, and reflectors).

\footnotetext{
${ }^{2}$ See Appendix F, "Related EIA Publications on Energy Consumption," for a listing of publications from the Commercial Buildings Energy Consumption Survey and from other EIA consumption surveys.
} 
This report also includes estimates of energy intensities. Energy intensities provide a measure for controlling or adjusting the amount of energy consumed for the effects of various building characteristics, such as size or number of workers. The intensities in this report are all conditional (fuel-specific) intensities; for example, the intensity per square foot for electricity is calculated by dividing electricity consumption by the floorspace of only those buildings that use electricity. The adjustment facilitates comparisons of energy consumption across energy sources and building types.

Many of the questions that were asked in the 1992 CBECS were asked in the FBSS to provide a comparison with the CBECS. Prior to the telephone interview, the energy managers of the sample buildings were contacted by the survey contractor to schedule the telephone appointment and provide advance questions that might require research as follows:

\section{- Building Square Footage}

- Motors Data: Age and number of motors 10 or more horsepower, and maintenance practices

- Energy Consumption: Fiscal Year 1993 (October 1992 - September 1993)

\section{- Energy Expenditures: Fiscal Year 1993}

The statistics published in this report are from a systematically selected sample of Federal buildings in Federal Regions 3,6 , and 9. Interviews were completed at 881 of these sampled Federal buildings. EIA worked closely with the Federal regions to ensure that respondent burden was minimized in the sampled buildings. (See Appendix E, "Outreach Efforts," for more information.)

EIA gratefully acknowledges the cooperation of respondents for providing the information used to produce the estimates in this report. 


\section{At a Glance - Highlights on Federal Buildings}

This section of Federal Buildings Supplemental Survey 1993 provides synopses of selected energy-related characteristics. Extensive analysis of the data was not conducted because this report represents the 881 responding buildings (buildings for which interviews were completed) and cannot be used to generalize about Federal buildings in each region. Crosstabulations of the data from the 881 buildings are provided in the Detailed Tables section.

- Energy Consumption: In FY 1993, the 881 responding Federal commercial buildings in Federal Regions 3, 6, and 9 consumed about 22 trillion Btu of electricity, natural gas, fuel oil, and district heat (Table 2.1).

- Energy Expenditures: Expenditures for the 22 trillion Btu of energy consumed in the responding Federal commercial buildings totaled about $\$ 308$ million dollars (Table 2.1).

- Energy Intensity: The major fuel energy consumption intensity was 125.79 thousand Btu per square foot for the 881 buildings (Table 2.1).

- Principal Building Activity: Principal building activity, the activity that occupies the most floorspace in the building, indicates the diversity of the Federal commercial building population. In the 881 responding buildings in Federal Regions 3, 6, and 9, office buildings accounted for the greatest number of buildings, 33 percent, and about 48 percent of the floorspace. Although health care buildings were only about 14 percent of the buildings in the sample, they constituted 21 percent of the floorspace (Table 3.7).

- Selected Characteristics by Agency: In a commercial building, building size and number of workers impact the consumption, expenditures and the associated energy intensities. Table 2.1 provides these data on an agency level. In FY 1993, of the total Federal expenditures for major fuel consumption in the 881 responding buildings in Federal Regions 3, 6, and 9 ( $\$ 308$ million), the General Services Administration (GSA) spent about $\$ 105$ million. Overall, the GSA consumed about 27 percent of the total energy consumed in the sampled buildings in Federal Regions 3, 6, and 9 in FY 1993. The Veterans Administration was the next largest consumer of energy among the participating FBSS agencies. 
Table 2.1. Selected Energy-Related Statistics by Federal Agency, 1993

\begin{tabular}{|c|c|c|c|c|c|c|c|}
\hline $\begin{array}{l}\text { Federal Agencies in FBSS - } \\
\text { Federal Regions 3, 6, and } 9\end{array}$ & $\begin{array}{c}\text { Number } \\
\text { of } \\
\text { Buildings }\end{array}$ & $\begin{array}{l}\text { Floorspace } \\
\text { (thousand } \\
\text { square feet) }\end{array}$ & $\begin{array}{c}\text { Number } \\
\text { of } \\
\text { Workers }\end{array}$ & $\mid \begin{array}{c}\text { Sum of Major } \\
\text { Fuel } \\
\text { Consumption } \\
\text { (billion Btu) }\end{array}$ & $\begin{array}{c}\text { Major Fuel } \\
\text { Intensity } \\
\text { (thousand Btu } \\
\text { per square } \\
\text { foot) }\end{array}$ & $\begin{array}{c}\text { Sum of Major } \\
\text { Fuel } \\
\text { Expenditures } \\
\text { (thousand } \\
\text { dollars) }\end{array}$ & $\begin{array}{c}\text { Major Fuel } \\
\text { Expenditures } \\
\text { per square } \\
\text { foot (dollars) }\end{array}$ \\
\hline Responding Agencies & 881 & 175,012 & 492,172 & 22,014 & 125.79 & 308,401 & 1.76 \\
\hline Department of Agriculture ...... & 14 & 720 & 1,156 & 148 & 205.98 & 2,008 & 2.79 \\
\hline Department of Commerce ...... & 17 & 2,390 & 3,579 & 682 & 285.31 & 9,481 & 3.97 \\
\hline Department of Defense ........ & 122 & 24,145 & 60,534 & 3,192 & 132.20 & 43,274 & 1.79 \\
\hline Department of Education ....... & 2 & 80 & 131 & 6 & 78.88 & 55 & .69 \\
\hline Department of Energy ........ & 86 & 7,990 & 14,001 & 2,473 & 309.53 & 32,400 & 4.05 \\
\hline Department of Justice $\ldots \ldots \ldots$ & 20 & 1,212 & 1,947 & 231 & 190.57 & 3,065 & 2.53 \\
\hline Department of Labor ......... & 7 & 729 & 577 & 54 & 74.07 & 666 & .91 \\
\hline Department of the Interior ...... & 30 & 971 & 1,378 & 63 & 65.15 & 963 & .99 \\
\hline Department of the Treasury ..... & 2 & 800 & 1,580 & 59 & 73.41 & 1,003 & 1.25 \\
\hline Department of Transportation ... & 13 & 1,426 & 4,909 & 189 & 132.19 & 2,882 & 2.02 \\
\hline $\begin{array}{l}\text { Environmental Protection } \\
\quad \text { Agency } \ldots \ldots \ldots \ldots \ldots \ldots\end{array}$ & 1 & 50 & 145 & 13 & 251.87 & 176 & 3.53 \\
\hline $\begin{array}{l}\text { Federal Emergency } \\
\quad \text { Management Agency ......... }\end{array}$ & 2 & 81 & 103 & 7 & 86.68 & 75 & .92 \\
\hline $\begin{array}{l}\text { General Accounting Office ....... } \\
\text { General Services }\end{array}$ & 1 & 1,844 & 2,588 & 106 & 57.28 & 1,827 & .99 \\
\hline Administration & 157 & 66,598 & 220,080 & 5,919 & 88.88 & 105,118 & 1.58 \\
\hline Government Printing Office ..... & 2 & 1,420 & 4,100 & 220 & 155.22 & 2,777 & 1.96 \\
\hline Health and Human Services ... & 23 & 2,042 & 4,243 & 760 & 372.11 & 7,940 & 3.89 \\
\hline $\begin{array}{l}\text { National Aeronautics and Space } \\
\text { Administration ............ }\end{array}$ & 52 & 4,180 & 9,878 & 913 & 218.52 & 11,881 & 2.84 \\
\hline National Science Foundation & 1 & 23 & 20 & 27 & $1,164.35^{\mathrm{a}}$ & 476 & 20.70 \\
\hline $\begin{array}{l}\text { United States Postal } \\
\text { Service } \ldots \ldots \ldots\end{array}$ & 181 & 24,806 & 103,458 & 2,373 & 95.66 & 32,732 & 1.32 \\
\hline Veterans Administration & 148 & 33,504 & 57,765 & 4,579 & 136.67 & 49,603 & 1.48 \\
\hline
\end{tabular}

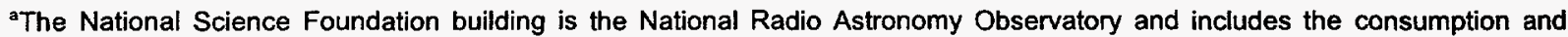
expenditures for the observatory as well as the office floorspace.

Notes: - These data are from 881 federally owned buildings having the following criteria: (1) located in Federal Regions 3, 6, or 9; (2) larger than 10,000 square feet; and (3) used for a commercial purpose, other than warehouse and storage. In addition, 9 out of 10 selected buildings were from agencies other than the Department of Defense. - Totals may not equal sum of components due to independent rounding. - Data are for Fiscal Year 1993 (October 1, 1992 through September 30, 1993).

Source: Energy Information Administration, Office of Energy Markets and End Use, 1993 Federal Buildings Supplemental Survey. 


\section{Conservation and Energy Management}

In response to OFEMP's request that EIA provide detailed information on energy efficiency and energy management for Federal buildings, the FBSS included a number of questions about energy efficiency features, energy management practices, and the reduction of equipment use during off-hours. Federal agencies have a variety of strategies available for implementation to reduce their energy consumption including energy-efficient equipment retrofits.

There are various sponsors and assistance programs available to retrofit equipment. The following synopsis provides data for retrofitting equipment practices for the FBSS.

- Of the 881 Federal commercial buildings that responded to the FBSS, about 39 percent reported using boilers as their heating equipment and 36 percent reported using district steam. In about 70 percent of the buildings using boilers and 84 percent of buildings using district steam, the equipment was 10 years or older (Table 2.2).

- Less than 10 percent of FBSS buildings reported using heat pumps as their heating equipment, and as might be expected, the majority of the heat pumps were less than 10 years old. Buildings with packaged units for heating were about evenly divided between those less than 10 years old and those 10 years or older.

- In buildings with water heating equipment, approximately 62 percent of that equipment was 10 years or older.

- The vast majority of building respondents reported purchasing new equipment rather than retrofitting existing equipment within the past 10 years.

- Lighting equipment was an overwhelming "favorite" for retrofitting or purchasing (56 percent of the 871 buildings with lighting equipment) followed by retrofits or purchases for heating equipment, water-heating equipment and central chillers. Of the 487 building respondents reporting either the retrofit or purchase of their lighting equipment, about 39 percent reported retrofitting rather than purchasing.

- With the exception of retrofitting lighting equipment, the majority of the 881 Federal buildings that were surveyed did not report the purchase or retrofit of energy-using equipment within the last 10 years. 
Table 2.2. Age of Equipment and Whether Retrofitted or Purchased in Past Ten Years, Number of FBSS Buildings, 1993

\begin{tabular}{|c|c|c|c|c|c|c|c|c|c|}
\hline \multirow[b]{2}{*}{$\begin{array}{l}\text { Type of Equipment } \\
\text { (more than one may } \\
\text { apply) }\end{array}$} & \multirow[b]{2}{*}{$\begin{array}{c}\text { All } \\
\text { Buildings } \\
\text { with Each } \\
\text { Type } \\
\text { of } \\
\text { Equipment }\end{array}$} & \multicolumn{4}{|c|}{ Age of Equipment } & \multicolumn{4}{|c|}{$\begin{array}{c}\text { Retrofitted or Purchased Equipment in Past } \\
\text { Ten Years }\end{array}$} \\
\hline & & $\begin{array}{l}\text { Less } \\
\text { Than } \\
\text { Ten } \\
\text { Years } \\
\text { Old }\end{array}$ & $\begin{array}{c}\text { Ten } \\
\text { Years } \\
\text { or } \\
\text { Older }\end{array}$ & $\begin{array}{c}\text { Some Less } \\
\text { Than Ten } \\
\text { Years, } \\
\text { Some More } \\
\text { than Ten } \\
\text { Years }\end{array}$ & $\begin{array}{c}\text { Don't } \\
\text { Know/Not } \\
\text { Ascertained }\end{array}$ & Retrofitted & Purchased & $\begin{array}{l}\text { Both } \\
\text { Retrofitted } \\
\text { and } \\
\text { Purchased }\end{array}$ & $\begin{array}{c}\text { Don't } \\
\text { Know/Not } \\
\text { Ascertained }\end{array}$ \\
\hline \multicolumn{10}{|l|}{$\begin{array}{l}\text { Heating } \\
\text { (more than one may } \\
\text { apply) }\end{array}$} \\
\hline Furnace $\ldots \ldots \ldots$ & 59 & 25 & 32 & 0 & 2 & 1 & 16 & 0 & 1 \\
\hline Heat Pump & 84 & 55 & 28 & 0 & 1 & 2 & 36 & 0 & 2 \\
\hline Space Heater & 138 & 70 & 64 & 0 & 4 & 2 & 53 & 0 & 2 \\
\hline District Steam . . . . & 320 & 45 & 269 & 3 & 3 & 10 & 25 & 0 & 3 \\
\hline Boiler .......... & 341 & 83 & 238 & 4 & 16 & 13 & 54 & 0 & 17 \\
\hline Packaged Unit ... & 151 & 66 & 67 & 3 & 15 & 3 & 46 & 0 & 15 \\
\hline Central Chillers .... & 419 & 128 & 257 & 18 & 16 & 15 & 98 & 2 & 20 \\
\hline Water-Heating $\ldots$. & 857 & 285 & 528 & 4 & 36 & 23 & 199 & 0 & 38 \\
\hline Lighting...$\ldots \ldots$ & 871 & NA & NA & NA & NA & 190 & 288 & 9 & 46 \\
\hline Refrigeration .... . . & 242 & 90 & 136 & 3 & 13 & 8 & 58 & 1 & 5 \\
\hline
\end{tabular}

NA $=$ Not Applicable.

Notes: - Only buildings in which the equipment was less than ten years old or the age was unknown were asked whether it was retrofitted or purchased (buildings answering no are not provided). - These data are from 881 federally owned buildings having the following criteria: (1) located in Federal Regions 3,6, or 9; (2) larger than 10,000 square feet; and (3) used for a commercial purpose, other than warehouse and storage. In addition, 9 out of 10 selected buildings were from agencies other than the Department of Defense. - Data are for Fiscal Year 1993 (October 1, 1992 through September 30, 1993).

Source: Energy Information Administration, Office of Energy Markets and End Use, 1993 Federal Buildings Supplemental Survey. 
Sponsors of programs aimed at assisting buildings in purchasing new equipment or retrofitting existing equipment include utilities, the Federal government (under the Federal Energy Management Program (FEMP)), and third-parties such as an energy service company (ESCO). Buildings can also institute energy-saving programs in-house (Table 2.3).

- Of the buildings that had retrofitted or purchased equipment, the majority did so within programs that were sponsored in-house.

- Of the buildings that reported receiving assistance from FEMP, most reported assistance in the area of lighting equipment.

Table 2.3. Sponsor of Retrofit or Purchase of Equipment, Number of FBSS Buildings, 1993

\begin{tabular}{|c|c|c|c|c|c|c|c|}
\hline \multirow[b]{2}{*}{$\begin{array}{l}\text { Type of Equipment } \\
\text { (More than one may apply) }\end{array}$} & \multirow{2}{*}{$\begin{array}{l}\text { Buildings } \\
\text { that } \\
\text { Retrofitted or } \\
\text { Purchased } \\
\text { Equipment in } \\
\text { Past Ten } \\
\text { Years }\end{array}$} & \multicolumn{6}{|c|}{ Sponsor of Retrofit or Purchase (More than one may apply) } \\
\hline & & $\begin{array}{l}\text { Electric } \\
\text { Utility }\end{array}$ & In-House & $\begin{array}{l}\text { Third } \\
\text { Party }\end{array}$ & Other & FEMP & $\begin{array}{l}\text { Don't } \\
\text { Know/Not } \\
\text { Ascertained }\end{array}$ \\
\hline
\end{tabular}

Heating(more than one may

apply)

Furnace ...............

Heat Pump ..............

Space Heater

District Steam ............

Boiler ..................

Packaged Unit ..........

Central Chillers ...........

Water-Heating

Lighting

\begin{tabular}{|c|c|}
\hline 17 & 0 \\
\hline 38 & 0 \\
\hline 55 & 0 \\
\hline 35 & 0 \\
\hline 67 & 3 \\
\hline 49 & 0 \\
\hline 115 & 3 \\
\hline 222 & 3 \\
\hline 487 & 46 \\
\hline
\end{tabular}

13

29

48

27

45

42

84

190

387

4
7
5
8
9
5
18
24
49

0
0
2
0
3
2
3
1
5

1

0

0

3

0

3

1

15

0

1

0

1

Note: - These data are from 881 federally owned buildings having the following criteria: (1) located in Federal Regions 3, 6, or 9; (2) larger than 10,000 square feet; and (3) used for a commercial purpose, other than warehouse and storage. In addition, 9 out of 10 selected buildings were from agencies other than the Department of Defense. • Data are for Fiscal Year 1993 (October 1, 1992 through September 30, 1993).

Source: Energy Information Administration, Office of Energy Markets and End Use, 1993 Federal Buildings Supplemental Survey.

Federal buildings have access to various types of programs aimed at saving energy. Among the programs that assist in either the purchase of new energy-using equipment or the retrofit of existing equipment are: incentives that offer monetary or non-monetary awards such as low-interest loans, rebates, and direct installation of low-cost measures; the Federal Energy Efficiency Fund (FEEF), sponsored by FEMP, that provides grants to Federal agencies to assist them in meeting energy efficiency and water conservation requirements; and alternative energy rates offered by utilities that are intended to reduce consumer bills and shift hours of operation of equipment from on-peak to off-peak periods (Table 2.4). 
- For the most part, those buildings that had purchased or retrofitted equipment did not receive assistance in upgrading their equipment.

- Incentives were the most often reported type of assistance. This was followed by assistance from the FEEF and use of alternatives rates.

Table 2.4. Type of Assistance Received for Retrofit or Purchase of Equipment, Number of FBSS Buildings, 1993

\begin{tabular}{|c|c|c|c|c|c|c|c|}
\hline \multirow[b]{2}{*}{$\begin{array}{c}\text { Type of Equipment } \\
\text { (more than one may apply) }\end{array}$} & \multicolumn{7}{|c|}{ Types of Assistance (More than one may apply) } \\
\hline & $\begin{array}{c}\text { that } \\
\text { Retrofitted or } \\
\text { Purchased } \\
\text { Equipment } \\
\text { in Past } \\
\text { Ten Years }\end{array}$ & $\begin{array}{c}\text { Federal } \\
\text { Energy } \\
\text { Efficiency } \\
\text { Fund }\end{array}$ & Incentives & $\begin{array}{c}\text { Alternative } \\
\text { Rates }\end{array}$ & $\begin{array}{c}\text { Fuel } \\
\text { Switching }\end{array}$ & $\begin{array}{l}\text { Otherl } \\
\text { None }\end{array}$ & $\begin{array}{c}\text { Don't } \\
\text { Know/Not } \\
\text { Ascertained }\end{array}$ \\
\hline \multicolumn{8}{|l|}{$\begin{array}{l}\text { Heating(more than one may } \\
\text { apply) }\end{array}$} \\
\hline Furnace & 17 & 0 & 0 & 0 & 2 & 12 & 3 \\
\hline Heat Pump ........... & 38 & 1 & 2 & 2 & 2 & 29 & 5 \\
\hline Space Heater . . . . . . . . . . & 55 & 1 & 0 & 1 & 0 & 52 & 1 \\
\hline District Steam $\ldots \ldots \ldots \ldots \ldots$ & 35 & 0 & 0 & 0 & 0 & 33 & 2 \\
\hline Boiler $\ldots \ldots \ldots \ldots \ldots \ldots \ldots$ & 67 & 2 & 1 & 5 & 1 & 47 & 11 \\
\hline Packaged Unit $\ldots \ldots \ldots \ldots \ldots$ & 49 & 1 & 0 & 2 & 0 & 44 & 3 \\
\hline Central Chillers $\ldots \ldots \ldots \ldots \ldots$ & 115 & 1 & 1 & 6 & 0 & 98 & 11 \\
\hline Water-Heating ... & 222 & 3 & 2 & 5 & 1 & 191 & 20 \\
\hline Lighting Equipment $\ldots \ldots \ldots \ldots$ & 487 & 24 & 88 & 18 & 1 & 344 & 23 \\
\hline
\end{tabular}

Note: - These data are from 881 federally owned buildings having the following criteria: (1) located in Federal Regions 3,6 , or 9 ; (2) larger than 10,000 square feet; and (3) used for a commercial purpose, other than warehouse and storage. In addition, 9 out of 10 selected buildings were from agencies other than the Department of Defense. - Data are for Fiscal Year 1993 (October 1, 1992 through September 30, 1993).

Source: Energy Information Administration, Office of Energy Markets and End Use, 1993 Federal Buildings Supplemental Survey. 
The FBSS collected detailed data on the number, type, and age of motors in Federal commercial buildings in addition to information about how many were rewound and how many were considered energy efficient (Table 2.5)

- Most buildings reported between one to five motors that were 10 or more horsepower in their equipment.

- With the exception of buildings using motors for their heat pumps, most buildings reported having equipment with motors that were 10 years or older.

Table 2.5. Motor Characteristics by Equipment Type, Number of FBSS Buildings, 1993

\begin{tabular}{|c|c|c|c|c|c|c|c|c|}
\hline \multirow[b]{2}{*}{$\begin{array}{c}\text { Motors } \\
\text { Characteristics }\end{array}$} & \multicolumn{8}{|c|}{ Type of Equipment with Motors 10 or More Horsepower (more than one may apply) } \\
\hline & Chillers & $\begin{array}{l}\text { Heat } \\
\text { Pumps }\end{array}$ & Fans & $\begin{array}{c}\text { Air } \\
\text { Compressors }\end{array}$ & $\begin{array}{l}\text { Water } \\
\text { Pumps }\end{array}$ & Elevators & Escalators & Refrigeration \\
\hline All Buildings & 381 & 23 & 461 & 294 & 374 & 411 & 35 & 86 \\
\hline \multicolumn{9}{|l|}{ Number of Motors } \\
\hline One to Five $\ldots \ldots \ldots$ & 320 & 18 & 201 & 221 & 194 & 280 & 21 & 44 \\
\hline Six to Ten ........ & 39 & 2 & 89 & 47 & 92 & 61 & 6 & 16 \\
\hline $\begin{array}{l}\text { Ten to } 100 \ldots . . . \\
\text { Don't Know/Not }\end{array}$ & 16 & 2 & 160 & 18 & 76 & 62 & 4 & 16 \\
\hline Ascertained ...... & 6 & 1 & 11 & 8 & 12 & 8 & 4 & 10 \\
\hline \multicolumn{9}{|l|}{$\begin{array}{l}\text { Age of Motors } \\
\text { Less Than Ten }\end{array}$} \\
\hline $\begin{array}{l}\text { Years Old ........ } \\
\text { Ten Years or }\end{array}$ & 127 & 12 & 119 & 109 & 96 & 79 & 6 & 23 \\
\hline $\begin{array}{l}\text { Older .......... } \\
\text { Some Less Than } \\
\text { Ten Years, } \\
\text { Some More Than }\end{array}$ & 179 & 8 & 245 & 132 & 192 & 272 & 24 & 41 \\
\hline $\begin{array}{c}\text { Ten Years ....... } \\
\text { Don't Know/Not }\end{array}$ & 32 & 0 & 39 & 17 & 33 & 22 & 0 & 11 \\
\hline Ascertained. & 43 & 3 & 58 & 36 & 53 & 38 & 5 & 11 \\
\hline
\end{tabular}

Notes: -These data are from 881 federally owned buildings having the following criteria: (1) located in Federal Regions 3,6 , or 9 ; (2) larger than 10,000 square feet; and (3) used for a commercial purpose, other than warehouse and storage. In addition, 9 out of 10 selected buildings were from agencies other than the Department of Defense. - Totals may not equal sum of components due to independent rounding. - Data are for Fiscal Year 1993 (October 1, 1992 through September 30, 1993).

Source: Energy Information Administration, Office of Energy Markets and End Use, 1993 Federal Buildings Supplemental Survey.

- Forty-two percent of 331 buildings that had equipment with motors less than 10 years old had fewer than 25 percent energy-efficient motors (Figure 2.1a).

- A higher percent ( 82 percent) of 331 buildings that had equipment with motors less than 10 years old had fewer than 25 percent of their motors rewound (Figure 2.1b). Sixty-five percent or 214 of the 331 building respondents said none of their motors were rewound (Figure 2.1c.) This suggests that Federal buildings could target the purchase of energy efficient motors for future conservation improvement. 
Figure 2.1 Selected Characteristics of Motors, 1993

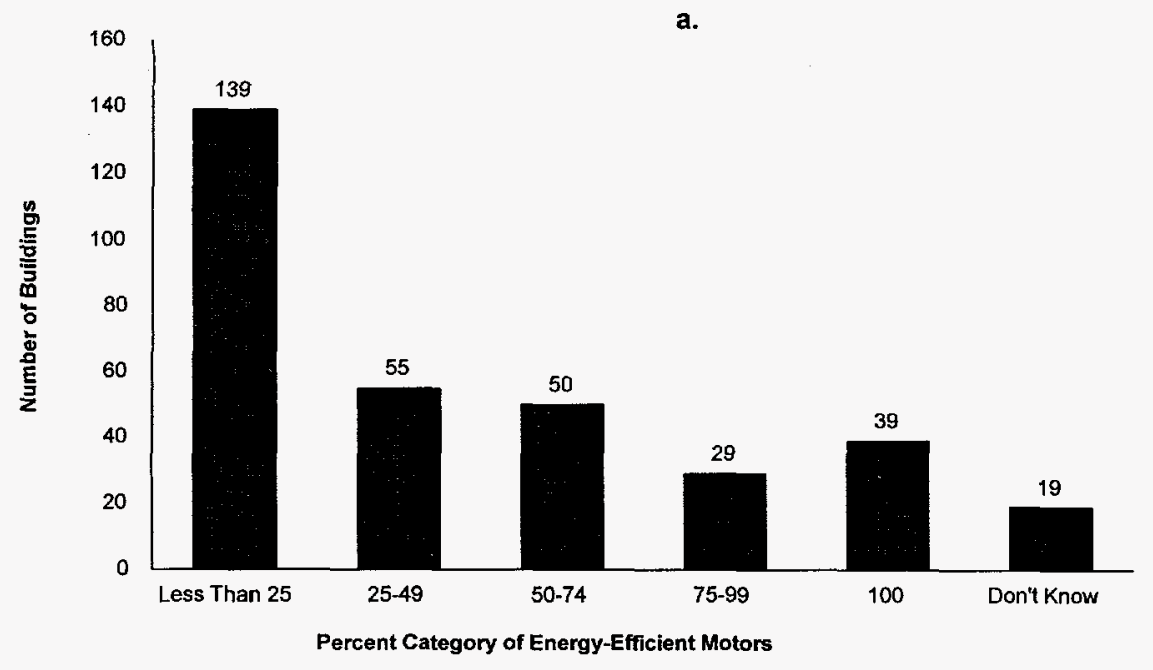

b.
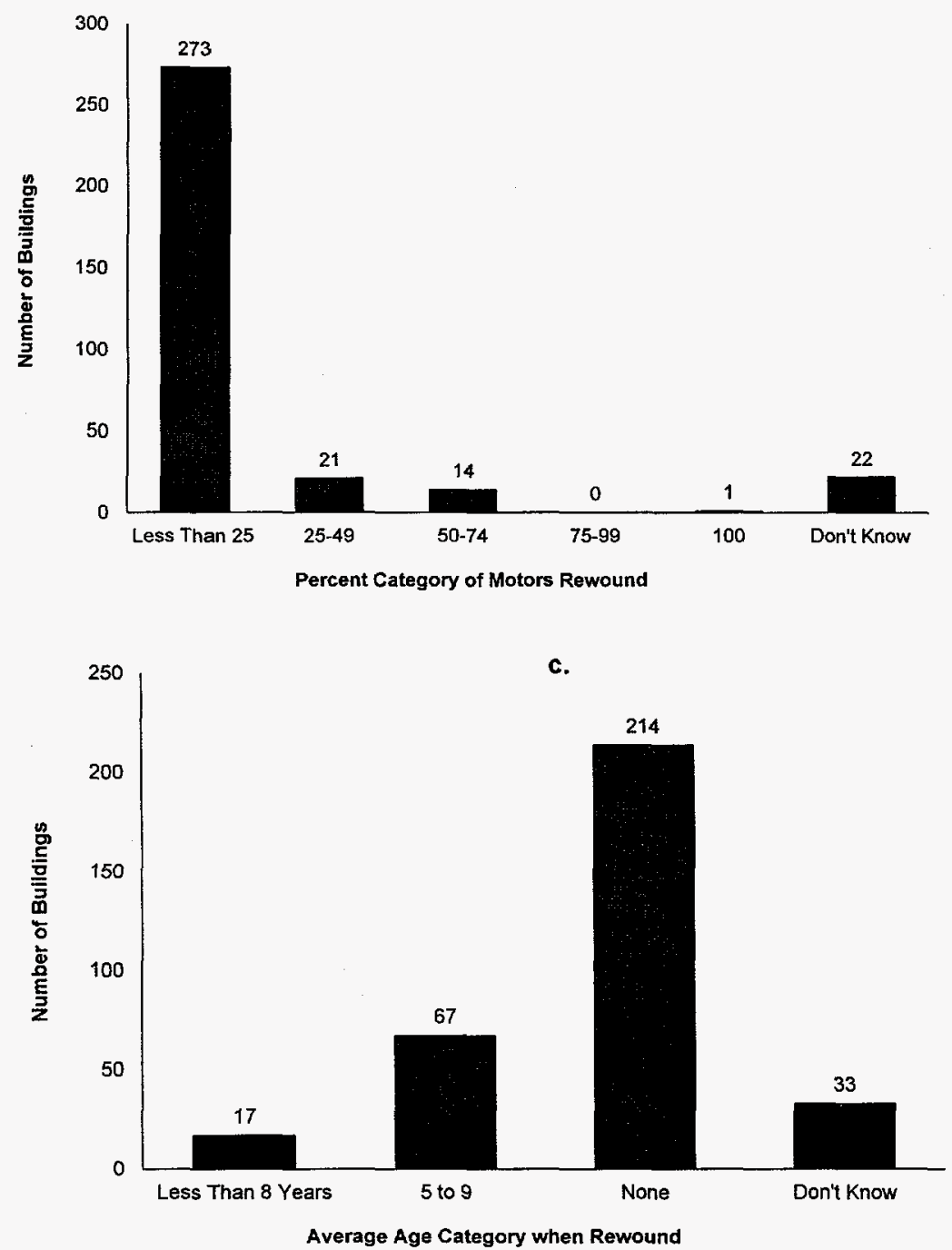

Note: These graphs are based on 331 buildings of the 881 responding buildings that had equipment with motors less than 10 years old.

Source: Energy Information Administration, Office of Energy Markets and End Use, 1993 Federal Buildings Supplemental Survey. 


\section{Detailed Tables}

There were 881 completed interviews for the FBSS. The population consisted of all Federal buildings in Federal Regions 3, 6, and 9 in the commercial sector. Based upon the sample, the population of interest includes 11,032 buildings. Because the sample was not a simple random sample, descriptions of the sample do not correspond to descriptions of the population. Of particular importance, larger buildings were sampled at a higher rate than smaller buildings and non-Department of Defense (DOD) buildings were sampled at a higher rate than DOD buildings. Hence, the sample contains a higher proportion of large buildings and a higher proportion of non-DOD buildings than the population. Sampling weights are used to account for differences in the sampling rates. Estimates of population percentages and population means can be obtained from the sample data using the sampling weights. Table 3.1 below shows the differences between the sample frequencies and the weighted frequencies by size and type (DOD versus nonDOD) of building.

Table 3.1. Sample and Weighted Totals in FBSS Buildings in Regions 3, 6, and 9, 1993

\begin{tabular}{|c|c|c|c|c|c|c|}
\hline \multirow[b]{3}{*}{ Federal Agency } & \multicolumn{3}{|c|}{ Sample Frequencies } & \multicolumn{3}{|c|}{ Weighted Frequencies } \\
\hline & \multicolumn{3}{|c|}{ Size of Building } & \multicolumn{3}{|c|}{ Size of Building } \\
\hline & Small & Medium & Large & Small & Medium & Large \\
\hline Total Buildings $\ldots \ldots \ldots \ldots$ & 349 & 326 & 206 & 8,548 & 2,144 & 340 \\
\hline $\begin{array}{l}\text { Department of Defense } \\
\text { Number of Buildings } \ldots \ldots \\
\text { Percent of Buildings } \ldots \ldots\end{array}$ & $\begin{array}{l}67 \\
19\end{array}$ & $\begin{array}{l}39 \\
12\end{array}$ & $\begin{array}{r}16 \\
8\end{array}$ & $\begin{array}{r}6,332 \\
74\end{array}$ & $\begin{array}{r}1,490 \\
69\end{array}$ & $\begin{array}{l}81 \\
24\end{array}$ \\
\hline $\begin{array}{l}\text { Non-Department of Defense } \\
\text { Number of Buildings } \ldots \ldots \\
\text { Percent of Buildings } \ldots \ldots\end{array}$ & $\begin{array}{r}282 \\
81\end{array}$ & $\begin{array}{r}287 \\
88\end{array}$ & $\begin{array}{r}190 \\
92\end{array}$ & $\begin{array}{r}2,217 \\
26\end{array}$ & $\begin{array}{r}654 \\
31\end{array}$ & $\begin{array}{r}258 \\
76\end{array}$ \\
\hline $\begin{array}{c}\text { Total Floorspace (thousand } \\
\text { square feet) } \ldots \ldots \ldots \ldots\end{array}$ & 9,014 & 35,395 & 130,604 & 188,150 & 186,186 & 201,663 \\
\hline $\begin{array}{l}\text { Department of Defense } \\
\text { Floorspace (thousand } \\
\text { square feet) } \ldots \ldots \ldots \\
\text { Percent of Floorspace..... }\end{array}$ & $\begin{array}{r}1,819 \\
20\end{array}$ & $\begin{array}{r}3,732 \\
11\end{array}$ & $\begin{array}{r}18,595 \\
14\end{array}$ & $\begin{array}{r}141,667 \\
75\end{array}$ & $\begin{array}{r}122,874 \\
66\end{array}$ & $\begin{array}{r}53,274 \\
26\end{array}$ \\
\hline $\begin{array}{l}\text { Non-Department of Defense } \\
\text { Floorspace (thousand } \\
\text { square feet) } \ldots \ldots \ldots \\
\text { Percent of Floorspace . . . }\end{array}$ & $\begin{array}{r}7,195 \\
80\end{array}$ & $\begin{array}{r}31,663 \\
89\end{array}$ & $\begin{array}{r}112,009 \\
86\end{array}$ & $\begin{array}{r}46,483 \\
25\end{array}$ & $\begin{array}{r}63,312 \\
34\end{array}$ & $\begin{array}{r}148,389 \\
74\end{array}$ \\
\hline
\end{tabular}

Notes: - Small $=10,000$ to 50,000 square feet; Medium $=50,000$ to 200,000 sqaure feet; and Large $=$ Over 200,000 square feet. - These sample frequencies provide data from 881 federally owned buildings having the following criteria: (1) located in Federal Regions 3,6 , or 9 ; (2) larger than 10,000 square feet; and (3) used for a commercial purpose, other than warehouse and storage. In addition, 9 out of 10 selected buildings were from agencies other than the Department of Defense. These weighted frequencies provide regionallevel estimates. - Totals may not equal sum of components due to independent rounding. - Data are for Fiscal Year 1993 (October 1 , 1992 through September 30, 1993).

Source: Energy Information Administration, Office of Energy Markets and End Use,1993 Federal Buildings Supplemental Survey. 


\section{Regional-level Estimates and Confidence Intervals}

Per the request of the Office of Federal Energy Management Programs, the data presented in this service report represent the 881 specifically responding buildings and cannot be used to generalize for Federal buildings in each region. However, the FBSS sample design permits regional-level estimates to be obtained by weighting the building-level data. Tables 3.2 through 3.4 provide regional-level estimates (weighted building-level data) for selected building characteristics and consumption and expenditures. Because these regional-level estimates are based on the sample surveyed, they are subject to sampling error. To help the reader interpret the regional-level estimates, 95-percent confidence intervals have been constructed along with the estimates. A confidence interval is an interval around an

Table 3.2. Regional-Level Estimates and Confidence Intervals for Selected Building Characteristics, Number of FBSS Buildings, 1993

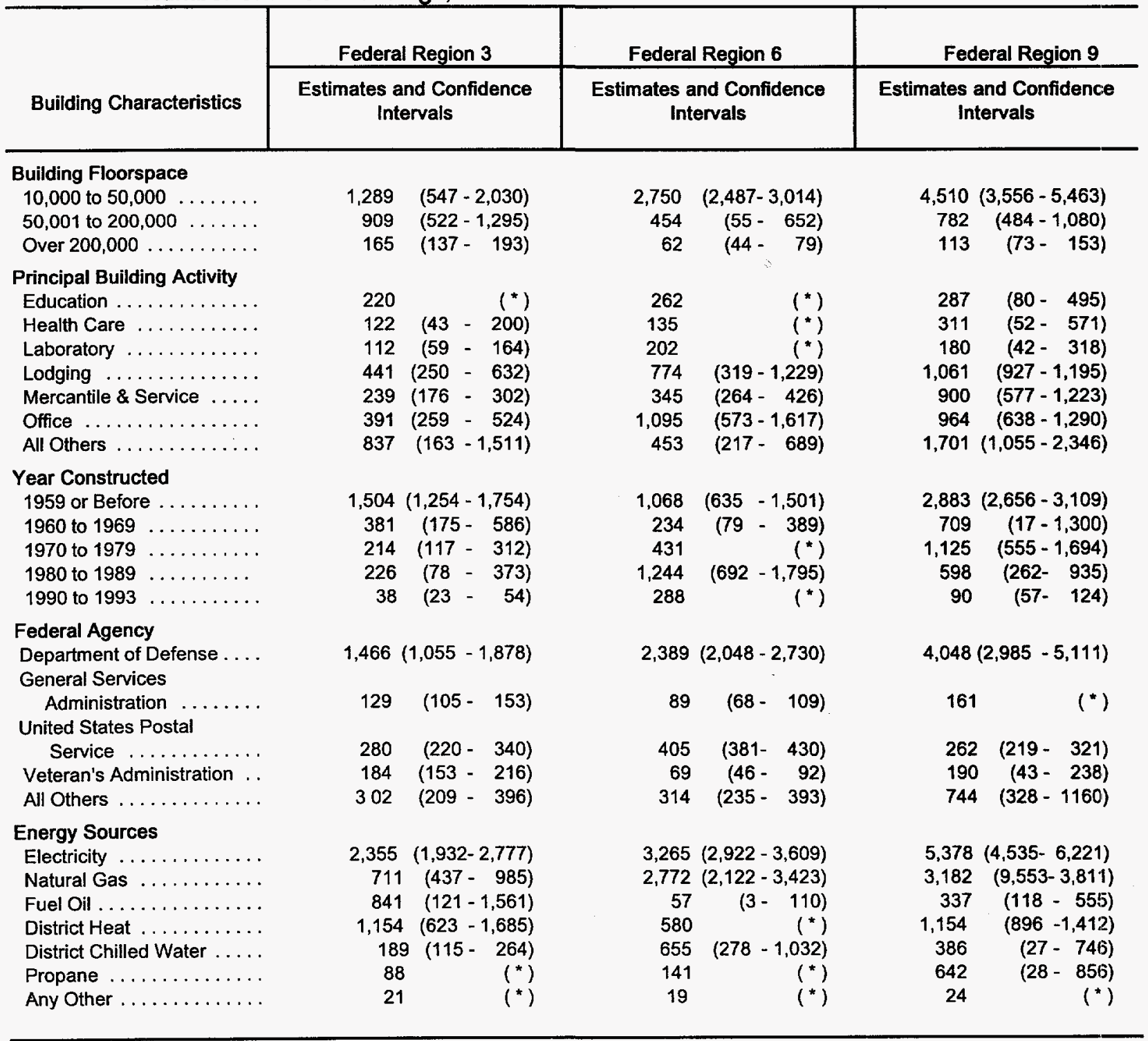

*Data withheld because variances are too large.

Notes: - These data are weighted to provide regional-level estimates. - Data are for Fiscal Year 1993 (October 1, 1992 through September 30, 1993).

Source: Energy Information Administration, Office of Energy Markets and End Use, 1993 Federal Buildings Supplemental Survey. 
estimate, which by virtue of the way it is constructed, "contains" the true (but unknown) population value of interest for 95 percent of all possible samples. Confidence intervals have lower bounds and upper bounds that provide minimum and maximum values expected for the estimate. For example, Table 3.2 provides an estimate of 122 health care buildings in Federal Region 3 with a lower bound of 43 and an upper bound of 200 . This means that for 95 percent of all possible samples, the estimate of the number of health care buildings in Federal Region 3 would be between 43 and 200 buildings. The confidence interval serves as a measure of the level of variability in the survey estimate. The wider the confidence interval, the higher the sampling variability of the survey estimate. For an estimate with high sampling variability, confidence intervals are not provided.

Selected estimates and their confidence intervals are provided as approximations and as such confidence intervals must also be regarded as only approximate.

Table 3.3. Regional-Level Estimates and Confidence Intervals for Selected Building Characteristics, FBSS Building Floorspace, 1993 (Thousand Square Feet)

\begin{tabular}{|c|c|c|c|c|c|}
\hline \multirow[b]{2}{*}{ Building Characteristics } & \multicolumn{2}{|c|}{ Federal Region 3} & Federal Region 6 & \multicolumn{2}{|c|}{ Federal Region 9} \\
\hline & \multicolumn{2}{|c|}{$\begin{array}{c}\text { Estimates and Confidence } \\
\text { Intervals } \\
\end{array}$} & $\begin{array}{c}\text { Estimates and Confidence } \\
\text { Intervals } \\
\end{array}$ & \multicolumn{2}{|c|}{$\begin{array}{c}\text { Estimates and Confidence } \\
\text { Intervals }\end{array}$} \\
\hline \multicolumn{6}{|l|}{ Building Floorspace } \\
\hline 10,000 to 50,000 & 28,502 & $(16,526-40,477)$ & $59,272(45,133-73,411)$ & 100,376 & $(80,933-119,819)$ \\
\hline 50,001 to $200,000 \ldots$ & 80,079 & $(61,678-98,479)$ & $38,844(25,597-52,092)$ & 67,263 & $(44,235-90,291)$ \\
\hline Over $200,000 \ldots \ldots$ & 122,544 & $(95,549-149,540)$ & $29,680(19,326-40,034)$ & 49,438 & $(34,706-64,170)$ \\
\hline \multicolumn{6}{|l|}{ Principal Building Activity } \\
\hline Education ............ & 14,602 & $(*)$ & 6,589 & 14,527 & $(*)$ \\
\hline Health Care & 25,466 & $(16,072-34,859)$ & $24,073 \quad(7,909-40,238)$ & 19,316 & $(14,164-24,467)$ \\
\hline Laboratory & 9,328 & $(8,174-10,481)$ & $8,286 \quad(1,341-15,230)$ & 8,064 & $(3,650-12,477)$ \\
\hline$\ldots \ldots \ldots \ldots \ldots$ & 33,012 & $(10,720-55,305)$ & $20,418(13,111-27,726)$ & 34,863 & $(24,637-45,088)$ \\
\hline Mercantile \& Service ...... & 20,142 & $(10,235-30,049)$ & $16,702(11,881-21,524)$ & 33,760 & $(18,231-49,290)$ \\
\hline Office $\ldots \ldots \ldots \ldots$ & 89,325 & $(65,588-113,063)$ & $34,714(22,574-46,855)$ & 52,742 & $(38,734-66,750)$ \\
\hline All Others & 39,250 & $(20,184-58,315)$ & $17,013 \quad(6,606-27,421)$ & 53,806 & $(40,287-67,324)$ \\
\hline \multicolumn{6}{|l|}{ Year Constructed } \\
\hline 1959 or Before & 107,102 & $(82,826-131,377)$ & $43,362(27,288-59,437)$ & 99,013 & $(86,649-111,377)$ \\
\hline 1960 to $1969 \ldots$ & 47,026 & $(15,092-78,960)$ & $16,015 \quad(6,342-25,689)$ & 33,064 & $(17,555-48,574)$ \\
\hline 1970 to 1979 & 42,666 & $(17,911-67,422)$ & $23,146 \quad(5,488-40,805)$ & 47,267 & $(29,343-65,192)$ \\
\hline 1980 to 1989 & 27,056 & $(8,634-45,478)$ & $34,926(14,483-55,369)$ & 31,566 & $(18,265-44,867)$ \\
\hline 1990 to 1993 . & 7,275 & $(5,408-9,141)$ & 10,346 & 6,167 & $(1,399-10,935)$ \\
\hline \multicolumn{6}{|l|}{ Federal Agency } \\
\hline $\begin{array}{l}\text { Department of Defense } \\
\text { General Services }\end{array}$ & 99,413 & $(88,447-110,380)$ & $73,132(44,214-102,051)$ & 145,269 & $(131,017-159,520)$ \\
\hline Administration .. & 65,382 & $(43,053-86,711)$ & $10,277 \quad(7,175-13,379)$ & 5,945 & $(10,433-21,457)$ \\
\hline \multicolumn{6}{|l|}{ United States Postal } \\
\hline Service & 22,436 & $(13,100-31,772)$ & $17,094 \quad(16,078-18,109)$ & 16,187 & $(12,137-20,137)$ \\
\hline Veterans Administration ... & 22,664 & $(21,979-23,348)$ & $12,758 \quad(11,665-13,852)$ & 15,641 & $(15,010-16,273)$ \\
\hline All Others $\ldots \ldots \ldots \ldots$ & 21,229 & $(18,925-23,532)$ & $14,535 \quad(10,906-18,165)$ & 24,035 & $(17,112-30,959)$ \\
\hline \multicolumn{6}{|l|}{ Energy Sources } \\
\hline Electricity .. & 225,630 & $(202,611-248,650)$ & $127,797(98,761-156,832)$ & 216,545 & $(202,386-230,703)$ \\
\hline Natural Gas & 126,202 & $(97,906-154,498)$ & $103,091(74,463-131,719)$ & 128,406 & $(117,871-138,940)$ \\
\hline Fuel Oil & 87,096 & $(58,257-115,935)$ & $9,643(2,270-17,016)$ & 24,784 & $(15,523-34,045)$ \\
\hline District Heat . & 133,203 & $(98,279-168,126)$ & $29,739(13,754-45,724)$ & 57,104 & $(41,754-72,455)$ \\
\hline District Chilled Water ..... & 40,202 & $(36,942-\quad 3,461)$ & $24,859(16,964-33,753)$ & 21,052 & $(8,917-33,186)$ \\
\hline Propane $\ldots \ldots \ldots \ldots \ldots$ & 6,125 & $(*)$ & 3,853 & 13,776 & $(8,862-18,690)$ \\
\hline Any Other $\ldots \ldots \ldots \ldots$ & 5,549 & $(5,053-\quad 6,045)$ & $3,878 \quad(2,087-5,670)$ & 3,766 & $(1,517-\quad 6,016)$ \\
\hline
\end{tabular}

*Data withheld because variances were too large.

Notes: - These data are weighted to provide regional-level estimates. - Data are for Fiscal Year 1993 (October 1, 1992 through September 30, 1993).

Source: Energy Information Administration, Office of Energy Markets and End Use, 1993 Federal Buildings Supplemental Survey. 
Table 3.4. Regional-Level Estimates and Confidence Intervals for Major Fuels, FBSS Consumption and Expenditures, 1993

\begin{tabular}{|c|c|c|c|c|c|c|}
\hline \multirow[b]{2}{*}{ Major Fuels } & \multicolumn{2}{|c|}{ Federal Region 3} & \multicolumn{2}{|c|}{ Federal Region 6} & \multicolumn{2}{|c|}{ Federal Region 9} \\
\hline & Estimates & $\begin{array}{l}\text { Confidence } \\
\text { Intervals }\end{array}$ & Estimates & $\begin{array}{l}\text { Confidence } \\
\text { Intervals }\end{array}$ & Estimates & $\begin{array}{l}\text { Confidence } \\
\text { Intervals }\end{array}$ \\
\hline \multicolumn{7}{|l|}{ Consumption (billion Btu) } \\
\hline All Major Fueis . . . . . & 33,322 & $(25,233-39,410)$ & 20,716 & $(11,383-30,049)$ & 24,424 & $(20,523-28,324)$ \\
\hline Electricity .... & 16,033 & $(13,068-18,998)$ & 9,655 & $(7,063-12,246)$ & 14,469 & $(10,854-18,085)$ \\
\hline Natural Gas & 5,594 & $(3,164-18,024)$ & 8,225 & $(2,922-13,529)$ & 5,594 & $(4,531-6,657)$ \\
\hline Fuel Oil . ... & 1,517 & $(437-2,598)$ & 20 & $(1-40)$ & 233 & $(9-457)$ \\
\hline District Heat . . . . . . & 9,177 & $(4,933-13,422)$ & 2,815 & $(694-4,936)$ & 4,127 & $(3,707-4,547)$ \\
\hline \multicolumn{7}{|l|}{$\begin{array}{l}\text { Expenditures } \\
\text { (million dollars) }\end{array}$} \\
\hline All Major Fuels & 413 & $(352-\quad 475)$ & 203 & $(143-264)$ & 397 & $(345-448)$ \\
\hline Electricity .... & 273 & $(3-304)$ & 146 & $(117-176)$ & 322 & $(269-\quad 376)$ \\
\hline Natural Gas ... & 23 & $(15-$ & 27 & $(11-43)$ & 27 & $(23-$ \\
\hline Fuel Oil ....... & 8 & 13) & 0 & $10-$ & 1 & $10-$ \\
\hline District Heat ... & 110 & $(63-157)$ & 30 & 55) & 45 & $(37-$ \\
\hline
\end{tabular}

Notes: - These data are weighted to provide regional-level estimates. - Data are for Fiscal Year 1993 (October 1, 1992 through September 30, 1993).

Source: Energy Information Administration, Office of Energy Markets and End Use, 1993 Federal Buildings Supplemental Survey.

\section{Table Organization}

The following 48 detailed tables in this section present unweighted cross-tabulations of commercial buildings' characteristics and consumption and expenditure data for the 881 responding Federal buildings in Federal Regions 3 , 6 , and 9. This section provides assistance in reading the detailed tables by explaining some of the data categories and some of the headings for the data categories.

\section{Overall Organization}

The detailed tables have been grouped to make it easier to find related information. Table 3.7 summarizes the total number of buildings, square footage, and workers and the average square footage per building for all the buildings interviewed in Federal Regions 3, 6, and 9 and the number of buildings and square footage for each of the three Federal regions in the FBSS. After Table 3.7, the tables are grouped by Federal region for building characteristics data (Tables 3.8 through 3.42) and consumption and expenditure data (Tables 3.43 through 3.54). Tables 3.8 through 3.33 contain data about the energy sources and equipment in Federal buildings. Tables 3.34 through 3.42 contain information about conservation measures and programs in the Federal buildings. Tables 3.43 through 3.54 contain consumption and expenditures data for electricity, natural gas, and district heat. There is a Quick Reference Guide to the tables on page 18 to help the reader quickly locate specific tables of interest.

Generally, for each Federal region there are two tables for each topic, one giving the number of buildings in each cross tabulation cell, the other giving the floorspace in those buildings. For example, for the topic "Heating Equipment," Tables 3.10 through 3.12 provide the number of buildings for Federal Regions 3, 6, and 9, respectively, while Tables 3.13 through 3.15 provide the floorspace for those regions for this same topic. For some smaller tables, the number of buildings and floorspace appear together in a single table.

Data in the tables are presented in column categories (at the top of each table) and row categories (in the far left column of each tables.) 


\section{Core Categories}

The following core set of categories appear in the same order in all basic tables: floorspace, principal building activity, year constructed, and Federal agency. Additional categories are included on the tables when they relate to specific overall topic areas. Table 3.7, the summary table, contains the core categories as well as most of the additional categories.

The "Sum of Major Fuels" is the sum of site electricity, natural gas, fuel oil, and district heat. Statistics in this category exclude data from "Primary Electricity." Although electricity is technically not a fuel, "Major Fuel" rather than "Major Energy Source," was retained as the title of this category to remain consistent with previous CBECS reports. Although fuel oil is considered a major fuel, Federal building respondents reported very little use of fuel oil; therefore, an individual consumption and expenditures table is not included.

\section{Exclusive/Nonexclusive Categories}

There are two types of categories, those that indicate exclusive, nonoverlapping categories and those that indicate nonexclusive, overlapping subsets of categories. For example, "building floorspace" is a set of exclusive categories; a given building belongs in only one of these. "Energy sources," on the other hand, is a set of nonexclusive categories; a given building may be included in more than one line under this category since the building may use more than one energy source. The phrase, "more than one may apply," indicates that the categories under this row header are overlapping. Both exclusive and overlapping categories may be nonexhaustive; that is, there may be some buildings that do not fall into any of the listed categories.

Nonexclusive Categories. For the exclusive nonoverlapping categories, the response option "don't know" is included on the detailed tables whenever applicable. However, in the nonexclusive overlapping categories, each line had the possibility of being answered "don't know;" these were not included in the detailed tables. Tables 3.5 and 3.6 provide the number of buildings and floorspace, respectively, for respondents that answered "don't know" for each line in the nonexclusive overlapping categories. 


\begin{tabular}{|c|c|c|c|c|}
\hline Building Characteristics & $\begin{array}{c}\text { Regions } 3,6, \\
\text { and } 9\end{array}$ & Region 3 & Region 6 & Region 9 \\
\hline \multicolumn{5}{|l|}{ Heating Equipment } \\
\hline Heat Pumps $\ldots \ldots \ldots \ldots \ldots \ldots \ldots$ & 6 & 0 & 1 & 5 \\
\hline Furnaces $\ldots \ldots \ldots \ldots \ldots \ldots \ldots$ & 5 & 0 & 0 & 5 \\
\hline Individual Space Heaters . . . . . . . . . . & 6 & 1 & 1 & 4 \\
\hline District Heat $\ldots \ldots \ldots \ldots \ldots \ldots \ldots$ & 5 & 0 & 1 & 4 \\
\hline Boilers $\ldots \ldots \ldots \ldots \ldots \ldots \ldots \ldots$ & 4 & 0 & 0 & 4 \\
\hline Packaged Heating Units $\ldots \ldots \ldots$ & 8 & 1 & 2 & 5 \\
\hline \multicolumn{5}{|l|}{ Cooling Equipment } \\
\hline Residential-Type Central AVC $\ldots \ldots \ldots$ & 2 & 0 & 1 & 1 \\
\hline Heat Pumps $\ldots \ldots \ldots \ldots \ldots \ldots \ldots$ & 4 & 0 & 2 & 2 \\
\hline Individual $A / C \ldots \ldots \ldots \ldots \ldots \ldots$ & 2 & 0 & 0 & 2 \\
\hline District Chilled Water . . . . . . . . . & 3 & 1 & 1 & 1 \\
\hline Central Chillers $\ldots \ldots \ldots \ldots \ldots \ldots$ & 1 & 0 & 0 & 1 \\
\hline Packaged AVC Units . . . . . . . . . & 3 & 0 & 1 & 2 \\
\hline Swamp Coolers ............... & 3 & 1 & 0 & 2 \\
\hline \multicolumn{5}{|l|}{ Lighting Equipment } \\
\hline Incandescent $\ldots \ldots \ldots$ & 6 & 2 & 1 & 3 \\
\hline Standard Fluorescent $\ldots \ldots \ldots \ldots \ldots$ & 3 & 0 & 0 & 3 \\
\hline Compact Fluorescent ............ & 50 & 3 & 39 & 8 \\
\hline High-Intensity Discharge $\ldots \ldots \ldots \ldots$ & 30 & 3 & 13 & 14 \\
\hline Electronic Ballast . . . . . . . . . . & 58 & 7 & 41 & 10 \\
\hline \multicolumn{5}{|l|}{ Building Shell Conservation Features } \\
\hline Roof or Ceiling Insulation $\ldots \ldots \ldots$ & 37 & 4 & 8 & 25 \\
\hline Wall Insulation $\ldots \ldots \ldots \ldots \ldots$ & 65 & 12 & 21 & 32 \\
\hline Storm or Multiple Glazing $\ldots \ldots \ldots \ldots$ & 39 & 3 & 8 & 28 \\
\hline \multicolumn{5}{|l|}{ Tinted or Reflective Glass or } \\
\hline Shading Film .......... & 38 & 0 & 8 & 30 \\
\hline \multicolumn{5}{|l|}{ Exterior or Interior Shading or } \\
\hline Awnings $\ldots \ldots \ldots \ldots \ldots \ldots \ldots$ & 37 & 1 & 11 & 25 \\
\hline \multicolumn{5}{|l|}{ HVAC Conservation Features } \\
\hline VAV System ............. & 25 & 9 & 2 & 14 \\
\hline Economizer Cycle $\ldots \ldots \ldots \ldots \ldots \ldots$ & 22 & 5 & 5 & 12 \\
\hline HVAC Maintenance $\ldots \ldots \ldots \ldots \ldots$ & 3 & 2 & 0 & 1 \\
\hline \multicolumn{5}{|l|}{ Lighting Conservation Features } \\
\hline Specular Reflectors $\ldots . \ldots \ldots \ldots$ & 7 & 0 & 2 & 5 \\
\hline Natural Lighting Control Sensors ..... & 4 & 0 & 0 & 4 \\
\hline Occupancy Sensors $\ldots \ldots \ldots \ldots \ldots$ & 6 & 0 & 1 & 5 \\
\hline Time Clock . . . . . . . . . . . . & 5 & 0 & 1 & 4 \\
\hline Manual Dimmer Switches . . . . . . . . & 20 & 2 & 14 & 4 \\
\hline \multicolumn{5}{|l|}{ Energy Management Practices } \\
\hline 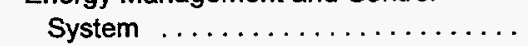 & 42 & 7 & 12 & 23 \\
\hline Energy Conservation Programs ...... & 29 & 11 & 7 & 11 \\
\hline Energy Audit $\ldots \ldots \ldots \ldots \ldots \ldots$ & 57 & 16 & 11 & 30 \\
\hline HVAC Maintenance Staff ........... & 32 & 2 & 27 & 3 \\
\hline \multicolumn{5}{|l|}{ Off-Hours Reduction in Equipment } \\
\hline Heating $\ldots \ldots \ldots \ldots \ldots \ldots \ldots$ & 32 & 2 & 25 & 5 \\
\hline Cooling $\ldots \ldots \ldots \ldots \ldots \ldots \ldots$ & 32 & 1 & 25 & 6 \\
\hline Hot Water . . . . . . . . . . . . & 45 & 8 & 27 & 10 \\
\hline Lighting $\ldots \ldots \ldots \ldots \ldots \ldots \ldots$ & 28 & 1 & 23 & 4 \\
\hline
\end{tabular}

Notes: - These data are from 881 federally owned buildings having the following criteria: (1) located in Federal Regions 3,6 , or 9 ; (2) larger than 10,000 square feet; and (3) used for a commercial purpose, other than warehouse and storage. In addition, 9 out of 10 selected buildings were from agencies other than the Department of Defense. - Data are for Fiscal Year 1993 (October 1, 1992 through September 30, 1993).

Source: Energy Information Administration, Office of Energy Markets and End Use, 1993 Federal Buildings Supplemental Survey. 
Table 3.6. Floorspace of FBSS Buildings with "Don't Know" Responses, 1993 (Thousand Square Feet)

\begin{tabular}{|c|c|c|c|c|}
\hline Building Characteristics & $\begin{array}{c}\text { Regions } 3,6 \text {, } \\
\text { and } 9\end{array}$ & Region 3 & Region 6 & Region 9 \\
\hline \multicolumn{5}{|l|}{ Heating Equipment } \\
\hline Heat Pumps & 305 & 0 & 11 & 294 \\
\hline Furnaces $\ldots \ldots \ldots \ldots \ldots \ldots \ldots$ & 286 & 0 & 0 & 286 \\
\hline Individual Space Heaters $\ldots \ldots \ldots \ldots \ldots$ & 345 & 60 & 11 & 274 \\
\hline District Heat $\ldots \ldots \ldots \ldots \ldots \ldots \ldots$ & 285 & 0 & 11 & 274 \\
\hline Boilers $\ldots \ldots \ldots \ldots \ldots \ldots \ldots \ldots$ & 274 & 0 & 0 & 274 \\
\hline Packaged Heating Units . . . . . . . . . & 574 & 245 & 35 & 294 \\
\hline \multicolumn{5}{|l|}{ Cooling Equipment } \\
\hline Residential-Type Central AVC . . . . . . . . & 251 & 0 & 20 & 231 \\
\hline Heat Pumps $\ldots \ldots \ldots \ldots \ldots \ldots \ldots$ & 648 & 0 & 44 & 604 \\
\hline Individual $A / C \ldots \ldots$ & 260 & 0 & 0 & 260 \\
\hline District Chilled Water & 551 & 300 & 20 & 260 \\
\hline Central Chillers & 231 & 0 & 0 & 231 \\
\hline Packaged AVC Units $\ldots \ldots \ldots \ldots \ldots \ldots$ & 309 & 0 & 20 & 289 \\
\hline Swamp Coolers $\ldots \ldots \ldots \ldots \ldots \ldots$ & 729 & 300 & 0 & 429 \\
\hline \multicolumn{5}{|l|}{ Lighting Equipment } \\
\hline Incandescent & 1,010 & 727 & 20 & 263 \\
\hline Standard Fluorescent & 263 & 0 & 0 & 263 \\
\hline Compact Fluorescent & 6,918 & 792 & 4,522 & 1,604 \\
\hline High-Intensity Discharge $\ldots \ldots \ldots \ldots$ & 6,023 & 2,301 & 1,632 & 2,090 \\
\hline Electronic Ballast $\ldots \ldots \ldots \ldots \ldots \ldots$ & 5,299 & 500 & 4,454 & 345 \\
\hline \multicolumn{5}{|l|}{ Building Shell Conservation Features } \\
\hline Roof or Ceiling Insulation ...... & 5,305 & 1,473 & 1,462 & 2,370 \\
\hline Wall Insulation $\ldots \ldots \ldots \ldots \ldots \ldots \ldots$ & 9,071 & 3,291 & 2,744 & 3,036 \\
\hline Storm or Multiple Glazing $\ldots \ldots \ldots \ldots \ldots$ & 5,182 & 1,579 & 668 & 2,934 \\
\hline Tinted or Reflective Glass or & & & & \\
\hline Shading Film $\ldots \ldots \ldots \ldots$ & 4,189 & 0 & 1,151 & 2,370 \\
\hline Exterior or Interior Shading & & & & \\
\hline or Awnings $\ldots \ldots \ldots \ldots \ldots \ldots$ & 3,743 & 54 & 1,404 & 2,285 \\
\hline \multicolumn{5}{|l|}{ HVAC Conservation Features } \\
\hline VAV System .... & 3,129 & 1,377 & 67 & 1,685 \\
\hline Economizer Cycle & 2,137 & 683 & 170 & 1,284 \\
\hline HVAC Maintenance...$\ldots \ldots$ & 186 & 73 & 0 & 113 \\
\hline \multicolumn{5}{|l|}{ Lighting Conservation Features } \\
\hline Specular Reflectors & 1,161 & 0 & 43 & 1,118 \\
\hline Natural Lighting Control Sensors . . . . . . . & 1,088 & 0 & 632 & 1,088 \\
\hline Occupancy Sensors $\ldots \ldots \ldots \ldots \ldots \ldots$ & 2,313 & 0 & 632 & 1,681 \\
\hline Time Clock . . . . . . . . . . . . & 96 & 0 & 18 & 77 \\
\hline Manual Dimmer Switches $\ldots \ldots \ldots \ldots$ & 3,491 & 727 & 1,676 & 1,088 \\
\hline \multicolumn{5}{|l|}{ Energy Management Practices } \\
\hline \multicolumn{5}{|l|}{ Energy Management and Control } \\
\hline$\ldots \ldots \ldots \ldots \ldots \ldots$ & 4,490 & 933 & 1,596 & 1,961 \\
\hline Energy Conservation Programs .... & 5,045 & 3,110 & 1,239 & 696 \\
\hline Energy Audit $\ldots \ldots \ldots \ldots \ldots \ldots \ldots$ & 13,232 & 6,539 & 2,460 & 4,233 \\
\hline HVAC Maintenance Staff $\ldots \ldots \ldots \ldots \ldots$ & 4,403 & 87 & 4,163 & 152 \\
\hline \multicolumn{5}{|l|}{ Off-Hours Reduction in Equipment } \\
\hline Heating $\ldots \ldots \ldots \ldots \ldots \ldots \ldots$ & 3,501 & 226 & 2,651 & 624 \\
\hline Cooling $\ldots \ldots \ldots \ldots \ldots \ldots$ & 3,509 & 169 & 2,651 & 689 \\
\hline Hot Water $\ldots \ldots \ldots \ldots \ldots \ldots \ldots \ldots$ & 7,455 & $\mathbf{3 , 4 1 5}$ & 2,687 & 1,343 \\
\hline Lighting $\ldots \ldots \ldots \ldots \ldots \ldots \ldots \ldots \ldots$ & 2,974 & 169 & 2,552 & 251 \\
\hline
\end{tabular}

Notes: - These data are from 881 federally owned buildings having the following criteria: (1) located in Federal Regions 3,6 , or 9 ; (2) larger than 10,000 square feet; and (3) used for a commercial purpose, other than warehouse and storage. In addition, 9 out of 10 selected buildings were from agencies other than the Department of Defense. - Data are for Fiscal Year 1993 (October 1, 1992 through September 30, 1993).

Source: Energy Information Administration, Office of Energy Markets and End Use, 1993 Federal Buildings Supplemental Survey. 


\section{Quick-Reference Guide}

The "Quick-Reference Guide" lists by broad class the topic areas covered by the detailed tables and the table numbers for the different types of tables. To find a particular two-way breakdown of interest, the tables featuring both topics should be consulted.

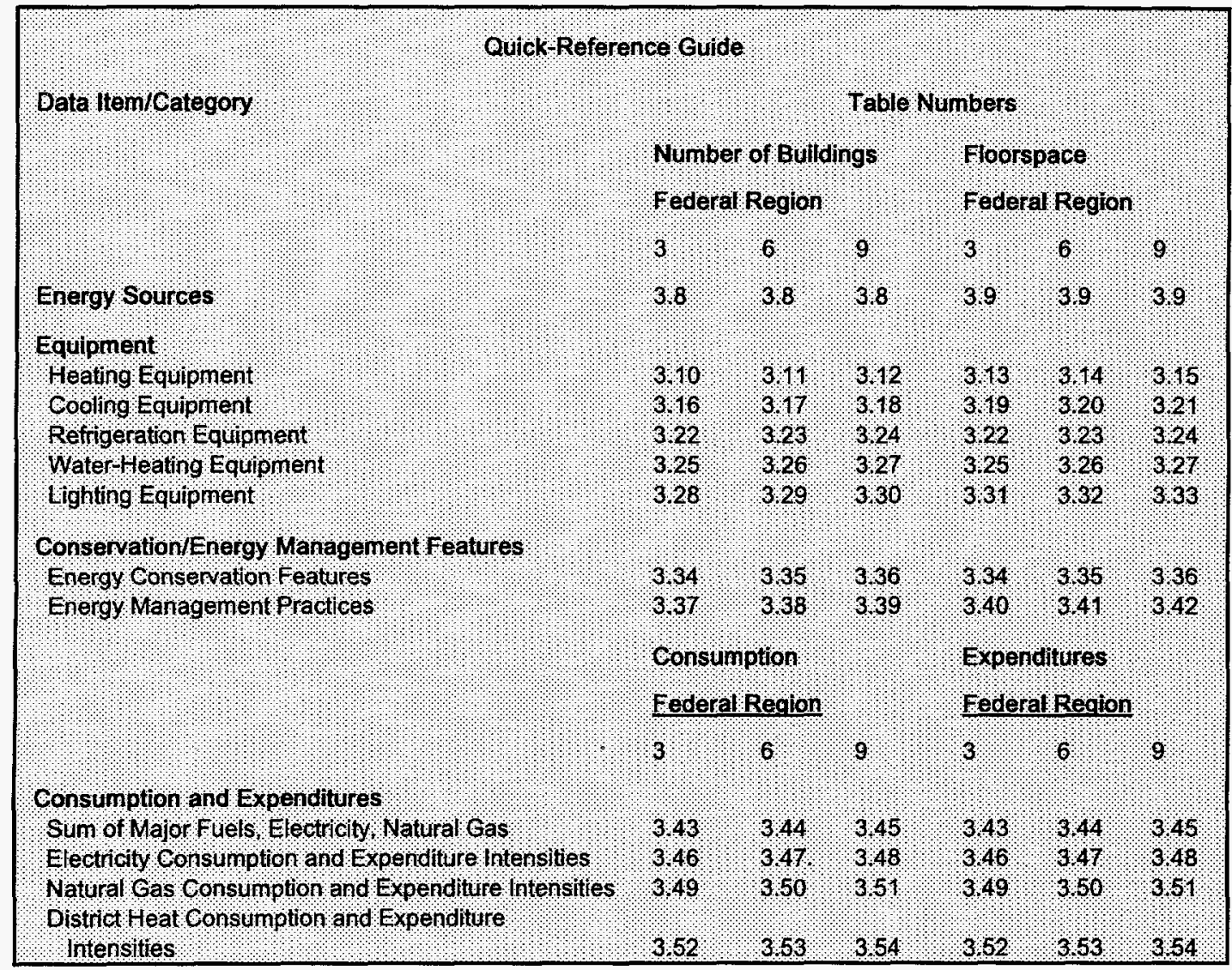

\section{Electronic Data Sets}

All the tables in this report are also available on diskette. The electronic files on the diskette are flat ASCII files. The diskette containing the files also contains a READ.ME ASCII text file with a table of contents. In addition, FBSS data are available on Public-Use Diskettes either in ASCII or dBase format (for details on obtaining the diskettes, see "Public-Use Data Preparation" section in Appendix A, "How the Survey Was Conducted," Public-Use Diskettes. 
Table 3.7. Summary of FBSS Buildings and Floorspace in Federal

Regions 3, 6, and 9, 1993

\begin{tabular}{|c|c|c|c|c|c|c|c|c|c|c|}
\hline \multirow{2}{*}{$\begin{array}{c}\text { Bullding } \\
\text { Characteristics }\end{array}$} & \multirow{2}{*}{$\begin{array}{c}\text { Total } \\
\text { Number of } \\
\text { Buildings }\end{array}$} & \multirow{2}{*}{$\begin{array}{c}\text { Total } \\
\text { Floorspace } \\
\text { (thousand } \\
\text { square } \\
\text { feet) }\end{array}$} & \multirow{2}{*}{$\begin{array}{c}\text { Total } \\
\text { Workers } \\
\text { (thousand) }\end{array}$} & \multirow{2}{*}{\begin{tabular}{|c|} 
Floorspace \\
per \\
Building \\
(thousand \\
square \\
feet)
\end{tabular}} & \multicolumn{3}{|c|}{$\begin{array}{l}\text { Number of Buildings } \\
\text { per Federal Region }\end{array}$} & \multicolumn{3}{|c|}{$\begin{array}{c}\text { Floorspace per } \\
\text { Federal Region } \\
\text { (thousand square feet) }\end{array}$} \\
\hline & & & & & 3 & 6 & 9 & 3 & 6 & 9 \\
\hline All Buildings & 881 & 175,012 & 492 & 198.7 & 312 & 243 & 326 & 94,880 & 35,816 & 44,316 \\
\hline \multicolumn{11}{|l|}{$\begin{array}{l}\text { Bullding Floorspace (square } \\
\text { feet) }\end{array}$} \\
\hline 10,000 to 50,000 & 349 & 9,013 & 21 & $25: 8$ & 94 & 107 & 148 & 2,564 & 2,591 & 3,858 \\
\hline 50,001 to 200,000 & 326 & 35,395 & 74 & 108.6 & 117 & 86 & 123 & 13,166 & 9,548 & 12,680 \\
\hline 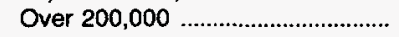 & 206 & 130,604 & 397 & 634.0 & 101 & 50 & 55 & 79,149 & 23,677 & 27,778 \\
\hline \multicolumn{11}{|l|}{ Principal Building Activity } \\
\hline 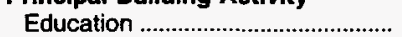 & 26 & 1,394 & 2 & 53.6 & 8 & 6 & 12 & 598 & 168 & 628 \\
\hline 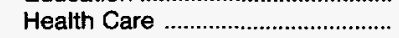 & 124 & 36,555 & 66 & 294.8 & 41 & 35 & 48 & 14,559 & 12,094 & 9,903 \\
\hline 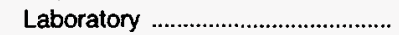 & 97 & 11,097 & 18 & 114.4 & 37 & 29 & 31 & 5,165 & 3,331 & 2,601 \\
\hline Lodging & 51 & 4,720 & 2 & 92.6 & 13 & 16 & 22 & 2,558 & 942 & 1,220 \\
\hline Mercantile and Service ................... & 158 & 22,397 & 99 & 141.8 & 46 & 49 & 63 & 7,966 & 6,236 & 8,194 \\
\hline 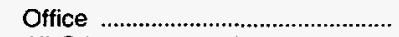 & 292 & 84,060 & 277 & 287.9 & 124 & 76 & 92 & 56,881 & 10,799 & 16,380 \\
\hline 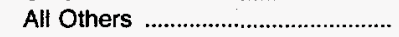 & 133 & 14,789 & 28 & 111.2 & 43 & 32 & 58 & 7,152 & 2,247 & 5,390 \\
\hline \multicolumn{11}{|l|}{ Year Constructed } \\
\hline 1959 or Before & 375 & 70,640 & 240 & 188.4 & 142 & 103 & 130 & 43,829 & 13,258 & 13,553 \\
\hline 1960 to 1969 & 163 & 35,033 & 89 & 214.9 & 70 & 38 & 55 & 19,564 & 6,386 & 9,082 \\
\hline 1970 to 1979 & 143 & 35,953 & 90 & 251.4 & 47 & 34 & 62 & 17,737 & 6,175 & 12,041 \\
\hline 1980 to 1989 & 160 & 23,833 & 59 & 149.0 & 38 & 57 & 65 & 8,975 & 6,903 & 7,955 \\
\hline 1990 to 1993 & 40 & 9,553 & 14 & 238.8 & 15 & 11 & 14 & 4,774 & 3,095 & 1,684 \\
\hline \multicolumn{11}{|l|}{ Federal Agency } \\
\hline Department of Defense ................. & 122 & 24,145 & 61 & 197.9 & 22 & 22 & 78 & 13,988 & 1,668 & 8,489 \\
\hline General Services Administration. & 157 & 66,598 & 220 & 424.2 & 83 & 35 & 39 & 46,205 & 7,888 & 12,505 \\
\hline United States Postal Service ....... & 181 & 24,806 & 103 & 137.0 & 57 & 61 & 63 & 9,392 & 7,027 & 8,387 \\
\hline 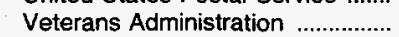 & 148 & 33,504 & 58 & 226.4 & 56 & 36 & 56 & 13,306 & 11,375 & 8,824 \\
\hline 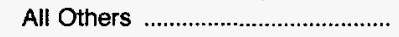 & 273 & 25,958 & 50 & 95.1 & 94 & 89 & 90 & 11,989 & 7,858 & 6,111 \\
\hline \multicolumn{11}{|l|}{$\begin{array}{l}\text { Energy Sources (more than one } \\
\text { may apply) }\end{array}$} \\
\hline 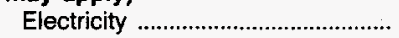 & 879 & 174,273 & 490 & 198.3 & 311 & 243 & 325 & 94,161 & 35,816 & 44,296 \\
\hline 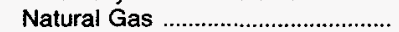 & 532 & 118,204 & 363 & 222.2 & 139 & 168 & 225 & 58,674 & 24,827 & 34,703 \\
\hline Fuel Oil & 161 & 55,167 & 135 & 342.7 & 89 & 18 & 54 & 39,300 & 4,740 & $+1,127$ \\
\hline 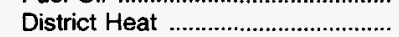 & 296 & 89,185 & 289 & 301.3 & 154 & 68 & 74 & 63,991 & 13,437 & 11,757 \\
\hline District Chilled Water & 114 & 37,263 & 95 & 326.9 & 57 & 30 & 27 & 22,922 & 7,980 & 6,362 \\
\hline 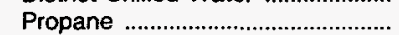 & 20 & 2,259 & 4 & 113.0 & 5 & 5 & 10 & 941 & 200 & 1,117 \\
\hline Any Other & 21 & 9,927 & 23 & 472.7 & 8 & 8 & 5 & 4,470 & 2,850 & 2,606 \\
\hline \multicolumn{11}{|l|}{$\begin{array}{l}\text { Energy End Uses (more than } \\
\text { one may apply) }\end{array}$} \\
\hline 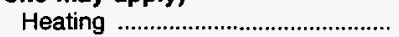 & 853 & 171,718 & 483 & 201.3 & 307 & 242 & 304 & 94,063 & 35,734 & 41,921 \\
\hline 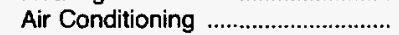 & 838 & 170,634 & 485 & 203.6 & 305 & 241 & 292 & 93,454 & 35,273 & 41,907 \\
\hline 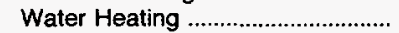 & 856 & 171,343 & 485 & 200.2 & 303 & 238 & 315 & 93,138 & 35,221 & 42,984 \\
\hline 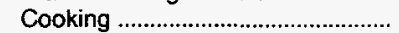 & 241 & 109,205 & 347 & 453.1 & 108 & 63 & 70 & 69,947 & 19,213 & 20,045 \\
\hline 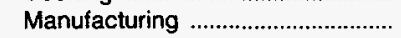 & 108 & 40,070 & 134 & 371.0 & 50 & 28 & 30 & 27,041 & 5,335 & 7,693 \\
\hline \multicolumn{11}{|l|}{ Workers (main shift) } \\
\hline Less than 50 & 234 & 10,335 & 5 & 44.2 & 71 & 70 & 93 & 4,102 & 2,548 & 3,684 \\
\hline 50 to 99 & 132 & 6,090 & 10 & 46.1 & 39 & 44 & 49 & 1,903 & 2,026 & 2,161 \\
\hline 100 to 499 & 321 & 36,678 & 69 & 114.3 & 107 & 80 & 134 & 13,632 & 9,029 & 14,017 \\
\hline 500 or More & 194 & 121,910 & 409 & 628.4 & 95 & 49 & 50 & 75,243 & 22,213 & 24,454 \\
\hline \multicolumn{11}{|l|}{ Weekly Operating Hours } \\
\hline 48 or Fewer & 170 & 17,257 & 33 & 101.5 & 51 & 39 & 80 & 9,694 & 2,740 & 4,822 \\
\hline 49 to 60 & 187 & 45,424 & 114 & 242.9 & 76 & 46 & 65 & 25,540 & 6,460 & 13,424 \\
\hline 61 to 167 & 206 & 25,749 & 58 & 125.0 & 60 & 78 & 68 & 11,993 & 8,051 & 5,706 \\
\hline 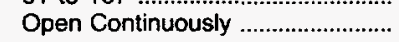 & 318 & 86,582 & 288 & 272.3 & 125 & 80 & 113 & 47,653 & 18,565 & 20,364 \\
\hline \multicolumn{11}{|l|}{$\begin{array}{l}\text { Predominant Exterior Wall } \\
\text { Material }\end{array}$} \\
\hline 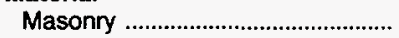 & 658 & 117,869 & 343 & 179.1 & 236 & 203 & 219 & 65,621 & 26,577 & 25,671 \\
\hline Other & 220 & 57,039 & 149 & 259.3 & 76 & 39 & 105 & 29,259 & 9,219 & 18,562 \\
\hline 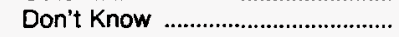 & 3 & 104 & $(*)$ & 34.6 & NC & 1 & 2 & NC & 20 & \\
\hline
\end{tabular}

See footnotes at end of table. 
Table 3.7. Summary of FBSS Buildings and Floorspace in Federal Regions 3, 6, and 9, 1993 (Continued)

\begin{tabular}{|c|c|c|c|c|c|c|c|c|c|c|}
\hline \multirow{2}{*}{$\begin{array}{c}\text { Building } \\
\text { Characteristics }\end{array}$} & \multirow{2}{*}{$\begin{array}{c}\text { Total } \\
\text { Number of } \\
\text { Buildings }\end{array}$} & \multirow{2}{*}{$\begin{array}{c}\text { Total } \\
\text { Floorspace } \\
\text { (thousand } \\
\text { square } \\
\text { feet) }\end{array}$} & \multirow{2}{*}{$\begin{array}{c}\text { Total } \\
\text { Workers } \\
\text { (thousand) }\end{array}$} & \multirow{2}{*}{ 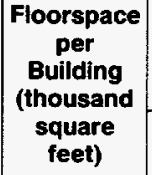 } & \multicolumn{3}{|c|}{$\begin{array}{l}\text { Number of Buildings } \\
\text { per Federal Region }\end{array}$} & \multicolumn{3}{|c|}{$\begin{array}{l}\text { Floorspace per } \\
\text { Federal Region } \\
\text { (thousand square feet) }\end{array}$} \\
\hline & & & & & 3 & 6 & 9 & 3 & 6 & 9 \\
\hline \multicolumn{11}{|l|}{ Predominant Roof Material } \\
\hline Built-Up & 499 & 102,996 & 249 & 206.4 & 156 & 143 & 200 & 47,882 & 24,341 & 30,773 \\
\hline 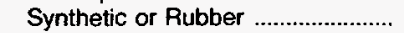 & 121 & 32,530 & 101 & 268.8 & 58 & 33 & 30 & 23,371 & 4,377 & 4,782 \\
\hline Other & 251 & 38,450 & 139 & 153.2 & 96 & 64 & 91 & 23,532 & 7,029 & 7,888 \\
\hline 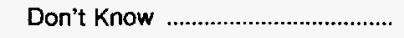 & 10 & 1,036 & 3 & 103.6 & 2 & 3 & 5 & 95 & 69 & 872 \\
\hline \multicolumn{11}{|l|}{ Floors } \\
\hline One & 233 & 14,045 & 32 & 60.3 & 56 & 74 & 103 & 4,414 & 4,504 & 5,127 \\
\hline Two & 159 & 17,213 & 44 & 108.3 & 51 & 30 & 78 & 6,307 & 2,622 & 8,284 \\
\hline Three & 162 & 18,239 & 45 & 112.6 & 60 & 40 & 62 & 7,440 & 3,598 & 7,201 \\
\hline Four to Nine & 236 & 80,032 & 239 & 339.1 & 108 & 60 & 68 & 48,067 & 15,186 & 16,779 \\
\hline 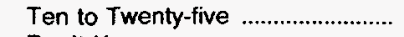 & 59 & 40,731 & 124 & 690.4 & 34 & 15 & 10 & 27,430 & 6,722 & 6,579 \\
\hline 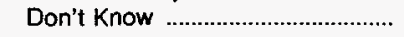 & 32 & 4,751 & 9 & 148.5 & 3 & 24 & 5 & 1,222 & 3,184 & 345 \\
\hline \multicolumn{11}{|l|}{ Percent Window Glass } \\
\hline 50 or Less & 771 & 146,270 & 428 & 189.7 & 269 & 204 & 298 & 79,002 & 29,836 & 37,432 \\
\hline 51 to 100 & 84 & 25,107 & 59 & 298.9 & 42 & 16 & 26 & 14,878 & 3,429 & 6,800 \\
\hline 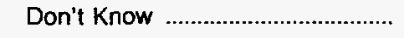 & 26 & 3,635 & 6 & 139.8 & 1 & 23 & 2 & 1,000 & 2,552 & 84 \\
\hline \multicolumn{11}{|l|}{ Multibuilding Facility } \\
\hline 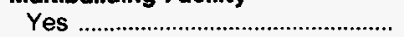 & 524 & 95,123 & 233 & 181.5 & 176 & 148 & 200 & 43,861 & 24,441 & 26,821 \\
\hline No & 357 & 79,889 & 259 & 223.8 & 136 & 95 & 126 & 51,018 & 11,375 & 17,495 \\
\hline \multicolumn{11}{|l|}{$\begin{array}{l}\text { Space-Heating Energy Sources } \\
\text { (more than one may apply) }\end{array}$} \\
\hline 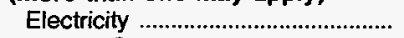 & 211 & 37,748 & 100 & 178.9 & 70 & 66 & 75 & 18,459 & 9,859 & 9,430 \\
\hline 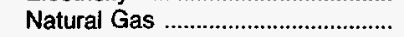 & 445 & 70,763 & 161 & 159.0 & 96 & 153 & 196 & 22,134 & 19,800 & 28,829 \\
\hline 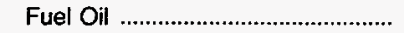 & 80 & 13,832 & 35 & 172.9 & 53 & 6 & 21 & 9,156 & 1,288 & 3,387 \\
\hline 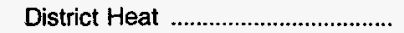 & 284 & 86,885 & 282 & 305.9 & 151 & 65 & 68 & 62,321 & 13,244 & 11,319 \\
\hline Propane & 11 & 322 & 1 & 29.3 & NG & 4 & 7 & NC & 65 & 257 \\
\hline Wood & NC & NC & NC & NC & NC & NC & NC & NC & NC & NC \\
\hline 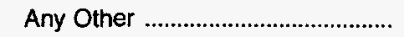 & 6 & 926 & 2 & 154.4 & 3 & 2 & 1 & 289 & 234 & 403 \\
\hline \multicolumn{11}{|l|}{$\begin{array}{l}\text { Main Space-Heating } \\
\text { Energy Source }\end{array}$} \\
\hline 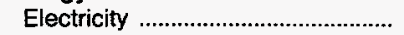 & 111 & 15,763 & 46 & 142.0 & 36 & 31 & 44 & 6,798 & 4,639 & 4,326 \\
\hline 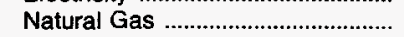 & 419 & 65,303 & 147 & 155.9 & 90 & 144 & 185 & 20,113 & 18,021 & 27,169 \\
\hline Fuel Oil & 44 & 5,885 & 13 & 133.7 & 34 & 1 & 9 & 5,083 & 11 & 790 \\
\hline 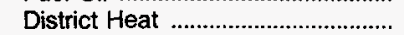 & 270 & 84,544 & 277 & 313.1 & 147 & 63 & 60 & 62,068 & 13,008 & 9,467 \\
\hline Propane & 9 & 223 & $(*)$ & 24.8 & NC & 3 & 6 & NC & 54 & 169 \\
\hline Wood & NC & $\mathrm{NC}$ & NC & NC & NC & NC & NC & NC & NC & NC \\
\hline Any Other & NC & $\mathrm{NC}$ & NC & NC & NC & NC & NC & NC & NC & NC \\
\hline \multicolumn{11}{|l|}{$\begin{array}{l}\text { Replacement Energy Source for } \\
\text { Main Heating }\end{array}$} \\
\hline 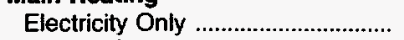 & 15 & 1,862 & 3 & 124.1 & 4 & 7 & 4 & 1,284 & 145 & 433 \\
\hline 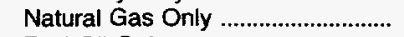 & 21 & 2,768 & 5 & 131.8 & 12 & 3 & 6 & 2,253 & 356 & 159 \\
\hline 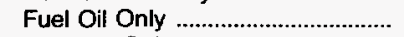 & 170 & 38,875 & 88 & 228.7 & 66 & 37 & 67 & 14,166 & 8,544 & 16,165 \\
\hline Propane Only & 25 & 2,354 & 4 & 94.2 & NC & 3 & 22 & NC & 184 & 2,170 \\
\hline Any Other Single Energy Source & 9 & 2,028 & 5 & 225.4 & 2 & 1 & 6 & 1,016 & 11 & 1,001 \\
\hline More than One Energy Source ... & 9 & 540 & 1 & 60.0 & 7 & 1 & 1 & 479 & 28 & 32 \\
\hline No Replacement Energy Source & 541 & 114,410 & 359 & 211.5 & 206 & 158 & 177 & 70,765 & 23,310 & 20,335 \\
\hline $\begin{array}{l}\text { Building Not Heated ............................. } \\
\text { Don't Know/ }\end{array}$ & 28 & 3,294 & 9 & 117.6 & 5 & 1 & 22 & 817 & 82 & 2,394 \\
\hline Not Ascertained ................................. & 63 & 8,881 & 19 & 141.0 & 10 & 32 & 21 & 4,100 & 3,157 & 1,625 \\
\hline \multicolumn{11}{|l|}{$\begin{array}{l}\text { Cooling Energy Sources (more } \\
\text { than one may apply) }\end{array}$} \\
\hline 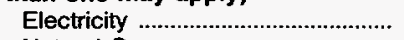 & 740 & 145,491 & 431 & 196.6 & 256 & 219 & 265 & 80,870 & 28,061 & 36,560 \\
\hline 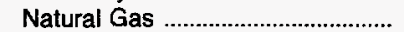 & 8 & 263 & 1 & 32.8 & 1 & 1 & 6 & 22 & 47 & 193 \\
\hline District Chilled Water ........................ & 109 & 29,667 & 67 & 272.2 & 55 & 29 & 25 & 15,695 & 7,742 & 6,230 \\
\hline $\begin{array}{l}\text { Water-Heating Energy Sources } \\
\text { (more than one may apply) }\end{array}$ & & & & & & & & & & \\
\hline 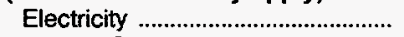 & 221 & 38,467 & 94 & 174.1 & 94 & 62 & 65 & 18,972 & 12,268 & 7,227 \\
\hline 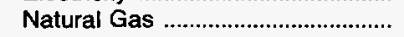 & 381 & 60,841 & 147 & 159.7 & 78 & 125 & 178 & 18,101 & 15,655 & 27,085 \\
\hline 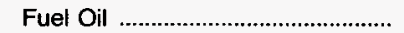 & 30 & 6,364 & 18 & 212.1 & 19 & 1 & 10 & 4,679 & 760 & 925 \\
\hline 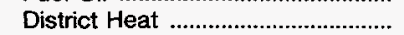 & 239 & 74,265 & 255 & 310.7 & 122 & 52 & 65 & 57,122 & 8,576 & 8,566 \\
\hline 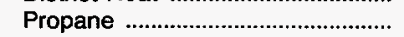 & 9 & 224 & $(*)$ & 24.8 & NC & 4 & 5 & NC & 65 & 158 \\
\hline
\end{tabular}

See footnotes at end of table. 
Table 3.7. Summary of FBSS Buildings and Floorspace in Federal Regions 3, 6, and 9, 1993 (Continued)

\begin{tabular}{|c|c|c|c|c|c|c|c|c|c|c|}
\hline \multirow{2}{*}{$\begin{array}{c}\text { Building } \\
\text { Characteristics }\end{array}$} & \multirow{2}{*}{$\begin{array}{c}\text { Total } \\
\text { Number of } \\
\text { Buildings }\end{array}$} & \multirow{2}{*}{$\begin{array}{c}\text { Total } \\
\text { Floorspace } \\
\text { (thousand } \\
\text { square } \\
\text { feet) }\end{array}$} & \multirow{2}{*}{$\begin{array}{c}\text { Total } \\
\text { Workers } \\
\text { (thousand) }\end{array}$} & \multirow{2}{*}{$\begin{array}{c}\text { Floorspace } \\
\text { per } \\
\text { Building } \\
\text { (thousand } \\
\text { square } \\
\text { feet) }\end{array}$} & \multicolumn{3}{|c|}{$\begin{array}{l}\text { Number of Buildings } \\
\text { per Federal Region }\end{array}$} & \multicolumn{3}{|c|}{$\begin{array}{l}\text { Floorspace per } \\
\text { Federal Region } \\
\text { (thousand square feet) }\end{array}$} \\
\hline & & & & & 3 & 6 & 9 & 3 & 6 & 9 \\
\hline \multicolumn{11}{|l|}{$\begin{array}{l}\text { Cooking Energy Sources (more } \\
\text { than one may apply) }\end{array}$} \\
\hline Electricity & 148 & 65,202 & 235 & 440.6 & 68 & 37 & 43 & 41,432 & 10,177 & 13,594 \\
\hline 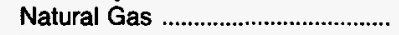 & 111 & 59,944 & 229 & 540.0 & 46 & 31 & 34 & 39,084 & 11,004 & 9,857 \\
\hline 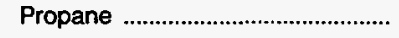 & 7 & 332 & (") & 47.4 & 2 & $\mathrm{NC}$ & 5 & & NC & 279 \\
\hline \multicolumn{11}{|l|}{$\begin{array}{l}\text { Manufacturing Energy Sources } \\
\text { (more than one may apply) }\end{array}$} \\
\hline Electricity & 94 & 31,563 & 118 & 335.8 & 43 & 26 & 25 & 20,521 & 4,838 & 6,205 \\
\hline 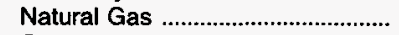 & 14 & 7,192 & 17 & 513.7 & 5 & 1 & 8 & 5,319 & 37 & 1,836 \\
\hline Other & 9 & 5,984 & 9 & 664.8 & 5 & 1 & 3 & 4,140 & 461 & 1,383 \\
\hline \multicolumn{11}{|l|}{ Percent of Floorspace Heated } \\
\hline Not Heated .......................................... & 28 & 3,294 & 9 & 117.6 & 5 & 1 & 22 & 817 & 82 & 2,394 \\
\hline 1 to 50 & 51 & 10,826 & 15 & 212.3 & 12 & 11 & 28 & 5,787 & 1,288 & 3,751 \\
\hline 51 to 100 & 802 & 160,892 & 468 & 200.6 & 295 & 231 & 276 & 88,275 & 34,446 & 38,170 \\
\hline \multicolumn{11}{|l|}{ Percent of Floorspace Cooled } \\
\hline 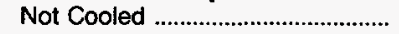 & 56 & 7,091 & 9 & 126.6 & 13 & 3 & 40 & 3,582 & 554 & 2,956 \\
\hline 1 to 50 & 111 & 19,129 & 34 & 172.3 & 30 & 26 & 55 & 9,874 & 1,951 & 7,304 \\
\hline 51 to 100 & 714 & 148,792 & 449 & 208.4 & 269 & 214 & 231 & 81,424 & 33,312 & 34,056 \\
\hline \multicolumn{11}{|l|}{ Percent Lit When Open } \\
\hline 1 to 50 & 46 & 3,572 & 4 & 77.6 & 11 & 15 & 20 & 663 & 1,062 & 1,847 \\
\hline 51 to 100 & 831 & 171,118 & 488 & 205.9 & 300 & 227 & 304 & 94,070 & 34,672 & 42,375 \\
\hline No Operating Hours .......................... & 3 & 258 & $(*)$ & 86.0 & 1 & 1 & 1 & 147 & 82 & 29 \\
\hline 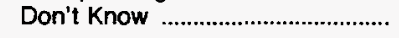 & 1 & 64 & $(*)$ & 64.4 & $\mathrm{NC}$ & NC & 1 & NC & NC & 64 \\
\hline \multicolumn{11}{|l|}{ Percent Lit When Closed } \\
\hline 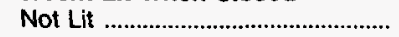 & 107 & 9,504 & 50 & 88.8 & 39 & 33 & 35 & 5,149 & 2,691 & 1,664 \\
\hline 1 to 50 & 564 & 103,736 & 289 & 183.9 & 194 & 165 & 205 & 57,953 & 20,495 & 25,288 \\
\hline 51 to 100 & 111 & 33,550 & 88 & 302.3 & 42 & 25 & 44 & 17,412 & 8,029 & 8,109 \\
\hline 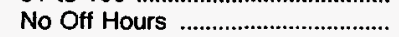 & 98 & 28,157 & 65 & 287.3 & 37 & 20 & 41 & 14,366 & 4,601 & 9,190 \\
\hline 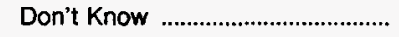 & 1 & 64 & $(*)$ & 64.4 & $\mathrm{NC}$ & NC & 1 & NC & NC & 64 \\
\hline \multicolumn{11}{|l|}{$\begin{array}{l}\text { Heating Equipment (more than } \\
\text { one may apply) }\end{array}$} \\
\hline Heat Pumps & 84 & 14,968 & 39 & 178.2 & 34 & 10 & 40 & 10,922 & 964 & 3,081 \\
\hline 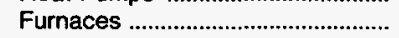 & 59 & 3,689 & 6 & 62.5 & 16 & 18 & 25 & 1,694 & 791 & 1,204 \\
\hline Individual Space Heaters ............... & 138 & 45,264 & 128 & 328.0 & 75 & 30 & 33 & 34,923 & 5,408 & 4,933 \\
\hline 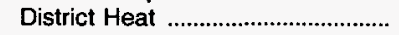 & 320 & 95,621 & 299 & 298.8 & 167 & 82 & 71 & 67,704 & 15,205 & 12,712 \\
\hline Boilers & 341 & 60,810 & 148 & 178.3 & 93 & 102 & 146 & 20,082 & 16,718 & 24,010 \\
\hline Packaged-Heating Units .................. & 151 & 19,576 & 50 & 129.6 & 25 & 59 & 67 & 6,006 & 7,574 & 5,997 \\
\hline \multicolumn{11}{|l|}{$\begin{array}{l}\text { Cooling Equipment (more than } \\
\text { one may apply) }\end{array}$} \\
\hline Residential-Type Central A/C ....... & 83 & 25,679 & 149 & 309.4 & 30 & 29 & 24 & 19,073 & 4,706 & 1,900 \\
\hline 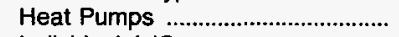 & 80 & 15,662 & 39 & 195.8 & 37 & 11 & 32 & 11,388 & 1,549 & 2,724 \\
\hline Individual A/C & 182 & 40,755 & 170 & 223.9 & 80 & 50 & 52 & 28,082 & 6,950 & 5,723 \\
\hline District Chilled Water ........................ & 147 & 46,698 & 116 & 317.7 & 72 & 44 & 31 & 28,194 & 10,856 & 7,647 \\
\hline Central Chillers & 419 & 113,233 & 361 & 270.2 & 158 & 122 & 139 & 63,914 & 21,596 & 27,723 \\
\hline 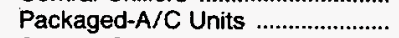 & 375 & 94,757 & 322 & 252.7 & 127 & 97 & 151 & 56,664 & 14,104 & 23,989 \\
\hline 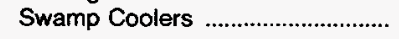 & 45 & 5,558 & 13 & 123.5 & 3 & 12 & 30 & 378 & 2,015 & 3,165 \\
\hline \multicolumn{11}{|l|}{$\begin{array}{l}\text { Lighting Equipment (more than } \\
\text { one may apply) }\end{array}$} \\
\hline 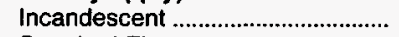 & 480 & 105,704 & 303 & 220.2 & 184 & 135 & 161 & 64,981 & 20,248 & 20,475 \\
\hline Standard Fluorescent ...................... & 844 & 169,893 & 457 & 201.3 & 300 & 235 & 309 & 91,986 & 35,057 & 42,850 \\
\hline Compact Fluorescent ....................... & 182 & 74,735 & 237 & 410.6 & 80 & 29 & 73 & 49,455 & 7,942 & 17,339 \\
\hline High-Intensity Discharge ................ & 250 & 87,517 & 303 & 350.1 & 111 & 64 & 75 & 52,593 & 16,188 & 18,736 \\
\hline 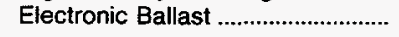 & 299 & 104,727 & 315 & 350.3 & 135 & 59 & 105 & 65,560 & 15,861 & 23,307 \\
\hline \multicolumn{11}{|l|}{$\begin{array}{l}\text { Water-Heating Equipment (more } \\
\text { than one may apply) }\end{array}$} \\
\hline 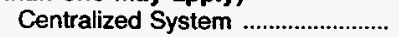 & 611 & 117,752 & 347 & 192.7 & 223 & 163 & 225 & 63,477 & 23,206 & 31,069 \\
\hline $\begin{array}{l}\text { Distributed System ........................... } \\
\text { Don't Know/ }\end{array}$ & 222 & 47,272 & 124 & 212.9 & 71 & 71 & 80 & 24,696 & 11,827 & 10,750 \\
\hline 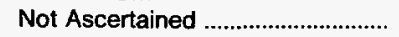 & 23 & 6,319 & 14 & 274.7 & 9 & 4 & 10 & 4,965 & 188 & 1,165 \\
\hline
\end{tabular}

See footnotes at end of table. 
Table 3.7. Summary of FBSS Buildings and Floorspace in Federal Regions 3, 6, and 9, 1993 (Continued)

\begin{tabular}{|c|c|c|c|c|c|c|c|c|c|c|}
\hline \multirow{2}{*}{$\begin{array}{c}\text { Building } \\
\text { Characteristics }\end{array}$} & \multirow{2}{*}{$\begin{array}{c}\text { Total } \\
\text { Number of } \\
\text { Buildings }\end{array}$} & \multirow{2}{*}{$\begin{array}{c}\text { Total } \\
\text { Floorspace } \\
\text { (thousand } \\
\text { square } \\
\text { feet) }\end{array}$} & \multirow{2}{*}{$\begin{array}{c}\text { Total } \\
\text { Workers } \\
\text { (thousand) }\end{array}$} & \multirow{2}{*}{$\begin{array}{c}\text { Floorspace } \\
\text { per } \\
\text { Building } \\
\text { (thousand } \\
\text { square } \\
\text { feet) }\end{array}$} & \multicolumn{3}{|c|}{$\begin{array}{l}\text { Number of Buildings } \\
\text { per Federal Region }\end{array}$} & \multicolumn{3}{|c|}{$\begin{array}{c}\text { Floorspace per } \\
\text { Federal Region } \\
\text { (thousand square feet) }\end{array}$} \\
\hline & & & & & 3 & 6 & 9 & 3 & 6 & 9 \\
\hline \multicolumn{11}{|l|}{$\begin{array}{l}\text { Commercial Refrigeration } \\
\text { Equipment (more than one may } \\
\text { apply) }\end{array}$} \\
\hline 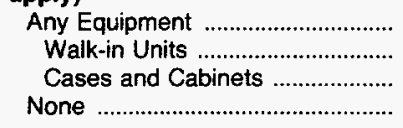 & $\begin{array}{l}242 \\
162 \\
181 \\
639\end{array}$ & $\begin{array}{l}98,429 \\
79,141 \\
82,507 \\
76,583\end{array}$ & $\begin{array}{l}322 \\
269 \\
204 \\
170\end{array}$ & $\begin{array}{l}406.7 \\
488.5 \\
455.8 \\
119.8\end{array}$ & $\begin{array}{r}109 \\
78 \\
87 \\
203\end{array}$ & $\begin{array}{r}70 \\
40 \\
44 \\
173\end{array}$ & $\begin{array}{r}63 \\
44 \\
50 \\
263\end{array}$ & $\begin{array}{l}61,731 \\
54,223 \\
53,454 \\
33,149\end{array}$ & $\begin{array}{l}17,917 \\
12,381 \\
14,125 \\
17,899\end{array}$ & $\begin{array}{l}18,781 \\
12,536 \\
14,928 \\
25,535\end{array}$ \\
\hline \multicolumn{11}{|l|}{$\begin{array}{l}\text { Retrofit or Purchase of any } \\
\text { Equipment Within Last Ten } \\
\text { Years (more than one may } \\
\text { apply) }\end{array}$} \\
\hline 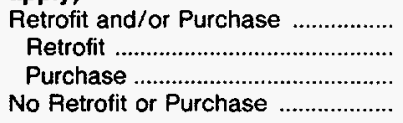 & $\begin{array}{l}604 \\
245 \\
462 \\
277\end{array}$ & $\begin{array}{r}144,849 \\
80,767 \\
104,046 \\
30,163\end{array}$ & $\begin{array}{r}437 \\
214 \\
332 \\
55\end{array}$ & $\begin{array}{l}239.8 \\
329.7 \\
225.2 \\
108.9\end{array}$ & $\begin{array}{r}229 \\
79 \\
188 \\
83\end{array}$ & $\begin{array}{r}162 \\
63 \\
126 \\
81\end{array}$ & $\begin{array}{l}213 \\
103 \\
148 \\
113\end{array}$ & $\begin{array}{l}81,286 \\
43,128 \\
62,995 \\
13,594\end{array}$ & $\begin{array}{r}29,015 \\
15,029 \\
21,409 \\
6,801\end{array}$ & $\begin{array}{r}34,547 \\
22,610 \\
19,642 \\
9,769\end{array}$ \\
\hline \multicolumn{11}{|l|}{$\begin{array}{l}\text { Energy Conservation Features } \\
\text { (more than one may apply) }\end{array}$} \\
\hline 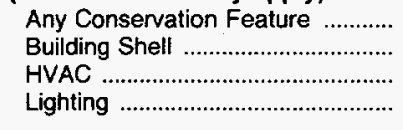 & $\begin{array}{l}870 \\
766 \\
846 \\
563\end{array}$ & $\begin{array}{l}174,119 \\
152,607 \\
172,084 \\
141,432\end{array}$ & $\begin{array}{l}492 \\
446 \\
489 \\
401\end{array}$ & $\begin{array}{l}200.1 \\
199.2 \\
203.4 \\
251.2\end{array}$ & $\begin{array}{l}309 \\
290 \\
303 \\
210\end{array}$ & $\begin{array}{l}242 \\
228 \\
236 \\
136\end{array}$ & $\begin{array}{l}319 \\
248 \\
307 \\
217\end{array}$ & $\begin{array}{l}94,361 \\
84,557 \\
93,543 \\
80,953\end{array}$ & $\begin{array}{l}35,734 \\
33,946 \\
35,425 \\
25,654\end{array}$ & $\begin{array}{l}44,023 \\
34,105 \\
43,116 \\
34,825\end{array}$ \\
\hline \multicolumn{11}{|l|}{$\begin{array}{l}\text { Building Shell Conservation } \\
\text { Features (more than one may } \\
\text { apply) } \\
\text { Roof or Ceiling }\end{array}$} \\
\hline $\begin{array}{l}\text { Insulation } \\
\text { Wall Insulation } \\
\text { Storm or Multiple }\end{array}$ & $\begin{array}{l}621 \\
352\end{array}$ & $\begin{array}{r}125,137 \\
69,887\end{array}$ & $\begin{array}{l}354 \\
154\end{array}$ & $\begin{array}{l}201.5 \\
198.5\end{array}$ & $\begin{array}{l}240 \\
117\end{array}$ & $\begin{array}{l}196 \\
122\end{array}$ & $\begin{array}{l}185 \\
113\end{array}$ & $\begin{array}{l}69,654 \\
31,876\end{array}$ & $\begin{array}{l}28,249 \\
20,550\end{array}$ & $\begin{array}{l}27,234 \\
17,462\end{array}$ \\
\hline Glazing & 326 & 74,340 & 227 & 228.0 & 173 & 96 & 57 & 46,141 & 17,939 & 10,260 \\
\hline $\begin{array}{l}\text { or Shading Film .......................... } \\
\text { Exterior or Interior Shading }\end{array}$ & 418 & 93,977 & 292 & 224.8 & 158 & 127 & 133 & 46,829 & 24,768 & 22,381 \\
\hline 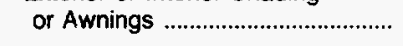 & 503 & 107,620 & 301 & 214.0 & 197 & 142 & 164 & 57,183 & 26,067 & 24,370 \\
\hline \multicolumn{11}{|l|}{$\begin{array}{l}\text { HVAC Conservation Features } \\
\text { (more than one may apply) }\end{array}$} \\
\hline 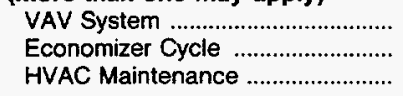 & $\begin{array}{l}224 \\
419 \\
841\end{array}$ & $\begin{array}{r}71,706 \\
110,998 \\
171,841\end{array}$ & $\begin{array}{l}211 \\
353 \\
489\end{array}$ & $\begin{array}{l}320.1 \\
264.9 \\
204.3\end{array}$ & $\begin{array}{r}84 \\
156 \\
301\end{array}$ & $\begin{array}{r}65 \\
123 \\
234\end{array}$ & $\begin{array}{r}75 \\
140 \\
306\end{array}$ & $\begin{array}{l}37,611 \\
61,089 \\
93,402\end{array}$ & $\begin{array}{l}18,518 \\
23,542 \\
35,341\end{array}$ & $\begin{array}{l}15,577 \\
26,367 \\
43,098\end{array}$ \\
\hline \multicolumn{11}{|l|}{$\begin{array}{l}\text { Lighting Conservation Features } \\
\text { (more than one may apply) }\end{array}$} \\
\hline $\begin{array}{l}\text { Specular Reflectors .......................... } \\
\text { Natural Lighting Control }\end{array}$ & 340 & 84,607 & 261 & 248.8 & 124 & 92 & 124 & 44,741 & 18,017 & 21,850 \\
\hline 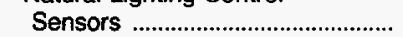 & 114 & 38,243 & 95 & 335.5 & 29 & 27 & 58 & 17,743 & 6,740 & 13,760 \\
\hline Occupancy Sensors & 262 & 103,941 & 314 & 396.7 & 95 & 58 & 109 & 62,261 & 16,435 & 25,245 \\
\hline 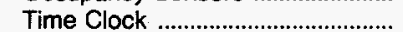 & 151 & 66,131 & 210 & 438.0 & 74 & 36 & 41 & 46,555 & 9,037 & 10,539 \\
\hline Manual Dimmer Switches ............. & 215 & 77,732 & 239 & 361.5 & 93 & 44 & 78 & 49,824 & 13,454 & 14,455 \\
\hline \multicolumn{11}{|l|}{$\begin{array}{l}\text { Energy Management Practices } \\
\text { (more than one may appiy) } \\
\text { Energy Management and Control }\end{array}$} \\
\hline $\begin{array}{l}\text { System ....................................... } \\
\text { Energy Conservation }\end{array}$ & 299 & 103,220 & 328 & 345.2 & 132 & 96 & 71 & 61,891 & 23,099 & 18,231 \\
\hline 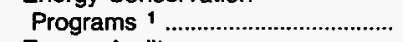 & 271 & 97,497 & 304 & 359.8 & 99 & 48 & 124 & 59,284 & 13,513 & 24,699 \\
\hline 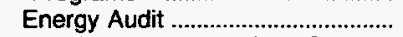 & 262 & 66,755 & 165 & 254.8 & 99 & 64 & 99 & 37,621 & $11,690^{\circ}$ & 17,444 \\
\hline HVAC Maintenance Staff 2 ........... & 206 & 83,032 & 287 & 403.1 & 97 & 57 & 52 & 52,924 & 15,538 & 14,570 \\
\hline \multicolumn{11}{|l|}{$\begin{array}{l}\text { Off-Hours Reduction in } \\
\text { Equipment (more than one may } \\
\text { apply) }\end{array}$} \\
\hline 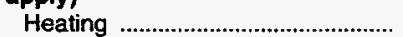 & 418 & 76,622 & 182 & 183.3 & 144 & 123 & 151 & 43,828 & 13,162 & 19,631 \\
\hline Cooling & 426 & 76,840 & 184 & 180.4 & 145 & 124 & 157 & 43,757 & 13,864 & 19,219 \\
\hline Hot Water & 178 & 44,187 & 105 & 248.2 & 77 & 33 & 68 & 26,018 & 4,554 & 13,614 \\
\hline 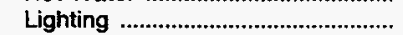 & 454 & 78,719 & 185 & 173.4 & 162 & 120 & 172 & 44,717 & 12,847 & 21,155 \\
\hline
\end{tabular}

See footnotes at end of table. 
Table 3.7. Summary of FBSS Buildings and Floorspace in Federal Regions 3, 6, and 9, 1993 (Continued)

\begin{tabular}{|c|c|c|c|c|c|c|c|c|c|c|}
\hline \multirow{2}{*}{$\begin{array}{c}\text { Building } \\
\text { Characteristics }\end{array}$} & \multirow{2}{*}{$\begin{array}{l}\text { Total } \\
\text { Number of } \\
\text { Buildings }\end{array}$} & \multirow{2}{*}{$\begin{array}{c}\text { Total } \\
\text { Floorspace } \\
\text { (thousand } \\
\text { square } \\
\text { feet) }\end{array}$} & \multirow{2}{*}{$\begin{array}{c}\text { Total } \\
\text { Workers } \\
\text { (thousand) }\end{array}$} & \multirow{2}{*}{$\begin{array}{c}\text { Floorspace } \\
\text { per } \\
\text { Building } \\
\text { (thousand } \\
\text { square } \\
\text { feet) }\end{array}$} & \multicolumn{3}{|c|}{$\begin{array}{l}\text { Number of Buildings } \\
\text { per Federal Region }\end{array}$} & \multicolumn{3}{|c|}{$\begin{array}{c}\text { Floorspace per } \\
\text { Federal Region } \\
\text { (thousand square feet) }\end{array}$} \\
\hline & & & & & 3 & 6 & 9 & 3 & 6 & 9 \\
\hline \multicolumn{11}{|l|}{ Building Generates Electricity } \\
\hline 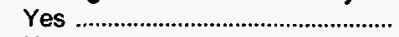 & 139 & 56,563 & 175 & 406.9 & 55 & 32 & 52 & 31,984 & 10,295 & 14,283 \\
\hline No & 742 & 118,449 & 318 & 159.6 & 257 & 211 & 274 & 62,896 & 25,521 & 30,032 \\
\hline \multicolumn{11}{|l|}{$\begin{array}{l}\text { Natural Gas Transported } \\
\text { for the Account of Others }\end{array}$} \\
\hline 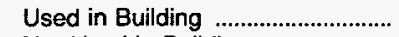 & 40 & 10,103 & 22 & 252.6 & 11 & 5 & 24 & 4,270 & 631 & 5,201 \\
\hline $\begin{array}{l}\text { Not Used in Building } \\
\text { Don't Know/................... }\end{array}$ & 438 & 101,624 & 326 & 232.0 & 124 & 140 & 174 & 52,636 & 22,202 & 26,787 \\
\hline 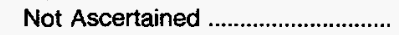 & 52 & 6,393 & 15 & 122.9 & 4 & 23 & 25 & 1,768 & 1,994 & 2,631 \\
\hline
\end{tabular}

1 Building participates in any programs sponsored by the Federal Energy Management Program, in-house, utility, or third party.

2 HVAC maintenance staff means at least one person spends at least half their working hours maintaining the heating/cooling equipment.

$(*)=$ Value rounds to zero in the units displayed.

NC $=$ No cases in responding sample.

Notes: - Total workers are the number of workers during the main shift. - See Glossary for explanation of abbreviations and definitions of terms used in this report. - These data are from 881 federally owned buildings having the following criteria: (1) located in Federal Regions 3,6 or 9; (2) larger than 10,000 square feet; and (3) used for a commercial purpose, other than warehouse and storage. In addition, 9 out of 10 selected buildings were from agencies other than the Department of Defense. - Statistics for the "energy end uses" represent consumption in buildings that have end use, not consumption for a particular fuel for a particular end use. $\bullet$ A/C = Air Conditioning. $\bullet$ FBSS = Federal Buildings Supplemental Survey. - HVAC = Heating, Ventilation, and Air Conditioning. - VAV = Variable-Air Volume. - Data are for Fiscal Year 1993 (October 1, 1992 through September 30, 1993). - Because of rounding, data may not sum to totals.

Source: Energy Information Administration, Office of Energy Markets and End Use, 1993 Federal Buildings Supplemental Survey. 
Table 3.8. Energy Sources in FBSS Buildings in Federal Regions 3, 6, and 9, Number of Buildings, 1993

\begin{tabular}{|c|c|c|c|c|c|c|c|c|c|}
\hline \multirow[b]{3}{*}{$\begin{array}{c}\text { Building } \\
\text { Characteristics }\end{array}$} & \multicolumn{3}{|c|}{$\begin{array}{l}\text { Number of Buildings } \\
\text { in Region } 3\end{array}$} & \multicolumn{3}{|c|}{$\begin{array}{l}\text { Number of Buildings } \\
\text { in Region } 6\end{array}$} & \multicolumn{3}{|c|}{$\begin{array}{l}\text { Number of Buildings } \\
\text { in Region } 9\end{array}$} \\
\hline & \multirow[b]{2}{*}{$\begin{array}{c}\text { All } \\
\text { Buildings }\end{array}$} & \multicolumn{2}{|c|}{$\begin{array}{l}\text { Energy Sources Used } \\
\text { in Region } 3 \text { (more } \\
\text { than one may apply) }\end{array}$} & \multirow[b]{2}{*}{$\begin{array}{c}\text { All } \\
\text { Buildings }\end{array}$} & \multicolumn{2}{|c|}{$\begin{array}{c}\text { Energy Sources Used } \\
\text { in Region } 6 \text { (more } \\
\text { than one may apply) }\end{array}$} & \multirow[b]{2}{*}{$\begin{array}{c}\text { All } \\
\text { Buildings }\end{array}$} & \multicolumn{2}{|c|}{$\begin{array}{l}\text { Energy Sources Used } \\
\text { in Region } 9 \text { (more } \\
\text { than one may apply) }\end{array}$} \\
\hline & & $\begin{array}{l}\text { Elec- } \\
\text { tricity }\end{array}$ & $\begin{array}{l}\text { Natural } \\
\text { Gas }\end{array}$ & & $\begin{array}{l}\text { Elec- } \\
\text { tricity }\end{array}$ & $\begin{array}{l}\text { Natural } \\
\text { Gas }\end{array}$ & & $\begin{array}{l}\text { Elec- } \\
\text { tricity }\end{array}$ & $\begin{array}{l}\text { Natural } \\
\text { Gas }\end{array}$ \\
\hline 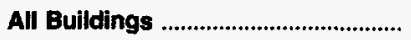 & 312 & 311 & 139 & 243 & 243 & 168 & 326 & 325 & 225 \\
\hline 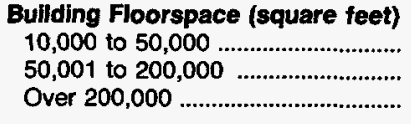 & $\begin{array}{r}94 \\
117 \\
101\end{array}$ & $\begin{array}{r}94 \\
117 \\
100\end{array}$ & $\begin{array}{l}37 \\
46 \\
56\end{array}$ & $\begin{array}{r}107 \\
86 \\
50\end{array}$ & $\begin{array}{r}107 \\
86 \\
50\end{array}$ & $\begin{array}{l}77 \\
57 \\
34\end{array}$ & $\begin{array}{r}148 \\
123 \\
55\end{array}$ & $\begin{array}{r}147 \\
123 \\
55\end{array}$ & $\begin{array}{l}93 \\
87 \\
45\end{array}$ \\
\hline 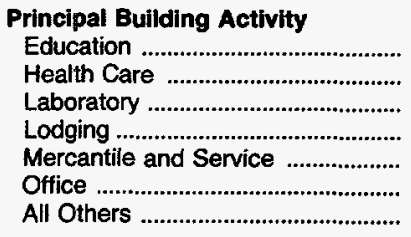 & $\begin{array}{r}8 \\
41 \\
37 \\
13 \\
46 \\
124 \\
43\end{array}$ & $\begin{array}{r}8 \\
41 \\
37 \\
13 \\
45 \\
124 \\
43\end{array}$ & $\begin{array}{r}3 \\
25 \\
8 \\
9 \\
27 \\
56 \\
11\end{array}$ & $\begin{array}{r}6 \\
35 \\
29 \\
16 \\
49 \\
76 \\
32\end{array}$ & $\begin{array}{r}6 \\
35 \\
29 \\
16 \\
49 \\
76 \\
32\end{array}$ & $\begin{array}{r}4 \\
25 \\
14 \\
11 \\
39 \\
54 \\
21\end{array}$ & $\begin{array}{l}12 \\
48 \\
31 \\
22 \\
63 \\
92 \\
58\end{array}$ & $\begin{array}{l}12 \\
48 \\
31 \\
22 \\
63 \\
92 \\
57\end{array}$ & $\begin{array}{r}7 \\
26 \\
29 \\
9 \\
47 \\
71 \\
36\end{array}$ \\
\hline 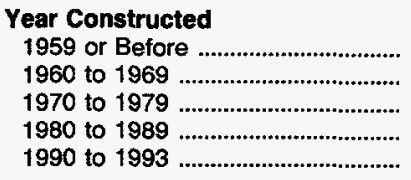 & $\begin{array}{r}142 \\
70 \\
47 \\
38 \\
15\end{array}$ & $\begin{array}{r}142 \\
70 \\
46 \\
38 \\
15\end{array}$ & $\begin{array}{r}63 \\
24 \\
26 \\
18 \\
8\end{array}$ & $\begin{array}{r}103 \\
38 \\
34 \\
57 \\
11\end{array}$ & $\begin{array}{r}103 \\
38 \\
34 \\
57 \\
11\end{array}$ & $\begin{array}{r}63 \\
30 \\
26 \\
41 \\
8\end{array}$ & $\begin{array}{r}130 \\
55 \\
62 \\
65 \\
14\end{array}$ & $\begin{array}{r}129 \\
55 \\
62 \\
65 \\
14\end{array}$ & $\begin{array}{l}78 \\
48 \\
45 \\
42 \\
12\end{array}$ \\
\hline 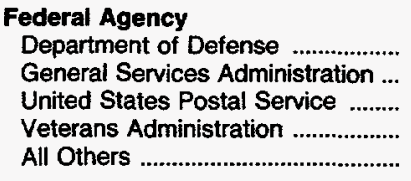 & $\begin{array}{l}22 \\
83 \\
57 \\
56 \\
94\end{array}$ & $\begin{array}{l}22 \\
83 \\
56 \\
56 \\
94\end{array}$ & $\begin{array}{r}9 \\
40 \\
35 \\
31 \\
24\end{array}$ & $\begin{array}{l}22 \\
35 \\
61 \\
36 \\
89\end{array}$ & $\begin{array}{l}22 \\
35 \\
61 \\
36 \\
89\end{array}$ & $\begin{array}{l}18 \\
29 \\
49 \\
24 \\
48\end{array}$ & $\begin{array}{l}78 \\
39 \\
63 \\
56 \\
90\end{array}$ & $\begin{array}{l}77 \\
39 \\
63 \\
56 \\
90\end{array}$ & $\begin{array}{l}37 \\
38 \\
53 \\
29 \\
68\end{array}$ \\
\hline $\begin{array}{l}\text { Energy End Uses (more than one } \\
\text { may apply) } \\
\text { Heating } \\
\text { Air Conditioning } \\
\text { Water Heating } \\
\text { Cooking } \\
\text { Manufacturing }\end{array}$ & $\begin{array}{r}307 \\
305 \\
303 \\
108 \\
50\end{array}$ & $\begin{array}{r}306 \\
305 \\
302 \\
108 \\
50\end{array}$ & $\begin{array}{r}139 \\
137 \\
137 \\
62 \\
28\end{array}$ & $\begin{array}{r}242 \\
241 \\
238 \\
63 \\
28\end{array}$ & $\begin{array}{r}242 \\
241 \\
238 \\
63 \\
28\end{array}$ & $\begin{array}{r}168 \\
168 \\
166 \\
48 \\
22\end{array}$ & $\begin{array}{r}304 \\
292 \\
315 \\
70 \\
30\end{array}$ & $\begin{array}{r}303 \\
292 \\
314 \\
70 \\
30\end{array}$ & $\begin{array}{r}223 \\
209 \\
223 \\
51 \\
21\end{array}$ \\
\hline $\begin{array}{l}\text { Workers (main shift) } \\
\text { Less than } 50 \\
50 \text { to } 99 \\
100 \text { to } 499 \\
500 \text { or More }\end{array}$ & $\begin{array}{r}71 \\
39 \\
107 \\
95\end{array}$ & $\begin{array}{r}71 \\
39 \\
107 \\
94\end{array}$ & $\begin{array}{l}23 \\
14 \\
46 \\
56\end{array}$ & $\begin{array}{l}70 \\
44 \\
80 \\
49\end{array}$ & $\begin{array}{l}70 \\
44 \\
80 \\
49\end{array}$ & $\begin{array}{l}46 \\
35 \\
56 \\
31\end{array}$ & $\begin{array}{r}93 \\
49 \\
134 \\
50\end{array}$ & $\begin{array}{r}92 \\
49 \\
134 \\
50\end{array}$ & $\begin{array}{l}55 \\
36 \\
94 \\
40\end{array}$ \\
\hline $\begin{array}{l}\text { Weekly Operating Hours } \\
48 \text { or Fewer } \\
49 \text { to } 60 \\
61 \text { to } 167 \\
\text { Open Continuously }\end{array}$ & $\begin{array}{r}51 \\
76 \\
60 \\
125\end{array}$ & $\begin{array}{r}51 \\
76 \\
60 \\
124\end{array}$ & $\begin{array}{l}13 \\
28 \\
28 \\
70\end{array}$ & $\begin{array}{l}39 \\
46 \\
78 \\
80\end{array}$ & $\begin{array}{l}39 \\
46 \\
78 \\
80\end{array}$ & $\begin{array}{l}30 \\
26 \\
54 \\
58\end{array}$ & $\begin{array}{r}80 \\
65 \\
68 \\
113\end{array}$ & $\begin{array}{r}80 \\
65 \\
68 \\
112\end{array}$ & $\begin{array}{l}43 \\
56 \\
57 \\
69\end{array}$ \\
\hline
\end{tabular}

Notes: - Total workers are the number of workers during the main shift. - See Glossary for explanation of abbreviations and definitions of terms used in this report. - These data are from 881 federally owned buildings having the following criteria: (1) located in Federal Regions 3,6 , or 9; (2) larger than 10,000 square feet; and (3) used for a commercial purpose, other than warehouse and storage. In addition, 9 out of 10 selected buildings were from agencies other than the Department of Defense. - Statistics for the "energy end uses" represent consumption in buildings that have end use, not consumption for a particular fuel for a particular end use. - FBSS = Federal Buildings Supplemental Survey.

- Data are for Fiscal Year 1993 (October 1, 1992 through September 30, 1993). - Because of rounding, data may not sum to totals.

Source: Energy Information Administration, Office of Energy Markets and End Use, 1993 Federal Buildings Supplemental Survey. 
Table 3.9. Energy Sources in FBSS Buildings in Federal Regions 3, 6, and 9, Floorspace, 1993

\begin{tabular}{|c|c|c|c|c|c|c|c|c|c|}
\hline \multirow[b]{3}{*}{$\begin{array}{c}\text { Bullding } \\
\text { Characteristics }\end{array}$} & \multicolumn{3}{|c|}{$\begin{array}{l}\text { Floorspace in Region } 3 \\
\text { (thousand square feet) }\end{array}$} & \multicolumn{3}{|c|}{$\begin{array}{l}\text { Floorspace in Region } 6 \\
\text { (thousand square feet) }\end{array}$} & \multicolumn{3}{|c|}{$\begin{array}{l}\text { Floorspace in Region } 9 \\
\text { (thousand square feet) }\end{array}$} \\
\hline & \multirow[b]{2}{*}{$\begin{array}{c}\text { All } \\
\text { Buildings }\end{array}$} & \multicolumn{2}{|c|}{$\begin{array}{l}\text { Energy Sources Used } \\
\text { in Region } 3 \text { (more } \\
\text { than one may apply) }\end{array}$} & \multirow[b]{2}{*}{$\begin{array}{l}\text { All } \\
\text { Buildings }\end{array}$} & \multicolumn{2}{|c|}{$\begin{array}{l}\text { Energy Sources Used } \\
\text { in Region } 6 \text { (more } \\
\text { than one may apply) }\end{array}$} & \multirow[b]{2}{*}{$\begin{array}{c}\text { All } \\
\text { Buildings }\end{array}$} & \multicolumn{2}{|c|}{$\begin{array}{l}\text { Energy Sources Used } \\
\text { in Region } 9 \text { (more } \\
\text { than one may apply) }\end{array}$} \\
\hline & & $\begin{array}{l}\text { Elec- } \\
\text { tricity }\end{array}$ & $\begin{array}{l}\text { Natural } \\
\text { Gas }\end{array}$ & & $\begin{array}{l}\text { Elec- } \\
\text { tricity }\end{array}$ & $\begin{array}{l}\text { Natural } \\
\text { Gas }\end{array}$ & & $\begin{array}{l}\text { Elec- } \\
\text { tricity }\end{array}$ & $\begin{array}{l}\text { Natural } \\
\text { Gas }\end{array}$ \\
\hline All Bulldings & 94,880 & 94,161 & 58,674 & 35,816 & 35,816 & 24,827 & 44,316 & 44,296 & 34,703 \\
\hline 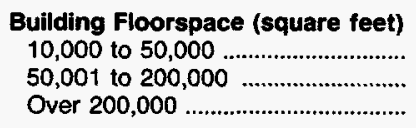 & $\begin{array}{r}2,564 \\
13,166 \\
79,149\end{array}$ & $\begin{array}{r}2,564 \\
13,166 \\
78,430\end{array}$ & $\begin{array}{r}1,024 \\
5,388 \\
52,262\end{array}$ & $\begin{array}{r}2,591 \\
9,548 \\
23,677\end{array}$ & $\begin{array}{r}2,591 \\
9,548 \\
23,677\end{array}$ & $\begin{array}{r}1,848 \\
6,079 \\
16,900\end{array}$ & $\begin{array}{r}3,858 \\
12,680 \\
27,778\end{array}$ & $\begin{array}{r}3,838 \\
12,680 \\
27,778\end{array}$ & $\begin{array}{r}2,304 \\
9,219 \\
23,180\end{array}$ \\
\hline 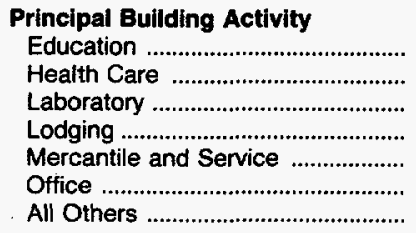 & $\begin{array}{r}598 \\
14,559 \\
5,165 \\
2,558 \\
7,966 \\
56,881 \\
7,152\end{array}$ & $\begin{array}{r}598 \\
14,559 \\
5,165 \\
2,558 \\
7,247 \\
56,881 \\
7,152\end{array}$ & $\begin{array}{r}212 \\
11,682 \\
2,305 \\
2,034 \\
6,152 \\
32,242 \\
4,047\end{array}$ & $\begin{array}{r}168 \\
12,094 \\
3,331 \\
942 \\
6,236 \\
10,799 \\
2,247\end{array}$ & $\begin{array}{r}168 \\
12,094 \\
3,331 \\
942 \\
6,236 \\
10,799 \\
2,247\end{array}$ & $\begin{array}{r}133 \\
9,552 \\
1,467 \\
665 \\
4,279 \\
7,594 \\
1,136\end{array}$ & $\begin{array}{r}628 \\
9,903 \\
2,601 \\
1,220 \\
8,194 \\
16,380 \\
5,390\end{array}$ & $\begin{array}{r}628 \\
9,903 \\
2,601 \\
1,220 \\
8,194 \\
16,380 \\
5,370\end{array}$ & $\begin{array}{r}272 \\
6,836 \\
2,547 \\
520 \\
6,630 \\
14,578 \\
3,320\end{array}$ \\
\hline 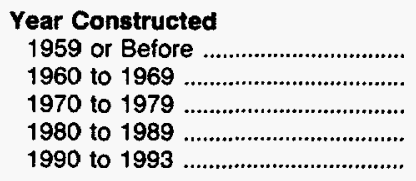 & $\begin{array}{r}43,829 \\
19,564 \\
17,737 \\
8,975 \\
4,774\end{array}$ & $\begin{array}{r}43,829 \\
19,564 \\
17,018 \\
8,975 \\
4,774\end{array}$ & $\begin{array}{r}24,528 \\
13,000 \\
12,961 \\
5,512 \\
2,673\end{array}$ & $\begin{array}{r}13,258 \\
6,386 \\
6,175 \\
6,903 \\
3,085\end{array}$ & $\begin{array}{r}13,258 \\
6,366 \\
6,175 \\
6,903 \\
3,095\end{array}$ & $\begin{array}{l}8,424 \\
4,889 \\
4,840 \\
3,688 \\
2,985\end{array}$ & $\begin{array}{r}13,553 \\
9,082 \\
12,041 \\
7,955 \\
1,684\end{array}$ & $\begin{array}{r}13,534 \\
9,082 \\
12,041 \\
7,955 \\
1,684\end{array}$ & $\begin{array}{l}9,153 \\
8,212 \\
9,847 \\
5,967 \\
1,524\end{array}$ \\
\hline $\begin{array}{l}\text { Federal Agency } \\
\text { Department of Defense ................. } \\
\text { General Services Administration ... } \\
\text { United States Postal Service ......... } \\
\text { Veterans Administration .................. } \\
\text { All Others .......................................... }\end{array}$ & $\begin{array}{r}13,988 \\
46,205 \\
9,392 \\
13,306 \\
11,989\end{array}$ & $\begin{array}{r}13,988 \\
46,205 \\
8,673 \\
13,306 \\
11,989\end{array}$ & $\begin{array}{r}12,480 \\
24,708 \\
7,197 \\
10,096 \\
4,192\end{array}$ & $\begin{array}{r}1,688 \\
7,888 \\
7,027 \\
11,375 \\
7,858\end{array}$ & $\begin{array}{r}1,668 \\
7,888 \\
7,027 \\
11,375 \\
7,858\end{array}$ & $\begin{array}{l}1,417 \\
6,293 \\
5,026 \\
8,716 \\
3,375\end{array}$ & $\begin{array}{r}8,489 \\
12,505 \\
8,387 \\
8,824 \\
6,111\end{array}$ & $\begin{array}{r}8,469 \\
12,505 \\
8,387 \\
8,824 \\
6,111\end{array}$ & $\begin{array}{r}4,763 \\
12,232 \\
7,070 \\
5,378 \\
5,260\end{array}$ \\
\hline \multicolumn{10}{|l|}{$\begin{array}{l}\text { Energy End Uses (more than one } \\
\text { may apply) }\end{array}$} \\
\hline 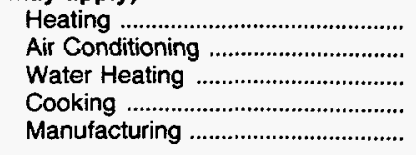 & $\begin{array}{l}94,063 \\
93,454 \\
93,138 \\
69,947 \\
27,041\end{array}$ & $\begin{array}{l}93,343 \\
93,454 \\
92,419 \\
69,947 \\
27,041\end{array}$ & $\begin{array}{l}\mathbf{5 8 , 6 7 4} \\
\mathbf{5 7 , 8 2 6} \\
\mathbf{5 8 , 5 6 0} \\
49,082 \\
21,235\end{array}$ & $\begin{array}{r}35,734 \\
35,273 \\
35,221 \\
19,213 \\
5,335\end{array}$ & $\begin{array}{r}35,734 \\
35,273 \\
35,221 \\
19,213 \\
5,335\end{array}$ & $\begin{array}{r}24,827 \\
24,827 \\
24,787 \\
15,802 \\
4,318\end{array}$ & $\begin{array}{r}41,921 \\
41,807 \\
42,984 \\
20,045 \\
7,693\end{array}$ & $\begin{array}{r}41,902 \\
41,907 \\
42,964 \\
20,045 \\
7,693\end{array}$ & $\begin{array}{r}34,045 \\
33,562 \\
34,379 \\
15,826 \\
5,438\end{array}$ \\
\hline \multicolumn{10}{|l|}{ Workers (main shift) } \\
\hline 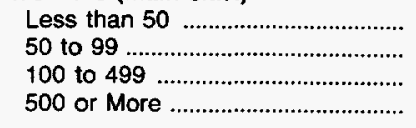 & $\begin{array}{r}4,102 \\
1,903 \\
13,632 \\
75,243\end{array}$ & $\begin{array}{r}4,102 \\
1,903 \\
13,632 \\
74,524\end{array}$ & $\begin{array}{r}872 \\
647 \\
5,674 \\
51,481\end{array}$ & $\begin{array}{r}2,548 \\
2,026 \\
9,029 \\
22,213\end{array}$ & $\begin{array}{r}2,546 \\
2,026 \\
9,029 \\
22,213\end{array}$ & $\begin{array}{r}1,557 \\
1,743 \\
5,755 \\
15,771\end{array}$ & $\begin{array}{r}3,684 \\
2,161 \\
14,017 \\
24,454\end{array}$ & $\begin{array}{r}3,665 \\
2,161 \\
14,017 \\
24,454\end{array}$ & $\begin{array}{r}2,025 \\
1,575 \\
10,930 \\
20,174\end{array}$ \\
\hline \multicolumn{10}{|l|}{ Weekly Operating Hours } \\
\hline 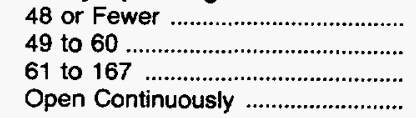 & $\begin{array}{r}9,694 \\
25,540 \\
11,993 \\
47,653\end{array}$ & $\begin{array}{r}9,694 \\
25,540 \\
11,993 \\
46,934\end{array}$ & $\begin{array}{r}2,996 \\
11,652 \\
7,186 \\
36,841\end{array}$ & $\begin{array}{r}2,740 \\
6,460 \\
8,051 \\
18,565\end{array}$ & $\begin{array}{r}2,740 \\
6,460 \\
8,051 \\
18,565\end{array}$ & $\begin{array}{r}1,540 \\
3,824 \\
4,718 \\
14,746\end{array}$ & $\begin{array}{r}4,822 \\
13,424 \\
5,706 \\
20,364\end{array}$ & $\begin{array}{r}4,822 \\
13,424 \\
5,706 \\
20,344\end{array}$ & $\begin{array}{r}3,003 \\
12,675 \\
5,329 \\
13,696\end{array}$ \\
\hline
\end{tabular}

Notes: - Total workers are the number of workers during the main shift. - See Glossary for explanation of abbreviations and definitions of terms used in this report. - These data are from 881 federally owned buildings having the following criteria: (1) located in Federal Regions 3,6 , or $9 ;$ (2) larger than 10,000 square feet; and (3) used for a commercial purpose, other than warehouse and storage. in addition, 9 out of 10 selected buildings were from agencies other than the Department of Defense. - Statistics for the "energy end uses" represent consumption in buildings that have end use, not consumption for a particular fuel for a particular end use. - FBSS = Federal Buildings Supplemental Survey.

- Data are for Fiscal Year 1993 (October 1, 1992 through September 30, 1993). - Because of rounding, data may not sum to totals.

Source: Energy Information Administration, Office of Energy Markets and End Use, 1993 Federal Buildings Supplemental Survey. 
Table 3.10. Heating Equipment in FBSS Buildings in Federal Region 3, Number of Buildings, 1993

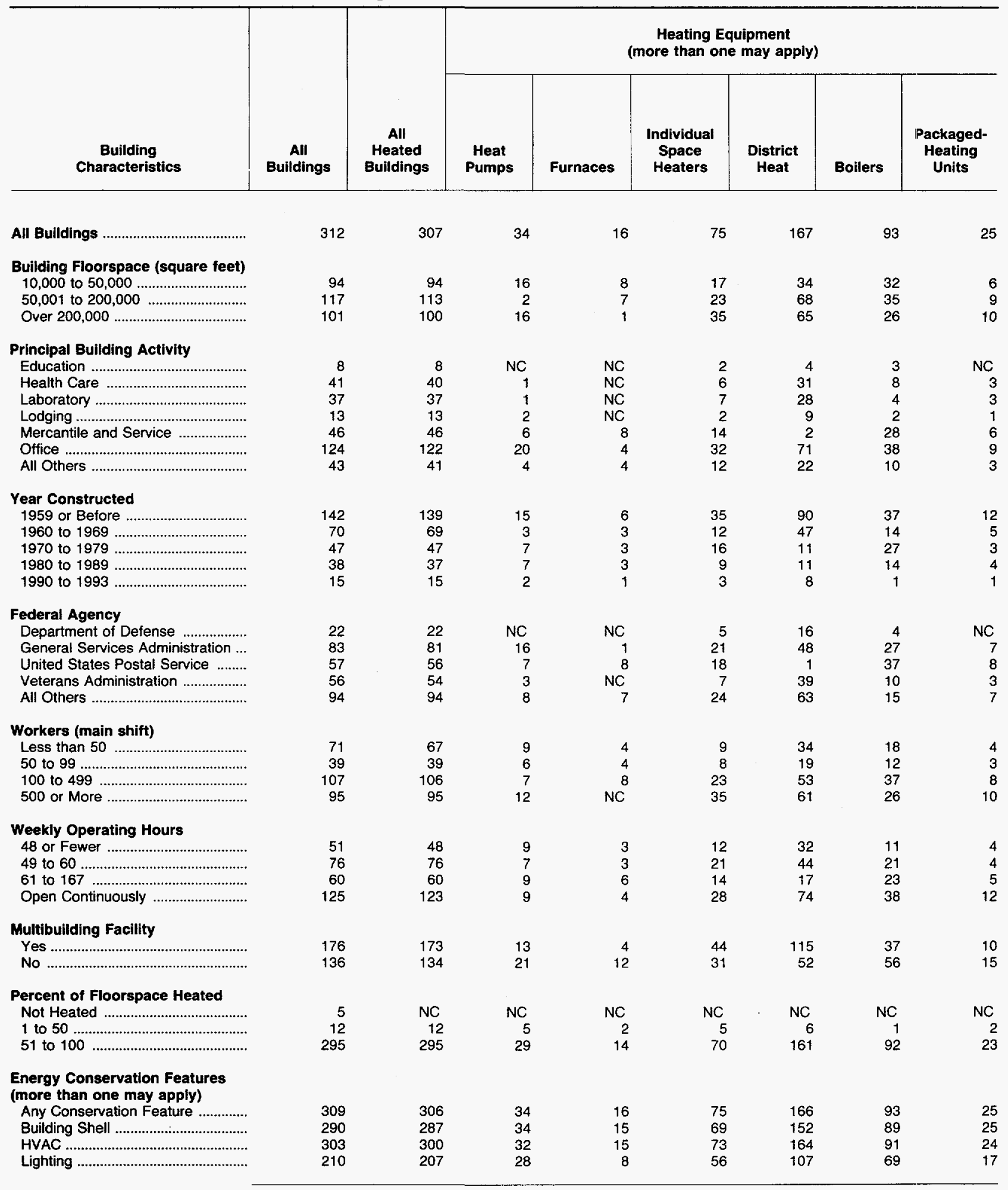

See footnotes at end of table. 
Table 3.10. Heating Equipment in FBSS Buildings in Federal Region 3, Number of Buildings, 1993 (Continued)

\begin{tabular}{|c|c|c|c|c|c|c|c|c|}
\hline \multirow[b]{2}{*}{$\begin{array}{l}\text { Building } \\
\text { Characteristics }\end{array}$} & \multirow[b]{2}{*}{$\begin{array}{c}\text { All } \\
\text { Buildings }\end{array}$} & \multirow[b]{2}{*}{$\begin{array}{c}\text { All } \\
\text { Heated } \\
\text { Buildings }\end{array}$} & \multicolumn{6}{|c|}{$\begin{array}{c}\text { Heating Equipment } \\
\text { (more than one may apply) }\end{array}$} \\
\hline & & & $\begin{array}{l}\text { Heat } \\
\text { Pumps }\end{array}$ & Furnaces & $\begin{array}{l}\text { Individual } \\
\text { Space } \\
\text { Heaters }\end{array}$ & $\begin{array}{l}\text { District } \\
\text { Heat }\end{array}$ & Boilers & $\begin{array}{l}\text { Packaged- } \\
\text { Heating } \\
\text { Units }\end{array}$ \\
\hline \multirow{2}{*}{\multicolumn{9}{|c|}{$\begin{array}{l}\text { Bullding Shell Conservation } \\
\text { Features (more than one may } \\
\text { apply) } \\
\text { Roof or Ceiling }\end{array}$}} \\
\hline & & & & & & & & \\
\hline 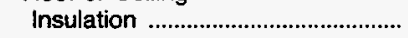 & 240 & 239 & 31 & 13 & 61 & 126 & 72 & 22 \\
\hline 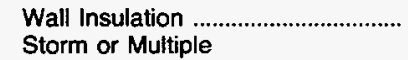 & 117 & 117 & 20 & 6 & 31 & 56 & 36 & 7 \\
\hline 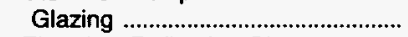 & 173 & 172 & 23 & 12 & 37 & 82 & 56 & 17 \\
\hline 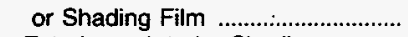 & 158 & 157 & 15 & 8 & 38 & 76 & 60 & 16 \\
\hline Exterior or Interior Shading & & & & & & & & \\
\hline 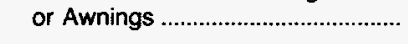 & 197 & 195 & 23 & 8 & 48 & 106 & 65 & 20 \\
\hline \multicolumn{9}{|l|}{$\begin{array}{l}\text { HVAC Conservation Features } \\
\text { (more than one may apply) }\end{array}$} \\
\hline 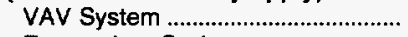 & 84 & 83 & 11 & 1 & 22 & 45 & 25 & 10 \\
\hline Economizer Cycle ................................ & 156 & 154 & 14 & 4 & 40 & 83 & 51 & 18 \\
\hline HVAC Maintenance .............................. & 301 & 299 & 32 & 15 & 73 & 164 & 90 & 24 \\
\hline \multicolumn{9}{|l|}{$\begin{array}{l}\text { Energy Management Practices } \\
\text { (more than one may apply) } \\
\text { Energy Management and Control }\end{array}$} \\
\hline $\begin{array}{l}\text { System ...................................................... } \\
\text { Energy Conservation }\end{array}$ & 132 & 132 & 19 & 1 & 39 & 80 & 40 & 11 \\
\hline Programs 1 & 99 & 98 & 13 & 1 & 29 & 58 & 28 & 7 \\
\hline 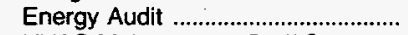 & 99 & 98 & 14 & 5 & 31 & 60 & 27 & 10 \\
\hline HVAC Maintenance Staff ${ }^{2}$.............. & 97 & 96 & 6 & 2 & 27 & 64 & 24 & 8 \\
\hline Heating & 144 & 144 & 21 & 9 & 41 & 73 & 47 & 12 \\
\hline Cooling & 145 & 144 & 20 & 7 & 39 & 73 & 47 & 12 \\
\hline Hot Water & 77 & 77 & 13 & 2 & 20 & 38 & 30 & 2 \\
\hline 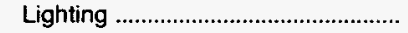 & 162 & 159 & 22 & 11 & 41 & 78 & 49 & 11 \\
\hline
\end{tabular}

1 Building participates in any programs sponsored by the Federal Energy Management Program, in-house, utility, or third party.

2 HVAC maintenance staff means at least one person spends at least half their working hours maintaining the heating/cooling equipment.

$\mathrm{NC}=$ No cases in responding sample.

Notes: - Total workers are the number of workers during the main shift. - See Glossary for explanation of abbreviations and definitions of terms used in this report. - These data are from 881 federally owned buildings having the following criteria: (1) located in Federal Regions 3 , 6 , or 9 ; (2) larger than 10,000 square feet; and (3) used for a commercial purpose, other than warehouse and storage. in addition, 9 out of 10 selected buildings were from agencies other than the Department of Defense. - Statistics for the "energy end uses" represent consumption in buildings that have end use, not consumption for a particular fuel for a particular end use. - FBSS = Federal Buildings Supplemental Survey. - HVAC = Heating, Ventilation, and Air Conditioning. - VAV = Variable-Air Volume. - Data are for Fiscal Year 1993 (October 1, 1992 through September 30, 1993). - Because of rounding, data may not sum to totals.

Source: Energy Information Administration, Office of Energy Markets and End Use, 1993 Federal Buildings Supplemental Survey. 
Table 3.11. Heating Equipment in FBSS Buildings in Federal Region 6, Number of Buildings, 1993

\begin{tabular}{|c|c|c|c|c|c|c|c|c|}
\hline \multirow[b]{2}{*}{$\begin{array}{c}\text { Building } \\
\text { Characteristics }\end{array}$} & \multirow[b]{2}{*}{$\begin{array}{c}\text { All } \\
\text { Buildings }\end{array}$} & \multirow[b]{2}{*}{$\begin{array}{c}\text { All } \\
\text { Heated } \\
\text { Buildings }\end{array}$} & \multicolumn{6}{|c|}{$\begin{array}{l}\text { Heating Equipment } \\
\text { (more than one may appiy) }\end{array}$} \\
\hline & & & $\begin{array}{l}\text { Heat } \\
\text { Pumps }\end{array}$ & Furnaces & $\begin{array}{l}\text { Individual } \\
\text { Space } \\
\text { Heaters }\end{array}$ & $\begin{array}{c}\text { District } \\
\text { Heat }\end{array}$ & Boilers & $\begin{array}{l}\text { Packaged- } \\
\text { Heating } \\
\text { Units }\end{array}$ \\
\hline All Bultdings & 243 & 242 & 10 & 18 & 30 & 82 & 102 & 59 \\
\hline 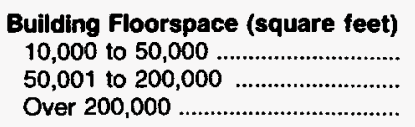 & $\begin{array}{r}107 \\
86 \\
50\end{array}$ & $\begin{array}{r}107 \\
85 \\
50\end{array}$ & $\begin{array}{l}6 \\
3 \\
1\end{array}$ & $\begin{array}{r}13 \\
5 \\
\mathrm{NC}\end{array}$ & $\begin{array}{r}9 \\
13 \\
8\end{array}$ & $\begin{array}{l}26 \\
36 \\
20\end{array}$ & $\begin{array}{l}36 \\
40 \\
26\end{array}$ & $\begin{array}{l}34 \\
15 \\
10\end{array}$ \\
\hline 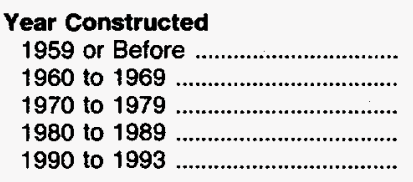 & $\begin{array}{r}103 \\
38 \\
34 \\
57 \\
11\end{array}$ & $\begin{array}{r}102 \\
38 \\
34 \\
57 \\
11\end{array}$ & $\begin{array}{l}3 \\
2 \\
1 \\
3 \\
1\end{array}$ & $\begin{array}{l}7 \\
3 \\
2 \\
4 \\
2\end{array}$ & $\begin{array}{r}11 \\
6 \\
5 \\
6 \\
2\end{array}$ & $\begin{array}{r}55 \\
9 \\
4 \\
12 \\
2\end{array}$ & $\begin{array}{r}38 \\
23 \\
23 \\
15 \\
3\end{array}$ & $\begin{array}{r}13 \\
10 \\
10 \\
24 \\
2\end{array}$ \\
\hline $\begin{array}{l}\text { Federal Agency } \\
\text { Department of Defense ................. } \\
\text { General Services Administration ... } \\
\text { United States Postal Service ........ } \\
\text { Veterans Administration ................... } \\
\text { All Others ......................................... }\end{array}$ & $\begin{array}{l}22 \\
35 \\
61 \\
36 \\
89\end{array}$ & $\begin{array}{l}22 \\
35 \\
61 \\
36 \\
88\end{array}$ & $\begin{array}{l}2 \\
1 \\
3 \\
1 \\
3\end{array}$ & $\begin{array}{r}2 \\
1 \\
7 \\
\text { NC } \\
8\end{array}$ & $\begin{array}{r}1 \\
5 \\
8 \\
5 \\
11\end{array}$ & $\begin{array}{r}4 \\
7 \\
\text { NC } \\
29 \\
42\end{array}$ & $\begin{array}{r}12 \\
26 \\
27 \\
5 \\
32\end{array}$ & $\begin{array}{r}7 \\
2 \\
29 \\
6 \\
15\end{array}$ \\
\hline 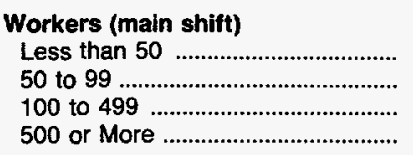 & $\begin{array}{l}70 \\
44 \\
80 \\
49\end{array}$ & $\begin{array}{l}69 \\
44 \\
80 \\
49\end{array}$ & $\begin{array}{l}2 \\
4 \\
3 \\
1\end{array}$ & $\begin{array}{r}8 \\
7 \\
3 \\
N C\end{array}$ & $\begin{array}{r}7 \\
3 \\
10 \\
10\end{array}$ & $\begin{array}{l}24 \\
11 \\
29 \\
18\end{array}$ & $\begin{array}{l}21 \\
18 \\
37 \\
26\end{array}$ & $\begin{array}{r}23 \\
7 \\
19 \\
10\end{array}$ \\
\hline 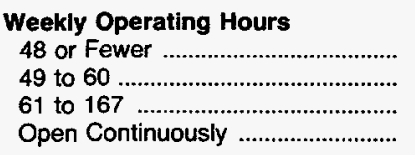 & $\begin{array}{l}39 \\
46 \\
78 \\
80\end{array}$ & $\begin{array}{l}38 \\
46 \\
78 \\
80\end{array}$ & $\begin{array}{l}2 \\
2 \\
3 \\
3\end{array}$ & $\begin{array}{l}4 \\
3 \\
7 \\
4\end{array}$ & $\begin{array}{r}5 \\
5 \\
9 \\
11\end{array}$ & $\begin{array}{l}13 \\
22 \\
18 \\
29\end{array}$ & $\begin{array}{l}16 \\
17 \\
32 \\
37\end{array}$ & $\begin{array}{r}6 \\
9 \\
25 \\
19\end{array}$ \\
\hline $\begin{array}{l}\text { Multibuilding Facility } \\
\text { Yes } \ldots \ldots \ldots \ldots \ldots \ldots\end{array}$ & $\begin{array}{r}148 \\
95\end{array}$ & $\begin{array}{r}147 \\
95\end{array}$ & $\begin{array}{l}5 \\
5\end{array}$ & $\begin{array}{r}5 \\
13\end{array}$ & $\begin{array}{l}17 \\
13\end{array}$ & $\begin{array}{r}80 \\
2\end{array}$ & $\begin{array}{l}51 \\
51\end{array}$ & $\begin{array}{l}20 \\
39\end{array}$ \\
\hline $\begin{array}{l}\text { Percent of Floorspace Heated } \\
\text { Not Heated } \\
1 \text { to } 50 \\
51 \text { to } 100 \ldots \ldots \ldots\end{array}$ & $\begin{array}{r}1 \\
11 \\
231\end{array}$ & $\begin{array}{l}\text { NC } \\
11 \\
231\end{array}$ & $\begin{array}{c}\text { NC } \\
\text { NC } \\
10\end{array}$ & $\begin{array}{r}\mathrm{NC} \\
2 \\
16\end{array}$ & $\begin{array}{r}\text { NC } \\
1 \\
29\end{array}$ & $\begin{array}{r}\text { NC } \\
2 \\
80\end{array}$ & $\begin{array}{r}\text { NC } \\
4 \\
98\end{array}$ & $\begin{array}{r}\text { NC } \\
2 \\
57\end{array}$ \\
\hline 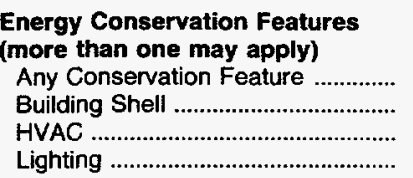 & $\begin{array}{l}242 \\
228 \\
236 \\
136\end{array}$ & $\begin{array}{l}242 \\
228 \\
236 \\
136\end{array}$ & $\begin{array}{r}10 \\
10 \\
9 \\
7\end{array}$ & $\begin{array}{l}18 \\
18 \\
16 \\
11\end{array}$ & $\begin{array}{l}30 \\
30 \\
28 \\
20\end{array}$ & $\begin{array}{l}82 \\
73 \\
82 \\
42\end{array}$ & $\begin{array}{r}102 \\
99 \\
101 \\
57\end{array}$ & $\begin{array}{l}59 \\
57 \\
58 \\
39\end{array}$ \\
\hline
\end{tabular}

See footnotes at end of table. 
Table 3.11. Heating Equipment in FBSS Buildings in Federal Region 6, Number of Buildings, 1993 (Continued)

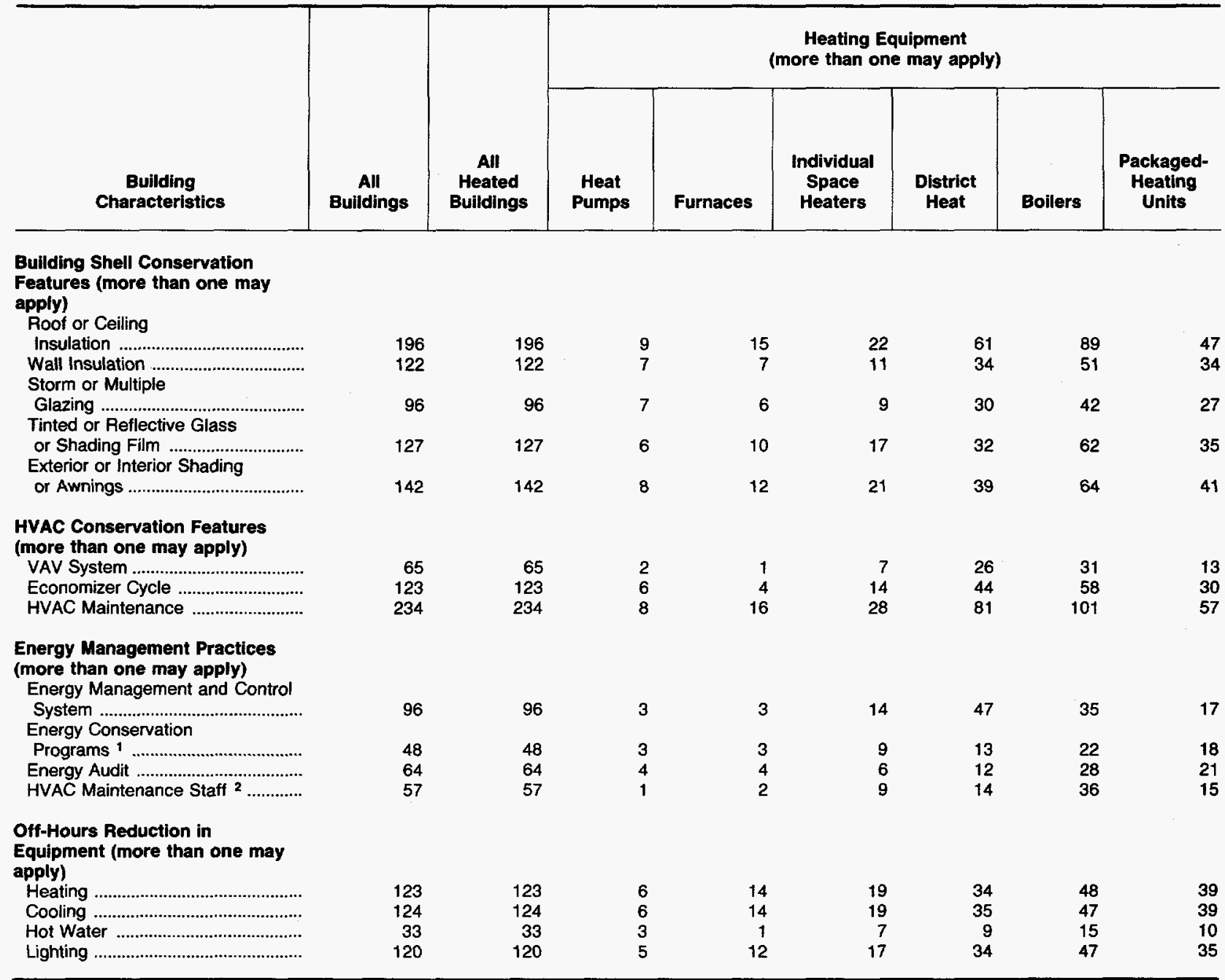

1 Building participates in any programs sponsored by the Federal Energy Management Program, in-house, utility, or third party.

2 HVAC maintenance staff means at least one person spends at least half their working hours maintaining the heating/cooling equipment.

NC $=$ No cases in responding sample.

Notes: - Total workers are the number of workers during the main shift. - See Glossary for explanation of abbreviations and definitions of terms used in this report. - These data are from 881 federally owned buildings having the following criteria: (1) located in Federal Regions 3, 6, or 9; (2) larger than 10,000 square feet; and (3) used for a commercial purpose, other than warehouse and storage. In addition, 9 out of 10 selected buildings were from agencies other than the Department of Defense. - Statistics for the "energy end uses" represent consumption in buildings that have end use, not consumption for a particular fuel for a particular end use. - FBSS = Federal Buildings Supplemental Survey. $\bullet$ HVAC = Heating, Ventilation, and Air Conditioning. - VAV = Variable-air Volume. - Data are for Fiscal Year 1993 (October 1, 1992 through September 30, 1993). Because of rounding, data may not sum to totals.

Source: Energy Information Administration, Office of Energy Markets and End Use, 1993 Federal Buildings Supplemental Survey. 
Table 3.12. Heating Equipment in FBSS Buildings in Federal Region 9, Number of Buildings, 1993

\begin{tabular}{|c|c|c|c|c|c|c|c|c|}
\hline \multirow[b]{2}{*}{$\begin{array}{c}\text { Building } \\
\text { Characteristics }\end{array}$} & \multirow[b]{2}{*}{$\begin{array}{l}\text { All } \\
\text { Buildings }\end{array}$} & \multirow[b]{2}{*}{$\begin{array}{c}\text { All } \\
\text { Heated } \\
\text { Buildings }\end{array}$} & \multicolumn{6}{|c|}{$\begin{array}{l}\text { Heating Equipment } \\
\text { (more than one may apply) }\end{array}$} \\
\hline & & & $\begin{array}{l}\text { Heat } \\
\text { Pumps }\end{array}$ & Furnaces & $\begin{array}{l}\text { Individual } \\
\text { Space } \\
\text { Heaters }\end{array}$ & $\begin{array}{c}\text { District } \\
\text { Heat }\end{array}$ & Boilers & $\begin{array}{l}\text { Packaged- } \\
\text { Heating } \\
\text { Units }\end{array}$ \\
\hline All Buildings & 326 & 304 & 40 & 25 & 33 & 71 & 146 & 67 \\
\hline 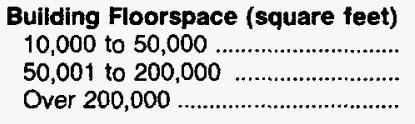 & $\begin{array}{r}148 \\
123 \\
55\end{array}$ & $\begin{array}{r}137 \\
116 \\
51\end{array}$ & $\begin{array}{r}23 \\
14 \\
3\end{array}$ & $\begin{array}{r}21 \\
3 \\
1\end{array}$ & $\begin{array}{r}18 \\
9 \\
6\end{array}$ & $\begin{array}{l}26 \\
30 \\
15\end{array}$ & $\begin{array}{l}49 \\
67 \\
30\end{array}$ & $\begin{array}{r}38 \\
23 \\
6\end{array}$ \\
\hline 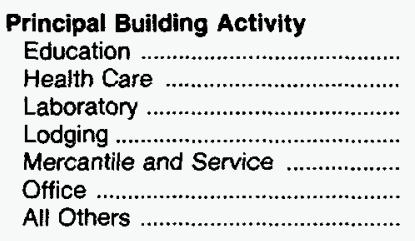 & $\begin{array}{l}12 \\
48 \\
31 \\
22 \\
63 \\
92 \\
58\end{array}$ & $\begin{array}{l}12 \\
47 \\
31 \\
19 \\
55 \\
87 \\
53\end{array}$ & $\begin{array}{r}2 \\
1 \\
8 \\
2 \\
10 \\
12 \\
12\end{array}$ & $\begin{array}{r}\text { NC } \\
\text { NC } \\
1 \\
1 \\
11 \\
8 \\
4\end{array}$ & $\begin{array}{r}\mathrm{NC} \\
1 \\
1 \\
1 \\
12 \\
12 \\
8 \\
10\end{array}$ & $\begin{array}{r}3 \\
37 \\
3 \\
9 \\
2 \\
6 \\
11\end{array}$ & $\begin{array}{r}4 \\
9 \\
9 \\
23 \\
6 \\
24 \\
57 \\
23\end{array}$ & $\begin{array}{r}3 \\
7 \\
5 \\
1 \\
18 \\
20 \\
13\end{array}$ \\
\hline $\begin{array}{l}\text { Year Constructed } \\
1959 \text { or Before } \\
1960 \text { to } 1969 . \ldots \ldots \ldots \\
1970 \text { to } 1979 \\
1980 \text { to } 1989 \\
1990 \text { to } 1993\end{array}$ & $\begin{array}{r}130 \\
55 \\
62 \\
65 \\
14\end{array}$ & $\begin{array}{r}118 \\
55 \\
59 \\
59 \\
13\end{array}$ & $\begin{array}{r}15 \\
7 \\
6 \\
9 \\
3\end{array}$ & $\begin{array}{r}10 \\
3 \\
4 \\
5 \\
3\end{array}$ & $\begin{array}{r}16 \\
6 \\
4 \\
6 \\
1\end{array}$ & $\begin{array}{r}33 \\
10 \\
17 \\
11 \\
\text { NC }\end{array}$ & $\begin{array}{r}54 \\
40 \\
25 \\
21 \\
6\end{array}$ & $\begin{array}{r}24 \\
9 \\
15 \\
14 \\
5\end{array}$ \\
\hline $\begin{array}{l}\text { Federal Agency } \\
\text { Department of Defense ................ } \\
\text { General Services Administration ... } \\
\text { United States Postal Service ........ } \\
\text { Veterans Administration .................. } \\
\text { All Others }\end{array}$ & $\begin{array}{l}78 \\
39 \\
63 \\
56 \\
90\end{array}$ & $\begin{array}{l}66 \\
39 \\
56 \\
55 \\
88\end{array}$ & $\begin{array}{r}\mathrm{NC}^{7} \\
9 \\
2 \\
22\end{array}$ & $\begin{array}{r}7 \\
3 \\
10 \\
\mathrm{NC} \\
5\end{array}$ & $\begin{array}{r}6 \\
3 \\
9 \\
1 \\
14\end{array}$ & $\begin{array}{r}20 \\
4 \\
\mathrm{NC} \\
43 \\
4\end{array}$ & $\begin{array}{r}22 \\
32 \\
26 \\
8 \\
58\end{array}$ & $\begin{array}{r}11 \\
2 \\
21 \\
7 \\
26\end{array}$ \\
\hline $\begin{array}{l}\text { Workers (main shift) } \\
\text { Less than } 50 \\
50 \text { to } 99 \\
100 \text { to } 499 \\
500 \text { or More }\end{array}$ & $\begin{array}{r}93 \\
49 \\
134 \\
50\end{array}$ & $\begin{array}{r}84 \\
47 \\
127 \\
46\end{array}$ & $\begin{array}{r}12 \\
6 \\
19 \\
3\end{array}$ & $\begin{array}{r}11 \\
5 \\
8 \\
1\end{array}$ & $\begin{array}{r}11 \\
4 \\
12 \\
6\end{array}$ & $\begin{array}{r}16 \\
4 \\
39 \\
12\end{array}$ & $\begin{array}{l}28 \\
25 \\
63 \\
30\end{array}$ & $\begin{array}{r}15 \\
13 \\
33 \\
6\end{array}$ \\
\hline $\begin{array}{l}\text { Weekly Operating Hours } \\
48 \text { or Fewer } \\
49 \text { to } 60 \\
61 \text { to } 167 \\
\text { Open Continuously }\end{array}$ & $\begin{array}{r}80 \\
65 \\
68 \\
113\end{array}$ & $\begin{array}{r}74 \\
63 \\
63 \\
104\end{array}$ & $\begin{array}{r}7 \\
7 \\
8 \\
18\end{array}$ & $\begin{array}{l}5 \\
7 \\
9 \\
4\end{array}$ & $\begin{array}{l}9 \\
6 \\
9 \\
9\end{array}$ & $\begin{array}{r}17 \\
13 \\
3 \\
38\end{array}$ & $\begin{array}{l}36 \\
37 \\
28 \\
45\end{array}$ & $\begin{array}{r}27 \\
5 \\
20 \\
15\end{array}$ \\
\hline $\begin{array}{l}\text { Multibuilding Facility } \\
\text { Yes }\end{array}$ & $\begin{array}{l}200 \\
126\end{array}$ & $\begin{array}{l}186 \\
118\end{array}$ & $\begin{array}{l}28 \\
12\end{array}$ & $\begin{array}{l}12 \\
13\end{array}$ & $\begin{array}{l}17 \\
16\end{array}$ & $\begin{array}{r}63 \\
8\end{array}$ & $\begin{array}{l}77 \\
69\end{array}$ & $\begin{array}{l}29 \\
38\end{array}$ \\
\hline $\begin{array}{l}\text { Percent of Floorspace Heated } \\
\text { Not Heated } \\
1 \text { to } 50 \\
51 \text { to } 100\end{array}$ & $\begin{array}{r}22 \\
28 \\
276\end{array}$ & $\begin{array}{l}\text { NC } \\
28 \\
276\end{array}$ & $\begin{array}{r}\text { NC } \\
3 \\
37\end{array}$ & $\begin{array}{r}\text { NC } \\
2 \\
23\end{array}$ & $\begin{array}{r}\mathrm{NC} \\
4 \\
29\end{array}$ & $\begin{array}{r}\text { NC } \\
3 \\
68\end{array}$ & $\begin{array}{r}\text { NC } \\
8 \\
138\end{array}$ & $\begin{array}{r}\text { NC } \\
6 \\
61\end{array}$ \\
\hline 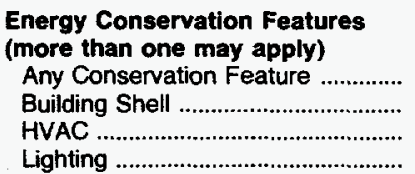 & $\begin{array}{l}319 \\
248 \\
307 \\
217\end{array}$ & $\begin{array}{l}299 \\
234 \\
290 \\
207\end{array}$ & $\begin{array}{l}40 \\
31 \\
39 \\
29\end{array}$ & $\begin{array}{l}25 \\
20 \\
22 \\
15\end{array}$ & $\begin{array}{l}33 \\
31 \\
32 \\
23\end{array}$ & $\begin{array}{l}69 \\
59 \\
69 \\
41\end{array}$ & $\begin{array}{l}145 \\
107 \\
143 \\
113\end{array}$ & $\begin{array}{l}67 \\
65 \\
65 \\
52\end{array}$ \\
\hline
\end{tabular}

See footnotes at end of table. 
Table 3.12. Heating Equipment in FBSS Buildings in Federal Region 9, Number of Buildings, 1993 (Continued)

\begin{tabular}{|c|c|c|c|c|c|c|c|c|}
\hline \multirow[b]{2}{*}{$\begin{array}{c}\text { Bullding } \\
\text { Characteristics }\end{array}$} & \multirow[b]{2}{*}{$\begin{array}{c}\text { All } \\
\text { Bulldings }\end{array}$} & \multirow[b]{2}{*}{$\begin{array}{c}\text { All } \\
\text { Heated } \\
\text { Bulldings }\end{array}$} & \multicolumn{6}{|c|}{$\begin{array}{l}\text { Heating Equipment } \\
\text { (more than one may apply) }\end{array}$} \\
\hline & & & $\begin{array}{l}\text { Heat } \\
\text { Pumps }\end{array}$ & Fumaces & $\begin{array}{l}\text { Individual } \\
\text { Space } \\
\text { Heaters }\end{array}$ & $\begin{array}{l}\text { District } \\
\text { Heat }\end{array}$ & Boilers & $\begin{array}{l}\text { Packaged- } \\
\text { Heating } \\
\text { Units }\end{array}$ \\
\hline \multicolumn{9}{|l|}{$\begin{array}{l}\text { Bullding Shell Conservation } \\
\text { Features (more than one may } \\
\text { apply) } \\
\text { Roof or Ceiling }\end{array}$} \\
\hline $\begin{array}{l}\text { Insulation } \\
\text { Wall Insulation } \\
\text { Storm or Multiple................................. }\end{array}$ & $\begin{array}{l}185 \\
113\end{array}$ & $\begin{array}{l}175 \\
111\end{array}$ & $\begin{array}{l}27 \\
13\end{array}$ & $\begin{array}{l}12 \\
13\end{array}$ & $\begin{array}{l}23 \\
12\end{array}$ & $\begin{array}{l}48 \\
32\end{array}$ & $\begin{array}{l}80 \\
41\end{array}$ & $\begin{array}{l}51 \\
27\end{array}$ \\
\hline $\begin{array}{l}\text { Glazing } \\
\text { Tinted or Reflective Glass }\end{array}$ & 57 & 52 & 11 & 5 & 10 & 14 & 19 & 17 \\
\hline $\begin{array}{l}\text { or Shading Film ......................... } \\
\text { Exterior or Interior Shading }\end{array}$ & 133 & 125 & 21 & 10 & 14 & 22 & 62 & 37 \\
\hline 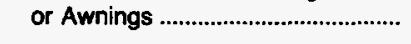 & 164 & 156 & 22 & 14 & 22 & 35 & 79 & 48 \\
\hline \multicolumn{9}{|l|}{$\begin{array}{l}\text { HVAC Conservation Features } \\
\text { (more than one may apply) }\end{array}$} \\
\hline 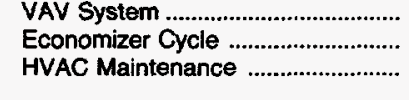 & $\begin{array}{r}75 \\
140 \\
306\end{array}$ & $\begin{array}{r}74 \\
138 \\
289\end{array}$ & $\begin{array}{r}9 \\
15 \\
39\end{array}$ & $\begin{array}{r}1 \\
7 \\
22\end{array}$ & $\begin{array}{l}11 \\
15 \\
32\end{array}$ & $\begin{array}{l}15 \\
28 \\
69\end{array}$ & $\begin{array}{r}48 \\
80 \\
143\end{array}$ & $\begin{array}{l}23 \\
40 \\
64\end{array}$ \\
\hline \multicolumn{9}{|l|}{$\begin{array}{l}\text { Energy Management Practices } \\
\text { (more than one may apply) } \\
\text { Energy Management and Control }\end{array}$} \\
\hline System & 71 & 67 & 12 & 2 & 8 & 15 & 40 & 13 \\
\hline 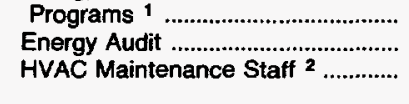 & $\begin{array}{r}124 \\
99 \\
52\end{array}$ & $\begin{array}{r}122 \\
92 \\
48\end{array}$ & $\begin{array}{r}23 \\
15 \\
3\end{array}$ & $\begin{array}{r}9 \\
10 \\
2\end{array}$ & $\begin{array}{r}17 \\
13 \\
3\end{array}$ & $\begin{array}{l}24 \\
20 \\
14\end{array}$ & $\begin{array}{l}67 \\
47 \\
25\end{array}$ & $\begin{array}{r}34 \\
13 \\
8\end{array}$ \\
\hline \multicolumn{9}{|l|}{$\begin{array}{l}\text { Off-Hours Reduction in } \\
\text { Equipment (more than one may } \\
\text { apply) }\end{array}$} \\
\hline $\begin{array}{l}\text { Heating } \\
\text { Cooling } \\
\text { Hot Water } \\
\text { Lighting }\end{array}$ & $\begin{array}{r}151 \\
157 \\
68 \\
172\end{array}$ & $\begin{array}{r}151 \\
147 \\
66 \\
161\end{array}$ & $\begin{array}{r}19 \\
18 \\
2 \\
19\end{array}$ & $\begin{array}{r}15 \\
11 \\
7 \\
18\end{array}$ & $\begin{array}{r}22 \\
17 \\
5 \\
24\end{array}$ & $\begin{array}{r}16 \\
20 \\
5 \\
23\end{array}$ & $\begin{array}{l}85 \\
84 \\
48 \\
85\end{array}$ & $\begin{array}{l}47 \\
45 \\
21 \\
45\end{array}$ \\
\hline
\end{tabular}

1 Building participates in any programs sponsored by the Federal Energy Management Program, in-house, utility, or third party.

2 HVAC maintenance staff means at least one person spends at least half their working hours maintaining the heating/cooling equipment.

NC $=$ No cases in responding sample.

Notes: - Total workers are the number of workers during the main shift. - See Glossary for explanation of abbreviations and definitions of terms used in this report. - These data are from 881 federally owned buildings having the following criteria: (1) located in Federal Regions 3,6 , or $9 ;$ (2) larger than 10,000 square feet; and (3) used for a commercial purpose, other than warehouse and storage. In addition, 9 out of 10 selected buildings were from agencies other than the Department of Defense. - Statistics for the "energy end uses" represent consumption in buildings that have end use, not consumption for a particular fuel for a particular end use. - FBSS = Federal Buildings Supplemental Survey. - HVAC = Heating, Ventilation, and Air Conditioning. - VAV = Variable-Air Volume. - Data are for Fiscal Year 1993 (October 1,1992 through September 30, 1993). Because of rounding, data may not sum to totals.

Source: Energy Information Administration, Office of Energy Markets and End Use, 1993 Federal Buildings Supplemental Survey. 
Table 3.13. Heating Equipment in FBSS Buildings in Federal Region 3, Floorspace, 1993

(Thousand Square Feet)

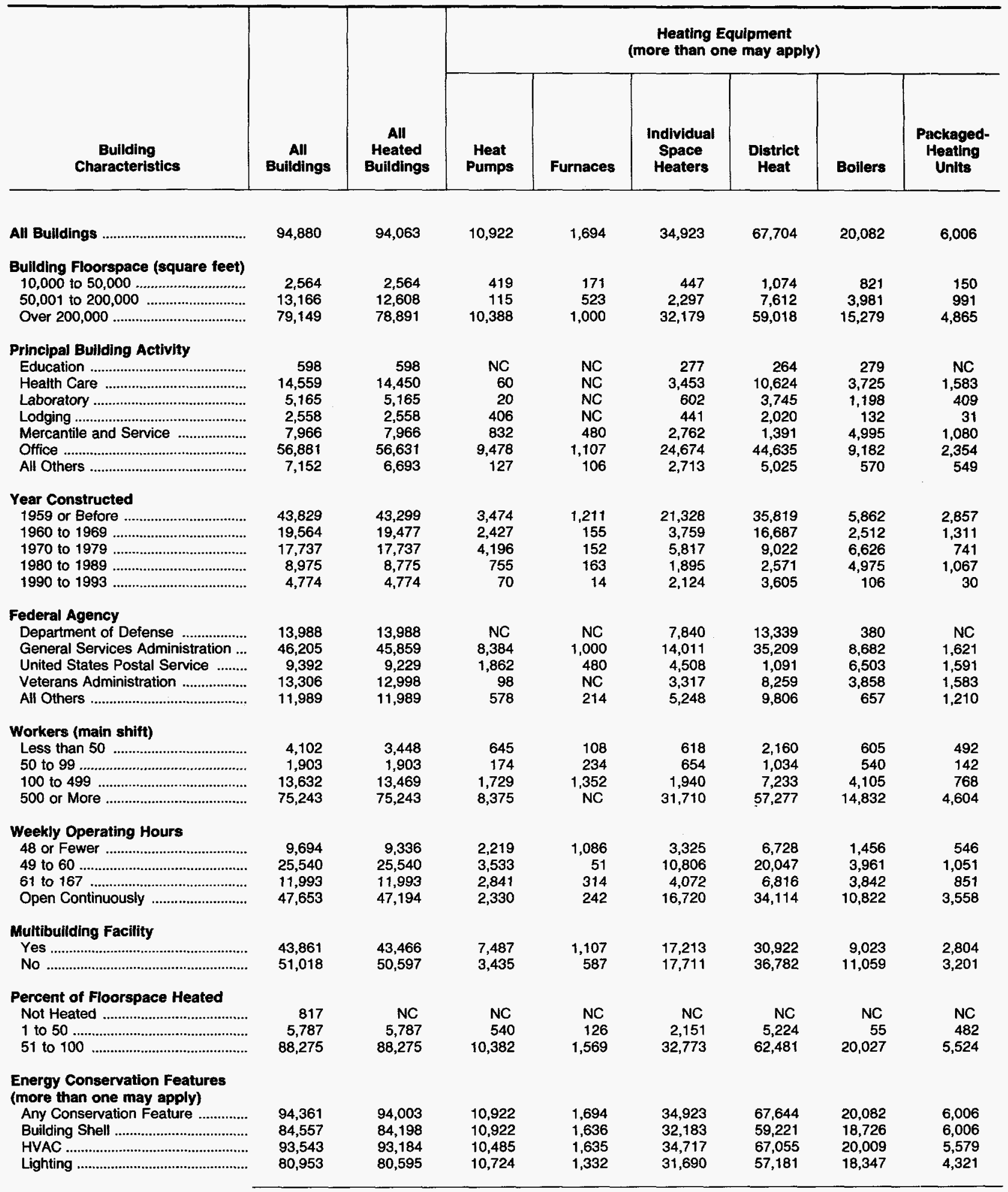

See footnotes at end of table. 
Table 3.13. Heating Equipment in FBSS Buildings in Federal Region 3, Floorspace, 1993 (Continued)

(Thousand Square Feet)

\begin{tabular}{|c|c|c|c|c|c|c|c|c|}
\hline \multirow[b]{2}{*}{$\begin{array}{c}\text { Building } \\
\text { Characteristics }\end{array}$} & \multirow[b]{2}{*}{$\begin{array}{c}\text { All } \\
\text { Bulldings }\end{array}$} & \multirow[b]{2}{*}{$\begin{array}{c}\text { All } \\
\text { Heated } \\
\text { Buildings }\end{array}$} & \multicolumn{6}{|c|}{$\begin{array}{l}\text { Heating Equipment } \\
\text { (more than one may apply) }\end{array}$} \\
\hline & & & $\begin{array}{l}\text { Heat } \\
\text { Pumps }\end{array}$ & Furnaces & $\begin{array}{l}\text { Individual } \\
\text { Space } \\
\text { Heaters }\end{array}$ & $\begin{array}{l}\text { District } \\
\text { Heat }\end{array}$ & Boilers & $\begin{array}{l}\text { Packaged- } \\
\text { Heating } \\
\text { Units }\end{array}$ \\
\hline \multicolumn{9}{|l|}{$\begin{array}{l}\text { Building Shell Conservation } \\
\text { Features (more than one may } \\
\text { apply) } \\
\text { Roof or Ceiling }\end{array}$} \\
\hline $\begin{array}{l}\text { Insulation } \\
\text { Wall Insulation } \\
\text { Storm or Multiple }\end{array}$ & $\begin{array}{l}69,654 \\
31,876\end{array}$ & $\begin{array}{l}69,567 \\
\mathbf{3 1 , 8 7 6}\end{array}$ & $\begin{array}{l}9,240 \\
6,084\end{array}$ & $\begin{array}{l}512 \\
174\end{array}$ & $\begin{array}{l}28,784 \\
11,695\end{array}$ & $\begin{array}{l}51,320 \\
20,857\end{array}$ & $\begin{array}{r}13,283 \\
8,687\end{array}$ & $\begin{array}{l}5,321 \\
1,433\end{array}$ \\
\hline Glazing & 46,141 & 46,032 & 5,371 & 511 & 18,186 & 30,197 & 11,784 & 3,114 \\
\hline $\begin{array}{l}\text { or Shading Film .......................... } \\
\text { Exterior or Interior Shading }\end{array}$ & 46,829 & 46,666 & 6,339 & 276 & 15,553 & 29,742 & 15,263 & 3,286 \\
\hline 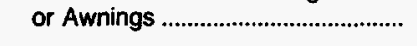 & 57,183 & 56,933 & 8,397 & 1,378 & 21,325 & 39,971 & 12,991 & 4,317 \\
\hline \multicolumn{9}{|l|}{$\begin{array}{l}\text { HVAC Conservation Features } \\
\text { (more than one may apply) }\end{array}$} \\
\hline 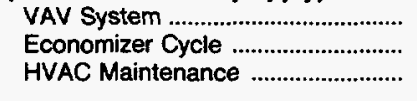 & $\begin{array}{l}37,611 \\
61,089 \\
93,402\end{array}$ & $\begin{array}{l}37,502 \\
60,894 \\
93,130\end{array}$ & $\begin{array}{r}4,125 \\
4,105 \\
10,485\end{array}$ & $\begin{array}{r}21 \\
176 \\
1,635\end{array}$ & $\begin{array}{l}13,828 \\
27,241 \\
34,717\end{array}$ & $\begin{array}{l}23,372 \\
44,513 \\
67,055\end{array}$ & $\begin{array}{l}11,640 \\
13,844 \\
19,955\end{array}$ & $\begin{array}{l}3,308 \\
4,651 \\
5,579\end{array}$ \\
\hline \multicolumn{9}{|l|}{$\begin{array}{l}\text { Energy Management Practices } \\
\text { (more than one may apply) } \\
\text { Energy Management and Control }\end{array}$} \\
\hline $\begin{array}{l}\text { System .................................................... } \\
\text { Energy Conservation }\end{array}$ & 61,891 & 61,891 & 8,197 & 107 & 26,031 & 45,017 & 14,594 & 3,994 \\
\hline 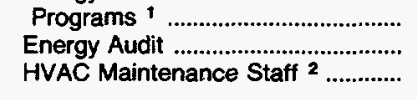 & $\begin{array}{l}59,284 \\
37,621 \\
52,924\end{array}$ & $\begin{array}{l}59,084 \\
37,421 \\
52,665\end{array}$ & $\begin{array}{l}6,244 \\
7,066 \\
4,762\end{array}$ & $\begin{array}{r}24 \\
1,233 \\
69\end{array}$ & $\begin{array}{l}25,674 \\
20,648 \\
22,670\end{array}$ & $\begin{array}{l}46,194 \\
27,770 \\
40,970\end{array}$ & $\begin{array}{l}8,858 \\
7,222 \\
9,644\end{array}$ & $\begin{array}{l}2,747 \\
1,745 \\
3,129\end{array}$ \\
\hline \multicolumn{9}{|l|}{$\begin{array}{l}\text { Off-Hours Reduction in } \\
\text { Equipment (more than one may } \\
\text { apply) }\end{array}$} \\
\hline 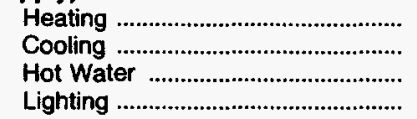 & $\begin{array}{l}43,828 \\
43,757 \\
26,018 \\
44,717\end{array}$ & $\begin{array}{l}43,828 \\
43,594 \\
26,018 \\
44,358\end{array}$ & $\begin{array}{l}8,477 \\
8,247 \\
6,649 \\
8,294\end{array}$ & $\begin{array}{l}1,292 \\
1,247 \\
1,050 \\
1,394\end{array}$ & $\begin{array}{r}17,564 \\
17,266 \\
9,995 \\
17,141\end{array}$ & $\begin{array}{l}31,731 \\
31,731 \\
17,684 \\
31,814\end{array}$ & $\begin{array}{l}8,745 \\
8,531 \\
5,680 \\
8,813\end{array}$ & $\begin{array}{r}2,396 \\
2,396 \\
716 \\
2,255\end{array}$ \\
\hline
\end{tabular}

1 Building participates in any programs sponsored by the Federal Energy Management Program, in-house, utility, or third party.

2 HVAC maintenance staff means at least one person spends at least half their working hours maintaining the heating/cooling equipment.

NC $=$ No cases in responding sample.

Notes: - Total workers are the number of workers during the main shift. - See Glossary for explanation of abbreviations and definitions of terms used in this report. - These data are from 881 federally owned buildings having the following criteria: (1) located in Federal Regions 3 , 6 , or 9; (2) larger than 10,000 square feet; and (3) used for a commercial purpose, other than warehouse and storage. In addition, 9 out of 10 selected buildings were from agencies other than the Department of Defense. - Statistics for the "energy end uses" represent consumption in buildings that have end use, not consumption for a particular fuel for a particular end use. - FBSS = Federal Buildings Supplemental Survey. - HVAC = Heating, Ventilation, and Air Conditioning. - VAV = Variable-Air Volume. - Data are for Fiscal Year 1993 (October 1, 1992 through September 30, 1993). Because of rounding, data may not sum to totals.

Source: Energy Information Administration, Office of Energy Markets and End Use, 1993 Federal Buildings Supplemental Survey. 
Table 3.14. Heating Equipment in FBSS Buildings in Federal Region 6, Floorspace, 1993

(Thousand Square Feet)

\begin{tabular}{|c|c|c|c|c|c|c|c|c|}
\hline \multirow[b]{2}{*}{$\begin{array}{c}\text { Building } \\
\text { Characteristics }\end{array}$} & \multirow[b]{2}{*}{$\begin{array}{c}\text { All } \\
\text { Buildings }\end{array}$} & \multirow[b]{2}{*}{$\begin{array}{l}\text { All } \\
\text { Heated } \\
\text { Buildings }\end{array}$} & \multicolumn{6}{|c|}{$\begin{array}{l}\text { Heating Equipment } \\
\text { (more than one may apply) }\end{array}$} \\
\hline & & & $\begin{array}{l}\text { Heat } \\
\text { Pumps }\end{array}$ & Furnaces & $\begin{array}{l}\text { Individual } \\
\text { Space } \\
\text { Heaters }\end{array}$ & $\begin{array}{c}\text { District } \\
\text { Heat }\end{array}$ & Boilers & $\begin{array}{l}\text { Packaged- } \\
\text { Heating } \\
\text { Units }\end{array}$ \\
\hline 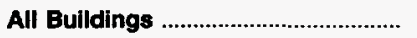 & 35,816 & 35,734 & 964 & 791 & 5,408 & 15,205 & 16,718 & 7,574 \\
\hline Building Floorspace (square feet) & & & & & & & & \\
\hline 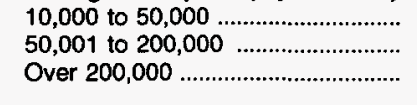 & $\begin{array}{r}2,591 \\
9,548 \\
23,677\end{array}$ & $\begin{array}{r}2,591 \\
9,466 \\
23,677\end{array}$ & $\begin{array}{l}140 \\
307 \\
517\end{array}$ & $\begin{array}{l}265 \\
526 \\
\text { NC }\end{array}$ & $\begin{array}{r}195 \\
1,694 \\
3,519\end{array}$ & $\begin{array}{r}713 \\
4,114 \\
10,378\end{array}$ & $\begin{array}{r}922 \\
4,359 \\
11,437\end{array}$ & $\begin{array}{r}719 \\
1,448 \\
5,407\end{array}$ \\
\hline 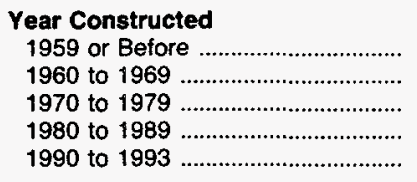 & $\begin{array}{r}13,258 \\
6,386 \\
6,175 \\
6,903 \\
3,095\end{array}$ & $\begin{array}{r}13,176 \\
6,386 \\
6,175 \\
6,903 \\
3,095\end{array}$ & $\begin{array}{r}199 \\
529 \\
102 \\
109 \\
25\end{array}$ & $\begin{array}{r}323 \\
109 \\
202 \\
115 \\
42\end{array}$ & $\begin{array}{r}1,947 \\
2,266 \\
391 \\
644 \\
159\end{array}$ & $\begin{array}{r}7,006 \\
1,539 \\
976 \\
2,926 \\
2,758\end{array}$ & $\begin{array}{r}6,178 \\
3,893 \\
4,189 \\
2,382 \\
75\end{array}$ & $\begin{array}{r}2,841 \\
1,349 \\
2,062 \\
1,254 \\
67\end{array}$ \\
\hline $\begin{array}{l}\text { Federal Agency } \\
\text { Department of Defense ................. } \\
\text { General Services Administration ... } \\
\text { United States Postal Service } \\
\text { Veterans Administration ................... } \\
\text { All Others ........................................ }\end{array}$ & $\begin{array}{r}1,668 \\
7,888 \\
7,027 \\
11,375 \\
7,858\end{array}$ & $\begin{array}{r}1,668 \\
7,888 \\
7,027 \\
11,375 \\
7,776\end{array}$ & $\begin{array}{r}88 \\
12 \\
563 \\
142 \\
159\end{array}$ & $\begin{array}{l}105 \\
12 \\
448 \\
N C \\
225\end{array}$ & $\begin{array}{r}20 \\
1,978 \\
854 \\
1,467 \\
1,088\end{array}$ & $\begin{array}{c}285 \\
1,643 \\
N C \\
8,140 \\
5,137\end{array}$ & $\begin{array}{l}1,218 \\
5,738 \\
4,335 \\
3,242 \\
2,184\end{array}$ & $\begin{array}{r}895 \\
491 \\
2,637 \\
2,400 \\
1,151\end{array}$ \\
\hline 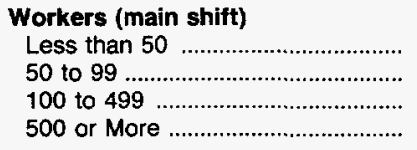 & $\begin{array}{r}2,548 \\
2,026 \\
9,029 \\
22,213\end{array}$ & $\begin{array}{r}2,466 \\
2,026 \\
9,029 \\
22,213\end{array}$ & $\begin{array}{r}170 \\
87 \\
190 \\
517\end{array}$ & $\begin{array}{l}143 \\
274 \\
374 \\
\text { NC }\end{array}$ & $\begin{array}{r}251 \\
152 \\
1,103 \\
3,901\end{array}$ & $\begin{array}{r}1,092 \\
680 \\
4,321 \\
9,112\end{array}$ & $\begin{array}{r}951 \\
800 \\
3,911 \\
11,057\end{array}$ & $\begin{array}{r}566 \\
259 \\
1,342 \\
5,407\end{array}$ \\
\hline 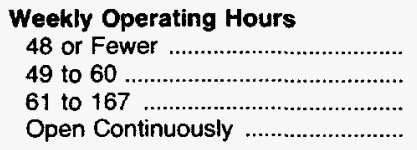 & $\begin{array}{r}2,740 \\
6,460 \\
8,051 \\
18,565\end{array}$ & $\begin{array}{r}2,658 \\
6,460 \\
8,051 \\
18,565\end{array}$ & $\begin{array}{r}154 \\
57 \\
101 \\
652\end{array}$ & $\begin{array}{r}65 \\
81 \\
488 \\
158\end{array}$ & $\begin{array}{r}226 \\
467 \\
2,206 \\
2,509\end{array}$ & $\begin{array}{l}1,135 \\
3,330 \\
3,041 \\
7,699\end{array}$ & $\begin{array}{l}1,052 \\
3,174 \\
3,859 \\
8,633\end{array}$ & $\begin{array}{r}233 \\
518 \\
1,312 \\
5,511\end{array}$ \\
\hline 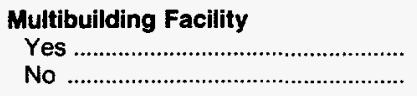 & $\begin{array}{l}24,441 \\
11,375\end{array}$ & $\begin{array}{l}24,359 \\
11,375\end{array}$ & $\begin{array}{l}364 \\
600\end{array}$ & $\begin{array}{l}159 \\
632\end{array}$ & $\begin{array}{l}3,672 \\
1,736\end{array}$ & $\begin{array}{r}15,000 \\
205\end{array}$ & $\begin{array}{l}8,520 \\
8,197\end{array}$ & $\begin{array}{l}2,691 \\
4,883\end{array}$ \\
\hline $\begin{array}{l}\text { Percent of Floorspace Heated } \\
\text { Not Heated } \\
1 \text { to } 50 \\
51 \text { to } 100\end{array}$ & $\begin{array}{r}82 \\
1,288 \\
34,446\end{array}$ & $\begin{array}{c}N C \\
1,288 \\
34,446\end{array}$ & $\begin{array}{l}\text { NC } \\
\text { NC } \\
964\end{array}$ & $\begin{array}{r}\text { NC } \\
66 \\
725\end{array}$ & $\begin{array}{r}N C \\
19 \\
5,388\end{array}$ & $\begin{array}{r}N C \\
278 \\
14,927\end{array}$ & $\begin{array}{r}N C \\
674 \\
16,043\end{array}$ & $\begin{array}{r}\text { NC } \\
325 \\
7,249\end{array}$ \\
\hline 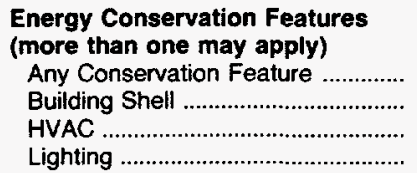 & $\begin{array}{l}35,734 \\
33,946 \\
35,425 \\
25,654\end{array}$ & $\begin{array}{l}35,734 \\
33,946 \\
35,425 \\
25,654\end{array}$ & $\begin{array}{l}964 \\
964 \\
939 \\
910\end{array}$ & $\begin{array}{l}791 \\
791 \\
680 \\
429\end{array}$ & $\begin{array}{l}5,408 \\
5,408 \\
5,377 \\
4,370\end{array}$ & $\begin{array}{l}15,205 \\
14,057 \\
15,205 \\
10,979\end{array}$ & $\begin{array}{l}16,718 \\
16,100 \\
16,576 \\
11,447\end{array}$ & $\begin{array}{l}7,574 \\
7,550 \\
7,563 \\
5,574\end{array}$ \\
\hline
\end{tabular}

See footnotes at end of table. 


\section{Table 3.14. Heating Equipment in FBSS Buildings in Federal Region 6, Floorspace, 1993 (Continued) (Thousand Square Feet)}

\begin{tabular}{|c|c|c|c|c|c|c|c|c|}
\hline \multirow[b]{2}{*}{$\begin{array}{c}\text { Building } \\
\text { Characteristics }\end{array}$} & \multirow[b]{2}{*}{$\begin{array}{l}\text { All } \\
\text { Buildings }\end{array}$} & \multirow[b]{2}{*}{$\begin{array}{c}\text { All } \\
\text { Heated } \\
\text { Buildings }\end{array}$} & \multicolumn{6}{|c|}{$\begin{array}{l}\text { Heating Equipment } \\
\text { (more than one may apply) }\end{array}$} \\
\hline & & & $\begin{array}{l}\text { Heat } \\
\text { Pumps }\end{array}$ & Furnaces & $\begin{array}{l}\text { Individual } \\
\text { Space } \\
\text { Heaters }\end{array}$ & $\begin{array}{l}\text { District } \\
\text { Heat }\end{array}$ & Boilers & $\begin{array}{l}\text { Packaged. } \\
\text { Heating } \\
\text { Units }\end{array}$ \\
\hline \multicolumn{9}{|l|}{$\begin{array}{l}\text { Building Shell Conservation } \\
\text { Features (more than one may } \\
\text { apply) } \\
\text { Roof or Ceiling }\end{array}$} \\
\hline 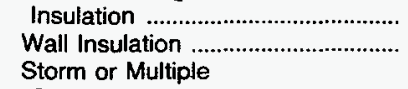 & $\begin{array}{l}28,249 \\
20,550\end{array}$ & $\begin{array}{l}28,249 \\
20,550\end{array}$ & $\begin{array}{l}932 \\
782\end{array}$ & $\begin{array}{l}629 \\
248\end{array}$ & $\begin{array}{l}3,936 \\
2,303\end{array}$ & $\begin{array}{r}11,866 \\
8,820\end{array}$ & $\begin{array}{r}13,412 \\
9,161\end{array}$ & $\begin{array}{l}5,269 \\
4,508\end{array}$ \\
\hline Glazing .................................... & 17,939 & 17,939 & 422 & 261 & 1,029 & 8,950 & 8,045 & 3,968 \\
\hline $\begin{array}{l}\text { or Shading Film } \\
\text { Exterior or Interior Shading }\end{array}$ & 24,768 & 24,768 & 732 & 463 & 4,758 & 10,361 & 11,373 & 5,597 \\
\hline 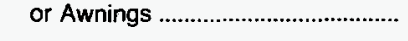 & 26,067 & 26,067 & 434 & 592 & 4,282 & 9,915 & 13,567 & 6,807 \\
\hline \multicolumn{9}{|l|}{$\begin{array}{l}\text { HVAC Conservation Features } \\
\text { (more than one may apply) }\end{array}$} \\
\hline 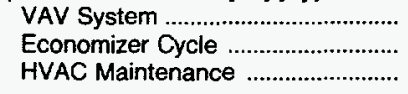 & $\begin{array}{l}18,518 \\
23,542 \\
35,341\end{array}$ & $\begin{array}{l}18,518 \\
23,542 \\
35,341\end{array}$ & $\begin{array}{l}170 \\
376 \\
876\end{array}$ & $\begin{array}{r}82 \\
189 \\
680\end{array}$ & $\begin{array}{l}1,713 \\
3,509 \\
5,377\end{array}$ & $\begin{array}{r}8,353 \\
10,756 \\
15,183\end{array}$ & $\begin{array}{r}9,135 \\
11,332 \\
16,576\end{array}$ & $\begin{array}{l}4,408 \\
5,842 \\
7,500\end{array}$ \\
\hline \multicolumn{9}{|l|}{$\begin{array}{l}\text { Energy Management Practices } \\
\text { (more than one may apply) } \\
\text { Energy Management and Control }\end{array}$} \\
\hline System ............................................ & 23,099 & 23,099 & 199 & 87 & 3,592 & 11,254 & 10,580 & 4,982 \\
\hline 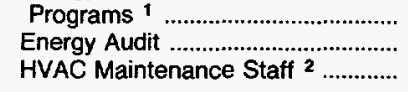 & $\begin{array}{l}13,513 \\
11,690 \\
15,538\end{array}$ & $\begin{array}{l}13,513 \\
11,690 \\
15,538\end{array}$ & $\begin{array}{r}100 \\
618 \\
12\end{array}$ & $\begin{array}{r}45 \\
69 \\
202\end{array}$ & $\begin{array}{l}2,576 \\
1,610 \\
1,881\end{array}$ & $\begin{array}{l}5,546 \\
2,221 \\
5,867\end{array}$ & $\begin{array}{l}7,178 \\
7,806 \\
8,854\end{array}$ & $\begin{array}{l}4,016 \\
3,421 \\
4,743\end{array}$ \\
\hline \multicolumn{9}{|l|}{$\begin{array}{l}\text { Off-Hours Reduction in } \\
\text { Equipment (more than one may } \\
\text { apply) }\end{array}$} \\
\hline 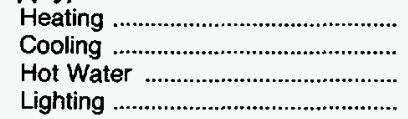 & $\begin{array}{r}13,162 \\
13,864 \\
4,554 \\
12,847\end{array}$ & $\begin{array}{r}13,162 \\
13,864 \\
4,554 \\
12,847\end{array}$ & $\begin{array}{l}299 \\
299 \\
117 \\
255\end{array}$ & $\begin{array}{l}633 \\
633 \\
190 \\
585\end{array}$ & $\begin{array}{l}2,898 \\
2,898 \\
1,379 \\
2,859\end{array}$ & $\begin{array}{l}5,002 \\
5,405 \\
1,471 \\
4,444\end{array}$ & $\begin{array}{l}6,937 \\
6,913 \\
2,408 \\
6,949\end{array}$ & $\begin{array}{l}2,044 \\
2,044 \\
1,058 \\
1,981\end{array}$ \\
\hline
\end{tabular}

1 Building participates in any programs sponsored by the Federal Energy Management Program, in-house, utility, or third party.

2 HVAC maintenance staff means at least one person spends at least half their working hours maintaining the heating/cooling equipment.

$\mathrm{NC}=$ No cases in responding sample.

Notes: - Total workers are the number of workers during the main shift. - See Glossary for explanation of abbreviations and definitions of terms used in this report. - These data are from 881 federally owned buildings having the following criteria: (1) located in Federal Regions 3,6 , or $9 ;(2)$ larger than 10,000 square feet; and (3) used for a commercial purpose, other than warehouse and storage. In addition, 9 out of 10 selected buildings were from agencies other than the Department of Defense. - Statistics for the "energy end uses" represent consumption in buildings that have end use, not consumption for a particular fuel for a particular end use. - FBSS = Federal Buildings Supplemental Survey. - HVAC = Heating, Ventilation, and Air Conditioning. - VAV = Variable-Air Volume. - Data are for Fiscal Year 1993 (October 1, 1992 through September 30, 1993). Because of rounding, data may not sum to totals.

Source: Energy Information Administration, Office of Energy Markets and End Use, 1993 Federal Buildings Supplemental Survey. 
Table 3.15. Heating Equipment in FBSS Buildings in Federal Region 9, Floorspace, 1993

(Thousand Square Feet)

\begin{tabular}{|c|c|c|c|c|c|c|c|c|}
\hline \multirow[b]{2}{*}{$\begin{array}{l}\text { Building } \\
\text { Characteristics }\end{array}$} & \multirow[b]{2}{*}{$\begin{array}{c}\text { All } \\
\text { Buildings }\end{array}$} & \multirow[b]{2}{*}{$\begin{array}{c}\text { All } \\
\text { Heated } \\
\text { Buildings }\end{array}$} & \multicolumn{6}{|c|}{$\begin{array}{l}\text { Heating Equipment } \\
\text { (more than one may apply) }\end{array}$} \\
\hline & & & $\begin{array}{l}\text { Heat } \\
\text { Pumps }\end{array}$ & Furnaces & $\begin{array}{l}\text { Individual } \\
\text { Space } \\
\text { Heaters }\end{array}$ & $\begin{array}{l}\text { District } \\
\text { Heat }\end{array}$ & Boilers & $\begin{array}{l}\text { Packaged- } \\
\text { Heating } \\
\text { Units }\end{array}$ \\
\hline All Buildings & 44,316 & 41,921 & 3,081 & 1,204 & 4,933 & 12,712 & 24,010 & 5,997 \\
\hline 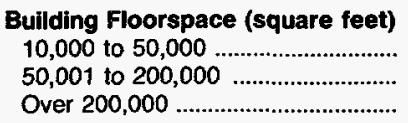 & $\begin{array}{r}3,858 \\
12,680 \\
27,778\end{array}$ & $\begin{array}{r}3,626 \\
12,016 \\
26,279\end{array}$ & $\begin{array}{r}566 \\
1,346 \\
1,169\end{array}$ & $\begin{array}{l}496 \\
242 \\
466\end{array}$ & $\begin{array}{r}511 \\
879 \\
3,542\end{array}$ & $\begin{array}{r}810 \\
3,191 \\
8,711\end{array}$ & $\begin{array}{r}1,321 \\
6,911 \\
15,778\end{array}$ & $\begin{array}{l}1,040 \\
2,439 \\
2,518\end{array}$ \\
\hline 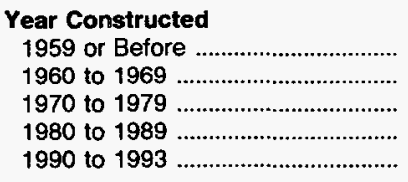 & $\begin{array}{r}13,553 \\
9,082 \\
12,041 \\
7,955 \\
1,684\end{array}$ & $\begin{array}{r}12,957 \\
9,082 \\
11,355 \\
6,986 \\
1,541\end{array}$ & $\begin{array}{r}1,324 \\
639 \\
623 \\
449 \\
46\end{array}$ & $\begin{array}{r}422 \\
107 \\
77 \\
94 \\
503\end{array}$ & $\begin{array}{r}1,598 \\
2,019 \\
514 \\
335 \\
466\end{array}$ & $\begin{array}{c}3,924 \\
1,437 \\
4,765 \\
2,586 \\
\text { NC }\end{array}$ & $\begin{array}{l}5,689 \\
7,535 \\
6,326 \\
3,291 \\
1,169\end{array}$ & $\begin{array}{r}2,409 \\
591 \\
1,590 \\
740 \\
667\end{array}$ \\
\hline $\begin{array}{l}\text { Federal Agency } \\
\text { Department of Defense ................ } \\
\text { General Services Administration ... } \\
\text { United States Postal Service ......... } \\
\text { Veterans Administration ................ } \\
\text { All Others ......................................... }\end{array}$ & $\begin{array}{r}8,489 \\
12,505 \\
8,387 \\
8,824 \\
6,111\end{array}$ & $\begin{array}{r}7,815 \\
12,505 \\
6,844 \\
8,681 \\
6,077\end{array}$ & $\begin{array}{c}260 \\
N C \\
1,171 \\
490 \\
1,160\end{array}$ & $\begin{array}{c}322 \\
82 \\
710 \\
\text { NC } \\
90\end{array}$ & $\begin{array}{r}551 \\
1,453 \\
2,023 \\
28 \\
877\end{array}$ & $\begin{array}{c}3,963 \\
1,500 \\
N C \\
6,803 \\
447\end{array}$ & $\begin{array}{r}1,701 \\
11,218 \\
4,675 \\
1,598 \\
4,819\end{array}$ & $\begin{array}{r}949 \\
605 \\
1,502 \\
1,186 \\
1,755\end{array}$ \\
\hline $\begin{array}{l}\text { Workers (main shift) } \\
\text { Less than } 50 \\
50 \text { to } 99 \\
100 \text { to } 499 \\
500 \text { or More }\end{array}$ & $\begin{array}{r}3,684 \\
2,161 \\
14,017 \\
24,454\end{array}$ & $\begin{array}{r}3,272 \\
2,122 \\
13,572 \\
22,956\end{array}$ & $\begin{array}{r}225 \\
231 \\
1,657 \\
967\end{array}$ & $\begin{array}{r}239 \\
99 \\
400 \\
466\end{array}$ & $\begin{array}{r}327 \\
227 \\
1,038 \\
3,340\end{array}$ & $\begin{array}{r}805 \\
247 \\
4,322 \\
7,338\end{array}$ & $\begin{array}{r}996 \\
1,349 \\
6,816 \\
14,850\end{array}$ & $\begin{array}{r}347 \\
424 \\
3,622 \\
1,604\end{array}$ \\
\hline 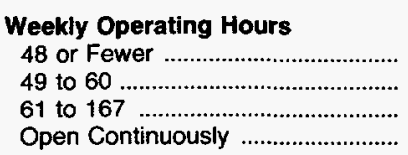 & $\begin{array}{r}4,822 \\
13,424 \\
5,706 \\
20,364\end{array}$ & $\begin{array}{r}4,498 \\
13,278 \\
5,513 \\
18,634\end{array}$ & $\begin{array}{r}659 \\
482 \\
331 \\
1,610\end{array}$ & $\begin{array}{l}159 \\
275 \\
265 \\
505\end{array}$ & $\begin{array}{r}732 \\
1,943 \\
525 \\
1,732\end{array}$ & $\begin{array}{r}1,045 \\
2,207 \\
477 \\
8,984\end{array}$ & $\begin{array}{r}2,513 \\
10,496 \\
2,917 \\
8,085\end{array}$ & $\begin{array}{r}1,304 \\
779 \\
1,772 \\
2,142\end{array}$ \\
\hline $\begin{array}{l}\text { Multibuilding Facility } \\
\text { Yes } \\
\text { No }\end{array}$ & $\begin{array}{l}26,821 \\
17,495\end{array}$ & $\begin{array}{l}25,713 \\
16,208\end{array}$ & $\begin{array}{l}1,859 \\
1,223\end{array}$ & $\begin{array}{l}896 \\
308\end{array}$ & $\begin{array}{l}1,606 \\
3,327\end{array}$ & $\begin{array}{r}10,888 \\
1,824\end{array}$ & $\begin{array}{l}11,611 \\
12,399\end{array}$ & $\begin{array}{l}3,000 \\
2,997\end{array}$ \\
\hline $\begin{array}{l}\text { Percent of Floorspace Heated } \\
\text { Not Heated } \\
1 \text { to } 50 \\
51 \text { to } 100\end{array}$ & $\begin{array}{r}2,394 \\
3,751 \\
38,170\end{array}$ & $\begin{array}{r}\mathrm{NC} \\
3,751 \\
38,170\end{array}$ & $\begin{array}{c}N C \\
132 \\
2,949\end{array}$ & $\begin{array}{r}N C \\
30 \\
1,174\end{array}$ & $\begin{array}{c}N C \\
116 \\
4,817\end{array}$ & $\begin{array}{r}\text { NC } \\
1,471 \\
11,241\end{array}$ & $\begin{array}{r}N C \\
499 \\
23,511\end{array}$ & $\begin{array}{c}\text { NC } \\
741 \\
5,256\end{array}$ \\
\hline 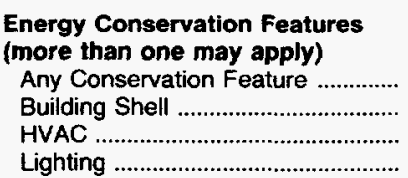 & $\begin{array}{l}44,023 \\
34,105 \\
43,116 \\
34,825\end{array}$ & $\begin{array}{l}41,775 \\
32,838 \\
41,197 \\
32,968\end{array}$ & $\begin{array}{l}3,081 \\
2,731 \\
3,060 \\
2,684\end{array}$ & $\begin{array}{r}1,204 \\
1,037 \\
1,026 \\
816\end{array}$ & $\begin{array}{l}4,933 \\
4,907 \\
4,894 \\
3,962\end{array}$ & $\begin{array}{r}12,659 \\
10,381 \\
12,659 \\
8,815\end{array}$ & $\begin{array}{l}23,976 \\
17,304 \\
23,697 \\
20,724\end{array}$ & $\begin{array}{l}5,997 \\
5,848 \\
5,939 \\
4,692\end{array}$ \\
\hline
\end{tabular}

See footnotes at end of table. 


\section{Table 3.15. Heating Equipment in FBSS Buildings in Federal Region 9, Floorspace, 1993 (Continued) \\ (Thousand Square Feet)}

\begin{tabular}{|c|c|c|c|c|c|c|c|c|}
\hline \multirow[b]{2}{*}{$\begin{array}{c}\text { Building } \\
\text { Characteristics }\end{array}$} & \multirow[b]{2}{*}{$\begin{array}{c}\text { All } \\
\text { Bulldings }\end{array}$} & \multirow[b]{2}{*}{$\begin{array}{c}\text { All } \\
\text { Heated } \\
\text { Buildings }\end{array}$} & \multicolumn{6}{|c|}{$\begin{array}{l}\text { Heating Equipment } \\
\text { (more than one may apply) }\end{array}$} \\
\hline & & & $\begin{array}{l}\text { Heat } \\
\text { Pumps }\end{array}$ & Furnaces & $\begin{array}{l}\text { Indlvidual } \\
\text { Space } \\
\text { Heaters }\end{array}$ & $\begin{array}{l}\text { District } \\
\text { Heat }\end{array}$ & Boilers & $\begin{array}{l}\text { Packaged- } \\
\text { Heating } \\
\text { Units }\end{array}$ \\
\hline \multicolumn{9}{|l|}{$\begin{array}{l}\text { Building Shell Conservation } \\
\text { Features (more than one may } \\
\text { apply) } \\
\text { Roof or Ceiling }\end{array}$} \\
\hline 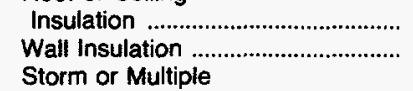 & $\begin{array}{l}27,234 \\
17,462\end{array}$ & $\begin{array}{l}26,350 \\
17,416\end{array}$ & $\begin{array}{r}2,512 \\
935\end{array}$ & $\begin{array}{l}805 \\
851\end{array}$ & $\begin{array}{l}4,165 \\
2,800\end{array}$ & $\begin{array}{l}9,869 \\
6,865\end{array}$ & $\begin{array}{r}13,175 \\
8,136\end{array}$ & $\begin{array}{l}4,603 \\
2,761\end{array}$ \\
\hline $\begin{array}{l}\text { or Shading Film ............................... } \\
\text { Exterior or Interior Shading }\end{array}$ & 22,381 & 21,601 & 2,395 & 803 & 4,082 & 5,227 & 12,523 & 3,980 \\
\hline 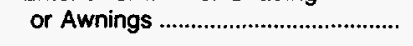 & 24,370 & 24,142 & 2,259 & 894 & 4,405 & 7,330 & 13,447 & 4,161 \\
\hline \multicolumn{9}{|l|}{$\begin{array}{l}\text { HVAC Conservation Features } \\
\text { (more than one may apply) }\end{array}$} \\
\hline 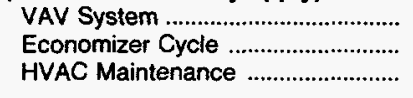 & $\begin{array}{l}15,577 \\
26,367 \\
43,098\end{array}$ & $\begin{array}{l}15,562 \\
26,050 \\
41,179\end{array}$ & $\begin{array}{r}746 \\
2,040 \\
3,060\end{array}$ & $\begin{array}{r}466 \\
654 \\
1,026\end{array}$ & $\begin{array}{l}3,221 \\
3,750 \\
4,894\end{array}$ & $\begin{array}{r}4,948 \\
5,461 \\
12,659\end{array}$ & $\begin{array}{r}9,605 \\
18,047 \\
23,697\end{array}$ & $\begin{array}{l}1,824 \\
4,160 \\
5,921\end{array}$ \\
\hline \multicolumn{9}{|l|}{$\begin{array}{l}\text { Energy Management Practices } \\
\text { (more than one may apply) } \\
\text { Energy Management and Control }\end{array}$} \\
\hline $\begin{array}{l}\text { System ............................................... } \\
\text { Energy Conservation }\end{array}$ & 18,231 & 16,963 & 1,477 & 507 & 1,634 & 6,515 & 9,045 & 1,712 \\
\hline 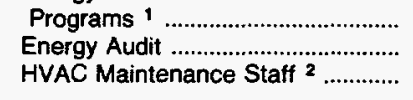 & $\begin{array}{l}24,699 \\
17,444 \\
14,570\end{array}$ & $\begin{array}{l}24,668 \\
16,711 \\
13,679\end{array}$ & $\begin{array}{r}2,456 \\
894 \\
412\end{array}$ & $\begin{array}{l}722 \\
763 \\
510\end{array}$ & $\begin{array}{l}3,373 \\
2,609 \\
1,923\end{array}$ & $\begin{array}{l}6,866 \\
4,926 \\
3,981\end{array}$ & $\begin{array}{r}15,566 \\
11,129 \\
8,573\end{array}$ & $\begin{array}{r}4,294 \\
863 \\
1,504\end{array}$ \\
\hline \multicolumn{9}{|l|}{$\begin{array}{l}\text { Off-Hours Reduction in } \\
\text { Equipment (more than one may } \\
\text { apply) }\end{array}$} \\
\hline 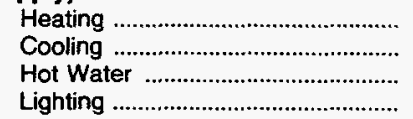 & $\begin{array}{l}19,631 \\
19,219 \\
13,614 \\
21,155\end{array}$ & $\begin{array}{l}19,631 \\
18,834 \\
13,574 \\
20,626\end{array}$ & $\begin{array}{r}1,285 \\
1,086 \\
162 \\
1,154\end{array}$ & $\begin{array}{l}481 \\
337 \\
218 \\
558\end{array}$ & $\begin{array}{l}3,076 \\
2,577 \\
1,664 \\
3,201\end{array}$ & $\begin{array}{l}2,449 \\
2,595 \\
1,641 \\
2,839\end{array}$ & $\begin{array}{l}14,670 \\
13,976 \\
11,615 \\
14,778\end{array}$ & $\begin{array}{l}3,697 \\
3,608 \\
1,887 \\
3,685\end{array}$ \\
\hline
\end{tabular}

1 Building participates in any programs sponsored by the Federal Energy Management Program, in-house, utility, or third party.

2 HVAC maintenance staff means at least one person spends at least half their working hours maintaining the heating/cooling equipment.

$\mathrm{NC}=$ No cases in responding sample.

Notes: - Total workers are the number of workers during the main shift. - See Glossary for explanation of abbreviations and definitions of terms used in this report. - These data are from 881 federally owned buildings having the following criteria: (1) located in Federal Regions 3 , 6 , or 9 ; (2) larger than 10,000 square feet; and (3) used for a commercial purpose, other than warehouse and storage. In addition, 9 out of 10 selected buildings were from agencies other than the Department of Defense. - Statistics for the "energy end uses" represent consumption in buildings that have end use, not consumption for a particular fuel for a particular end use. - FBSS = Federal Buildings Supplemental Survey. - HVAC = Heating, Ventilation, and Air Conditioning. - VAV = Variable-Air Volume. • Data are for Fiscal Year 1993 (October 1,1992 through September 30, 1993). - Because of rounding, data may not sum to totals.

Source: Energy Information Administration, Office of Energy Markets and End Use, 1993 Federal Buildings Supplemental Survey. 
Table 3.16. Cooling Equipment in FBSS Buildings in Federal Region 3, Number of Buildings, 1993

\begin{tabular}{|c|c|c|c|c|c|c|c|c|}
\hline \multirow[b]{2}{*}{$\begin{array}{l}\text { Building } \\
\text { Characteristics }\end{array}$} & \multirow[b]{2}{*}{$\begin{array}{c}\text { All } \\
\text { Buildings }\end{array}$} & \multirow[b]{2}{*}{$\begin{array}{c}\text { All } \\
\text { Cooled } \\
\text { Buildings }\end{array}$} & \multicolumn{6}{|c|}{$\begin{array}{c}\text { Cooling Equipment } \\
\text { (more than one may apply) }\end{array}$} \\
\hline & & & $\begin{array}{c}\text { Residential- } \\
\text { Type } \\
\text { Central Air } \\
\text { Conditioners }\end{array}$ & $\begin{array}{l}\text { Heat } \\
\text { Pumps }\end{array}$ & $\begin{array}{c}\begin{array}{c}\text { Individual } \\
\text { Air }\end{array} \\
\text { Conditioners }\end{array}$ & $\begin{array}{l}\text { District } \\
\text { Chilled } \\
\text { Water }\end{array}$ & $\begin{array}{l}\text { Central } \\
\text { Chillers }\end{array}$ & $\begin{array}{l}\text { Packaged- } \\
\text { Air- } \\
\text { Condlitioning } \\
\text { Units }\end{array}$ \\
\hline All Bulldings . & 312 & 305 & 30 & 37 & 80 & 72 & 158 & 127 \\
\hline \multicolumn{9}{|l|}{ Building Floorspace (square feet) } \\
\hline 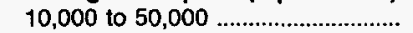 & 94 & 92 & 8 & 20 & 20 & 10 & 25 & 37 \\
\hline 50,001 to 200,000 & 117 & 114 & 6 & 1 & 23 & 38 & 61 & 34 \\
\hline Over 200,000 & 101 & 99 & 16 & 16 & 37 & 24 & 72 & 56 \\
\hline \multicolumn{9}{|l|}{ Principal Building Activity } \\
\hline 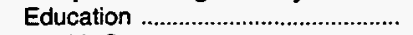 & 8 & 8 & NC & NC & 2 & NC & 4 & 3 \\
\hline 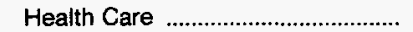 & 41 & 40 & 3 & 1 & 10 & 22 & 15 & 16 \\
\hline 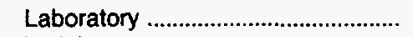 & 37 & 37 & 2 & 1 & 5 & 14 & 16 & 7 \\
\hline 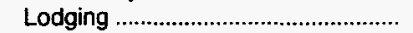 & 13 & 12 & 1 & 1 & 3 & 1 & 5 & 6 \\
\hline Mercantile and Service ...................... & 46 & 44 & 4 & 6 & 9 & NC & 22 & 20 \\
\hline 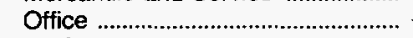 & 124 & 123 & 16 & 24 & 39 & 25 & 82 & 61 \\
\hline All Others & 43 & 41 & 4 & 4 & 12 & 10 & 14 & 14 \\
\hline \multicolumn{9}{|l|}{ Year Constructed } \\
\hline 1959 or Before & 142 & 140 & 19 & 20 & 61 & 30 & 69 & 66 \\
\hline 1960 to 1969 & 70 & 69 & 6 & 2 & 5 & 28 & 31 & 17 \\
\hline 1970 to 1979 & 47 & 44 & 3 & 5 & 9 & 3 & 30 & 22 \\
\hline 1980 to 1989 & 38 & 37 & 2 & 8 & 4 & 6 & 21 & 17 \\
\hline 1990 to 1993 & 15 & 15 & NC & 2 & 1 & 5 & 7 & 5 \\
\hline \multicolumn{9}{|l|}{ Federal Agency } \\
\hline Department of Detense & 22 & 22 & 2 & NC & 6 & 9 & 5 & 5 \\
\hline General Services Administration ... & 83 & 81 & 12 & 18 & 29 & 9 & 69 & 42 \\
\hline United States Postal Service ......... & 57 & 55 & 6 & 8 & 13 & NC & 30 & 28 \\
\hline Veterans Administration ....................... & 56 & 54 & 3 & 3 & 12 & 24 & 19 & 20 \\
\hline 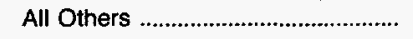 & 94 & 93 & 7 & 8 & 20 & 30 & 35 & 32 \\
\hline \multicolumn{9}{|l|}{ Workers (main shift) } \\
\hline Less than 50 & 71 & 66 & 7 & 11 & 18 & 12 & 17 & 21 \\
\hline 50 to 99 & 39 & 39 & 2 & 8 & 9 & 7 & 10 & 14 \\
\hline 100 to 499 & 107 & 106 & 5 & 5 & 18 & 30 & 58 & 38 \\
\hline 500 or More & 95 & 94 & 16 & 13 & 35 & 23 & 73 & 54 \\
\hline \multicolumn{9}{|l|}{ Weekly Operating Hours } \\
\hline 48 or Fewer & 51 & 49 & 9 & 11 & 15 & 8 & 22 & 23 \\
\hline 49 to 60 & 76 & 76 & 5 & 8 & 23 & 15 & 47 & 31 \\
\hline 61 to 167 & 60 & 59 & 7 & 12 & 11 & 8 & 27 & 23 \\
\hline 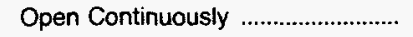 & 125 & 121 & 9 & 6 & 31 & 41 & 62 & 50 \\
\hline \multicolumn{9}{|l|}{ Multibuilding Facility } \\
\hline 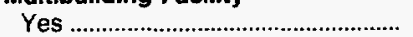 & 176 & 171 & 10 & 14 & 40 & 63 & 74 & 64 \\
\hline No & 136 & 134 & 20 & 23 & 40 & 9 & 84 & 63 \\
\hline \multicolumn{9}{|l|}{ Percent of Floorspace Cooled } \\
\hline Not Cooled & 13 & 6 & NC & NC & NC & NG & NC & NC \\
\hline 1 to 50 & 30 & 30 & 2 & 5 & 16 & 4 & 11 & 17 \\
\hline 51 to 100 & 269 & 269 & $2 \overline{8}$ & 32 & 64 & 68 & 147 & 110 \\
\hline \multicolumn{9}{|l|}{$\begin{array}{l}\text { Energy Conservation Features } \\
\text { (more than one may apply) }\end{array}$} \\
\hline Any Conservation Feature ................ & 309 & 304 & 30 & 37 & 80 & 72 & 158 & 127 \\
\hline 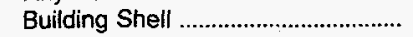 & 290 & 285 & 25 & 34 & 72 & 71 & 146 & 117 \\
\hline 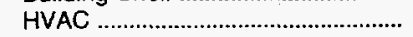 & 303 & 298 & 29 & 35 & 79 & 72 & 155 & 125 \\
\hline Lighting & 210 & 207 & 19 & 29 & 60 & 45 & 120 & 94 \\
\hline
\end{tabular}

See footnotes at end of table. 
Table 3.16. Cooling Equipment in FBSS Buildings in Federal Region 3, Number of Buildings, 1993 (Continued)

\begin{tabular}{|c|c|c|c|c|c|c|c|c|}
\hline \multirow[b]{2}{*}{$\begin{array}{c}\text { Building } \\
\text { Characteristics }\end{array}$} & \multirow[b]{2}{*}{$\begin{array}{c}\text { All } \\
\text { Buildings }\end{array}$} & \multirow[b]{2}{*}{$\begin{array}{c}\text { All } \\
\text { Cooled } \\
\text { Buildings }\end{array}$} & \multicolumn{6}{|c|}{$\begin{array}{c}\text { Cooling Equipment } \\
\text { (more than one may apply) }\end{array}$} \\
\hline & & & $\begin{array}{c}\text { Residential- } \\
\text { Type } \\
\text { Central Air } \\
\text { Conditioners }\end{array}$ & $\begin{array}{c}\text { Heat } \\
\text { Pumps }\end{array}$ & $\begin{array}{c}\text { Individual } \\
\text { Air } \\
\text { Conditioners }\end{array}$ & $\begin{array}{l}\text { District } \\
\text { Chilled } \\
\text { Water }\end{array}$ & $\begin{array}{l}\text { Central } \\
\text { Chillers }\end{array}$ & $\begin{array}{c}\text { Packaged- } \\
\text { Air- } \\
\text { Conditioning } \\
\text { Units }\end{array}$ \\
\hline
\end{tabular}

Building Shell Conservation

Features (more than one may

apply)

Roof or Ceiling

Insulation .....

Wall Insulation

Storm or Multiple

Glazing

Tinted or Reflective Glass

or Shading Film

Exterior or Interior Shading

or Awnings

$\begin{array}{ll}240 & 236 \\ 117 & 116 \\ 173 & 170 \\ 158 & 156 \\ 197 & 196\end{array}$

$\begin{array}{rr}21 & 32 \\ 9 & 20 \\ 16 & 23 \\ 12 & 14 \\ 18 & 25\end{array}$

61
29
40
32
55

64
26
38
42
55

$\begin{array}{rr}117 & 93 \\ 58 & 51 \\ 89 & 68 \\ 84 & 66 \\ 102 & 86\end{array}$

HVAC Conservation Features

(more than one may apply)

VAV System

Economizer Cycle

HVAC Maintenance

$84 \quad 82$

$\begin{array}{rr}84 & 82 \\ 156 & 154\end{array}$

$\begin{array}{ll}156 & 154 \\ 301 & 297\end{array}$

$\begin{array}{rr}9 & 8 \\ 16 & 19 \\ 29 & 35\end{array}$

$\begin{array}{ll}132 & 131 \\ 99 & 95 \\ 99 & 97 \\ 97 & 96\end{array}$

$\begin{array}{rr}13 & 1 \\ 8 & 1 \\ 10 & 1 \\ 13 & \end{array}$

19
15
14
9

36
28
32
28

$\begin{array}{ll}38 & 80 \\ 20 & 70 \\ 17 & 61 \\ 26 & 70\end{array}$

80
70
61
70

65

50
43

HVAC Maintenance Staff ${ }^{2}$...............

Off-Hours Reduction in

Equipment (more than one may

apply)

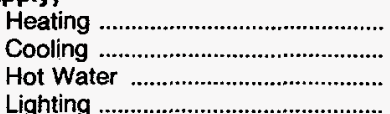

$\begin{array}{rr}144 & 143 \\ 145 & 145 \\ 77 & 77 \\ 162 & 159\end{array}$

$\begin{array}{rr}19 & 24 \\ 19 & 24 \\ 8 & 11 \\ 18 & 28\end{array}$

39
42
23
44

22
22
7
23

82
83
51
91

66

66

35

1 Building participates in any programs sponsored by the Federal Energy Management Program, in-house, utility, or third party.

2 HVAC maintenance staff means at least one person spends at least half their working hours maintaining the heating/cooling equipment.

$\mathrm{NC}=$ No cases in responding sample.

Notes: - Total workers are the number of workers during the main shift. - See Glossary for explanation of abbreviations and definitions of terms used in this report. - These data are from 881 federally owned buildings having the following criteria: (1) located in Federal Regions 3,6 , or 9; (2) larger than 10,000 square feet; and (3) used for a commercial purpose, other than warehouse and storage. In addition, 9 out of 10 selected buildings were from agencies other than the Department of Defense. - Statistics for the "energy end uses" represent consumption in buildings that have end use, not consumption for a particular fuel for a particular end use. $\bullet$ FBSS = Federal Buildings Supplemental Survey. $\bullet$ HVAC = Heating, Ventilation, and Air Conditioning. - VAV = Variable-Air Volume. - Data are for Fiscal Year 1993 (October 1, 1992 through September 30 , 1993). - Because of rounding, data may not sum to totals.

Source: Energy Information Administration, Office of Energy Markets and End Use, 1993 Federal Buildings Supplemental Survey. 
Table 3.17. Cooling Equipment in FBSS Buildings in Federal Region 6, Number of Buildings, 1993

\begin{tabular}{|c|c|c|c|c|c|c|c|c|}
\hline \multirow[b]{2}{*}{$\begin{array}{c}\text { Building } \\
\text { Characteristics }\end{array}$} & \multirow[b]{2}{*}{$\begin{array}{c}\text { All } \\
\text { Buildings }\end{array}$} & \multirow[b]{2}{*}{$\begin{array}{c}\text { All } \\
\text { Cooled } \\
\text { Buildings }\end{array}$} & \multicolumn{6}{|c|}{$\begin{array}{l}\text { Cooling Equipment } \\
\text { (more than one may apply) }\end{array}$} \\
\hline & & & $\begin{array}{c}\text { Residential- } \\
\text { Type } \\
\text { Central Air } \\
\text { Conditioners }\end{array}$ & $\begin{array}{l}\text { Heat } \\
\text { Pumps }\end{array}$ & $\begin{array}{c}\begin{array}{c}\text { Individual } \\
\text { Air }\end{array} \\
\text { Conditioners }\end{array}$ & $\begin{array}{l}\text { District } \\
\text { Chilled } \\
\text { Water }\end{array}$ & $\begin{array}{l}\text { Central } \\
\text { Chiliers }\end{array}$ & $\begin{array}{c}\text { Packaged- } \\
\text { Air- } \\
\text { Conditioning } \\
\text { Units }\end{array}$ \\
\hline 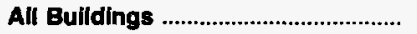 & 243 & 241 & 29 & 11 & 50 & 44 & 122 & 97 \\
\hline \multicolumn{9}{|l|}{ Building Floorspace (square feet) } \\
\hline 10,000 to 50,000 & 107 & 107 & to & 4 & 19 & 12 & 31 & 54 \\
\hline 50,001 to 200,000 & 86 & 85 & 12 & 5 & 23 & 16 & 58 & 21 \\
\hline Over 200,000 & 50 & 49 & 7 & 2 & 8 & 16 & 33 & 22 \\
\hline \multicolumn{9}{|l|}{ Principal Building Activity } \\
\hline 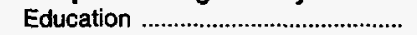 & 6 & 6 & 1 & NC & NC & NC & 3 & 3 \\
\hline Health Care & 35 & 35 & 3 & 2 & 10 & 16 & 18 & 15 \\
\hline 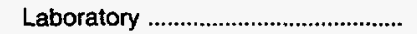 & 29 & 29 & NC & NC & 7 & 5 & 17 & 7 \\
\hline Lodging & 16 & 16 & 2 & NC & 5 & 4 & 4 & 5 \\
\hline Mercantile and Service ....................... & 49 & 48 & $\overline{8}$ & 4 & 5 & NC & 22 & 31 \\
\hline Office & 76 & 76 & 11 & 3 & 15 & 13 & 47 & 24 \\
\hline All Others & 32 & 31 & 4 & 2 & 8 & 6 & 11 & 12 \\
\hline \multicolumn{9}{|l|}{ Year Constructed } \\
\hline 1959 or Before & 103 & 102 & 15 & 3 & 30 & 26 & 47 & 34 \\
\hline 1960 to 1969 & 38 & 38 & 6 & 2 & 11 & 6 & 27 & 15 \\
\hline 1970 to 1979 & 34 & 33 & 5 & 2 & 6 & 5 & 22 & 14 \\
\hline 1980 to 1989 & 57 & 57 & 3 & 3 & 2 & 6 & 22 & 30 \\
\hline 1990 to 1993 & 11 & 11 & NC & 1 & 1 & 1 & 4 & 4 \\
\hline \multicolumn{9}{|l|}{ Federal Agency } \\
\hline Department of Defense .................... & 22 & 22 & 1 & 2 & 3 & 3 & 8 & 8 \\
\hline General Services Administration ... & 35 & 35 & 5 & 1 & 4 & 8 & 27 & 10 \\
\hline United States Postal Service ......... & 61 & 60 & 11 & 3 & 6 & NC & 29 & 35 \\
\hline Veterans Administration ................... & 36 & 36 & 4 & 1 & 10 & 18 & 16 & 14 \\
\hline All Others & 89 & 88 & 8 & 4 & 27 & 15 & 42 & 30 \\
\hline \multicolumn{9}{|l|}{ Workers (main shift) } \\
\hline Less than 50 & 70 & 69 & 12 & 2 & 17 & 10 & 19 & 33 \\
\hline 50 to 99 & 44 & 44 & 5 & 2 & 7 & 3 & 21 & 16 \\
\hline 100 to 499 & 80 & 80 & 5 & 5 & 15 & 16 & 48 & 26 \\
\hline 500 or More & 49 & 48 & 7 & 2 & 11 & 15 & 34 & 22 \\
\hline \multicolumn{9}{|l|}{ Weekly Operating Hours } \\
\hline 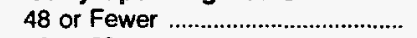 & 39 & 38 & 6 & 2 & 9 & 4 & 20 & 11 \\
\hline 49 to 60 & 46 & 46 & 3 & 2 & 7 & 14 & 22 & 19 \\
\hline 61 to 167 & 78 & 78 & 10 & 3 & 16 & 7 & 30 & 35 \\
\hline Open Continuously & 80 & 79 & 10 & 4 & 18 & 19 & 50 & 32 \\
\hline \multicolumn{9}{|l|}{ Multibuilding Facility } \\
\hline 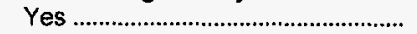 & 148 & 147 & 15 & 7 & 39 & 44 & 73 & 47 \\
\hline No & 95 & 94 & 14 & 4 & 11 & NC & 49 & 50 \\
\hline \multicolumn{9}{|l|}{ Percent of Fioorspace Cooled } \\
\hline Not Cooled & 3 & 1 & NC & NC & NC & NC & NC & NC \\
\hline 1 to 50 & 26 & 26 & 1 & 1 & 13 & 2 & 4 & 13 \\
\hline 51 to 100 & 214 & 214 & 28 & 10 & 37 & 42 & 118 & 84 \\
\hline \multicolumn{9}{|l|}{$\begin{array}{l}\text { Energy Conservation Features } \\
\text { (more than one may apply) }\end{array}$} \\
\hline Any Conservation Feature ................. & 242 & 241 & 29 & 11 & 50 & 44 & 122 & 97 \\
\hline 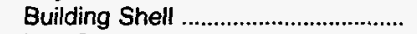 & 228 & 227 & 28 & 11 & 46 & 40 & 116 & 93 \\
\hline HVAC & 236 & 235 & 27 & 10 & 48 & 44 & 121 & 96 \\
\hline 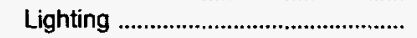 & 136 & 135 & 19 & 9 & 23 & 24 & 72 & 59 \\
\hline
\end{tabular}

See footnotes at end of table. 

Table 3.17. Cooling Equipment in FBSS Buildings in Federal Region 6,
Number of Buildings, 1993 (Continued)

\begin{tabular}{|c|c|c|c|c|c|c|c|c|}
\hline \multirow[b]{2}{*}{$\begin{array}{c}\text { Building } \\
\text { Characteristics }\end{array}$} & \multirow[b]{2}{*}{$\begin{array}{l}\text { All } \\
\text { Buildings }\end{array}$} & \multirow[b]{2}{*}{$\begin{array}{c}\text { All } \\
\text { Cooled } \\
\text { Bulldings }\end{array}$} & \multicolumn{6}{|c|}{$\begin{array}{l}\text { Cooling Equipment } \\
\text { (more than one may apply) }\end{array}$} \\
\hline & & & $\begin{array}{c}\text { Residential- } \\
\text { Type } \\
\text { Central Air } \\
\text { Conditioners }\end{array}$ & $\begin{array}{l}\text { Heat } \\
\text { Pumps }\end{array}$ & \begin{tabular}{c|} 
Individual \\
Air \\
Conditioners
\end{tabular} & $\begin{array}{l}\text { District } \\
\text { Chilled } \\
\text { Water }\end{array}$ & $\begin{array}{l}\text { Central } \\
\text { Chillers }\end{array}$ & $\begin{array}{l}\text { Packaged- } \\
\text { Alr- } \\
\text { Conditioning } \\
\text { Units }\end{array}$ \\
\hline \multicolumn{9}{|l|}{$\begin{array}{l}\text { Bullding Shell Conservation } \\
\text { Features (more than one may } \\
\text { apply) } \\
\text { Roof or Ceiling }\end{array}$} \\
\hline 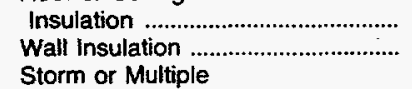 & $\begin{array}{l}196 \\
122\end{array}$ & $\begin{array}{l}195 \\
121\end{array}$ & $\begin{array}{r}23 \\
9\end{array}$ & $\begin{array}{r}11 \\
8\end{array}$ & $\begin{array}{l}39 \\
22\end{array}$ & $\begin{array}{l}29 \\
13\end{array}$ & $\begin{array}{r}101 \\
63\end{array}$ & $\begin{array}{l}77 \\
51\end{array}$ \\
\hline 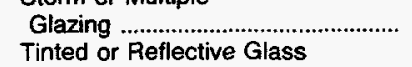 & 96 & 96 & 10 & 8 & 16 & 20 & 48 & 43 \\
\hline $\begin{array}{l}\text { or Shading Film ........................... } \\
\text { Exterior or Interior Shading }\end{array}$ & 127 & 126 & 16 & 8 & 23 & 22 & 73 & 59 \\
\hline 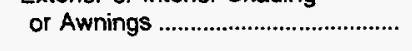 & 142 & 141 & 20 & 9 & 29 & 25 & 74 & 65 \\
\hline \multicolumn{9}{|l|}{$\begin{array}{l}\text { HVAC Conservation Features } \\
\text { (more than one may apply) }\end{array}$} \\
\hline 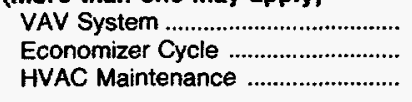 & $\begin{array}{r}65 \\
123 \\
234\end{array}$ & $\begin{array}{r}65 \\
122 \\
233\end{array}$ & $\begin{array}{r}6 \\
13 \\
27\end{array}$ & $\begin{array}{l}3 \\
8 \\
9\end{array}$ & $\begin{array}{r}8 \\
26 \\
48\end{array}$ & $\begin{array}{l}18 \\
27 \\
43\end{array}$ & $\begin{array}{r}41 \\
67 \\
121\end{array}$ & $\begin{array}{l}22 \\
51 \\
95\end{array}$ \\
\hline \multicolumn{9}{|l|}{$\begin{array}{l}\text { Energy Management Practices } \\
\text { (more than one may apply) } \\
\text { Energy Management and Control }\end{array}$} \\
\hline Enstem ................................................ & 96 & 95 & 10 & 6 & 17 & 31 & 54 & 37 \\
\hline 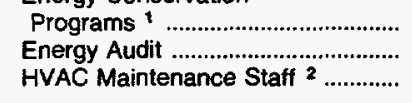 & $\begin{array}{l}48 \\
64 \\
57\end{array}$ & $\begin{array}{l}47 \\
64 \\
57\end{array}$ & $\begin{array}{l}7 \\
8 \\
3\end{array}$ & $\begin{array}{l}2 \\
4 \\
1\end{array}$ & $\begin{array}{r}6 \\
10 \\
9\end{array}$ & $\begin{array}{r}12 \\
8 \\
13\end{array}$ & $\begin{array}{l}23 \\
32 \\
40\end{array}$ & $\begin{array}{l}28 \\
29 \\
21\end{array}$ \\
\hline \multicolumn{9}{|l|}{$\begin{array}{l}\text { Off-Hours Reduction in } \\
\text { Equipment (more than one may } \\
\text { apply) }\end{array}$} \\
\hline $\begin{array}{l}\text { Heating } \\
\text { Cooling } \\
\text { Hot Water } \\
\text { Lighting }\end{array}$ & $\begin{array}{r}123 \\
124 \\
33 \\
120\end{array}$ & $\begin{array}{r}123 \\
124 \\
33 \\
120\end{array}$ & $\begin{array}{r}18 \\
17 \\
2 \\
13\end{array}$ & $\begin{array}{l}6 \\
6 \\
3 \\
5\end{array}$ & $\begin{array}{r}17 \\
17 \\
1 \\
20\end{array}$ & $\begin{array}{r}17 \\
18 \\
8 \\
17\end{array}$ & $\begin{array}{l}53 \\
54 \\
17 \\
56\end{array}$ & $\begin{array}{l}54 \\
54 \\
14 \\
53\end{array}$ \\
\hline
\end{tabular}

1 Building participates in any programs sponsored by the Federal Energy Management Program, in-house, utility, or third party.

2 HVAC maintenance staff means at least one person spends at least half their working hours maintaining the heating/cooling equipment.

$\mathrm{NC}=$ No cases in responding sample.

Notes: - Total workers are the number of workers during the main shift. - See Glossary for explanation of abbreviations and definitions of terms used in this report. - These data are from 881 federally owned buildings having the following criteria: (1) located in Federal Regions 3,6 , or 9; (2) larger than 10,000 square feet; and (3) used for a commercial purpose, other than warehouse and storage. In addition, 9 out of 10 selected buildings were from agencies other than the Department of Defense. - Statistics for the "energy end uses" represent consumption in buildings that have end use, not consumption for a particular fuel for a particular end use. FBSS = Federal Buildings Supplemental Survey. - HVAC = Heating, Ventilation, and Air Conditioning. - VAV = Variable-Air Volume. - Data are for Fiscal Year 1993 (October 1, 1992 through September 30 , 1993). - Because of rounding, data may not sum to totals.

Source: Energy Information Administration, Office of Energy Markets and End Use, 1993 Federal Buildings Supplemental Survey. 
Table 3.18. Cooling Equipment in FBSS Buildings in Federal Region 9, Number of Buildings, 1993

\begin{tabular}{|c|c|c|c|c|c|c|c|c|}
\hline \multirow[b]{2}{*}{$\begin{array}{c}\text { Building } \\
\text { Characteristics }\end{array}$} & \multirow[b]{2}{*}{$\begin{array}{c}\text { All } \\
\text { Buildings }\end{array}$} & \multirow[b]{2}{*}{$\begin{array}{c}\text { All } \\
\text { Cooled } \\
\text { Buildings }\end{array}$} & \multicolumn{6}{|c|}{$\begin{array}{l}\text { Cooling Equipment } \\
\text { (more than one may apply) }\end{array}$} \\
\hline & & & $\begin{array}{c}\text { Residential- } \\
\text { Type } \\
\text { Central Air } \\
\text { Conditioners }\end{array}$ & $\begin{array}{l}\text { Heat } \\
\text { Pumps }\end{array}$ & $\begin{array}{c}\begin{array}{c}\text { Individual } \\
\text { Air }\end{array} \\
\text { Conditioners }\end{array}$ & $\begin{array}{l}\text { District } \\
\text { Chilled } \\
\text { Water }\end{array}$ & $\begin{array}{l}\text { Central } \\
\text { Chillers }\end{array}$ & $\begin{array}{c}\text { Packaged- } \\
\text { Air- } \\
\text { Conditioning } \\
\text { Units }\end{array}$ \\
\hline 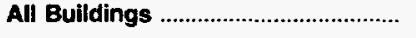 & 326 & 292 & 24 & 32 & 52 & 31 & 139 & 151 \\
\hline \multicolumn{9}{|l|}{ Building Floorspace (square feet) } \\
\hline 10,000 to 50,000 & 148 & 126 & 17 & 15 & 25 & 7 & 40 & 59 \\
\hline 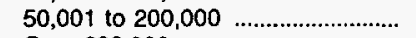 & 123 & 114 & 5 & 15 & 18 & 15 & 61 & 63 \\
\hline Over 200,000 & 55 & 52 & 2 & 2 & 9 & 9 & 38 & 29 \\
\hline \multicolumn{9}{|l|}{ Principal Building Activity } \\
\hline 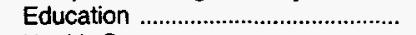 & 12 & 8 & NC & 1 & NC & NC & 2 & 3 \\
\hline 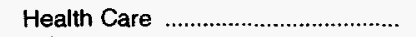 & 48 & 45 & 3 & 2 & 9 & 17 & 15 & 22 \\
\hline 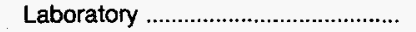 & 31 & 29 & 3 & 7 & 5 & 1 & 24 & 17 \\
\hline 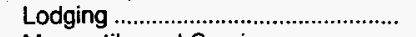 & 22 & 16 & 1 & 1 & 4 & 5 & 3 & 5 \\
\hline Mercantile and Service ................... & 63 & 60 & 7 & 8 & 11 & 1 & 30 & 30 \\
\hline Office & 92 & 88 & 8 & 9 & 16 & 5 & 45 & 54 \\
\hline 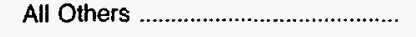 & 58 & 46 & 2 & 4 & 7 & 2 & 20 & 20 \\
\hline \multicolumn{9}{|l|}{ Year Constructed } \\
\hline 1959 or Before & 130 & 112 & 10 & 14 & 35 & 12 & 49 & 55 \\
\hline 1960 to 1969 & 55 & 50 & 3 & 5 & 9 & 2 & 32 & 29 \\
\hline 1970 to 1979 & 62 & 58 & 5 & 5 & 7 & 5 & 28 & 35 \\
\hline 1980 to 1989 & 65 & 59 & 4 & 5 & 1 & 11 & 27 & 25 \\
\hline 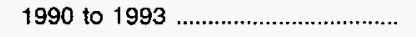 & 14 & 13 & 2 & 3 & NC & 1 & 3 & 7 \\
\hline \multicolumn{9}{|l|}{ Federal Agency } \\
\hline Department of Defense .................. & 78 & 64 & 6 & 4 & 18 & 6 & 25 & 22 \\
\hline General Services Administration ... & 39 & 38 & 2 & NC & 7 & NC & 26 & 25 \\
\hline United States Postal Service ........ & 63 & 62 & 8 & 7 & 7 & 2 & 29 & 32 \\
\hline Veterans Administration ...................... & 56 & 51 & 3 & 3 & 10 & 18 & 12 & 26 \\
\hline 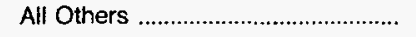 & 90 & 77 & 5 & 18 & 10 & 5 & 47 & 46 \\
\hline \multicolumn{9}{|l|}{ Workers (main shift) } \\
\hline Less than 50 & 93 & 70 & 9 & 7 & 16 & 5 & 17 & 24 \\
\hline 50 to 99 & 49 & 47 & 6 & 6 & 4 & 1 & 21 & 30 \\
\hline 100 to 499 & 134 & 128 & 7 & 17 & 25 & 17 & 62 & 69 \\
\hline 500 or More & 50 & 47 & 2 & 2 & 7 & 8 & 39 & 28 \\
\hline \multicolumn{9}{|l|}{ Weekly Operating Hours } \\
\hline 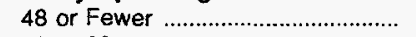 & 80 & 68 & 6 & 5 & 16 & 4 & 26 & 38 \\
\hline 49 to 60 & 65 & 61 & 3 & 5 & 14 & 4 & 36 & 37 \\
\hline 61 to 167 & 68 & 61 & 8 & 7 & 7 & 2 & 26 & 30 \\
\hline 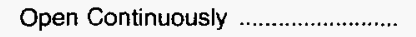 & 113 & 102 & 7 & +5 & 15 & 21 & 51 & 46 \\
\hline \multicolumn{9}{|l|}{ Multibuilding Facility } \\
\hline 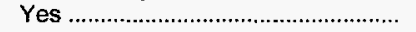 & 200 & 171 & 14 & 24 & 34 & 30 & 73 & 85 \\
\hline 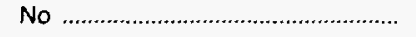 & 126 & 121 & 10 & 8 & 18 & 1 & 66 & 66 \\
\hline \multicolumn{9}{|l|}{ Percent of Floorspace Cooled } \\
\hline 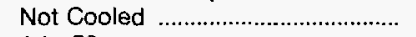 & 40 & 6 & NC & NC & NC & NC & $\mathrm{NC}$ & NC \\
\hline 1 to 50 & 55 & 55 & 6 & 5 & 27 & 3 & 15 & 25 \\
\hline 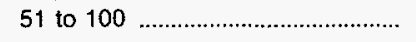 & 231 & 231 & 18 & 27 & 25 & 28 & 124 & 126 \\
\hline \multicolumn{9}{|l|}{$\begin{array}{l}\text { Energy Conservation Features } \\
\text { (more than one may apply) }\end{array}$} \\
\hline Any Conservation Feature .............. & 319 & 291 & 24 & 32 & 52 & 31 & 139 & 151 \\
\hline 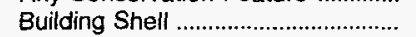 & 248 & 228 & 21 & 26 & 38 & 27 & 106 & 120 \\
\hline 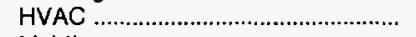 & 307 & 282 & 22 & 32 & 49 & 31 & 136 & 147 \\
\hline 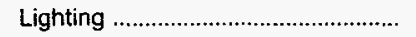 & 217 & 205 & 15 & 25 & 32 & 21 & 114 & 121 \\
\hline
\end{tabular}

See footnotes at end of table. 
Table 3.18. Cooling Equipment in FBSS Buildings in Federal Region 9, Number of Buildings, 1993 (Continued)

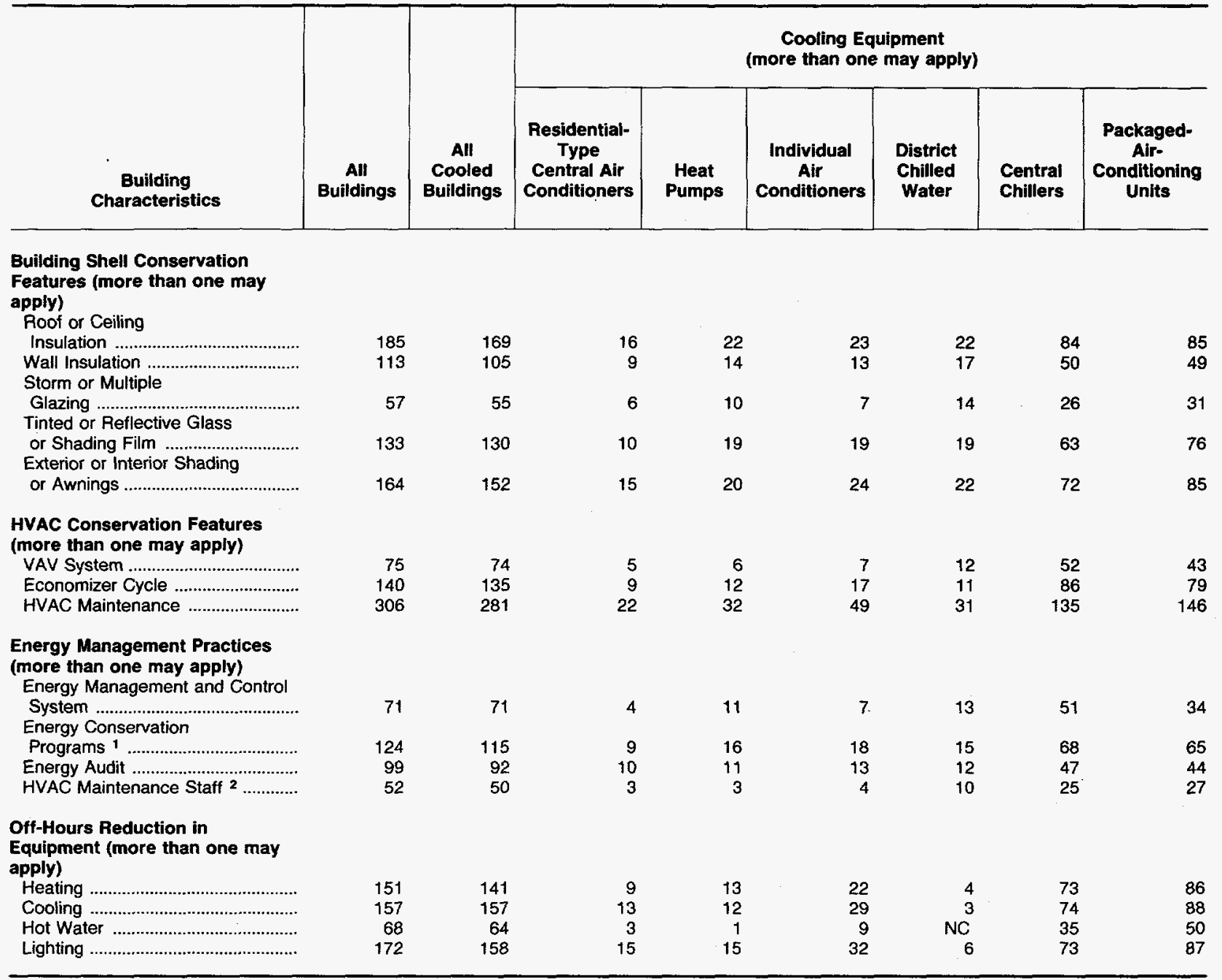

1 Building participates in any programs sponsored by the Federal Energy Management Program, in-house, utility, or third party.

2 HVAC maintenance staff means at least one person spends at least half their working hours maintaining the heating/cooling equipment.

NC $=$ No cases in responding sample.

Notes: - Total workers are the number of workers during the main shift. - See Glossary for explanation of abbreviations and definitions of terms used in this report. - These data are from 881 federally owned buildings having the following criteria: (1) located in Federal Regions 3,6 , or 9; (2) larger than 10,000 square feet; and (3) used for a commercial purpose, other than warehouse and storage: In addition, 9 out of 10 selected buildings were from agencies other than the Department of Defense. - Statistics for the "energy end uses" represent consumption in buildings that have end use, not consumption for a particular fuel for a particular end use. - FBSS = Federal Buildings Supplemental Survey. $\bullet$ HVAC = Heating, Ventilation, and Air Conditioning. - VAV = Variable-Air Volume. • Data are for Fiscal Year 1993 (October 1, 1992 through September 30, 1993). Because of rounding, data may not sum to totals.

Source: Energy Information Administration, Office of Energy Markets and End Use, 1993 Federal Buildings Supplemental Survey. 
Table 3.19. Cooling Equipment in FBSS Buildings in Federal Region 3, Floorspace, 1993

(Thousand Square Feet)

\begin{tabular}{|c|c|c|c|c|c|c|c|c|}
\hline \multirow[b]{2}{*}{$\begin{array}{c}\text { Building } \\
\text { Characteristics }\end{array}$} & \multirow[b]{2}{*}{$\begin{array}{l}\text { All } \\
\text { Buildings }\end{array}$} & \multirow[b]{2}{*}{$\begin{array}{c}\text { All } \\
\text { Cooled } \\
\text { Buildings }\end{array}$} & \multicolumn{6}{|c|}{$\begin{array}{c}\text { Cooling Equipment } \\
\text { (more than one may apply) }\end{array}$} \\
\hline & & & $\begin{array}{c}\text { Residential- } \\
\text { Type } \\
\text { Central Air } \\
\text { Conditioners }\end{array}$ & $\begin{array}{l}\text { Heat } \\
\text { Pumps }\end{array}$ & $\begin{array}{c}\text { Individual } \\
\text { Air } \\
\text { Conditioners }\end{array}$ & $\begin{array}{l}\text { District } \\
\text { Chilled } \\
\text { Water }\end{array}$ & $\begin{array}{l}\text { Central } \\
\text { Chillers }\end{array}$ & $\begin{array}{l}\text { Packaged- } \\
\text { Air- } \\
\text { Conditioning } \\
\text { Units }\end{array}$ \\
\hline 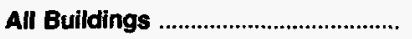 & 94,880 & 93,454 & 19,073 & 11,388 & 28,082 & 28,194 & 63,914 & 56,664 \\
\hline \multicolumn{9}{|l|}{ Building Floorspace (square feet) } \\
\hline 10,000 to 50,000 & 2,564 & 2,512 & 222 & 514 & 561 & 320 & 750 & 1,005 \\
\hline 50,001 to 200,000 & 13,166 & 12,771 & 691 & 60 & 2,589 & 4,169 & 7,591 & 4,096 \\
\hline 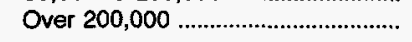 & 79,149 & 78,171 & 18,161 & 10,814 & 24,932 & 23,706 & 55,573 & 51,562 \\
\hline \multicolumn{9}{|l|}{ Principal Building Activity } \\
\hline Education & 598 & 598 & NC & NC & 258 & NC & 210 & 317 \\
\hline 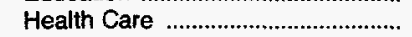 & 14,559 & 14,450 & 1,702 & 60 & 4,115 & 7,721 & 7,471 & 9,246 \\
\hline 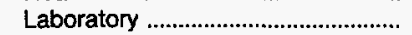 & 5,165 & 5,165 & 569 & 20 & 501 & 2,081 & 2,873 & 506 \\
\hline 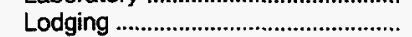 & 2,558 & 2,529 & 36 & 31 & 442 & 66 & 816 & 541 \\
\hline Mercantile and Service ..................... & 7,966 & 7,224 & 1,311 & 124 & 2,482 & NC & 5,759 & 4,086 \\
\hline 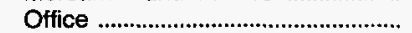 & 56,881 & 56,794 & 15,310 & 11,053 & 19,255 & 17,649 & 41,892 & 37,522 \\
\hline 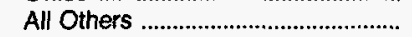 & 7,152 & 6,693 & 145 & 101 & 1,029 & 677 & 4,893 & 4,446 \\
\hline \multicolumn{9}{|l|}{ Year Constructed } \\
\hline 1959 or Before & 43,829 & 43,462 & 15,136 & 5,577 & 19,969 & 16,274 & 26,489 & 30,974 \\
\hline 1960 to 1969 & 19,564 & 19,477 & 1,286 & 2,182 & 1,608 & 5,296 & 13,536 & 6,958 \\
\hline 1970 to 1979 & 17,737 & 16,966 & 1,652 & 3,102 & 3,857 & 3,593 & 13,559 & 11,450 \\
\hline 1980 to 1989 & 8,975 & 8,775 & 999 & 458 & 1,011 & 1,787 & 6,735 & 4,831 \\
\hline 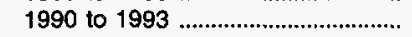 & 4,774 & 4,774 & NC & 70 & 1,638 & 1,243 & 3,595 & 2,451 \\
\hline \multicolumn{9}{|l|}{ Federal Agency } \\
\hline Department of Defense .................... & 13,988 & 13,988 & 6,836 & NC & 390 & 11,568 & 454 & 9,681 \\
\hline General Services Administration ... & 46,205 & 45,859 & 8,083 & 9,904 & 15,376 & 8,147 & 41,067 & 27,650 \\
\hline United States Postal Service ......... & 9,392 & 8,650 & 1,488 & 1,174 & 4,030 & NC & 7,635 & 6,126 \\
\hline Veterans Administration ................... & 13,306 & 12,998 & 1,702 & 98 & 4,166 & 4,896 & 8,243 & 7,196 \\
\hline 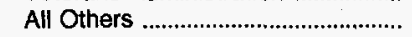 & 11,989 & 11,960 & 965 & 212 & 4,119 & 3,582 & 6,515 & 6,011 \\
\hline \multicolumn{9}{|l|}{ Workers (main shift) } \\
\hline Less than 50 & 4,102 & 3,419 & 198 & 685 & 918 & 685 & 1,132 & 1,200 \\
\hline 50 to 99 & 1,903 & 1,903 & 163 & 229 & 533 & 421 & 399 & 755 \\
\hline 100 to 499 & 13,632 & 13,608 & 552 & 1,299 & 2,527 & 4,132 & 7,825 & 5,116 \\
\hline 500 or More & 75,243 & 74,524 & 18,161 & 9,176 & 24,104 & 22,956 & 54,558 & 49,592 \\
\hline \multicolumn{9}{|l|}{ Weekly Operating Hours } \\
\hline 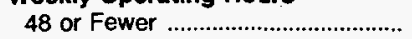 & 9,694 & 9,499 & 3,943 & 3,732 & 3,561 & 1,164 & 6,286 & 7,577 \\
\hline 49 to 60 & 25,540 & 25,540 & 2,461 & 3,575 & 9,528 & 7,076 & 20,682 & 14,457 \\
\hline 61 to 167 & 11,993 & 11,969 & 2,377 & 2,901 & 3,956 & 938 & 10,252 & 6,419 \\
\hline 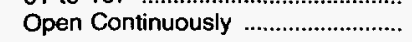 & 47,653 & 46,446 & 10,293 & 1,181 & 11,037 & 19,016 & 26,695 & 28,210 \\
\hline \multicolumn{9}{|l|}{ Multibuilding Facility } \\
\hline 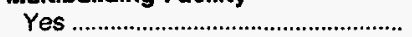 & 43,861 & 42,718 & 6,223 & 7,434 & 12,523 & 18,875 & 24,994 & 22,887 \\
\hline No & 51,018 & 50,736 & 12,851 & 3,954 & 15,559 & 9,319 & 38,921 & 33,777 \\
\hline \multicolumn{9}{|l|}{ Percent of Floorspace Cooled } \\
\hline Not Cooled & 3,582 & 2,156 & NC & NC & NC & NC & NC & NC \\
\hline 1 to 50 & 9,874 & 9,874 & 78 & 1,439 & 5,230 & 5,301 & 5,180 & 9,078 \\
\hline 51 to $100 \ldots \ldots$ & 81,424 & 81,424 & 18,995 & 9,949 & 22,852 & 22,893 & 58,734 & 47,586 \\
\hline \multicolumn{9}{|l|}{$\begin{array}{l}\text { Energy Conservation Features } \\
\text { (more than one may apply) }\end{array}$} \\
\hline Any Conservation Feature .............. & 94,361 & 93,394 & 19,073 & 11,388 & 28,082 & 28,194 & 63,914 & 56,664 \\
\hline 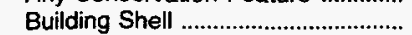 & 84,557 & 83,590 & 16,160 & 9,517 & 25,938 & 28,046 & 56,118 & 51,507 \\
\hline HVAC & 93,543 & 92,576 & 19,060 & 10,951 & 28,023 & 28,194 & 63,281 & 56,075 \\
\hline 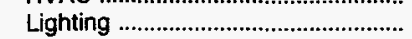 & 80,953 & 80,039 & 16,883 & 11,170 & 23,933 & 23,915 & 57,368 & 51,246 \\
\hline
\end{tabular}

See footnotes at end of table. 
Table 3.19. Cooling Equipment in FBSS Buildings in Federal Region 3, Floorspace, 1993 (Continued)

(Thousand Square Feet)

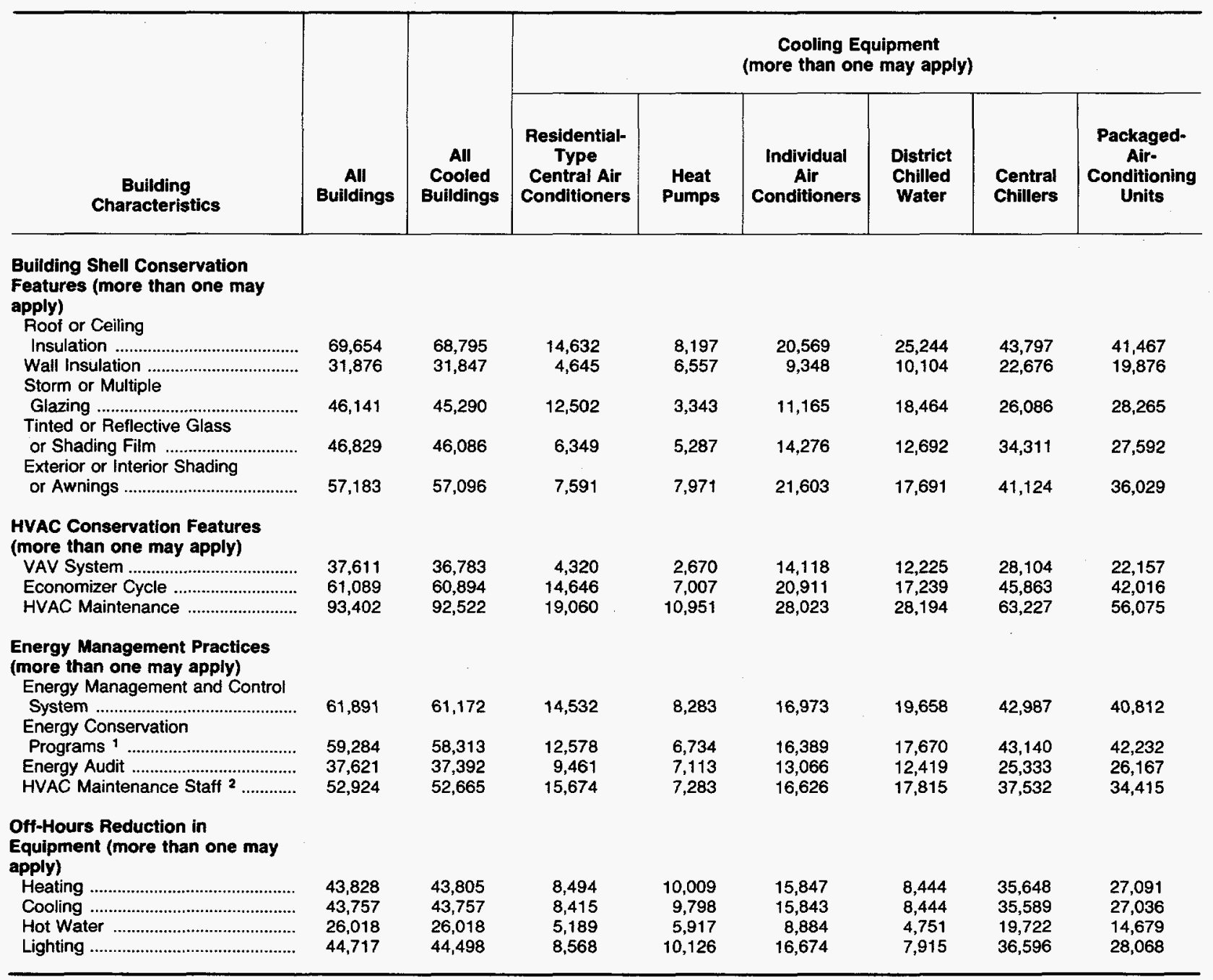

1 Building participates in any programs sponsored by the Federal Energy Management Program, in-house, utility, or third party.

2 HVAC maintenance staff means at least one person spends at least half their working hours maintaining the heating/cooling equipment.

$\mathrm{NC}=$ No cases in responding sample.

Notes: - Total workers are the number of workers during the main shift. See Glossary for explanation of abbreviations and definitions of terms used in this report. - These data are from 881 federally owned buildings having the following criteria: (1) located in Federal Regions 3,6 , or 9; (2) larger than 10,000 square feet; and (3) used for a commercial purpose, other than warehouse and storage. In addition, 9 out of 10 selected buildings were from agencies other than the Department of Defense. - Statistics for the "energy end uses" represent consumption in buildings that have end use, not consumption for a particular fuel for a particular end use. - FBSS = Federal Buildings Supplemental Survey. - HVAC = Heating, Ventilation, and Air Conditioning. - VAV = Variable-Air Volume. - Data are for Fiscal Year 1993 (October 1, 1992 through September 30 , 1993). Because of rounding, data may not sum to totals.

Source: Energy Information Administration, Office of Energy Markets and End Use, 1993 Federal Buildings Supplemental Survey. 
Table 3.20. Cooling Equipment in FBSS Buildings in Federal Region 6, Floorspace, 1993

(Thousand Square Feet)

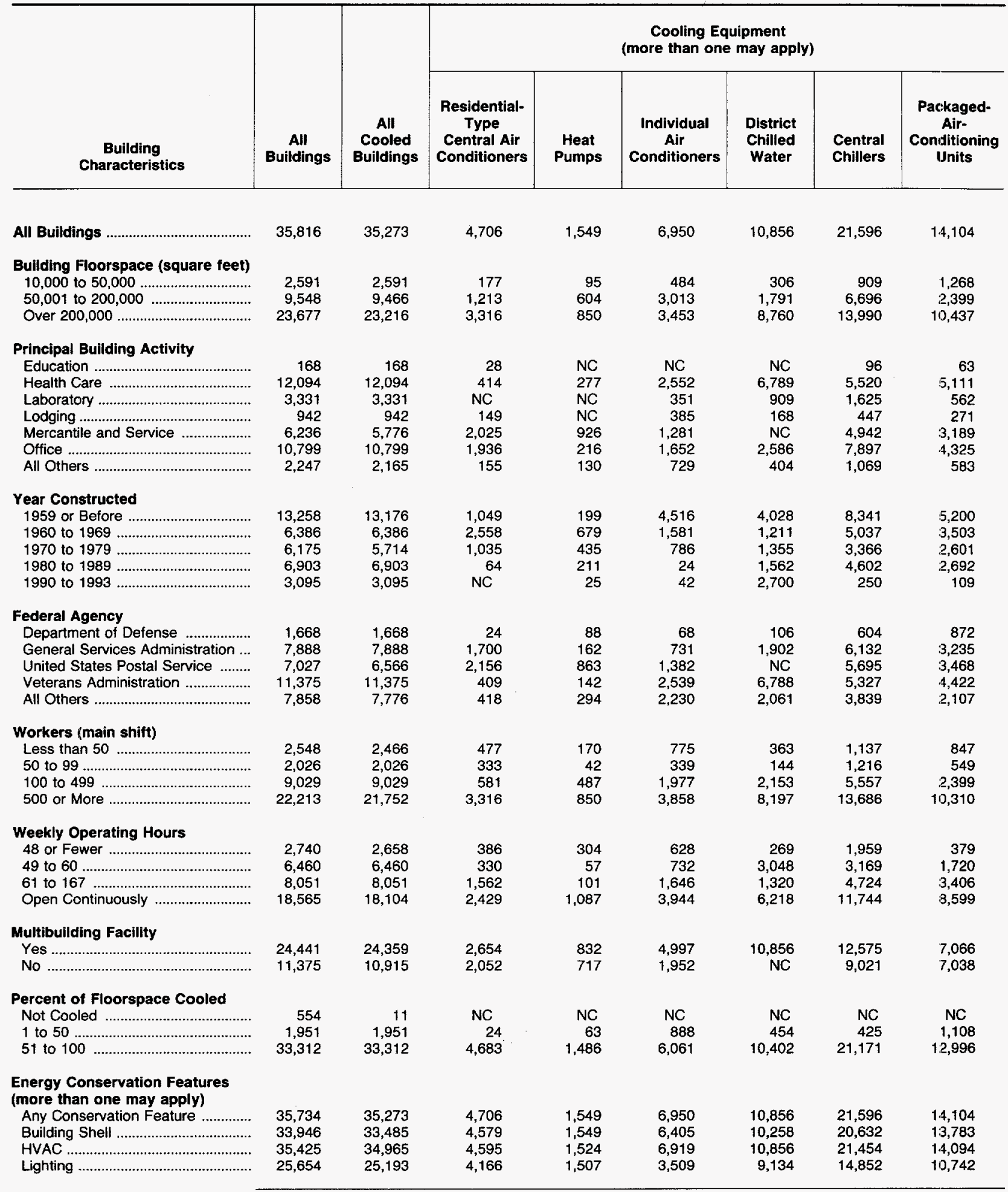

See footnotes at end of table. 
Table 3.20. Cooling Equipment in FBSS Buildings in Federal Region 6, Floorspace, 1993 (Continued)

(Thousand Square Feet)

\begin{tabular}{|c|c|c|c|c|c|c|c|c|}
\hline \multirow[b]{2}{*}{$\begin{array}{l}\text { Building } \\
\text { Characteristics }\end{array}$} & \multirow[b]{2}{*}{$\begin{array}{l}\text { All } \\
\text { Buildings }\end{array}$} & \multirow[b]{2}{*}{$\begin{array}{l}\text { All } \\
\text { Cooled } \\
\text { Buildings }\end{array}$} & \multicolumn{6}{|c|}{$\begin{array}{l}\text { Cooling Equipment } \\
\text { (more than one may apply) }\end{array}$} \\
\hline & & & $\begin{array}{l}\text { Residential- } \\
\text { Type } \\
\text { Central Air } \\
\text { Conditioners }\end{array}$ & $\begin{array}{l}\text { Heat } \\
\text { Pumps }\end{array}$ & \begin{tabular}{|c|}
$\begin{array}{c}\text { Individual } \\
\text { Air } \\
\text { Conditioners }\end{array}$ \\
\end{tabular} & $\begin{array}{l}\text { District } \\
\text { Chilled } \\
\text { Water }\end{array}$ & $\begin{array}{l}\text { Central } \\
\text { Chillers }\end{array}$ & $\begin{array}{l}\text { Packaged- } \\
\text { Alr- } \\
\text { Conditioning } \\
\text { Units }\end{array}$ \\
\hline \multicolumn{9}{|l|}{$\begin{array}{l}\text { Bullding Shell Conservation } \\
\text { Features (more than one may } \\
\text { apply) } \\
\text { Roof or Ceiling }\end{array}$} \\
\hline 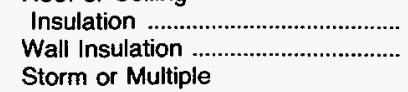 & $\begin{array}{l}28,249 \\
20,550\end{array}$ & $\begin{array}{l}27,789 \\
20,089\end{array}$ & $\begin{array}{l}3,715 \\
1,922\end{array}$ & $\begin{array}{l}1,549 \\
1,046\end{array}$ & $\begin{array}{l}4,623 \\
1,632\end{array}$ & $\begin{array}{l}8,150 \\
5,793\end{array}$ & $\begin{array}{l}17,119 \\
11,673\end{array}$ & $\begin{array}{r}10,265 \\
7,286\end{array}$ \\
\hline Glazing & 17,939 & 17,939 & 960 & 858 & 2,937 & 7,518 & 9,559 & 7,045 \\
\hline $\begin{array}{l}\text { or Shading Film ........................ } \\
\text { Exterior or Interior Shading }\end{array}$ & 24,768 & 24,307 & 4,010 & 1,350 & 3,972 & 8,554 & 14,981 & 10,341 \\
\hline 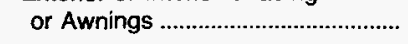 & 26,067 & 25,606 & 3,685 & 1,019 & 5,014 & 8,430 & 15,968 & 11,876 \\
\hline \multicolumn{9}{|l|}{$\begin{array}{l}\text { HVAC Conservation Features } \\
\text { (more than one may apply) }\end{array}$} \\
\hline 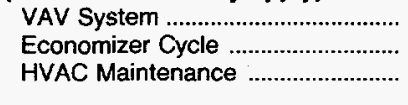 & $\begin{array}{l}18,518 \\
23,542 \\
35,341\end{array}$ & $\begin{array}{l}18,518 \\
23,081 \\
34,880\end{array}$ & $\begin{array}{l}1,443 \\
2,507 \\
4,595\end{array}$ & $\begin{array}{r}305 \\
994 \\
1,461\end{array}$ & $\begin{array}{l}2,105 \\
4,263 \\
6,919\end{array}$ & $\begin{array}{r}6,902 \\
8,463 \\
10,834\end{array}$ & $\begin{array}{l}10,897 \\
13,305 \\
21,454\end{array}$ & $\begin{array}{r}7,170 \\
10,331 \\
14,031\end{array}$ \\
\hline \multicolumn{9}{|l|}{$\begin{array}{l}\text { Energy Management Practices } \\
\text { (more than one may apply) } \\
\text { Energy Management and Control }\end{array}$} \\
\hline $\begin{array}{l}\text { System } \\
\text { Energy Conservation }\end{array}$ & 23,099 & 22,638 & 2,379 & 829 & 4,000 & 9,349 & 13,285 & 9,541 \\
\hline 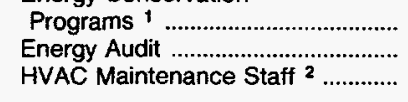 & $\begin{array}{l}13,513 \\
11,690 \\
15,538\end{array}$ & $\begin{array}{l}13,052 \\
11,690 \\
15,538\end{array}$ & $\begin{array}{r}2,207 \\
1,630 \\
673\end{array}$ & $\begin{array}{r}88 \\
618 \\
162\end{array}$ & $\begin{array}{l}1,009 \\
1,946 \\
2,121\end{array}$ & $\begin{array}{l}5,258 \\
2,347 \\
5,840\end{array}$ & $\begin{array}{l}7,116 \\
8,219 \\
9,365\end{array}$ & $\begin{array}{l}6,929 \\
6,103 \\
6,418\end{array}$ \\
\hline \multicolumn{9}{|l|}{$\begin{array}{l}\text { Off-Hours Reduction in } \\
\text { Equipment (more than one may } \\
\text { apply) }\end{array}$} \\
\hline 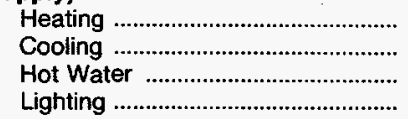 & $\begin{array}{r}13,162 \\
13,864 \\
4,554 \\
12,847\end{array}$ & $\begin{array}{r}13,162 \\
13,864 \\
4,554 \\
12,847\end{array}$ & $\begin{array}{r}2,260 \\
2,237 \\
180 \\
2,115\end{array}$ & $\begin{array}{l}449 \\
449 \\
117 \\
405\end{array}$ & $\begin{array}{r}1,868 \\
1,868 \\
190 \\
1,998\end{array}$ & $\begin{array}{l}3,760 \\
4,163 \\
1,668 \\
3,185\end{array}$ & $\begin{array}{l}7,813 \\
8,135 \\
2,659 \\
8,199\end{array}$ & $\begin{array}{l}4,832 \\
4,832 \\
1,764 \\
4,940\end{array}$ \\
\hline
\end{tabular}

1 Building participates in any programs sponsored by the Federal Energy Management Program, in-house, utility, or third party.

2 HVAC maintenance staff means at least one person spends at least half their working hours maintaining the heating/cooling equipment. $\mathrm{NC}=$ No cases in responding sample.

Notes: - Total workers are the number of workers during the main shift. - See Glossary for explanation of abbreviations and definitions of terms used in this report. - These data are from 881 federally owned buildings having the following criteria: (1) located in Federal Regions 3 , 6 , or 9; (2) larger than 10,000 square feet; and (3) used for a commercial purpose, other than warehouse and storage. In addition, 9 out of 10 selected buildings were from agencies other than the Department of Defense. - Statistics for the "energy end uses" represent consumption in buildings that have end use, not consumption for a particular fuel for a particular end use. $\bullet$ FBSS = Federal Buildings Supplemental Survey. $\bullet$ HVAC = Heating, Ventilation, and Air Conditioning. - VAV = Variable-Air Volume. - Data are for Fiscal Year 1993 (October 1,1992 through September 30, 1993). Because of rounding, data may not sum to totals.

Source: Energy Information Administration, Office of Energy Markets and End Use, 1993 Federal Buildings Supplemental Survey. 
Table 3.21. Cooling Equipment in FBSS Buildings in Federal Region 9, Floorspace, 1993

(Thousand Square Feet)

\begin{tabular}{|c|c|c|c|c|c|c|c|c|}
\hline \multirow[b]{2}{*}{$\begin{array}{c}\text { Building } \\
\text { Characteristics }\end{array}$} & \multirow[b]{2}{*}{$\begin{array}{c}\text { All } \\
\text { Buildings }\end{array}$} & \multirow[b]{2}{*}{$\begin{array}{c}\text { All } \\
\text { Cooled } \\
\text { Buildings }\end{array}$} & \multicolumn{6}{|c|}{$\begin{array}{l}\text { Cooling Equipment } \\
\text { (more than one may apply) }\end{array}$} \\
\hline & & & $\begin{array}{l}\text { Residential- } \\
\text { Type } \\
\text { Central Air } \\
\text { Conditioners }\end{array}$ & $\begin{array}{l}\text { Heat } \\
\text { Pumps }\end{array}$ & $\begin{array}{c}\text { Individual } \\
\text { Air } \\
\text { Conditioners }\end{array}$ & $\begin{array}{l}\text { District } \\
\text { Chilled } \\
\text { Water }\end{array}$ & $\begin{array}{l}\text { Central } \\
\text { Chillers }\end{array}$ & $\begin{array}{l}\text { Packaged- } \\
\text { Alr- } \\
\text { Conditioning } \\
\text { Units }\end{array}$ \\
\hline 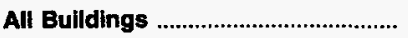 & 44,316 & 41,907 & 1,900 & 2,724 & 5,723 & 7,647 & 27,723 & 23,989 \\
\hline \multicolumn{9}{|l|}{ Building Floorspace (square feet) } \\
\hline 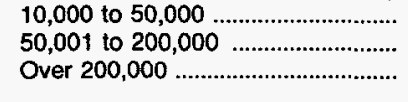 & $\begin{array}{r}3,858 \\
12,680 \\
27,778\end{array}$ & $\begin{array}{r}3,277 \\
11,875 \\
26,754\end{array}$ & $\begin{array}{l}387 \\
674 \\
839\end{array}$ & $\begin{array}{r}371 \\
1,615 \\
738\end{array}$ & $\begin{array}{r}607 \\
1,884 \\
3,232\end{array}$ & $\begin{array}{r}244 \\
1,641 \\
5,763\end{array}$ & $\begin{array}{r}1,084 \\
6,933 \\
19,706\end{array}$ & $\begin{array}{r}1,622 \\
6,530 \\
15,837\end{array}$ \\
\hline 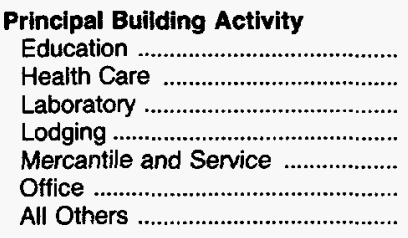 & $\begin{array}{r}628 \\
9,903 \\
2,601 \\
1,220 \\
8,194 \\
16,380 \\
5,390\end{array}$ & $\begin{array}{r}488 \\
9,711 \\
2,570 \\
982 \\
8,079 \\
15,551 \\
4,526\end{array}$ & $\begin{array}{l}\text { NC } \\
249 \\
243 \\
15 \\
587 \\
417 \\
388\end{array}$ & $\begin{array}{r}38 \\
208 \\
657 \\
59 \\
1,140 \\
464 \\
159\end{array}$ & $\begin{array}{r}\text { NC } \\
650 \\
677 \\
79 \\
1,524 \\
2,466 \\
326\end{array}$ & $\begin{array}{r}\text { NC } \\
4,898 \\
198 \\
165 \\
466 \\
448 \\
1,472\end{array}$ & $\begin{array}{r}128 \\
4,137 \\
2,240 \\
310 \\
6,663 \\
12,001 \\
2,245\end{array}$ & $\begin{array}{r}205 \\
5,080 \\
1,689 \\
208 \\
4,637 \\
10,923 \\
1,246\end{array}$ \\
\hline 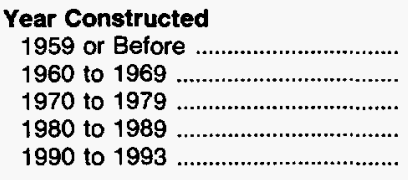 & $\begin{array}{r}13,553 \\
9,082 \\
12,041 \\
7,955 \\
1,684\end{array}$ & $\begin{array}{r}12,111 \\
8,892 \\
11,601 \\
7,761 \\
1,541\end{array}$ & $\begin{array}{r}1,002 \\
43 \\
285 \\
92 \\
478\end{array}$ & $\begin{array}{r}1,210 \\
574 \\
603 \\
290 \\
46\end{array}$ & $\begin{array}{r}2,910 \\
1,901 \\
898 \\
14 \\
N C\end{array}$ & $\begin{array}{r}2,601 \\
445 \\
1,585 \\
2,551 \\
466\end{array}$ & $\begin{array}{r}6,210 \\
6,874 \\
9,075 \\
4,892 \\
672\end{array}$ & $\begin{array}{r}5,923 \\
5,839 \\
7,645 \\
3,758 \\
823\end{array}$ \\
\hline 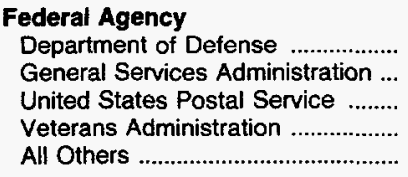 & $\begin{array}{r}8,489 \\
12,505 \\
8,387 \\
8,824 \\
6,111\end{array}$ & $\begin{array}{r}7,225 \\
12,146 \\
8,375 \\
8,469 \\
5,691\end{array}$ & $\begin{array}{l}515 \\
202 \\
615 \\
289 \\
280\end{array}$ & $\begin{array}{r}161 \\
\mathrm{NC} \\
1,089 \\
266 \\
1,207\end{array}$ & $\begin{array}{r}1,222 \\
1,457 \\
1,452 \\
772 \\
821\end{array}$ & $\begin{array}{r}3,069 \\
\text { NC } \\
626 \\
3,504 \\
448\end{array}$ & $\begin{array}{r}2,706 \\
10,369 \\
6,693 \\
3,815 \\
4,140\end{array}$ & $\begin{array}{l}1,382 \\
8,924 \\
4,763 \\
5,078 \\
3,843\end{array}$ \\
\hline $\begin{array}{l}\text { Workers (main shift) } \\
\text { Less than } 50 \\
50 \text { to } 99 \\
100 \text { to } 499 \\
500 \text { or More }\end{array}$ & $\begin{array}{r}3,684 \\
2,161 \\
14,017 \\
24,454\end{array}$ & $\begin{array}{r}2,830 \\
2,032 \\
13,382 \\
23,663\end{array}$ & $\begin{array}{l}170 \\
171 \\
720 \\
839\end{array}$ & $\begin{array}{r}112 \\
409 \\
1,667 \\
536\end{array}$ & $\begin{array}{r}732 \\
205 \\
2,633 \\
2,153\end{array}$ & $\begin{array}{r}165 \\
76 \\
2,026 \\
5,380\end{array}$ & $\begin{array}{r}492 \\
1,050 \\
7,527 \\
18,654\end{array}$ & $\begin{array}{r}1,025 \\
1,396 \\
6,942 \\
14,625\end{array}$ \\
\hline 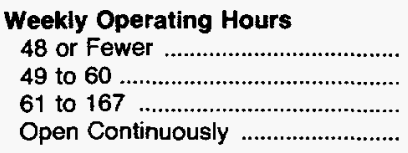 & $\begin{array}{r}4,822 \\
13,424 \\
5,706 \\
20,364\end{array}$ & $\begin{array}{r}4,322 \\
12,875 \\
5,264 \\
19,446\end{array}$ & $\begin{array}{l}171 \\
589 \\
210 \\
930\end{array}$ & $\begin{array}{r}721 \\
412 \\
306 \\
1,285\end{array}$ & $\begin{array}{l}1,185 \\
1,683 \\
1,378 \\
1,477\end{array}$ & $\begin{array}{r}372 \\
742 \\
172 \\
6,361\end{array}$ & $\begin{array}{r}1,813 \\
10,962 \\
1 \quad 3,394 \\
11,554\end{array}$ & $\begin{array}{l}2,524 \\
9,267 \\
2,388 \\
9,810\end{array}$ \\
\hline $\begin{array}{l}\text { Multibuilding Facility } \\
\text { Yes }\end{array}$ & $\begin{array}{l}26,821 \\
17,495\end{array}$ & $\begin{array}{l}24,882 \\
17,025\end{array}$ & $\begin{array}{r}1,673 \\
227\end{array}$ & $\begin{array}{l}1,696 \\
1,028\end{array}$ & $\begin{array}{l}2,956 \\
2,767\end{array}$ & $\begin{array}{r}7,487 \\
160\end{array}$ & $\begin{array}{l}14,574 \\
13,149\end{array}$ & $\begin{array}{l}13,236 \\
10,753\end{array}$ \\
\hline 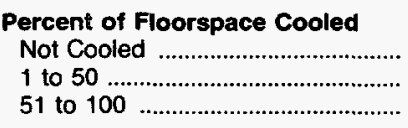 & $\begin{array}{r}2,956 \\
7,304 \\
34,056\end{array}$ & $\begin{array}{r}547 \\
7,304 \\
34,056\end{array}$ & $\begin{array}{c}\text { NC } \\
531 \\
1,369\end{array}$ & $\begin{array}{c}\text { NC } \\
412 \\
2,312\end{array}$ & $\begin{array}{c}N C \\
2,253 \\
3,471\end{array}$ & $\begin{array}{c}N C \\
1,436 \\
6,212\end{array}$ & $\begin{array}{r}\mathrm{NC} \\
3,430 \\
24,293\end{array}$ & $\begin{array}{c}\text { NC } \\
3,059 \\
20,930\end{array}$ \\
\hline 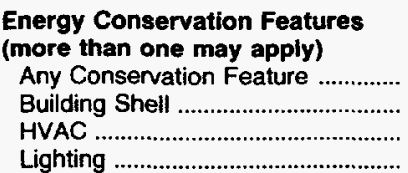 & $\begin{array}{l}44,023 \\
34,105 \\
43,116 \\
34,825\end{array}$ & $\begin{array}{l}41,894 \\
32,754 \\
41,282 \\
33,846\end{array}$ & $\begin{array}{l}1,900 \\
1,670 \\
1,847 \\
1,693\end{array}$ & $\begin{array}{l}2,724 \\
2,320 \\
2,724 \\
2,439\end{array}$ & $\begin{array}{l}5,723 \\
4,149 \\
5,520 \\
4,449\end{array}$ & $\begin{array}{l}7,647 \\
7,342 \\
7,647 \\
5,559\end{array}$ & $\begin{array}{l}27,723 \\
20,592 \\
27,301 \\
24,098\end{array}$ & $\begin{array}{l}23,989 \\
18,416 \\
23,777 \\
20,584\end{array}$ \\
\hline
\end{tabular}

See footnotes at end of table. 

Table 3.21. Cooling Equipment in FBSS Buildings in Federal Region 9,
Floorspace, 1993 (Continued)

(Thousand Square Feet)

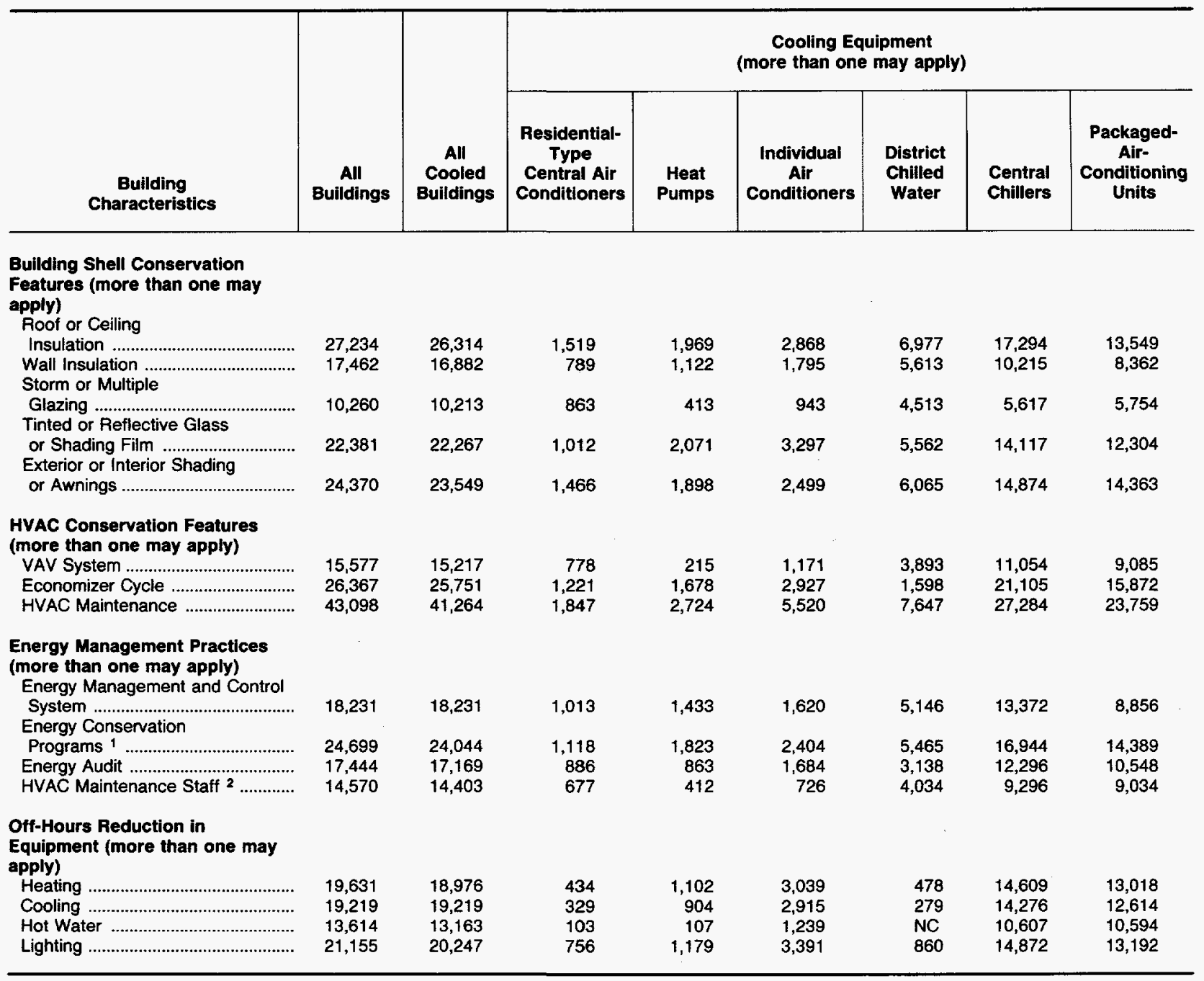

Building participates in any programs sponsored by the Federal Energy Management Program, in-house, utility, or third party.

2 HVAC maintenance staff means at least one person spends at least half their working hours maintaining the heating/cooling equipment. $\mathrm{NC}=$ No cases in responding sample.

Notes: - Total workers are the number of workers during the main shift. - See Glossary for explanation of abbreviations and definitions of terms used in this report. These data are from 881 federally owned buildings having the following criteria: (1) located in Federal Regions 3,6 , or 9; (2) larger than 10,000 square feet; and (3) used for a commercial purpose, other than warehouse and storage. In addition, 9 out of 10 selected buildings were from agencies other than the Department of Defense. - Statistics for the "energy end uses" represent consumption in buildings that have end use, not consumption for a particular fuel for a particular end use. - FBSS = Federal Buildings Supplemental Survey. - HVAC = Heating, Ventilation, and Air Conditioning. - VAV = Variable-Air Volume. - Data are for Fiscal Year 1993 (October 1, 1992 through September 30, 1993). - Because of rounding, data may not sum to totals.

Source: Energy Information Administration, Office of Energy Markets and End Use, 1993 Federal Buildings Supplemental Survey. 
Table 3.22. Refrigeration Equipment in FBSS Buildings in Federal Region 3, Number of Buildings and Floorspace, 1993

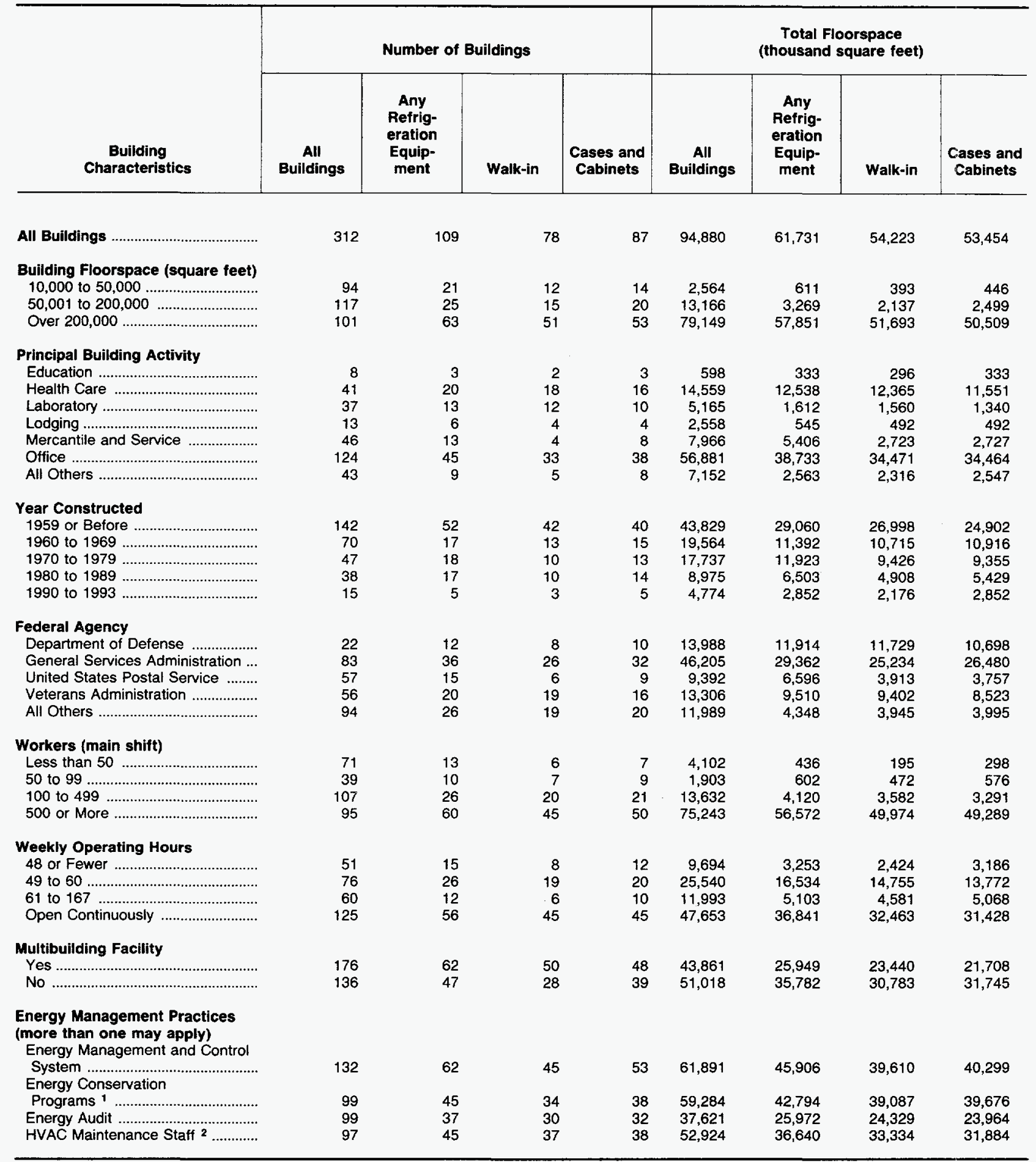

1 Building participates in any programs sponsored by the Federal Energy Management Program, in-house, utility, or third party.

2 HVAC maintenance staff means at least one person spends at least half their working hours maintaining the heating/cooling equipment.

Notes: - Total workers are the number of workers during the main shift. - See Glossary for explanation of abbreviations and definitions of terms used in this report. - These data are from 881 federally owned buildings having the following criteria: (1) located in Federal Regions 3 , 6 , or $9 ;$ (2) larger than 10,000 square feet; and (3) used for a commercial purpose, other than warehouse and storage. In addition, 9 out of 10 selected buildings were from agencies other than the Department of Defense. - Statistics for the "energy end uses" represent consumption in buildings that have end use, not consumption for a particular fuel for a particular end use. - FBSS = Federal Buildings Supplemental Survey. - HVAC = Heating, Ventilation, and Air Conditioning. - Data are for Fiscal Year 1993 (October 1, 1992 through September 30, 1993). $\bullet$ Because of rounding, data may not sum to totals.

Source: Energy Information Administration, Office of Energy Markets and End Use, 1993 Federal Buildings Supplemental Survey. 
Table 3.23. Refrigeration Equipment in FBSS Buildings in Federal Region 6, Number of Buildings and Floorspace, 1993

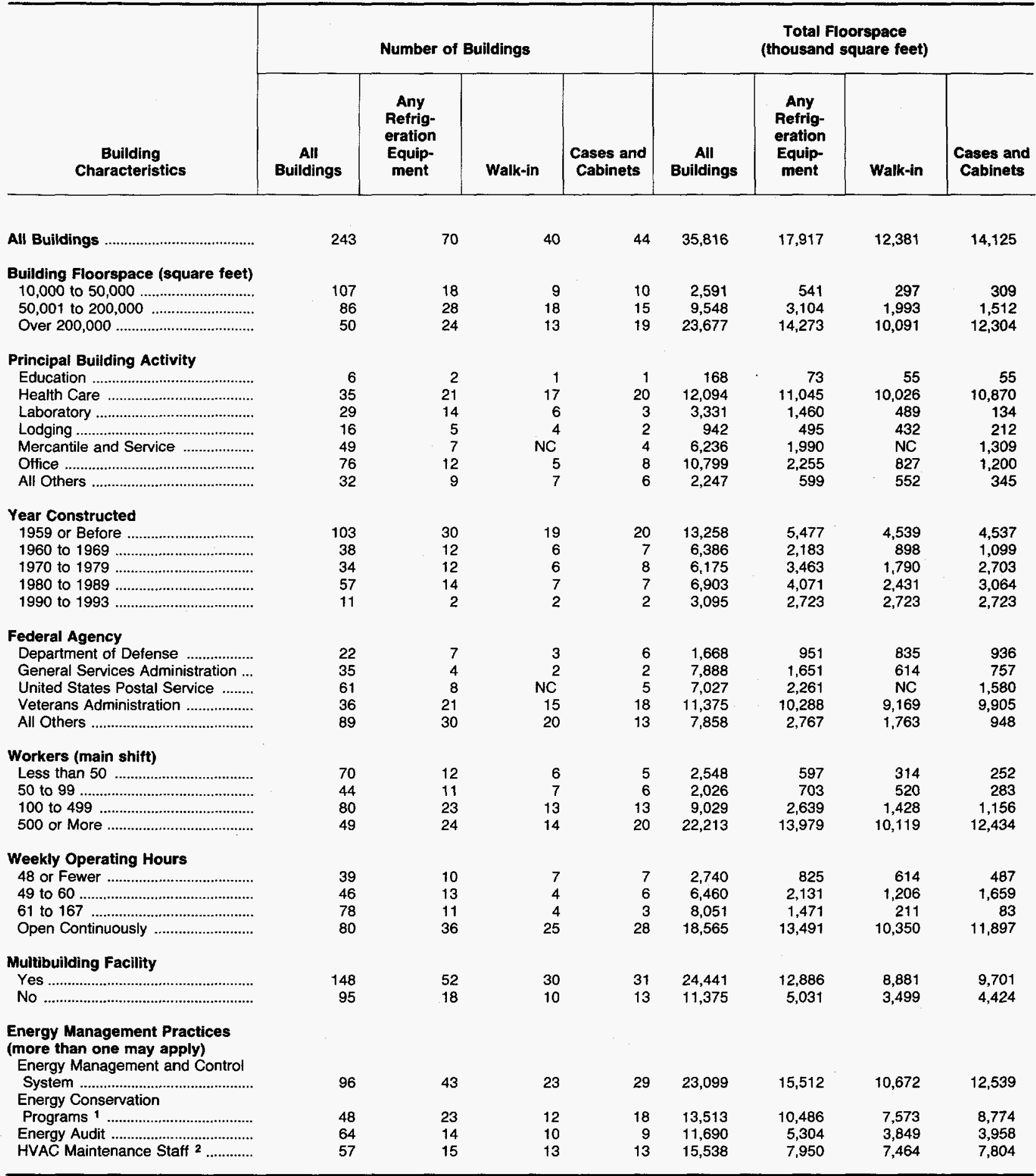

1 Building participates in any programs sponsored by the Federal Energy Management Program, in-house, utility, or third party.

2 HVAC maintenance staff means at least one person spends at least half their working hours maintaining the heating/cooling equipment.

NC $=$ No cases in responding sample.

Notes: - Total workers are the number of workers during the main shift. - See Glossary for explanation of abbreviations and definitions of terms used in this report. - These data are from 881 federally owned buildings having the following criteria: (1) located in Federal Regions 3, 6, or 9; (2) larger than 10,000 square feet; and (3) used for a commercial purpose, other than warehouse and storage. In addition, 9 out of 10 selected buildings were from agencies other than the Department of Defense. - Statistics for the "energy end uses" represent consumption in buildings that have end use, not consumption for a particular fuel for a particular end use. - FBSS = Federal Buildings Supplemental Survey. - HVAC = Heating, Ventilation, and Air Conditioning. - Data are for Fiscal Year 1993 (October 1, 1992 through September 30, 1993). - Because of rounding, data may not sum to totals.

Source: Energy Information Administration, Office of Energy Markets and End Use, 1993 Federal Buildings Supplemental Survey. 
Table 3.24. Refrigeration Equipment in FBSS Buildings in Federal Region 9, Number of Buildings and Floorspace, 1993

\begin{tabular}{|c|c|c|c|c|c|c|c|c|}
\hline \multirow[b]{2}{*}{$\begin{array}{c}\text { Bullding } \\
\text { Characteristics }\end{array}$} & \multicolumn{4}{|c|}{ Number of Bulldings } & \multicolumn{4}{|c|}{$\begin{array}{l}\text { Total Floorspace } \\
\text { (thousand square feet) }\end{array}$} \\
\hline & $\begin{array}{c}\text { All } \\
\text { Buildings }\end{array}$ & $\begin{array}{l}\text { Any } \\
\text { Refrig- } \\
\text { eration } \\
\text { Equip- } \\
\text { ment }\end{array}$ & Walk-in & $\begin{array}{c}\text { Cases and } \\
\text { Cabinets }\end{array}$ & $\begin{array}{l}\text { All } \\
\text { Buildings }\end{array}$ & $\begin{array}{c}\text { Any } \\
\text { Refrig- } \\
\text { eration } \\
\text { Equip- } \\
\text { ment }\end{array}$ & Walk-in & $\begin{array}{c}\text { Cases and } \\
\text { Cabinets }\end{array}$ \\
\hline 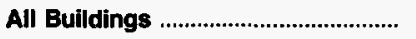 & 326 & 63 & 44 & 50 & 44,316 & 18,781 & 12,536 & 14,928 \\
\hline \multicolumn{9}{|l|}{ Bullding Floorspace (square feet) } \\
\hline 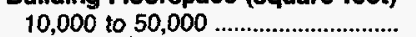 & 148 & 14 & 11 & 12 & 3,858 & 336 & 237 & 299 \\
\hline 50,001 to 200,000 & 123 & 22 & 17 & 19 & 12,680 & 2,380 & 1,883 & 2,188 \\
\hline Over $200,000 \ldots \ldots \ldots \ldots \ldots \ldots \ldots \ldots$ & 55 & 27 & 16 & 19 & 27,778 & 16,065 & 10,416 & 12,441 \\
\hline \multicolumn{9}{|l|}{ Principal Building Activity } \\
\hline 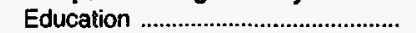 & 12 & 2 & 2 & 2 & 628 & 94 & 94 & 94 \\
\hline 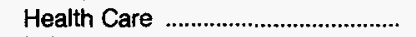 & 48 & $1 \overline{8}$ & 15 & 15 & 9,903 & 7,062 & 6,811 & 6,349 \\
\hline 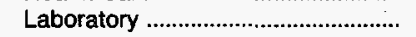 & 31 & 4 & 4 & 4 & 2,601 & 325 & 325 & 325 \\
\hline 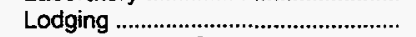 & 22 & 4 & 4 & 3 & 1,220 & 131 & 131 & 72 \\
\hline Mercantile and Service ................... & 63 & 11 & 6 & 8 & 8,194 & 3,921 & 2,372 & 2,793 \\
\hline 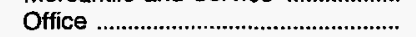 & 92 & 11 & 3 & 9 & 16,380 & 4,880 & 1,919 & 4,435 \\
\hline All Others & 58 & 13 & 10 & 9 & 5,390 & 2,368 & 885 & 860 \\
\hline \multicolumn{9}{|l|}{ Year Constructed } \\
\hline 1959 or Before & 130 & 19 & 14 & 15 & 13,553 & 3,577 & 1,552 & 1,725 \\
\hline 1960 to 1969 & 55 & 13 & 8 & 11 & 9,082 & 3,410 & 2,083 & 3,018 \\
\hline 1970 to 1979 & 62 & 14 & 12 & 12 & 12,041 & 6,298 & 4,796 & 5,904 \\
\hline 1980 to 1989 & 65 & 14 & 9 & 11 & 7,955 & 4,647 & 3,788 & 3,963 \\
\hline 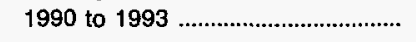 & 14 & 3 & 1 & 1 & 1,684 & 848 & $(*)$ & $\left(^{*}\right)$ \\
\hline \multicolumn{9}{|l|}{ Federal Agency } \\
\hline 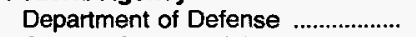 & 78 & 12 & 7 & 9 & 8,489 & 3,726 & 1,758 & 2,187 \\
\hline General Services Administration ... & 39 & 8 & 3 & 7 & 12,505 & 4,332 & 1,867 & 3,955 \\
\hline United States Postal Service ......... & 63 & 9 & 4 & 6 & 8,387 & 3,785 & 2,236 & 2,657 \\
\hline Veterans Administration .................... & 56 & 17 & 15 & 13 & 8,824 & 5,394 & 5,228 & 4,623 \\
\hline All Others & 90 & 17 & 15 & 15 & 6,111 & 1,544 & 1,448 & 1,507 \\
\hline \multicolumn{9}{|l|}{ Workers (main shift) } \\
\hline Less than 50 & 93 & 10 & 10 & 8 & 3,684 & 234 & 234 & 197 \\
\hline 50 to 99 & 49 & 2 & 1 & 2 & 2,161 & 66 & 54 & 66 \\
\hline 100 to 499 & 134 & 24 & 16 & 20 & 14,017 & 3,029 & 2,054 & 2,525 \\
\hline 500 or More & 50 & 27 & 17 & 20 & 24,454 & 15,451 & 10,194 & 12,140 \\
\hline \multicolumn{9}{|l|}{ Weekly Operating Hours } \\
\hline 48 or Fewer & 80 & 8 & 4 & 6 & 4,822 & 762 & 208 & 629 \\
\hline 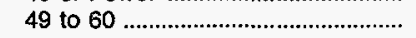 & 65 & 13 & 6 & 12 & 13,424 & 4,675 & 1,785 & 4,302 \\
\hline 61 to 167 & 68 & 6 & 4 & 5 & 5,706 & 1,091 & 672 & 714 \\
\hline 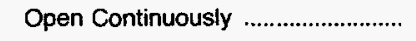 & 113 & 36 & 30 & 27 & 20,364 & 12,253 & 9,871 & 9,282 \\
\hline \multicolumn{9}{|l|}{ Multibuilding Facility } \\
\hline 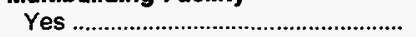 & 200 & 43 & 33 & 33 & 26,821 & 13,420 & 10,412 & 9,956 \\
\hline 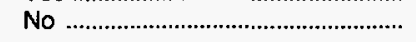 & 126 & 20 & 11 & 17 & 17,495 & 5,361 & 2,125 & 4,972 \\
\hline \multicolumn{9}{|l|}{$\begin{array}{l}\text { Energy Management Practices } \\
\text { (more than one may apply) }\end{array}$} \\
\hline 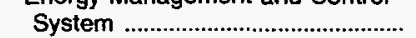 & 71 & 25 & 14 & 17 & 18,231 & 12,657 & 8,327 & 9,589 \\
\hline Energy Conservation & & & & & & & & \\
\hline Programs 1 & 124 & 34 & 22 & 29 & 24,699 & 12,274 & 7,606 & 9,921 \\
\hline 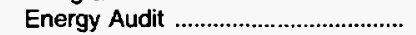 & 99 & 26 & 17 & 23 & 17,444 & 9,337 & 6,259 & 7,420 \\
\hline HVAC Maintenance Staff 2 ................ & 52 & 20 & 14 & 14 & 14,570 & 8,294 & 6,029 & 6,417 \\
\hline
\end{tabular}

1 Building participates in any programs sponsored by the Federal Energy Management Program, in-house, utility, or third party.

2 HVAC maintenance staff means at least one person spends at least half their working hours maintaining the heating/cooling equipment.

(*) = Value rounds to zero in the units displayed.

Notes: - Total workers are the number of workers during the main shift. - See Glossary for explanation of abbreviations and definitions of terms used in this report. These data are from $\mathbf{8 8 1}$ federally owned buildings having the following criteria: (1) located in Federal Regions 3 , 6, or 9; (2) larger than 10,000 square feet; and (3) used for a commercial purpose, other than warehouse and storage. In addition, 9 out of 10 selected buildings were from agencies other than the Department of Defense. - Statistics for the "energy end uses" represent consumption in buildings that have end use, not consumption for a particular fuel for a particular end use. - FBSS = Federal Buildings Supplemental Survey. - HVAC = Heating, Ventilation, and Air Conditioning. - Data are for Fiscal Year 1993 (October 1, 1992 through September 30, 1993). • Because of rounding, data may not sum to totals.

Source: Energy Information Administration, Office of Energy Markets and End Use, 1993 Federal Buildings Supplemental Survey. 
Table 3.25. Water-Heating Equipment in FBSS Buildings in Federal Region 3, Number of Buildings and Floorspace, 1993

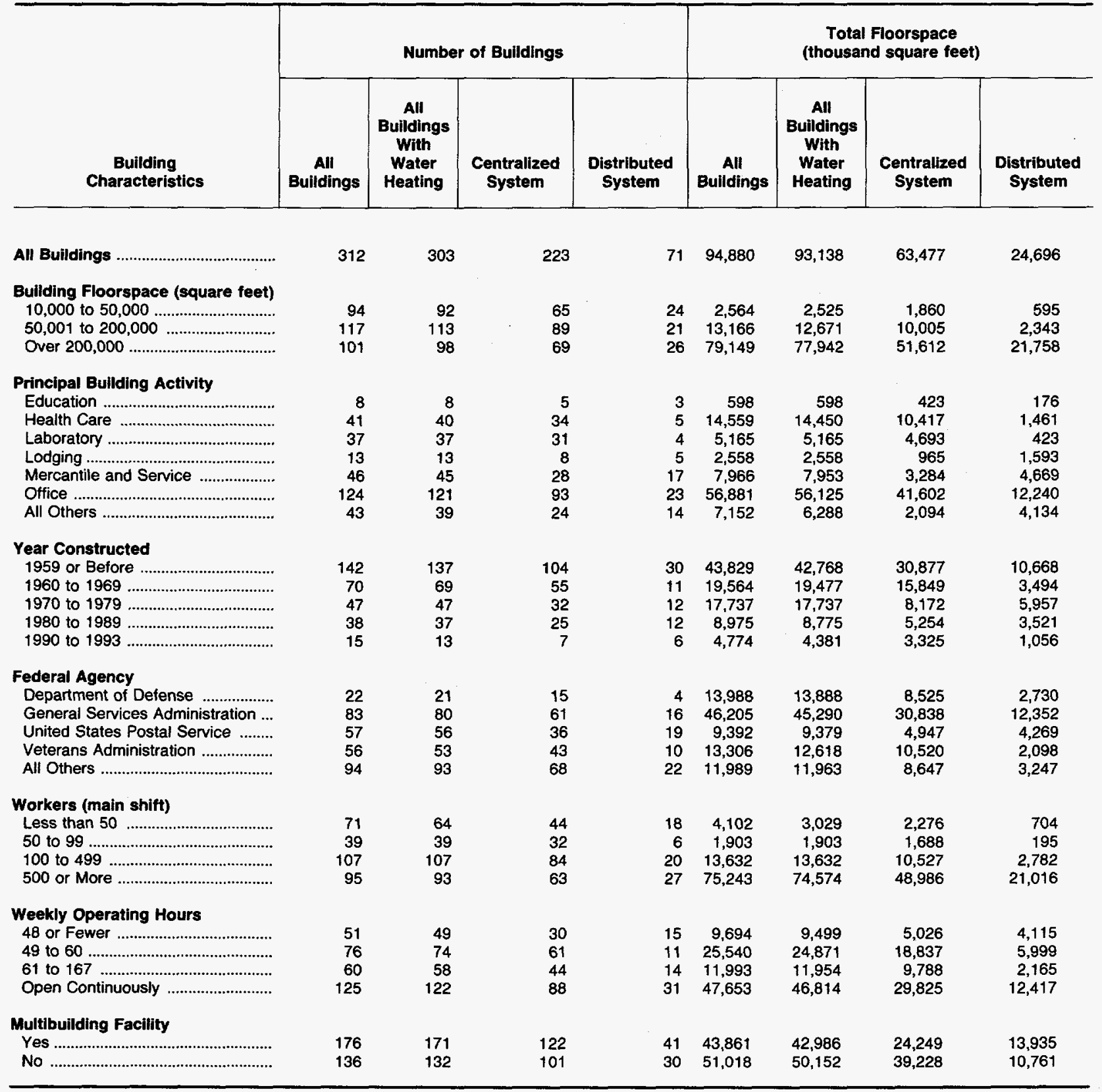

Notes: - Total workers are the number of workers during the main shift. - See Glossary for explanation of abbreviations and definitions of terms used in this report. - These data are from 881 federally owned buildings having the following criteria: (1) located in Federal Regions 3 , 6 , or 9 ; (2) larger than 10,000 square feet; and (3) used for a commercial purpose, other than warehouse and storage. In addition, 9 out of 10 selected buildings were from agencies other than the Department of Defense. - Statistics for the "energy end uses" represent consumption in buildings that have end use, not consumption for a particular fuel for a particular end use. - FBSS = Federal Buildings Supplemental Survey.

- Data are for Fiscal Year 1993 (October 1, 1992 through September 30, 1993). - Because of rounding, data may not sum to totals.

Source: Energy Information Administration, Office of Energy Markets and End Use, 1993 Federal Buildings Supplemental Survey. 
Table 3.26. Water-Heating Equipment in FBSS Buildings in Federal Region 6, Number of Buildings and Floorspace, 1993

\begin{tabular}{|c|c|c|c|c|c|c|c|c|}
\hline \multirow[b]{2}{*}{$\begin{array}{c}\text { Building } \\
\text { Characteristics }\end{array}$} & \multicolumn{4}{|c|}{ Number of Buildings } & \multicolumn{4}{|c|}{$\begin{array}{c}\text { Total Floorspace } \\
\text { (thousand square feet) }\end{array}$} \\
\hline & $\begin{array}{c}\text { All } \\
\text { Buildings }\end{array}$ & $\begin{array}{c}\text { All } \\
\text { Buildings } \\
\text { With } \\
\text { Water } \\
\text { Heating }\end{array}$ & $\begin{array}{l}\text { Centralized } \\
\text { System }\end{array}$ & $\begin{array}{l}\text { Distributed } \\
\text { System }\end{array}$ & $\begin{array}{c}\text { All } \\
\text { Buildings }\end{array}$ & \begin{tabular}{|c} 
All \\
Buildings \\
With \\
Water \\
Heating
\end{tabular} & $\begin{array}{l}\text { Centralized } \\
\text { System }\end{array}$ & $\begin{array}{l}\text { Distributed } \\
\text { System }\end{array}$ \\
\hline 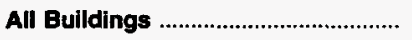 & 243 & 238 & 163 & 71 & 35,816 & 35,221 & 23,206 & 11,827 \\
\hline 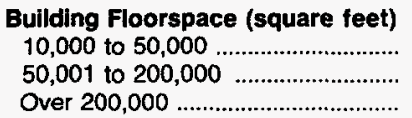 & $\begin{array}{r}107 \\
86 \\
50\end{array}$ & $\begin{array}{r}104 \\
85 \\
49\end{array}$ & $\begin{array}{l}70 \\
68 \\
25\end{array}$ & $\begin{array}{l}31 \\
16 \\
24\end{array}$ & $\begin{array}{r}2,591 \\
9,548 \\
23,677\end{array}$ & $\begin{array}{r}2,539 \\
9,466 \\
23,216\end{array}$ & $\begin{array}{r}1,675 \\
7,426 \\
14,105\end{array}$ & $\begin{array}{r}811 \\
1,905 \\
9,111\end{array}$ \\
\hline 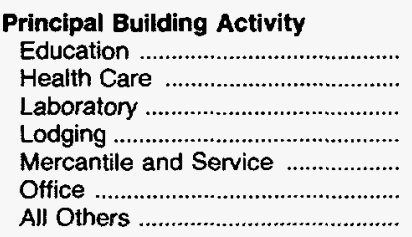 & $\begin{array}{r}6 \\
35 \\
29 \\
16 \\
49 \\
76 \\
32\end{array}$ & $\begin{array}{r}6 \\
35 \\
29 \\
16 \\
47 \\
75 \\
30\end{array}$ & $\begin{array}{r}5 \\
27 \\
18 \\
15 \\
21 \\
58 \\
19\end{array}$ & $\begin{array}{r}1 \\
6 \\
11 \\
1 \\
25 \\
16 \\
11\end{array}$ & $\begin{array}{r}168 \\
12,094 \\
3,331 \\
942 \\
6,236 \\
10,799 \\
2,247\end{array}$ & $\begin{array}{r}168 \\
12,094 \\
3,331 \\
942 \\
5,763 \\
10,779 \\
2,146\end{array}$ & $\begin{array}{r}143 \\
10,341 \\
2,211 \\
815 \\
1,528 \\
6,697 \\
1,472\end{array}$ & $\begin{array}{r}25 \\
1,607 \\
1,120 \\
127 \\
4,210 \\
4,064 \\
674\end{array}$ \\
\hline 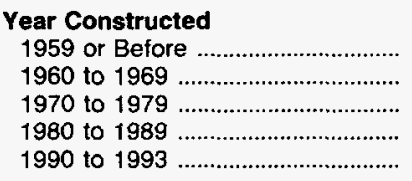 & $\begin{array}{r}103 \\
38 \\
34 \\
57 \\
11\end{array}$ & $\begin{array}{r}102 \\
37 \\
32 \\
56 \\
11\end{array}$ & $\begin{array}{r}75 \\
26 \\
22 \\
36 \\
4\end{array}$ & $\begin{array}{r}26 \\
11 \\
10 \\
19 \\
5\end{array}$ & $\begin{array}{r}13,258 \\
6,386 \\
6,175 \\
6,903 \\
3,095\end{array}$ & $\begin{array}{r}13,176 \\
6,367 \\
5,694 \\
6,890 \\
3,095\end{array}$ & $\begin{array}{l}8,536 \\
3,770 \\
3,153 \\
4,855 \\
2,892\end{array}$ & $\begin{array}{r}4,622 \\
2,597 \\
2,540 \\
1,900 \\
167\end{array}$ \\
\hline 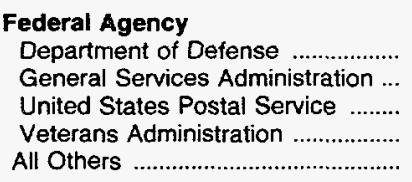 & $\begin{array}{l}22 \\
35 \\
61 \\
36 \\
89\end{array}$ & $\begin{array}{l}21 \\
35 \\
59 \\
36 \\
87\end{array}$ & $\begin{array}{l}18 \\
26 \\
32 \\
27 \\
60\end{array}$ & $\begin{array}{r}3 \\
9 \\
26 \\
8 \\
25\end{array}$ & $\begin{array}{r}1,668 \\
7,888 \\
7,027 \\
11,375 \\
7,858\end{array}$ & $\begin{array}{r}1,648 \\
7,888 \\
6,553 \\
11,375 \\
7,757\end{array}$ & $\begin{array}{r}928 \\
4,821 \\
2,077 \\
10,349 \\
5,031\end{array}$ & $\begin{array}{r}721 \\
3,067 \\
4,451 \\
1,014 \\
2,574\end{array}$ \\
\hline 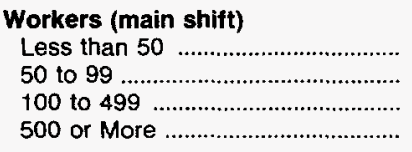 & $\begin{array}{l}70 \\
44 \\
80 \\
49\end{array}$ & $\begin{array}{l}67 \\
43 \\
80 \\
48\end{array}$ & $\begin{array}{l}50 \\
25 \\
62 \\
26\end{array}$ & $\begin{array}{l}16 \\
17 \\
16 \\
22\end{array}$ & $\begin{array}{r}2,548 \\
2,026 \\
9,029 \\
22,213\end{array}$ & $\begin{array}{r}2,427 \\
2,013 \\
9,029 \\
21,752\end{array}$ & $\begin{array}{r}1,748 \\
1,278 \\
6,462 \\
13,718\end{array}$ & $\begin{array}{r}662 \\
710 \\
2,421 \\
8,034\end{array}$ \\
\hline 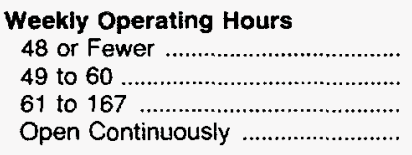 & $\begin{array}{l}39 \\
46 \\
78 \\
80\end{array}$ & $\begin{array}{l}37 \\
45 \\
77 \\
79\end{array}$ & $\begin{array}{l}29 \\
35 \\
45 \\
54\end{array}$ & $\begin{array}{r}8 \\
10 \\
30 \\
23\end{array}$ & $\begin{array}{r}2,740 \\
6,460 \\
8,051 \\
18,565\end{array}$ & $\begin{array}{r}2,639 \\
6,440 \\
8,038 \\
18,104\end{array}$ & $\begin{array}{r}2,181 \\
3,966 \\
5,458 \\
11,600\end{array}$ & $\begin{array}{r}457 \\
2,473 \\
2,538 \\
6,358\end{array}$ \\
\hline 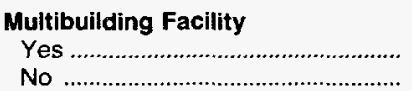 & $\begin{array}{r}148 \\
95\end{array}$ & $\begin{array}{r}146 \\
92\end{array}$ & $\begin{array}{l}98 \\
65\end{array}$ & $\begin{array}{l}45 \\
26\end{array}$ & $\begin{array}{l}24,441 \\
11,375\end{array}$ & $\begin{array}{l}24,339 \\
10,881\end{array}$ & $\begin{array}{r}16,457 \\
6,749\end{array}$ & $\begin{array}{l}7,7 y 9 \\
4,108\end{array}$ \\
\hline
\end{tabular}

Notes: - Total workers are the number of workers during the main shift. - See Glossary for explanation of abbreviations and definitions of terms used in this report. - These data are from 881 federally owned buildings having the following criteria: (1) located in Federal Regions 3 , 6 , or 9 ; (2) larger than 10,000 square feet; and (3) used for a commercial purpose, other than warehouse and storage. In addition, 9 out of 10 selected buildings were from agencies other than the Department of Defense. - Statistics for the "energy end uses" represent consumption in buildings that have end use, not consumption for a particular fuel for a particular end use. - FBSS = Federal Buildings Supplemental Survey.

- Data are for Fiscal Year 1993 (October 1, 1992 through September 30, 1993). - Because of rounding, data may not sum to totals.

Source: Energy information Administration, Office of Energy Markets and End Use, 1993 Federal Buildings Supplemental Survey. 
Table 3.27. Water-Heating Equipment in FBSS Buildings in Federal Region 9, Number of Buildings and Floorspace, 1993

\begin{tabular}{|c|c|c|c|c|c|c|c|c|}
\hline \multirow[b]{2}{*}{$\begin{array}{l}\text { Building } \\
\text { Characteristics }\end{array}$} & \multicolumn{4}{|c|}{ Number of Buildings } & \multicolumn{4}{|c|}{$\begin{array}{l}\text { Total Floorspace } \\
\text { (thousand square feet) }\end{array}$} \\
\hline & $\begin{array}{c}\text { All } \\
\text { Buildings }\end{array}$ & $\begin{array}{c}\text { All } \\
\text { Buildings } \\
\text { With } \\
\text { Water } \\
\text { Heating }\end{array}$ & $\begin{array}{l}\text { Centralized } \\
\text { System }\end{array}$ & $\begin{array}{l}\text { Distributed } \\
\text { System }\end{array}$ & $\begin{array}{c}\text { All } \\
\text { Buildings }\end{array}$ & $\begin{array}{c}\text { All } \\
\text { Bulldings } \\
\text { With } \\
\text { Water } \\
\text { Heating }\end{array}$ & $\begin{array}{l}\text { Centralized } \\
\text { System }\end{array}$ & $\begin{array}{l}\text { Distributed } \\
\text { System }\end{array}$ \\
\hline All Buildings & 326 & 315 & 225 & 80 & 44,316 & 42,984 & 31,069 & 10,750 \\
\hline \multicolumn{9}{|l|}{ Bullding Floorspace (square feet) } \\
\hline 10,000 to 50,000 & 148 & 142 & 101 & 37 & 3,858 & 3,728 & 2,612 & 1,044 \\
\hline 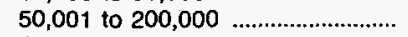 & 123 & 120 & 87 & 29 & 12,680 & 12,325 & 8,929 & 3,024 \\
\hline Over 200,000 & 55 & 53 & 37 & 14 & 27,778 & 26,931 & 19,528 & 6,681 \\
\hline \multicolumn{9}{|l|}{ Principal Building Activity } \\
\hline Education & 12 & 12 & 11 & 1 & 628 & 628 & 590 & 38 \\
\hline 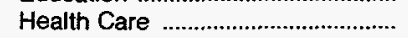 & 48 & 47 & 41 & 4 & 9,903 & 9,888 & 8,985 & 732 \\
\hline 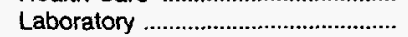 & 31 & 30 & 23 & 7 & 2,601 & 2,582 & 1,612 & 970 \\
\hline Lodging & 22 & 22 & 17 & 5 & 1,220 & 1,220 & 988 & 232 \\
\hline Mercantile and Service .................... & 63 & 60 & 34 & 22 & 8,194 & 7,562 & 3,947 & 3,081 \\
\hline 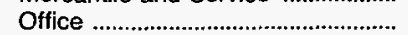 & 92 & 90 & 64 & 26 & 16,380 & 16,329 & 12,672 & 3,657 \\
\hline All Others & 58 & 54 & 35 & 15 & 5,390 & 4,775 & 2,275 & 2,040 \\
\hline \multicolumn{9}{|l|}{ Year Constructed } \\
\hline 1959 or Before & 130 & 125 & 83 & 38 & 13,553 & 13,321 & 7,763 & 5,050 \\
\hline 1960 to 1969 & 55 & 55 & 45 & 10 & 9,082 & 9,082 & 6,873 & 2,210 \\
\hline 1970 to 1979 & 62 & 61 & 44 & 14 & 12,041 & 11,736 & 9,014 & 2,323 \\
\hline 1980 to 1989 & 65 & 61 & 43 & 16 & 7,955 & 7,303 & 5,963 & 1,140 \\
\hline 1990 to 1993 & 14 & 13 & 10 & 2 & 1,684 & 1,541 & 1,457 & 26 \\
\hline \multicolumn{9}{|l|}{ Federal Agency } \\
\hline Department of Defense ................... & 78 & 73 & 49 & 22 & 8,489 & 7,928 & 4,447 & 2,995 \\
\hline General Services Administration ... & 39 & 39 & 34 & 5 & 12,505 & 12,505 & 10,625 & 1,880 \\
\hline United States Postal Service ........ & 63 & 60 & 35 & 22 & 8,387 & 7,813 & 4,266 & 3,126 \\
\hline Veterans Administration .................. & 56 & 55 & 48 & 5 & 8,824 & 8,681 & 7,729 & 780 \\
\hline All Others & 90 & 88 & 59 & 26 & 6,111 & 6,057 & 4,002 & 1,968 \\
\hline \multicolumn{9}{|l|}{ Workers (main shift) } \\
\hline Less than 50 & 93 & 87 & 64 & 20 & 3,684 & 3,319 & 2,219 & 1,014 \\
\hline 50 to 99 & 49 & 48 & 34 & 14 & 2,161 & 2,150 & 1,477 & 674 \\
\hline 100 to 499 & 134 & 131 & 92 & 34 & 14,017 & 13,603 & 9,812 & 3,434 \\
\hline 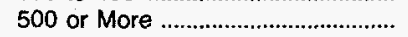 & 50 & 49 & 35 & 12 & 24,454 & 23,912 & 17,561 & 5,629 \\
\hline \multicolumn{9}{|l|}{ Weekly Operating Hours } \\
\hline 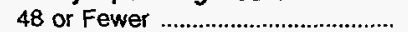 & 80 & 76 & 48 & 27 & 4,822 & 4,611 & 2,520 & 2,080 \\
\hline 49 to 60 & 65 & 64 & 51 & 11 & 13,424 & 13,344 & 10,505 & 2,353 \\
\hline 61 to 167 & 68 & 65 & 46 & 14 & 5,706 & 5,369 & 3,717 & 1,344 \\
\hline 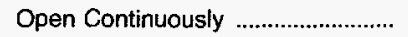 & 113 & 110 & 80 & 28 & 20,364 & 19,660 & 14,327 & 4,972 \\
\hline \multicolumn{9}{|l|}{ Multibuilding Facility } \\
\hline 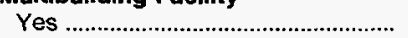 & 200 & 192 & 146 & 40 & 26,821 & 26,063 & 20,291 & 5,039 \\
\hline 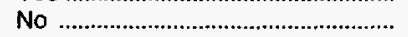 & 126 & 123 & 79 & 40 & 17,495 & 16,921 & 10,778 & 5,711 \\
\hline
\end{tabular}

Notes: - Total workers are the number of workers during the main shift. - See Glossary for explanation of abbreviations and definitions of terms used in this report. - These data are from 881 federally owned buildings having the following criteria: (1) located in Federal Regions 3, 6 , or 9 ; (2) larger than 10,000 square feet; and (3) used for a commercial purpose, other than warehouse and storage. in addition, 9 out of 10 selected buildings were from agencies other than the Department of Defense. - Statistics for the "energy end uses" represent consumption in buildings that have end use, not consumption for a particular fuel for a particular end use. - FBSS = Federal Buildings Supplemental Survey.

- Data are for Fiscal Year 1993 (October 1, 1992 through September 30, 1993). - Because of rounding, data may not sum to totals.

Source: Energy Information Administration, Office of Energy Markets and End Use, 1993 Federal Buildings Supplemental Survey. 
Table 3.28. Lighting Equipment in FBSS Buildings in Federal Region 3, Number of Buildings, 1993

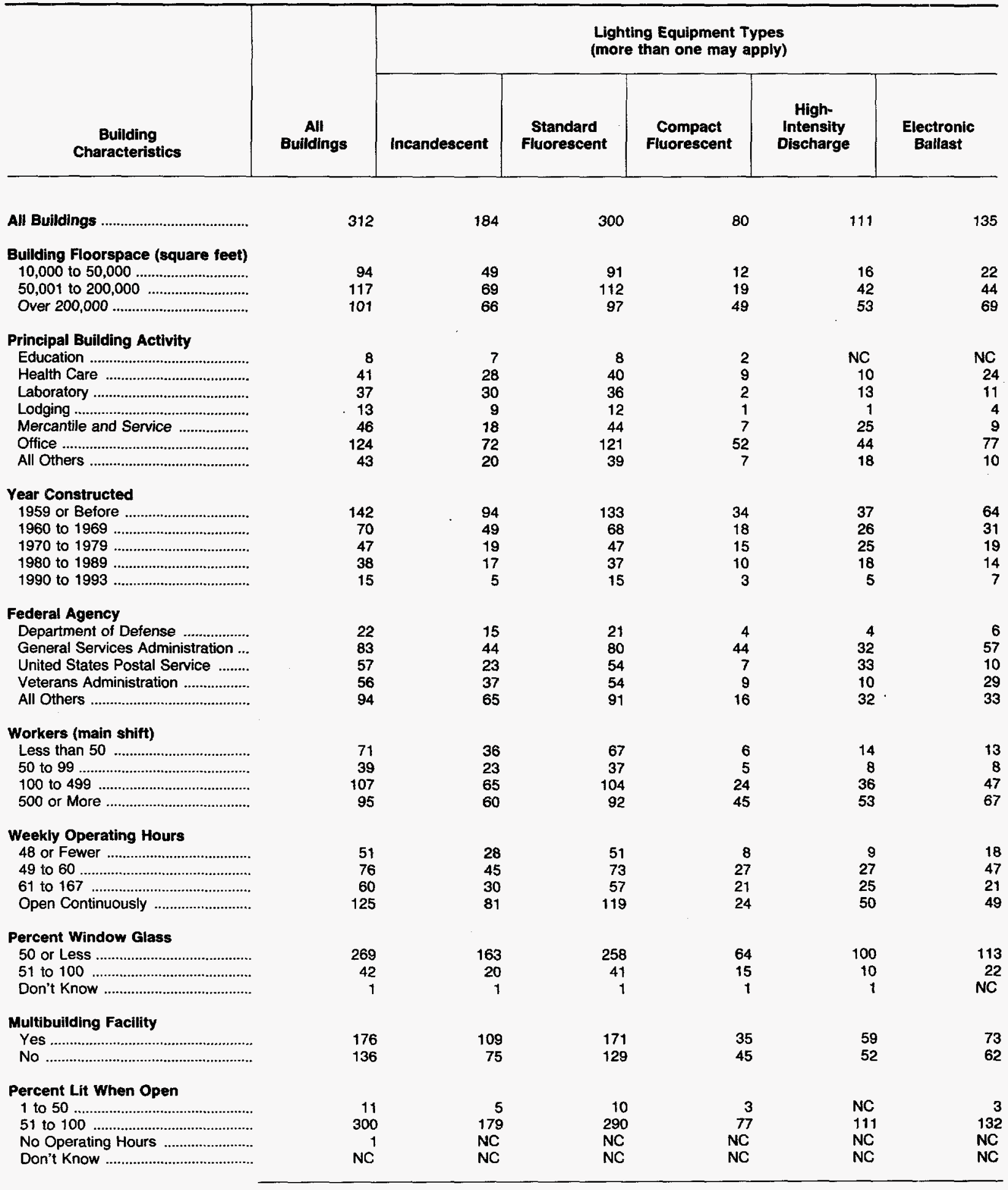

See footnotes at end of table. 
Table 3.28. Lighting Equipment in FBSS Buildings in Federal Region 3, Number of Buildings, 1993 (Continued)

\begin{tabular}{|c|c|c|c|c|c|c|}
\hline \multirow[b]{2}{*}{$\begin{array}{c}\text { Building } \\
\text { Characteristics }\end{array}$} & \multirow[b]{2}{*}{$\begin{array}{c}\text { All } \\
\text { Euildings }\end{array}$} & \multicolumn{5}{|c|}{$\begin{array}{l}\text { Lighting Equipment Types } \\
\text { (more than one may apply) }\end{array}$} \\
\hline & & Incandescent & $\begin{array}{l}\text { Standard } \\
\text { Fluorescent }\end{array}$ & $\begin{array}{l}\text { Compact } \\
\text { Fluorescent }\end{array}$ & $\begin{array}{l}\text { High- } \\
\text { Intensity } \\
\text { Discharge }\end{array}$ & $\begin{array}{l}\text { Electronic } \\
\text { Ballast }\end{array}$ \\
\hline \multicolumn{7}{|l|}{ Percent Lit When Closed } \\
\hline 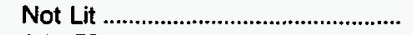 & 39 & 18 & 37 & 6 & 12 & 10 \\
\hline 1 to 50 & 194 & 113 & 189 & 58 & 67 & 93 \\
\hline 51 to 100 & 42 & 29 & 40 & 7 & 20 & 19 \\
\hline 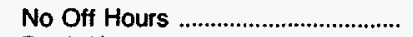 & 37 & 24 & 34 & 9 & 12 & 13 \\
\hline 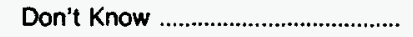 & NC & NC & NC & NC & NC & NC \\
\hline \multicolumn{7}{|l|}{$\begin{array}{l}\text { Lighting Conservation Features } \\
\text { (more than one may apply) }\end{array}$} \\
\hline $\begin{array}{l}\text { Specular Reflectors ............................ } \\
\text { Natural Lighting Control }\end{array}$ & 124 & 76 & 120 & 43 & 46 & 72 \\
\hline 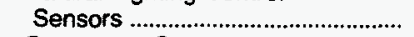 & 29 & 20 & 29 & 17 & 15 & 23 \\
\hline Occupancy Sensors ........................... & 95 & 57 & 94 & 51 & 41 & 68 \\
\hline 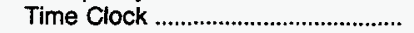 & 74 & 48 & 73 & 35 & 31 & 46 \\
\hline Manual Dimmer Switches ............... & 93 & 73 & 90 & 47 & 37 & 62 \\
\hline \multicolumn{7}{|l|}{$\begin{array}{l}\text { Energy Management Practices } \\
\text { (more than one may apply) } \\
\text { Energy Management and Control }\end{array}$} \\
\hline $\begin{array}{l}\text { System } \\
\text { Energy Conservation }\end{array}$ & 132 & 77 & 130 & 43 & 59 & 82 \\
\hline Programs ${ }^{1}$ & 99 & 64 & 97 & 44 & 37 & 66 \\
\hline 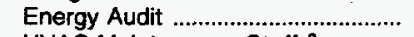 & 99 & 60 & 98 & 31 & 36 & 53 \\
\hline HVAC Maintenance Staff ${ }^{2} \ldots \ldots \ldots \ldots$ & 97 & 61 & 94 & 33 & 41 & 57 \\
\hline \multicolumn{7}{|l|}{$\begin{array}{l}\text { Off-Hours Reduction in } \\
\text { Equipment (more than one may } \\
\text { apply) }\end{array}$} \\
\hline 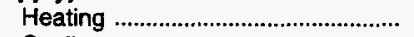 & 144 & 80 & 142 & 50 & 51 & 75 \\
\hline 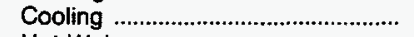 & 145 & 84 & 141 & 51 & 51 & 76 \\
\hline 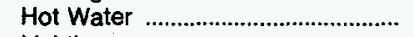 & 77 & 43 & 76 & 32 & 27 & 39 \\
\hline Lighting & 162 & 90 & 157 & 51 & 50 & 76 \\
\hline
\end{tabular}

1 Building participates in any programs sponsored by the Federal Energy Management Program, in-house, utility, or third party.

2 HVAC maintenance staff means at least one person spends at least half their working hours maintaining the heating/cooling equipment. $\mathrm{NC}=$ No cases in responding sample.

Notes: - Total workers are the number of workers during the main shift. - See Glossary for explanation of abbreviations and definitions of terms used in this report. - These data are from 881 federally owned buildings having the following criteria: (1) located in Federal Regions 3,6 , or 9 ; (2) larger than 10,000 square feet; and (3) used for a commercial purpose, other than warehouse and storage. In addition, 9 out of 10 selected buildings were from agencies other than the Department of Defense. - Statistics for the "energy end uses" represent consumption in buildings that have end use, not consumption for a particular fuel for a particular end use. - FBSS = Federal Buildings Supplemental Survey.

- HVAC = Heating. Ventilation, and Air Conditioning. - Data are for Fiscal Year 1993 (October 1, 1992 through September 30, 1993). Because of rounding, data may not sum to totals.

Source: Energy Information Administration, Office of Energy Markets and End Use, 1993 Federal Buildings Supplemental Survey. 
Table 3.29. Lighting Equipment in FBSS Buildings in Federal Region 6, Number of Buildings, 1993

\begin{tabular}{|c|c|c|c|c|c|c|}
\hline \multirow[b]{2}{*}{$\begin{array}{c}\text { Building } \\
\text { Characteristics }\end{array}$} & \multirow[b]{2}{*}{$\begin{array}{l}\text { All } \\
\text { Buildings }\end{array}$} & \multicolumn{5}{|c|}{$\begin{array}{l}\text { Lighting Equipment Types } \\
\text { (more than one may apply) }\end{array}$} \\
\hline & & Incandescent & $\begin{array}{l}\text { Standard } \\
\text { Fluorescent }\end{array}$ & $\begin{array}{c}\text { Compact } \\
\text { Fluorescent }\end{array}$ & $\begin{array}{c}\text { High- } \\
\text { Intensity } \\
\text { Discharge }\end{array}$ & $\begin{array}{l}\text { Electronic } \\
\text { Ballast }\end{array}$ \\
\hline All Bulldings & 243 & 135 & 235 & 29 & 64 & 59 \\
\hline \multicolumn{7}{|l|}{ Building Floorspace (square feet) } \\
\hline 10,000 to 50,000 & 107 & 42 & 103 & 7 & 17 & 15 \\
\hline 50,001 to 200,000 & 86 & 62 & 83 & 5 & 19 & 22 \\
\hline 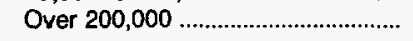 & 50 & 31 & 49 & 17 & 28 & 22 \\
\hline \multicolumn{7}{|l|}{ Principal Building Activity } \\
\hline 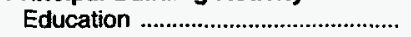 & 6 & 2 & 6 & 1 & 2 & 1 \\
\hline 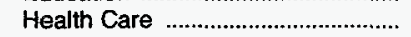 & 35 & 20 & 35 & 5 & 9 & 16 \\
\hline 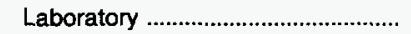 & 29 & 21 & 28 & 1 & 10 & 1 \\
\hline 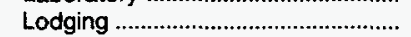 & 16 & 9 & 15 & NC & 1 & 4 \\
\hline Mercantile and Service ................... & 49 & 22 & 48 & 5 & 15 & 9 \\
\hline 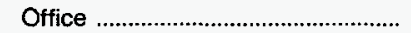 & 76 & 43 & 75 & 17 & 19 & 23 \\
\hline 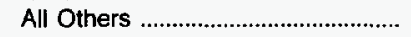 & 32 & 18 & 28 & NC & 8 & 5 \\
\hline \multicolumn{7}{|l|}{ Year Constructed } \\
\hline 1959 or Before & 103 & 67 & 98 & 13 & 23 & 23 \\
\hline 1960 to 1969 & 38 & 25 & 37 & 7 & 13 & 14 \\
\hline 1970 to 1979 & 34 & 17 & 34 & 4 & 14 & 11 \\
\hline 1980 to 1989 & 57 & 22 & 55 & 4 & 13 & 9 \\
\hline 1990 to $1993 \ldots \ldots$ & 11 & 4 & 11 & 1 & 1 & 2 \\
\hline \multicolumn{7}{|l|}{ Federal Agency } \\
\hline Department of Defense ................... & 22 & 12 & 20 & 1 & 6 & 3 \\
\hline General Services Administration ... & 35 & 21 & 34 & 15 & 12 & 19 \\
\hline United States Postal Service ......... & 61 & 28 & 59 & 6 & 16 & 10 \\
\hline Veterans Administration ................... & 36 & 14 & 35 & 2 & 6 & 19 \\
\hline 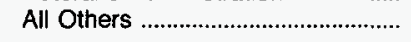 & 89 & 60 & 87 & 5 & 24 & 8 \\
\hline \multicolumn{7}{|l|}{ Workers (main shift) } \\
\hline Less than 50 & 70 & 29 & 66 & 3 & 10 & 7 \\
\hline 50 to 99 & 44 & 22 & 43 & 2 & 8 & 7 \\
\hline 100 to 499 & 80 & 53 & 77 & $\overline{9}$ & 19 & 23 \\
\hline 500 or More & 49 & 31 & 49 & 15 & 27 & 22 \\
\hline \multicolumn{7}{|l|}{ Weekly Operating Hours } \\
\hline 48 or Fewer & 39 & 18 & 37 & 4 & 5 & 10 \\
\hline 49 to $60^{\circ}$ & 46 & 22 & 45 & 11 & 9 & 10 \\
\hline 61 to 167 & 78 & 47 & 76 & 6 & 26 & 14 \\
\hline 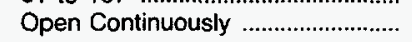 & 80 & 48 & 77 & 8 & 24 & 25 \\
\hline \multicolumn{7}{|l|}{ Percent Window Glass } \\
\hline 50 or Less & 204 & 103 & 196 & 25 & 44 & 51 \\
\hline 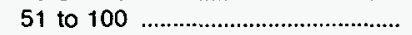 & 16 & 10 & 16 & 4 & 4 & 8 \\
\hline 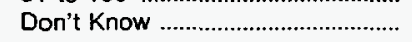 & 23 & 22 & 23 & NC & 16 & $\mathrm{NC}$ \\
\hline \multicolumn{7}{|l|}{ Multibuilding Facility } \\
\hline 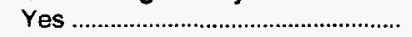 & 148 & 93 & 144 & 15 & 39 & 35 \\
\hline No & 95 & 42 & 91 & 14 & 25 & 24 \\
\hline \multicolumn{7}{|l|}{ Percent Lit When Open } \\
\hline 1 to 50 & 15 & 9 & 14 & NC & 1 & 1 \\
\hline 51 to 100 & 227 & 126 & 221 & 29 & 63 & 58 \\
\hline 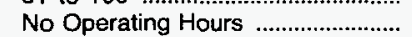 & 1 & NC & NC & $\mathrm{NC}$ & NC & NC \\
\hline Don't Know & NC & NC & NC & NC & NC & NC \\
\hline
\end{tabular}

See footnotes at end of table. 
Table 3.29. Lighting Equipment in FBSS Buildings in Federal Region 6, Number of Buildings, 1993 (Continued)

\begin{tabular}{|c|c|c|c|c|c|c|}
\hline \multirow[b]{2}{*}{$\begin{array}{l}\text { Building } \\
\text { Characteristics }\end{array}$} & \multirow[b]{2}{*}{$\begin{array}{l}\text { All } \\
\text { Buildings }\end{array}$} & \multicolumn{5}{|c|}{$\begin{array}{l}\text { Lighting Equipment Types } \\
\text { (more than one may apply) }\end{array}$} \\
\hline & & Incandescent & $\begin{array}{l}\text { Standard } \\
\text { Fluorescent }\end{array}$ & $\begin{array}{l}\text { Compact } \\
\text { Fluorescent }\end{array}$ & $\begin{array}{l}\text { High- } \\
\text { Intensity } \\
\text { Discharge }\end{array}$ & $\begin{array}{l}\text { Electronic } \\
\text { Ballast }\end{array}$ \\
\hline \multicolumn{7}{|l|}{ Percent Lit When Closed } \\
\hline Not Lit & 33 & 10 & 30 & 4 & 11 & 8 \\
\hline 1 to 50 & 165 & 97 & 162 & 21 & 38 & 34 \\
\hline 51 to 100 & 25 & 18 & 25 & 4 & 10 & 9 \\
\hline 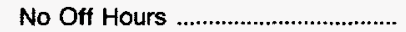 & 20 & 10 & 18 & NC & 5 & 8 \\
\hline 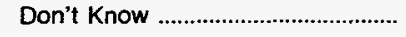 & NC & NC & $\mathrm{NC}$ & NC & NC & NC \\
\hline \multicolumn{7}{|l|}{$\begin{array}{l}\text { Lighting Conservation Features } \\
\text { (more than one may apply) }\end{array}$} \\
\hline $\begin{array}{l}\text { Specular Reflectors .......................... } \\
\text { Natural Lighting Control }\end{array}$ & 92 & 44 & 89 & 16 & 24 & 39 \\
\hline 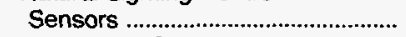 & 27 & 13 & 26 & 8 & 12 & 16 \\
\hline Occupancy Sensors .......................... & 58 & 29 & 56 & 17 & 20 & 34 \\
\hline Time Clock & 36 & 18 & 35 & 9 & 14 & 18 \\
\hline Manual Dimmer Switches ............... & 44 & 26 & 41 & 16 & 11 & 20 \\
\hline \multicolumn{7}{|l|}{$\begin{array}{l}\text { Energy Management Practices } \\
\text { (more than one may apply) } \\
\text { Energy Management and Control }\end{array}$} \\
\hline 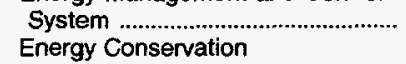 & 96 & 52 & 92 & 18 & 29 & 34 \\
\hline 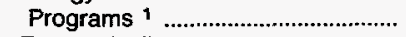 & 48 & 25 & 47 & 8 & 16 & 21 \\
\hline Energy Audit & 64 & 27 & 60 & 10 & 16 & 22 \\
\hline HVAC Maintenance Staff ${ }^{2}$.............. & 57 & 35 & 56 & 15 & 19 & 25 \\
\hline \multicolumn{7}{|l|}{$\begin{array}{l}\text { Off-Hours Reduction in } \\
\text { Equipment (more than one may } \\
\text { apply) }\end{array}$} \\
\hline 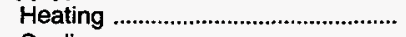 & 123 & 57 & 119 & 21 & 23 & 30 \\
\hline Cooling & 124 & 58 & 121 & 21 & 22 & 31 \\
\hline 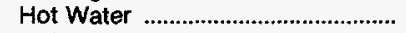 & 33 & 14 & 33 & 7 & 9 & 10 \\
\hline 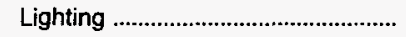 & 120 & 61 & 117 & 19 & 21 & 33 \\
\hline
\end{tabular}

1 Building participates in any programs sponsored by the Federal Energy Management Program, in-house, utility, or third party.

2 HVAC maintenance staff means at least one person spends at least half their working hours maintaining the heating/cooling equipment

NC $=$ No cases in responding sample.

Notes: - Total workers are the number of workers during the main shift. - See Glossary for explanation of abbreviations and definitions of terms used in this report. - These data are from 881 federally owned buildings having the following criteria: (1) located in Federal Regions 3 , 6, or 9; (2) larger than 10,000 square feet; and (3) used for a commercial purpose, other than warehouse and storage. In addition, 9 out of 10 selected buildings were from agencies other than the Department of Defense. - Statistics for the "energy end uses" represent consumption in buildings that have end use, not consumption for a particular fuel for a particular end use. - FBSS = Federal Buildings Supplemental Survey. - HVAC = Heating, Ventilation, and Air Conditioning. - Data are for Fiscal Year 1993 (October 1, 1992 through September 30 , 1993). Because of rounding, data may not sum to totals.

Source: Energy Information Administration, Office of Energy Markets and End Use, 1993 Federal Buildings Supplemental Survey. 
Table 3.30. Lighting Equipment in FBSS Buildings in Federal Region 9, Number of Bulldings, 1993

\begin{tabular}{|c|c|c|c|c|c|c|}
\hline \multirow[b]{2}{*}{$\begin{array}{c}\text { Building } \\
\text { Characteristics }\end{array}$} & \multirow[b]{2}{*}{$\begin{array}{c}\text { All } \\
\text { Bulldings }\end{array}$} & \multicolumn{5}{|c|}{$\begin{array}{l}\text { Lighting Equipment Types } \\
\text { (more than one may apply) }\end{array}$} \\
\hline & & Incandescent & $\begin{array}{l}\text { Standard } \\
\text { Fluorescent }\end{array}$ & $\begin{array}{l}\text { Compact } \\
\text { Fluorescent }\end{array}$ & $\begin{array}{l}\text { High- } \\
\text { Intensity } \\
\text { Discharge }\end{array}$ & $\begin{array}{l}\text { Electronic } \\
\text { Ballast }\end{array}$ \\
\hline 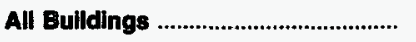 & 326 & 161 & 309 & 73 & 75 & 105 \\
\hline 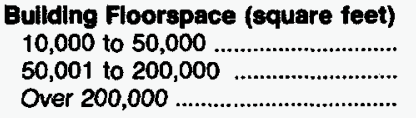 & $\begin{array}{r}148 \\
123 \\
55\end{array}$ & $\begin{array}{l}73 \\
68 \\
20\end{array}$ & $\begin{array}{r}140 \\
117 \\
52\end{array}$ & $\begin{array}{l}16 \\
35 \\
22\end{array}$ & $\begin{array}{l}20 \\
30 \\
25\end{array}$ & $\begin{array}{l}33 \\
45 \\
27\end{array}$ \\
\hline 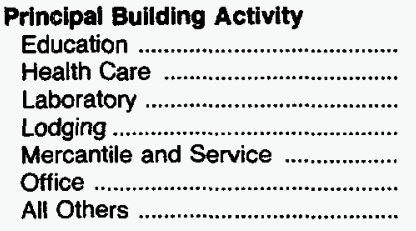 & $\begin{array}{l}12 \\
48 \\
31 \\
22 \\
63 \\
92 \\
58\end{array}$ & $\begin{array}{r}9 \\
20 \\
21 \\
15 \\
28 \\
37 \\
31\end{array}$ & $\begin{array}{l}12 \\
48 \\
29 \\
19 \\
61 \\
88 \\
52\end{array}$ & $\begin{array}{r}\text { NC } \\
4 \\
16 \\
2 \\
11 \\
34 \\
6\end{array}$ & $\begin{array}{r}4 \\
6 \\
4 \\
2 \\
27 \\
14 \\
18\end{array}$ & $\begin{array}{r}1 \\
19 \\
7 \\
2 \\
21 \\
40 \\
15\end{array}$ \\
\hline 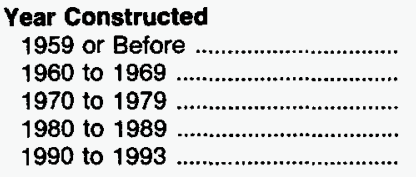 & $\begin{array}{r}130 \\
55 \\
62 \\
65 \\
14\end{array}$ & $\begin{array}{r}70 \\
28 \\
27 \\
31 \\
5\end{array}$ & $\begin{array}{r}124 \\
54 \\
56 \\
62 \\
13\end{array}$ & $\begin{array}{r}27 \\
13 \\
19 \\
11 \\
3\end{array}$ & $\begin{array}{r}27 \\
9 \\
16 \\
20 \\
3\end{array}$ & $\begin{array}{r}34 \\
21 \\
27 \\
21 \\
2\end{array}$ \\
\hline 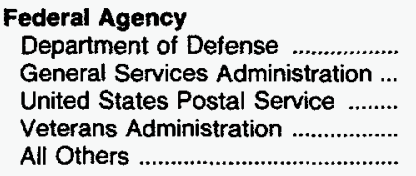 & $\begin{array}{l}78 \\
39 \\
63 \\
56 \\
90\end{array}$ & $\begin{array}{l}33 \\
11 \\
28 \\
19 \\
70\end{array}$ & $\begin{array}{l}73 \\
37 \\
61 \\
55 \\
83\end{array}$ & $\begin{array}{r}9 \\
29 \\
11 \\
4 \\
20\end{array}$ & $\begin{array}{r}19 \\
10 \\
22 \\
7 \\
17\end{array}$ & $\begin{array}{l}14 \\
26 \\
22 \\
24 \\
19\end{array}$ \\
\hline $\begin{array}{l}\text { Workers (main shift) } \\
\text { Less than } 50 \\
50 \text { to } 99 \\
100 \text { to } 499 \\
500 \text { or More }\end{array}$ & $\begin{array}{r}93 \\
49 \\
134 \\
50\end{array}$ & $\begin{array}{l}54 \\
22 \\
67 \\
18\end{array}$ & $\begin{array}{r}83 \\
48 \\
129 \\
49\end{array}$ & $\begin{array}{r}10 \\
7 \\
35 \\
21\end{array}$ & $\begin{array}{l}17 \\
10 \\
25 \\
23\end{array}$ & $\begin{array}{l}14 \\
15 \\
46 \\
30\end{array}$ \\
\hline 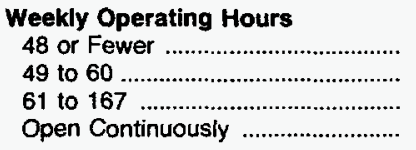 & $\begin{array}{r}80 \\
65 \\
68 \\
113\end{array}$ & $\begin{array}{l}40 \\
23 \\
34 \\
64\end{array}$ & $\begin{array}{r}73 \\
64 \\
65 \\
107\end{array}$ & $\begin{array}{r}5 \\
28 \\
10 \\
30\end{array}$ & $\begin{array}{l}15 \\
15 \\
19 \\
26\end{array}$ & $\begin{array}{l}12 \\
32 \\
27 \\
34\end{array}$ \\
\hline 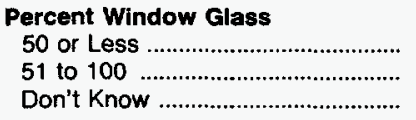 & $\begin{array}{r}298 \\
26 \\
2\end{array}$ & $\begin{array}{r}150 \\
11 \\
N C\end{array}$ & $\begin{array}{r}284 \\
25 \\
\mathrm{NC}\end{array}$ & $\begin{array}{r}58 \\
15 \\
N C\end{array}$ & $\begin{array}{r}72 \\
3 \\
\mathrm{NC}\end{array}$ & $\begin{array}{r}90 \\
15 \\
N C\end{array}$ \\
\hline 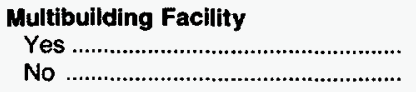 & $\begin{array}{l}200 \\
126\end{array}$ & $\begin{array}{r}103 \\
58\end{array}$ & $\begin{array}{l}189 \\
120\end{array}$ & $\begin{array}{l}41 \\
32\end{array}$ & $\begin{array}{l}46 \\
29\end{array}$ & $\begin{array}{l}64 \\
41\end{array}$ \\
\hline 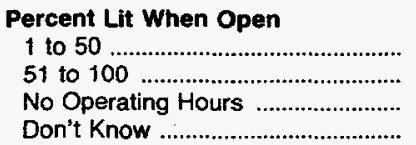 & $\begin{array}{r}20 \\
304 \\
1 \\
1\end{array}$ & $\begin{array}{l}11 \\
150 \\
\text { NC } \\
\text { NC }\end{array}$ & $\begin{array}{l}18 \\
291 \\
\text { NC } \\
\text { NC }\end{array}$ & $\begin{array}{r}1 \\
72 \\
\mathrm{NC} \\
\mathrm{NC}\end{array}$ & $\begin{array}{r}2 \\
73 \\
N C \\
N C\end{array}$ & $\begin{array}{l}1 \\
104 \\
\text { NC } \\
\text { NC }\end{array}$ \\
\hline
\end{tabular}

See footnotes at end of table. 
Table 3.30. Lighting Equipment in FBSS Buildings in Federal Region 9, Number of Buildings, 1993 (Continued)

\begin{tabular}{|c|c|c|c|c|c|c|}
\hline \multirow[b]{2}{*}{$\begin{array}{c}\text { Building } \\
\text { Characteristics }\end{array}$} & \multirow[b]{2}{*}{$\begin{array}{c}\text { All } \\
\text { Buildings }\end{array}$} & \multicolumn{5}{|c|}{$\begin{array}{l}\text { Lighting Equipment Types } \\
\text { (more than one may apply) }\end{array}$} \\
\hline & & Incandescent & $\begin{array}{l}\text { Standard } \\
\text { Fluorescent }\end{array}$ & $\begin{array}{l}\text { Compact } \\
\text { Fluorescent }\end{array}$ & $\begin{array}{l}\text { High- } \\
\text { Intensity } \\
\text { Discharge }\end{array}$ & $\begin{array}{l}\text { Electronic } \\
\text { Ballast }\end{array}$ \\
\hline \multicolumn{7}{|l|}{ Percent Lit When Closed } \\
\hline 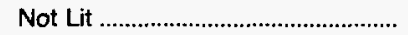 & 35 & 10 & 33 & 3 & 6 & 10 \\
\hline 1 to 50 & 205 & 107 & 197 & 46 & 45 & 71 \\
\hline 51 to 100 & 44 & 32 & 41 & 22 & 9 & 11 \\
\hline 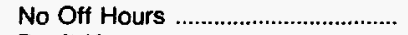 & 41 & 12 & 38 & 2 & 15 & 13 \\
\hline 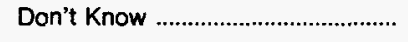 & 1 & NC & NC & NC & NC & NC \\
\hline \multicolumn{7}{|l|}{$\begin{array}{l}\text { Lighting Conservation Features } \\
\text { (more than one may apply) }\end{array}$} \\
\hline $\begin{array}{l}\text { Specular Reflectors ......................... } \\
\text { Natural Lighting Control }\end{array}$ & 124 & 59 & 119 & 42 & 31 & 59 \\
\hline 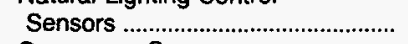 & 58 & 21 & 57 & 23 & 17 & 33 \\
\hline 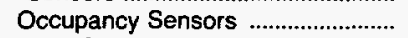 & 109 & 54 & 108 & 55 & 30 & 51 \\
\hline 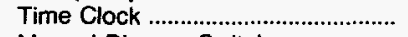 & 41 & 25 & 40 & 20 & 16 & 24 \\
\hline Manual Dimmer Switches ............... & 78 & 54 & 76 & 29 & 19 & 35 \\
\hline \multicolumn{7}{|l|}{$\begin{array}{l}\text { Energy Management Practices } \\
\text { (more than one may apply) } \\
\text { Energy Management and Control }\end{array}$} \\
\hline System & 71 & 34 & 69 & 24 & 25 & 31 \\
\hline Energy Conservation & & & & & & \\
\hline 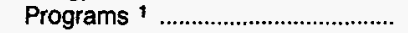 & 124 & 64 & 119 & 43 & 34 & 63 \\
\hline 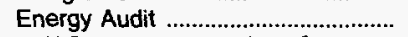 & 99 & 48 & 95 & 24 & 24 & 45 \\
\hline HVAC Maintenance Staff 2 ............. & 52 & 27 & 52 & 20 & 17 & 28 \\
\hline \multicolumn{7}{|l|}{$\begin{array}{l}\text { Off-Hours Reduction in } \\
\text { Equipment (more than one may } \\
\text { apply) }\end{array}$} \\
\hline 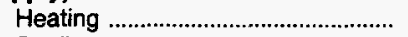 & 151 & 71 & 142 & 40 & 30 & 60 \\
\hline 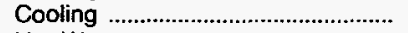 & 157 & 73 & 149 & 37 & 32 & 56 \\
\hline 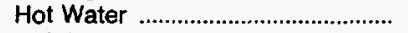 & 68 & 30 & 62 & 26 & 11 & 31 \\
\hline 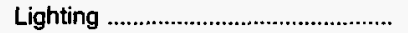 & 172 & 80 & 164 & 38 & 36 & 65 \\
\hline
\end{tabular}

1 Building participates in any programs sponsored by the Federal Energy Management Program, in-house, utility, or third party.

2 HVAC maintenance staff means at least one person spends at least half their working hours maintaining the heating/cooling equipment. $\mathrm{NC}=$ No cases in responding sample.

Notes: - Total workers are the number of workers during the main shift - See Glossary for explanation of abbreviations and definitions of terms used in this report. - These data are from 881 federally owned buildings having the following criteria: (1) located in Federal Regions 3 , 6 , or 9 ; (2) larger than 10,000 square feet; and (3) used for a commercial purpose, other than warehouse and storage. In addition, 9 out of 10 selected buildings were from agencies other than the Department of Defense. - Statistics for the "energy end uses" represent consumption in buildings that have end use, not consumption for a particular fuel for a particular end use. - FBSS = Federal Buildings Supplemental Survey. $\quad$ HVAC = Heating, Ventilation, and Air Conditioning. - Data are for Fiscal Year 1993 (October 1 , 1992 through September 30, 1993). Because of rounding, data may not sum to totals.

Source: Energy Information Administration, Office of Energy Markets and End Use, 1993 Federal Buildings Supplemental Survey. 
Table 3.31. Lighting Equipment in FBSS Building in Federal Region 3, Floorspace, 1993

(Thousand Square Feet)

\begin{tabular}{|c|c|c|c|c|c|c|}
\hline \multirow[b]{2}{*}{$\begin{array}{c}\text { Building } \\
\text { Characteristics }\end{array}$} & \multirow[b]{2}{*}{$\begin{array}{c}\text { All } \\
\text { Buildings }\end{array}$} & \multicolumn{5}{|c|}{$\begin{array}{l}\text { Lighting Equipment Types } \\
\text { (more than one may apply) }\end{array}$} \\
\hline & & Incandescent & $\begin{array}{l}\text { Standard } \\
\text { Fluorescent }\end{array}$ & $\begin{array}{l}\text { Compact } \\
\text { Fluorescent }\end{array}$ & $\begin{array}{l}\text { High- } \\
\text { Intensity } \\
\text { Discharge }\end{array}$ & $\begin{array}{c}\text { Electronic } \\
\text { Ballast }\end{array}$ \\
\hline All Buildings & 94,880 & 64,981 & 91,986 & 49,455 & 52,593 & 65,560 \\
\hline 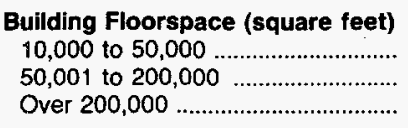 & $\begin{array}{r}2,564 \\
13,166 \\
79,149\end{array}$ & $\begin{array}{r}1,398 \\
7,539 \\
56,045\end{array}$ & $\begin{array}{r}2,494 \\
12,661 \\
76,831\end{array}$ & $\begin{array}{r}349 \\
2,180 \\
46,925\end{array}$ & $\begin{array}{r}368 \\
5,287 \\
46,938\end{array}$ & $\begin{array}{r}640 \\
5,285 \\
59,634\end{array}$ \\
\hline 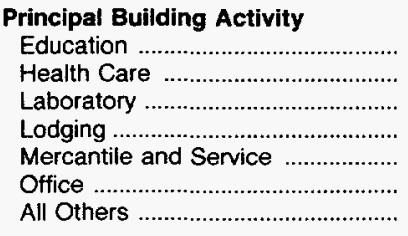 & $\begin{array}{r}598 \\
14,559 \\
5,165 \\
2,558 \\
7,966 \\
56,881 \\
7,152\end{array}$ & $\begin{array}{r}571 \\
10,992 \\
4,603 \\
2,431 \\
2,821 \\
40,025 \\
3,538\end{array}$ & $\begin{array}{r}598 \\
14,412 \\
5,112 \\
2,508 \\
6,851 \\
55,892 \\
6,613\end{array}$ & $\begin{array}{r}162 \\
7,501 \\
1,162 \\
31 \\
1,433 \\
36,601 \\
2,565\end{array}$ & $\begin{array}{r}\text { NC } \\
8,509 \\
2,654 \\
375 \\
7,031 \\
30,658 \\
3,366\end{array}$ & $\begin{array}{r}\mathrm{NC} \\
11,161 \\
2,555 \\
364 \\
2,168 \\
45,520 \\
3,791\end{array}$ \\
\hline 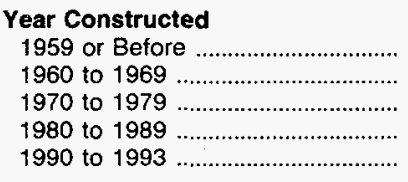 & $\begin{array}{r}43,829 \\
19,564 \\
17,737 \\
8,975 \\
4,774\end{array}$ & $\begin{array}{r}32,805 \\
13,197 \\
11,535 \\
5,688 \\
1,756\end{array}$ & $\begin{array}{r}42,104 \\
18,595 \\
17,737 \\
8,775 \\
4,774\end{array}$ & $\begin{array}{r}23,915 \\
7,637 \\
12,283 \\
4,537 \\
1,082\end{array}$ & $\begin{array}{r}23,514 \\
6,697 \\
14,377 \\
6,424 \\
1,581\end{array}$ & $\begin{array}{r}33,695 \\
10,765 \\
13,909 \\
5,163 \\
2,027\end{array}$ \\
\hline 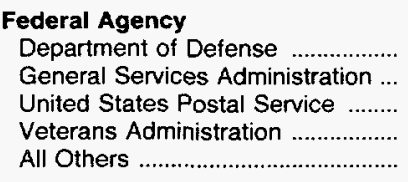 & $\begin{array}{r}13,988 \\
46,205 \\
9,392 \\
13,306 \\
11,989\end{array}$ & $\begin{array}{r}13,431 \\
29,825 \\
3,693 \\
8,926 \\
9,106\end{array}$ & $\begin{array}{r}13,938 \\
44,977 \\
8,256 \\
12,959 \\
11,856\end{array}$ & $\begin{array}{r}10,688 \\
30,330 \\
1,153 \\
5,291 \\
1,993\end{array}$ & $\begin{array}{r}11,155 \\
21,578 \\
8,637 \\
5,702 \\
5,522\end{array}$ & $\begin{array}{r}10,819 \\
36,094 \\
2,918 \\
9,535 \\
6,194\end{array}$ \\
\hline 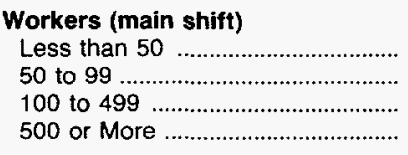 & $\begin{array}{r}4,102 \\
1,903 \\
13,632 \\
75,243\end{array}$ & $\begin{array}{r}1,741 \\
1,203 \\
8,871 \\
53,166\end{array}$ & $\begin{array}{r}3,593 \\
1,833 \\
13,377 \\
73,183\end{array}$ & $\begin{array}{r}365 \\
236 \\
3,870 \\
44,983\end{array}$ & $\begin{array}{r}1,166 \\
412 \\
5,702 \\
45,313\end{array}$ & $\begin{array}{r}814 \\
310 \\
6,753 \\
57,682\end{array}$ \\
\hline 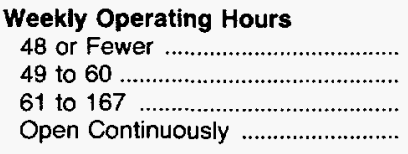 & $\begin{array}{r}9,694 \\
25,540 \\
11,993 \\
47,653\end{array}$ & $\begin{array}{r}6,878 \\
17,434 \\
7,802 \\
32,867\end{array}$ & $\begin{array}{r}9,694 \\
24,519 \\
11,922 \\
45,851\end{array}$ & $\begin{array}{r}4,841 \\
13,371 \\
8,519 \\
22,724\end{array}$ & $\begin{array}{r}2,164 \\
12,454 \\
8,062 \\
29,913\end{array}$ & $\begin{array}{r}5,933 \\
22,383 \\
8,530 \\
28,714\end{array}$ \\
\hline $\begin{array}{l}\text { Percent Window Glass } \\
50 \text { or Less } \\
51 \text { to } 100 \\
\text { Don't Know }\end{array}$ & $\begin{array}{r}79,002 \\
14,878 \\
1,000\end{array}$ & $\begin{array}{r}54,231 \\
9,750 \\
1,000\end{array}$ & $\begin{array}{r}76,558 \\
14,428 \\
1,000\end{array}$ & $\begin{array}{r}38,208 \\
10,247 \\
1,000\end{array}$ & $\begin{array}{r}46,067 \\
5,526 \\
1,000\end{array}$ & $\begin{array}{r}53,692 \\
11,867 \\
\text { NC }\end{array}$ \\
\hline 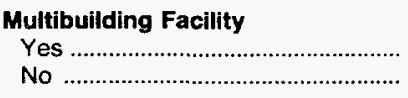 & $\begin{array}{l}43,861 \\
51,018\end{array}$ & $\begin{array}{l}28,586 \\
36,395\end{array}$ & $\begin{array}{l}42,321 \\
49,665\end{array}$ & $\begin{array}{l}18,115 \\
31,339\end{array}$ & $\begin{array}{l}24,187 \\
28,406\end{array}$ & $\begin{array}{l}27,847 \\
37,713\end{array}$ \\
\hline 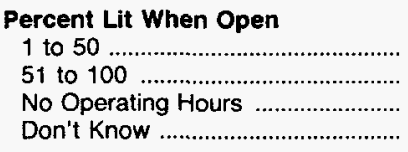 & $\begin{array}{r}663 \\
94,070 \\
147 \\
\mathrm{NC}\end{array}$ & $\begin{array}{r}229 \\
64,752 \\
\mathrm{NC} \\
\mathrm{NC}\end{array}$ & $\begin{array}{l}643 \\
91,343 \\
\text { NC } \\
\text { NC }\end{array}$ & $\begin{array}{l}291 \\
49,163 \\
\text { NC } \\
\text { NC }\end{array}$ & $\begin{array}{c}\text { NC } \\
52,593 \\
N C \\
N C\end{array}$ & $\begin{array}{c}266 \\
65,293 \\
\text { NC } \\
\text { NC }\end{array}$ \\
\hline 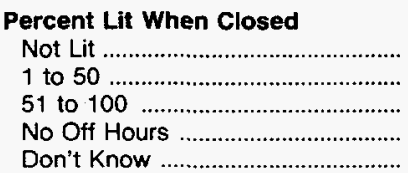 & $\begin{array}{r}5,149 \\
57,953 \\
17,412 \\
14,366 \\
\text { NC }\end{array}$ & $\begin{array}{r}2,079 \\
36,667 \\
13,955 \\
12,280 \\
\text { NC }\end{array}$ & $\begin{array}{l}4,039 \\
56,880 \\
17,161 \\
13,906 \\
\text { NC }\end{array}$ & $\begin{array}{l}1,708 \\
28,192 \\
10,197 \\
9,357 \\
\mathrm{NC}\end{array}$ & $\begin{array}{l}3,311 \\
26,092 \\
14,864 \\
8,325 \\
\mathrm{NC}\end{array}$ & $\begin{array}{r}2,244 \\
40,357 \\
13,725 \\
9,234 \\
\text { NC }\end{array}$ \\
\hline
\end{tabular}

See footnotes at end of table. 

Table 3.31. Lighting Equipment in FBSS Building in Federal Region 3,
Floorspace, 1993 (Continued)

(Thousand Square Feet)

\begin{tabular}{|c|c|c|c|c|c|c|}
\hline \multirow[b]{2}{*}{$\begin{array}{c}\text { Building } \\
\text { Characteristics }\end{array}$} & \multirow[b]{2}{*}{$\begin{array}{l}\text { All } \\
\text { Buildings }\end{array}$} & \multicolumn{5}{|c|}{$\begin{array}{l}\text { Lighting Equipment Types } \\
\text { (more than one may apply) }\end{array}$} \\
\hline & & Incandescent & $\begin{array}{l}\text { Standard } \\
\text { Fluorescent }\end{array}$ & $\begin{array}{l}\text { Compact } \\
\text { Fluorescent }\end{array}$ & $\begin{array}{l}\text { High- } \\
\text { Intensity } \\
\text { Discharge }\end{array}$ & $\begin{array}{c}\text { Electronic } \\
\text { Ballast }\end{array}$ \\
\hline \multicolumn{7}{|l|}{$\begin{array}{l}\text { Lighting Conservation Features } \\
\text { (more than one may apply) }\end{array}$} \\
\hline $\begin{array}{l}\text { Specular Reflectors ............................. } \\
\text { Natural Lighting Control }\end{array}$ & 44,741 & 32,688 & 44,126 & 27,412 & 24,739 & 36,255 \\
\hline 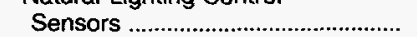 & 17,743 & 15,216 & 17,743 & 14,476 & 9,237 & 16,076 \\
\hline 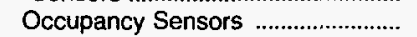 & 62,261 & 45,486 & 61,742 & 43,991 & 37,666 & 52,610 \\
\hline Time Clock & 46,555 & 39,907 & 46,036 & 36,171 & 29,709 & 39,466 \\
\hline Manual Dimmer Switches ................ & 49,824 & 42,097 & 49,259 & 37,377 & 28,905 & 43,797 \\
\hline \multicolumn{7}{|l|}{$\begin{array}{l}\text { Energy Management Practices } \\
\text { (more than one may apply) } \\
\text { Energy Management and Control }\end{array}$} \\
\hline 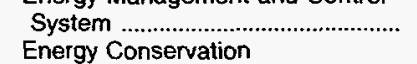 & 61,891 & 42,602 & 60,750 & 33,756 & 35,944 & 46,236 \\
\hline 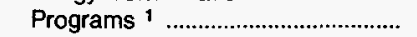 & 59,284 & 42,505 & 58,634 & 37,128 & 32,936 & 48,576 \\
\hline 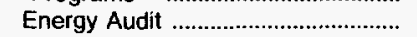 & 37,621 & 28,368 & 37,421 & 21,471 & 22,966 & 30,043 \\
\hline HVAC Maintenance Staff ${ }^{2}$................. & 52,924 & 39,568 & 51,125 & 30,245 & 29,904 & 40,191 \\
\hline \multicolumn{7}{|l|}{$\begin{array}{l}\text { Off-Hours Reduction in } \\
\text { Equipment (more than one may } \\
\text { apply) }\end{array}$} \\
\hline 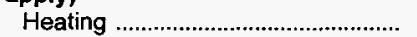 & 43,828 & 29,832 & 42,859 & 26,438 & 21,851 & 35,668 \\
\hline 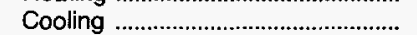 & 43,757 & 30,067 & 42,742 & 26,261 & 21,774 & 35,491 \\
\hline Hot Water & 26,018 & 19,184 & 25,499 & 17,164 & 10,462 & 20,798 \\
\hline 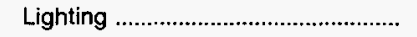 & 44,717 & 30,343 & 43,678 & 26,105 & 21,391 & 35,670 \\
\hline
\end{tabular}

1 Building participates in any programs sponsored by the Federal Energy Management Program, in-house, utility, or third party.

2 HVAC maintenance staff means at least one person spends at least half their working hours maintaining the heating/cooling equipment. $\mathrm{NC}=$ No cases in responding sample.

Notes: - Total workers are the number of workers during the main shift. - See Glossary for explanation of abbreviations and definitions of terms used in this report. - These data are from 881 federally owned buildings having the following criteria: (1) located in Federal Regions 3 , 6 , or 9; (2) larger than 10,000 square feet; and (3) used for a commercial purpose, other than warehouse and storage. In addition, 9 out of 10 selected buildings were from agencies other than the Department of Delense. - Statistics for the "energy end uses" represent consumption in buildings that have end use, not consumption for a particular fuel for a particular end use. - FBSS = Federal Buildings Supplemental Survey. - HVAC = Heating, Ventilation, and Air Conditioning. - Data are for Fiscal Year 1993 (October 1, 1992 through September 30 , 1993). - Because of rounding, data may not sum to totals.

Source: Energy Information Administration, Office of Energy Markets and End Use, 1993 Federal Buildings Supplemental Survey. 
Table 3.32. Lighting Equipment in FBSS Buildings in Federal Region 6, Floorspace, 1993

(Thousand Square Feet)

\begin{tabular}{|c|c|c|c|c|c|c|}
\hline \multirow[b]{2}{*}{$\begin{array}{c}\text { Building } \\
\text { Characteristics }\end{array}$} & \multirow[b]{2}{*}{$\begin{array}{c}\text { All } \\
\text { Buildings }\end{array}$} & \multicolumn{5}{|c|}{$\begin{array}{l}\text { Lighting Equipment Types } \\
\text { (more than one may apply) }\end{array}$} \\
\hline & & Incandescent & $\begin{array}{l}\text { Standard } \\
\text { Fluorescent }\end{array}$ & $\begin{array}{l}\text { Compact } \\
\text { Fluorescent }\end{array}$ & $\begin{array}{c}\text { High- } \\
\text { Intensity } \\
\text { Discharge }\end{array}$ & $\begin{array}{c}\text { Electronic } \\
\text { Ballast }\end{array}$ \\
\hline All Buildings & 35,816 & 20,248 & 35,057 & 7,942 & 16,188 & 15,861 \\
\hline \multicolumn{7}{|l|}{ Bullding Floorspace (square feet) } \\
\hline 10,000 to 50,000 & 2,591 & 1,099 & 2,478 & 164 & 406 & 440 \\
\hline 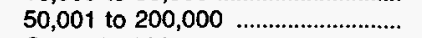 & 9,548 & 7,017 & 9,146 & 602 & 2,343 & 2,620 \\
\hline Over 200,000 & 23,677 & 12,133 & 23,433 & 7,175 & 13,439 & 12,801 \\
\hline \multicolumn{7}{|l|}{ Principal Building Activity } \\
\hline 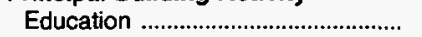 & 168 & 43 & 168 & 18 & 53 & 55 \\
\hline 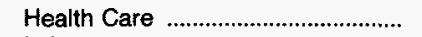 & 12,094 & 4,168 & 12,094 & 2,076 & 5,304 & $B, 444$ \\
\hline 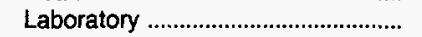 & 3,331 & 2,639 & 3,087 & 256 & 1,323 & 50 \\
\hline 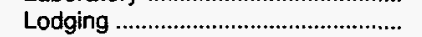 & 942 & 607 & 783 & NC & 21 & 432 \\
\hline Mercantile and Service ................... & 6,236 & 3,927 & 6,192 & 671 & 4,060 & 1,299 \\
\hline Office & 10,799 & 7,256 & 10,637 & 4,920 & 4,915 & 5,247 \\
\hline 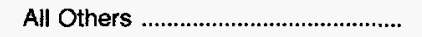 & 2,247 & 1,608 & 2,096 & NC & 512 & 334 \\
\hline \multicolumn{7}{|l|}{ Year Constructed } \\
\hline 1959 or Before & 13,258 & 9,746 & 12,831 & 3,350 & 5,968 & 5,886 \\
\hline 1960 to 1969 & 6,386 & 5,302 & 6,225 & 1,938 & 2,989 & 2,891 \\
\hline 1970 to 1979 & 6,175 & 1,749 & 6,175 & 2,087 & 4,716 & 2,400 \\
\hline 1980 to 1989 & 6,903 & 3,338 & 6,731 & 546 & 2,493 & 1,972 \\
\hline 1990 to 1993 & 3,095 & 114 & 3,095 & 19 & 23 & 2,711 \\
\hline \multicolumn{7}{|l|}{ Federal Agency } \\
\hline Department of Defense ..................... & 1,668 & 665 & 1,612 & 633 & 992 & 165 \\
\hline General Services Administration ... & 7,888 & 4,809 & 7,727 & 4,719 & 4,068 & 5,053 \\
\hline United States Postal Service ......... & 7,027 & 4,607 & 6,970 & 897 & 4,169 & 1,435 \\
\hline Veterans Administration ................... & 11,375 & 3,669 & 11,216 & 1,339 & 4,552 & 8,628 \\
\hline All Others & 7,858 & 6,499 & 7,532 & 354 & 2,407 & 580 \\
\hline \multicolumn{7}{|l|}{ Workers (main shift) } \\
\hline Less than 50 & 2,548 & 1,601 & 2,397 & 48 & 506 & 319 \\
\hline 50 to 99 & 2,026 & 1,016 & 1,867 & 124 & 263 & 588 \\
\hline 100 to 499 & 9,029 & 6,640 & 8,580 & 1,145 & 2,964 & 2,371 \\
\hline 500 or More & 22,213 & 10,992 & 22,213 & 6,625 & 12,456 & 12,582 \\
\hline \multicolumn{7}{|l|}{ Weekly Operating Hours } \\
\hline 48 or Fewer & 2,740 & 1,280 & 2,497 & 174 & 361 & 1,046 \\
\hline 49 to 60 & 6,460 & 2,878 & 6,447 & 2,968 & 2,314 & 1,834 \\
\hline 61 to 167 & 8,051 & 6,731 & 7,995 & 1,783 & 4,117 & 2,472 \\
\hline 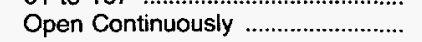 & 18,565 & 9,359 & 18,118 & 3,016 & 9,396 & 10,508 \\
\hline \multicolumn{7}{|l|}{ Percent Window Glass } \\
\hline 50 or Less & 29,836 & 16,321 & 29,076 & 6,021 & 12,127 & $1: 3,265$ \\
\hline 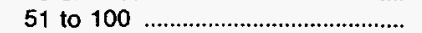 & 3,429 & 1,390 & 3,429 & 1,920 & 1,920 & 2,596 \\
\hline 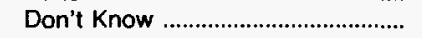 & 2,552 & 2,538 & 2,552 & NC & 2,141 & NC \\
\hline \multicolumn{7}{|l|}{ Multibuilding Facility } \\
\hline 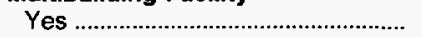 & 24,441 & 14,792 & 23,933 & 4,014 & 9,206 & 10,340 \\
\hline No & 11,375 & 5,457 & 11,124 & 3,927 & 6,983 & 5,521 \\
\hline \multicolumn{7}{|l|}{ Percent Lit When Open } \\
\hline 1 to 50 & 1,062 & 744 & 1,049 & NC & 183 & 75 \\
\hline 51 to 100 & 34,672 & 19,505 & 34,008 & 7,942 & 16,005 & 15,786 \\
\hline No Operating Hours .............................. & 82 & NC & NC & $\mathrm{NC}$ & NC & NC \\
\hline 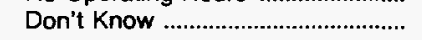 & NC & NG & NC & NC & NC & NC \\
\hline \multicolumn{7}{|l|}{ Percent Lit When Closed } \\
\hline 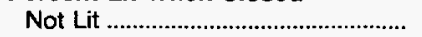 & 2,691 & 1,147 & 2,424 & 1,201 & 1,496 & 724 \\
\hline 1 to 50 & 20,495 & 13,218 & 20,406 & 5,814 & 8,833 & 7,195 \\
\hline 51 to 100 & 8,029 & 4,121 & 8,029 & 927 & 3,308 & 5,239 \\
\hline 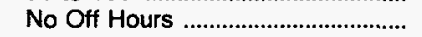 & 4,601 & 1,763 & 4,199 & NC & 2,552 & 2,702 \\
\hline Don't Know & NC & NC & NC & NC & NC & NC \\
\hline
\end{tabular}

See footnotes at end of table. 
Table 3.32. Lighting Equipment in FBSS Buildings in Federal Region 6, Floorspace, 1993 (Continued)

(Thousand Square Feet)

\begin{tabular}{|c|c|c|c|c|c|c|}
\hline \multirow[b]{2}{*}{$\begin{array}{c}\text { Building } \\
\text { Characteristics }\end{array}$} & \multirow[b]{2}{*}{$\begin{array}{c}\text { All } \\
\text { Buildings }\end{array}$} & \multicolumn{5}{|c|}{$\begin{array}{l}\text { Lighting Equipment Types } \\
\text { (more than one may apply) }\end{array}$} \\
\hline & & Incandescent & $\begin{array}{l}\text { Standard } \\
\text { Fluorescent }\end{array}$ & $\begin{array}{l}\text { Compact } \\
\text { Fluorescent }\end{array}$ & $\begin{array}{l}\text { High- } \\
\text { Intensity } \\
\text { Discharge }\end{array}$ & $\begin{array}{c}\text { Electronic } \\
\text { Ballast }\end{array}$ \\
\hline \multicolumn{7}{|l|}{$\begin{array}{l}\text { Lighting Conservation Features } \\
\text { (more than one may apply) }\end{array}$} \\
\hline $\begin{array}{l}\text { Specular Reflectors ........................... } \\
\text { Natural Lighting Control }\end{array}$ & 18,017 & 9,240 & 17,664 & 4,173 & 7,863 & 11,662 \\
\hline 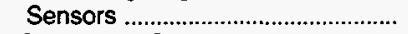 & 6,740 & 2,524 & 6,581 & 2,025 & 2,658 & 5,669 \\
\hline 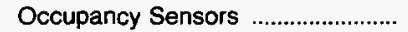 & 16,435 & 8,859 & 16,114 & 4,909 & 8,217 & 11,542 \\
\hline 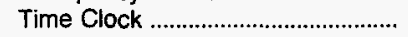 & 9,037 & 3,236 & 8,878 & 2,875 & 3,191 & 6,225 \\
\hline Manual Dimmer Switches ................ & 13,454 & 6,641 & 13,249 & 4,457 & 4,567 & 8,110 \\
\hline \multicolumn{7}{|l|}{$\begin{array}{l}\text { Energy Management Practices } \\
\text { (more than one may apply) } \\
\text { Energy Management and Control }\end{array}$} \\
\hline $\begin{array}{l}\text { System ............................................... } \\
\text { Energy Conservation }\end{array}$ & 23,099 & 10,360 & 22,511 & 6,385 & 11,491 & 12,411 \\
\hline Programs ${ }^{1} \ldots \ldots \ldots \ldots \ldots \ldots \ldots \ldots$ & 13,513 & 6,777 & 13,480 & 3,388 & 6,805 & 9,798 \\
\hline 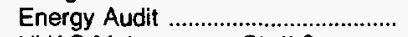 & 11,690 & 5,719 & 11,251 & 4,430 & 6,194 & 5,856 \\
\hline HVAC Maintenance Staff 2 ............... & 15,538 & 7,664 & 15,376 & 4,995 & 6,374 & 9,417 \\
\hline \multicolumn{7}{|l|}{$\begin{array}{l}\text { Off-Hours Reduction in } \\
\text { Equipment (more than one may } \\
\text { apply) }\end{array}$} \\
\hline 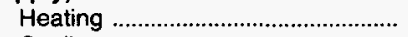 & 13,162 & 7,434 & 12,932 & 4,926 & 4,639 & 4,923 \\
\hline 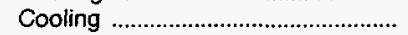 & 13,864 & 7,837 & 13,657 & 4,926 & 4,615 & 5,246 \\
\hline 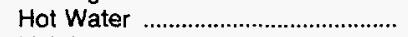 & 4,554 & 2,503 & 4,554 & 1,780 & 2,266 & 1,387 \\
\hline 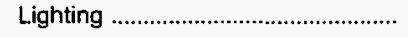 & 12,847 & 7,991 & 12,640 & 4,892 & 4,577 & 5,342 \\
\hline
\end{tabular}

1 Building participates in any programs sponsored by the Federal Energy Management Program, in-house, utility, or third party.

2 HVAC maintenance staff means at least one person spends at least half their working hours maintaining the heating/cooling equipment. NC $=$ No cases in responding sample.

Notes: - Total workers are the number of workers during the main shift. - See Glossary for explanation of abbreviations and definitions of terms used in this report. - These data are from 881 federally owned buildings having the following criteria: (1) located in Federal Regions 3 , 6 , or 9; (2) larger than 10,000 square feet; and (3) used for a commercial purpose, other than warehouse and storage. In addition, 9 out of 10 selected buildings were from agencies other than the Department of Defense. - Statistics for the "energy end uses" represent consumption in buildings that have end use, not consumption for a particular fuel for a particular end use. - FBSS = Federal Buildings Supplemental Survey. - HVAC = Heating, Ventilation, and Air Conditioning. - Data are for Fiscal Year 1993 (October 1, 1992 through September 30, 1993). - Because of rounding, data may not sum to totals.

Source: Energy Information Administration, Office of Energy Markets and End Use, 1993 Federal Buildings Supplemental Survey. 


\section{Table 3.33. Lighting Equipment in FBSS Buildings in Federal Region 9, Floorspace, 1993 \\ (Thousand Square Feet)}

\begin{tabular}{|c|c|c|c|c|c|c|}
\hline \multirow[b]{2}{*}{$\begin{array}{c}\text { Bullding } \\
\text { Characteristics }\end{array}$} & \multirow[b]{2}{*}{$\begin{array}{l}\text { All } \\
\text { Bulldings }\end{array}$} & \multicolumn{5}{|c|}{$\begin{array}{l}\text { Lighting Equipment Types } \\
\text { (more than one may apply) }\end{array}$} \\
\hline & & Incandescent & $\begin{array}{l}\text { Standard } \\
\text { Fluorescent }\end{array}$ & $\begin{array}{l}\text { Compact } \\
\text { Fluorescent }\end{array}$ & $\begin{array}{c}\text { High- } \\
\text { Intensity } \\
\text { Discharge }\end{array}$ & $\begin{array}{c}\text { Electronic } \\
\text { Ballast }\end{array}$ \\
\hline All Buildings & 44,316 & 20,475 & 42,850 & 17,339 & 18,736 & 23,307 \\
\hline \multicolumn{7}{|l|}{ Building Floorspace (square feet) } \\
\hline 10,000 to 50,000 & 3,858 & 1,864 & 3,663 & 415 & 562 & 1,032 \\
\hline 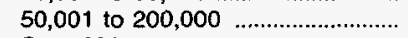 & 12,680 & 7,322 & 12,228 & 4,155 & 3,503 & 5,236 \\
\hline 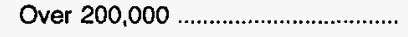 & 27,778 & 11,289 & 26,960 & 12,769 & 14,671 & 17,038 \\
\hline \multicolumn{7}{|l|}{ Principal Building Activity } \\
\hline 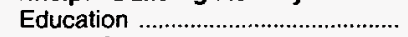 & 628 & 450 & 628 & $\mathrm{NC}$ & 262 & 85 \\
\hline 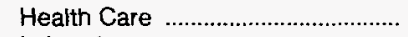 & 9,903 & 4,574 & 9,903 & 2,484 & 2,472 & 4,961 \\
\hline 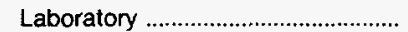 & 2,601 & 1,960 & 2,527 & 1,592 & 213 & 631 \\
\hline Lodging & 1,220 & 667 & 897 & 83 & 62 & 73 \\
\hline Mercantile and Service .................... & 8,194 & 3,716 & 8,111 & 859 & 6,475 & 4,237 \\
\hline Office & 16,380 & 6,522 & 15,725 & 11,151 & 5,590 & 10,762 \\
\hline 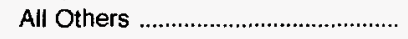 & 5,390 & 2,586 & 5,060 & 1,170 & 3,662 & 2,558 \\
\hline \multicolumn{7}{|l|}{ Year Constructed } \\
\hline 1959 or Before & 13,553 & 6,594 & 12,818 & 4,673 & 4,693 & $6,82.4$ \\
\hline 1960 to 1969 & 9,082 & 4,486 & 9,027 & 5,204 & 3,997 & 5,592 \\
\hline 1970 to 1979 & 12,041 & 5,426 & 11,622 & 4,454 & 5,379 & 7,411 \\
\hline 1980 to 1989 & 7,955 & 3,097 & 7,842 & 2,750 & 3,741 & 3,241 \\
\hline 1990 to 1993 & 1,684 & 870 & 1,541 & 258 & 926 & 239 \\
\hline \multicolumn{7}{|l|}{ Federal Agency } \\
\hline Department of Defense ..................... & 8,489 & 2,849 & 7,866 & 2,943 & 3,537 & $2,86: 8$ \\
\hline General Services Administration ... & 12,505 & 4,354 & 12,165 & 10,436 & 5,024 & 9,527 \\
\hline United States Postal Service ......... & 8,387 & 3,827 & 8,304 & 1,001 & 6,229 & 4,292 \\
\hline Veterans Administration ................... & 8,824 & 4,269 & 8,681 & 1,043 & 2,615 & 5,302 \\
\hline All Others & 6,111 & 5,175 & 5,834 & 1,916 & 1,330 & 1,318 \\
\hline \multicolumn{7}{|l|}{ Workers (main shift) } \\
\hline Less than 50 & 3,684 & 2,242 & 2,945 & 719 & 930 & 753 \\
\hline 50 to 99 & 2,161 & 1,100 & 2,142 & 437 & 604 & 705 \\
\hline 100 to 499 & 14,017 & 7,305 & 13,540 & 4,446 & 3,734 & 5,095 \\
\hline 500 or More & 24,454 & 9,828 & 24,223 & 11,738 & 13,468 & 16,753 \\
\hline \multicolumn{7}{|l|}{ Weekly Operating Hours } \\
\hline 48 or Fewer & 4,822 & 2,719 & 4,276 & 454 & 1,172 & 955 \\
\hline 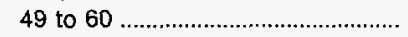 & 13,424 & 4,499 & 13,369 & 9,532 & $5,13 \overline{3}$ & 9,185 \\
\hline 61 to 167 & 5,706 & 3,301 & 5,558 & 1,877 & 2,905 & 3,217 \\
\hline 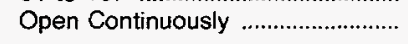 & 20,364 & 9,955 & 19,647 & 5,476 & 9,526 & 9,950 \\
\hline \multicolumn{7}{|l|}{ Percent Window Glass } \\
\hline 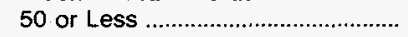 & 37,432 & 18,276 & 36,105 & 12,394 & 16,372 & 17,762 \\
\hline 51 to 100 & 6,800 & 2,199 & 6,745 & 4,945 & 2,363 & 5,545 \\
\hline 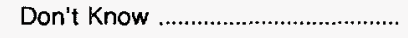 & 84 & NC & NC & NC & NC & NC: \\
\hline \multicolumn{7}{|l|}{ Multibuilding Facility } \\
\hline Yes ....................... & 26,821 & 14,046 & 26,038 & 8,337 & 10,972 & 12,980 \\
\hline No & 17,495 & 6,429 & 16,812 & 9,002 & 7,763 & 10,327 \\
\hline \multicolumn{7}{|l|}{ Percent Lit When Open } \\
\hline 1 to 50 & 1,847 & 931 & 1,260 & 78 & 69 & $12: 3$ \\
\hline 51 to $100 \ldots \ldots \ldots \ldots$ & 42,375 & 19,543 & 41,590 & 17,261 & 18,667 & $23,18 \cdot 4$ \\
\hline No Operating Hours .......................... & 29 & NC & NC & $\mathrm{NC}$ & NC & NC \\
\hline 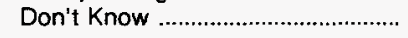 & 64 & NC & NC & NC & NC & NC \\
\hline \multicolumn{7}{|l|}{ Percent Lit When Closed } \\
\hline 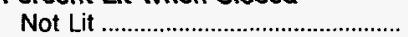 & 1,664 & 222 & 1,600 & 317 & 417 & 701 \\
\hline 1 to 50 & 25,288 & 12,656 & 24,667 & 12,447 & 9,589 & 14,273 \\
\hline 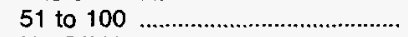 & 8,109 & 4,994 & 7,801 & 3,640 & 2,615 & 2,684 \\
\hline No Off Hours .................................... & 9,190 & 2,603 & 8,781 & 935 & 6,115 & 5,648 \\
\hline 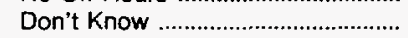 & 64 & NC & NC & NC & NC & NC \\
\hline
\end{tabular}

See footnotes at end of table. 
Table 3.33. Lighting Equipment in FBSS Buildings in Federal Region 9, Floorspace, 1993 (Continued)

(Thousand Square Feet)

\begin{tabular}{|c|c|c|c|c|c|c|}
\hline \multirow[b]{2}{*}{$\begin{array}{c}\text { Building } \\
\text { Characteristics }\end{array}$} & \multirow[b]{2}{*}{$\begin{array}{c}\text { All } \\
\text { Buildings }\end{array}$} & \multicolumn{5}{|c|}{$\begin{array}{l}\text { Lighting Equipment Types } \\
\text { (more than one may apply) }\end{array}$} \\
\hline & & Incandescent & $\begin{array}{l}\text { Standard } \\
\text { Fluorescent }\end{array}$ & $\begin{array}{l}\text { Compact } \\
\text { Fluorescent }\end{array}$ & $\begin{array}{l}\text { High- } \\
\text { Intensity } \\
\text { Discharge }\end{array}$ & $\begin{array}{c}\text { Electronic } \\
\text { Ballast }\end{array}$ \\
\hline \multicolumn{7}{|l|}{$\begin{array}{l}\text { Lighting Conservation Features } \\
\text { (more than one may apply) }\end{array}$} \\
\hline $\begin{array}{l}\text { Specular Reflectors ....................... } \\
\text { Natural Lighting Control }\end{array}$ & 21,850 & 9.554 & 21,637 & 12,265 & 8,640 & 13,517 \\
\hline 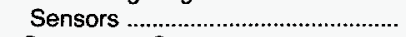 & 13,760 & 4,011 & 13,617 & 7,935 & 6,074 & 8,258 \\
\hline 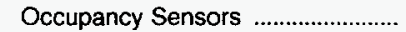 & 25,245 & 11,469 & 25,180 & 15,083 & 12,036 & 16,127 \\
\hline Time Clock & 10,539 & 6,482 & 10,484 & 7,210 & 6,584 & 6,656 \\
\hline Manual Dimmer Switches ................ & 14,455 & 10,224 & 14,371 & 7,795 & 6,245 & 7,293 \\
\hline \multicolumn{7}{|l|}{$\begin{array}{l}\text { Energy Management Practices } \\
\text { (more than one may apply) } \\
\text { Energy Management and Control }\end{array}$} \\
\hline $\begin{array}{l}\text { System ........................................... } \\
\text { Energy Conservation }\end{array}$ & $18,23 i$ & 7,727 & 17,903 & 7,441 & 10,875 & 11,378 \\
\hline Programs 1 & 24,699 & 11,924 & 24,511 & 13,891 & 10,570 & 16,181 \\
\hline Energy Audit & 17,444 & 9,157 & 16,789 & 6,254 & 9,828 & 12,008 \\
\hline HVAC Maintenance Staff 2 ............. & 14,570 & 8,130 & 14,570 & 8,282 & 6,651 & 8,859 \\
\hline \multicolumn{7}{|l|}{$\begin{array}{l}\text { Off-Hours Reduction in } \\
\text { Equipment (more than one may } \\
\text { apply) }\end{array}$} \\
\hline Heating & 19,631 & 9,172 & 19,015 & 11,608 & 6,905 & 12,166 \\
\hline 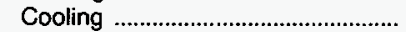 & 19,219 & 8,636 & 18,599 & 10,787 & 6,834 & 11,521 \\
\hline 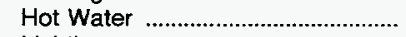 & 13,614 & 5,154 & 13,155 & 9,541 & 4,876 & 9,004 \\
\hline 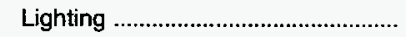 & 21,155 & 9,503 & 20,567 & 11,605 & 7,825 & 12,556 \\
\hline
\end{tabular}

1 Building participates in any programs sponsored by the Federal Energy Management Program, in-house, utility, or third party.

2 HVAC maintenance staff means at least one person spends at least half their working hours maintaining the heating/cooling equipment $\mathrm{NC}=$ No cases in responding sample.

Notes: - Total workers are the number of workers during the main shift. - See Glossary for explanation of abbreviations and definitions of terms used in this report. - These data are from 881 federally owned buildings having the following criteria: (1) located in Federal Regions 3 , 6 , or 9; (2) larger than 10,000 square feet; and (3) used for a commercial purpose, other than warehouse and storage. In addition, 9 out of 10 selected buildings were from agencies other than the Department of Defense. - Statistics for the "energy end uses" represent consumption in buildings that have end use, not consumption for a particular fuel for a particular end use. - FBSS = Federal Buildings Supplemental Survey. - HVAC = Heating, Ventilation, and Air Conditioning. - Data are for Fiscal Year 1993 (October 1, 1992 through September 30, 1993). Because of rounding, data may not sum to totals.

Source: Energy Information Administration, Office of Energy Markets and End Use, 1993 Federal Buildings Supplemental Survey. 
Table 3.34. Energy Conservation Features in FBSS Building in Federal Region 3, Number of Buildings and Floorspace, 1993

\begin{tabular}{|c|c|c|c|c|c|c|c|c|c|c|}
\hline \multirow[b]{2}{*}{$\begin{array}{c}\text { Building } \\
\text { Characteristics }\end{array}$} & \multicolumn{5}{|c|}{ Number of Buildings } & \multicolumn{5}{|c|}{$\begin{array}{l}\text { Total Floorspace } \\
\text { (thousand square feet) }\end{array}$} \\
\hline & $\begin{array}{c}\text { All } \\
\text { Buildings }\end{array}$ & $\begin{array}{l}\text { Any } \\
\text { Conser- } \\
\text { vation } \\
\text { Feature }\end{array}$ & $\begin{array}{c}\text { Building } \\
\text { Shell }\end{array}$ & HVAC & Lighting & $\begin{array}{c}\text { All } \\
\text { Buildings }\end{array}$ & $\begin{array}{c}\text { Any } \\
\text { Conser- } \\
\text { vation } \\
\text { Feature }\end{array}$ & $\begin{array}{l}\text { Building } \\
\text { Shell }\end{array}$ & HVAC & Lighting \\
\hline 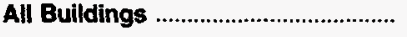 & 312 & 309 & 290 & 303 & 210 & 94,880 & 94,361 & 84,557 & 93,543 & 30,953 \\
\hline \multicolumn{11}{|l|}{ Building Floorspace (square feet) } \\
\hline 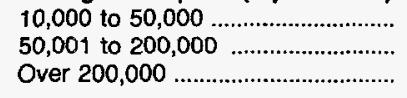 & $\begin{array}{r}94 \\
117 \\
101\end{array}$ & $\begin{array}{r}94 \\
115 \\
100\end{array}$ & $\begin{array}{r}91 \\
110 \\
89\end{array}$ & $\begin{array}{r}92 \\
112 \\
99\end{array}$ & $\begin{array}{l}52 \\
74 \\
84\end{array}$ & $\begin{array}{r}2,564 \\
13,166 \\
79,149\end{array}$ & $\begin{array}{r}2,564 \\
12,906 \\
78,891\end{array}$ & $\begin{array}{r}2,451 \\
12,381 \\
69,724\end{array}$ & $\begin{array}{r}2,541 \\
12,538 \\
78,464\end{array}$ & $\begin{array}{r}1,401 \\
8,315 \\
71,238\end{array}$ \\
\hline \multicolumn{11}{|l|}{ Principal Building Activity } \\
\hline 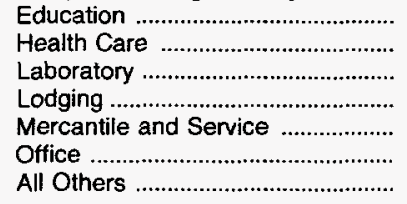 & $\begin{array}{r}8 \\
41 \\
37 \\
13 \\
46 \\
124 \\
43\end{array}$ & $\begin{array}{r}8 \\
41 \\
37 \\
13 \\
46 \\
124 \\
40\end{array}$ & $\begin{array}{r}8 \\
41 \\
37 \\
12 \\
44 \\
111 \\
37\end{array}$ & $\begin{array}{r}8 \\
40 \\
37 \\
13 \\
45 \\
120 \\
40\end{array}$ & $\begin{array}{r}5 \\
31 \\
13 \\
6 \\
29 \\
103 \\
23\end{array}$ & $\begin{array}{r}598 \\
14,559 \\
5,165 \\
2,558 \\
7,966 \\
56,881 \\
7,152\end{array}$ & $\begin{array}{r}598 \\
14,559 \\
5,165 \\
2,558 \\
7,966 \\
56,881 \\
6,633\end{array}$ & $\begin{array}{r}598 \\
14,559 \\
5,165 \\
1,077 \\
7,863 \\
48,877 \\
6,418\end{array}$ & $\begin{array}{r}598 \\
14,412 \\
5,165 \\
2,558 \\
7,907 \\
56,269 \\
6,633\end{array}$ & $\begin{array}{r}459 \\
12,538 \\
2,654 \\
605 \\
6,050 \\
53,853 \\
4,795\end{array}$ \\
\hline 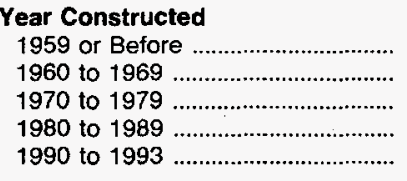 & $\begin{array}{r}142 \\
70 \\
47 \\
38 \\
15\end{array}$ & $\begin{array}{r}140 \\
70 \\
47 \\
37 \\
15\end{array}$ & $\begin{array}{r}128 \\
65 \\
46 \\
37 \\
14\end{array}$ & $\begin{array}{r}136 \\
69 \\
47 \\
36 \\
15\end{array}$ & $\begin{array}{l}98 \\
41 \\
32 \\
29 \\
10\end{array}$ & $\begin{array}{r}43,829 \\
19,564 \\
17,737 \\
8,975 \\
4,774\end{array}$ & $\begin{array}{r}43,510 \\
19,564 \\
17,737 \\
8,775 \\
4,774\end{array}$ & $\begin{array}{r}37,203 \\
16,273 \\
17,679 \\
8,775 \\
4,626\end{array}$ & $\begin{array}{r}43,129 \\
19,138 \\
17,737 \\
8,765 \\
4,774\end{array}$ & $\begin{array}{r}37,505 \\
14,596 \\
16,630 \\
8,058 \\
4,163\end{array}$ \\
\hline 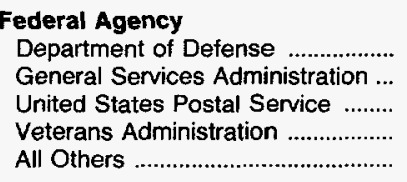 & $\begin{array}{l}22 \\
83 \\
57 \\
56 \\
94\end{array}$ & $\begin{array}{l}21 \\
82 \\
57 \\
55 \\
94\end{array}$ & $\begin{array}{l}20 \\
69 \\
55 \\
54 \\
92\end{array}$ & $\begin{array}{l}21 \\
81 \\
55 \\
53 \\
93\end{array}$ & $\begin{array}{l}14 \\
73 \\
36 \\
40 \\
47\end{array}$ & $\begin{array}{r}13,988 \\
46,205 \\
9,392 \\
13,306 \\
11,989\end{array}$ & $\begin{array}{r}13,928 \\
45,946 \\
9,392 \\
13,106 \\
11,989\end{array}$ & $\begin{array}{r}12,447 \\
37,942 \\
9,289 \\
12,958 \\
11,921\end{array}$ & $\begin{array}{r}13,928 \\
45,519 \\
9,319 \\
12,949 \\
11,827\end{array}$ & $\begin{array}{r}12,088 \\
43,692 \\
6,866 \\
10,241 \\
8,067\end{array}$ \\
\hline \multicolumn{11}{|l|}{$\begin{array}{l}\text { Energy Sources (more than one } \\
\text { may apply) }\end{array}$} \\
\hline 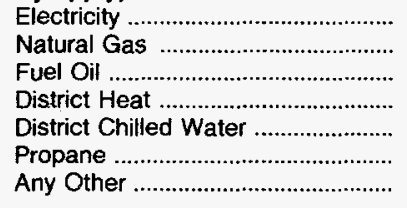 & $\begin{array}{r}311 \\
139 \\
89 \\
154 \\
57 \\
5 \\
8\end{array}$ & $\begin{array}{r}308 \\
139 \\
89 \\
153 \\
57 \\
5 \\
8\end{array}$ & $\begin{array}{r}289 \\
130 \\
86 \\
142 \\
57 \\
5 \\
6\end{array}$ & $\begin{array}{r}302 \\
136 \\
88 \\
151 \\
56 \\
5 \\
8\end{array}$ & $\begin{array}{r}209 \\
100 \\
63 \\
103 \\
35 \\
4 \\
6\end{array}$ & $\begin{array}{r}94,161 \\
58,674 \\
39,300 \\
63,991 \\
22,922 \\
941 \\
4,470\end{array}$ & $\begin{array}{r}93,642 \\
58,674 \\
39,300 \\
63,931 \\
22,922 \\
941 \\
4,470\end{array}$ & $\begin{array}{r}83,838 \\
53,375 \\
37,719 \\
57,611 \\
22,922 \\
941 \\
2,941\end{array}$ & $\begin{array}{r}92,824 \\
58,454 \\
39,153 \\
63,342 \\
22,495 \\
941 \\
4,470\end{array}$ & $\begin{array}{r}80,234 \\
52,264 \\
35,096 \\
56,414 \\
19,908 \\
905 \\
4,293\end{array}$ \\
\hline \multicolumn{11}{|l|}{$\begin{array}{l}\text { Energy End Uses (more than one } \\
\text { may apply) }\end{array}$} \\
\hline 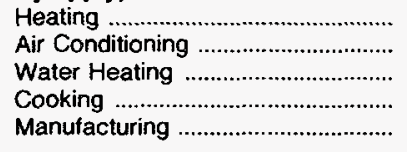 & $\begin{array}{r}307 \\
305 \\
303 \\
108 \\
50\end{array}$ & $\begin{array}{r}306 \\
304 \\
302 \\
108 \\
49\end{array}$ & $\begin{array}{r}287 \\
285 \\
284 \\
103 \\
48\end{array}$ & $\begin{array}{r}300 \\
298 \\
296 \\
107 \\
48\end{array}$ & $\begin{array}{r}207 \\
207 \\
205 \\
91 \\
35\end{array}$ & $\begin{array}{l}94,063 \\
93,454 \\
93,138 \\
69,947 \\
27,041\end{array}$ & $\begin{array}{l}94,003 \\
93,394 \\
93,078 \\
69,947 \\
26,981\end{array}$ & $\begin{array}{l}84,198 \\
83,590 \\
83,299 \\
64,665 \\
26,923\end{array}$ & $\begin{array}{l}93,184 \\
92,576 \\
92,260 \\
69,520 \\
26,922\end{array}$ & $\begin{array}{l}80,595 \\
80,039 \\
80,064 \\
63,750 \\
24,375\end{array}$ \\
\hline \multicolumn{11}{|l|}{ Workers (main shift) } \\
\hline $\begin{array}{l}\text { Less than } 50 \\
50 \text { to } 99 \\
100 \text { to } 499 \\
500 \text { or More }\end{array}$ & $\begin{array}{r}71 \\
39 \\
107 \\
95\end{array}$ & $\begin{array}{r}69 \\
39 \\
106 \\
95\end{array}$ & $\begin{array}{r}64 \\
38 \\
103 \\
85\end{array}$ & $\begin{array}{r}66 \\
39 \\
103 \\
95\end{array}$ & $\begin{array}{l}35 \\
26 \\
68 \\
81\end{array}$ & $\begin{array}{r}4,102 \\
1,903 \\
13,632 \\
75,243\end{array}$ & $\begin{array}{r}3,643 \\
1,903 \\
13,572 \\
75,243\end{array}$ & $\begin{array}{r}3,113 \\
1,858 \\
13,300 \\
66,285\end{array}$ & $\begin{array}{r}3,194 \\
1,903 \\
13,203 \\
75,243\end{array}$ & $\begin{array}{r}1,978 \\
1,354 \\
8,973 \\
68,648\end{array}$ \\
\hline 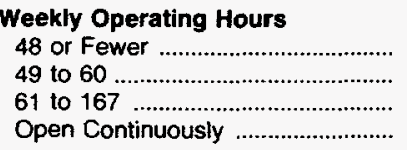 & $\begin{array}{r}51 \\
76 \\
60 \\
125\end{array}$ & $\begin{array}{r}50 \\
76 \\
60 \\
123\end{array}$ & $\begin{array}{r}44 \\
73 \\
54 \\
119\end{array}$ & $\begin{array}{r}47 \\
76 \\
59 \\
121\end{array}$ & $\begin{array}{l}35 \\
55 \\
40 \\
80\end{array}$ & $\begin{array}{r}9,694 \\
25,540 \\
11,993 \\
47,653\end{array}$ & $\begin{array}{r}9,634 \\
25,540 \\
11,993 \\
47,194\end{array}$ & $\begin{array}{r}5,910 \\
24,449 \\
8,720 \\
45,477\end{array}$ & $\begin{array}{r}9,036 \\
25,540 \\
11,980 \\
46,987\end{array}$ & $\begin{array}{r}8,377 \\
23,182 \\
10,939 \\
38,455\end{array}$ \\
\hline
\end{tabular}

See footnotes at end of table. 
Table 3.34. Energy Conservation Features in FBSS Building in Federal Region 3, Number of Buildings and Floorspace, 1993 (Continued)

\begin{tabular}{|c|c|c|c|c|c|c|c|c|c|c|}
\hline \multirow[b]{2}{*}{$\begin{array}{c}\text { Building } \\
\text { Characteristics }\end{array}$} & \multicolumn{5}{|c|}{ Number of Buildings } & \multicolumn{5}{|c|}{$\begin{array}{l}\text { Total Floorspace } \\
\text { (thousand square feet) }\end{array}$} \\
\hline & \begin{tabular}{c|} 
All \\
Buildings
\end{tabular} & $\begin{array}{c}\text { Any } \\
\text { Conser- } \\
\text { vation } \\
\text { Feature }\end{array}$ & $\begin{array}{l}\text { Building } \\
\text { Shell }\end{array}$ & HVAC & Lighting & $\begin{array}{c}\text { All } \\
\text { Bulldings }\end{array}$ & $\begin{array}{l}\text { Any } \\
\text { Conser- } \\
\text { vation } \\
\text { Feature }\end{array}$ & $\begin{array}{c}\text { Building } \\
\text { Shell }\end{array}$ & HVAC & Lighting \\
\hline \multicolumn{11}{|l|}{ Percent Window Glass } \\
\hline $\begin{array}{l}50 \text { or Less } \\
51 \text { to } 100 \\
\text { Don't Know }\end{array}$ & $\begin{array}{r}269 \\
42 \\
1\end{array}$ & $\begin{array}{r}266 \\
42 \\
1\end{array}$ & $\begin{array}{r}249 \\
40 \\
1\end{array}$ & $\begin{array}{r}261 \\
41 \\
1\end{array}$ & $\begin{array}{r}173 \\
36 \\
1\end{array}$ & $\begin{array}{r}79,002 \\
14,878 \\
1,000\end{array}$ & $\begin{array}{r}78,483 \\
14,878 \\
1,000\end{array}$ & $\begin{array}{r}69,326 \\
14,231 \\
1,000\end{array}$ & $\begin{array}{r}78,091 \\
14,452 \\
1,000\end{array}$ & $\begin{array}{r}67,002 \\
12,952 \\
1,000\end{array}$ \\
\hline \multicolumn{11}{|l|}{ Multibuilding Facility } \\
\hline Yes & $\begin{array}{l}176 \\
136\end{array}$ & $\begin{array}{l}174 \\
135\end{array}$ & $\begin{array}{l}163 \\
127\end{array}$ & $\begin{array}{l}170 \\
133\end{array}$ & $\begin{array}{l}110 \\
100\end{array}$ & $\begin{array}{l}43,861 \\
51,018\end{array}$ & $\begin{array}{l}43,601 \\
50,760\end{array}$ & $\begin{array}{l}38,067 \\
46,490\end{array}$ & $\begin{array}{l}42,806 \\
50,737\end{array}$ & $\begin{array}{l}33,289 \\
47,664\end{array}$ \\
\hline 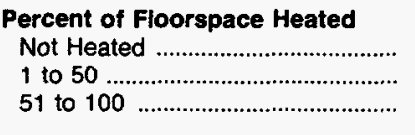 & $\begin{array}{r}5 \\
12 \\
295\end{array}$ & $\begin{array}{r}3 \\
12 \\
294\end{array}$ & $\begin{array}{r}3 \\
11 \\
276\end{array}$ & $\begin{array}{r}3 \\
10 \\
290\end{array}$ & $\begin{array}{r}3 \\
7 \\
200\end{array}$ & $\begin{array}{r}817 \\
5,787 \\
88,275\end{array}$ & $\begin{array}{r}359 \\
5,787 \\
88,215\end{array}$ & $\begin{array}{r}359 \\
5,639 \\
78,559\end{array}$ & $\begin{array}{r}359 \\
5,350 \\
87,834\end{array}$ & $\begin{array}{r}359 \\
5,127 \\
75,468\end{array}$ \\
\hline 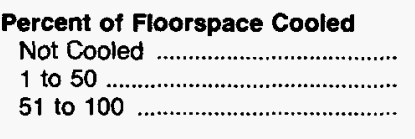 & $\begin{array}{r}13 \\
30 \\
269\end{array}$ & $\begin{array}{r}11 \\
30 \\
268\end{array}$ & $\begin{array}{r}10 \\
25 \\
255\end{array}$ & $\begin{array}{r}11 \\
29 \\
263\end{array}$ & $\begin{array}{r}7 \\
18 \\
185\end{array}$ & $\begin{array}{r}3,582 \\
9,874 \\
81,424\end{array}$ & $\begin{array}{r}3,123 \\
9,874 \\
81,364\end{array}$ & $\begin{array}{r}1,641 \\
9,351 \\
73,565\end{array}$ & $\begin{array}{r}3,123 \\
9,864 \\
80,556\end{array}$ & $\begin{array}{r}1,209 \\
9,047 \\
70,697\end{array}$ \\
\hline 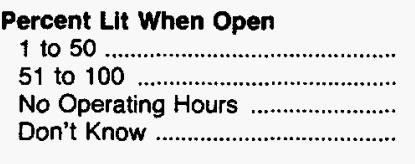 & $\begin{array}{r}11 \\
300 \\
1 \\
N C\end{array}$ & $\begin{array}{r}11 \\
297 \\
1 \\
N C\end{array}$ & $\begin{array}{r}9 \\
280 \\
1 \\
N C\end{array}$ & $\begin{array}{l}10 \\
293 \\
\text { NC } \\
\text { NC }\end{array}$ & $\begin{array}{l}5 \\
205 \\
\text { NC } \\
\text { NC }\end{array}$ & $\begin{array}{r}663 \\
94,070 \\
147 \\
\mathrm{NC}\end{array}$ & $\begin{array}{r}663 \\
93,551 \\
147 \\
\mathrm{NC}\end{array}$ & $\begin{array}{r}515 \\
83,895 \\
147 \\
N C\end{array}$ & $\begin{array}{c}653 \\
92,890 \\
N C \\
N C\end{array}$ & $\begin{array}{c}297 \\
80,656 \\
\text { NC } \\
\text { NC }\end{array}$ \\
\hline \multicolumn{11}{|l|}{$\begin{array}{l}\text { Heating Equipment (more than } \\
\text { one may apply) }\end{array}$} \\
\hline 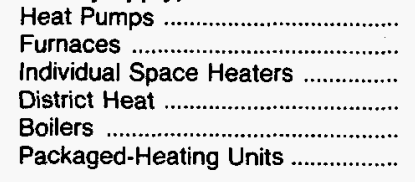 & $\begin{array}{r}34 \\
16 \\
75 \\
167 \\
93 \\
25\end{array}$ & $\begin{array}{r}34 \\
16 \\
75 \\
166 \\
93 \\
25\end{array}$ & $\begin{array}{r}34 \\
15 \\
69 \\
152 \\
89 \\
25\end{array}$ & $\begin{array}{r}32 \\
15 \\
73 \\
164 \\
91 \\
24\end{array}$ & $\begin{array}{r}28 \\
8 \\
56 \\
107 \\
69 \\
17\end{array}$ & $\begin{array}{r}10,922 \\
1,694 \\
34,923 \\
67,704 \\
20,082 \\
6,006\end{array}$ & $\begin{array}{r}10,922 \\
1,694 \\
34,923 \\
67,644 \\
20,082 \\
6,006\end{array}$ & $\begin{array}{r}10,922 \\
1,636 \\
32,183 \\
59,221 \\
18,726 \\
6,006\end{array}$ & $\begin{array}{r}10,485 \\
1,635 \\
34,717 \\
67,055 \\
20,009 \\
5,579\end{array}$ & $\begin{array}{r}10,724 \\
1,332 \\
31,690 \\
57,181 \\
18,347 \\
4,321\end{array}$ \\
\hline \multicolumn{11}{|l|}{$\begin{array}{l}\text { Cooling Equipment (more than } \\
\text { one may apply) }\end{array}$} \\
\hline 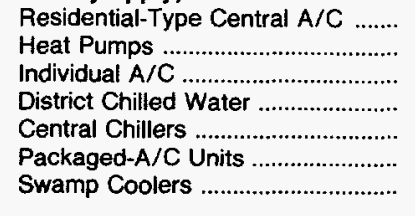 & $\begin{array}{r}30 \\
37 \\
80 \\
72 \\
158 \\
127 \\
3\end{array}$ & $\begin{array}{r}30 \\
37 \\
80 \\
72 \\
158 \\
127 \\
3\end{array}$ & $\begin{array}{r}25 \\
34 \\
72 \\
71 \\
146 \\
117 \\
3\end{array}$ & $\begin{array}{r}29 \\
35 \\
79 \\
72 \\
155 \\
125 \\
3\end{array}$ & $\begin{array}{r}19 \\
29 \\
60 \\
45 \\
120 \\
94 \\
3\end{array}$ & $\begin{array}{r}19,073 \\
11,388 \\
28,082 \\
28,194 \\
63,914 \\
56,664 \\
378\end{array}$ & $\begin{array}{r}19,073 \\
11,388 \\
28,082 \\
28,194 \\
63,914 \\
56,664 \\
378\end{array}$ & $\begin{array}{r}16,160 \\
9,517 \\
25,938 \\
28,046 \\
56,118 \\
51,507 \\
378\end{array}$ & $\begin{array}{r}19,060 \\
10,951 \\
28,023 \\
28,194 \\
63,281 \\
56,075 \\
378\end{array}$ & $\begin{array}{r}16,883 \\
11,170 \\
23,933 \\
23,915 \\
57,368 \\
51,246 \\
378\end{array}$ \\
\hline \multicolumn{11}{|l|}{$\begin{array}{l}\text { Lighting Equipment (more than } \\
\text { one may apply) }\end{array}$} \\
\hline $\begin{array}{l}\text { Incandescent ................................... } \\
\text { Standard Fluorescent .................... } \\
\text { Compact Fluorescent .................... } \\
\text { High-Intensity Discharge ................. } \\
\text { Electronic Ballast .......................... }\end{array}$ & $\begin{array}{r}184 \\
300 \\
80 \\
111 \\
135\end{array}$ & $\begin{array}{r}182 \\
299 \\
80 \\
109 \\
135\end{array}$ & $\begin{array}{r}170 \\
281 \\
72 \\
102 \\
126\end{array}$ & $\begin{array}{r}180 \\
294 \\
80 \\
109 \\
135\end{array}$ & $\begin{array}{r}133 \\
205 \\
72 \\
82 \\
115\end{array}$ & $\begin{array}{l}64,981 \\
91,986 \\
49,455 \\
52,593 \\
65,560\end{array}$ & $\begin{array}{l}64,662 \\
91,926 \\
49,455 \\
52,134 \\
65,560\end{array}$ & $\begin{array}{l}57,532 \\
82,147 \\
42,933 \\
48,685 \\
60,177\end{array}$ & $\begin{array}{l}64,593 \\
91,255 \\
49,455 \\
52,134 \\
65,560\end{array}$ & $\begin{array}{l}57,794 \\
80,314 \\
48,012 \\
47,858 \\
62,397\end{array}$ \\
\hline \multicolumn{11}{|l|}{$\begin{array}{l}\text { Energy Management Practices } \\
\text { (more than one may apply) } \\
\text { Energy Management and Control }\end{array}$} \\
\hline Enstem ....................................... & 132 & 132 & 127 & 132 & 105 & 61,891 & 61,891 & 57,447 & 61,891 & 55,202 \\
\hline 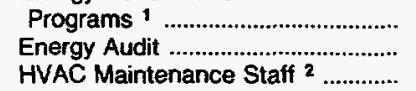 & $\begin{array}{l}99 \\
99 \\
97\end{array}$ & $\begin{array}{l}97 \\
98 \\
96\end{array}$ & $\begin{array}{l}87 \\
90 \\
84\end{array}$ & $\begin{array}{l}97 \\
98 \\
96\end{array}$ & $\begin{array}{l}84 \\
74 \\
67\end{array}$ & $\begin{array}{l}59,284 \\
37,621 \\
52,924\end{array}$ & $\begin{array}{l}59,024 \\
37,421 \\
52,665\end{array}$ & $\begin{array}{l}51,419 \\
33,287 \\
43,488\end{array}$ & $\begin{array}{l}59,024 \\
37,421 \\
52,665\end{array}$ & $\begin{array}{l}56,297 \\
34,439 \\
44,519\end{array}$ \\
\hline
\end{tabular}

See footnotes at end of table. 
Table 3.34. Energy Conservation Features in FBSS Building in Federal Region 3, Number of Buildings and Floorspace, 1993 (Continued)

\begin{tabular}{|c|c|c|c|c|c|c|c|c|c|c|}
\hline \multirow[b]{2}{*}{$\begin{array}{c}\text { Building } \\
\text { Characteristics }\end{array}$} & \multicolumn{5}{|c|}{ Number of Buildings } & \multicolumn{5}{|c|}{$\begin{array}{c}\text { Total Floorspace } \\
\text { (thousand square feet) }\end{array}$} \\
\hline & $\begin{array}{c}\text { All } \\
\text { Buildings }\end{array}$ & $\begin{array}{l}\text { Any } \\
\text { Conser- } \\
\text { vation } \\
\text { Feature }\end{array}$ & $\begin{array}{c}\text { Building } \\
\text { Shell }\end{array}$ & HVAC & Lighting & $\begin{array}{c}\text { All } \\
\text { Buildings }\end{array}$ & $\begin{array}{l}\text { Any } \\
\text { Conser- } \\
\text { vation } \\
\text { Feature }\end{array}$ & $\begin{array}{c}\text { Building } \\
\text { Shell }\end{array}$ & HVAC & Lighting \\
\hline \multicolumn{11}{|l|}{$\begin{array}{l}\text { Off-Hours Reduction in } \\
\text { Equipment (more than one may } \\
\text { apply) }\end{array}$} \\
\hline 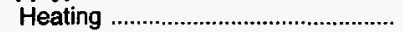 & 144 & 144 & 130 & 140 & 107 & 43,828 & 43,828 & 35,766 & 43,217 & 40,223 \\
\hline 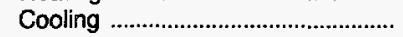 & 145 & 145 & 130 & 141 & 109 & 43,757 & 43,757 & 35,669 & 43,145 & 40,171 \\
\hline 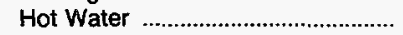 & 77 & 77 & 69 & 76 & 65 & 26,018 & 26,018 & 21,230 & 25,591 & 24,187 \\
\hline Lighting & 162 & 162 & 148 & 159 & 119 & 44,717 & 44,717 & 36,688 & 44,118 & 41,127 \\
\hline
\end{tabular}

1 Building participates in any programs sponsored by the Federal Energy Management Program, in-house, utility, or third party.

2 HVAC maintenance staff means at least one person spends at least half their working hours maintaining the heating/cooling equipment NC $=$ No cases in responding sample.

Notes: - Total workers are the number of workers during the main shift. - See Glossary for explanation of abbreviations and definitions of terms used in this report. - These data are from 881 federally owned buildings having the following criteria: (1) located in Federal Regions 3 , 6 , or 9 ; (2) larger than 10,000 square feet; and (3) used for a commercial purpose, other than warehouse and storage. In addition, 9 out of 10 selected buildings were from agencies other than the Department of Defense. - Statistics for the "energy end uses" represent consumption in buildings that have end use, not consumption for a particular fuel for a particular end use. $\bullet$ A/C = Air Conditioning. $\bullet$ FBSS = Federal Buildings Supplemental Survey. - HVAC $=$ Heating, Ventilation, and Air Conditioning. - Data are for Fiscal Year 1993 (October 1, 1992 through September 30, 1993). - Because of rounding, data may not sum to totals.

Source: Energy Information Administration, Office of Energy Markets and End Use, 1993 Federal Buildings Supplemental Survey. 
Table 3.35. Energy Conservation Features in FBSS Buildings in Federal Region 6, Number of Buildings and Floorspace, 1993

\begin{tabular}{|c|c|c|c|c|c|c|c|c|c|c|}
\hline \multirow[b]{2}{*}{$\begin{array}{c}\text { Building } \\
\text { Characteristics }\end{array}$} & \multicolumn{5}{|c|}{ Number of Buildings } & \multicolumn{5}{|c|}{$\begin{array}{l}\text { Total Floorspace } \\
\text { (thousand square feet) }\end{array}$} \\
\hline & $\begin{array}{c}\text { All } \\
\text { Buildings }\end{array}$ & $\begin{array}{c}\text { Any } \\
\text { Conser- } \\
\text { vation } \\
\text { Feature }\end{array}$ & $\begin{array}{c}\text { Building } \\
\text { Shell }\end{array}$ & HVAC & Lighting & $\begin{array}{c}\text { All } \\
\text { Buildings }\end{array}$ & $\begin{array}{c}\text { Any } \\
\text { Conser- } \\
\text { vation } \\
\text { Feature }\end{array}$ & $\begin{array}{c}\text { Building } \\
\text { Shell }\end{array}$ & HVAC & Lighting \\
\hline 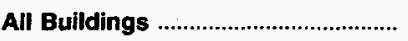 & 243 & 242 & 228 & 236 & 136 & 35,816 & 35,734 & 33,946 & 35,425 & 25,654 \\
\hline \multicolumn{11}{|l|}{ Building Floorspace (square feet) } \\
\hline 10,000 to 50,000 & 107 & 107 & 102 & 103 & 55 & 2,591 & 2,591 & 2,454 & 2,525 & 1,450 \\
\hline 50,001 to 200,000 & 86 & 85 & 79 & 83 & 42 & 9,548 & 9,466 & 8,763 & 9,224 & 4,717 \\
\hline Over $200,000 \ldots \ldots \ldots \ldots \ldots \ldots \ldots \ldots \ldots \ldots \ldots \ldots \ldots \ldots \ldots \ldots \ldots$ & 50 & 50 & 47 & 50 & 39 & 23,677 & 23,677 & 22,728 & 23,677 & 19,487 \\
\hline \multicolumn{11}{|l|}{ Principal Building Activity } \\
\hline 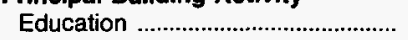 & 6 & 6 & 6 & 6 & 6 & 168 & 168 & 168 & 168 & 168 \\
\hline 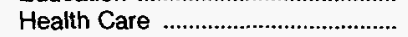 & 35 & 35 & 32 & 34 & 24 & 12,094 & 12,094 & 11,738 & 11,951 & 10,284 \\
\hline Laboratory & 29 & 29 & 27 & 29 & 8 & 3,331 & 3,331 & 3,131 & 3,331 & 715 \\
\hline Lodging & 16 & 16 & 14 & 16 & 11 & 942 & 942 & 757 & 942 & 704 \\
\hline Mercantile and Service ..................... & 49 & 49 & 48 & 48 & 30 & 6,236 & 6,236 & 5,791 & 6,136 & 4,474 \\
\hline Office & 76 & 76 & 73 & 73 & 43 & 10,799 & 10,799 & 10,616 & 10,752 & 8,453 \\
\hline 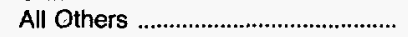 & 32 & 31 & 28 & 30 & 14 & 2,247 & 2,165 & 1,745 & 2,146 & 856 \\
\hline \multicolumn{11}{|l|}{ Year Constructed } \\
\hline 1959 or Before & 103 & 102 & 94 & 100 & 52 & 13,258 & 13,176 & 11,805 & 13,023 & 7,543 \\
\hline 1960 to 1969 & 38 & 38 & 36 & 36 & 26 & 6,386 & 6,386 & 6,107 & 6,356 & 5,577 \\
\hline 1970 to 1979 & 34 & 34 & 34 & 33 & 20 & 6,175 & 6,175 & 6,175 & 6,075 & 4,743 \\
\hline 1980 to 1989 & 57 & 57 & 54 & 57 & 31 & 6,903 & 6,903 & 6,822 & 6,903 & 4,785 \\
\hline 1990 to 1993 & 11 & 11 & 10 & 10 & 7 & 3,095 & 3,095 & 3,037 & 3,070 & 3,006 \\
\hline \multicolumn{11}{|l|}{ Federal Agency } \\
\hline Department of Defense ..................... & 22 & 22 & 21 & 20 & 12 & 1,668 & 1,668 & 1,658 & 1,501 & 1,042 \\
\hline General Services Administration ... & 35 & 35 & 35 & 35 & 28 & 7,888 & 7,888 & 7,888 & 7,888 & 7,128 \\
\hline United States Postal Service ......... & 61 & 61 & 57 & 59 & 37 & 7,027 & 7,027 & 6,396 & 6,916 & 4,947 \\
\hline Veterans Administration .................... & 36 & 36 & 33 & 36 & 24 & 11,375 & 11,375 & 11,019 & 11,375 & 9,709 \\
\hline 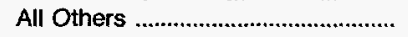 & 89 & 88 & 82 & 86 & 35 & 7,858 & 7,776 & 6,984 & 7,746 & 2,828 \\
\hline \multicolumn{11}{|l|}{$\begin{array}{l}\text { Energy Sources (more than one } \\
\text { may apply) }\end{array}$} \\
\hline 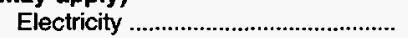 & 243 & 242 & 228 & 236 & 136 & 35,816 & 35,734 & 33,946 & 35,425 & 25,654 \\
\hline 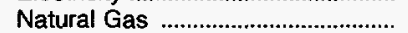 & 168 & 168 & 163 & 163 & 98 & 24,827 & 24,827 & 24,186 & 24,544 & 18,117 \\
\hline Fuel Oil & 18 & 18 & 17 & 17 & 14 & 4,740 & 4,740 & 4,294 & 4,598 & 4,492 \\
\hline 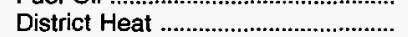 & 68 & 68 & 59 & 68 & 33 & 13,437 & 13,437 & 12,289 & 13,437 & 9,527 \\
\hline 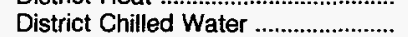 & 30 & 30 & 27 & 30 & 21 & 7,980 & 7,980 & 7,624 & 7,980 & 7,497 \\
\hline Propane & 5 & 5 & 5 & 5 & 1 & 200 & 200 & 200 & 200 & 135 \\
\hline 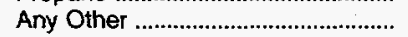 & 8 & 8 & 8 & 8 & 7 & 2,850 & 2,850 & 2,850 & 2,850 & 2,709 \\
\hline \multicolumn{11}{|l|}{$\begin{array}{l}\text { Energy End Uses (more than one } \\
\text { may apply) }\end{array}$} \\
\hline 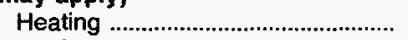 & 242 & 242 & 228 & 236 & 136 & 35,734 & 35,734 & 33,946 & 35,425 & 25,654 \\
\hline 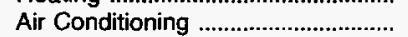 & 241 & 241 & 227 & 235 & 135 & 35,273 & 35,273 & 33,485 & 34,965 & 25,193 \\
\hline 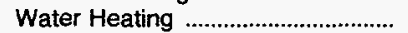 & 238 & 238 & 224 & 233 & 133 & 35,221 & 35,221 & 33,432 & 34,932 & 25,154 \\
\hline 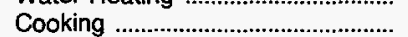 & 63 & 63 & 62 & 63 & 48 & 19,213 & 19,213 & 19,203 & 19,213 & 16,821 \\
\hline 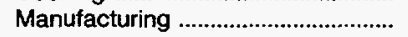 & 28 & 28 & 27 & 27 & 22 & 5,335 & 5,335 & 4,890 & 5,316 & 4,471 \\
\hline \multicolumn{11}{|l|}{ Workers (main shift) } \\
\hline Less than 50 & 70 & 69 & 63 & 66 & 34 & 2,548 & 2,466 & 2,074 & 2,425 & 1,226 \\
\hline 50 to 99 & 44 & 44 & 41 & 43 & 24 & 2,026 & 2,026 & 1,874 & 1,926 & 1,253 \\
\hline 100 to 499 & 80 & 80 & 76 & 78 & 39 & 9,029 & 9,029 & 8,231 & 8,862 & 4,083 \\
\hline 500 or More & 49 & 49 & 48 & 49 & 39 & 22,213 & 22,213 & 21,767 & 22,213 & 19,091 \\
\hline \multicolumn{11}{|l|}{ Weekly Operating Hours } \\
\hline 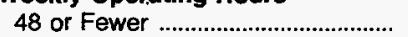 & 39 & 38 & 33 & 35 & 22 & 2,740 & 2,658 & 2,245 & 2,486 & 1,802 \\
\hline 49 to $60 \ldots . . . . . . . .$. & 46 & 46 & 45 & 46 & 28 & 6,460 & 6,460 & 6,447 & 6,460 & 5,279 \\
\hline 61 to 167 & 78 & 78 & 75 & 75 & 32 & 8,051 & 8,051 & 7,636 & 7,915 & 3,672 \\
\hline 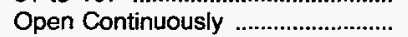 & 80 & 80 & 75 & 80 & 54 & 18,565 & 18,565 & 17,618 & 18,565 & 14,901 \\
\hline
\end{tabular}

See footnotes at end of table. 
Table 3.35. Energy Conservation Features in FBSS Buildings in Federal Region 6, Number of Buildings and Floorspace, 1993 (Continued)

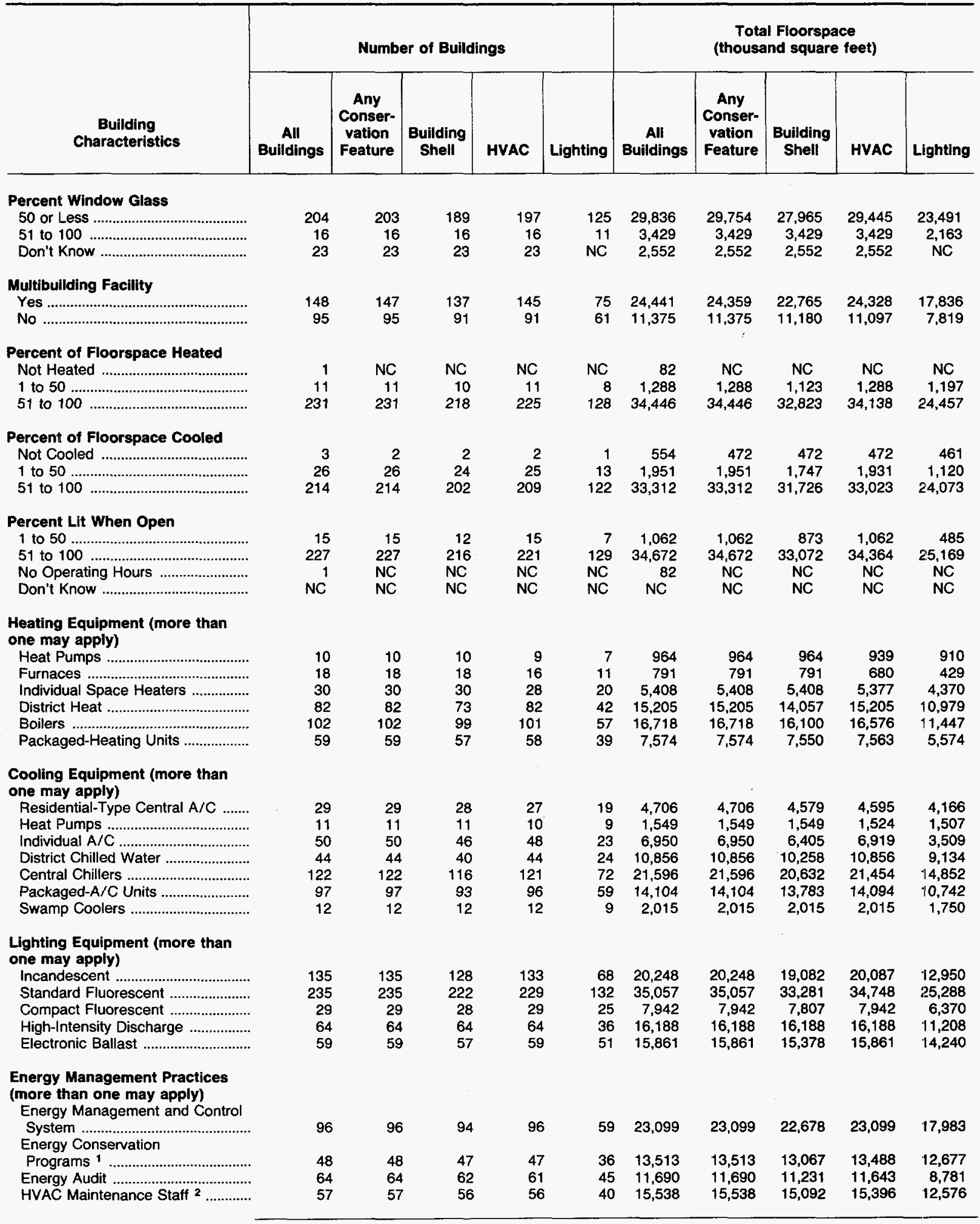

See footnotes at end of table. 


\section{Table 3.35. Energy Conservation Features in FBSS Buildings in Federal Region 6, Number of Buildings and Floorspace, 1993 (Continued)}

\begin{tabular}{|c|c|c|c|c|c|c|c|c|c|c|}
\hline \multirow[b]{2}{*}{$\begin{array}{c}\text { Building } \\
\text { Characteristics }\end{array}$} & \multicolumn{5}{|c|}{ Number of Buildings } & \multicolumn{5}{|c|}{$\begin{array}{l}\text { Total Floorspace } \\
\text { (thousand square feet) }\end{array}$} \\
\hline & $\begin{array}{c}\text { All } \\
\text { Buildings }\end{array}$ & $\begin{array}{l}\text { Any } \\
\text { Conser- } \\
\text { vation } \\
\text { Feature }\end{array}$ & $\begin{array}{l}\text { Building } \\
\text { Shell }\end{array}$ & HVAC & Lighting & $\begin{array}{l}\text { All } \\
\text { Buildings }\end{array}$ & $\begin{array}{l}\text { Any } \\
\text { Conser- } \\
\text { vation } \\
\text { Feature }\end{array}$ & $\begin{array}{c}\text { Bullding } \\
\text { Shell }\end{array}$ & HVAC & Lighting \\
\hline \multicolumn{11}{|l|}{$\begin{array}{l}\text { Off-Hours Reduction in } \\
\text { Equipment (more than one may } \\
\text { apply) }\end{array}$} \\
\hline 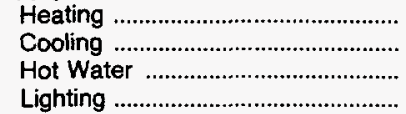 & $\begin{array}{r}123 \\
124 \\
33 \\
120\end{array}$ & $\begin{array}{r}123 \\
124 \\
33 \\
120\end{array}$ & $\begin{array}{r}115 \\
116 \\
33 \\
112\end{array}$ & $\begin{array}{r}117 \\
118 \\
31 \\
114\end{array}$ & $\begin{array}{l}75 \\
77 \\
21 \\
74\end{array}$ & $\begin{array}{r}13,162 \\
13,864 \\
4,554 \\
12,847\end{array}$ & $\begin{array}{r}13,162 \\
13,864 \\
4,554 \\
12,847\end{array}$ & $\begin{array}{r}12,360 \\
13,061 \\
4,554 \\
12,045\end{array}$ & $\begin{array}{r}12,854 \\
13,555 \\
4,518 \\
12,538\end{array}$ & $\begin{array}{r}9,864 \\
10,589 \\
3,532 \\
9,586\end{array}$ \\
\hline
\end{tabular}

t Building participates in any programs sponsored by the Federal Energy Management Program, in-house, utility, or third party.

2 HVAC maintenance staff means at least one person spends at least half their working hours maintaining the heating/cooling equipment.

$\mathrm{NC}=$ No cases in responding sample.

Notes: - Total workers are the number of workers during the main shift. - See Glossary for explanation of abbreviations and definitions of terms used in this report. - These data are from 881 federally owned buildings having the following criteria: (1) located in Federal Regions 3 , 6 , or 9 ; (2) larger than 10,000 square feet; and (3) used for a commercial purpose, other than warehouse and storage. In addition, 9 out of 10 selected buildings were from agencies other than the Department of Defense. - Statistics for the "energy end uses" represent consumption in buildings that have end use, not consumption for a particular fuel for a particular end use. $\bullet$ A/C = Air Conditioning. $\bullet$ FBSS = Federal Buildings Supplemental Survey. - HVAC = Heating, Ventilation, and Air Conditioning. - Data are for Fiscal Year 1993 (October 1 , 1992 through September 30, 1993). - Because of rounding, data may not sum to totals.

Source: Energy Information Administration, Office of Energy Markets and End Use, 1993 Federal Buildings Supplemental Survey. 
Table 3.36. Energy Conservation Features in FBSS Buildings in Federal Region 9, Number of Buildings and Floorspace, 1993

\begin{tabular}{|c|c|c|c|c|c|c|c|c|c|c|}
\hline \multirow[b]{2}{*}{$\begin{array}{c}\text { Building } \\
\text { Characteristics }\end{array}$} & \multicolumn{5}{|c|}{ Number of Buildings } & \multicolumn{5}{|c|}{$\begin{array}{l}\text { Total Floorspace } \\
\text { (thousand square feet) }\end{array}$} \\
\hline & $\begin{array}{c}\text { All } \\
\text { Buildings }\end{array}$ & $\begin{array}{c}\text { Any } \\
\text { Conser- } \\
\text { vation } \\
\text { Feature }\end{array}$ & $\begin{array}{l}\text { Building } \\
\text { Shell }\end{array}$ & HVAC & Lighting & $\begin{array}{c}\text { All } \\
\text { Buildings }\end{array}$ & $\begin{array}{c}\text { Any } \\
\text { Conser- } \\
\text { vation } \\
\text { Feature }\end{array}$ & $\begin{array}{l}\text { Building } \\
\text { Shell }\end{array}$ & HVAC & Lighting \\
\hline All Bulldings & 326 & 319 & 248 & 307 & 217 & 44,316 & 44,023 & 34,105 & 43,116 & 34,825 \\
\hline \multicolumn{11}{|l|}{ Building Floorspace (square feet) } \\
\hline 10,000 to 50,000 & 148 & 143 & 116 & 136 & 81 & 3,858 & 3,711 & 3,079 & 3,506 & 2,086 \\
\hline 50,001 to 200,000 & 123 & 121 & 90 & 117 & 91 & 12,680 & 12,534 & 9,234 & 12,092 & 9,914 \\
\hline 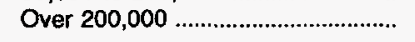 & 55 & 55 & 42 & 54 & 45 & 27,778 & 27,778 & 21,791 & 27,518 & 22,824 \\
\hline \multicolumn{11}{|l|}{ Principal Building Activity } \\
\hline 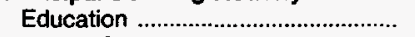 & 12 & 12 & 8 & 12 & 4 & 628 & 628 & 434 & 628 & 234 \\
\hline 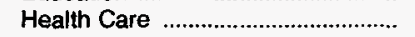 & 48 & 47 & 42 & 47 & 35 & 9,903 & 9,890 & 9,618 & 9,890 & 8,631 \\
\hline 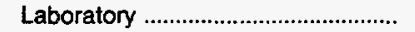 & 31 & 31 & 15 & 31 & 25 & 2,601 & 2,601 & 911 & 2,601 & 2,266 \\
\hline 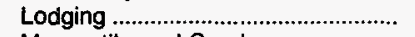 & 22 & 19 & 19 & 17 & 6 & 1,220 & 1,116 & 1,116 & 832 & 357 \\
\hline Mercantile and Service ................... & 63 & 62 & 60 & 58 & 45 & 8,194 & 8,115 & 7,525 & 7,916 & 6,681 \\
\hline Office & 92 & 91 & 59 & 89 & 66 & 16,380 & 16,314 & 9,826 & 16,223 & 13,419 \\
\hline 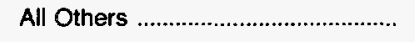 & 58 & 57 & 45 & 53 & 36 & 5,390 & 5,360 & 4,674 & 5,026 & 3,236 \\
\hline \multicolumn{11}{|l|}{ Year Constructed } \\
\hline 1959 or Before & 130 & 126 & 97 & 121 & 71 & 13,553 & 13,404 & 10,689 & 13,047 & 8,530 \\
\hline 1960 to 1969 & 55 & 55 & 41 & 53 & 44 & 9,082 & 9,082 & 7,024 & 9,014 & 8,462 \\
\hline 1970 to 1979 & 62 & 60 & 46 & 58 & 45 & 12,041 & 11,978 & 8,200 & 11,702 & 9,358 \\
\hline 1980 to 1989 & 65 & 64 & 52 & 62 & 46 & 7,955 & 7,875 & 6,668 & 7,812 & 7,082 \\
\hline 1990 to 1993 & 14 & 14 & 12 & 13 & 11 & 1,684 & 1,684 & 1,524 & 1,541 & 1,392 \\
\hline \multicolumn{11}{|l|}{ Federal Agency } \\
\hline Department of Defense .................. & 78 & 71 & 56 & 61 & 31 & 8,489 & 8,196 & 6,964 & 7,448 & 4,260 \\
\hline General Services Administration ... & 39 & 39 & 18 & 39 & 35 & 12,505 & 12,505 & 6,919 & 12,505 & 11,109 \\
\hline United States Postal Service ......... & 63 & 63 & 62 & 62 & 45 & 8,387 & 8,387 & 7,845 & 8,371 & 7,011 \\
\hline Veterans Administration .................. & 56 & 56 & 49 & 55 & 42 & 8,824 & 8,824 & 8,326 & 8,681 & 7,466 \\
\hline 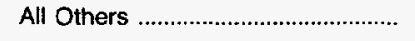 & 90 & 90 & 63 & 90 & 64 & 6,111 & 6,111 & 4,050 & 6,111 & 4,978 \\
\hline \multicolumn{11}{|l|}{$\begin{array}{l}\text { Energy Sources (more than one } \\
\text { may apply) }\end{array}$} \\
\hline 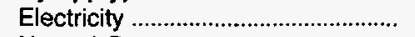 & 325 & 318 & 247 & 306 & 216 & 44,296 & 44,004 & 34,085 & 43,096 & 34,805 \\
\hline 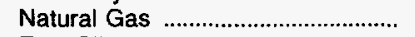 & 225 & 224 & 175 & 216 & 169 & 34,703 & 34,669 & 27,083 & 34,130 & 29,144 \\
\hline 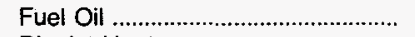 & 54 & 53 & 37 & 53 & 40 & 11,127 & 11,060 & 9,415 & 11,060 & 9,379 \\
\hline 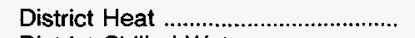 & 74 & 71 & 61 & 71 & 45 & 11,757 & 11,675 & 9,705 & 11,675 & 9,252 \\
\hline District Chilled Water ........................ & 27 & 27 & 24 & 27 & 20 & 6,362 & 6,362 & 5,629 & 6,362 & 5,484 \\
\hline 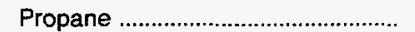 & 10 & 10 & 9 & 10 & 3 & 1,117 & 1,117 & 1,106 & 1,117 & 815 \\
\hline 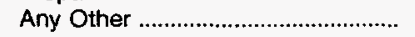 & 5 & 5 & 3 & 5 & 4 & 2,606 & 2,606 & 2,537 & 2,606 & 2,595 \\
\hline \multicolumn{11}{|l|}{$\begin{array}{l}\text { Energy End Uses (more than one } \\
\text { may apply) }\end{array}$} \\
\hline 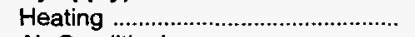 & 304 & 299 & 234 & 290 & 207 & 41,921 & 41,775 & 32,838 & 41,197 & 32,968 \\
\hline 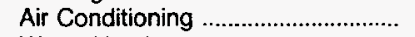 & 292 & 291 & 228 & 282 & 205 & 41,907 & 41,894 & 32,754 & 41,282 & 33,846 \\
\hline Water Heating . ...................................... & 315 & 309 & 244 & 299 & 214 & 42,984 & 42,771 & 33,733 & 42,140 & 34,007 \\
\hline Cooking & 70 & 70 & 63 & 68 & 51 & 20,045 & 20,045 & 17,163 & 19,972 & 17,125 \\
\hline 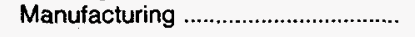 & 30 & 30 & 25 & 30 & 22 & 7,693 & 7,693 & 6,948 & 7,693 & 5,539 \\
\hline \multicolumn{11}{|l|}{ Workers (main shift) } \\
\hline Less than 50 & 93 & 89 & 66 & 83 & 44 & 3,684 & 3,579 & 2,334 & 3,184 & 1,841 \\
\hline 50 to 99 & 49 & 48 & 38 & 46 & 31 & 2,161 & 2,120 & 1,414 & 2,083 & 1,429 \\
\hline 100 to 499 & 134 & 132 & 104 & 128 & 98 & 14,017 & 13,871 & 10,879 & 13,395 & 10,897 \\
\hline 500 or More & 50 & 50 & 40 & 50 & 44 & 24,454 & 24,454 & 19,477 & 24,454 & 20,658 \\
\hline \multicolumn{11}{|l|}{ Weekly Operating Hours } \\
\hline 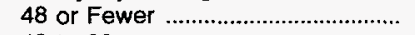 & 80 & 77 & 58 & 73 & 40 & 4,822 & 4,751 & 3,485 & 4,507 & 2,935 \\
\hline 49 to 60 & 65 & 63 & 42 & 62 & 52 & 13,424 & 13,278 & 8,153 & 13,165 & 11,802 \\
\hline 61 to 167 & 68 & 68 & 64 & 64 & 45 & 5,706 & 5,706 & 5,331 & 5,582 & 4,033 \\
\hline Open Continuously ............................... & 113 & 111 & 84 & 108 & 80 & 20,364 & 20,289 & 17,136 & 19,862 & 16,054 \\
\hline
\end{tabular}

See footnotes at end of table. 
Table 3.36. Energy Conservation Features in FBSS Buildings in Federal Region 9, Number of Buildings and Floorspace, 1993 (Continued)

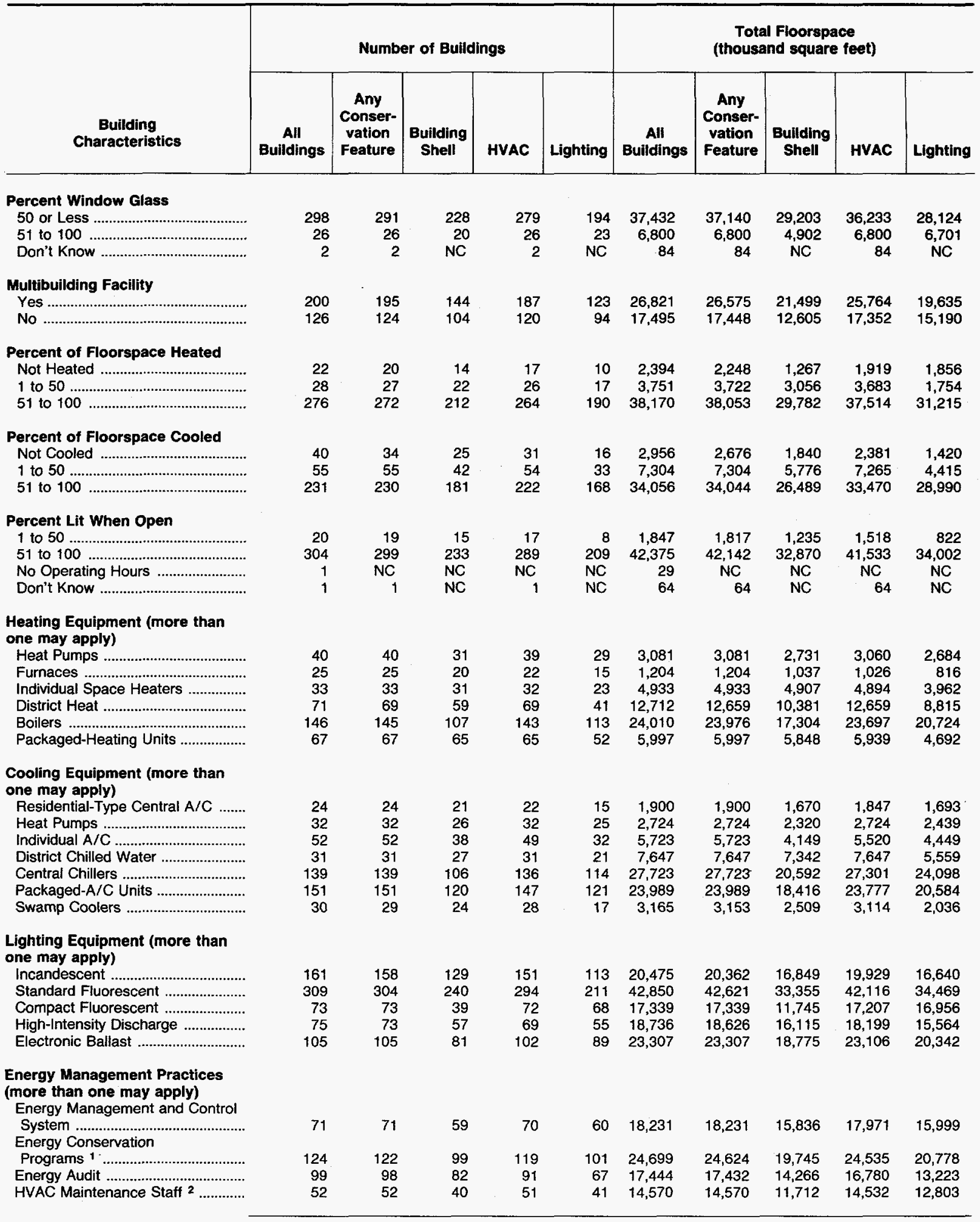

See footnotes at end of table. 


\section{Table 3.36. Energy Conservation Features in FBSS Buildings in Federal Region 9, Number of Buildings and Floorspace, 1993 (Continued)}

\begin{tabular}{|c|c|c|c|c|c|c|c|c|c|c|}
\hline \multirow[b]{2}{*}{$\begin{array}{c}\text { Building } \\
\text { Characteristics }\end{array}$} & \multicolumn{5}{|c|}{ Number of Buildings } & \multicolumn{5}{|c|}{$\begin{array}{c}\text { Total Floorspace } \\
\text { (thousand square feet) }\end{array}$} \\
\hline & $\begin{array}{c}\text { All } \\
\text { Buildings }\end{array}$ & $\begin{array}{c}\text { Any } \\
\text { Conser- } \\
\text { vation } \\
\text { Feature }\end{array}$ & $\begin{array}{l}\text { Building } \\
\text { Shell }\end{array}$ & HVAC & Lighting & $\begin{array}{c}\text { All } \\
\text { Buildings }\end{array}$ & $\begin{array}{l}\text { Any } \\
\text { Conser- } \\
\text { vation } \\
\text { Feature }\end{array}$ & $\begin{array}{l}\text { Building } \\
\text { Shell }\end{array}$ & HVAC & Lighting \\
\hline \multicolumn{11}{|l|}{$\begin{array}{l}\text { Off-Hours Reduction in } \\
\text { Equipment (more than one may } \\
\text { apply) }\end{array}$} \\
\hline 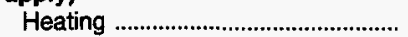 & 151 & 149 & 120 & 144 & 106 & 19,631 & 19,590 & 13,808 & 19,425 & 16,304 \\
\hline 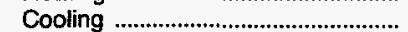 & 157 & 156 & 124 & 151 & 108 & 19,219 & 19,207 & 13,253 & 19,007 & 15,693 \\
\hline Hot Water & 68 & 67 & 46 & 66 & 57 & 13,614 & 13,585 & 8,258 & 13,566 & 11,978 \\
\hline 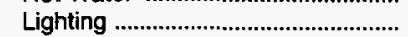 & 172 & 170 & 134 & 163 & 116 & 21,155 & 21,063 & 14,822 & 20,711 & 17,272 \\
\hline
\end{tabular}

1 Building participates in any programs sponsored by the Federal Energy Management Program, in-house, utility, or third party.

2 HVAC maintenance staff means at least one person spends at least half their working hours maintaining the heating/cooling equipment $\mathrm{NC}=$ No cases in responding sample.

Notes: - Total workers are the number of workers during the main shift. - See Glossary for explanation of abbreviations and definitions of terms used in this report. - These data are from 881 federally owned buildings having the following criteria: (1) located in Federal Fiegions 3 , 6 , or 9 ; (2) larger than 10,000 square feet; and (3) used for a commercial purpose, other than warehouse and storage. In addition, 9 out of 10 selected buildings were from agencies other than the Department of Defense. - Statistics for the "energy end uses" represent consumption in buildings that have end use, not consumption for a particular fuel for a particular end use. $\bullet$ A/C = Air Conditioning. - FBSS = Federa Buildings Supplemental Survey. - HVAC = Heating, Ventilation, and Air Conditioning. - Data are for Fiscal Year 1993 (October 1, 1992 through September 30,1993$)$. Because of rounding, data may not sum to totals.

Source: Energy Information Administration, Office of Energy Markets and End Use, 1993 Federal Buildings Supplemental Survey. 
Table 3.37. Energy Management Practices in FBSS Buildings in Federal Region 3, Number of Buildings, 1993

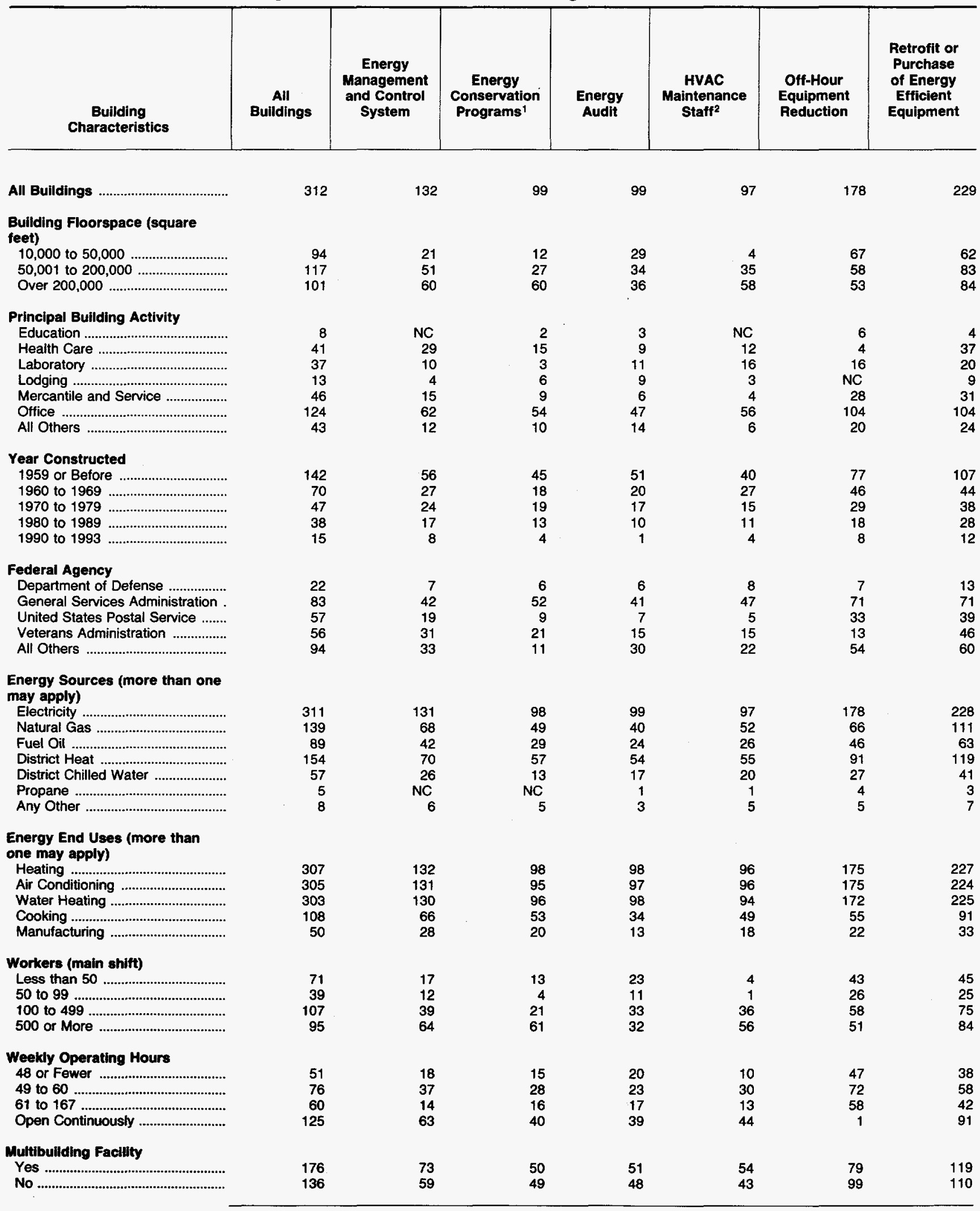

See footnotes at end of table. 
Table 3.37. Energy Management Practices in FBSS Buildings in Federal Region 3, Number of Buildings, 1993 (Continued)

\begin{tabular}{|c|c|c|c|c|c|c|c|}
\hline $\begin{array}{c}\text { Building } \\
\text { Characteristics }\end{array}$ & $\begin{array}{c}\text { All } \\
\text { Buildings }\end{array}$ & $\begin{array}{c}\text { Energy } \\
\text { Management } \\
\text { and Control } \\
\text { System }\end{array}$ & $\begin{array}{c}\text { Energy } \\
\text { Conservation } \\
\text { Programs }{ }^{1}\end{array}$ & $\begin{array}{c}\text { Energy } \\
\text { Audit }\end{array}$ & $\begin{array}{c}\text { HVAC } \\
\text { Maintenance } \\
\text { Staif }{ }^{2}\end{array}$ & $\begin{array}{l}\text { Off-Hour } \\
\text { Equipment } \\
\text { Reduction }\end{array}$ & $\begin{array}{l}\text { Retrofit or } \\
\text { Purchase } \\
\text { of Energy } \\
\text { Efficient } \\
\text { Equipment }\end{array}$ \\
\hline \multicolumn{8}{|l|}{ Percent of Floorspace Heated } \\
\hline 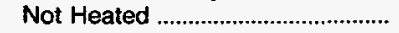 & 5 & NC & 1 & 1 & 1 & 3 & 2 \\
\hline 1 to 50 & 12 & 3 & 4 & NC & 2 & 6 & 8 \\
\hline 51 to 100 & 295 & 129 & 94 & 98 & $9 \overline{4}$ & 169 & 219 \\
\hline \multicolumn{8}{|l|}{ Percent of Floorspace Cooled } \\
\hline Not Cooled & 13 & 2 & 6 & 5 & 3 & 5 & 7 \\
\hline 1 to 50 & 30 & 8 & 6 & 10 & 5 & 21 & 19 \\
\hline 51 to 100 & 269 & 122 & 87 & 84 & 89 & 152 & 203 \\
\hline \multicolumn{8}{|l|}{ Percent Lit When Open } \\
\hline 1 to 50 & 11 & 1 & 3 & 4 & 2 & 8 & 6 \\
\hline 51 to 100 & 300 & 131 & 96 & 95 & 95 & 170 & 223 \\
\hline No Operating Hours ......................... & 1 & $\mathrm{NC}$ & NC & NC & $\mathrm{NC}$ & NC & NC \\
\hline 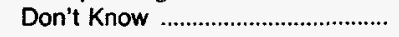 & NC & NC & NC & NC & $\mathrm{NC}$ & $\mathrm{NC}$ & NC \\
\hline \multicolumn{8}{|l|}{$\begin{array}{l}\text { Heating Equipment (more than } \\
\text { one may apply) }\end{array}$} \\
\hline Heat Pumps & 34 & 19 & 13 & 14 & 6 & 25 & 29 \\
\hline 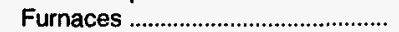 & 16 & 1 & 1 & 5 & 2 & 12 & 12 \\
\hline Individual Space Heaters .............. & 75 & 39 & 29 & 31 & 27 & 44 & 60 \\
\hline 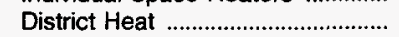 & 167 & 80 & 58 & 60 & 64 & 88 & 131 \\
\hline 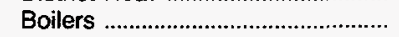 & 93 & 40 & 28 & 27 & 24 & 54 & 67 \\
\hline Packaged-Heating Units ................. & 25 & 11 & 7 & 10 & 8 & 12 & 21 \\
\hline \multicolumn{8}{|l|}{$\begin{array}{l}\text { Cooling Equipment (more than } \\
\text { one may apply) }\end{array}$} \\
\hline Residential-Type Central A/C ...... & 30 & 13 & 8 & 10 & 13 & 21 & 25 \\
\hline Heat Pumps & 37 & 19 & 15 & 14 & 9 & 30 & 32 \\
\hline 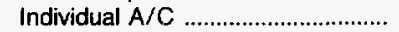 & 80 & 36 & 28 & 32 & 28 & 47 & 61 \\
\hline District Chilled Water ...................... & 72 & 38 & 20 & 17 & 26 & 30 & 52 \\
\hline 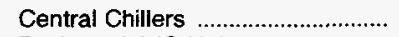 & 158 & 80 & 70 & 61 & 70 & 93 & 128 \\
\hline Packaged-A/C Units ...................... & 127 & 65 & 50 & 43 & 44 & 74 & 101 \\
\hline Swamp Coolers & 3 & 2 & NC & 2 & 2 & 1 & 3 \\
\hline \multicolumn{8}{|l|}{$\begin{array}{l}\text { Lighting Equipment (more than } \\
\text { one may apply) }\end{array}$} \\
\hline 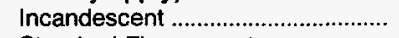 & 184 & 77 & 64 & 60 & 61 & 100 & 132 \\
\hline 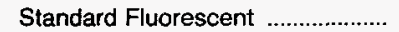 & 300 & 130 & 97 & 98 & 94 & 173 & 223 \\
\hline Compact Fluorescent ...................... & 80 & 43 & 44 & 31 & 33 & 56 & 73 \\
\hline High-Intensity Discharge ................. & 111 & 59 & 37 & 36 & 41 & 58 & 87 \\
\hline 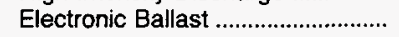 & 135 & 82 & 66 & 53 & 57 & 84 & 125 \\
\hline \multicolumn{8}{|l|}{$\begin{array}{l}\text { Water-Heating Equipment (more } \\
\text { than one may apply) }\end{array}$} \\
\hline Centralized System ............................ & 223 & 95 & 69 & 75 & 76 & 130 & 165 \\
\hline $\begin{array}{l}\text { Distributed System ................................ } \\
\text { Don't Know/ }\end{array}$ & 71 & 29 & 25 & 23 & 18 & 37 & 57 \\
\hline Not Ascertained ............................... & 9 & 6 & 2 & NC & NC & 5 & 3 \\
\hline \multicolumn{8}{|l|}{$\begin{array}{l}\text { Energy Conservation Features } \\
\text { (more than one may apply) }\end{array}$} \\
\hline Any Conservation Feature ............ & 309 & 132 & 97 & 98 & 96 & 178 & 228 \\
\hline Building Shell & 290 & 127 & 87 & 90 & 84 & 163 & 215 \\
\hline HVAC & 303 & 132 & 97 & 98 & 96 & 174 & 227 \\
\hline 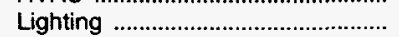 & 210 & 105 & 84 & 74 & 67 & 128 & 175 \\
\hline
\end{tabular}

See footnotes at end of table. 
Table 3.37. Energy Management Practices in FBSS Buildings in Federal Region 3, Number of Buildings, 1993 (Continued)

\begin{tabular}{|c|c|c|c|c|c|c|c|}
\hline $\begin{array}{c}\text { Building } \\
\text { Characteristics }\end{array}$ & $\begin{array}{c}\text { All } \\
\text { Buildings }\end{array}$ & $\begin{array}{l}\text { Energy } \\
\text { Management } \\
\text { and Control } \\
\text { System }\end{array}$ & $\begin{array}{l}\text { Energy } \\
\text { Conservation } \\
\text { Programs } 1\end{array}$ & $\begin{array}{l}\text { Energy } \\
\text { Audit }\end{array}$ & $\begin{array}{l}\text { HVAC } \\
\text { Maintenance } \\
\text { Staff }^{2}\end{array}$ & $\begin{array}{l}\text { Off-Hour } \\
\text { Equipment } \\
\text { Reduction }\end{array}$ & $\begin{array}{l}\text { Retrofit or } \\
\text { Purchase } \\
\text { of Energy } \\
\text { Efficient } \\
\text { Equipment }\end{array}$ \\
\hline \multicolumn{8}{|l|}{$\begin{array}{l}\text { Building Shell Conservation } \\
\text { Features (more than one may } \\
\text { apply) } \\
\text { Roof or Ceiling }\end{array}$} \\
\hline 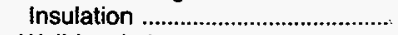 & 240 & 110 & 70 & 75 & 69 & 135 & 176 \\
\hline $\begin{array}{l}\text { Wall Insulation } \\
\text { Storm or Multiple............................ }\end{array}$ & 117 & 61 & 31 & 29 & 29 & 69 & 87 \\
\hline Glazing & 173 & 87 & 49 & 49 & 36 & 91 & 139 \\
\hline $\begin{array}{l}\text { or Shading Film ............................ } \\
\text { Exterior or Interior Shading }\end{array}$ & 158 & 83 & 47 & 40 & 56 & 94 & 117 \\
\hline or Awnings & 197 & 97 & 57 & 58 & 55 & 115 & 145 \\
\hline \multicolumn{8}{|l|}{$\begin{array}{l}\text { HVAC Conservation Features } \\
\text { (more than one may apply) }\end{array}$} \\
\hline 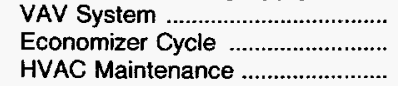 & $\begin{array}{r}84 \\
156 \\
301\end{array}$ & $\begin{array}{r}59 \\
93 \\
132\end{array}$ & $\begin{array}{l}33 \\
59 \\
97\end{array}$ & $\begin{array}{l}25 \\
50 \\
98\end{array}$ & $\begin{array}{l}37 \\
60 \\
96\end{array}$ & $\begin{array}{r}37 \\
92 \\
173\end{array}$ & $\begin{array}{r}75 \\
131 \\
227\end{array}$ \\
\hline \multicolumn{8}{|l|}{$\begin{array}{l}\text { Lighting Conservation Features } \\
\text { (more than one may apply) }\end{array}$} \\
\hline $\begin{array}{l}\text { Specular Reflectors } \\
\text { Natural Lighting Control }\end{array}$ & 124 & 64 & 49 & 52 & 43 & 69 & 101 \\
\hline Sensors . & 29 & 18 & 17 & 9 & 13 & 14 & 26 \\
\hline Occupancy Sensors .......................... & 95 & 55 & 54 & 40 & 44 & 66 & 86 \\
\hline 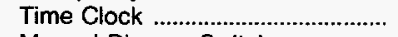 & 74 & 39 & 36 & 28 & 32 & 47 & 64 \\
\hline Manual Dimmer Switches ............... & 93 & 50 & 45 & 29 & 32 & 64 & 81 \\
\hline
\end{tabular}

1 Building participates in any programs sponsored by the Federal Energy Management Program, in-house, utility, or third party.

2 HVAC maintenance staff means at least one person spends at least half their working hours maintaining the heating/cooling equipment.

$\mathrm{NC}=$ No cases in responding sample.

Notes: - Total workers are the number of workers during the main shift. - See Glossary for explanation of abbreviations and definitions of terms used in this report. - These data are from 881 federally owned buildings having the following criteria: (1) located in Federal Regions 3 , 6 , or 9; (2) larger than 10,000 square feet; and (3) used for a commercial purpose, other than warehouse and storage. In addition, 9 out of 10 selected buildings were from agencies other than the Department of Defense. - Statistics for the "energy end uses" represent consumption in buildings that have end use, not consumption for a particular fuel for a particular end use. $\bullet$ A/C = Air Conditioning. - FBSS $=$ Federal Buildings Supplemental Survey.

- HVAC = Heating, Ventilation, and Air Conditioning. - VAV = Variable-Air Volume. - Data are for Fiscal Year 1993 (October 1, 1992 through September 30,1993$)$. - Because of rounding, data may not sum to totals.

Source: Energy Information Administration, Office of Energy Markets and End Use, 1993 Federal Buildings Supplemental Survey. 
Table 3.38. Energy Management Practices in FBSS Buildings in Federal Region 6, Number of Buildings, 1993

\begin{tabular}{|c|c|c|c|c|c|c|c|}
\hline $\begin{array}{c}\text { Building } \\
\text { Characteristics }\end{array}$ & $\begin{array}{l}\text { All } \\
\text { Buildings }\end{array}$ & $\begin{array}{l}\text { Energy } \\
\text { Management } \\
\text { and Control } \\
\text { System }\end{array}$ & $\begin{array}{l}\text { Energy } \\
\text { Conservation } \\
\text { Programs }{ }^{1}\end{array}$ & $\begin{array}{l}\text { Energy } \\
\text { Audit }\end{array}$ & $\begin{array}{l}\text { HVAC } \\
\text { Maintenance } \\
\text { Staff }^{2}\end{array}$ & $\begin{array}{l}\text { Off-Hour } \\
\text { Equipment } \\
\text { Reduction }\end{array}$ & $\begin{array}{l}\text { Reitrofit or } \\
\text { Purchase } \\
\text { of Energy } \\
\text { Efficient } \\
\text { Equipment }\end{array}$ \\
\hline 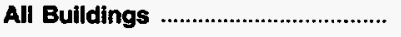 & 243 & 96 & 48 & 64 & 57 & 136 & 162 \\
\hline \multicolumn{8}{|l|}{$\begin{array}{l}\text { Building Floorspace (square } \\
\text { feet) }\end{array}$} \\
\hline 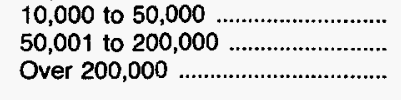 & $\begin{array}{r}107 \\
86 \\
50\end{array}$ & $\begin{array}{l}21 \\
40 \\
35\end{array}$ & $\begin{array}{r}18 \\
9 \\
21\end{array}$ & $\begin{array}{l}28 \\
16 \\
20\end{array}$ & $\begin{array}{l}14 \\
20 \\
23\end{array}$ & $\begin{array}{l}74 \\
43 \\
19\end{array}$ & $\begin{array}{l}65 \\
54 \\
43\end{array}$ \\
\hline 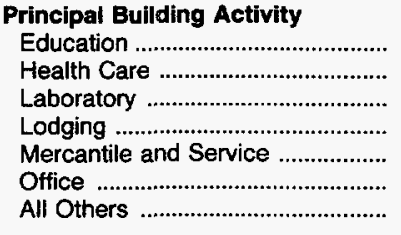 & $\begin{array}{r}6 \\
35 \\
29 \\
16 \\
49 \\
76 \\
32\end{array}$ & $\begin{array}{r}1 \\
28 \\
10 \\
5 \\
17 \\
30 \\
5\end{array}$ & $\begin{array}{r}\text { NC } \\
9 \\
3 \\
5 \\
14 \\
16 \\
1\end{array}$ & $\begin{array}{r}\text { NC } \\
6 \\
1 \\
7 \\
19 \\
25 \\
6\end{array}$ & $\begin{array}{r}1 \\
10 \\
3 \\
2 \\
8 \\
30 \\
3\end{array}$ & $\begin{array}{r}6 \\
6 \\
14 \\
\text { NC } \\
30 \\
61 \\
19\end{array}$ & $\begin{array}{r}3 \\
31 \\
9 \\
13 \\
39 \\
50 \\
17\end{array}$ \\
\hline 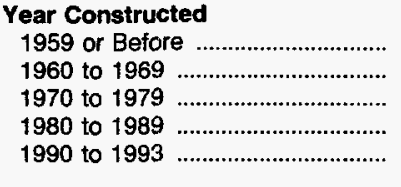 & $\begin{array}{r}103 \\
38 \\
34 \\
57 \\
11\end{array}$ & $\begin{array}{r}44 \\
12 \\
15 \\
21 \\
4\end{array}$ & $\begin{array}{r}14 \\
11 \\
11 \\
9 \\
3\end{array}$ & $\begin{array}{r}22 \\
14 \\
9 \\
16 \\
3\end{array}$ & $\begin{array}{r}24 \\
12 \\
9 \\
10 \\
2\end{array}$ & $\begin{array}{r}57 \\
25 \\
14 \\
33 \\
7\end{array}$ & $\begin{array}{r}70 \\
28 \\
22 \\
36 \\
6\end{array}$ \\
\hline 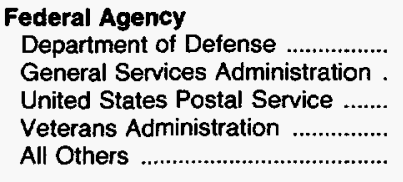 & $\begin{array}{l}22 \\
35 \\
61 \\
36 \\
89\end{array}$ & $\begin{array}{r}6 \\
19 \\
19 \\
30 \\
22\end{array}$ & $\begin{array}{r}5 \\
12 \\
15 \\
10 \\
6\end{array}$ & $\begin{array}{r}10 \\
17 \\
21 \\
8 \\
8\end{array}$ & $\begin{array}{r}6 \\
24 \\
10 \\
6 \\
11\end{array}$ & $\begin{array}{l}16 \\
32 \\
38 \\
10 \\
40\end{array}$ & $\begin{array}{l}13 \\
31 \\
45 \\
33 \\
40\end{array}$ \\
\hline \multicolumn{8}{|l|}{$\begin{array}{l}\text { Energy Sources (more than one } \\
\text { may apply) }\end{array}$} \\
\hline 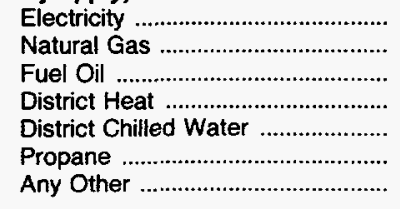 & $\begin{array}{r}243 \\
168 \\
18 \\
68 \\
30 \\
5 \\
8\end{array}$ & $\begin{array}{r}96 \\
65 \\
10 \\
36 \\
22 \\
1 \\
4\end{array}$ & $\begin{array}{r}48 \\
34 \\
8 \\
11 \\
12 \\
2 \\
3\end{array}$ & $\begin{array}{r}64 \\
55 \\
7 \\
5 \\
5 \\
\mathrm{NC} \\
\mathrm{NC}\end{array}$ & $\begin{array}{r}57 \\
45 \\
9 \\
12 \\
10 \\
2 \\
2\end{array}$ & $\begin{array}{r}136 \\
95 \\
9 \\
36 \\
16 \\
4 \\
1\end{array}$ & $\begin{array}{r}162 \\
121 \\
15 \\
40 \\
25 \\
1 \\
8\end{array}$ \\
\hline \multicolumn{8}{|l|}{$\begin{array}{l}\text { Energy End Uses (more than } \\
\text { one may apply) }\end{array}$} \\
\hline 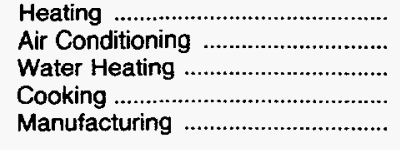 & $\begin{array}{r}242 \\
241 \\
238 \\
63 \\
28\end{array}$ & $\begin{array}{l}96 \\
95 \\
95 \\
37 \\
13\end{array}$ & $\begin{array}{r}48 \\
47 \\
47 \\
20 \\
8\end{array}$ & $\begin{array}{l}64 \\
64 \\
62 \\
24 \\
14\end{array}$ & $\begin{array}{r}57 \\
57 \\
56 \\
21 \\
9\end{array}$ & $\begin{array}{r}136 \\
136 \\
133 \\
27 \\
16\end{array}$ & $\begin{array}{r}162 \\
161 \\
160 \\
51 \\
21\end{array}$ \\
\hline \multicolumn{8}{|l|}{ Workers (main shift) } \\
\hline $\begin{array}{l}\text { Less than } 50 \\
50 \text { to } 99 \\
100 \text { to } 499 \\
500 \text { or More }\end{array}$ & $\begin{array}{l}70 \\
44 \\
80 \\
49\end{array}$ & $\begin{array}{l}10 \\
15 \\
36 \\
35\end{array}$ & $\begin{array}{r}10 \\
4 \\
11 \\
23\end{array}$ & $\begin{array}{r}19 \\
9 \\
14 \\
22\end{array}$ & $\begin{array}{r}10 \\
9 \\
13 \\
25\end{array}$ & $\begin{array}{l}43 \\
27 \\
47 \\
19\end{array}$ & $\begin{array}{l}41 \\
26 \\
50 \\
45\end{array}$ \\
\hline $\begin{array}{l}\text { Weekly Operating Hours } \\
48 \text { or Fewer } \\
49 \text { to } 60 \\
61 \text { to } 167 \\
\text { Open Continuously }\end{array}$ & $\begin{array}{l}39 \\
46 \\
78 \\
80\end{array}$ & $\begin{array}{l}11 \\
27 \\
12 \\
46\end{array}$ & $\begin{array}{r}4 \\
10 \\
11 \\
23\end{array}$ & $\begin{array}{l}10 \\
14 \\
17 \\
23\end{array}$ & $\begin{array}{r}10 \\
18 \\
9 \\
20\end{array}$ & $\begin{array}{r}36 \\
46 \\
54 \\
\text { NC }\end{array}$ & $\begin{array}{l}24 \\
29 \\
42 \\
67\end{array}$ \\
\hline 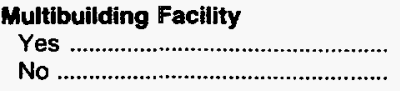 & $\begin{array}{r}148 \\
95\end{array}$ & $\begin{array}{l}69 \\
27\end{array}$ & $\begin{array}{l}29 \\
19\end{array}$ & $\begin{array}{l}33 \\
31\end{array}$ & $\begin{array}{l}29 \\
28\end{array}$ & $\begin{array}{l}64 \\
72\end{array}$ & $\begin{array}{l}95 \\
67\end{array}$ \\
\hline
\end{tabular}

See footnotes at end of table. 
Table 3.38. Energy Management Practices in FBSS Buildings in Federal Region 6, Number of Buildings, 1993 (Continued)

\begin{tabular}{|c|c|c|c|c|c|c|c|}
\hline $\begin{array}{c}\text { Building } \\
\text { Characteristics }\end{array}$ & $\begin{array}{c}\text { All } \\
\text { Buildings }\end{array}$ & $\begin{array}{c}\text { Energy } \\
\text { Management } \\
\text { and Control } \\
\text { System }\end{array}$ & $\begin{array}{c}\text { Energy } \\
\text { Conservation } \\
\text { Programs }{ }^{1}\end{array}$ & $\begin{array}{c}\text { Energy } \\
\text { Audit }\end{array}$ & $\begin{array}{c}\text { HVAC } \\
\text { Maintenance } \\
\text { Staff }^{2}\end{array}$ & $\begin{array}{l}\text { Off-Hour } \\
\text { Equipment } \\
\text { Reduction }\end{array}$ & $\begin{array}{l}\text { Retrofit or } \\
\text { Purchase } \\
\text { of Energy } \\
\text { Efficient } \\
\text { Equipment }\end{array}$ \\
\hline
\end{tabular}

Percent of Floorspace Heated

Not Heated.

1 to 50

51 to 100

Percent of Floorspace Cooled

Not Cooled

1 to 50

51 to 100

1 to 50

51 to 100

No Operating Hours

Don't Know

Heating Equipment (more than

one may apply)

Heat Pumps

Furnaces

Individual Space Heaters

District Heat

Boilers

Packaged-Heating Units

Cooling Equipment (more than

one may apply)

Residential-Type Central A/C .....

Heat Pumps

Individual $\mathrm{A} / \mathrm{C}$

District Chilled Water

Central Chillers

Packaged-A/C Ur

Swamp Coolers

Lighting Equipment (more than one may apply)

Incandescent.

Standard Fluorescent

Compact Fluorescent

High-intensity Discharge

Electronic Ballast

Water-Heating Equipment (more

than one may apply)

Centralized System

Distributed System .........................

Don't Know/

Not Ascertained

Energy Conservation Features

(more than one may apply)

Any Conservation Feature

Building Shell

HVAC

Lighting

See footnotes at end of table.

10
18
30
82
102
59

3
3
14
47
35
17

29

11

50

122

12

135

235

29

64

59

163

71

4

242

228

236

136
10
6
17
31
54
37
6

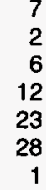

8
4
10
8
32
29
1

27

60

10

16

22

39

22

1

64

62

61

45

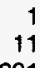

23

NC

5

$\mathrm{NC}$
2

2

NC

26
214

2
4

90

15
227

NC

54

NC

128

NC

154

NC

$\begin{array}{rr}14 & 14 \\ 122 & 146\end{array}$

$\begin{array}{lr}10 & 10 \\ 26 & 152 \\ \text { NC } & \text { NC } \\ \text { NC } & \text { NC }\end{array}$

NC

4
92
$N C$
$N C$

1
47
NC
NC

3
61
$N C$

NC

10

NC

NC

6
14
23
53
66
45 
Table 3.38. Energy Management Practices in FBSS Buildings in Federal Region 6, Number of Buildings, 1993 (Continued)

\begin{tabular}{|c|c|c|c|c|c|c|c|}
\hline $\begin{array}{c}\text { Building } \\
\text { Characteristics }\end{array}$ & $\begin{array}{c}\text { All } \\
\text { Buildings }\end{array}$ & $\begin{array}{l}\text { Energy } \\
\text { Management } \\
\text { and Control } \\
\text { System }\end{array}$ & $\begin{array}{c}\text { Energy } \\
\text { Conservation } \\
\text { Programs }\end{array}$ & $\begin{array}{c}\text { Energy } \\
\text { Audit }\end{array}$ & $\begin{array}{l}\text { HVAC } \\
\text { Maintenance } \\
\text { Staff }^{2}\end{array}$ & $\begin{array}{l}\text { Off-Hour } \\
\text { Equipment } \\
\text { Reduction }\end{array}$ & $\begin{array}{l}\text { Retrofit or } \\
\text { Purchase } \\
\text { of Energy } \\
\text { Efficient } \\
\text { Equipment }\end{array}$ \\
\hline \multicolumn{8}{|l|}{$\begin{array}{l}\text { Bullding Shell Conservation } \\
\text { Features (more than one may } \\
\text { apply) } \\
\text { Roof or Ceiling }\end{array}$} \\
\hline 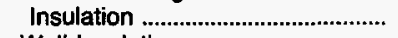 & 196 & 82 & 42 & 53 & 43 & 106 & 129 \\
\hline $\begin{array}{l}\text { Wall Insulation } \\
\text { Storm or Multiple }\end{array}$ & 122 & 49 & 26 & 35 & 25 & 58 & 76 \\
\hline $\begin{array}{l}\text { Glazing } \\
\text { Tinted or Reflective Glass }\end{array}$ & 96 & 48 & 21 & 22 & 22 & 53 & 66 \\
\hline $\begin{array}{l}\text { or Shading Film ........................... } \\
\text { Exterior or Interior Shading }\end{array}$ & 127 & 59 & 39 & 41 & 42 & 69 & 98 \\
\hline 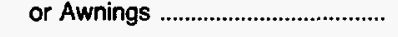 & 142 & 68 & 33 & 47 & 46 & 84 & 114 \\
\hline \multicolumn{8}{|l|}{$\begin{array}{l}\text { HVAC Conservation Features } \\
\text { (more than one may apply) }\end{array}$} \\
\hline VAV System & 65 & 40 & 16 & 20 & 25 & 32 & 47 \\
\hline Economizer Cycle ............................... & 123 & 62 & 28 & 32 & 30 & 55 & 87 \\
\hline HVAC Maintenance .......................... & 234 & 95 & 46 & 60 & 56 & 129 & 156 \\
\hline \multicolumn{8}{|l|}{$\begin{array}{l}\text { Lighting Conservation Features } \\
\text { (more than one may apply) }\end{array}$} \\
\hline $\begin{array}{l}\text { Specular Reflectors ........................ } \\
\text { Natural Lighting Control }\end{array}$ & 92 & 36 & 30 & 33 & 28 & 55 & 76 \\
\hline 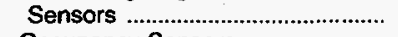 & 27 & 12 & 9 & 9 & 13 & 14 & 23 \\
\hline Occupancy Sensors ........................... & 58 & 31 & 18 & 28 & 24 & 35 & 49 \\
\hline 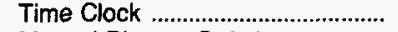 & 36 & 18 & 14 & 15 & 16 & 22 & 29 \\
\hline Manual Dimmer Switches .............. & 44 & 29 & 16 & 17 & 14 & 27 & 39 \\
\hline
\end{tabular}

1 Building participates in any programs sponsored by the Federal Energy Management Program, in-house, utility, or third party.

2 HVAC maintenance staff means at least one person spends at least half their working hours maintaining the heating/cooling equipment.

NC $=$ No cases in responding sample.

Notes: - Total workers are the number of workers during the main shift. - See Glossary for explanation of abbreviations and definitions of terms used in this report. - These data are from 881 federally owned buildings having the following criteria: (1) located in Federal Regions 3 , 6, or 9; (2) larger than 10,000 square feet; and (3) used for a commercial purpose, other than warehouse and storage. in addition, 9 out of 10 selected buildings were from agencies other than the Department of Defense. - Statistics for the "energy end uses" represent consumption in buildings that have end use, not consumption for a particular fuel for a particular end use. $\bullet$ A/C = Air Conditioning. $\bullet$ FBSS = Federal Buildings Supplemental Survey.

- HVAC = Heating, Ventilation, and Air Conditioning. - VAV = Variable-Air Volume. - Data are for Fiscal Year 1993 (October 1, 1992 through September 30,1993$)$. Because of rounding, data may not sum to totals.

Source: Energy Information Administration, Office of Energy Markets and End Use, 1993 Federal Buildings Supplemental Survey. 
Table 3.39. Energy Management Practices in FBSS Buildings in Federal Region 9, Number of Buildings, 1993

\begin{tabular}{|c|c|c|c|c|c|c|c|}
\hline $\begin{array}{c}\text { Building } \\
\text { Characteristics }\end{array}$ & $\begin{array}{l}\text { All } \\
\text { Buildings }\end{array}$ & $\begin{array}{c}\text { Energy } \\
\text { Management } \\
\text { and Control } \\
\text { System }\end{array}$ & $\begin{array}{c}\text { Energy } \\
\text { Conservation } \\
\text { Programs }\end{array}$ & $\begin{array}{l}\text { Energy } \\
\text { Audit }\end{array}$ & $\begin{array}{c}\text { HVAC } \\
\text { Maintenance } \\
\text { Staft }^{2}\end{array}$ & $\begin{array}{l}\text { Off-Hour } \\
\text { Equipment } \\
\text { Reduction }\end{array}$ & $\begin{array}{l}\text { Retroftit or } \\
\text { Purchase } \\
\text { of Energy } \\
\text { Efficient } \\
\text { Equipment }\end{array}$ \\
\hline All Buildings & 326 & 71 & 124 & 99 & 52 & 189 & 213 \\
\hline \multicolumn{8}{|l|}{$\begin{array}{l}\text { Building Floorspace (square } \\
\text { feet) }\end{array}$} \\
\hline 10,000 to 50,000 & 148 & 15 & 46 & 40 & 11 & 96 & 84 \\
\hline 50,001 to $200,000 \ldots \ldots \ldots \ldots$ & 123 & 34 & 50 & 42 & 22 & 67 & 85 \\
\hline Over 200,000 & 55 & 22 & 28 & 17 & 19 & 26 & 44 \\
\hline \multicolumn{8}{|l|}{ Principal Building Activity } \\
\hline 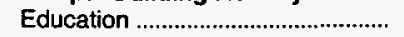 & 12 & NC & 1 & 2 & 1 & 7 & 4 \\
\hline Health Care & 48 & 14 & 18 & 14 & 16 & 15 & 30 \\
\hline 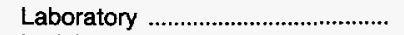 & 31 & 12 & 9 & 9 & 1 & 13 & 25 \\
\hline 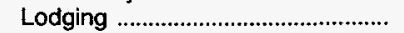 & 22 & 2 & 8 & 8 & 1 & 1 & 11 \\
\hline Mercantile and Service ................... & 63 & 20 & 25 & 21 & 8 & 43 & 47 \\
\hline Office & 92 & 18 & 43 & 27 & 17 & 75 & 65 \\
\hline 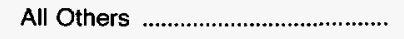 & 58 & 5 & 20 & 18 & 8 & 35 & 31 \\
\hline \multicolumn{8}{|l|}{ Year Constructed } \\
\hline 1959 or Before & 130 & 20 & 37 & 36 & 18 & 86 & 77 \\
\hline 1960 to 1969 & 55 & 11 & 30 & 16 & 6 & 36 & 35 \\
\hline 1970 to 1979 & 62 & 13 & 29 & 24 & 13 & 30 & 46 \\
\hline 1980 to 1989 & 65 & 23 & 25 & 18 & 12 & 29 & 44 \\
\hline 1990 to 1993 & 14 & 4 & 3 & 5 & 3 & 8 & 11 \\
\hline \multicolumn{8}{|l|}{ Federal Agency } \\
\hline Department of Defense & 78 & 14 & 17 & 26 & 6 & 52 & 36 \\
\hline General Services Administration . & 39 & 5 & 27 & 12 & 14 & 35 & 33 \\
\hline United States Postal Service ....... & 63 & 21 & 24 & 22 & 9 & 43 & 49 \\
\hline 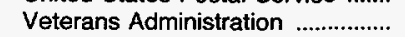 & 56 & 15 & 16 & 15 & 15 & 18 & 36 \\
\hline 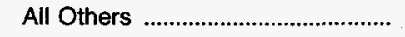 & 90 & 16 & 40 & 24 & 8 & 41 & 59 \\
\hline \multicolumn{8}{|l|}{$\begin{array}{l}\text { Energy Sources (more than one } \\
\text { may apply) }\end{array}$} \\
\hline 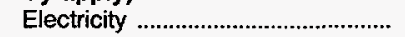 & 325 & 71 & 123 & 98 & 52 & 189 & 212 \\
\hline 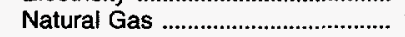 & 225 & 57 & 98 & 67 & 39 & 141 & 162 \\
\hline 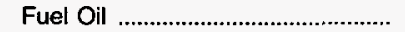 & 54 & 17 & 23 & 11 & 12 & 14 & 46 \\
\hline 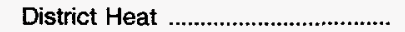 & 74 & 13 & 21 & 24 & 15 & 29 & 39 \\
\hline District Chilled Water ......................... & 27 & 6 & 6 & 14 & 8 & 10 & 15 \\
\hline 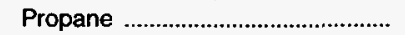 & 10 & 1 & 4 & 2 & 1 & 4 & 5 \\
\hline Any Other & 5 & 2 & 1 & 3 & NC & 2 & 3 \\
\hline \multicolumn{8}{|l|}{$\begin{array}{l}\text { Energy End Uses (more than } \\
\text { one may apply) }\end{array}$} \\
\hline Heating & 304 & 67 & 122 & 92 & 48 & 177 & 202 \\
\hline 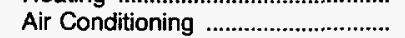 & 292 & 71 & 115 & 92 & 50 & 174 & 201 \\
\hline Water Heating & 315 & 70 & 123 & 97 & 51 & 182 & 209 \\
\hline 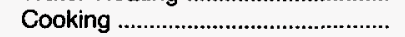 & 70 & 23 & 42 & 25 & 22 & 25 & 54 \\
\hline 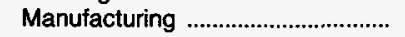 & 30 & 15 & 14 & 13 & 6 & 15 & 23 \\
\hline \multicolumn{8}{|l|}{ Workers (main shift) } \\
\hline Less than 50 & 93 & 8 & 20 & 27 & 7 & 51 & 43 \\
\hline 50 to 99 & 49 & 5 & 14 & 14 & 2 & 39 & 30 \\
\hline 100 to 499 & 134 & 36 & 58 & 41 & 20 & 79 & 98 \\
\hline 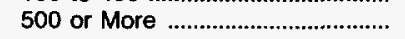 & 50 & 22 & 32 & 17 & 23 & 20 & 42 \\
\hline \multicolumn{8}{|l|}{ Weekly Operating Hours } \\
\hline 48 or Fewer & 80 & 8 & 20 & 21 & 3 & 67 & 29 \\
\hline 49 to 60 & 65 & 9 & 27 & 18 & 14 & 59 & 45 \\
\hline 61 to 167 & 68 & 14 & 26 & 26 & 8 & 63 & 52 \\
\hline 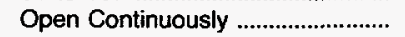 & 113 & 40 & 51 & 34 & 27 & NC & 87 \\
\hline \multicolumn{8}{|l|}{ Multibuilding Facility } \\
\hline Yes & 200 & 48 & 69 & 64 & 35 & 95 & 128 \\
\hline No & 126 & 23 & 55 & 35 & 17 & 94 & 85 \\
\hline
\end{tabular}

See footnotes at end of table. 
Table 3.39. Energy Management Practices in FBSS Buildings in Federal Region 9, Number of Buildings, 1993 (Continued)

\begin{tabular}{|c|c|c|c|c|c|c|c|}
\hline $\begin{array}{c}\text { Building } \\
\text { Characteristics }\end{array}$ & $\begin{array}{c}\text { All } \\
\text { Bulldings }\end{array}$ & $\begin{array}{c}\text { Energy } \\
\text { Management } \\
\text { and Control } \\
\text { System }\end{array}$ & $\begin{array}{c}\text { Energy } \\
\text { Conservation } \\
\text { Programs }^{1}\end{array}$ & $\begin{array}{c}\text { Energy } \\
\text { Audit }\end{array}$ & $\begin{array}{c}\text { HVAC } \\
\text { Maintenance } \\
\text { Staff }^{2}\end{array}$ & $\begin{array}{l}\text { Off-Hour } \\
\text { Equipment } \\
\text { Reduction }\end{array}$ & $\begin{array}{l}\text { Retrofit or } \\
\text { Purchase } \\
\text { of Energy } \\
\text { Efficient } \\
\text { Equipment }\end{array}$ \\
\hline \multicolumn{8}{|l|}{ Percent of Floorspace Heated } \\
\hline Not Heated & 22 & 4 & 2 & 7 & 4 & 12 & 11 \\
\hline 1 to 50 & 28 & 6 & 6 & 11 & 3 & 20 & 18 \\
\hline 51 to 100 & 276 & 61 & 116 & 81 & 45 & 157 & 184 \\
\hline \multicolumn{8}{|l|}{ Percent of Floorspace Cooled } \\
\hline Not Cooled & 40 & 1 & 12 & 8 & 4 & 19 & 16 \\
\hline 1 to 50 & 55 & 9 & 14 & 13 & 7 & 32 & 39 \\
\hline 51 to 100 & 231 & 61 & 98 & 78 & 41 & 138 & 158 \\
\hline \multicolumn{8}{|l|}{ Percent Lit When Open } \\
\hline 1 to 50 & 20 & 1 & 5 & 6 & 3 & 10 & 12 \\
\hline 51 to 100 & 304 & 70 & 119 & 93 & 49 & 178 & 201 \\
\hline No Operating Hours ......................... & 1 & NC & NC & NC & NC & 1 & NC \\
\hline 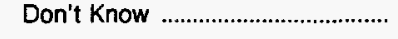 & 1 & NC & NC & NC & NC & NC & NC \\
\hline \multicolumn{8}{|l|}{$\begin{array}{l}\text { Heating Equiprnent (more than } \\
\text { one may apply) }\end{array}$} \\
\hline Heat Pumps .................................... & 40 & 12 & 23 & 15 & 3 & 21 & 34 \\
\hline Furnaces & 25 & 2 & 9 & 10 & 2 & 18 & 16 \\
\hline Individual Space Heaters .............. & 33 & 8 & 17 & 13 & 3 & 24 & 28 \\
\hline 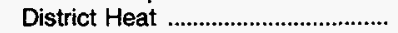 & 71 & 15 & 24 & 20 & 14 & 28 & 41 \\
\hline 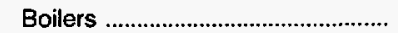 & 146 & 40 & 67 & 47 & 25 & 92 & 102 \\
\hline Packaged-Heating Units .................. & 67 & 13 & 34 & 13 & 8 & 49 & 47 \\
\hline \multicolumn{8}{|l|}{$\begin{array}{l}\text { Cooling Equipment (more than } \\
\text { one may apply) }\end{array}$} \\
\hline Residential-Type Central A/C ...... & 24 & 4 & 9 & 10 & 3 & 16 & 12 \\
\hline Heat Pumps & 32 & 11 & 16 & 11 & 3 & 17 & 28 \\
\hline 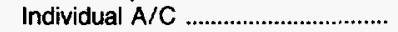 & 52 & 7 & 18 & 13 & 4 & 35 & 38 \\
\hline District Chilled Water ....................... & 31 & 13 & 15 & 12 & 10 & 7 & 17 \\
\hline 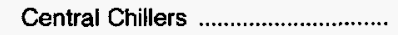 & 139 & 51 & 68 & 47 & 25 & 81 & 105 \\
\hline 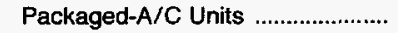 & 151 & 34 & 65 & 44 & 27 & 99 & 112 \\
\hline 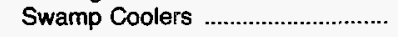 & 30 & 8 & 10 & 12 & 5 & 16 & 16 \\
\hline \multicolumn{8}{|l|}{$\begin{array}{l}\text { Lighting Equipment (more than } \\
\text { one may apply) }\end{array}$} \\
\hline 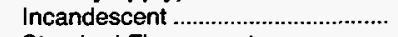 & 161 & 34 & 64 & 48 & 27 & 84 & 106 \\
\hline Standard Fluorescent & 309 & 69 & 119 & 95 & 52 & 179 & 206 \\
\hline Compact Fluorescent ........................ & 73 & 24 & 43 & 24 & 20 & 41 & 70 \\
\hline High-Intensity Discharge ................ & 75 & 25 & 34 & 24 & 17 & 40 & 56 \\
\hline Electronic Ballast ............................... & 105 & 31 & 63 & 45 & 28 & 69 & 95 \\
\hline \multicolumn{8}{|l|}{$\begin{array}{l}\text { Water-Heating Equipment (more } \\
\text { than one may apply) }\end{array}$} \\
\hline Centralized System ............................ & 225 & 50 & 88 & 73 & 43 & 131 & 148 \\
\hline $\begin{array}{l}\text { Distributed System .............................. } \\
\text { Don't Know/ }\end{array}$ & 80 & 17 & 34 & 21 & 5 & 45 & 55 \\
\hline Not Ascertained ............................... & 10 & 3 & 1 & 3 & 3 & 6 & 6 \\
\hline \multicolumn{8}{|l|}{$\begin{array}{l}\text { Energy Conservation Features } \\
\text { (more than one may apply) }\end{array}$} \\
\hline Any Conservation Feature ............ & $3+9$ & 71 & 122 & 98 & 52 & 186 & 213 \\
\hline Building Shell & 248 & 59 & 99 & 82 & 40 & 147 & 166 \\
\hline 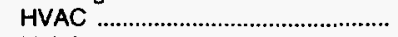 & 307 & 70 & 119 & 91 & 51 & 179 & 210 \\
\hline Lighting & 217 & 60 & 101 & 67 & 41 & 126 & 162 \\
\hline
\end{tabular}

See footnotes at end of table. 
Table 3.39. Energy Management Practices in FBSS Buildings in Federal Region 9, Number of Buildings, 1993 (Continued)

\begin{tabular}{|c|c|c|c|c|c|c|c|}
\hline $\begin{array}{c}\text { Building } \\
\text { Characteristics }\end{array}$ & $\begin{array}{c}\text { All } \\
\text { Buildings }\end{array}$ & $\begin{array}{l}\text { Energy } \\
\text { Management } \\
\text { and Control } \\
\text { System }\end{array}$ & $\begin{array}{l}\text { Energy } \\
\text { Conservation } \\
\text { Programs }^{1}\end{array}$ & $\begin{array}{l}\text { Energy } \\
\text { Audit }\end{array}$ & $\begin{array}{l}\text { HVAC } \\
\text { Maintenance } \\
\text { Staff }\end{array}$ & $\begin{array}{l}\text { Off-Hour } \\
\text { Equipment } \\
\text { Reduction }\end{array}$ & $\begin{array}{l}\text { Retrotit or } \\
\text { Purchase } \\
\text { of Energy } \\
\text { Efficient } \\
\text { Equipment }\end{array}$ \\
\hline \multicolumn{8}{|l|}{$\begin{array}{l}\text { Bullding Shell Conservation } \\
\text { Features (more than one may } \\
\text { apply) } \\
\text { Roof or Ceiling }\end{array}$} \\
\hline 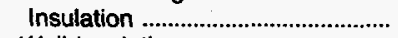 & 185 & 51 & 78 & 60 & 31 & 99 & 124 \\
\hline $\begin{array}{l}\text { Wall Insulation } \\
\text { Storm or Multiple }\end{array}$ & 113 & 35 & 41 & 41 & 18 & 59 & 73 \\
\hline Glazing & 57 & 18 & 30 & 15 & 13 & 26 & 48 \\
\hline $\begin{array}{l}\text { or Shading Film .............................. } \\
\text { Exterior or Interior Shading }\end{array}$ & 133 & 34 & 63 & 50 & 24 & 78 & 101 \\
\hline 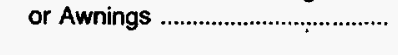 & 164 & 41 & 73 & 51 & 31 & 90 & 117 \\
\hline \multicolumn{8}{|l|}{$\begin{array}{l}\text { HVAC Conservation Features } \\
\text { (more than one may apply) }\end{array}$} \\
\hline 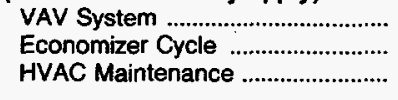 & $\begin{array}{r}75 \\
140 \\
306\end{array}$ & $\begin{array}{l}33 \\
45 \\
69\end{array}$ & $\begin{array}{r}39 \\
79 \\
118\end{array}$ & $\begin{array}{l}25 \\
44 \\
91\end{array}$ & $\begin{array}{l}18 \\
31 \\
51\end{array}$ & $\begin{array}{r}37 \\
86 \\
178\end{array}$ & $\begin{array}{r}64 \\
107 \\
209\end{array}$ \\
\hline \multicolumn{8}{|l|}{$\begin{array}{l}\text { Lighting Conservation Features } \\
\text { (more than one may apply) }\end{array}$} \\
\hline $\begin{array}{l}\text { Specular Reflectors .......................... } \\
\text { Natural Lighting Control }\end{array}$ & 124 & 26 & 75 & 32 & 30 & 85 & 96 \\
\hline 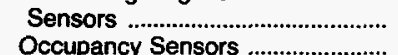 & $\begin{array}{r}58 \\
109\end{array}$ & $\begin{array}{l}15 \\
38\end{array}$ & $\begin{array}{l}26 \\
48\end{array}$ & $\begin{array}{l}20 \\
42\end{array}$ & $\begin{array}{l}16 \\
25\end{array}$ & $\begin{array}{l}35 \\
52\end{array}$ & $\begin{array}{l}45 \\
92\end{array}$ \\
\hline 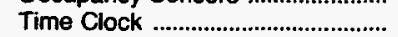 & 41 & 12 & 21 & 19 & 8 & 28 & 34 \\
\hline Manual Dimmer Switches .............. & 78 & 28 & 34 & 28 & 21 & 29 & 62 \\
\hline
\end{tabular}

1 Building participates in any programs sponsored by the Federal Energy Management Program, in-house, utility, or third party.

2 HVAC maintenance staff means at least one person spends at least half their working hours maintaining the heating/cooling equipment.

NC $=$ No cases in responding sample.

Notes: - Total workers are the number of workers during the main shift. - See Glossary for explanation of abbreviations and definitions of terms used in this report. - These data are from 881 federally owned buildings having the following criteria: (1) looated in Federal Regions 3 , 6 , or 9 ; (2) larger than 10,000 square feet; and (3) used for a commercial purpose, other than warehouse and storage. In addition, 9 out of 10 selected buildings were from agencies other than the Department of Defense. - Statistics for the "energy end uses" represent consumption in buildings that have end use, not consumption for a particular fuel for a particular end use. $\bullet$ A $/ C=$ Air Conditioning. $\bullet$ FBS $=$ Federal Buildings Supplemental Survey.

- HVAC = Heating, Ventilation, and Air Conditioning. - VAV = Variable-Air Volume. - Data are for Fiscal Year 1993 (October 1, 1992 through September 30,1993$)$. Because of rounding, data may not sum to totals.

Source: Energy Information Administration, Office of Energy Markets and End Use, 1993 Federal Buildings Supplemental Survey. 
Table 3.40. Energy Management Practices in FBSS Buildings in Federal Region 3, Floorspace, 1993

(Thousand Square Feet)

\begin{tabular}{|c|c|c|c|c|c|c|c|}
\hline $\begin{array}{l}\text { Builiding } \\
\text { Characteristics }\end{array}$ & $\begin{array}{l}\text { All } \\
\text { Buildings }\end{array}$ & $\begin{array}{c}\text { Energy } \\
\text { Management } \\
\text { and Control } \\
\text { System }\end{array}$ & $\begin{array}{c}\text { Energy } \\
\text { Conservation } \\
\text { Programs } 1\end{array}$ & $\begin{array}{l}\text { Energy } \\
\text { Audit }\end{array}$ & $\begin{array}{c}\text { HVAC } \\
\text { Maintenance } \\
\text { Staff }^{2}\end{array}$ & $\begin{array}{l}\text { Off-Hour } \\
\text { Equipment } \\
\text { Reduction }\end{array}$ & $\begin{array}{l}\text { Retrofit or } \\
\text { Purchase } \\
\text { of Energy } \\
\text { Efficient } \\
\text { Equipment }\end{array}$ \\
\hline All Buildings & 94,880 & 61,891 & 59,284 & 37,621 & 52,924 & 46,595 & 81,286 \\
\hline \multicolumn{8}{|l|}{ Building Floorspace (square feet) } \\
\hline 10,000 to 50,000 & 2,564 & 604 & 361 & 868 & 141 & 1,819 & 1,617 \\
\hline 50,001 to 200,000 & 13,166 & 5,984 & 3,395 & 3,545 & 4,785 & 6,455 & 9,405 \\
\hline Over 200,000 & 79,149 & 55,303 & 55,528 & 33,208 & 47,998 & 38,322 & 70,264 \\
\hline \multicolumn{8}{|l|}{ Principal Building Activity } \\
\hline 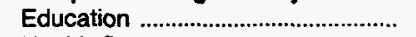 & 598 & NC & 171 & 298 & NC & 462 & 264 \\
\hline 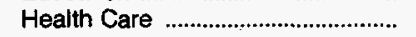 & 14,559 & 12,399 & 8,137 & 1,597 & 6,289 & 206 & 13,789 \\
\hline 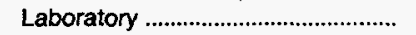 & 5,165 & 1,827 & 361 & 1,969 & 3,891 & 2,512 & 3,238 \\
\hline Lodging & 2,558 & 2,157 & 784 & 2,373 & 1,840 & NC & 908 \\
\hline Mercantile and Service ................... & 7,966 & 6,058 & 3,124 & 1,454 & 2,233 & 1,300 & 6,387 \\
\hline Office & 56,881 & 36,854 & 42,364 & 28,924 & 37,641 & 40,781 & 51,485 \\
\hline 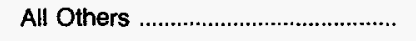 & 7,152 & 2,596 & 4,343 & 1,006 & 1,030 & 1,335 & 5,215 \\
\hline \multicolumn{8}{|l|}{ Year Constructed } \\
\hline 1959 or Before & 43,829 & 25,341 & 29,902 & 20,038 & 29,624 & 23,359 & 39,006 \\
\hline 1960 to 1969 & 19,564 & 11,821 & 11,618 & 7,852 & 12,049 & 11,333 & 14,706 \\
\hline 1970 to 1979 & 17,737 & 14,405 & 10,803 & 6,179 & 5,099 & 8,812 & 15,377 \\
\hline 1980 to 1989 & 8,975 & 6,446 & 5,230 & 2,874 & 5,035 & 2,085 & 8,308 \\
\hline 1990 to 1993 & 4,774 & 3,877 & 1,732 & 678 & 1,117 & 1,006 & 3,889 \\
\hline \multicolumn{8}{|l|}{ Federal Agency } \\
\hline Department of Defense .................. & 13,988 & 11,136 & 10,164 & 9,261 & 10,573 & 1,615 & 11,708 \\
\hline General Services Administration ... & 46,205 & 26,744 & 35,701 & 20,539 & 27,705 & 35,547 & 40,941 \\
\hline United States Postal Service ......... & 9,392 & 7,143 & 2,343 & 2,255 & 2,382 & 2,398 & 7,910 \\
\hline 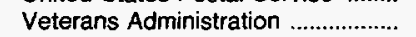 & 13,306 & 10,289 & 6,427 & 1,687 & 6,748 & 956 & 11,866 \\
\hline All Others & 11,989 & 6,579 & 4,650 & 3,880 & 5,515 & 6,079 & 8,861 \\
\hline \multicolumn{8}{|l|}{$\begin{array}{l}\text { Energy Sources (more than one } \\
\text { may apply) }\end{array}$} \\
\hline 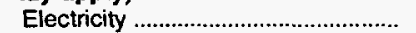 & 94,161 & 61,172 & 58,565 & 37,621 & 52,924 & 46,595 & 80,567 \\
\hline 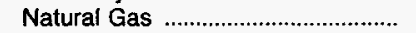 & 58,674 & 44,873 & 38,182 & 20,605 & 32,344 & 21,566 & 51,875 \\
\hline 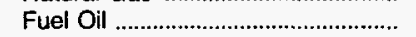 & 39,300 & 30,851 & 24,941 & 16,293 & 20,200 & 11,751 & 33,574 \\
\hline 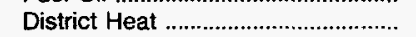 & 63,991 & 41,010 & 46,603 & 25,813 & 37,204 & 34,089 & 58,464 \\
\hline District Chilled Water ........................ & 22,922 & 14,652 & 15,190 & 12,406 & 15,253 & 8,564 & 20,544 \\
\hline 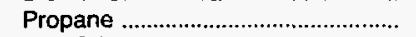 & 941 & NC & NC & 222 & 148 & 793 & 683 \\
\hline 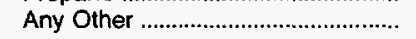 & 4,470 & 3,059 & 3,462 & 2,761 & 4,410 & 4,273 & $4,4.41$ \\
\hline \multicolumn{8}{|l|}{$\begin{array}{l}\text { Energy End Uses (more than one } \\
\text { may apply) }\end{array}$} \\
\hline 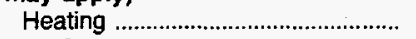 & 94,063 & 61,891 & 59,084 & 37,421 & 52,665 & 46,237 & 80,919 \\
\hline 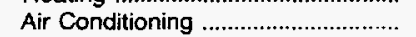 & 93,454 & 61,172 & 58,313 & 37,392 & 52,665 & 46,376 & 80,147 \\
\hline 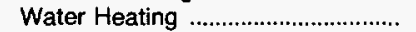 & 93,138 & 61,222 & 58,136 & 37,421 & 51,717 & 45,692 & 80,336 \\
\hline 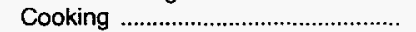 & 69,947 & 52,300 & 49,012 & 28,107 & 40,466 & 31,295 & 62,196 \\
\hline 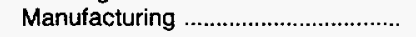 & 27,041 & 23,953 & 18,390 & 13,925 & 16,741 & 9,251 & 23,383 \\
\hline \multicolumn{8}{|l|}{ Workers (main shift) } \\
\hline Less than 50 & 4,102 & 816 & 1,085 & 1,308 & 837 & 2,115 & 1,818 \\
\hline 50 to 99 & 1,903 & 501 & 248 & 687 & 19 & 1,198 & 1,175 \\
\hline 100 to 499 & 13,632 & 5,390 & 2,879 & 4,455 & 5,263 & 6,874 & 10,165 \\
\hline 500 or More & 75,243 & 55,184 & 55,073 & 31,171 & 46,804 & 36,409 & 68,128 \\
\hline \multicolumn{8}{|l|}{ Weekly Operating Hours } \\
\hline 48 or Fewer & 9,694 & 4,600 & 6,042 & 3,534 & 5,139 & 9,367 & 8,337 \\
\hline 49 to 60 & 25,540 & 14,948 & 17,947 & 12,556 & 15,985 & 25,233 & 22,537 \\
\hline 61 to 167 & 11,993 & 5,270 & 6,234 & 6,552 & 8,344 & 11,948 & 10,868 \\
\hline 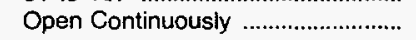 & 47,653 & 37,072 & 29,062 & 14,979 & 23,457 & 47 & 39,545 \\
\hline
\end{tabular}

See footnotes at end of table. 
Table 3.40. Energy Management Practices in FBSS Buildings in Federal Region 3, Floorspace, 1993 (Continued)

(Thousand Square Feet)

\begin{tabular}{|c|c|c|c|c|c|c|c|}
\hline $\begin{array}{c}\text { Building } \\
\text { Characteristics }\end{array}$ & $\begin{array}{l}\text { All } \\
\text { Buildings }\end{array}$ & $\begin{array}{l}\text { Energy } \\
\text { Management } \\
\text { and Control } \\
\text { System }\end{array}$ & $\begin{array}{l}\text { Energy } \\
\text { Conservation } \\
\text { Programs } 1\end{array}$ & $\begin{array}{l}\text { Energy } \\
\text { Audit }\end{array}$ & $\begin{array}{l}\text { HVAC } \\
\text { Maintenance } \\
\text { Staff }^{2}\end{array}$ & $\begin{array}{l}\text { Off-Hour } \\
\text { Equipment } \\
\text { Reduction }\end{array}$ & $\begin{array}{l}\text { Retrofit or } \\
\text { Purchase } \\
\text { of Energy } \\
\text { Efficlent } \\
\text { Equipment }\end{array}$ \\
\hline \multicolumn{8}{|l|}{ Multibuilding Facility } \\
\hline Yes No & $\begin{array}{l}43,861 \\
51,018\end{array}$ & $\begin{array}{l}25,196 \\
36,695\end{array}$ & $\begin{array}{l}22,808 \\
36,476\end{array}$ & $\begin{array}{l}16,938 \\
20,683\end{array}$ & $\begin{array}{l}21,997 \\
30,927\end{array}$ & $\begin{array}{l}19,405 \\
27,190\end{array}$ & $\begin{array}{l}32,434 \\
48,852\end{array}$ \\
\hline \multicolumn{8}{|l|}{ Percent of Floorspace Heated } \\
\hline 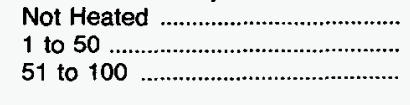 & $\begin{array}{r}817 \\
5,787 \\
88,275\end{array}$ & $\begin{array}{c}\text { NC } \\
4,358 \\
57,532\end{array}$ & $\begin{array}{r}200 \\
3,361 \\
55,723\end{array}$ & $\begin{array}{l}200 \\
\mathrm{NC} \\
37,421\end{array}$ & $\begin{array}{r}259 \\
761 \\
51,904\end{array}$ & $\begin{array}{r}359 \\
974 \\
45,263\end{array}$ & $\begin{array}{r}367 \\
4,913 \\
76,006\end{array}$ \\
\hline $\begin{array}{l}\text { Percent of Floorspace Cooled } \\
\text { Not Cooled } \\
1 \text { to } 50 \\
51 \text { to } 100\end{array}$ & $\begin{array}{r}3,582 \\
9,874 \\
81,424\end{array}$ & $\begin{array}{r}2,200 \\
4,917 \\
54,773\end{array}$ & $\begin{array}{r}1,407 \\
5,325 \\
52,552\end{array}$ & $\begin{array}{r}1,784 \\
3,964 \\
31,873\end{array}$ & $\begin{array}{r}2,120 \\
2,880 \\
47,924\end{array}$ & $\begin{array}{r}286 \\
5,171 \\
41,138\end{array}$ & $\begin{array}{r}1,360 \\
8,668 \\
71,258\end{array}$ \\
\hline 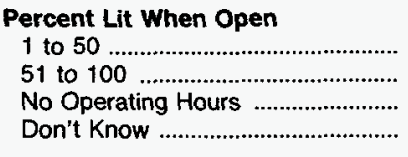 & $\begin{array}{r}663 \\
94,070 \\
147 \\
\mathrm{NC}\end{array}$ & $\begin{array}{l}213 \\
61,677 \\
\text { NC } \\
\text { NC }\end{array}$ & $\begin{array}{l}267 \\
59,017 \\
\text { NC } \\
\text { NG }\end{array}$ & $\begin{array}{l}404 \\
37,218 \\
\text { NC } \\
\text { NC }\end{array}$ & $\begin{array}{l}199 \\
52,725 \\
\text { NC } \\
\text { NC }\end{array}$ & $\begin{array}{l}497 \\
46,098 \\
\text { NC } \\
\text { NC }\end{array}$ & $\begin{array}{l}373 \\
80,913 \\
\text { NC } \\
\text { NC }\end{array}$ \\
\hline 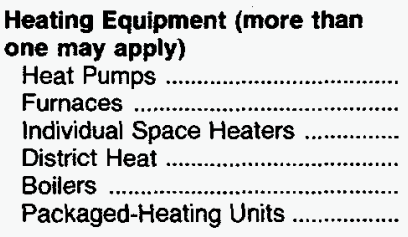 & $\begin{array}{r}10,922 \\
1,694 \\
34,923 \\
67,704 \\
20,082 \\
6,006\end{array}$ & $\begin{array}{r}8,197 \\
107 \\
26,031 \\
45,017 \\
14,594 \\
3,994\end{array}$ & $\begin{array}{r}6,244 \\
24 \\
25,674 \\
46,194 \\
8,858 \\
2,747\end{array}$ & $\begin{array}{r}7,066 \\
1,233 \\
20,648 \\
27,770 \\
7,222 \\
1,745\end{array}$ & $\begin{array}{r}4,762 \\
69 \\
22,670 \\
40,970 \\
9,644 \\
3,129\end{array}$ & $\begin{array}{r}8,592 \\
1,452 \\
17,774 \\
33,405 \\
9,068 \\
2,396\end{array}$ & $\begin{array}{r}9,016 \\
1,467 \\
31,921 \\
60,246 \\
15,822 \\
5,406\end{array}$ \\
\hline 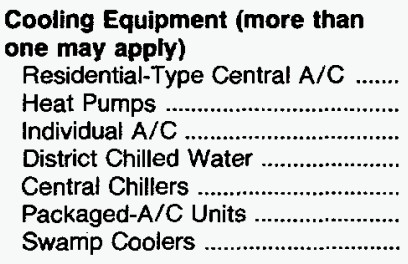 & $\begin{array}{r}19,073 \\
11,388 \\
28,082 \\
28,194 \\
63,914 \\
56,664 \\
378\end{array}$ & $\begin{array}{r}14,532 \\
8,283 \\
16,973 \\
19,658 \\
42,987 \\
40,812 \\
358\end{array}$ & $\begin{array}{r}12,578 \\
6,734 \\
16,389 \\
17,670 \\
43,140 \\
42,232 \\
\text { NC }\end{array}$ & $\begin{array}{r}9,461 \\
7,113 \\
13,066 \\
12,419 \\
25,333 \\
26,167 \\
358\end{array}$ & $\begin{array}{r}15,674 \\
7,283 \\
16,626 \\
17,815 \\
37,532 \\
34,415 \\
358\end{array}$ & $\begin{array}{r}8,781 \\
10,179 \\
16,959 \\
9,149 \\
36,983 \\
28,362 \\
20\end{array}$ & $\begin{array}{r}17,818 \\
10,453 \\
25,361 \\
23,639 \\
57,948 \\
53,394 \\
378\end{array}$ \\
\hline 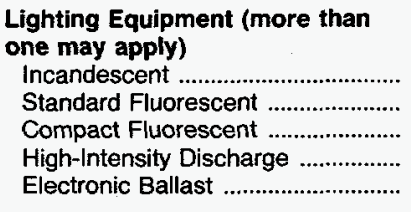 & $\begin{array}{l}64,981 \\
91,986 \\
49,455 \\
52,593 \\
65,560\end{array}$ & $\begin{array}{l}42,602 \\
60,750 \\
33,756 \\
35,944 \\
46,236\end{array}$ & $\begin{array}{l}42,505 \\
58,634 \\
37,128 \\
32,936 \\
48,576\end{array}$ & $\begin{array}{l}28,368 \\
37,421 \\
21,471 \\
22,966 \\
30,043\end{array}$ & $\begin{array}{l}39,568 \\
51,125 \\
30,245 \\
29,904 \\
40,191\end{array}$ & $\begin{array}{l}31,825 \\
45,556 \\
26,730 \\
22,390 \\
36,741\end{array}$ & $\begin{array}{l}55,041 \\
79,292 \\
45,161 \\
47,085 \\
61,700\end{array}$ \\
\hline \multicolumn{8}{|l|}{$\begin{array}{l}\text { Water-Heating Equipment (more } \\
\text { than one may apply) }\end{array}$} \\
\hline 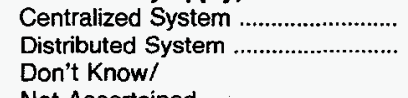 & $\begin{array}{l}63,477 \\
24,696\end{array}$ & $\begin{array}{l}41,930 \\
14,649\end{array}$ & $\begin{array}{l}41,517 \\
13,987\end{array}$ & $\begin{array}{r}27,532 \\
9,890\end{array}$ & $\begin{array}{l}39,892 \\
11,824\end{array}$ & $\begin{array}{l}33,365 \\
11,994\end{array}$ & $\begin{array}{l}56,221 \\
21,429\end{array}$ \\
\hline 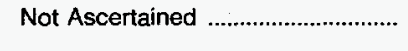 & 4,965 & 4,642 & 2,632 & $\mathrm{NC}$ & NC & 333 & 2,686 \\
\hline 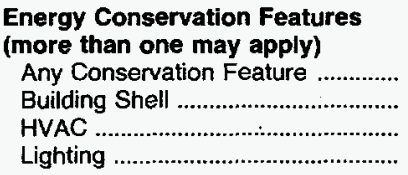 & $\begin{array}{l}94,361 \\
84,557 \\
93,543 \\
80,953\end{array}$ & $\begin{array}{l}61,891 \\
57,447 \\
61,891 \\
55,202\end{array}$ & $\begin{array}{l}59,024 \\
51,419 \\
59,024 \\
56,297\end{array}$ & $\begin{array}{l}37,421 \\
33,287 \\
37,421 \\
34,439\end{array}$ & $\begin{array}{l}52,665 \\
43,488 \\
52,665 \\
44,519\end{array}$ & $\begin{array}{l}46,595 \\
38,508 \\
45,983 \\
42,120\end{array}$ & $\begin{array}{l}81,027 \\
73,116 \\
80,865 \\
73,667\end{array}$ \\
\hline
\end{tabular}

See footnotes at end of table. 
Table 3.40. Energy Management Practices in FBSS Buildings in Federal Region 3, Floorspace, 1993 (Continued)

(Thousand Square Feet)

\begin{tabular}{|c|c|c|c|c|c|c|c|}
\hline $\begin{array}{c}\text { Bullding } \\
\text { Characteristics }\end{array}$ & $\begin{array}{c}\text { All } \\
\text { Bulldings }\end{array}$ & $\begin{array}{l}\text { Energy } \\
\text { Management } \\
\text { and Control } \\
\text { System }\end{array}$ & $\begin{array}{l}\text { Energy } \\
\text { Conservation } \\
\text { Programs } 1\end{array}$ & $\begin{array}{l}\text { Energy } \\
\text { Audit }\end{array}$ & $\begin{array}{l}\text { HVAC } \\
\text { Maintenance } \\
\text { Staff }^{2}\end{array}$ & $\begin{array}{l}\text { Off-Hour } \\
\text { Equipment } \\
\text { Reduction }\end{array}$ & $\begin{array}{l}\text { Retrofit or } \\
\text { Purchase } \\
\text { of Energy } \\
\text { Efficient } \\
\text { Equipment }\end{array}$ \\
\hline \multicolumn{8}{|l|}{$\begin{array}{l}\text { Buliding Shell Conservation } \\
\text { Features (more than one may } \\
\text { apply) } \\
\text { Roof or Ceiling }\end{array}$} \\
\hline Insulation & 69,654 & 49,683 & 42,066 & 26,983 & 33,849 & 29,888 & 59,342 \\
\hline $\begin{array}{l}\text { Wall Insulation ......................................... } \\
\text { Storm or Multiple }\end{array}$ & 31,876 & 26,169 & 16,376 & 9,623 & 12,884 & 14,838 & 28,026 \\
\hline Glazing ............................................. & 46,141 & 33,757 & 26,289 & 15,709 & 20,126 & $.11,659$ & 39,905 \\
\hline $\begin{array}{l}\text { or Shading Film ............................... } \\
\text { Exterior or Interior Shading }\end{array}$ & 46,829 & 36,581 & 24,607 & 15,439 & 25,852 & 22,644 & 39,533 \\
\hline 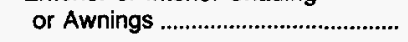 & 57,183 & 38,723 & 32,446 & 20,681 & 23,889 & 32,193 & 47,638 \\
\hline \multicolumn{8}{|l|}{$\begin{array}{l}\text { HVAC Conservation Features } \\
\text { (more than one may apply) }\end{array}$} \\
\hline 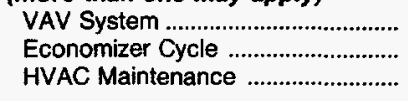 & $\begin{array}{l}37,611 \\
61,089 \\
93,402\end{array}$ & $\begin{array}{l}29,150 \\
44,488 \\
61,891\end{array}$ & $\begin{array}{l}22,577 \\
40,658 \\
59,024\end{array}$ & $\begin{array}{l}14,320 \\
25,654 \\
37,421\end{array}$ & $\begin{array}{l}19,649 \\
35,957 \\
52,665\end{array}$ & $\begin{array}{l}13,711 \\
29,237 \\
45,896\end{array}$ & $\begin{array}{l}33,689 \\
57,386 \\
80,865\end{array}$ \\
\hline \multicolumn{8}{|l|}{$\begin{array}{l}\text { Lighting Conservation Features } \\
\text { (more than one may apply) }\end{array}$} \\
\hline $\begin{array}{l}\text { Specular Reflectors ......................... } \\
\text { Natural Lighting Control }\end{array}$ & 44,741 & 32,503 & 29,046 & 23,785 & 27,006 & 22,524 & 39,714 \\
\hline 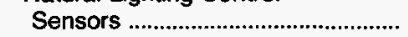 & 17,743 & 12,598 & 12,151 & 5,843 & 10,858 & 9,442 & 16,630 \\
\hline 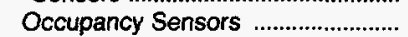 & 62,261 & 42,365 & 47,987 & 29,458 & 37,976 & 36,119 & 57,610 \\
\hline Time Clock & 46,555 & 34,709 & 32,755 & 20,406 & 27,052 & 25,802 & 42,629 \\
\hline 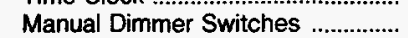 & 49,824 & 37,475 & 38,239 & 23,774 & 29,434 & 26,104 & 48,332 \\
\hline
\end{tabular}

1 Building participates in any programs sponsored by the Federal Energy Management Program, in-house, utility, or third party.

2 HVAC maintenance staff means at least one person spends at least half their working hours maintaining the heating/cooling equipment.

NC $=$ No cases in responding sample.

Notes: - Total workers are the number of workers during the main shift. • See Glossary for explanation of abbreviations and definitions of terms used in this report. - These data are from 881 federally owned buildings having the following criteria: (1) located in Federal Regions 3 , 6, or 9; (2) larger than 10,000 square feet; and (3) used for a commercial purpose, other than warehouse and storage. In addition, 9 out of 10 selected buildings were from agencies other than the Department of Defense. - Statistics for the "energy end uses" represent consumption in buildings that have end use, not consumption for a particular fuel for a particular end use. - A/C = Air Conditioning. - FBSS = Federal Buildings Supplemental Survey. - HVAC = Heating, Ventilation, and Air Conditioning. • VAV = Variable-Air Volume. $\bullet$ Data are for Fiscal Year 1993 (October 1, 1992 through September 30,1993$)$. Because of rounding, data may not sum to totals.

Source: Energy Information Administration, Office of Energy Markets and End Use, 1993 Federal Buildings Supplemental Survey. 
Table 3.41. Energy Management Practices in FBSS Buildings in Federal Region 6, Floorspace, 1993

(Thousand Square Feet)

\begin{tabular}{|c|c|c|c|c|c|c|c|}
\hline $\begin{array}{c}\text { Building } \\
\text { Characteristics }\end{array}$ & $\begin{array}{c}\text { All } \\
\text { Bulldings }\end{array}$ & $\begin{array}{l}\text { Energy } \\
\text { Management } \\
\text { and Control } \\
\text { System }\end{array}$ & $\begin{array}{l}\text { Energy } \\
\text { Conservation } \\
\text { Programs }\end{array}$ & $\begin{array}{c}\text { Energy } \\
\text { Audit }\end{array}$ & $\begin{array}{l}\text { HVAC } \\
\text { Maintenance } \\
\text { Staff }^{2}\end{array}$ & $\begin{array}{l}\text { Off-Hour } \\
\text { Equipment } \\
\text { Reduction }\end{array}$ & $\begin{array}{l}\text { Retrofit or } \\
\text { Purchase } \\
\text { of Energy } \\
\text { Efficient } \\
\text { Equipment }\end{array}$ \\
\hline 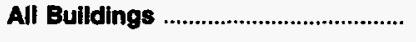 & 35,816 & 23,099 & 13,513 & 11,690 & 15,538 & 14,304 & 29,015 \\
\hline 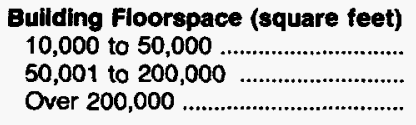 & $\begin{array}{r}2,591 \\
9,548 \\
23,677\end{array}$ & $\begin{array}{r}638 \\
4,762 \\
17,698\end{array}$ & $\begin{array}{r}430 \\
1,094 \\
11,989\end{array}$ & $\begin{array}{r}626 \\
1,965 \\
9,099\end{array}$ & $\begin{array}{r}264 \\
2,412 \\
12,862\end{array}$ & $\begin{array}{l}1,783 \\
4,851 \\
7,670\end{array}$ & $\begin{array}{r}1,582 \\
6,105 \\
21,328\end{array}$ \\
\hline 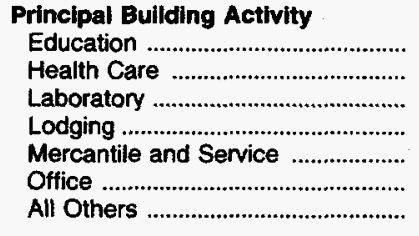 & $\begin{array}{r}168 \\
12,094 \\
3,331 \\
942 \\
6,236 \\
10,799 \\
2,247\end{array}$ & $\begin{array}{r}17 \\
11,662 \\
1,633 \\
369 \\
3,528 \\
5,607 \\
283\end{array}$ & $\begin{array}{r}\mathrm{NC} \\
6,935 \\
99 \\
270 \\
2,455 \\
3,720 \\
33\end{array}$ & $\begin{array}{r}\text { NC } \\
2,754 \\
244 \\
535 \\
2,559 \\
5,237 \\
361\end{array}$ & $\begin{array}{r}55 \\
6,770 \\
72 \\
128 \\
1,625 \\
6,615 \\
274\end{array}$ & $\begin{array}{r}168 \\
1,435 \\
979 \\
N C \\
1,199 \\
9,274 \\
1,250\end{array}$ & $\begin{array}{r}63 \\
11,812 \\
1,264 \\
597 \\
5,440 \\
8,868 \\
972\end{array}$ \\
\hline 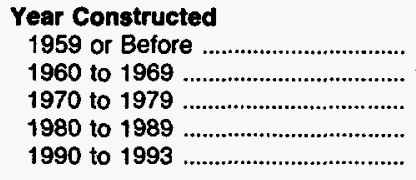 & $\begin{array}{r}13,258 \\
6,386 \\
6,175 \\
6,903 \\
3,095\end{array}$ & $\begin{array}{l}8,070 \\
2,422 \\
4,655 \\
5,082 \\
2,870\end{array}$ & $\begin{array}{l}3,456 \\
2,588 \\
3,147 \\
1,571 \\
2,750\end{array}$ & $\begin{array}{r}3,609 \\
3,428 \\
2,771 \\
1,692 \\
190\end{array}$ & $\begin{array}{l}5,896 \\
2,562 \\
1,921 \\
2,447 \\
2,711\end{array}$ & $\begin{array}{r}5,517 \\
3,887 \\
1,747 \\
2,852 \\
300\end{array}$ & $\begin{array}{r}10,053 \\
5,759 \\
5,459 \\
4,941 \\
2,803\end{array}$ \\
\hline $\begin{array}{l}\text { Federal Agency } \\
\text { Department of Defense ................. } \\
\text { General Services Administration ... } \\
\text { United States Postal Service ........ } \\
\text { Veterans Administration .................. } \\
\text { All Others ......................................... }\end{array}$ & $\begin{array}{r}1,668 \\
7,888 \\
7,027 \\
11,375 \\
7,858\end{array}$ & $\begin{array}{r}848 \\
5,034 \\
3,692 \\
11,178 \\
2,347\end{array}$ & $\begin{array}{r}779 \\
3,391 \\
2,686 \\
6,403 \\
255\end{array}$ & $\begin{array}{r}1,129 \\
4,819 \\
2,791 \\
2,346 \\
605\end{array}$ & $\begin{array}{r}1,133 \\
5,570 \\
2,000 \\
5,912 \\
923\end{array}$ & $\begin{array}{r}793 \\
7,258 \\
1,808 \\
1,505 \\
2,940\end{array}$ & $\begin{array}{r}1,277 \\
7,317 \\
5,854 \\
11,127 \\
3,441\end{array}$ \\
\hline 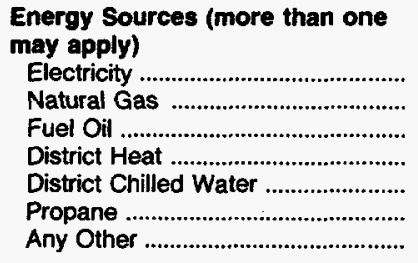 & $\begin{array}{r}35,816 \\
24,827 \\
4,740 \\
13,437 \\
7,980 \\
200 \\
2,850\end{array}$ & $\begin{array}{r}23,099 \\
17,179 \\
3,293 \\
9,499 \\
6,984 \\
135 \\
2,458\end{array}$ & $\begin{array}{r}13,513 \\
11,257 \\
2,833 \\
5,031 \\
5,258 \\
24 \\
1,436\end{array}$ & $\begin{array}{c}11,690 \\
10,680 \\
2,753 \\
1,376 \\
1,818 \\
\text { NC } \\
\text { NC }\end{array}$ & $\begin{array}{r}15,538 \\
11,936 \\
2,039 \\
5,836 \\
4,647 \\
24 \\
1,114\end{array}$ & $\begin{array}{r}14,304 \\
8,809 \\
2,214 \\
5,034 \\
2,669 \\
65 \\
1,022\end{array}$ & $\begin{array}{r}29,015 \\
21,277 \\
4,581 \\
9,972 \\
7,577 \\
15 \\
2,850\end{array}$ \\
\hline 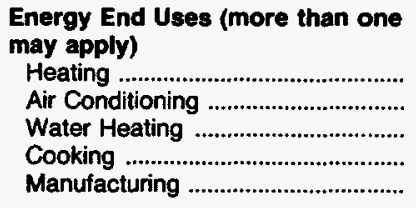 & $\begin{array}{r}35,734 \\
35,273 \\
35,221 \\
19,213 \\
5,335\end{array}$ & $\begin{array}{r}23,099 \\
22,638 \\
22,638 \\
15,694 \\
3,545\end{array}$ & $\begin{array}{r}13,513 \\
13,052 \\
13,052 \\
10,071 \\
2,597\end{array}$ & $\begin{array}{r}11,690 \\
11,690 \\
11,657 \\
8,148 \\
2,957\end{array}$ & $\begin{array}{r}15,538 \\
15,538 \\
15,518 \\
10,470 \\
2,378\end{array}$ & $\begin{array}{r}14,304 \\
14,304 \\
14,251 \\
5,934 \\
2,549\end{array}$ & $\begin{array}{r}29,015 \\
28,555 \\
28,535 \\
18,533 \\
5,140\end{array}$ \\
\hline $\begin{array}{l}\text { Workers (main shift) } \\
\text { Less than } 50 \\
50 \text { to } 99 \\
100 \text { to } 499 \\
500 \text { or More }\end{array}$ & $\begin{array}{r}2,548 \\
2,026 \\
9,029 \\
22,213\end{array}$ & $\begin{array}{r}415 \\
944 \\
4,647 \\
17,093\end{array}$ & $\begin{array}{r}204 \\
231 \\
730 \\
12,348\end{array}$ & $\begin{array}{r}498 \\
577 \\
1,275 \\
9,341\end{array}$ & $\begin{array}{r}247 \\
566 \\
1,774 \\
12,951\end{array}$ & $\begin{array}{l}1,291 \\
1,044 \\
4,652 \\
7,316\end{array}$ & $\begin{array}{r}1,510 \\
1,241 \\
5,211 \\
21,052\end{array}$ \\
\hline 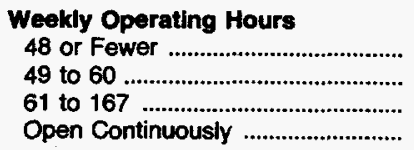 & $\begin{array}{r}2,740 \\
6,460 \\
8,051 \\
18,565\end{array}$ & $\begin{array}{r}1,111 \\
5,027 \\
1,898 \\
15,063\end{array}$ & $\begin{array}{r}256 \\
1,764 \\
1,512 \\
9,982\end{array}$ & $\begin{array}{r}860 \\
2,891 \\
1,557 \\
6,381\end{array}$ & $\begin{array}{l}1,087 \\
4,399 \\
2,148 \\
7,904\end{array}$ & $\begin{array}{c}2,600 \\
6,460 \\
5,244 \\
\text { NC }\end{array}$ & $\begin{array}{r}2,018 \\
5,375 \\
4,435 \\
17,189\end{array}$ \\
\hline
\end{tabular}

See footnotes at end of table. 
Table 3.41. Energy Management Practices in FBSS Buildings in Federal Region 6, Floorspace, 1993 (Continued)

(Thousand Square Feet)

\begin{tabular}{|c|c|c|c|c|c|c|c|}
\hline $\begin{array}{l}\text { Building } \\
\text { Characteristics }\end{array}$ & $\begin{array}{c}\text { All } \\
\text { Buildings }\end{array}$ & $\begin{array}{c}\text { Energy } \\
\text { Management } \\
\text { and Control } \\
\text { System }\end{array}$ & $\begin{array}{c}\text { Energy } \\
\text { Conservation } \\
\text { Programs }^{1}\end{array}$ & $\begin{array}{l}\text { Energy } \\
\text { Audit }\end{array}$ & $\begin{array}{c}\text { HVAC } \\
\text { Maintenance } \\
\text { Staff }^{2}\end{array}$ & $\begin{array}{l}\text { Otf-Hour } \\
\text { Equipment } \\
\text { Reduction }\end{array}$ & $\begin{array}{l}\text { Relrofit or } \\
\text { Purchase } \\
\text { of Energy } \\
\text { Efficient } \\
\text { Equipment }\end{array}$ \\
\hline \multicolumn{8}{|l|}{ Multibuliding Facility } \\
\hline 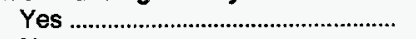 & 24,441 & 16,370 & 9,126 & 6,726 & 9,701 & 9,504 & 19,477 \\
\hline 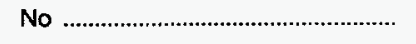 & 11,375 & 6,729 & 4,387 & 4,963 & 5,837 & 4,800 & 9,539 \\
\hline \multicolumn{8}{|l|}{ Percent of Floorspace Heated } \\
\hline Not Heated ........................................... & 82 & NC & NC & NC & NC & NC & NC \\
\hline 1 to 50 & 1,288 & 671 & 363 & 594 & 291 & 914 & 998 \\
\hline 51 to 100 & 34,446 & 22,428 & 13,150 & 11,095 & 15,247 & 13,390 & 28,017 \\
\hline \multicolumn{8}{|l|}{ Percent of Floorspace Cooled } \\
\hline 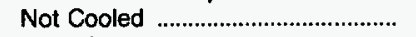 & 554 & 472 & 461 & NC & 11 & NC & 472 \\
\hline 1 to 50 & 1,951 & 524 & 74 & 568 & 272 & 1,385 & 1,192 \\
\hline 51 to 100 & 33,312 & 22,103 & 12,978 & 11,122 & 15,254 & 12,919 & 27,351 \\
\hline \multicolumn{8}{|l|}{ Percent Lit When Open } \\
\hline 1 to 50 & 1,062 & 326 & 183 & 102 & 272 & 742 & 687 \\
\hline 51 to 100 & 34,672 & 22,773 & 13,330 & 11,587 & 15,265 & 13,562 & 28,328 \\
\hline No Operating Hours ........................ & & NC & NC & NC & NC & NC & NC \\
\hline 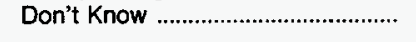 & NC & $\mathrm{NC}$ & NC & NC & NC & NC & NC \\
\hline \multicolumn{8}{|l|}{$\begin{array}{l}\text { Heating Equipment (more than } \\
\text { one may apply) }\end{array}$} \\
\hline 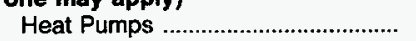 & 964 & 199 & 100 & 618 & 12 & 313 & 877 \\
\hline Furnaces & 791 & 87 & 45 & 69 & 202 & 633 & 584 \\
\hline Individual Space Heaters ................. & 5,408 & 3,592 & 2,576 & 1,610 & 1,881 & 2,898 & 4,846 \\
\hline District Heat & 15,205 & 11,254 & 5,546 & 2,221 & 5,867 & 5,657 & 11,614 \\
\hline 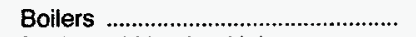 & 16,718 & 10,580 & 7,178 & 7,806 & 8,854 & 7,088 & 14,190 \\
\hline Packaged-Heating Units .................... & 7,574 & 4,982 & 4,016 & 3,421 & 4,743 & 2,044 & 6,566 \\
\hline \multicolumn{8}{|l|}{$\begin{array}{l}\text { Cooling Equipment (more than } \\
\text { one may apply) }\end{array}$} \\
\hline Residential-Type Central A/C ....... & 4,706 & 2,379 & 2,207 & 1,630 & 673 & 2,260 & 4,432 \\
\hline 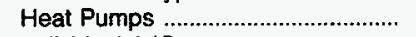 & 1,549 & 829 & 88 & 618 & 162 & 462 & 1,372 \\
\hline Individual A/C & 6,950 & 4,000 & 1,009 & 1,946 & 2,121 & 1,998 & 5,338 \\
\hline District Chilled Water ....................... & 10,856 & 9,349 & 5,258 & 2,347 & 5,840 & 4,328 & 9,701 \\
\hline 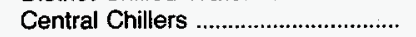 & 21,596 & 13,285 & 7,116 & 8,219 & 9,365 & 8,337 & 17,893 \\
\hline 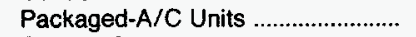 & 14,104 & 9,541 & 6,929 & 6,103 & 6,418 & 5,049 & 12,808 \\
\hline 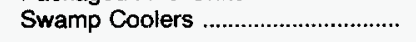 & 2,015 & 1,559 & 148 & 148 & 1,114 & 1,194 & 1,725 \\
\hline \multicolumn{8}{|l|}{$\begin{array}{l}\text { Lighting Equipment (more than } \\
\text { one may apply) }\end{array}$} \\
\hline 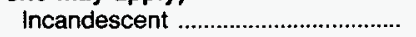 & 20,248 & 10,360 & 6,777 & 5,719 & 7,664 & 8,096 & 14,588 \\
\hline Standard Fluorescent ..................... & 35,057 & 22,511 & 13,480 & 11,251 & 15,376 & 14,073 & 28,529 \\
\hline Compact Fluorescent ....................... & 7,942 & 6,385 & 3,388 & 4,430 & 4,995 & 4,926 & 7,516 \\
\hline High-Intensity Discharge ................. & 16,188 & 11,491 & 6,805 & 6,194 & 6,374 & 4,651 & 13,662 \\
\hline Electronic Ballast ............................... & 15,861 & 12,411 & 9,798 & 5,856 & 9,417 & 5,353 & 15,050 \\
\hline \multicolumn{8}{|l|}{$\begin{array}{l}\text { Water-Heating Equipment (more } \\
\text { than one may apply) }\end{array}$} \\
\hline 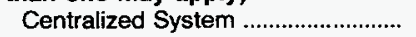 & 23,206 & 15,886 & 8,690 & 6,361 & 9,660 & 9,882 & 18,644 \\
\hline 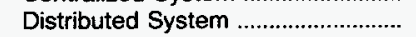 & 11,827 & 6,606 & 4,337 & 5,271 & 5,846 & 4,344 & 9,855 \\
\hline Don't Know/ & & & & & & & \\
\hline 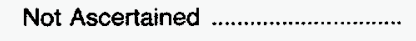 & 188 & 146 & 25 & 25 & 11 & 25 & 36 \\
\hline \multicolumn{8}{|l|}{$\begin{array}{l}\text { Energy Conservation Features } \\
\text { (more than one may apply) }\end{array}$} \\
\hline Any Conservation Feature ............... & 35,734 & 23,099 & 13,513 & 11,690 & 15,538 & 14,304 & 29,015 \\
\hline 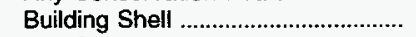 & 33,946 & 22,678 & 13,067 & 11,231 & 15,092 & 13,501 & 27,876 \\
\hline HVAC & 35,425 & 23,099 & 13,488 & 11,643 & 15,396 & 13,995 & 28,826 \\
\hline 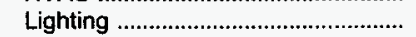 & 25,654 & 17,983 & 12,677 & 8,781 & 12,576 & 10,753 & 24,105 \\
\hline
\end{tabular}

See footnotes at end of table. 


\section{Table 3.41. Energy Management Practices in FBSS Buildings in Federal Region 6, Floorspace, 1993 (Continued) (Thousand Square Feet)}

\begin{tabular}{|c|c|c|c|c|c|c|c|}
\hline $\begin{array}{c}\text { Building } \\
\text { Characteristics }\end{array}$ & $\begin{array}{c}\text { All } \\
\text { Buildings }\end{array}$ & $\begin{array}{l}\text { Energy } \\
\text { Management } \\
\text { and Control } \\
\text { System }\end{array}$ & $\begin{array}{l}\text { Energy } \\
\text { Conservation } \\
\text { Programs }\end{array}$ & $\begin{array}{l}\text { Energy } \\
\text { Audit }\end{array}$ & $\begin{array}{l}\text { HVAC } \\
\text { Maintenance } \\
\text { Staff }^{2}\end{array}$ & $\begin{array}{l}\text { Off-Hour } \\
\text { Equipment } \\
\text { Reduction }\end{array}$ & $\begin{array}{l}\text { Retroflt or } \\
\text { Purchase } \\
\text { of Energy } \\
\text { Efficient } \\
\text { Equipment }\end{array}$ \\
\hline \multicolumn{8}{|l|}{$\begin{array}{l}\text { Building Shefl Conservation } \\
\text { Features (more than one may } \\
\text { apply) } \\
\text { Roof or Ceiling }\end{array}$} \\
\hline Insulation & 28,249 & 19,380 & 11,688 & 8,794 & 11,081 & 10,088 & 23,169 \\
\hline $\begin{array}{l}\text { Wall Insulation } \\
\text { Storm or Multiple }\end{array}$ & 20,550 & 14,492 & 9,928 & 6,991 & 8,650 & 5,384 & 16,527 \\
\hline $\begin{array}{l}\text { Glazing } \\
\text { Tinted or Reflective Glass }\end{array}$ & 17,939 & 14,959 & 7,870 & 5,711 & 9,429 & 5,920 & 15,562 \\
\hline $\begin{array}{l}\text { or Shading Film ........................... } \\
\text { Exterior or Interior Shading }\end{array}$ & 24,768 & 17,244 & 11,836 & 8,557 & 12,800 & 10,141 & 21,955 \\
\hline 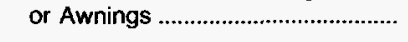 & 26,067 & 19,444 & 11,662 & 9,676 & 14,311 & 11,197 & 23,959 \\
\hline \multicolumn{8}{|l|}{$\begin{array}{l}\text { HVAC Conservation Features } \\
\text { (more than one may apply) }\end{array}$} \\
\hline VAV System & 18,518 & 14,894 & 8,839 & 6,867 & 11,160 & 6,306 & 15,889 \\
\hline 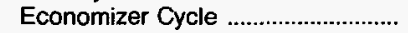 & 23,542 & 17,962 & 11,116 & 7,928 & 10,448 & 6,681 & 20,313 \\
\hline HVAC Maintenance ............................ & 35,341 & 23,077 & 13,425 & 11,580 & 15,396 & 13,932 & 28,741 \\
\hline \multicolumn{8}{|l|}{$\begin{array}{l}\text { Lighting Conservation Features } \\
\text { (more than one may apply) }\end{array}$} \\
\hline $\begin{array}{l}\text { Specular Reflectors .............................. } \\
\text { Natural Lighting Control }\end{array}$ & 18,017 & 12,007 & 10,434 & 6,629 & 9,331 & 7,294 & 17,117 \\
\hline 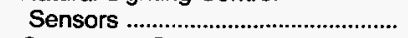 & 6,740 & 5,062 & 4,790 & 2,044 & 5,036 & 1,564 & 6,473 \\
\hline 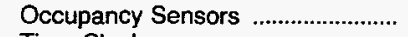 & 16,435 & 11,571 & 9,690 & 7,220 & 9,031 & 6,824 & 15,479 \\
\hline 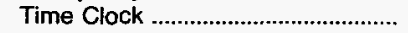 & 9,037 & 6,776 & 6,265 & 3,459 & 6,078 & 3,094 & 8,529 \\
\hline Manual Dimmer Switches ................ & 13,454 & 10,952 & 8,056 & 4,184 & 7,723 & 5,764 & 12,865 \\
\hline
\end{tabular}

1 Building participates in any programs sponsored by the Federal Energy Management Program, in-house, utility, or third party.

2 HVAC maintenance staff means at least one person spends at least half their working hours maintaining the heating/cooling equipment.

NC $=$ No cases in responding sample.

Notes: - Total workers are the number of workers during the main shift. - See Glossary for explanation of abbreviations and definitions of terms used in this report. - These data are from 881 federally owned buildings having the following criteria: (1) located in Federal Regions 3 , 6 , or 9 ; (2) larger than 10,000 square feet; and (3) used for a commercial purpose, other than warehouse and storage. In addition, 9 out of 10 selected buildings were from agencies other than the Department of Defense. - Statistics for the "energy end uses" represent consumption in buildings that have end use, not consumption for a particular fuel for a particular end use. - A/C = Air Conditioning. • FBSS = Federal Buildings Supplemental Survey.

- HVAC = Heating, Ventilation, and Air Conditioning. - VAV = Variable-Air Volume. - Data are for Fiscal Year 1993 (October 1 , 1992 through September 30,1993$)$. Because of rounding, data may not sum to totals.

Source: Energy Information Administration, Office of Energy Markets and End Use, 1993 Federal Buildings Supplemental Survey. 
Table 3.42. Energy Management Practices in FBSS Buildings in Federal Region 9, Floorspace, 1993

(Thousand Square Feet)

\begin{tabular}{|c|c|c|c|c|c|c|c|}
\hline $\begin{array}{c}\text { Building } \\
\text { Characteristics }\end{array}$ & $\begin{array}{c}\text { All } \\
\text { Buildings }\end{array}$ & $\begin{array}{l}\text { Energy } \\
\text { Management } \\
\text { and Control } \\
\text { System }\end{array}$ & $\begin{array}{l}\text { Energy } \\
\text { Conservation } \\
\text { Programs }\end{array}$ & $\begin{array}{l}\text { Energy } \\
\text { Audit }\end{array}$ & $\begin{array}{l}\text { HVAC } \\
\text { Maintenance } \\
\text { Staff }^{2}\end{array}$ & $\begin{array}{l}\text { Off-Hour } \\
\text { Equipment } \\
\text { Reduction }\end{array}$ & $\begin{array}{l}\text { Retrofit or } \\
\text { Purchase } \\
\text { of Einergy } \\
\text { Efficient } \\
\text { Equipment }\end{array}$ \\
\hline 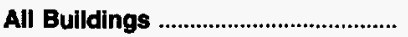 & 44,316 & 18,231 & 24,699 & 17,444 & 14,570 & 21,974 & 34,547 \\
\hline 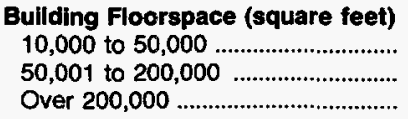 & $\begin{array}{r}3,858 \\
12,680 \\
27,778\end{array}$ & $\begin{array}{r}436 \\
4,094 \\
13,701\end{array}$ & $\begin{array}{r}1,386 \\
5,859 \\
17,454\end{array}$ & $\begin{array}{r}1,069 \\
4,233 \\
12,142\end{array}$ & $\begin{array}{r}300 \\
2,738 \\
11,533\end{array}$ & $\begin{array}{r}2,485 \\
6,395 \\
13,095\end{array}$ & $\begin{array}{r}2,297 \\
9,384 \\
22,867\end{array}$ \\
\hline 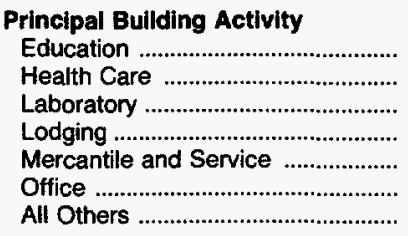 & $\begin{array}{r}628 \\
9,903 \\
2,601 \\
1,220 \\
8,194 \\
16,380 \\
5,390\end{array}$ & $\begin{array}{r}\text { NC } \\
5,388 \\
961 \\
308 \\
5,503 \\
3,868 \\
2,203\end{array}$ & $\begin{array}{r}38 \\
5,402 \\
733 \\
421 \\
3,193 \\
11,694 \\
3,219\end{array}$ & $\begin{array}{r}157 \\
3,398 \\
438 \\
616 \\
4,125 \\
6,572 \\
2,138\end{array}$ & $\begin{array}{r}21 \\
4,851 \\
198 \\
59 \\
2,155 \\
6,660 \\
627\end{array}$ & $\begin{array}{r}388 \\
1,121 \\
862 \\
29 \\
2,210 \\
14,700 \\
2,664\end{array}$ & $\begin{array}{r}200 \\
7,886 \\
2,371 \\
608 \\
6,960 \\
14,196 \\
2,327\end{array}$ \\
\hline 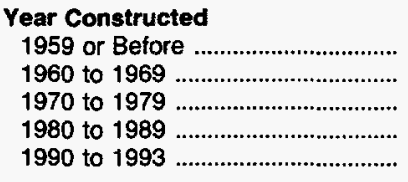 & $\begin{array}{r}13,553 \\
9,082 \\
12,041 \\
7,955 \\
1,684\end{array}$ & $\begin{array}{r}3,502 \\
2,592 \\
5,705 \\
5,825 \\
607\end{array}$ & $\begin{array}{r}5,791 \\
6,668 \\
7,827 \\
3,750 \\
664\end{array}$ & $\begin{array}{r}3,575 \\
3,934 \\
6,295 \\
2,818 \\
821\end{array}$ & $\begin{array}{r}3,030 \\
2,470 \\
4,538 \\
3,570 \\
964\end{array}$ & $\begin{array}{r}7,954 \\
6,759 \\
5,016 \\
1,581 \\
664\end{array}$ & $\begin{array}{l}9,006 \\
7,807 \\
9,904 \\
6,772 \\
1,058\end{array}$ \\
\hline 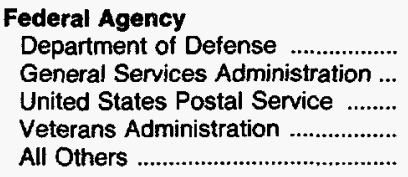 & $\begin{array}{r}8,489 \\
12,505 \\
8,387 \\
8,824 \\
6,111\end{array}$ & $\begin{array}{l}4,442 \\
2,654 \\
5,667 \\
4,000 \\
1,469\end{array}$ & $\begin{array}{r}4,072 \\
10,566 \\
3,291 \\
3,668 \\
3,104\end{array}$ & $\begin{array}{l}2,738 \\
5,688 \\
4,169 \\
3,412 \\
1,437\end{array}$ & $\begin{array}{l}1,850 \\
6,245 \\
2,221 \\
3,179 \\
1,076\end{array}$ & $\begin{array}{r}3,720 \\
11,989 \\
2,356 \\
1,410 \\
2,500\end{array}$ & $\begin{array}{r}4,555 \\
11,490 \\
7,332 \\
6,664 \\
4,506\end{array}$ \\
\hline 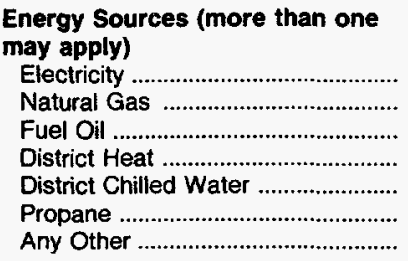 & $\begin{array}{r}44,296 \\
34,703 \\
11,127 \\
11,757 \\
6,362 \\
1,117 \\
2,606\end{array}$ & $\begin{array}{r}18,231 \\
14,480 \\
3,891 \\
5,426 \\
3,286 \\
752 \\
2,134\end{array}$ & $\begin{array}{r}24,680 \\
21,884 \\
6,989 \\
5,771 \\
3,259 \\
878 \\
1,234\end{array}$ & $\begin{array}{r}17,424 \\
13,331 \\
3,202 \\
4,039 \\
1,737 \\
841 \\
2,192\end{array}$ & $\begin{array}{r}14,570 \\
12,586 \\
4,972 \\
4,204 \\
4,042 \\
752 \\
\text { NC }\end{array}$ & $\begin{array}{r}21,974 \\
19,537 \\
3,376 \\
3,160 \\
1,073 \\
86 \\
1,245\end{array}$ & $\begin{array}{r}34,527 \\
29,791 \\
10,692 \\
8,528 \\
5,462 \\
813 \\
1,648\end{array}$ \\
\hline 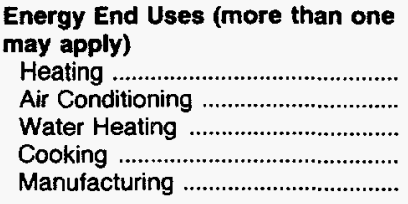 & $\begin{array}{r}41,921 \\
41,907 \\
42,984 \\
20,045 \\
7,693\end{array}$ & $\begin{array}{r}16,963 \\
18,231 \\
17,689 \\
11,783 \\
5,481\end{array}$ & $\begin{array}{r}24,668 \\
24,044 \\
24,395 \\
14,043 \\
4,723\end{array}$ & $\begin{array}{r}16,711 \\
17,169 \\
17,290 \\
10,482 \\
4,029\end{array}$ & $\begin{array}{r}13,679 \\
14,403 \\
14,029 \\
9,567 \\
2,286\end{array}$ & $\begin{array}{r}21,377 \\
21,037 \\
21,651 \\
7,179 \\
1,638\end{array}$ & $\begin{array}{r}32,765 \\
33,718 \\
33,938 \\
16,241 \\
5,395\end{array}$ \\
\hline $\begin{array}{l}\text { Workers (main shift) } \\
\text { Less than } 50 \\
50 \text { to } 99 \\
100 \text { to } 499 \\
500 \text { or More }\end{array}$ & $\begin{array}{r}3,684 \\
2,161 \\
14,017 \\
24,454\end{array}$ & $\begin{array}{r}371 \\
145 \\
4,852 \\
12,863\end{array}$ & $\begin{array}{r}816 \\
567 \\
6,555 \\
16,762\end{array}$ & $\begin{array}{r}1,371 \\
596 \\
3,828 \\
11,649\end{array}$ & $\begin{array}{r}308 \\
61 \\
2,400 \\
11,801\end{array}$ & $\begin{array}{r}2,001 \\
1,534 \\
7,606 \\
10,833\end{array}$ & $\begin{array}{r}1,871 \\
1,294 \\
10,947 \\
20,435\end{array}$ \\
\hline $\begin{array}{l}\text { Weekly Operating Hours } \\
48 \text { or Fewer } \\
49 \text { to } 60 \\
61 \text { to } 167 \\
\text { Open Continuously }\end{array}$ & $\begin{array}{r}4,822 \\
13,424 \\
5,706 \\
20,364\end{array}$ & $\begin{array}{r}949 \\
3,181 \\
1,804 \\
12,297\end{array}$ & $\begin{array}{r}1,537 \\
9,623 \\
3,153 \\
10,387\end{array}$ & $\begin{array}{l}1,504 \\
5,773 \\
1,914 \\
8,252\end{array}$ & $\begin{array}{r}369 \\
5,427 \\
1,311 \\
7,463\end{array}$ & $\begin{array}{r}4,176 \\
12,945 \\
4,853 \\
\text { NC }\end{array}$ & $\begin{array}{r}1,937 \\
11,669 \\
5,019 \\
15,922\end{array}$ \\
\hline
\end{tabular}

See footnotes at end of tabie. 
Table 3.42. Energy Management Practices in FBSS Buildings in Federal Region 9, Floorspace, 1993 (Continued) (Thousand Square Feet)

\begin{tabular}{|c|c|c|c|c|c|c|c|}
\hline $\begin{array}{c}\text { Building } \\
\text { Characteristics }\end{array}$ & $\begin{array}{c}\text { All } \\
\text { Buildings }\end{array}$ & $\begin{array}{l}\text { Energy } \\
\text { Management } \\
\text { and Control } \\
\text { System }\end{array}$ & $\begin{array}{l}\text { Energy } \\
\text { Conservation } \\
\text { Programs }\end{array}$ & $\begin{array}{l}\text { Energy } \\
\text { Audit }\end{array}$ & $\begin{array}{l}\text { HVAC } \\
\text { Maintenance } \\
\text { Staff }^{2}\end{array}$ & $\begin{array}{l}\text { Off-Hour } \\
\text { Equipment } \\
\text { Reduction }\end{array}$ & $\begin{array}{l}\text { Retrofit or } \\
\text { Purchase } \\
\text { of Energy } \\
\text { Efficient } \\
\text { Equipment }\end{array}$ \\
\hline Multibuilding Facility & & & & & & & \\
\hline 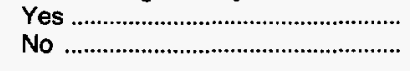 & $\begin{array}{l}26,821 \\
17,495\end{array}$ & $\begin{array}{r}13,401 \\
4,830\end{array}$ & $\begin{array}{l}13,884 \\
10,816\end{array}$ & $\begin{array}{r}11,814 \\
5,630\end{array}$ & $\begin{array}{l}8,369 \\
6,201\end{array}$ & $\begin{array}{r}9,385 \\
12,589\end{array}$ & $\begin{array}{l}19,618 \\
14,930\end{array}$ \\
\hline $\begin{array}{l}\text { Percent of Floorspace Heated } \\
\text { Not Heated } \\
1 \text { to } 50 \\
51 \text { to } 100\end{array}$ & $\begin{array}{r}2,394 \\
3,751 \\
38,170\end{array}$ & $\begin{array}{r}1,268 \\
2,120 \\
14,843\end{array}$ & $\begin{array}{r}32 \\
1,913 \\
22,755\end{array}$ & $\begin{array}{r}733 \\
2,011 \\
14,700\end{array}$ & $\begin{array}{r}891 \\
253 \\
13,426\end{array}$ & $\begin{array}{r}597 \\
1,923 \\
19,453\end{array}$ & $\begin{array}{r}1,782 \\
1,899 \\
30,866\end{array}$ \\
\hline 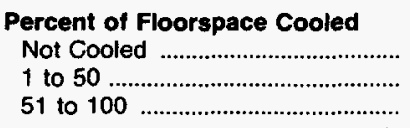 & $\begin{array}{r}2,956 \\
7,304 \\
34,056\end{array}$ & $\begin{array}{r}193 \\
3,000 \\
15,038\end{array}$ & $\begin{array}{r}942 \\
2,539 \\
21,218\end{array}$ & $\begin{array}{r}455 \\
1,949 \\
15,039\end{array}$ & $\begin{array}{r}371 \\
995 \\
13,204\end{array}$ & $\begin{array}{r}1,293 \\
2,868 \\
17,814\end{array}$ & $\begin{array}{r}1,308 \\
5,383 \\
27,857\end{array}$ \\
\hline 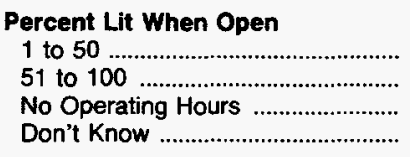 & $\begin{array}{r}1,847 \\
42,375 \\
29 \\
64\end{array}$ & $\begin{array}{l}260 \\
17,971 \\
\text { NC } \\
\text { NC }\end{array}$ & $\begin{array}{l}604 \\
24,096 \\
\text { NC } \\
\text { NC }\end{array}$ & $\begin{array}{l}848 \\
16,596 \\
\text { NC } \\
\text { NC }\end{array}$ & $\begin{array}{l}283 \\
14,288 \\
\text { NC } \\
\text { NC }\end{array}$ & $\begin{array}{r}857 \\
21,088 \\
29 \\
N C\end{array}$ & $\begin{array}{l}952 \\
33,595 \\
\text { NC } \\
\text { NC }\end{array}$ \\
\hline 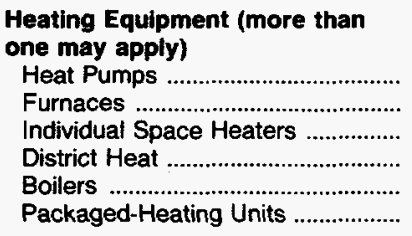 & $\begin{array}{r}3,081 \\
1,204 \\
4,933 \\
12,712 \\
24,010 \\
5,997\end{array}$ & $\begin{array}{r}1,477 \\
507 \\
1,634 \\
6,515 \\
9,045 \\
1,712\end{array}$ & $\begin{array}{r}2,456 \\
722 \\
3,373 \\
6,866 \\
15,566 \\
4,294\end{array}$ & $\begin{array}{r}894 \\
763 \\
2,609 \\
4,926 \\
11,129 \\
863\end{array}$ & $\begin{array}{r}412 \\
510 \\
1,923 \\
3,981 \\
8,573 \\
1,504\end{array}$ & $\begin{array}{r}1,414 \\
558 \\
3,201 \\
3,194 \\
15,082 \\
3,783\end{array}$ & $\begin{array}{r}2,941 \\
499 \\
4,180 \\
8,460 \\
20,352 \\
4,976\end{array}$ \\
\hline 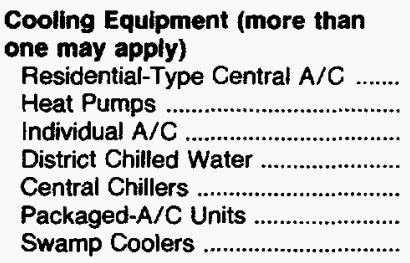 & $\begin{array}{r}1,900 \\
2,724 \\
5,723 \\
7,647 \\
27,723 \\
23,989 \\
3,165\end{array}$ & $\begin{array}{r}1,013 \\
1,433 \\
1,620 \\
5,146 \\
13,372 \\
8,856 \\
1,592\end{array}$ & $\begin{array}{r}1,118 \\
1,823 \\
2,404 \\
5,465 \\
16,944 \\
14,389 \\
1,636\end{array}$ & $\begin{array}{r}886 \\
863 \\
1,684 \\
3,138 \\
12,296 \\
10,548 \\
1,301\end{array}$ & $\begin{array}{r}677 \\
412 \\
726 \\
4,034 \\
9,296 \\
9,034 \\
1,142\end{array}$ & $\begin{array}{r}954 \\
1,439 \\
3,678 \\
1,058 \\
15,384 \\
13,817 \\
758\end{array}$ & $\begin{array}{r}807 \\
2,485 \\
4,953 \\
4,932 \\
23,266 \\
20,197 \\
2,285\end{array}$ \\
\hline 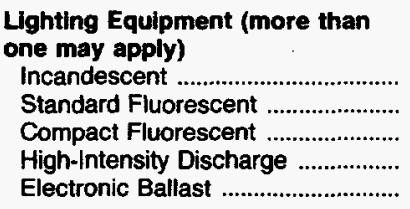 & $\begin{array}{l}20,475 \\
42,850 \\
17,339 \\
18,736 \\
23,307\end{array}$ & $\begin{array}{r}7,727 \\
17,903 \\
7,441 \\
10,875 \\
11,378\end{array}$ & $\begin{array}{l}11,924 \\
24,511 \\
13,891 \\
10,570 \\
16,181\end{array}$ & $\begin{array}{r}9,157 \\
16,789 \\
6,254 \\
9,828 \\
12,008\end{array}$ & $\begin{array}{r}8,130 \\
14,570 \\
8,282 \\
6,651 \\
8,859\end{array}$ & $\begin{array}{r}9,724 \\
21,289 \\
11,741 \\
7,978 \\
12,845\end{array}$ & $\begin{array}{l}17,342 \\
34,047 \\
16,985 \\
14,352 \\
20,703\end{array}$ \\
\hline $\begin{array}{l}\text { Water-Heating Equipment (more } \\
\text { than one may apply) }\end{array}$ & & & & & & & \\
\hline 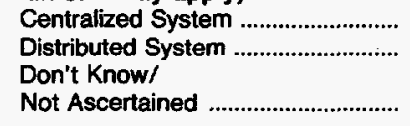 & $\begin{array}{r}31,069 \\
10,750 \\
1,165\end{array}$ & $\begin{array}{r}13,213 \\
3,696 \\
781\end{array}$ & $\begin{array}{r}18,305 \\
6,054 \\
36\end{array}$ & $\begin{array}{r}12,457 \\
4,312 \\
521\end{array}$ & $\begin{array}{r}11,891 \\
1,955 \\
183\end{array}$ & $\begin{array}{r}16,096 \\
4,928 \\
628\end{array}$ & $\begin{array}{r}25,491 \\
7,843 \\
604\end{array}$ \\
\hline $\begin{array}{l}\text { Energy Conservation Features } \\
\text { (more than one may apply) } \\
\text { Any Conservation Feature .............. } \\
\text { Building Shell } \\
\text { HVAC }\end{array}$ & $\begin{array}{l}44,023 \\
34,105 \\
43,116 \\
34,825\end{array}$ & $\begin{array}{l}18,231 \\
15,836 \\
17,971 \\
15,999\end{array}$ & $\begin{array}{l}24,624 \\
19,745 \\
24,535 \\
20,778\end{array}$ & $\begin{array}{l}17,432 \\
14,266 \\
16,780 \\
13,223\end{array}$ & $\begin{array}{l}14,570 \\
11,712 \\
14,532 \\
12,803\end{array}$ & $\begin{array}{l}21,853 \\
15,481 \\
21,502 \\
17,791\end{array}$ & $\begin{array}{l}34,547 \\
26,983 \\
34,455 \\
29,501\end{array}$ \\
\hline
\end{tabular}

See footnotes at end of table. 
Table 3.42. Energy Management Practices in FBSS Buildings in Federal Region 9, Floorspace, 1993 (Continued) (Thousand Square Feet)

\begin{tabular}{|c|c|c|c|c|c|c|c|}
\hline $\begin{array}{c}\text { Building } \\
\text { Characteristics }\end{array}$ & $\begin{array}{c}\text { All } \\
\text { Buildings }\end{array}$ & $\begin{array}{l}\text { Energy } \\
\text { Management } \\
\text { and Control } \\
\text { System }\end{array}$ & $\begin{array}{c}\text { Energy } \\
\text { Conservation } \\
\text { Programs }\end{array}$ & $\begin{array}{l}\text { Energy } \\
\text { Audit }\end{array}$ & $\begin{array}{l}\text { HVAC } \\
\text { Maintenance } \\
\text { Staff }^{2}\end{array}$ & $\begin{array}{l}\text { Off-Hour } \\
\text { Equipment } \\
\text { Reduction }\end{array}$ & $\begin{array}{l}\text { Retrofit or } \\
\text { Purchase } \\
\text { of Energy } \\
\text { Efficient } \\
\text { Equipment }\end{array}$ \\
\hline \multicolumn{8}{|l|}{$\begin{array}{l}\text { Bullding Shell Conservation } \\
\text { Features (more than one may } \\
\text { apply) } \\
\text { Roof or Ceiling }\end{array}$} \\
\hline Insulation & 27,234 & 15,092 & 16,156 & 12,807 & 9,157 & 9,929 & 21,217 \\
\hline $\begin{array}{l}\text { Wall Insulation } \\
\text { Storm or Multiple............................... }\end{array}$ & 17,462 & 10,333 & 10,951 & 9,275 & 7,263 & 5,872 & 13,563 \\
\hline $\begin{array}{l}\text { Glazing .............................................. } \\
\text { Tinted or Reflective Glass }\end{array}$ & 10,260 & 4,429 & 7,098 & 3,871 & 5,672 & 3,397 & 9,417 \\
\hline $\begin{array}{l}\text { or Shading Film ........................... } \\
\text { Exterior or Interior Shading }\end{array}$ & 22,381 & 10,096 & 14,637 & 9,488 & 8,991 & 9,823 & 19,110 \\
\hline 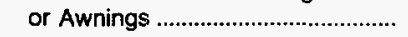 & 24,370 & 11,233 & 14,182 & 10,337 & 9,080 & 10,266 & 20,183 \\
\hline \multicolumn{8}{|l|}{$\begin{array}{l}\text { HVAC Conservation Features } \\
\text { (more than one may apply) }\end{array}$} \\
\hline 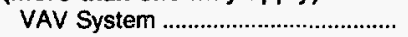 & 15,577 & 7,683 & 10,148 & 6,474 & 8,209 & 6,212 & 13,029 \\
\hline 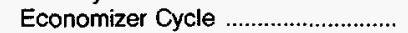 & 26,367 & 11,661 & 17,092 & 11,957 & 8,690 & 14,527 & 22,271 \\
\hline 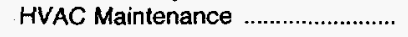 & 43,098 & 17,954 & 24,517 & 16,780 & 14,532 & 21,484 & 34,437 \\
\hline \multicolumn{8}{|l|}{$\begin{array}{l}\text { Lighting Conservation Features } \\
\text { (more than one may apply) }\end{array}$} \\
\hline $\begin{array}{l}\text { Specular Reflectors ............................ } \\
\text { Natural Lighting Control }\end{array}$ & 21,850 & 7,520 & 15,832 & 5,450 & 10,376 & 13,481 & 20,033 \\
\hline 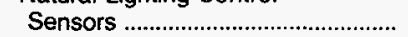 & 13,760 & 6,808 & 8,689 & 4,365 & 6,208 & 6,717 & 12,187 \\
\hline 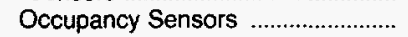 & 25,245 & 12,584 & 15,295 & 11,847 & 11,003 & 11,773 & 22,559 \\
\hline Time Clock & 10,539 & 5,499 & 8,426 & 5,292 & 5,304 & 5,810 & 9,778 \\
\hline Manual Dimmer Switches ............... & 14,455 & 7,269 & 9,461 & 5,939 & 7,711 & 5,206 & 12,377 \\
\hline
\end{tabular}

1 Building participates in any programs sponsored by the Federal Energy Management Program, in-house, utility, or third party.

2 HVAC maintenance staff means at least one person spends at least half their working hours maintaining the heating/cooling equipment. $\mathrm{NC}=$ No cases in responding sample.

Notes: - Total workers are the number of workers during the main shift. - See Glossary for explanation of abbreviations and definitions of terms used in this report. - These data are from 881 federally owned buildings having the following criteria: (1) located in Federal Regions 3,6 , or 9 ; (2) larger than 10,000 square feet; and (3) used for a commercial purpose, other than warehouse and storage. In addition, 9 out of 10 selected buildings were from agencies other than the Department of Defense. - Statistics for the "energy end uses" represent consumption in buildings that have end use, not consumption for a particular fuel for a particular end use. $\bullet$ A/C = Air Conditioning. $\bullet$ FBSS = Federal Buildings Supplemental Survey. - HVAC = Heating, Ventilation, and Air Conditioning. - VAV = Variable-Air Volume. - Data are for Fiscal Year 1993 (October 1 , 1992 through September 30,1993$)$. - Because of rounding, data may not sum to totals.

Source: Energy Information Administration, Office of Energy Markets and End Use, 1993 Federal Buildings Supplemental Survey. 
Table 3.43. Consumption and Expenditures for Sum of Major Fuels, Electricity, and Natural Gas in FBSS Buildings in Federal Region 3, 1993

\begin{tabular}{|c|c|c|c|c|c|c|c|c|c|}
\hline \multirow{2}{*}{$\begin{array}{c}\text { Building } \\
\text { Characteristics }\end{array}$} & \multirow{2}{*}{$\begin{array}{l}\text { Number of } \\
\text { Buildings }\end{array}$} & \multirow{2}{*}{$\begin{array}{c}\text { Floorspace } \\
\text { (thousand } \\
\text { square } \\
\text { feet) }\end{array}$} & \multirow{2}{*}{$\begin{array}{l}\text { Sum of } \\
\text { Major } \\
\text { Fuel } \\
\text { Consump- } \\
\text { tion } \\
\text { (billion } \\
\text { Btu) }\end{array}$} & \multirow{2}{*}{$\begin{array}{l}\text { Sum of } \\
\text { Major } \\
\text { Fuel } \\
\text { Expend- } \\
\text { itures } \\
\text { (million } \\
\text { dollars) }\end{array}$} & \multicolumn{2}{|c|}{$\begin{array}{l}\text { Electricity } \\
\text { Consumption } \\
\text { (billion Btu) }\end{array}$} & \multirow{2}{*}{$\begin{array}{l}\text { Electricity } \\
\text { Expend- } \\
\text { itures } \\
\text { (million } \\
\text { dollars) }\end{array}$} & \multirow{2}{*}{$\begin{array}{c}\text { Natural } \\
\text { Gas } \\
\text { Consump- } \\
\text { tion } \\
\text { (billion } \\
\text { Btu) }\end{array}$} & \multirow{2}{*}{$\begin{array}{l}\text { Natural } \\
\text { Gas } \\
\text { Expend- } \\
\text { itures } \\
\text { (million } \\
\text { dollars) }\end{array}$} \\
\hline & & & & & Primary & Site & & & \\
\hline All Buildings & 312 & 94,880 & 12,010 & 167 & 20,445 & 6,772 & 121 & 1,968 & 8 \\
\hline \multicolumn{10}{|l|}{$\begin{array}{l}\text { Building Floorspace (square } \\
\text { feet) }\end{array}$} \\
\hline 10,000 to 50,000 & 94 & 2,564 & 477 & 6 & 605 & 201 & 3 & 63 & $(*)$ \\
\hline 50,001 to 200,000 & 117 & 13,166 & 2,290 & 28 & 3,470 & 1,149 & 18 & 304 & 1 \\
\hline Over 200,000 & 101 & 79,149 & 9,243 & 133 & 16,370 & 5,422 & 99 & 1,602 & 7 \\
\hline \multicolumn{10}{|l|}{ Principal Building Activity } \\
\hline 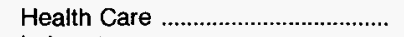 & 41 & 14,559 & 1,829 & 18 & 2,219 & 735 & 11 & 599 & 2 \\
\hline 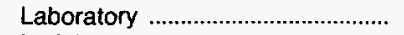 & 37 & 5,165 & 1,581 & 21 & 2,248 & 744 & 12 & 58 & $(*)$ \\
\hline 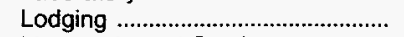 & 13 & 2,558 & 216 & 3 & 393 & 130 & 2 & 35 & $\left({ }^{*}\right)$ \\
\hline Mercantile and Service .................... & 46 & 7,966 & 1,166 & 12 & 1,339 & 444 & 8 & 635 & 3 \\
\hline Office & 124 & 56,881 & 6,074 & 97 & 12,044 & 3,989 & 75 & 598 & 2 \\
\hline 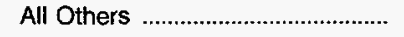 & 43 & 7,152 & 1,080 & 16 & 2,118 & 702 & 11 & 38 & $(")$ \\
\hline \multicolumn{10}{|l|}{ Year Constructed } \\
\hline 1959 or Before & 142 & 43,829 & 5,445 & 80 & 8,749 & 2,898 & 57 & 949 & 4 \\
\hline 1960 to 1969 & 70 & 19,564 & 2,695 & 40 & 4,675 & 1,548 & 27 & 162 & 1 \\
\hline 1970 to 1979 & 47 & 17,737 & 1,929 & 28 & 4,516 & 1,496 & 23 & 104 & $\left({ }^{*}\right)$ \\
\hline 1980 to 1989 & 38 & 8,975 & 1,494 & 14 & 1,732 & 574 & 9 & 717 & 3 \\
\hline 1990 to 1993 & 15 & 4,774 & 446 & 6 & 773 & 256 & 4 & 36 & $(*)$ \\
\hline \multicolumn{10}{|l|}{ Federal Agency } \\
\hline Department of Defense ..................... & 22 & 13,988 & 1,911 & 23 & 2,900 & 961 & 14 & 448 & 2 \\
\hline General Services Administration . & 83 & 46,205 & 4,607 & 82 & 10,082 & 3,340 & 67 & 184 & 1 \\
\hline United States Postal Service ........ & 57 & 9,392 & 1,312 & 14 & 1,713 & 568 & 10 & 649 & 3 \\
\hline \multicolumn{10}{|l|}{$\begin{array}{l}\text { Energy Sources (more than one } \\
\text { may apply) }\end{array}$} \\
\hline Electricity & 311 & 94,161 & 11,990 & 167 & 20,445 & 6,772 & 121 & 1,949 & 8 \\
\hline 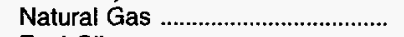 & 139 & 58,674 & 7,606 & 103 & 12,413 & 4,112 & 75 & 1,968 & 8 \\
\hline 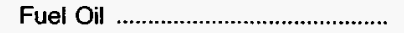 & 89 & 39,300 & 5,008 & 63 & 9,111 & 3,018 & 46 & 986 & 4 \\
\hline 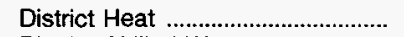 & 154 & 63,991 & 8,106 & 126 & 14,023 & 4,645 & 87 & 425 & 2 \\
\hline District Chilled Water .......................... & 57 & 22,922 & 3,305 & 43 & 4,940 & 1,636 & 26 & 384 & 1 \\
\hline 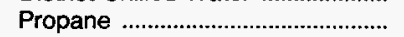 & 5 & 941 & 115 & 2 & 179 & 59 & 1 & 3 & $\left({ }^{*}\right)$ \\
\hline 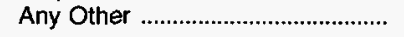 & 8 & 4,470 & 355 & 5 & 770 & 255 & 4 & 20 & $(*)$ \\
\hline \multicolumn{10}{|l|}{$\begin{array}{l}\text { Energy End Uses (more than } \\
\text { one may apply) }\end{array}$} \\
\hline 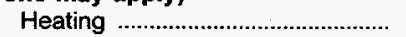 & 307 & 94,063 & 11,991 & 167 & 20,390 & 6,754 & 120 & 1,968 & 8 \\
\hline 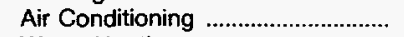 & 305 & 93,454 & 11,970 & 167 & 20,407 & 6,759 & 120 & 1,947 & 8 \\
\hline Water Heating & 303 & 93,138 & 11,924 & 166 & 20,246 & 6,706 & 119 & 1,963 & 8 \\
\hline 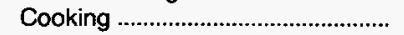 & 108 & 69,947 & 8,780 & 125 & 15,430 & 5,111 & 93 & 1,635 & 7 \\
\hline Manufacturing & 50 & 27,041 & 3,755 & 48 & 6,701 & 2,220 & 34 & 583 & 2 \\
\hline \multicolumn{10}{|l|}{ Workers (main shift) } \\
\hline Less than 50 & 71 & 4,102 & 444 & 5 & 548 & 181 & 3 & 61 & (*) \\
\hline 50 to 99 & 39 & 1,903 & 319 & 3 & 336 & 111 & 2 & 53 & (*) \\
\hline 100 to 499 & 107 & 13,632 & 2,321 & 29 & 3,460 & 1,146 & 18 & 358 & 2 \\
\hline 500 or More & 95 & 75,243 & 8,926 & 130 & 16,100 & 5,333 & 97 & 1,496 & 6 \\
\hline \multicolumn{10}{|l|}{ Weekly Operating Hours } \\
\hline 48 or Fewer & 51 & 9,694 & 854 & 21 & 1,310 & 434 & 16 & 40 & $(*)$ \\
\hline 49 to 60 & 76 & 25,540 & 2,496 & 41 & 4,935 & 1,635 & 30 & 76 & $(*)$ \\
\hline 61 to 167 & 60 & 11,993 & 1,308 & 18 & 2,650 & 878 & 15 & 131 & (*) \\
\hline 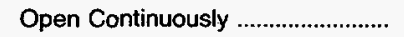 & 125 & 47,653 & 7,352 & 88 & 11,550 & 3,826 & 60 & 1,722 & 7 \\
\hline
\end{tabular}

See footnotes at end of table. 
Table 3.43. Consumption and Expenditures for Sum of Major Fuels, Electricity, and Natural Gas in FBSS Buildings in Federal Region 3, 1993 (Continued)

\begin{tabular}{|c|c|c|c|c|c|c|c|c|c|}
\hline \multirow{2}{*}{$\begin{array}{c}\text { Bullding } \\
\text { Characteristics }\end{array}$} & \multirow{2}{*}{$\begin{array}{l}\text { Number of } \\
\text { Buildings }\end{array}$} & \multirow{2}{*}{$\begin{array}{c}\text { Floorspace } \\
\text { (thousand } \\
\text { square } \\
\text { feet) }\end{array}$} & \multirow{2}{*}{$\begin{array}{l}\text { Sum of } \\
\text { Major } \\
\text { Fuel } \\
\text { Consump- } \\
\text { tion } \\
\text { (billion } \\
\text { Btu) }\end{array}$} & \multirow{2}{*}{$\begin{array}{l}\text { Sum of } \\
\text { Major } \\
\text { Fuel } \\
\text { Expend- } \\
\text { itures } \\
\text { (million } \\
\text { dollars) }\end{array}$} & \multicolumn{2}{|c|}{$\begin{array}{l}\text { Electricity } \\
\text { Consumption } \\
\text { (billion Btu) }\end{array}$} & \multirow{2}{*}{$\begin{array}{l}\text { Electricity } \\
\text { Expend- } \\
\text { itures } \\
\text { (million } \\
\text { dollars) }\end{array}$} & \multirow{2}{*}{$\begin{array}{l}\text { Natural } \\
\text { Gas } \\
\text { Consump- } \\
\text { tion } \\
\text { (billion } \\
\text { Btu) }\end{array}$} & \multirow{2}{*}{$\begin{array}{l}\text { Natural } \\
\text { Gas } \\
\text { Expend- } \\
\text { itures } \\
\text { (million } \\
\text { dollars) }\end{array}$} \\
\hline & & & & & Primary & Site & & & \\
\hline \multicolumn{10}{|l|}{ Multibuilding Facility } \\
\hline Yes & $\begin{array}{l}176 \\
136\end{array}$ & $\begin{array}{l}43,861 \\
51,018\end{array}$ & $\begin{array}{l}5,572 \\
6,437\end{array}$ & $\begin{array}{l}71 \\
96\end{array}$ & $\begin{array}{r}9,379 \\
11,066\end{array}$ & $\begin{array}{l}3,107 \\
3,666\end{array}$ & $\begin{array}{l}50 \\
71\end{array}$ & $\begin{array}{r}787 \\
1,182\end{array}$ & $\begin{array}{l}3 \\
5\end{array}$ \\
\hline 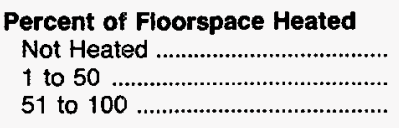 & $\begin{array}{r}5 \\
12 \\
295\end{array}$ & $\begin{array}{r}817 \\
5,787 \\
88,275\end{array}$ & $\begin{array}{r}18 \\
395 \\
11,596\end{array}$ & $\begin{array}{r}(*) \\
6 \\
161\end{array}$ & $\begin{array}{r}55 \\
722 \\
19,668\end{array}$ & $\begin{array}{r}18 \\
239 \\
6,515\end{array}$ & $\begin{array}{r}(*) \\
4 \\
116\end{array}$ & $\begin{array}{r}\text { NC } \\
17 \\
1,951\end{array}$ & $\begin{array}{r}\text { NC } \\
(") \\
8\end{array}$ \\
\hline 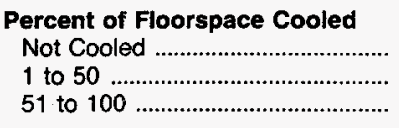 & $\begin{array}{r}13 \\
30 \\
269\end{array}$ & $\begin{array}{r}3,582 \\
9,874 \\
81,424\end{array}$ & $\begin{array}{r}180 \\
870 \\
10,960\end{array}$ & $\begin{array}{r}2 \\
13 \\
152\end{array}$ & $\begin{array}{r}334 \\
1,617 \\
18,494\end{array}$ & $\begin{array}{r}111 \\
536 \\
6,126\end{array}$ & $\begin{array}{r}2 \\
9 \\
109\end{array}$ & $\begin{array}{r}39 \\
36 \\
1,894\end{array}$ & $\begin{array}{l}(") \\
(")\end{array}$ \\
\hline 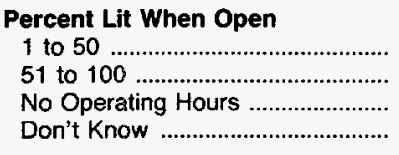 & $\begin{array}{r}11 \\
300 \\
1 \\
\mathrm{NC}\end{array}$ & $\begin{array}{r}663 \\
94,070 \\
147 \\
\text { NC }\end{array}$ & $\begin{array}{r}46 \\
11,939 \\
26 \\
N C\end{array}$ & $\begin{array}{r}1 \\
167 \\
\left({ }^{*}\right) \\
\text { NC }\end{array}$ & $\begin{array}{r}77 \\
20,340 \\
28 \\
\text { NC }\end{array}$ & $\begin{array}{r}26 \\
6,737 \\
9 \\
\mathrm{NC}\end{array}$ & $\begin{array}{r}1 \\
120 \\
\left({ }^{*}\right) \\
N C\end{array}$ & $\begin{array}{r}9 \\
1,944 \\
16 \\
\text { NC }\end{array}$ & $\begin{array}{r}(*) \\
8 \\
(*) \\
\mathrm{NC}\end{array}$ \\
\hline 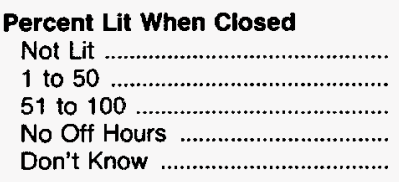 & $\begin{array}{r}39 \\
194 \\
42 \\
37 \\
\text { NC }\end{array}$ & $\begin{array}{r}5,149 \\
57,953 \\
17,412 \\
14,366 \\
\text { NC }\end{array}$ & $\begin{array}{r}449 \\
6,919 \\
2,786 \\
1,856 \\
\text { NC }\end{array}$ & $\begin{array}{r}7 \\
107 \\
32 \\
22 \\
\text { NC }\end{array}$ & $\begin{array}{r}879 \\
11,770 \\
4,134 \\
3,662 \\
\text { NC }\end{array}$ & $\begin{array}{r}291 \\
3,899 \\
1,369 \\
1,213 \\
\mathrm{NC}\end{array}$ & $\begin{array}{r}5 \\
78 \\
20 \\
17 \\
\mathrm{NC}\end{array}$ & $\begin{array}{r}44 \\
1,006 \\
655 \\
263 \\
\text { NC }\end{array}$ & $\begin{array}{r}(*) \\
5 \\
2 \\
1 \\
N C\end{array}$ \\
\hline 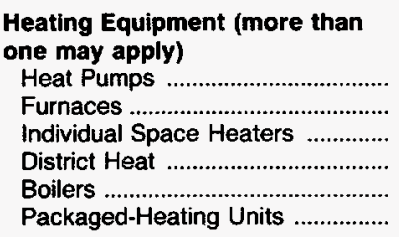 & $\begin{array}{r}34 \\
16 \\
75 \\
167 \\
93 \\
25\end{array}$ & $\begin{array}{r}10,922 \\
1,694 \\
34,923 \\
67,704 \\
20,082 \\
6,006\end{array}$ & $\begin{array}{r}935 \\
93 \\
4,433 \\
8,711 \\
2,640 \\
1,162\end{array}$ & $\begin{array}{r}13 \\
1 \\
57 \\
131 \\
26 \\
12\end{array}$ & $\begin{array}{r}2,168 \\
128 \\
6,910 \\
14,899 \\
3,950 \\
1,206\end{array}$ & $\begin{array}{r}718 \\
42 \\
2,289 \\
4,935 \\
1,308 \\
399\end{array}$ & $\begin{array}{r}11 \\
1 \\
39 \\
91 \\
20 \\
7\end{array}$ & $\begin{array}{r}34 \\
34 \\
1,164 \\
749 \\
1,111 \\
659\end{array}$ & $\begin{array}{l}\left({ }^{*}\right) \\
(*) \\
5 \\
3 \\
5 \\
3\end{array}$ \\
\hline 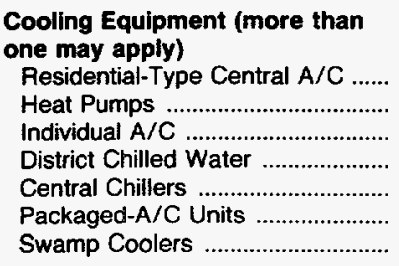 & $\begin{array}{r}30 \\
37 \\
80 \\
72 \\
158 \\
127 \\
3\end{array}$ & $\begin{array}{r}19,073 \\
11,388 \\
28,082 \\
28,194 \\
63,914 \\
56,664 \\
378\end{array}$ & $\begin{array}{r}2,655 \\
987 \\
3,276 \\
4,102 \\
7,621 \\
6,677 \\
99\end{array}$ & $\begin{array}{r}43 \\
15 \\
44 \\
51 \\
113 \\
98 \\
1\end{array}$ & $\begin{array}{r}4,292 \\
2,234 \\
5,084 \\
6,319 \\
13,867 \\
11,321 \\
195\end{array}$ & $\begin{array}{r}1,422 \\
740 \\
1,684 \\
2,093 \\
4,593 \\
3,750 \\
65\end{array}$ & $\begin{array}{r}31 \\
13 \\
31 \\
32 \\
86 \\
72 \\
1\end{array}$ & $\begin{array}{r}471 \\
11 \\
914 \\
586 \\
1,246 \\
1,481 \\
35\end{array}$ & $\begin{array}{l}2 \\
\text { (") } \\
4 \\
2 \\
6 \\
6 \\
\text { (*) }\end{array}$ \\
\hline \multicolumn{10}{|l|}{$\begin{array}{l}\text { Lighting Equipment (more than } \\
\text { one may apply) }\end{array}$} \\
\hline 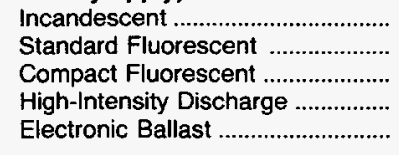 & $\begin{array}{r}184 \\
300 \\
80 \\
111 \\
135\end{array}$ & $\begin{array}{l}64,981 \\
91,986 \\
49,455 \\
52,593 \\
65,560\end{array}$ & $\begin{array}{r}8,811 \\
11,709 \\
5,583 \\
6,889 \\
7,548\end{array}$ & $\begin{array}{r}122 \\
162 \\
84 \\
88 \\
112\end{array}$ & $\begin{array}{l}14,418 \\
19,860 \\
10,301 \\
11,695 \\
13,264\end{array}$ & $\begin{array}{l}4,776 \\
6,578 \\
3,412 \\
3,874 \\
4,394\end{array}$ & $\begin{array}{r}85 \\
117 \\
63 \\
62 \\
82\end{array}$ & $\begin{array}{r}1,487 \\
1,946 \\
826 \\
1,445 \\
1,192\end{array}$ & $\begin{array}{l}7 \\
8 \\
3 \\
6 \\
5\end{array}$ \\
\hline \multicolumn{10}{|l|}{$\begin{array}{l}\text { Water-Heating Equipment (more } \\
\text { than one may apply) }\end{array}$} \\
\hline $\begin{array}{l}\text { Centralized System ........................ } \\
\text { Distributed System ....................... } \\
\text { Don't Know/ }\end{array}$ & $\begin{array}{r}223 \\
71\end{array}$ & $\begin{array}{l}63,477 \\
24,696\end{array}$ & $\begin{array}{l}8,916 \\
2,438\end{array}$ & $\begin{array}{r}116 \\
44\end{array}$ & $\begin{array}{r}14,310 \\
4,653\end{array}$ & $\begin{array}{l}4,740 \\
1,541\end{array}$ & $\begin{array}{l}80 \\
34\end{array}$ & $\begin{array}{r}1,730 \\
206\end{array}$ & $\begin{array}{l}7 \\
1\end{array}$ \\
\hline 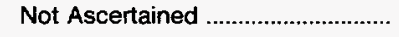 & 9 & 4,965 & 571 & 6 & 1,283 & 425 & 5 & 28 & $\left({ }^{*}\right)$ \\
\hline
\end{tabular}

See footnotes at end of table. 
Table 3.43. Consumption and Expenditures for Sum of Major Fuels, Electricity, and Natural Gas in FBSS Buildings in Federal Region 3, 1993 (Continued)

\begin{tabular}{|c|c|c|c|c|c|c|c|c|c|}
\hline \multirow{2}{*}{$\begin{array}{c}\text { Building } \\
\text { Characteristics }\end{array}$} & \multirow{2}{*}{$\begin{array}{l}\text { Number of } \\
\text { Buildings }\end{array}$} & \multirow{2}{*}{$\begin{array}{l}\text { Floorspace } \\
\text { (thousand } \\
\text { square } \\
\text { feet) }\end{array}$} & \multirow{2}{*}{$\begin{array}{c}\text { Sum of } \\
\text { Major } \\
\text { Fuel } \\
\text { Consump- } \\
\text { tion } \\
\text { (billion } \\
\text { Btu) }\end{array}$} & \multirow{2}{*}{$\begin{array}{l}\text { Sum of } \\
\text { Major } \\
\text { Fuel } \\
\text { Expend- } \\
\text { itures } \\
\text { (million } \\
\text { dollars) }\end{array}$} & \multicolumn{2}{|c|}{$\begin{array}{l}\text { Electricity } \\
\text { Consumption } \\
\text { (billion Btu) }\end{array}$} & \multirow{2}{*}{$\begin{array}{l}\text { Electricity } \\
\text { Expend- } \\
\text { itures } \\
\text { (million } \\
\text { dollars) }\end{array}$} & \multirow{2}{*}{$\begin{array}{l}\text { Natural } \\
\text { Gas } \\
\text { Consump- } \\
\text { tion } \\
\text { (billion } \\
\text { Btu) }\end{array}$} & \multirow{2}{*}{$\begin{array}{l}\text { Natural } \\
\text { Gas } \\
\text { Expend- } \\
\text { itures } \\
\text { (million } \\
\text { dollars) }\end{array}$} \\
\hline & & & & & Primary & Site & & & \\
\hline \multicolumn{10}{|l|}{$\begin{array}{l}\text { Commercial Refrigeration } \\
\text { Equipment (more than one may } \\
\text { apply) }\end{array}$} \\
\hline 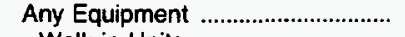 & 109 & 61,731 & 8,358 & 116 & 14,116 & 4,676 & 84 & 1,633 & 7 \\
\hline Walk-in Units & 78 & 54,223 & 7,473 & 105 & 12,314 & 4,079 & 75 & 1,543 & 7 \\
\hline Cases and Cabinets ....................... & 87 & 53,454 & 7,508 & 105 & 12,534 & 4,152 & 76 & 1,519 & 6 \\
\hline None & 203 & 33,149 & 3,652 & 51 & 6,329 & 2,096 & 36 & 336 & 1 \\
\hline \multicolumn{10}{|l|}{$\begin{array}{l}\text { Retrofit or Purchase of any } \\
\text { Equipment Within Last Ten } \\
\text { Years (more than one may } \\
\text { apply) }\end{array}$} \\
\hline Retrofit and/or Purchase & 229 & 81,286 & 9,984 & 142 & 16,613 & 5,503 & 102 & 1,843 & 8 \\
\hline 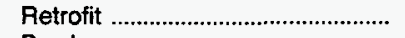 & 79 & 43,128 & 4,664 & 67 & 8,449 & 2,799 & 48 & 718 & 3 \\
\hline 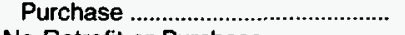 & 188 & 62,995 & 8,180 & 113 & 13,081 & 4,333 & 81 & 1,722 & 7 \\
\hline No Retrofit or Purchase ....................... & 83 & 13,594 & 2,025 & 26 & 3,832 & 1,269 & 18 & 125 & 1 \\
\hline \multicolumn{10}{|l|}{$\begin{array}{l}\text { Energy Conservation Features } \\
\text { (more than one may apply) }\end{array}$} \\
\hline Any Conservation Feature ............. & 309 & 94,361 & 11,986 & 167 & 20,407 & 6,760 & 120 & 1,968 & 8 \\
\hline 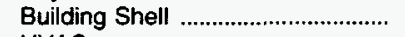 & 290 & 84,557 & 11,201 & 147 & 18,863 & 6,248 & 103 & 1,871 & 8 \\
\hline HVAC & 303 & 93,543 & 11,922 & 166 & 20,330 & 6,734 & 120 & 1,951 & 8 \\
\hline 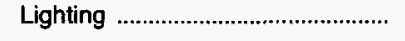 & 210 & 80,953 & 9,866 & 140 & 17,200 & 5,697 & 103 & 1,652 & 7 \\
\hline \multicolumn{10}{|l|}{$\begin{array}{l}\text { HVAC Conservation Features } \\
\text { (more than one may apply) }\end{array}$} \\
\hline 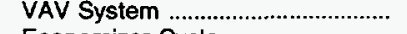 & 84 & 37,611 & 4,764 & 59 & 8,153 & 2,700 & 43 & 1,130 & 5 \\
\hline Economizer Cycle ................................. & 156 & 61,089 & 8,255 & 110 & 14,240 & 4,717 & 79 & 1,582 & 7 \\
\hline HVAC Maintenance ......................... & 301 & 93,402 & 11,917 & 166 & 20,316 & 6,729 & 120 & 1,951 & 8 \\
\hline \multicolumn{10}{|l|}{$\begin{array}{l}\text { Lighting Conservation Features } \\
\text { (more than one may apply) }\end{array}$} \\
\hline $\begin{array}{l}\text { Specular Reflectors .......................... } \\
\text { Natural Lighting Control }\end{array}$ & 124 & 44,741 & 5,880 & 73 & 9,465 & 3,135 & 51 & 1,346 & 6 \\
\hline 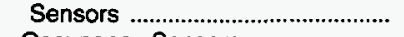 & 29 & 17,743 & 1,830 & 32 & 3,312 & 1,097 & 26 & 311 & 1 \\
\hline Occupancy Sensors ........................... & 95 & 62,261 & 6,564 & 103 & 12,728 & 4,216 & 79 & 749 & 3 \\
\hline Time Clock & 74 & 46,555 & 5,309 & 80 & 9,750 & 3,229 & 60 & 633 & 2 \\
\hline Manual Dimmer Switches .............. & 93 & 49,824 & 5,521 & 85 & 9,856 & 3,265 & 62 & 752 & 3 \\
\hline \multicolumn{10}{|l|}{$\begin{array}{l}\text { Energy Management Practices } \\
\text { (more than one may apply) } \\
\text { Energy Management and Control }\end{array}$} \\
\hline System & 132 & 61,891 & 7,959 & 109 & 13,645 & 4,520 & 81 & 1,692 & 7 \\
\hline 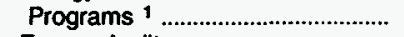 & 99 & 59,284 & 7,021 & 105 & 12,129 & 4,018 & 77 & 1,375 & 6 \\
\hline 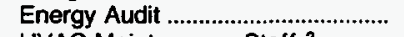 & 99 & 37,621 & 4,388 & 60 & 7,990 & 2,647 & 44 & 585 & 2 \\
\hline HVAC Maintenance Staff ${ }^{2}$........... & 97 & 52,924 & 6,266 & 97 & 11,252 & 3,727 & 72 & 930 & 4 \\
\hline \multicolumn{10}{|l|}{$\begin{array}{l}\text { Off-Hours Reduction in } \\
\text { Equipment (more than one may } \\
\text { apply) }\end{array}$} \\
\hline Heating & 144 & 43,828 & 4,127 & 73 & 8,085 & 2,678 & 56 & 199 & 1 \\
\hline 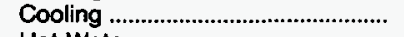 & 145 & 43,757 & 4,129 & 73 & 8,083 & 2,677 & 56 & 198 & 1 \\
\hline Hot Water & 77 & 26,018 & 2,407 & 45 & 4,552 & 1,508 & 35 & 115 & 1 \\
\hline 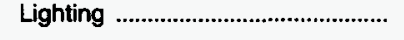 & 162 & 44,717 & 4,316 & 75 & 8,302 & 2,750 & 58 & 230 & 1 \\
\hline \multicolumn{10}{|l|}{$\begin{array}{l}\text { Sponsor of Program (more than } \\
\text { one may apply) }\end{array}$} \\
\hline FEMP & 3 & 1,848 & 208 & 3 & 339 & 112 & 2 & 7 & $\left({ }^{*}\right)$ \\
\hline Electric Utility ................................... & 29 & 20,357 & 2,466 & 33 & 4,103 & 1,359 & 24 & 687 & 3 \\
\hline $\begin{array}{l}\text { Natural Gas Utility } \\
\text { In-house }\end{array}$ & $\begin{array}{l}\mathrm{NC} \\
33\end{array}$ & $\begin{array}{r}\text { NC } \\
26,265\end{array}$ & $\begin{array}{c}\mathrm{NC} \\
3,221\end{array}$ & $\begin{array}{l}\text { NC } \\
45\end{array}$ & $\begin{array}{c}\mathrm{NC} \\
5,606\end{array}$ & $\begin{array}{c}\mathrm{NC} \\
\uparrow, 857\end{array}$ & $\begin{array}{l}\mathrm{NC} \\
32\end{array}$ & $\begin{array}{l}\text { NC } \\
510\end{array}$ & $\mathrm{NC}_{2}$ \\
\hline 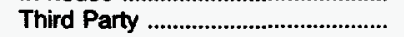 & 5 & 4,322 & 443 & 6 & 882 & 292 & 5 & 110 & $\left({ }^{*}\right)$ \\
\hline
\end{tabular}

See footnotes at end of table. 
Table 3.43. Consumption and Expenditures for Sum of Major Fuels, Electricity, and Natural Gas in FBSS Buildings in Federal Region 3, 1993 (Continued)

\begin{tabular}{|c|c|c|c|c|c|c|c|c|c|}
\hline \multirow{2}{*}{$\begin{array}{c}\text { Building } \\
\text { Characteristics }\end{array}$} & \multirow{2}{*}{$\begin{array}{l}\text { Number of } \\
\text { Buildings }\end{array}$} & \multirow{2}{*}{$\begin{array}{l}\text { Floorspace } \\
\text { (thousand } \\
\text { square } \\
\text { feet) }\end{array}$} & \multirow{2}{*}{$\begin{array}{l}\text { Sum of } \\
\text { Major } \\
\text { Fuel } \\
\text { Consump- } \\
\text { tion } \\
\text { (billion } \\
\text { Btu) }\end{array}$} & \multirow{2}{*}{$\begin{array}{l}\text { Sum of } \\
\text { Major } \\
\text { Fuel } \\
\text { Expend- } \\
\text { itures } \\
\text { (million } \\
\text { dollars) }\end{array}$} & \multicolumn{2}{|c|}{$\begin{array}{l}\text { Electricity } \\
\text { Consumption } \\
\text { (billion Btu) }\end{array}$} & \multirow{2}{*}{$\begin{array}{l}\text { Electricity } \\
\text { Expend- } \\
\text { itures } \\
\text { (million } \\
\text { dollars) }\end{array}$} & \multirow{2}{*}{$\begin{array}{l}\text { Natural } \\
\text { Gas } \\
\text { Consump- } \\
\text { tion } \\
\text { (billion } \\
\text { Btu) }\end{array}$} & \multirow{2}{*}{$\begin{array}{c}\text { Natural } \\
\text { Gas } \\
\text { Expend- } \\
\text { itures } \\
\text { (million } \\
\text { dollars) }\end{array}$} \\
\hline & & & & & Primary & Site & & & \\
\hline \multicolumn{10}{|l|}{$\begin{array}{l}\text { Type of Assistance (more than } \\
\text { one may apply) } \\
\text { Federal Energy Efficiency }\end{array}$} \\
\hline Fund & 4 & 962 & 128 & 1 & 123 & 41 & 1 & 9 & $(*)$ \\
\hline General Information ............................ & 7 & 6,729 & 573 & 8 & 1,135 & 376 & 6 & 109 & $(*)$ \\
\hline Site-Specific Information .................. & 9 & 16,219 & 2,292 & 30 & 4,028 & 1,334 & 20 & 486 & 2 \\
\hline 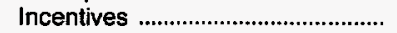 & 18 & 12,755 & 1,066 & 19 & 2,313 & 766 & 14 & 10 & $(*)$ \\
\hline 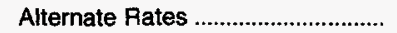 & 8 & 7,707 & 1,100 & 12 & 1,176 & 389 & 8 & 567 & 3 \\
\hline 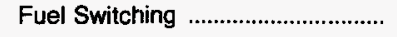 & 4 & 2,154 & 185 & 3 & 373 & 123 & 2 & 12 & $(*)$ \\
\hline Yes & 55 & 31,984 & 4,282 & 53 & 7,462 & 2,472 & 37 & 724 & 3 \\
\hline No & 257 & 62,896 & 7,728 & 115 & 12,983 & 4,301 & 83 & 1,244 & 6 \\
\hline \multicolumn{10}{|l|}{$\begin{array}{l}\text { Natural Gas Transported } \\
\text { for the Account of Others }\end{array}$} \\
\hline 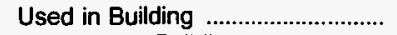 & 11 & 4,270 & 394 & 4 & 573 & 190 & 3 & 142 & 1 \\
\hline $\begin{array}{l}\text { Not Used in Building ......................... } \\
\text { Don't Know/ }\end{array}$ & 124 & 52,636 & 6,973 & 96 & 11,240 & 3,723 & 70 & 1,805 & 8 \\
\hline Not Ascertained & 4 & 1,768 & 239 & 2 & 601 & 199 & 2 & 21 & $\left(^{*}\right)$ \\
\hline
\end{tabular}

1 Building participates in any programs sponsored by the Federal Energy Management Program, in-house, utility, or third party.

2 HVAC maintenance staff means at least one person spends at least half their working hours maintaining the heating/cooling equipment

$\left(^{*}\right)=$ Value rounds to zero in the units displayed.

$\mathrm{NC}=$ No cases in responding sample.

Notes: - Total workers are the number of workers during the main shift. - See Glossary for explanation of abbreviations and definitions of terms used in this report. - These data are from 881 federally owned buildings having the following criteria: (1) located in Federal Regions 3 , 6 , or 9; (2) larger than 10,000 square feet; and (3) used for a commercial purpose, other than warehouse and storage. In addition, 9 out of 10 selected buildings were from agencies other than the Department of Defense. - Statistics for the "energy end uses" represent consumption in buildings that have end use, not consumption for a particular fuel for a particular end use. - Site electricity is the amount of electricity delivered to commercial buildings.

Primary electricity is site electricity plus the conversion losses in the electric generation process at the utility plant. $\bullet$ FBSS = Federal Buildings Supplemental Survey. - HVAC = Heating, Ventilation, and Air Conditioning. - Data are for Fiscal Year 1993 (October 1, 1992 through September 30 , 1993). - Because of rounding, data may not sum to totals.

Source: Energy Information Administration, Office of Energy Markets and End Use, 1993 Federal Buildings Supplemental Survey. 
Table 3.44. Consumption and Expenditures for Sum of Major Fuels, Electricity, and Natural Gas in FBSS Buildings in Federal Region 6, 1993

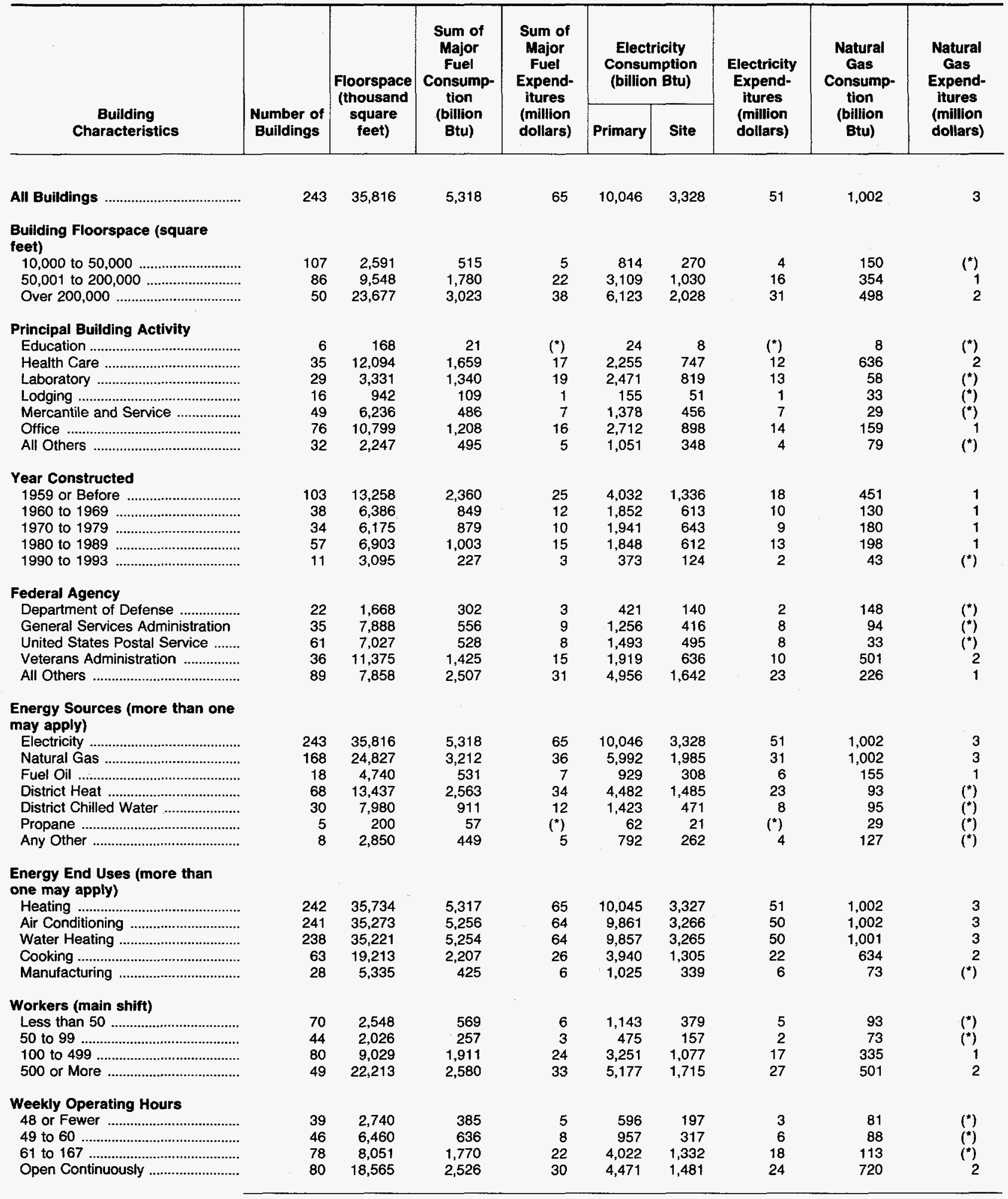

See footnotes at end of table. 
Table 3.44. Consumption and Expenditures for Sum of Major Fuels, Electricity, and Natural Gas in FBSS Buildings in Federal Region 6, 1993 (Continued)

\begin{tabular}{|c|c|c|c|c|c|c|c|c|c|}
\hline \multirow{2}{*}{$\begin{array}{c}\text { Building } \\
\text { Characteristics }\end{array}$} & \multirow{2}{*}{$\begin{array}{l}\text { Number of } \\
\text { Buildings }\end{array}$} & \multirow{2}{*}{$\begin{array}{c}\text { Floorspace } \\
\text { (thousand } \\
\text { square } \\
\text { feet) }\end{array}$} & \multirow{2}{*}{$\begin{array}{c}\text { Sum of } \\
\text { Major } \\
\text { Fuel } \\
\text { Consump- } \\
\text { tion } \\
\text { (billion } \\
\text { Btu) }\end{array}$} & \multirow{2}{*}{$\begin{array}{l}\text { Sum of } \\
\text { Major } \\
\text { Fuel } \\
\text { Expend- } \\
\text { itures } \\
\text { (million } \\
\text { dollars) }\end{array}$} & \multicolumn{2}{|c|}{$\begin{array}{l}\text { Electricity } \\
\text { Consumption } \\
\text { (billion Btu) }\end{array}$} & \multirow{2}{*}{$\begin{array}{l}\text { Electricity } \\
\text { Expend- } \\
\text { itures } \\
\text { (million } \\
\text { dollars) }\end{array}$} & \multirow{2}{*}{$\begin{array}{c}\text { Natural } \\
\text { Gas } \\
\text { Consump- } \\
\text { tion } \\
\text { (billion } \\
\text { Btu) }\end{array}$} & \multirow{2}{*}{$\begin{array}{l}\text { Natural } \\
\text { Gas } \\
\text { Expend- } \\
\text { itures } \\
\text { (million } \\
\text { dollars) }\end{array}$} \\
\hline & & & & & Primary & Site & & & \\
\hline \multicolumn{10}{|l|}{ Multibuilding Facility } \\
\hline Yes & 148 & 24,441 & 4,151 & 50 & 7,527 & 2,493 & 37 & 677 & 2 \\
\hline No & 95 & 11,375 & 1,167 & 15 & $2,5+9$ & 834 & 14 & 325 & 1 \\
\hline \multicolumn{10}{|l|}{ Percent of Floorspace Heated } \\
\hline 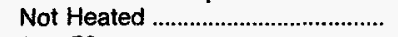 & 1 & 82 & $\left({ }^{*}\right)$ & $(*)$ & 1 & $(*)$ & $\left({ }^{*}\right)$ & NC & NC \\
\hline 1 to 50 & 11 & 1,288 & 104 & 1 & 228 & 76 & 1 & 24 & $(*)$ \\
\hline 51 to 100 & 231 & 34,446 & 5,213 & 64 & 9,817 & 3,252 & 50 & 978 & 3 \\
\hline \multicolumn{10}{|l|}{ Percent of Floorspace Cooled } \\
\hline 1 to 50 & 26 & 1,951 & 373 & 4 & 769 & 255 & 3 & 41 & $(*)$ \\
\hline 51 to 100 & 214 & 33,312 & 4,875 & 60 & 9,091 & 3,011 & 47 & 953 & 3 \\
\hline \multicolumn{10}{|l|}{ Percent Lit When Open } \\
\hline 1 to 50 & 15 & 1,062 & 83 & 1 & 190 & 63 & 1 & 13 & $\left({ }^{*}\right)$ \\
\hline 51 to 100 & 227 & 34,672 & 5,234 & 64 & 9,855 & 3,264 & 50 & 989 & 3 \\
\hline No Operating Hours ........................ & & 82 & $\left({ }^{*}\right)$ & $\left({ }^{*}\right)$ & & $(*)$ & $(*)$ & NC & NC \\
\hline 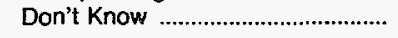 & NC & NC & NC & $\mathrm{NC}$ & NC & NC & NC & NC & NC \\
\hline \multicolumn{10}{|l|}{ Percent Lit When Closed } \\
\hline 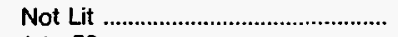 & 33 & 2,691 & 192 & 2 & 348 & 115 & 2 & 59 & $\left({ }^{\star}\right)$ \\
\hline 1 to 50 & 165 & 20,495 & 3,709 & $4 \overline{6}$ & 7,142 & 2,366 & $3 \overline{6}$ & 588 & 2 \\
\hline 51 to 100 & 25 & 8,029 & 797 & 9 & 1,400 & 464 & 7 & 192 & 1 \\
\hline No Off Hours .................................... & 20 & 4,601 & 619 & 7 & 1,156 & 383 & 6 & 163 & 1 \\
\hline 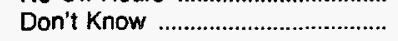 & $\mathrm{NC}$ & NC & NC & NC & NC & NC & NC & NC & NC \\
\hline \multicolumn{10}{|l|}{$\begin{array}{l}\text { Heating Equipment (more than } \\
\text { one may apply) }\end{array}$} \\
\hline 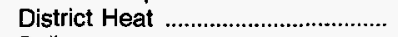 & 82 & 15,205 & 2,831 & 36 & 4,707 & 1,559 & 24 & 293 & 1 \\
\hline 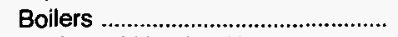 & 102 & 16,718 & 2,121 & 24 & 4,329 & 1,434 & 22 & 644 & 2 \\
\hline Packaged-Heating Units ............... & 59 & 7,574 & 1,099 & 13 & 2,049 & 679 & 11 & 338 & 1 \\
\hline \multicolumn{10}{|l|}{$\begin{array}{l}\text { Cooling Equipment (more than } \\
\text { one may apply) }\end{array}$} \\
\hline Residential-Type Central $A / C$...... & 29 & 4,706 & 454 & 6 & 984 & 326 & 6 & 92 & $(*)$ \\
\hline 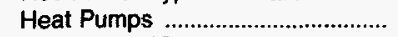 & 11 & 1,549 & 204 & 2 & 407 & 135 & 2 & 62 & $(*)$ \\
\hline Individual A/C & 50 & 6,950 & 1,246 & 13 & 2,482 & 822 & 10 & 226 & 1 \\
\hline District Chilled Water ...................... & 44 & 10,856 & 1,404 & 19 & 1,971 & 653 & 14 & 268 & 1 \\
\hline 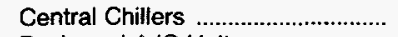 & 122 & 21,596 & 3,059 & 36 & 6,266 & 2,076 & 30 & 638 & 2 \\
\hline Packaged-A/C Units .......................... & 97 & 14,104 & 1,852 & 22 & 3,478 & 1,152 & 19 & 502 & 2 \\
\hline 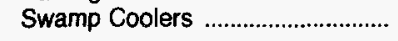 & 12 & 2,015 & 234 & 3 & 398 & 132 & 2 & 28 & $(*)$ \\
\hline \multicolumn{10}{|l|}{$\begin{array}{l}\text { Lighting Equipment (more than } \\
\text { one may apply) }\end{array}$} \\
\hline Incandescent & 135 & 20,248 & 3,539 & 44 & 7,068 & 2,341 & 34 & 556 & 2 \\
\hline Standard Fluorescent ..................... & 235 & 35,057 & 5,283 & 65 & 9,966 & 3,301 & 51 & 994 & 3 \\
\hline Compact Fluorescent ....................... & 29 & 7,942 & 741 & 11 & 1,467 & 486 & 9 & 173 & 1 \\
\hline High-Intensity Discharge ................. & 64 & 16,188 & 2,515 & 27 & 5,332 & 1,766 & 23 & 480 & 2 \\
\hline 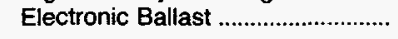 & 59 & 15,861 & 1,649 & 20 & 2,873 & 952 & 16 & 505 & 2 \\
\hline \multicolumn{10}{|l|}{$\begin{array}{l}\text { Water-Heating Equipment (more } \\
\text { than one may apply) }\end{array}$} \\
\hline Centralized System ............................ & 163 & 23,206 & 3,448 & 41 & 5,924 & 1,962 & 31 & 801 & 3 \\
\hline $\begin{array}{l}\text { Distributed System } \\
\text { Don't Know/....................... }\end{array}$ & 71 & 11,827 & 1,744 & 22 & 3,864 & 1,280 & 19 & 164 & 1 \\
\hline Not Ascertained & 4 & 188 & 63 & 1 & 69 & 23 & $(*)$ & 37 & $(*)$ \\
\hline
\end{tabular}

See footnotes at end of table. 
Table 3.44. Consumption and Expenditures for Sum of Major Fuels, Electricity, and Natural Gas in FBSS Buildings in Federal Region 6, 1993 (Continued)

\begin{tabular}{|c|c|c|c|c|c|c|c|c|c|}
\hline \multirow{2}{*}{$\begin{array}{c}\text { Building } \\
\text { Characteristics }\end{array}$} & \multirow[b]{2}{*}{$\begin{array}{l}\text { Number of } \\
\text { Buildings }\end{array}$} & \multirow{2}{*}{$\begin{array}{c}\text { Floorspace } \\
\text { (thousand } \\
\text { square } \\
\text { feet) }\end{array}$} & \multirow{2}{*}{$\begin{array}{l}\text { Sum of } \\
\text { Major } \\
\text { Fuel } \\
\text { Consump- } \\
\text { tion } \\
\text { (billion } \\
\text { Btu) }\end{array}$} & \multirow{2}{*}{$\begin{array}{l}\text { Sum of } \\
\text { Major } \\
\text { Fuel } \\
\text { Expend- } \\
\text { itures } \\
\text { (million } \\
\text { dollars) }\end{array}$} & \multicolumn{2}{|c|}{$\begin{array}{c}\text { Electricity } \\
\text { Consumption } \\
\text { (billion Btu) }\end{array}$} & \multirow{2}{*}{$\begin{array}{c}\text { Electricity } \\
\text { Expend- } \\
\text { itures } \\
\text { (million } \\
\text { dollars) }\end{array}$} & \multirow{2}{*}{$\begin{array}{l}\text { Natural } \\
\text { Gas } \\
\text { Consump- } \\
\text { tion } \\
\text { (billion } \\
\text { Btu) }\end{array}$} & \multirow{2}{*}{$\begin{array}{c}\text { Natural } \\
\text { Gas } \\
\text { Expend- } \\
\text { itures } \\
\text { (million } \\
\text { dollars) }\end{array}$} \\
\hline & & & & & Primary & Site & & & \\
\hline
\end{tabular}

Commercial Refrigeration

Equipment (more than one may apply)

Any Equipment

Walk-in Units

Cases and Cabinets .....................

None.

$\begin{array}{rrr}70 & 17,917 & 2,529 \\ 40 & 12,381 & 1,783 \\ 44 & 14,125 & 1,782 \\ 173 & 17,899 & 2,789\end{array}$

1,783

1,782
2,789

Retrofit or Purchase of any

Equipment Within Last Ten

Years (more than one may

apply)

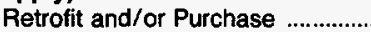

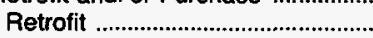

Purchase.

No Retrofit or Purchase

$63 \quad 15,029$

12621,409

21,409
6,801

3,517
1,542

2,732

Energy Conservation Features

(more than one may apply)

Any Conservation Feature

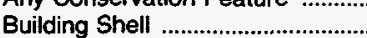

HVAC

Lighting

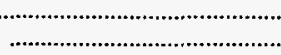

$228 \quad 33,946$

$236 \quad 35,425$

$136 \quad 25,654$

5,317

5,092

5,287

2,997

HVAC Conservation Features

(more than one may apply)

Economizer Cycle ...........................

$65 \quad 18,518$

2,319

HVAC Maintenance

35,341

3,459

Lighting Conservation Features

(more than one may apply)

Specular Reflectors ..............

Natural Lighting Control

Sensors .

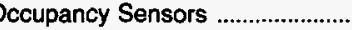

Time Clock

Manual Dimmer Switches ...............

$\begin{array}{rrr}92 & 18,017 & 2,263 \\ 27 & 6,740 & 652 \\ 58 & 16,435 & 1,639 \\ 36 & 9,037 & 861 \\ 44 & 13,454 & 1,475\end{array}$

$\begin{array}{rrr}43 & 6,462 & 2,140 \\ 20 & 2,772 & 918 \\ 32 & 5,039 & 1,669\end{array}$

$3,584 \quad 1,187$

$\begin{array}{ll}34 & 832 \\ 16 & 307 \\ 27 & 725\end{array}$

307
725

170

Energy Management Practices

(more than one may apply)

Energy Management and Control

System .

Energy Conservation

Programs 1

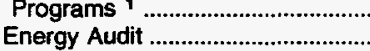

HVAC Maintenance Staff ${ }^{2}$

$96 \quad 23,099$

$48 \quad 13,513$

$64 \quad 11,690$

$57 \quad 15,538$

Off-Hours Reduction in

Equipment (more than one may apply)

Heating

Cooling

Hot Water

Lighting

$123 \quad 13,162$

$124 \quad 13,864$

$33 \quad 4,554$

$120 \quad 12,847$

1,356

1,413

401

1,383

2,889

1,481

1,177

1,542
Sponsor of Program (more than one may apply)

FEMP

Electric Utility .................

Natural Gas Utility

In-house .

Third Party

See footnotes at end of table.

\begin{tabular}{rrrrrrrrrr}
4 & 1,286 & 77 & 2 & 148 & 49 & 1 & 29 & ()$\left.^{*}\right)$ \\
4 & 1,257 & 63 & 1 & 111 & 37 & 1 & 14 & $\left(^{*}\right)$ \\
NC & NC & NC & NC & NC & NC & NC & NC & NC \\
19 & 6,915 & 741 & 9 & 1,277 & 423 & 8 & 181 & 1 \\
2 & 340 & 27 & $(*)$ & 62 & 20 & $\left(^{*}\right)$ & 6 & $\left(^{*}\right)$ \\
\hline
\end{tabular}


Table 3.44. Consumption and Expenditures for Sum of Major Fuels, Electricity, and Natural Gas in FBSS Buildings in Federal Region 6, 1993 (Continued)

\begin{tabular}{|c|c|c|c|c|c|c|c|c|c|}
\hline \multirow{2}{*}{$\begin{array}{c}\text { Building } \\
\text { Characteristics }\end{array}$} & \multirow{2}{*}{$\begin{array}{l}\text { Number of } \\
\text { Buildings }\end{array}$} & \multirow{2}{*}{$\begin{array}{l}\text { Floorspace } \\
\text { (thousand } \\
\text { square } \\
\text { feet) }\end{array}$} & \multirow{2}{*}{$\begin{array}{l}\text { Sum of } \\
\text { Major } \\
\text { Fuel } \\
\text { Consump- } \\
\text { tion } \\
\text { (billion } \\
\text { Btu) }\end{array}$} & \multirow{2}{*}{$\begin{array}{l}\text { Sum of } \\
\text { Major } \\
\text { Fuel } \\
\text { Expend- } \\
\text { itures } \\
\text { (million } \\
\text { dollars) }\end{array}$} & \multicolumn{2}{|c|}{$\begin{array}{l}\text { Electricity } \\
\text { Consumption } \\
\text { (billion Btu) }\end{array}$} & \multirow{2}{*}{$\begin{array}{c}\text { Electricity } \\
\text { Expend- } \\
\text { itures } \\
\text { (million } \\
\text { dollars) }\end{array}$} & \multirow{2}{*}{$\begin{array}{l}\text { Natural } \\
\text { Gas } \\
\text { Consump- } \\
\text { tion } \\
\text { (billion } \\
\text { Btu) }\end{array}$} & \multirow{2}{*}{$\begin{array}{c}\text { Natural } \\
\text { Gas } \\
\text { Expend- } \\
\text { itures } \\
\text { (million } \\
\text { dollars) }\end{array}$} \\
\hline & & & & & Primary & Site & & & \\
\hline \multicolumn{10}{|l|}{$\begin{array}{l}\text { Type of Assistance (more than } \\
\text { one may apply) } \\
\text { Federal Energy Efficiency }\end{array}$} \\
\hline Fund & 4 & 1,564 & 90 & 2 & 177 & 59 & 2 & 32 & $(*)$ \\
\hline 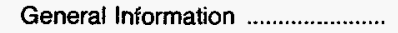 & 3 & 100 & 13 & $(*)$ & 27 & 9 & $\left({ }^{\star}\right)$ & 4 & $(*)$ \\
\hline Site-Specific Information ................... & 5 & 423 & 64 & 1 & 108 & 36 & 1 & 24 & $(*)$ \\
\hline 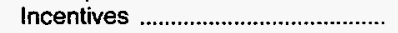 & 4 & 296 & 35 & $(*)$ & 59 & 19 & $(*)$ & 4 & $(*)$ \\
\hline 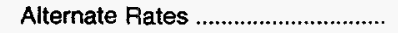 & NC & NC & NC & $\mathrm{NC}$ & $\mathrm{NC}$ & NC & NC & NC & NC \\
\hline 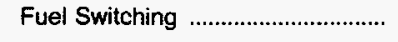 & 1 & 772 & 104 & 1 & 186 & 61 & 1 & 5 & $(*)$ \\
\hline \multicolumn{10}{|l|}{ Bullding Generates Electricity } \\
\hline Yes & 32 & 10,295 & 1,086 & 12 & 1,668 & 553 & 9 & 377 & 1 \\
\hline No & 211 & 25,521 & 4,232 & 53 & 8,378 & 2,775 & 42 & 625 & 2 \\
\hline \multicolumn{10}{|l|}{$\begin{array}{l}\text { Natural Gas Transported } \\
\text { for the Account of Others }\end{array}$} \\
\hline Used in Building & 5 & 631 & 160 & 1 & 160 & 53 & 1 & 69 & $(*)$ \\
\hline $\begin{array}{l}\text { Not Used in Building } \\
\text { Don't Know/..................... }\end{array}$ & 140 & 22,202 & 2,377 & 28 & 4,230 & 1,401 & 24 & 853 & 3 \\
\hline 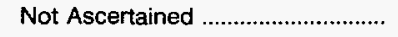 & 23 & 1,994 & 675 & 7 & 1,602 & 530 & 6 & 80 & $\left({ }^{*}\right)$ \\
\hline
\end{tabular}

1 Building participates in any programs sponsored by the Federal Energy Management Program, in-house, utility, or third party.

2 HVAC maintenance staff means at least one person spends at least half their working hours maintaining the heating/cooling equipment.

$(*)=$ Value rounds to zero in the units displayed.

NC $=$ No cases in responding sample.

Notes: - Total workers are the number of workers during the main shift. - See Glossany for explanation of abbreviations and definitions of terms used in this report. - These data are from 881 federally owned buildings having the following criteria: (1) located in Federal Regions 3 , 6 , or 9; (2) larger than 10,000 square feet; and (3) used for a commercial purpose, other than warehouse and storage. In addition, 9 out of 10 selected buildings were from agencies other than the Department of Defense. - Statistics for the "energy end uses" represent consumption in buildings that have end use, not consumption for a particular fuel for a particular end use. - Site electricity is the amount of electricity delivered to commercial buildings.

Primary electricity is site electricity plus the conversion losses in the electric generation process at the utility plant. $-\mathrm{FBSS}=$ Federal Buildings Supplemental Survey. - HVAC = Heating, Ventilation, and Air Conditioning. - Data are for Fiscal Year 1993 (October 1,1992 through September 30 , 1993). - Because of rounding, data may not sum to totals.

Source: Energy Information Administration, Office of Energy Markets and End Use, 1993 Federal Buildings Supplemental Survey. 
Table 3.45. Consumption and Expenditures for Sum of Major Fuels, Electricity, and Natural Gas in FBSS Buildings in Federal Region 9, 1993

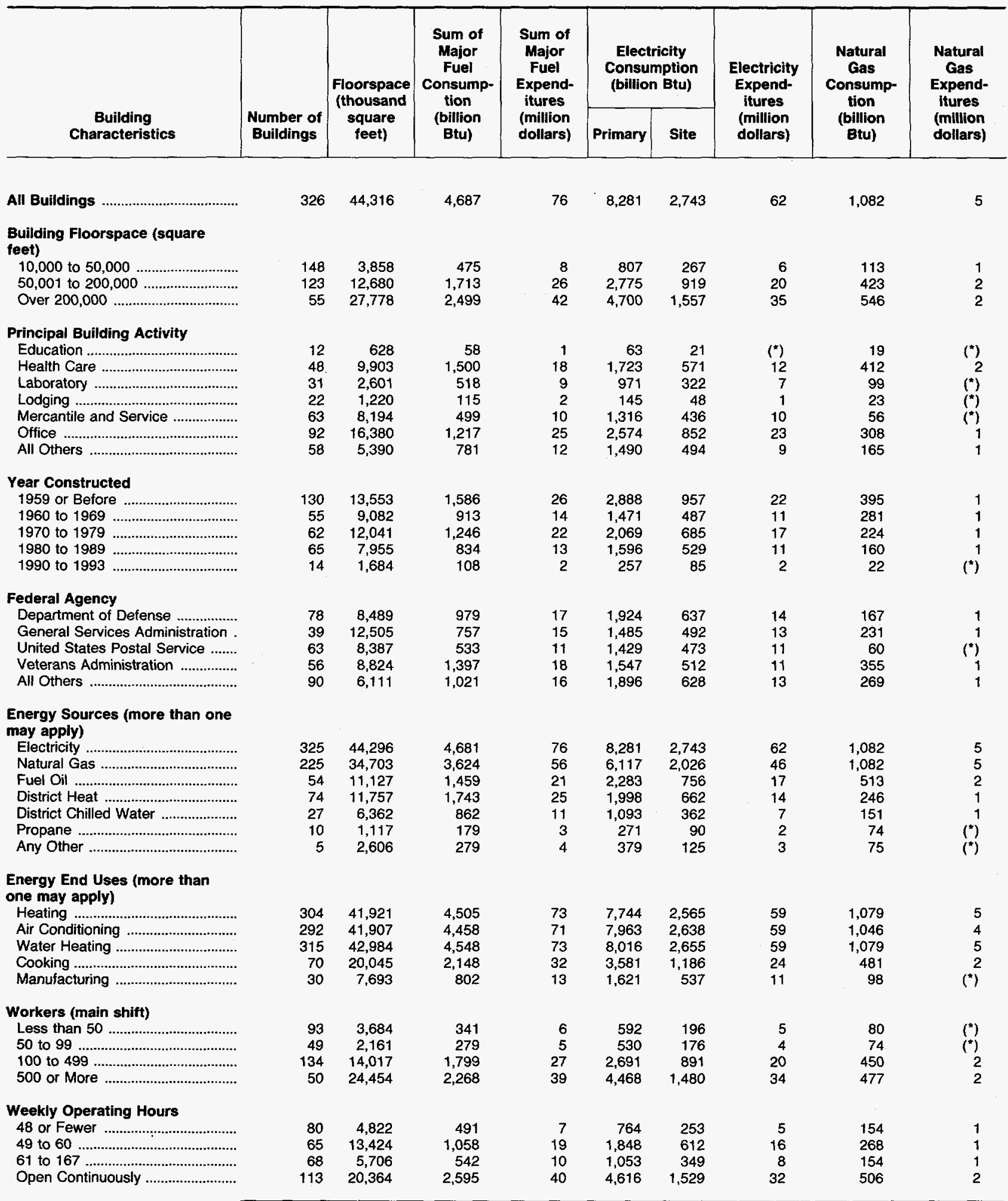

See footnotes at end of table. 
Table 3.45. Consumption and Expenditures for Sum of Major Fuels, Electricity, and Natural Gas in FBSS Buildings in Federal Region 9, 1993 (Continued)

\begin{tabular}{|c|c|c|c|c|c|c|c|c|c|}
\hline \multirow{2}{*}{$\begin{array}{l}\text { Building } \\
\text { Characteristics }\end{array}$} & \multirow{2}{*}{$\begin{array}{l}\text { Number of } \\
\text { Buildings }\end{array}$} & \multirow{2}{*}{$\begin{array}{c}\text { Floorspace } \\
\text { (thousand } \\
\text { square } \\
\text { feet) }\end{array}$} & \multirow{2}{*}{$\begin{array}{l}\text { Sum of } \\
\text { Major } \\
\text { Fuel } \\
\text { Consump- } \\
\text { tion } \\
\text { (billion } \\
\text { Btu) }\end{array}$} & \multirow{2}{*}{$\begin{array}{l}\text { Sum of } \\
\text { Major } \\
\text { Fuel } \\
\text { Expend- } \\
\text { itures } \\
\text { (million } \\
\text { dollars) }\end{array}$} & \multicolumn{2}{|c|}{$\begin{array}{l}\text { Electricity } \\
\text { Consumption } \\
\text { (billion Btu) }\end{array}$} & \multirow{2}{*}{$\begin{array}{l}\text { Electricity } \\
\text { Expend- } \\
\text { itures } \\
\text { (million } \\
\text { dollars) }\end{array}$} & \multirow{2}{*}{$\begin{array}{c}\text { Natural } \\
\text { Gas } \\
\text { Consump- } \\
\text { tion } \\
\text { (billion } \\
\text { Btu) }\end{array}$} & \multirow{2}{*}{$\begin{array}{c}\text { Natural } \\
\text { Gas } \\
\text { Expend- } \\
\text { itures } \\
\text { (million } \\
\text { dollars) }\end{array}$} \\
\hline & & & & & Primary & Site & & & \\
\hline \multicolumn{10}{|l|}{ Multibuilding Facility } \\
\hline Yes & 200 & 26,821 & 3,165 & 47 & 5,002 & 1,657 & 36 & 744 & 3 \\
\hline No & 126 & 17,495 & 1,522 & 29 & 3,280 & 1,086 & 26 & 338 & 1 \\
\hline \multicolumn{10}{|l|}{ Percent of Floorspace Heated } \\
\hline 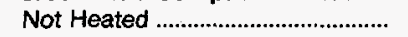 & 22 & 2,394 & 182 & 3 & 538 & 178 & 3 & 3 & $(")$ \\
\hline 1 to 50 & 28 & 3,751 & 462 & 9 & 1,119 & 371 & 8 & 78 & (*) \\
\hline 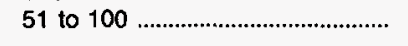 & 276 & 38,170 & 4,043 & 64 & 6,625 & 2,194 & 51 & 1,000 & 4 \\
\hline \multicolumn{10}{|l|}{ Percent of Floorspace Cooled } \\
\hline 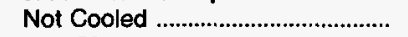 & 40 & 2,956 & 283 & 5 & 415 & 137 & 4 & 43 & $\left({ }^{*}\right)$ \\
\hline 1 to 50 & 55 & 7,304 & 920 & 13 & 1,396 & 462 & 9 & 240 & 1 \\
\hline 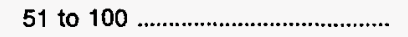 & 231 & 34,056 & 3,484 & 58 & 6,471 & 2,143 & 49 & 798 & 3 \\
\hline \multicolumn{10}{|l|}{ Percent Lit When Open } \\
\hline 1 to 50 & 20 & 1,847 & 157 & 2 & 221 & 73 & 2 & 56 & (") \\
\hline 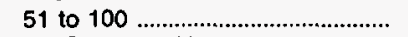 & 304 & 42,375 & 4,523 & $7 \overline{4}$ & 8,050 & 2,667 & 60 & 1,023 & 4 \\
\hline No Operating Hours ......................... & 1 & 29 & $(")$ & $(*)$ & $(*)$ & $(*)$ & (*) & NC & NC \\
\hline 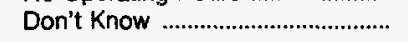 & 1 & 64 & 7 & $\left({ }^{*}\right)$ & 10 & 3 & ()$\left.^{*}\right)$ & 3 & $\left({ }^{*}\right)$ \\
\hline \multicolumn{10}{|l|}{ Percent Lit When Closed } \\
\hline 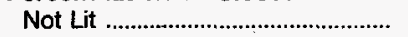 & 35 & 1,664 & $189^{\circ}$ & 3 & 337 & 112 & 3 & 47 & $\left({ }^{*}\right)$ \\
\hline 1 to 50 & 205 & 25,288 & 2,222 & 37 & 3,854 & 1,276 & 31 & 643 & 3 \\
\hline 51 to 100 & 44 & 8,109 & 929 & 15 & 1,652 & 547 & 12 & 168 & 1 \\
\hline 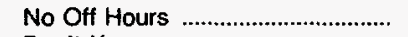 & 41 & 9,190 & 1,340 & 21 & 2,429 & 804 & 17 & 220 & 1 \\
\hline 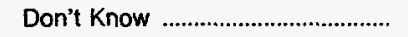 & 1 & 64 & 7 & $(*)$ & 10 & 3 & $\left({ }^{*}\right)$ & 3 & (*) \\
\hline \multicolumn{10}{|l|}{$\begin{array}{l}\text { Heating Equipment (more than } \\
\text { one may apply) }\end{array}$} \\
\hline Heat Pumps & 40 & 3,081 & 364 & 6 & 703 & 233 & 5 & 78 & (") \\
\hline 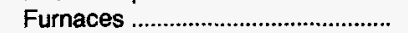 & 25 & 1,204 & 76 & 1 & 161 & 53 & 1 & 23 & (*) \\
\hline Individual Space Heaters .............. & 33 & 4,933 & 418 & 7 & 843 & 279 & 7 & 137 & 1 \\
\hline District Heat ....................................... & 71 & 12,712 & 1,861 & 26 & 2,437 & 807 & 16 & 265 & 1 \\
\hline 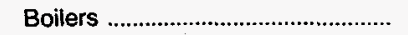 & 146 & 24,010 & 2,203 & 38 & 4,338 & 1,437 & 34 & 637 & 3 \\
\hline Packaged-Heating Units ................. & 67 & 5,997 & 675 & 9 & 1,054 & 349 & 7 & 273 & 1 \\
\hline \multicolumn{10}{|l|}{$\begin{array}{l}\text { Cooling Equipment (more than } \\
\text { one may apply) }\end{array}$} \\
\hline Residential-Type Central A/C ...... & 24 & 1,900 & 231 & 3 & 347 & 115 & 3 & 63 & (*) \\
\hline 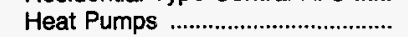 & 32 & 2,724 & 345 & 6 & 645 & 214 & 5 & 72 & $(*)$ \\
\hline 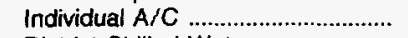 & 52 & 5,723 & 629 & 10 & 1,035 & 343 & 8 & 165 & 1 \\
\hline District Chilled Water ...................... & 31 & 7,647 & 1,044 & 14 & 1,751 & 580 & 10 & 157 & 1 \\
\hline Central Chillers ................................. & 139 & 27,723 & 2,710 & 45 & 5,139 & 1,702 & 39 & 675 & 3 \\
\hline 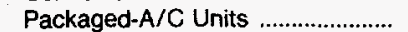 & 151 & 23,989 & 2,436 & 39 & 4,287 & 1,420 & 32 & 629 & 3 \\
\hline Swamp Coolers & 30 & 3,165 & 299 & 4 & 442 & 146 & 3 & 107 & $(")$ \\
\hline \multicolumn{10}{|l|}{$\begin{array}{l}\text { Lighting Equipment (more than } \\
\text { one may apply) }\end{array}$} \\
\hline 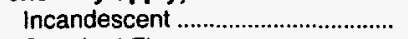 & 161 & 20,475 & 2,093 & 33 & 3,650 & 1,209 & 27 & 593 & 3 \\
\hline Standard Fluorescent ....................... & 309 & 42,850 & 4,520 & 71 & 7,862 & 2,604 & 58 & 1,063 & 5 \\
\hline 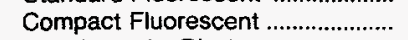 & 73 & 17,339 & 1,609 & 29 & 3,054 & 1,012 & 25 & 432 & 2 \\
\hline High-Intensity Discharge ............... & 75 & 18,736 & 1,699 & 28 & 3,392 & 1,123 & 23 & 319 & 1 \\
\hline 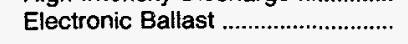 & 105 & 23,307 & 2,308 & 37 & 4,041 & 1,339 & 31 & 585 & 2 \\
\hline \multicolumn{10}{|l|}{$\begin{array}{l}\text { Water-Heating Equipment (more } \\
\text { than one may apply) }\end{array}$} \\
\hline Centralized System & 225 & 31,069 & 3,304 & 51 & 5,220 & 1,729 & 40 & 847 & 4 \\
\hline $\begin{array}{l}\text { Distributed System ........................... } \\
\text { Don't Know/ }\end{array}$ & 80 & 10,750 & 1,098 & 19 & 2,468 & 817 & 17 & 196 & 1 \\
\hline 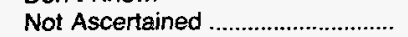 & 10 & 1,165 & 145 & 2 & 328 & 109 & 2 & 37 & $\left({ }^{*}\right)$ \\
\hline
\end{tabular}

See footnotes at end of table. 
Table 3.45. Consumption and Expenditures for Sum of Major Fuels, Electricity, and Natural Gas in FBSS Buildings in Federal Region 9, 1993 (Continued)

\begin{tabular}{|c|c|c|c|c|c|c|c|c|c|}
\hline \multirow{2}{*}{$\begin{array}{c}\text { Building } \\
\text { Characteristics }\end{array}$} & \multirow{2}{*}{$\begin{array}{l}\text { Number of } \\
\text { Buildings }\end{array}$} & \multirow{2}{*}{$\begin{array}{l}\text { Floorspace } \\
\text { (thousand } \\
\text { square } \\
\text { feet) }\end{array}$} & \multirow{2}{*}{\begin{tabular}{|c|} 
Sum of \\
Major \\
Fuel \\
Consump- \\
tion \\
(billion \\
Btu)
\end{tabular}} & \multirow{2}{*}{$\begin{array}{l}\text { Sum of } \\
\text { Major } \\
\text { Fuel } \\
\text { Expend- } \\
\text { itures } \\
\text { (million } \\
\text { dollars) }\end{array}$} & \multicolumn{2}{|c|}{$\begin{array}{l}\text { Electricity } \\
\text { Consumption } \\
\text { (billion Btu) }\end{array}$} & \multirow{2}{*}{$\begin{array}{l}\text { Electricity } \\
\text { Expend- } \\
\text { itures } \\
\text { (million } \\
\text { dollars) }\end{array}$} & \multirow{2}{*}{$\begin{array}{c}\text { Natural } \\
\text { Gas } \\
\text { Consump- } \\
\text { tion } \\
\text { (billion } \\
\text { Btu) }\end{array}$} & \multirow{2}{*}{$\begin{array}{l}\text { Natural } \\
\text { Gas } \\
\text { Expend- } \\
\text { itures } \\
\text { (million } \\
\text { dollars) }\end{array}$} \\
\hline & & & & & Primary & Site & & & \\
\hline \multicolumn{10}{|l|}{$\begin{array}{l}\text { Commercial Refrigeration } \\
\text { Equipment (more than one may } \\
\text { apply) }\end{array}$} \\
\hline 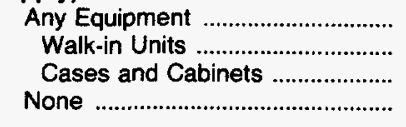 & $\begin{array}{r}63 \\
44 \\
50 \\
263\end{array}$ & $\begin{array}{l}18,781 \\
12,536 \\
14,928 \\
25,535\end{array}$ & $\begin{array}{l}2, C 16 \\
1,506 \\
1,589 \\
2,670\end{array}$ & $\begin{array}{l}31 \\
22 \\
25 \\
45\end{array}$ & $\begin{array}{l}3,565 \\
2,397 \\
2,558 \\
4,716\end{array}$ & $\begin{array}{r}1,181 \\
794 \\
847 \\
1,562\end{array}$ & $\begin{array}{l}24 \\
16 \\
19 \\
38\end{array}$ & $\begin{array}{l}397 \\
315 \\
345 \\
685\end{array}$ & $\begin{array}{l}2 \\
1 \\
2 \\
3\end{array}$ \\
\hline \multicolumn{10}{|l|}{$\begin{array}{l}\text { Retrofit or Purchase of any } \\
\text { Equipment Within Last Ten } \\
\text { Years (more than one may } \\
\text { apply) }\end{array}$} \\
\hline 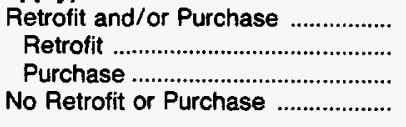 & $\begin{array}{l}213 \\
103 \\
148 \\
113\end{array}$ & $\begin{array}{r}34,547 \\
22,610 \\
19,642 \\
9,769\end{array}$ & $\begin{array}{l}3,592 \\
2,298 \\
2,263 \\
1,094\end{array}$ & $\begin{array}{l}59 \\
35 \\
39 \\
17\end{array}$ & $\begin{array}{l}6,291 \\
3,560 \\
4,276 \\
1,991\end{array}$ & $\begin{array}{r}2,084 \\
1,179 \\
1,416 \\
659\end{array}$ & $\begin{array}{l}49 \\
28 \\
33 \\
13\end{array}$ & $\begin{array}{l}905 \\
665 \\
511 \\
177\end{array}$ & $\begin{array}{l}4 \\
3 \\
2 \\
1\end{array}$ \\
\hline \multicolumn{10}{|l|}{$\begin{array}{l}\text { Energy Conservation Features } \\
\text { (more than one may apply) }\end{array}$} \\
\hline 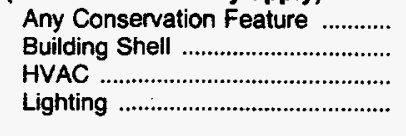 & $\begin{array}{l}319 \\
248 \\
307 \\
217\end{array}$ & $\begin{array}{l}44,023 \\
34,105 \\
43,116 \\
34,825\end{array}$ & $\begin{array}{l}4,676 \\
3,858 \\
4,610 \\
3,665\end{array}$ & $\begin{array}{l}76 \\
60 \\
74 \\
57\end{array}$ & $\begin{array}{l}8,262 \\
6,537 \\
8,112 \\
6,250\end{array}$ & $\begin{array}{l}2,737 \\
2,165 \\
2,687 \\
2,070\end{array}$ & $\begin{array}{l}62 \\
48 \\
61 \\
47\end{array}$ & $\begin{array}{r}1,080 \\
921 \\
1,063 \\
940\end{array}$ & $\begin{array}{l}5 \\
4 \\
5 \\
4\end{array}$ \\
\hline \multicolumn{10}{|l|}{$\begin{array}{l}\text { HYAC Conservation Features } \\
\text { (more than one may apply) }\end{array}$} \\
\hline 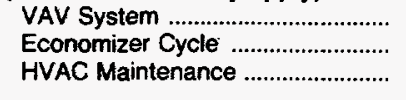 & $\begin{array}{r}75 \\
140 \\
306\end{array}$ & $\begin{array}{l}15,577 \\
26,367 \\
43,098\end{array}$ & $\begin{array}{l}1,690 \\
2,642 \\
4,607\end{array}$ & $\begin{array}{l}27 \\
42 \\
74\end{array}$ & $\begin{array}{l}3,013 \\
4,438 \\
8,108\end{array}$ & $\begin{array}{r}998 \\
1,470 \\
2,686\end{array}$ & $\begin{array}{l}22 \\
34 \\
61\end{array}$ & $\begin{array}{r}403 \\
696 \\
1,062\end{array}$ & $\begin{array}{l}2 \\
3 \\
5\end{array}$ \\
\hline \multicolumn{10}{|l|}{$\begin{array}{l}\text { Lighting Conservation Features } \\
\text { (more than one may apply) }\end{array}$} \\
\hline $\begin{array}{l}\text { Specular Reflectors .......................... } \\
\text { Natural Lighting Control }\end{array}$ & 124 & 21,850 & 2,085 & 32 & 3,417 & 1,132 & 26 & 555 & 2 \\
\hline 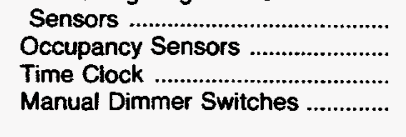 & $\begin{array}{r}58 \\
109 \\
41 \\
78\end{array}$ & $\begin{array}{l}13,760 \\
25,245 \\
10,539 \\
14,455\end{array}$ & $\begin{array}{r}1,174 \\
2,618 \\
953 \\
1,808\end{array}$ & $\begin{array}{l}19 \\
42 \\
14 \\
26\end{array}$ & $\begin{array}{l}1,919 \\
4,594 \\
1,450 \\
2,766\end{array}$ & $\begin{array}{r}636 \\
1,522 \\
480 \\
916\end{array}$ & $\begin{array}{l}16 \\
35 \\
11 \\
21\end{array}$ & $\begin{array}{l}298 \\
568 \\
278 \\
586\end{array}$ & $\begin{array}{l}1 \\
3 \\
1 \\
2\end{array}$ \\
\hline \multicolumn{10}{|l|}{$\begin{array}{l}\text { Energy Management Practices } \\
\text { (more than one may apply) } \\
\text { Energy Management and Control }\end{array}$} \\
\hline $\begin{array}{l}\text { System } \\
\text { Energy Conservation }\end{array}$ & 71 & 18,231 & 1,942 & 30 & 3,827 & 1,268 & 25 & 372 & 2 \\
\hline 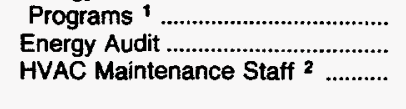 & $\begin{array}{r}124 \\
99 \\
52\end{array}$ & $\begin{array}{l}24,699 \\
17,444 \\
14,570\end{array}$ & $\begin{array}{l}2,623 \\
1,737 \\
1,473\end{array}$ & $\begin{array}{l}41 \\
29 \\
22\end{array}$ & $\begin{array}{l}4,616 \\
3,377 \\
2,509\end{array}$ & $\begin{array}{r}1,529 \\
1,118 \\
831\end{array}$ & $\begin{array}{l}33 \\
24 \\
18\end{array}$ & $\begin{array}{l}677 \\
356 \\
383\end{array}$ & $\begin{array}{l}3 \\
2 \\
2\end{array}$ \\
\hline \multicolumn{10}{|l|}{$\begin{array}{l}\text { Off-Hours Reduction in } \\
\text { Equipment (more than one may } \\
\text { apply) }\end{array}$} \\
\hline 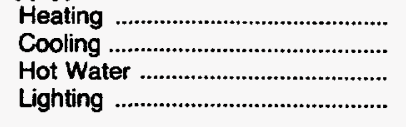 & $\begin{array}{r}151 \\
157 \\
68 \\
172\end{array}$ & $\begin{array}{l}19,631 \\
19,219 \\
13,614 \\
21,155\end{array}$ & $\begin{array}{r}1,547 \\
1,484 \\
922 \\
1,692\end{array}$ & $\begin{array}{l}26 \\
25 \\
17 \\
28\end{array}$ & $\begin{array}{l}2,777 \\
2,698 \\
1,796 \\
3,022\end{array}$ & $\begin{array}{r}920 \\
894 \\
595 \\
1,001\end{array}$ & $\begin{array}{l}22 \\
22 \\
15 \\
24\end{array}$ & $\begin{array}{l}468 \\
447 \\
279 \\
528\end{array}$ & $\begin{array}{l}2 \\
2 \\
1 \\
2\end{array}$ \\
\hline \multicolumn{10}{|l|}{$\begin{array}{l}\text { Sponsor of Program (more than } \\
\text { one may apply) }\end{array}$} \\
\hline 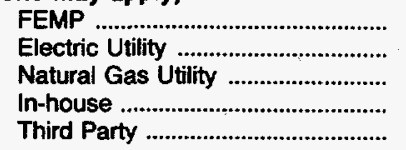 & $\begin{array}{r}1 \\
21 \\
2 \\
35 \\
3\end{array}$ & $\begin{array}{r}317 \\
6,670 \\
482 \\
6,695 \\
136\end{array}$ & $\begin{array}{r}20 \\
657 \\
19 \\
841 \\
18\end{array}$ & $\begin{array}{c}\left({ }^{*}\right) \\
10 \\
\left(^{*}\right) \\
12 \\
\left(^{\star}\right)\end{array}$ & $\begin{array}{r}54 \\
1,074 \\
44 \\
1,341 \\
19\end{array}$ & $\begin{array}{r}18 \\
356 \\
14 \\
444 \\
6\end{array}$ & $\begin{array}{c}(") \\
8 \\
(*) \\
9 \\
(")\end{array}$ & $\begin{array}{r}2 \\
247 \\
5 \\
194 \\
(")\end{array}$ & $\begin{array}{r}(*) \\
1 \\
\left.()^{*}\right) \\
1 \\
(*)\end{array}$ \\
\hline
\end{tabular}

See footnotes at end of table. 
Table 3.45. Consumption and Expenditures for Sum of Major Fuels, Electricity, and Natural Gas in FBSS Buildings in Federal Region 9, 1993 (Continued)

\begin{tabular}{|c|c|c|c|c|c|c|c|c|c|}
\hline \multirow{2}{*}{$\begin{array}{c}\text { Building } \\
\text { Characteristics }\end{array}$} & \multirow{2}{*}{$\begin{array}{l}\text { Number of } \\
\text { Buildings }\end{array}$} & \multirow{2}{*}{$\begin{array}{l}\text { Floorspace } \\
\text { (thousand } \\
\text { square } \\
\text { feet) }\end{array}$} & \multirow{2}{*}{$\begin{array}{l}\text { Sum of } \\
\text { Major } \\
\text { Fuel } \\
\text { Consump- } \\
\text { tion } \\
\text { (billion } \\
\text { Btu) }\end{array}$} & \multirow{2}{*}{$\begin{array}{l}\text { Sum of } \\
\text { Major } \\
\text { Fuel } \\
\text { Expend- } \\
\text { itures } \\
\text { (million } \\
\text { dollars) }\end{array}$} & \multicolumn{2}{|c|}{$\begin{array}{l}\text { Electricity } \\
\text { Consumption } \\
\text { (billion Btu) }\end{array}$} & \multirow{2}{*}{$\begin{array}{l}\text { Electricity } \\
\text { Expend- } \\
\text { itures } \\
\text { (million } \\
\text { dollars) }\end{array}$} & \multirow{2}{*}{$\begin{array}{c}\text { Natural } \\
\text { Gas } \\
\text { Consump- } \\
\text { tion } \\
\text { (billion } \\
\text { Btu) }\end{array}$} & \multirow{2}{*}{$\begin{array}{l}\text { Natural } \\
\text { Gas } \\
\text { Expend- } \\
\text { itures } \\
\text { (million } \\
\text { dollars) }\end{array}$} \\
\hline & & & & & Primary & Site & & & \\
\hline \multicolumn{10}{|l|}{$\begin{array}{l}\text { Type of Assistance (more than } \\
\text { one may apply) } \\
\text { Federal Energy Efficiency }\end{array}$} \\
\hline Fund & 3 & 1,725 & 236 & 4 & 695 & 230 & 4 & 6 & $\left({ }^{*}\right)$ \\
\hline 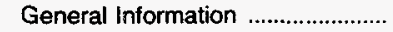 & 3 & 2,013 & 227 & 4 & 429 & 142 & 3 & 32 & (") \\
\hline Site-Specific Information ................. & 15 & 2,099 & 350 & 6 & 598 & 198 & 4 & 107 & 1 \\
\hline 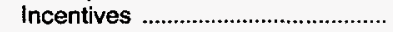 & 14 & 3,131 & 269 & 5 & 499 & 165 & 4 & 103 & (*) \\
\hline Alternate Rates & 13 & 3,071 & 273 & 3 & 408 & 135 & 2 & 76 & (*) \\
\hline Fuel Switching . ..................................... & 1 & 164 & 6 & $(*)$ & 10 & 3 & (*) & 2 & (*) \\
\hline Yes & 52 & 14,283 & 1,884 & 31 & 3,613 & 1,197 & 27 & 438 & 2 \\
\hline 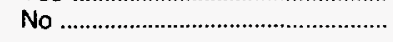 & 274 & 30,032 & 2,802 & 44 & 4,669 & 1.546 & 35 & 644 & 3 \\
\hline \multicolumn{10}{|l|}{$\begin{array}{l}\text { Natural Gas Transported } \\
\text { for the Account of Others }\end{array}$} \\
\hline Used in Building & 24 & 5,201 & 659 & 9 & 821 & 272 & 6 & 230 & 1 \\
\hline $\begin{array}{l}\text { Not Used in Building } \\
\text { Don't Know/................... }\end{array}$ & 174 & 26,787 & 2,595 & 42 & 4,706 & 1,559 & 35 & 760 & 3 \\
\hline Not Ascertained & 25 & 2,631 & 353 & 6 & 575 & 190 & 4 & 88 & $(*)$ \\
\hline
\end{tabular}

1 Building participates in any programs sponsored by the Federal Energy Management Program, in-house, utility, or third party.

2 HVAC maintenance staff means at least one person spends at least half their working hours maintaining the heating/cooling equipment.

$(*)=$ Value rounds to zero in the units displayed.

NC $=$ No cases in responding sample.

Notes: - Total workers are the number of workers during the main shift. - See Glossary for explanation of abbreviations and definitions of terms used in this report. - These data are from 881 federally owned buildings having the following criteria: (1) located in Federal Regions 3 , 6 , or $9 ;$ (2) larger than 10,000 square feet; and (3) used for a commercial purpose, other than warehouse and storage. In addition, 9 out of 10 selected buildings were from agencies other than the Department of Defense. - Statistics for the "energy end uses" represent consumption in buildings that have end use, not consumption for a particular fuel for a particular end use. - Site electricity is the amount of electricity delivered to commercial buildings.

Primary electricity is site electricity plus the conversion losses in the electric generation process at the utility plant. $\bullet$ FBSS = Federal Buildings Supplemental Survey. - HVAC = Heating, Ventilation, and Air Conditioning. - Data are for Fiscal Year 1993 (October 1, 1992 through September 30, 1993). Because of rounding, data may not sum to totals.

Source: Energy Information Administration, Office of Energy Markets and End Use, 1993 Federal Buildings Supplemental Survey. 
Table 3.46. Electricity Consumption and Expenditure Intensities in FBSS Buildings in Federal Region 3, 1993

\begin{tabular}{|c|c|c|c|c|c|c|c|c|c|c|}
\hline \multirow{2}{*}{$\begin{array}{c}\text { Building } \\
\text { Characteristics }\end{array}$} & \multicolumn{7}{|c|}{ Electricity Consumption } & \multicolumn{3}{|c|}{ Electricity Expenditures } \\
\hline & $\begin{array}{l}\text { Total } \\
\text { (million } \\
\text { kWh) }\end{array}$ & $\begin{array}{c}\text { per } \\
\text { Building } \\
\text { (thousand } \\
\text { kWh) }\end{array}$ & $\begin{array}{c}\text { per } \\
\text { square } \\
\text { Foot } \\
(\mathbf{k W h})\end{array}$ & $\begin{array}{c}\text { per } \\
\text { Worker } \\
\text { (thousand } \\
\text { kWh) }\end{array}$ & \multicolumn{3}{|c|}{$\begin{array}{l}\text { Distribution of } \\
\text { Building-Level Intensities } \\
\text { (kWh/square foot) }\end{array}$} & $\begin{array}{c}\text { per } \\
\text { Building } \\
\text { (thousand } \\
\text { dollars) }\end{array}$ & $\begin{array}{c}\text { per } \\
\text { Square } \\
\text { Foot } \\
\text { (dollars) }\end{array}$ & $\begin{array}{l}\text { per kWh } \\
\text { (dollars) }\end{array}$ \\
\hline 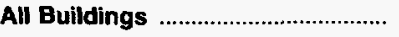 & 1,985 & 6,382 & 21.1 & 6.4 & 11.8 & 17.3 & 27.4 & 387.5 & 1.28 & 0.06 \\
\hline \multicolumn{11}{|l|}{$\begin{array}{l}\text { Building Floorspace (square } \\
\text { feet) }\end{array}$} \\
\hline 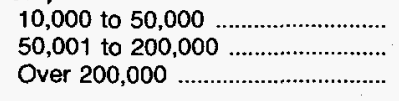 & $\begin{array}{r}59 \\
337 \\
1,589\end{array}$ & $\begin{array}{r}625 \\
2,879 \\
15,892\end{array}$ & $\begin{array}{l}22.9 \\
25.6 \\
20.3\end{array}$ & $\begin{array}{r}9.5 \\
11.3 \\
5.8\end{array}$ & $\begin{array}{l}12.0 \\
11.4 \\
12.4\end{array}$ & $\begin{array}{l}17.1 \\
18.1 \\
17.0\end{array}$ & $\begin{array}{l}25.5 \\
31.7 \\
23.4\end{array}$ & $\begin{array}{r}37.0 \\
156.5 \\
987.3\end{array}$ & $\begin{array}{l}1.36 \\
1.39 \\
1.26\end{array}$ & $\begin{array}{l}.06 \\
.05 \\
.06\end{array}$ \\
\hline 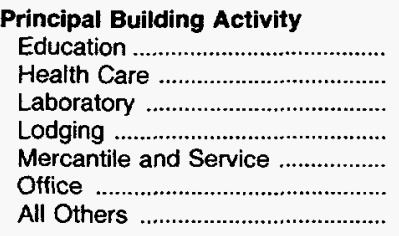 & $\begin{array}{r}8 \\
215 \\
218 \\
38 \\
130 \\
1,169 \\
206\end{array}$ & $\begin{array}{l}1,026 \\
5,255 \\
5,897 \\
2,933 \\
2,889 \\
9,429 \\
4,781\end{array}$ & $\begin{array}{l}13.7 \\
14.8 \\
42.2 \\
14.9 \\
17.9 \\
20.6 \\
28.7\end{array}$ & $\begin{array}{r}7.8 \\
9.2 \\
22.5 \\
31.4 \\
2.5 \\
5.7 \\
12.0\end{array}$ & $\begin{array}{r}6.7 \\
11.8 \\
31.7 \\
12.0 \\
7.7 \\
12.1 \\
13.2\end{array}$ & $\begin{array}{l}12.2 \\
13.2 \\
39.2 \\
18.5 \\
12.8 \\
16.9 \\
18.8\end{array}$ & $\begin{array}{l}28.3 \\
18.1 \\
49.7 \\
22.0 \\
18.3 \\
25.7 \\
28.0\end{array}$ & $\begin{array}{r}51.8 \\
280.4 \\
321.5 \\
152.7 \\
174.7 \\
608.0 \\
267.0\end{array}$ & $\begin{array}{r}.69 \\
.79 \\
2.30 \\
.78 \\
1.08 \\
1.33 \\
1.61\end{array}$ & $\begin{array}{l}.05 \\
.05 \\
.05 \\
.05 \\
.06 \\
.06 \\
.06\end{array}$ \\
\hline 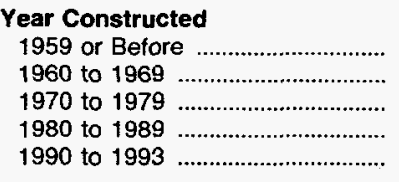 & $\begin{array}{r}849 \\
454 \\
438 \\
168 \\
75\end{array}$ & $\begin{array}{l}5,981 \\
6,483 \\
9,532 \\
4,425 \\
5,003\end{array}$ & $\begin{array}{l}19.4 \\
23.2 \\
25.8 \\
18.7 \\
15.7\end{array}$ & $\begin{array}{r}4.4 \\
10.0 \\
10.0 \\
7.0 \\
11.2\end{array}$ & $\begin{array}{l}11.4 \\
17.3 \\
12.0 \\
13.1 \\
12.5\end{array}$ & $\begin{array}{l}14.4 \\
24.2 \\
19.1 \\
18.1 \\
14.3\end{array}$ & $\begin{array}{l}20.9 \\
38.4 \\
28.0 \\
23.4 \\
18.5\end{array}$ & $\begin{array}{l}403.7 \\
383.8 \\
497.2 \\
241.8 \\
284.4\end{array}$ & $\begin{array}{r}1.31 \\
1.37 \\
1.34 \\
1.02 \\
.89\end{array}$ & $\begin{array}{l}.07 \\
.06 \\
.05 \\
.05 \\
.06\end{array}$ \\
\hline \multicolumn{11}{|l|}{$\begin{array}{l}\text { Energy Sources (more than one } \\
\text { may apply) }\end{array}$} \\
\hline 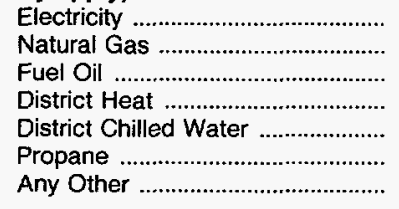 & $\begin{array}{r}1,985 \\
1,205 \\
885 \\
1,361 \\
480 \\
17 \\
75\end{array}$ & $\begin{array}{l}6,382 \\
8,732 \\
9,938 \\
8,840 \\
8,413 \\
3,483 \\
9,346\end{array}$ & $\begin{array}{l}21.1 \\
20.8 \\
22.5 \\
21.3 \\
20.9 \\
18.5 \\
16.7\end{array}$ & $\begin{array}{r}6.4 \\
5.2 \\
9.1 \\
5.6 \\
7.4 \\
19.1 \\
6.3\end{array}$ & $\begin{array}{l}11.8 \\
11.2 \\
11.7 \\
12.5 \\
13.2 \\
12.7 \\
11.7\end{array}$ & $\begin{array}{l}17.3 \\
16.5 \\
18.2 \\
18.3 \\
22.4 \\
21.0 \\
16.3\end{array}$ & $\begin{array}{l}27.4 \\
22.9 \\
23.9 \\
31.7 \\
38.4 \\
22.3 \\
24.8\end{array}$ & $\begin{array}{l}387.5 \\
544.3 \\
521.7 \\
564.8 \\
447.9 \\
298.3 \\
539.9\end{array}$ & $\begin{array}{r}1.28 \\
1.30 \\
1.18 \\
1.36 \\
1.11 \\
1.58 \\
.97\end{array}$ & $\begin{array}{l}.06 \\
.06 \\
.05 \\
.06 \\
.05 \\
.09 \\
.06\end{array}$ \\
\hline \multicolumn{11}{|l|}{$\begin{array}{l}\text { Energy End Uses (more than } \\
\text { one may apply) }\end{array}$} \\
\hline 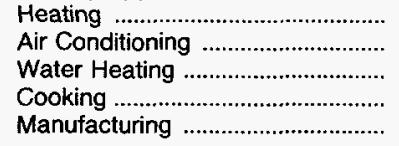 & $\begin{array}{r}1,979 \\
1,981 \\
1,965 \\
1,498 \\
651\end{array}$ & $\begin{array}{r}6,469 \\
6,495 \\
6,508 \\
13,870 \\
13,010\end{array}$ & $\begin{array}{l}21.2 \\
21.2 \\
21.3 \\
21.4 \\
24.1\end{array}$ & $\begin{array}{l}6.4 \\
6.4 \\
6.4 \\
5.9 \\
6.5\end{array}$ & $\begin{array}{l}12.0 \\
12.3 \\
12.0 \\
12.8 \\
12.7\end{array}$ & $\begin{array}{l}17.5 \\
17.6 \\
17.6 \\
17.9 \\
18.9\end{array}$ & $\begin{array}{l}27.5 \\
27.5 \\
27.5 \\
27.1 \\
28.5\end{array}$ & $\begin{array}{l}392.5 \\
394.3 \\
394.6 \\
857.7 \\
686.3\end{array}$ & $\begin{array}{l}1.29 \\
1.29 \\
1.29 \\
1.32 \\
1.27\end{array}$ & $\begin{array}{l}.06 \\
.06 \\
.06 \\
.06 \\
.05\end{array}$ \\
\hline \multicolumn{11}{|l|}{ Workers (main shift) } \\
\hline $\begin{array}{l}\text { Less than } 50 \\
50 \text { to } 99 \\
100 \text { to } 499 \\
500 \text { or More }\end{array}$ & $\begin{array}{r}53 \\
33 \\
336 \\
1,563\end{array}$ & $\begin{array}{r}749 \\
837 \\
3,140 \\
16,628\end{array}$ & $\begin{array}{l}13.0 \\
17.2 \\
24.6 \\
21.0\end{array}$ & $\begin{array}{r}33.6 \\
12.4 \\
12.8 \\
5.6\end{array}$ & $\begin{array}{l}10.0 \\
11.4 \\
12.3 \\
13.1\end{array}$ & $\begin{array}{l}13.2 \\
18.0 \\
19.8 \\
17.7\end{array}$ & $\begin{array}{l}19.6 \\
31.7 \\
34.7 \\
26.2\end{array}$ & $\begin{array}{r}44.7 \\
48.3 \\
171.7 \\
1,033.0\end{array}$ & $\begin{array}{r}.77 \\
.99 \\
1.35 \\
1.30\end{array}$ & $\begin{array}{l}.06 \\
.06 \\
.05 \\
.06\end{array}$ \\
\hline \multicolumn{11}{|l|}{ Weekly Operating Hours } \\
\hline 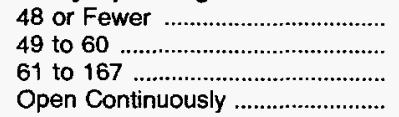 & $\begin{array}{r}127 \\
479 \\
257 \\
1,121\end{array}$ & $\begin{array}{l}2,493 \\
6,304 \\
4,287 \\
9,043\end{array}$ & $\begin{array}{l}13.1 \\
18.8 \\
21.4 \\
23.9\end{array}$ & $\begin{array}{l}6.2 \\
7.1 \\
9.3 \\
5.7\end{array}$ & $\begin{array}{r}11.3 \\
12.3 \\
9.4 \\
12.6\end{array}$ & $\begin{array}{l}14.3 \\
16.4 \\
17.4 \\
18.5\end{array}$ & $\begin{array}{l}21.0 \\
27.5 \\
25.2 \\
34.0\end{array}$ & $\begin{array}{l}323.4 \\
391.8 \\
244.6 \\
480.5\end{array}$ & $\begin{array}{l}1.70 \\
1.17 \\
1.22 \\
1.27\end{array}$ & $\begin{array}{l}.13 \\
.06 \\
.06 \\
.05\end{array}$ \\
\hline
\end{tabular}

See footnotes at end of table. 


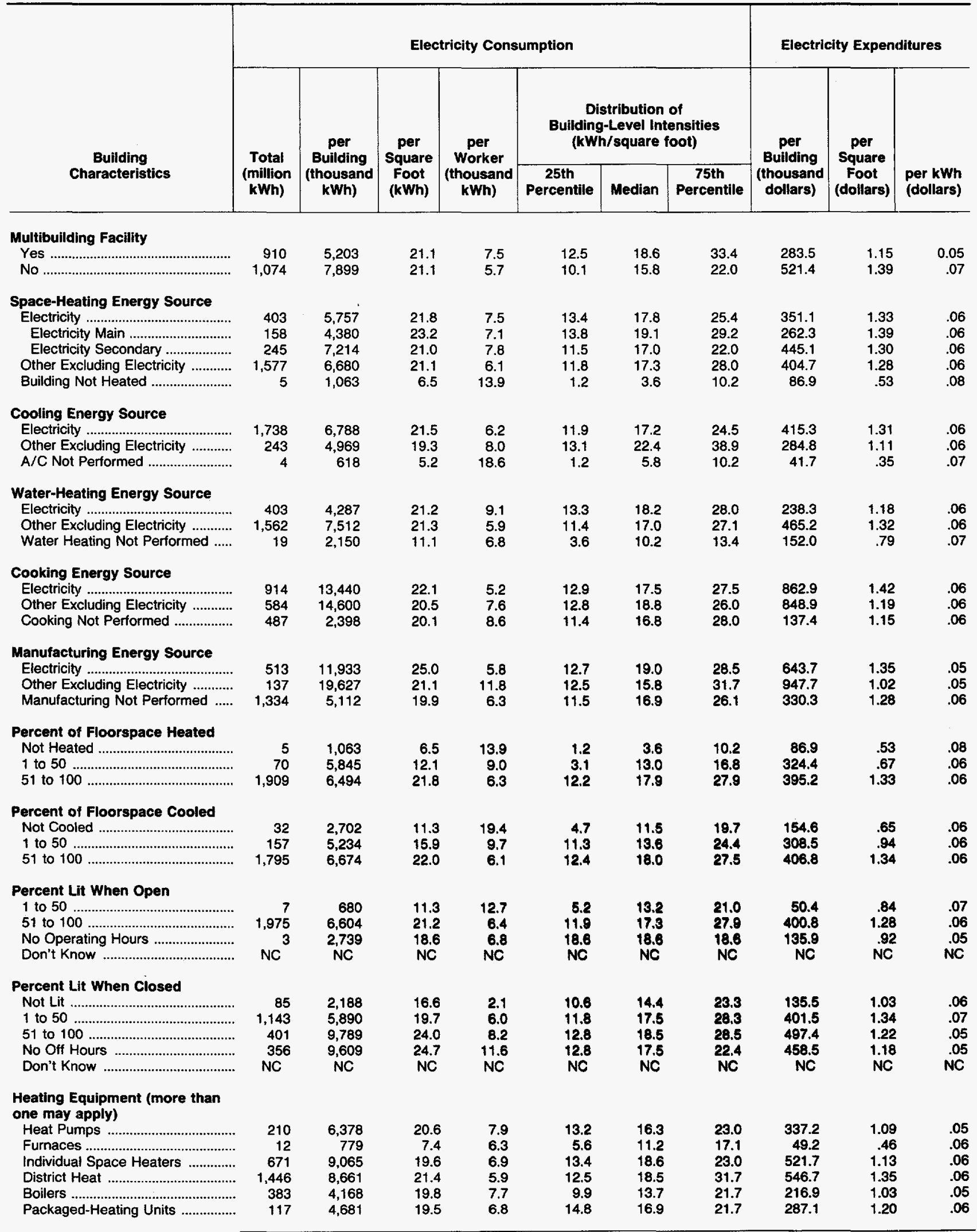

See footnotes at end of table. 
Table 3.46. Electricity Consumption and Expenditure Intensities in FBSS Buildings in Federal Region 3, 1993 (Continued)

\begin{tabular}{|c|c|c|c|c|c|c|c|c|c|c|}
\hline \multirow{3}{*}{$\begin{array}{c}\text { Building } \\
\text { Characteristics }\end{array}$} & \multicolumn{7}{|c|}{ Electricity Consumption } & \multicolumn{3}{|c|}{ Electricity Expenditures } \\
\hline & \multirow{2}{*}{$\begin{array}{l}\text { Total } \\
\text { (million } \\
\text { kWh) }\end{array}$} & \multirow{2}{*}{$\begin{array}{c}\text { per } \\
\text { Building } \\
\text { (thousand } \\
\text { kWh) }\end{array}$} & \multirow{2}{*}{$\begin{array}{c}\text { per } \\
\text { Square } \\
\text { Foot } \\
\text { (kWh) }\end{array}$} & \multirow{2}{*}{$\begin{array}{c}\text { per } \\
\text { Worker } \\
\text { (thousand } \\
\text { kWh) }\end{array}$} & \multicolumn{3}{|c|}{$\begin{array}{l}\text { Distribution of } \\
\text { Bullding-Level Intensities } \\
\text { (kWh/square foot) }\end{array}$} & \multirow{2}{*}{$\begin{array}{c}\text { per } \\
\text { Building } \\
\text { (thousand } \\
\text { dollars) }\end{array}$} & \multirow{2}{*}{\begin{tabular}{|} 
per \\
Square \\
Foot \\
(dollars)
\end{tabular}} & \multirow[b]{2}{*}{$\begin{array}{l}\text { per kWh } \\
\text { (dollars) }\end{array}$} \\
\hline & & & & & $\begin{array}{c}\text { 25th } \\
\text { Percentile }\end{array}$ & Median & $\begin{array}{c}\text { 75th } \\
\text { Percentile }\end{array}$ & & & \\
\hline \multicolumn{11}{|l|}{$\begin{array}{l}\text { Cooling Equipment (more than } \\
\text { one may apply) }\end{array}$} \\
\hline Residential-Type Central A/C ...... & 417 & 13,890 & 21.8 & 3.2 & 10.5 & 15.1 & 17.6 & $1,031.4$ & 1.62 & 0.07 \\
\hline 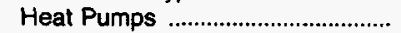 & 217 & 5,862 & 19.0 & 7.7 & 10.6 & 15.1 & 23.0 & 339.2 & 1.10 & .06 \\
\hline Individual $\mathrm{A} / \mathrm{C}$ & 494 & 6,169 & 17.6 & 3.5 & 11.4 & 15.6 & 21.0 & 393.6 & 1.12 & .06 \\
\hline District Chilled Water ...................... & 613 & 8,520 & 21.8 & 7.8 & 13.2 & 19.0 & 38.3 & 444.0 & 1.13 & .05 \\
\hline Packaged-A/C Units ......................... & 1,099 & 8,654 & 19.4 & 4.9 & 12.0 & 17.6 & 22.7 & 567.0 & 1.27 & .07 \\
\hline 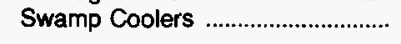 & & 6,319 & 50.2 & 22.2 & 41.0 & 49.7 & 51.8 & 431.9 & 3.43 & .07 \\
\hline \multicolumn{11}{|l|}{$\begin{array}{l}\text { Lighting Equipment (more than } \\
\text { one may apply) }\end{array}$} \\
\hline 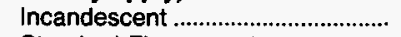 & 1,400 & 7,607 & 21.5 & 6.7 & 11.8 & 17.2 & 31.6 & 460.6 & 1.30 & .06 \\
\hline Standard Fluorescent ...................... & 1,928 & 6,448 & 21.1 & 6.9 & 11.8 & 16.9 & 27.5 & 389.7 & 1.28 & .06 \\
\hline Compact Fluorescent ..................... & 1,000 & 12,501 & 20.2 & 5.5 & 11.5 & 16.5 & 23.2 & 787.1 & 1.27 & .06 \\
\hline High-Intensity Discharge ................ & 1,135 & 10,321 & 21.9 & 5.2 & 13.4 & 18.6 & 28.3 & 564.6 & 1.20 & .05 \\
\hline Electronic Ballast ............................... & 1,288 & 9,610 & 19.9 & 5.9 & 12.4 & 16.8 & 23.4 & 612.7 & 1.27 & .06 \\
\hline \multicolumn{11}{|l|}{$\begin{array}{l}\text { Water-Heating Equipment (more } \\
\text { than one may apply) }\end{array}$} \\
\hline 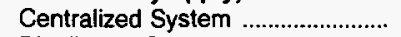 & 1,389 & 6,230 & 21.9 & 6.0 & 11.7 & 16.9 & 27.4 & 356.7 & 1.25 & .06 \\
\hline $\begin{array}{l}\text { Distributed System } \\
\text { Don't Know......................... }\end{array}$ & 452 & 6,453 & 18.8 & 6.9 & 12.8 & 18.7 & 27.5 & 491.9 & 1.44 & .08 \\
\hline 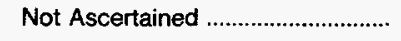 & 125 & 13,834 & 25.1 & 12.0 & 12.8 & 21.8 & 42.7 & 576.2 & 1.04 & .04 \\
\hline \multicolumn{11}{|l|}{$\begin{array}{l}\text { Commercial Refrigeration } \\
\text { Equipment (more than one may } \\
\text { apply) }\end{array}$} \\
\hline Any Equipment & 1,370 & 12,689 & 22.5 & 5.8 & 12.9 & 19.4 & 33.0 & 780.3 & 1.38 & .06 \\
\hline 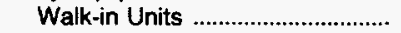 & 1,195 & 15,526 & 22.3 & 5.6 & 13.5 & 20.4 & 32.0 & 969.7 & 1.40 & .06 \\
\hline 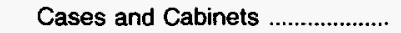 & 1,217 & 14,149 & 23.1 & 8.9 & 13.1 & 20.3 & 33.4 & 885.4 & 1.44 & .06 \\
\hline None & 614 & 3,027 & 18.5 & 8.2 & 11.4 & 16.3 & 24.4 & 178.6 & 1.09 & .06 \\
\hline \multicolumn{11}{|l|}{$\begin{array}{l}\text { Retrofit or Purchase of any } \\
\text { Equipment Within Last Ten } \\
\text { Years (more than one may } \\
\text { apply) }\end{array}$} \\
\hline Retrofit and/or Purchase ................... & 1,613 & 7,074 & 20.0 & 5.6 & 11.9 & 16.7 & 24.1 & 447.9 & 1.27 & .06 \\
\hline 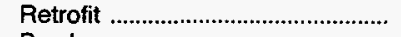 & 820 & 10,516 & 19.3 & 6.8 & 11.9 & 16.6 & 26.1 & 610.5 & 1.12 & .06 \\
\hline 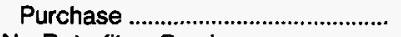 & 1,270 & 6,791 & 20.4 & 5.5 & 11.4 & 16.8 & 23.3 & 434.0 & 1.30 & .06 \\
\hline No Retrofit or Purchase ...................... & 372 & 4,482 & 27.4 & 14.5 & 11.5 & 18.8 & 38.2 & 221.8 & 1.35 & .05 \\
\hline \multicolumn{11}{|l|}{$\begin{array}{l}\text { HVAC Conservation Features } \\
\text { (more than one may apply) }\end{array}$} \\
\hline VAV System & 791 & 9,536 & 21.5 & 5.9 & 12.8 & 18.1 & 28.3 & 518.0 & 1.17 & .05 \\
\hline 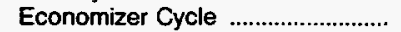 & 1,382 & 8,862 & 22.6 & 5.8 & 12.4 & 18.1 & 25.5 & 506.3 & 1.29 & .06 \\
\hline HVAC Maintenance ......................... & 1,972 & 6,574 & 21.3 & 6.4 & 12.2 & 17.5 & 27.8 & 399.6 & 1.29 & .06 \\
\hline \multicolumn{11}{|l|}{$\begin{array}{l}\text { Lighting Conservation Features } \\
\text { (more than one may apply) }\end{array}$} \\
\hline $\begin{array}{l}\text { Specular Reflectors .......................... } \\
\text { Natural Lighting Control }\end{array}$ & 919 & 7,470 & 20.9 & 5.6 & 11.4 & 14.8 & 22.0 & 413.2 & 1.15 & .06 \\
\hline 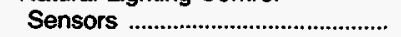 & 322 & 11,087 & 18.1 & 6.6 & 10.6 & 15.8 & 18.8 & 882.7 & 1.44 & .08 \\
\hline 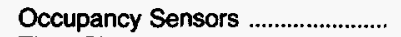 & 1,236 & 13,145 & 20.1 & 5.9 & 10.8 & 15.3 & 22.5 & 836.2 & 1.28 & .06 \\
\hline 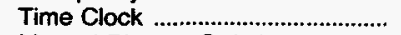 & 947 & 12,791 & 20.3 & 5.7 & 11.9 & 16.3 & 23.4 & 808.3 & 1.28 & .06 \\
\hline Manual Dimmer Switches ................ & 957 & 10,400 & 19.5 & 5.4 & 12.6 & 18.7 & 24.6 & 671.5 & 1.26 & .06 \\
\hline
\end{tabular}

See footnotes at end of table. 
Table 3.46. Electricity Consumption and Expenditure Intensities in FBSS Buildings in Federal Region 3, 1993 (Continued)

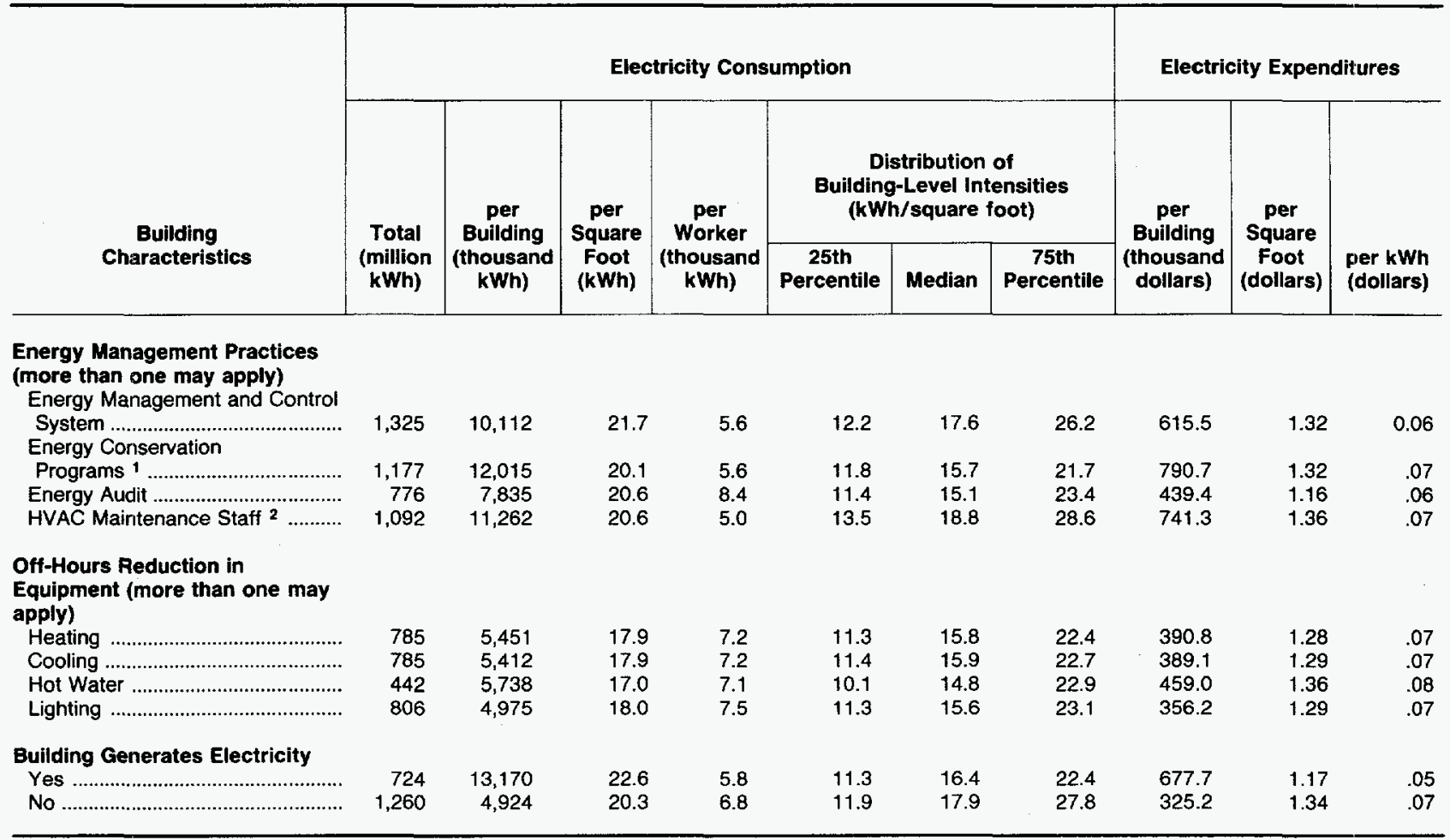

1 Building participates in any programs sponsored by the Federal Energy Management Program, in-house, utility, or third party.

2 HVAC maintenance staff means at least one person spends at least half their working hours maintaining the heating/cooling equipment.

Notes: - Total workers are the number of workers during the main shift. - See Glossary for explanation of abbreviations and definitions of terms used in this report. - These data are from 881 federally owned buildings having the following criteria: (1) located in Federal Regions 3,6 , or 9 ; (2) larger than 10,000 square feet; and (3) used for a commercial purpose, other than warehouse and storage. In addition, 9 out of 10 selected buildings were from agencies other than the Department of Defense. - Statistics for the "energy end uses" represent consumption in buildings that have end use, not consumption for a particular fuel for a particular end use. - A/C = Air Conditioning. - FBSS = Federal Buildings Supplemental Suivey. - HVAC = Heating, Ventilation, and Air Conditioning. • VAV = Variabie-Air Volume. $\bullet \mathrm{KWH}=\mathrm{Kilowatthour.} \bullet$ Data are for Fiscal Year 1993 (October 1, 1992 through September 30, 1993). - Because of rounding, data may not sum to totals.

Source: Energy Information Administration, Office of Energy Markets and End Use, 1993 Federal Buildings Supplemental Survey. 
Table 3.47. Electricity Consumption and Expenditure Intensities in FBSS Buildings in Federal Region 6, 1993

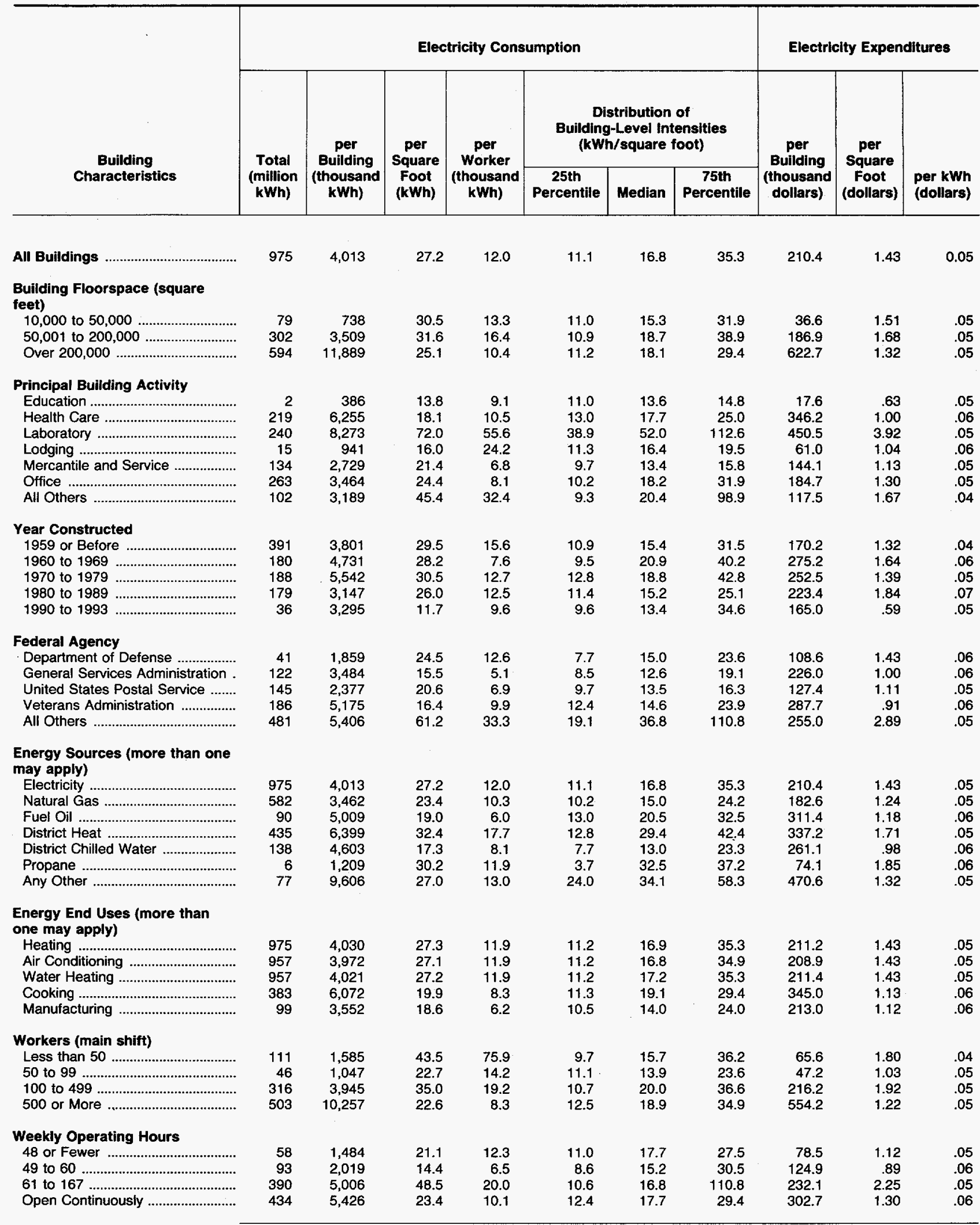

See footnotes at end of table. 
Table 3.47. Electricity Consumption and Expenditure Intensities in FBSS Buildings in Federal Region 6, 1993 (Continued)

\begin{tabular}{|c|c|c|c|c|c|c|c|c|c|c|}
\hline \multirow{3}{*}{$\begin{array}{c}\text { Building } \\
\text { Characteristics }\end{array}$} & \multicolumn{7}{|c|}{ Electricity Consumption } & \multicolumn{3}{|c|}{ Electricity Expenditures } \\
\hline & \multirow{2}{*}{$\begin{array}{l}\text { Total } \\
\text { (million } \\
\text { kWh) }\end{array}$} & \multirow{2}{*}{$\begin{array}{c}\text { per } \\
\text { Building } \\
\text { (thousand } \\
\text { kWh) }\end{array}$} & \multirow{2}{*}{$\begin{array}{c}\text { per } \\
\text { Square } \\
\text { Foot } \\
(\mathbf{k W h})\end{array}$} & \multirow{2}{*}{$\begin{array}{c}\text { per } \\
\text { Worker } \\
\text { (thousand } \\
\text { kWh) }\end{array}$} & \multicolumn{3}{|c|}{$\begin{array}{l}\text { Distribution of } \\
\text { Building-Level Intensities } \\
\text { (kWh/square foot) }\end{array}$} & \multirow{2}{*}{$\begin{array}{c}\text { per } \\
\text { Euilding } \\
\text { (thousand } \\
\text { dollars) }\end{array}$} & \multirow{2}{*}{$\begin{array}{c}\text { per } \\
\text { Square } \\
\text { Foot } \\
\text { (dollars) }\end{array}$} & \multirow[b]{2}{*}{$\begin{array}{l}\text { per kWh } \\
\text { (dollars) }\end{array}$} \\
\hline & & & & & $\begin{array}{c}\text { 25th } \\
\text { Percentile }\end{array}$ & Median & $\begin{array}{c}\text { 75th } \\
\text { Percentile }\end{array}$ & & & \\
\hline \multicolumn{11}{|l|}{ Multibuilding Facility } \\
\hline 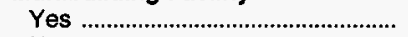 & 731 & 4,937 & 29.9 & 13.9 & 13.1 & 23.0 & 41.8 & 252.9 & 1.53 & 0.05 \\
\hline No & 245 & 2,574 & 21.5 & 8.5 & 9.6 & 13.2 & 20.1 & 144.2 & 1.20 & .06 \\
\hline \multicolumn{11}{|l|}{ Space-Heating Energy Source } \\
\hline 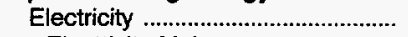 & 360 & 5,450 & 36.5 & 13.6 & 12.5 & 16.8 & 38.8 & 227.9 & 1.53 & .04 \\
\hline Electricity Secondary .................... & 239 & 6,826 & 45.8 & 19.2 & 9.7 & 16.9 & 108.5 & 250.6 & 1.68 & .04 \\
\hline Other Excluding Electricity ............ & 615 & 3,497 & 23.8 & 11.2 & 10.8 & 17.6 & 33.1 & 205.0 & 1.39 & .06 \\
\hline Building Not Heated ........................... & $(*)$ & 81 & 1.0 & (1) & 1.0 & 1.0 & 1.0 & 5.9 & .07 & .07 \\
\hline \multicolumn{11}{|l|}{ Cooling Energy Source } \\
\hline 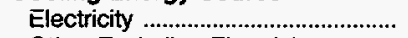 & 850 & 3,880 & 30.3 & 12.7 & 11.4 & 17.4 & 35.8 & 202.0 & 1.58 & .05 \\
\hline Other Excluding Electricity ............. & 108 & 4,893 & 14.9 & 8.1 & 7.7 & 12.8 & 20.4 & 276.9 & .84 & .06 \\
\hline A/C Not Performed ............................. & 18 & 8,987 & 33.1 & 15.0 & 1.0 & 19.9 & 38.8 & 395.2 & 1.46 & .04 \\
\hline \multicolumn{11}{|l|}{ Water-Heating Energy Source } \\
\hline Electricity & 285 & 4,595 & 23.2 & 9.2 & 9.5 & 14.5 & 31.9 & 244.7 & 1.24 & .05 \\
\hline Other Excluding Electricity ............. & 672 & 3,819 & 29.3 & 13.6 & 12.4 & 19.0 & 35.6 & 199.7 & 1.53 & .05 \\
\hline Water Heating Not Performed ..... & 18 & 3,659 & 30.7 & 14.3 & 1.9 & 5.0 & 13.9 & 162.7 & 1.37 & .04 \\
\hline \multicolumn{11}{|l|}{ Cooking Energy Source } \\
\hline 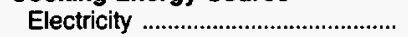 & 182 & 4,916 & 17.9 & 6.5 & 11.2 & 16.7 & 25.1 & 279.4 & 1.02 & .06 \\
\hline Other Excluding Electricity ............. & 201 & 7,718 & 22.2 & 11.0 & 17.7 & 23.6 & 32.5 & 438.2 & 1.26 & .06 \\
\hline Cooking Not Performed ................... & 593 & 3,293 & 35.7 & 16.8 & 10.3 & 15.2 & 36.7 & 163.3 & 1.77 & .05 \\
\hline \multicolumn{11}{|l|}{ Manufacturing Energy Source } \\
\hline Other Excluding Electricity ........... & 19 & 9,489 & 38.1 & 15.3 & 29.4 & 34.1 & 38.8 & 419.3 & 1.69 & .04 \\
\hline Manufacturing Not Performed ...... & 876 & 4,073 & 28.7 & 13.4 & 11.2 & 17.4 & 35.8 & 210.0 & 1.48 & .05 \\
\hline Percent of Floorspace Heated & & & & & & & & & & \\
\hline 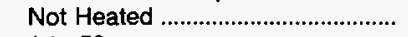 & (*) & 81 & 1.0 & (1) & 1.0 & 1.0 & 1.0 & 5.9 & .07 & .07 \\
\hline 1 to 50 & 22 & 2,013 & 17.2 & 10.1 & 8.2 & 13.5 & 21.0 & 119.9 & 1.02 & .06 \\
\hline 51 to $100 \ldots \ldots \ldots \ldots \ldots \ldots \ldots$ & 953 & 4,126 & 27.7 & 12.0 & 11.2 & 17.6 & 35.3 & 215.6 & 1.45 & .05 \\
\hline Percent of Floorspace Cooled & & & & & & & & & & \\
\hline 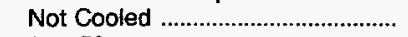 & 18 & 6,021 & 32.6 & 13.4 & 1.0 & 8.2 & 38.8 & 265.6 & 1.44 & .04 \\
\hline 1 to 50 & 75 & 2,870 & 38.3 & 22.9 & 10.9 & 22.3 & 111.0 & 110.4 & 1.47 & .04 \\
\hline 51 to 100 & 883 & 4,124 & 26.5 & 11.5 & 11.2 & 16.1 & 32.4 & 221.8 & 1.42 & .05 \\
\hline Percent Lit When Open & & & & & & & & & & \\
\hline 1 to 50 & 18 & 1,228 & 17.4 & 14.4 & 8.7 & 11.4 & 21.0 & 71.4 & 1.01 & .06 \\
\hline 51 to 100 & 957 & 4,215 & 27.6 & 11.9 & 11.4 & 17.6 & 35.7 & 220.5 & 1.44 & .05 \\
\hline No Operating Hours ........................ & $\left({ }^{*}\right)$ & 81 & 1.0 & (1) & 1.0 & 1.0 & 1.0 & 5.9 & .07 & .07 \\
\hline 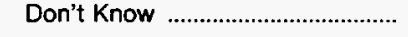 & NC & NC & NC & NC & NC & NC & NC & NC & NC & NC \\
\hline Percent Lit When Closed & & & & & & & & & & \\
\hline Not Lit & 34 & 1,025 & 12.6 & 5.6 & 7.5 & 13.9 & 16.9 & 64.8 & .79 & .06 \\
\hline 1 to 50 & 693 & 4,202 & 33.8 & 15.5 & 12.0 & 19.1 & 38.9 & 218.1 & 1.76 & .05 \\
\hline 51 to 100 & 136 & 5,435 & 16.9 & 6.8 & 11.4 & 15.4 & 23.9 & 288.6 & .90 & .05 \\
\hline No Off Hours . .............................. & 112 & 5,611 & 24.4 & 10.3 & 11.6 & 19.4 & 30.4 & 289.2 & 1.26 & .05 \\
\hline 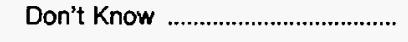 & NC & NC & NC & NC & NC & NC & NC & NC & NC & NC \\
\hline $\begin{array}{l}\text { Heating Equipment (more than } \\
\text { one may apply) }\end{array}$ & & & & & & & & & & \\
\hline 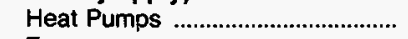 & 32 & 3,205 & 33.2 & 8.8 & 14.2 & 25.5 & 32.4 & 164.3 & 1.70 & .05 \\
\hline 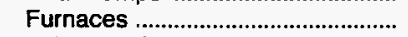 & 11 & 610 & 13.9 & 7.9 & 6.8 & 14.9 & 19.1 & 35.0 & .80 & .06 \\
\hline Individual Space Heaters ............. & 124 & 4,121 & 22.9 & 6.9 & 8.7 & 12.8 & 29.4 & 349.3 & 1.94 & .08 \\
\hline 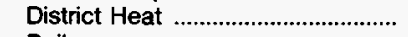 & 457 & 5,572 & 30.1 & 16.0 & 13.0 & 21.0 & 38.9 & 295.2 & 1.59 & .05 \\
\hline 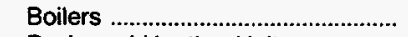 & 420 & 4,120 & 25.1 & 10.0 & 10.8 & 18.2 & 36.1 & 213.7 & 1.30 & .05 \\
\hline Packaged.Heating Units ................. & 199 & 3,371 & 26.3 & 10.1 & 9.8 & 14.8 & 25.0 & 194.3 & 1.51 & .06 \\
\hline
\end{tabular}

See footnotes at end of table. 


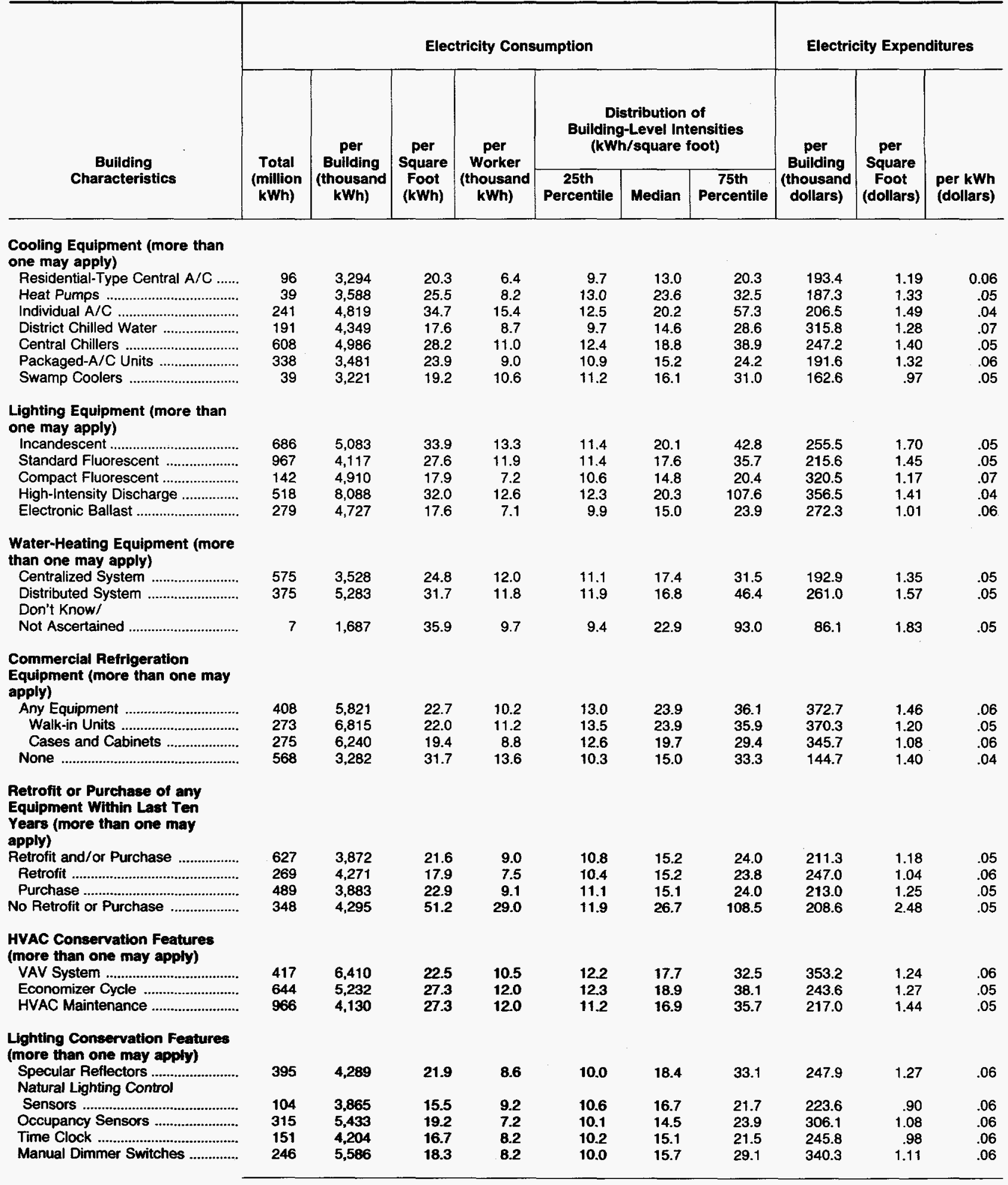

See footnotes at end of table. 
Table 3.47. Electricity Consumption and Expenditure Intensities in FBSS Buildings in Federal Region 6, 1993 (Continued)

\begin{tabular}{|c|c|c|c|c|c|c|c|c|c|c|}
\hline \multirow{3}{*}{$\begin{array}{c}\text { Building } \\
\text { Characteristics }\end{array}$} & \multicolumn{7}{|c|}{ Electricity Consumption } & \multicolumn{3}{|c|}{ Electricity Expenditures } \\
\hline & \multirow{2}{*}{$\begin{array}{l}\text { Total } \\
\text { (million } \\
\text { kWh) }\end{array}$} & \multirow{2}{*}{$\begin{array}{c}\text { per } \\
\text { Building } \\
\text { (thousand } \\
\mathbf{k W h} \text { ) }\end{array}$} & \multirow{2}{*}{$\begin{array}{l}\text { per } \\
\text { Square } \\
\text { Foot } \\
\text { (kWh) }\end{array}$} & \multirow{2}{*}{$\begin{array}{c}\text { per } \\
\text { Worker } \\
\text { (thousand } \\
\text { kWh) }\end{array}$} & \multicolumn{3}{|c|}{$\begin{array}{l}\text { Distribution of } \\
\text { Building-Level Intensities } \\
\text { (kWh/square foot) }\end{array}$} & \multirow{2}{*}{$\begin{array}{c}\text { per } \\
\text { Building } \\
\text { (thousand } \\
\text { dollars) }\end{array}$} & \multirow{2}{*}{$\begin{array}{c}\text { per } \\
\text { Square } \\
\text { Foot } \\
\text { (dollars) }\end{array}$} & \multirow[b]{2}{*}{$\begin{array}{l}\text { per kWh } \\
\text { (dollars) }\end{array}$} \\
\hline & & & & & $\begin{array}{c}\text { 25th } \\
\text { Percentile }\end{array}$ & Median & $\begin{array}{c}\text { 75th } \\
\text { Percentile }\end{array}$ & & & \\
\hline $\begin{array}{l}\text { Energy Management Practices } \\
\text { (more than one may apply) }\end{array}$ & & & & & & & & & & \\
\hline $\begin{array}{l}\text { System } \\
\text { Energy Conservation }\end{array}$ & 459 & 4,780 & 19.9 & 9.2 & 10.4 & 15.1 & 25.0 & 273.9 & 1.14 & 0.06 \\
\hline 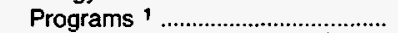 & 259 & 5,399 & 19.2 & 7.1 & 12.7 & 17.9 & 28.9 & 307.7 & 1.09 & .06 \\
\hline HVAC Maintenance Staff ${ }^{2}$ & 291 & 5,112 & 18.8 & 8.5 & 9.5 & 13.3 & 21.7 & 298.3 & 1.09 & .06 \\
\hline \multicolumn{11}{|l|}{$\begin{array}{l}\text { Off-Hours Reduction in } \\
\text { Equipment (more than one may } \\
\text { apply) }\end{array}$} \\
\hline 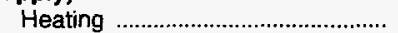 & 226 & 1,836 & 17.2 & 7.3 & 9.5 & 13.7 & 23.6 & 138.5 & 1.29 & .08 \\
\hline 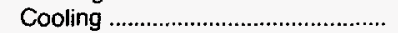 & 236 & 1,907 & 17.1 & 7.1 & 9.5 & 13.8 & 23.4 & 142.0 & 1.27 & .07 \\
\hline 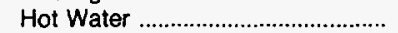 & 83 & 2,518 & 18.2 & 7.2 & 9.5 & 12.8 & 30.5 & 152.9 & 1.11 & .06 \\
\hline 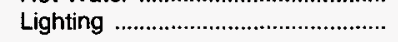 & 232 & 1,934 & 18.1 & 7.2 & 9.6 & 14.7 & 23.4 & 144.3 & 1.35 & .07 \\
\hline \multicolumn{11}{|l|}{ Building Generates Electricity } \\
\hline Yes & 162 & 5,061 & 15.7 & 7.6 & 10.9 & 17.5 & 25.9 & 281.5 & .87 & .06 \\
\hline No & 813 & 3,855 & 31.9 & 13.5 & 11.2 & 16.8 & 35.8 & 199.6 & 1.65 & .05 \\
\hline
\end{tabular}

1 Data or computation error.

1 Building participates in any programs sponsored by the Federal Energy Management Program, in-house, utility, or third party.

2 HVAC maintenance staff means at least one person spends at least half their working hours maintaining the heating/cooling equipment.

$\mathrm{NC}=$ No cases in responding sample.

Notes: - Total workers are the number of workers during the main shift. - See Glossary for explanation of abbreviations and definitions of terms used in this report. - These data are from 881 federally owned buildings having the following criteria: (1) located in Federal Regions 3 , 6 , or 9; (2) larger than 10,000 square feet; and (3) used for a commercial purpose, other than warehouse and storage. In addition, 9 out of 10 selected buildings were from agencies other than the Department of Defense. - Statistics for the "energy end uses" represent consumption in buildings that have end use, not consumption for a particular fuel for a particular end use. $\bullet A / C=$ Air Conditioning. $\bullet$ FBSS = Federal Buildings Supplemental Survey. - HVAC = Heating, Ventilation, and Air Conditioning. - VAV = Variable-Air Volume. - KWH = Kilowatthour. $\bullet$ Data are for Fiscal Year 1993 (October 1, 1992 through September 30, 1993). - Because of rounding, data may not sum to totals.

Source: Energy Information Administration, Office of Energy Markets and End Use, 1993 Federal Buildings Supplemental Survey. 
Table 3.48. Electricity Consumption and Expenditure Intensities in FBSS Buildings in Federal Region 9, 1993

\begin{tabular}{|c|c|c|c|c|c|c|c|c|c|c|}
\hline \multirow{2}{*}{$\begin{array}{c}\text { Building } \\
\text { Characteristics }\end{array}$} & \multicolumn{7}{|c|}{ Electricity Consumption } & \multicolumn{3}{|c|}{ Electricity Expenditures } \\
\hline & $\begin{array}{l}\text { Total } \\
\text { (million } \\
\text { kWh) }\end{array}$ & $\begin{array}{c}\text { per } \\
\text { Building } \\
\text { (thousand } \\
\text { kWh) }\end{array}$ & $\begin{array}{c}\text { per } \\
\text { Square } \\
\text { Foot } \\
\text { (kWh) }\end{array}$ & $\begin{array}{c}\text { per } \\
\text { Worker } \\
\text { (thousand } \\
\text { kWh) }\end{array}$ & \multicolumn{3}{|c|}{$\begin{array}{l}\text { Distribution of } \\
\text { Building-Level Intensities } \\
\text { (kWh/square foot) }\end{array}$} & $\begin{array}{c}\text { per } \\
\text { Building } \\
\text { (thousand } \\
\text { dollars) }\end{array}$ & $\begin{array}{c}\text { per } \\
\text { Square } \\
\text { Foot } \\
\text { (dollars) }\end{array}$ & $\begin{array}{l}\text { per kWh } \\
\text { (dollars) }\end{array}$ \\
\hline 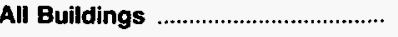 & 804 & 2,474 & 18.1 & 8.2 & 9.5 & 15.9 & 23.0 & 190.9 & 1.40 & 0.08 \\
\hline $\begin{array}{l}\text { Building Floorspace (square } \\
\text { feet) }\end{array}$ & & & & & & & & & & \\
\hline 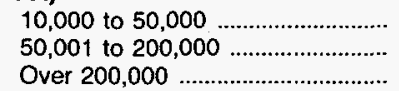 & $\begin{array}{r}78 \\
269 \\
456\end{array}$ & $\begin{array}{r}533 \\
2,190 \\
8,296\end{array}$ & $\begin{array}{l}20.4 \\
21.2 \\
16.4\end{array}$ & $\begin{array}{r}8.5 \\
10.4 \\
7.3\end{array}$ & $\begin{array}{l}9.2 \\
9.8 \\
8.5\end{array}$ & $\begin{array}{l}15.3 \\
19.1 \\
13.0\end{array}$ & $\begin{array}{l}22.9 \\
24.4 \\
18.0\end{array}$ & $\begin{array}{r}42.7 \\
165.7 \\
643.1\end{array}$ & $\begin{array}{l}1.63 \\
1.61 \\
1.27\end{array}$ & $\begin{array}{l}.08 \\
.08 \\
.08\end{array}$ \\
\hline 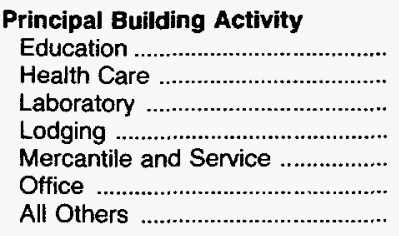 & $\begin{array}{r}6 \\
167 \\
94 \\
14 \\
128 \\
250 \\
145\end{array}$ & $\begin{array}{r}512 \\
3,484 \\
3,041 \\
640 \\
2,027 \\
2,716 \\
2,538\end{array}$ & $\begin{array}{r}9.8 \\
16.9 \\
36.2 \\
11.5 \\
15.6 \\
15.3 \\
26.9\end{array}$ & $\begin{array}{r}9.6 \\
7.7 \\
24.8 \\
21.6 \\
5.2 \\
6.4 \\
19.5\end{array}$ & $\begin{array}{r}1.4 \\
12.3 \\
20.2 \\
2.9 \\
9.4 \\
8.7 \\
10.1\end{array}$ & $\begin{array}{r}3.8 \\
17.1 \\
26.6 \\
13.7 \\
13.9 \\
15.1 \\
18.5\end{array}$ & $\begin{array}{r}8.8 \\
20.8 \\
47.6 \\
18.7 \\
19.3 \\
21.9 \\
25.3\end{array}$ & $\begin{array}{r}31.0 \\
243.5 \\
229.5 \\
53.6 \\
153.3 \\
246.0 \\
164.7\end{array}$ & $\begin{array}{r}.59 \\
1.18 \\
2.74 \\
.97 \\
1.18 \\
1.38 \\
1.75\end{array}$ & $\begin{array}{l}.06 \\
.07 \\
.08 \\
.08 \\
.08 \\
.09 \\
.06\end{array}$ \\
\hline 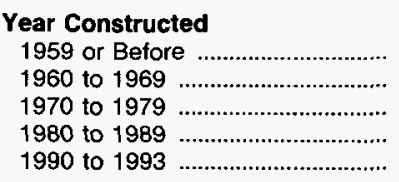 & $\begin{array}{r}280 \\
143 \\
201 \\
155 \\
25\end{array}$ & $\begin{array}{l}2,173 \\
2,597 \\
3,240 \\
2,384 \\
1,784\end{array}$ & $\begin{array}{l}20.7 \\
15.7 \\
16.7 \\
19.5 \\
14.8\end{array}$ & $\begin{array}{r}11.6 \\
7.0 \\
6.9 \\
7.5 \\
8.0\end{array}$ & $\begin{array}{r}8.3 \\
8.3 \\
9.5 \\
10.8 \\
13.7\end{array}$ & $\begin{array}{l}17.1 \\
15.9 \\
15.0 \\
16.2 \\
17.0\end{array}$ & $\begin{array}{l}24.4 \\
23.9 \\
20.4 \\
22.9 \\
24.1\end{array}$ & $\begin{array}{l}167.8 \\
197.7 \\
268.7 \\
166.1 \\
147.1\end{array}$ & $\begin{array}{l}1.60 \\
1.20 \\
1.38 \\
1.36 \\
1.22\end{array}$ & $\begin{array}{l}.08 \\
.08 \\
.08 \\
.07 \\
.08\end{array}$ \\
\hline $\begin{array}{l}\text { Federal Agency } \\
\text { Department of Defense ............... } \\
\text { General Services Administration. } \\
\text { United States Postal Service ....... } \\
\text { Veterans Administration } \\
\text { All Others }\end{array}$ & $\begin{array}{l}187 \\
144 \\
139 \\
150 \\
184\end{array}$ & $\begin{array}{l}2,426 \\
3,698 \\
2,203 \\
2,682 \\
2,045\end{array}$ & $\begin{array}{l}22.1 \\
11.5 \\
16.5 \\
17.0 \\
30.1\end{array}$ & $\begin{array}{r}13.5 \\
5.0 \\
5.7 \\
7.7 \\
17.0\end{array}$ & $\begin{array}{r}6.6 \\
7.2 \\
9.2 \\
14.4 \\
12.7\end{array}$ & $\begin{array}{l}13.0 \\
10.6 \\
15.2 \\
18.3 \\
23.6\end{array}$ & $\begin{array}{l}18.7 \\
16.2 \\
19.6 \\
21.8 \\
32.1\end{array}$ & $\begin{array}{l}186.2 \\
339.5 \\
168.6 \\
193.7 \\
144.3\end{array}$ & $\begin{array}{l}1.69 \\
1.06 \\
1.27 \\
1.23 \\
2.12\end{array}$ & $\begin{array}{l}.08 \\
.09 \\
.08 \\
.07 \\
.07\end{array}$ \\
\hline 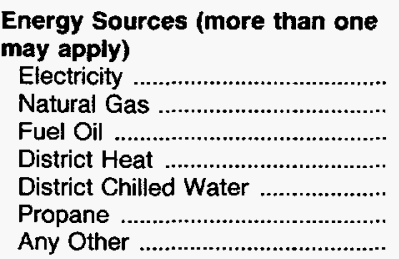 & $\begin{array}{r}804 \\
594 \\
222 \\
194 \\
106 \\
26 \\
37\end{array}$ & $\begin{array}{l}2,474 \\
2,639 \\
4,103 \\
2,657 \\
3,929 \\
2,633 \\
7,350\end{array}$ & $\begin{array}{l}18.1 \\
17.1 \\
19.9 \\
16.5 \\
16.7 \\
23.6 \\
14.1\end{array}$ & $\begin{array}{r}8.2 \\
7.9 \\
9.7 \\
8.4 \\
8.3 \\
12.2 \\
6.8\end{array}$ & $\begin{array}{r}9.5 \\
9.6 \\
12.0 \\
12.3 \\
15.3 \\
.7 \\
.8\end{array}$ & $\begin{array}{r}15.9 \\
15.9 \\
17.1 \\
17.2 \\
20.3 \\
4.8 \\
14.2\end{array}$ & $\begin{array}{l}23.0 \\
23.9 \\
31.4 \\
20.7 \\
25.0 \\
16.3 \\
14.4\end{array}$ & $\begin{array}{l}190.9 \\
203.9 \\
321.5 \\
198.0 \\
262.4 \\
212.5 \\
526.8\end{array}$ & $\begin{array}{l}1.40 \\
1.32 \\
1.56 \\
1.23 \\
1.11 \\
1.90 \\
1.01\end{array}$ & $\begin{array}{l}.08 \\
.08 \\
.08 \\
.07 \\
.07 \\
.08 \\
.07\end{array}$ \\
\hline 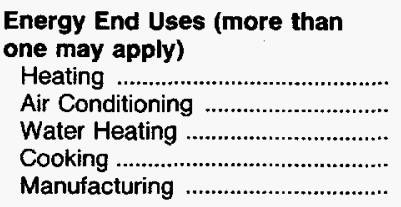 & $\begin{array}{l}752 \\
773 \\
778 \\
348 \\
157\end{array}$ & $\begin{array}{l}2,481 \\
2,647 \\
2,478 \\
4,967 \\
5,245\end{array}$ & $\begin{array}{l}17.9 \\
18.4 \\
18.1 \\
17.3 \\
20.5\end{array}$ & $\begin{array}{l}8.5 \\
8.3 \\
8.3 \\
7.5 \\
8.9\end{array}$ & $\begin{array}{r}9.5 \\
10.7 \\
9.4 \\
9.1 \\
12.3\end{array}$ & $\begin{array}{l}16.0 \\
16.8 \\
16.0 \\
14.6 \\
15.8\end{array}$ & $\begin{array}{l}23.4 \\
23.9 \\
23.4 \\
19.8 \\
23.0\end{array}$ & $\begin{array}{l}193.9 \\
201.7 \\
189.3 \\
349.3 \\
372.5\end{array}$ & $\begin{array}{l}1.40 \\
1.41 \\
1.38 \\
1.22 \\
1.45\end{array}$ & $\begin{array}{l}.08 \\
.08 \\
.08 \\
.07 \\
.07\end{array}$ \\
\hline $\begin{array}{l}\text { Workers (main shift) } \\
\text { Less than } 50 \\
50 \text { to } 99 \\
100 \text { to } 499 \\
500 \text { or More }\end{array}$ & $\begin{array}{r}57 \\
51 \\
261 \\
434\end{array}$ & $\begin{array}{r}624 \\
1,051 \\
1,950 \\
8,676\end{array}$ & $\begin{array}{l}15.7 \\
23.8 \\
18.6 \\
17.7\end{array}$ & $\begin{array}{r}37.2 \\
14.1 \\
9.8 \\
6.6\end{array}$ & $\begin{array}{r}5.2 \\
9.5 \\
11.1 \\
10.4\end{array}$ & $\begin{array}{l}13.3 \\
16.2 \\
16.9 \\
15.8\end{array}$ & $\begin{array}{l}23.0 \\
24.3 \\
23.4 \\
20.7\end{array}$ & $\begin{array}{r}50.1 \\
80.9 \\
148.5 \\
671.1\end{array}$ & $\begin{array}{l}1.26 \\
1.83 \\
1.42 \\
1.37\end{array}$ & $\begin{array}{l}.08 \\
.08 \\
.08 \\
.08\end{array}$ \\
\hline 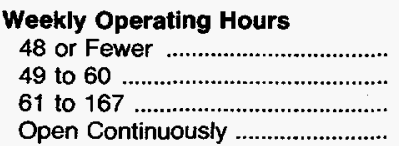 & $\begin{array}{r}74 \\
179 \\
102 \\
448\end{array}$ & $\begin{array}{r}927 \\
2,760 \\
1,504 \\
4,001\end{array}$ & $\begin{array}{l}15.4 \\
13.4 \\
17.9 \\
22.0\end{array}$ & $\begin{array}{l}9.8 \\
5.6 \\
9.6 \\
9.5\end{array}$ & $\begin{array}{r}7.8 \\
7.7 \\
9.3 \\
12.0\end{array}$ & $\begin{array}{l}14.8 \\
13.0 \\
15.7 \\
18.5\end{array}$ & $\begin{array}{l}24.4 \\
18.7 \\
23.4 \\
24.3\end{array}$ & $\begin{array}{r}66.5 \\
246.0 \\
123.8 \\
288.4\end{array}$ & $\begin{array}{l}1.10 \\
1.19 \\
1.48 \\
1.59\end{array}$ & $\begin{array}{l}.07 \\
.09 \\
.08 \\
.07\end{array}$ \\
\hline
\end{tabular}

See footnotes at end of table. 
Table 3.48. Electricity Consumption and Expenditure Intensities in FBSS Buildings in Federal Region 9, 1993 (Continued)

\begin{tabular}{|c|c|c|c|c|c|c|c|c|c|c|}
\hline \multirow{3}{*}{$\begin{array}{c}\text { Building } \\
\text { Characteristics }\end{array}$} & \multicolumn{7}{|c|}{ Electricity Consumption } & \multicolumn{3}{|c|}{ Electricity Expendiltures } \\
\hline & \multirow{2}{*}{$\begin{array}{l}\text { Total } \\
\text { (million } \\
\text { kWh) }\end{array}$} & \multirow{2}{*}{$\begin{array}{c}\text { per } \\
\text { Building } \\
\text { (thousand } \\
\text { kWh) }\end{array}$} & \multirow{2}{*}{$\begin{array}{c}\text { per } \\
\text { Square } \\
\text { Foot } \\
\text { (kWh) }\end{array}$} & \multirow{2}{*}{$\begin{array}{c}\text { per } \\
\text { Worker } \\
\text { (thousand } \\
\text { kWh) }\end{array}$} & \multicolumn{3}{|c|}{$\begin{array}{l}\text { Distribution of } \\
\text { Building-Level Intensities } \\
\text { (kWh/square foot) }\end{array}$} & \multirow{2}{*}{$\begin{array}{l}\text { per } \\
\text { Building } \\
\text { (thousand } \\
\text { dollars) }\end{array}$} & \multirow{2}{*}{$\begin{array}{c}\text { per } \\
\text { Square } \\
\text { Foot } \\
\text { (dollars) }\end{array}$} & \multirow[b]{2}{*}{$\begin{array}{l}\text { per kWh } \\
\text { (dollars) }\end{array}$} \\
\hline & & & & & $\begin{array}{c}25 \text { th } \\
\text { Percentile }\end{array}$ & Median & $\begin{array}{c}\text { 75th } \\
\text { Percentile }\end{array}$ & & & \\
\hline \multicolumn{11}{|l|}{ Multibuilding Facility } \\
\hline 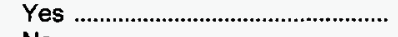 & 486 & 2,440 & 18.1 & 8.6 & 9.7 & 15.9 & 22.9 & 179.2 & 1.33 & 0.07 \\
\hline No & 318 & 2,527 & 18.2 & 7.8 & 9.1 & 16.0 & 24.2 & 209.2 & 1.51 & .08 \\
\hline \multicolumn{11}{|l|}{ Space-Heating Energy Source } \\
\hline 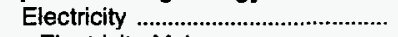 & 212 & 2,824 & 22.5 & 10.6 & 9.6 & 16.6 & 24.1 & 232.6 & 1.85 & .08 \\
\hline Electricity Secondary ................... & 71 & 2,279 & 13.8 & 6.7 & 7.5 & 13.8 & 20.1 & 213.3 & 1.30 & .09 \\
\hline Other Excluding Electricity ............ & 540 & 2,368 & 16.6 & 7.9 & 9.4 & 16.0 & 23.0 & 181.2 & 1.27 & .08 \\
\hline Building Not Heated ....................... & 52 & 2,373 & 21.8 & 5.9 & 8.3 & 12.7 & 19.5 & 148.3 & 1.36 & .06 \\
\hline \multicolumn{11}{|l|}{ Cooling Energy Source } \\
\hline 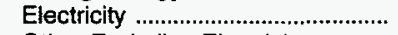 & 683 & 2,578 & 18.7 & 8.2 & 10.6 & 16.5 & 23.6 & 199.0 & 1.44 & .08 \\
\hline Other Excluding Electricity ............. & 90 & 3,333 & 16.8 & 8.5 & 12.3 & 20.3 & 25.0 & 227.7 & 1.15 & .07 \\
\hline A/C Not Performed ............................. & 31 & 937 & 12.9 & 7.9 & 2.0 & 9.0 & 10.2 & 95.1 & 1.31 & .10 \\
\hline \multicolumn{11}{|l|}{ Water-Heating Energy Source } \\
\hline 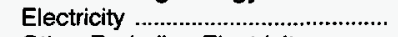 & 189 & 2,911 & 26.2 & 10.3 & 8.0 & 13.1 & 21.1 & 214.5 & 1.93 & .07 \\
\hline Other Excluding Electricity ............. & 589 & 2,365 & 16.5 & 7.8 & 10.1 & 16.5 & 23.8 & 182.7 & 1.27 & .08 \\
\hline Water Heating Not Performed ..... & 26 & 2,345 & 19.4 & 7.6 & 9.7 & 10.6 & 20.8 & 235.3 & 1.94 & .10 \\
\hline \multicolumn{11}{|l|}{ Cooking Energy Source } \\
\hline 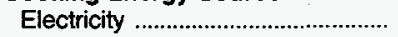 & 239 & 5,569 & 17.6 & 8.0 & 10.1 & 15.3 & 19.5 & 390.2 & 1.23 & .07 \\
\hline Other Excluding Electricity .............. & 108 & 4,008 & 16.8 & 6.5 & 7.6 & 12.3 & 21.5 & 284.1 & 1.19 & .07 \\
\hline Cooking Not Performed ................... & 456 & 1,789 & 18.8 & 8.9 & 9.6 & 16.6 & 23.9 & 147.4 & 1.55 & .08 \\
\hline \multicolumn{11}{|l|}{ Manufacturing Energy Source } \\
\hline Other Excluding Electricity ............ & 22 & 4,307 & 14.5 & 6.2 & 12.3 & 15.6 & 16.0 & 301.0 & 1.01 & .07 \\
\hline Manufacturing Not Performed ..... & 647 & 2,192 & 17.7 & 8.1 & 9.2 & 15.9 & 23.2 & 172.4 & 1.39 & .08 \\
\hline Percent of Floorspace Heated & & & & & & & & & & \\
\hline 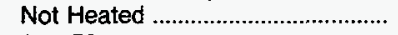 & 52 & 2,373 & 21.8 & 5.9 & 8.3 & 12.7 & 19.5 & 148.3 & 1.36 & .06 \\
\hline 1 to 50 & 109 & 3,879 & 29.0 & 20.9 & 3.8 & 19.8 & 25.6 & 295.1 & 2.20 & .08 \\
\hline 51 to 100 & 643 & 2,339 & 16.9 & 7.7 & 9.6 & 15.9 & 22.9 & 183.6 & 1.32 & .08 \\
\hline Percent of Floorspace Cooled & & & & & & & & & & \\
\hline 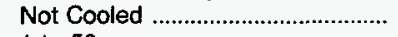 & 40 & 1,032 & 13.7 & 9.7 & 2.0 & 9.0 & 13.5 & 96.2 & 1.28 & .09 \\
\hline 1 to 50 & 136 & 2,464 & 18.6 & 9.4 & 10.2 & 15.3 & 19.2 & 168.2 & 1.27 & .07 \\
\hline 51 to 100 & 628 & 2,719 & 18.4 & 8.0 & 11.1 & 17.6 & 24.3 & 212.2 & 1.44 & .08 \\
\hline Percent Lit When Open & & & & & & & & & & \\
\hline 1 to 50 & 21 & 1,072 & 11.6 & 11.7 & 1.1 & 11.2 & 17.2 & 78.0 & .84 & .07 \\
\hline 51 to 100 & 782 & 2,579 & 18.5 & 8.2 & 9.8 & 16.2 & 23.4 & 199.3 & 1.43 & .08 \\
\hline No Operating Hours ........................... & $(*)$ & & .1 & NC & .1 & .1 & .1 & .1 & (") & .03 \\
\hline 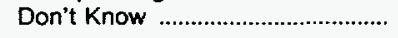 & 1 & 982 & 15.3 & 31.7 & 15.3 & 15.3 & 15.3 & 83.7 & 1.30 & .09 \\
\hline Percent Lit When Closed & & & & & & & & & & \\
\hline 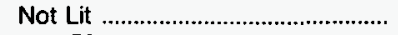 & 33 & 935 & 19.7 & 15.8 & 6.6 & 13.4 & 24.3 & 72.3 & 1.52 & .08 \\
\hline 1 to 50 & 374 & 1,834 & 14.8 & 6.9 & 9.2 & 15.2 & 20.9 & 149.7 & 1.21 & .08 \\
\hline 51 to 100 & 160 & 3,645 & 19.8 & 9.0 & 12.6 & 21.6 & 41.6 & 275.3 & 1.49 & .08 \\
\hline 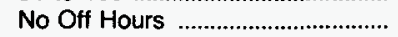 & 236 & 5,751 & 25.7 & 10.2 & 10.6 & 17.3 & 22.1 & 408.8 & 1.82 & .07 \\
\hline 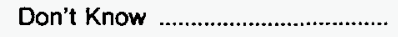 & 1 & 982 & 15.3 & 31.7 & 15.3 & 15.3 & 15.3 & 83.7 & 1.30 & .09 \\
\hline $\begin{array}{l}\text { Heating Equipment (more than } \\
\text { one may apply) }\end{array}$ & & & & & & & & & & \\
\hline 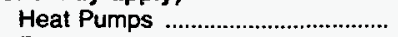 & 68 & 1,705 & 22.1 & 9.4 & 11.4 & 19.4 & 24.1 & 134.2 & 1.74 & .08 \\
\hline 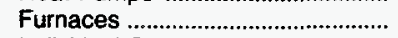 & 16 & 624 & 13.0 & 5.6 & 8.2 & 12.7 & 17.8 & 51.8 & 1.07 & .08 \\
\hline Individual Space Heaters .............. & 82 & 2,480 & 16.6 & 7.5 & 9.1 & 13.0 & 19.8 & 203.5 & 1.36 & .08 \\
\hline District Heat ...................................... & 237 & 3,380 & 18.6 & 9.8 & 12.0 & 16.3 & 20.3 & 227.5 & 1.25 & .07 \\
\hline Boilers & 421 & 2,884 & 17.5 & 7.8 & 10.1 & 17.5 & 24.4 & 232.8 & 1.42 & .08 \\
\hline Packaged-Heating Units ................. & 102 & 1,528 & 17.1 & 7.6 & 9.5 & 15.3 & 24.4 & 108.5 & 1.21 & .07 \\
\hline
\end{tabular}

See footnotes at end of table. 
Table 3.48. Electricity Consumption and Expenditure Intensities in FBSS Buildings in Federal Region 9, 1993 (Continued)

\begin{tabular}{|c|c|c|c|c|c|c|c|c|c|c|}
\hline \multirow{3}{*}{$\begin{array}{c}\text { Building } \\
\text { Characteristics }\end{array}$} & \multicolumn{7}{|c|}{ Electricity Consumption } & \multicolumn{3}{|c|}{ Electricity Expenditures } \\
\hline & \multirow{2}{*}{$\begin{array}{l}\text { Total } \\
\text { (million } \\
\text { kWh) }\end{array}$} & \multirow{2}{*}{$\begin{array}{c}\text { per } \\
\text { Bullding } \\
\text { (thousand } \\
\mathbf{k W h} \text { ) }\end{array}$} & \multirow{2}{*}{$\begin{array}{c}\text { per } \\
\text { Square } \\
\text { Foot } \\
\text { (kWh) }\end{array}$} & \multirow{2}{*}{$\begin{array}{c}\text { per } \\
\text { Worker } \\
\text { (thousand } \\
\text { kWh) }\end{array}$} & \multicolumn{3}{|c|}{$\begin{array}{c}\text { Distribution of } \\
\text { Building-Level Intensities } \\
\text { (kWh/square foot) }\end{array}$} & \multirow{2}{*}{$\begin{array}{c}\text { per } \\
\text { Building } \\
\text { (thousand } \\
\text { dollars) }\end{array}$} & \multirow{2}{*}{$\begin{array}{c}\text { per } \\
\text { Square } \\
\text { Foot } \\
\text { (dollars) }\end{array}$} & \multirow[b]{2}{*}{$\begin{array}{l}\text { per kWh } \\
\text { (dollars) }\end{array}$} \\
\hline & & & & & $\begin{array}{c}\text { 25th } \\
\text { Percentile }\end{array}$ & Median & $\begin{array}{c}\text { 75th } \\
\text { Percentile }\end{array}$ & & & \\
\hline \multicolumn{11}{|l|}{$\begin{array}{l}\text { Cooling Equipment (more than } \\
\text { one may apply) }\end{array}$} \\
\hline Residential-Type Central A/C ...... & 34 & 1,404 & 17.7 & 6.9 & 9.1 & 14.3 & 20.6 & 104.4 & 1.32 & 0.07 \\
\hline 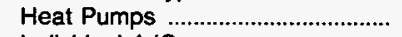 & 63 & 1,958 & 23.0 & 11.1 & 12.9 & 20.5 & 30.2 & 156.5 & 1.84 & .08 \\
\hline Individual A/C & 100 & 1,932 & 17.6 & 8.4 & 10.1 & 17.0 & 19.7 & 153.7 & 1.40 & .08 \\
\hline District Chilled Water ...................... & $\$ 70$ & 5,484 & 22.2 & 11.6 & 15.3 & 19.5 & 22.5 & 332.5 & 1.35 & .06 \\
\hline 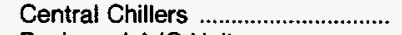 & 499 & 3,589 & 18.0 & 7.4 & 11.5 & 17.8 & 24.4 & 278.4 & 1.40 & .08 \\
\hline Packaged-A/C Units ........................ & 416 & 2,756 & 17.3 & 6.9 & 11.4 & 17.0 & 24.4 & 214.3 & 1.35 & .08 \\
\hline 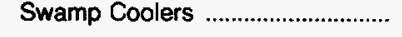 & 43 & 1,430 & 13.6 & 5.0 & 6.6 & 11.4 & 18.7 & 111.0 & 1.05 & .08 \\
\hline \multicolumn{11}{|l|}{$\begin{array}{l}\text { Lighting Equipment (more than } \\
\text { one may apply) }\end{array}$} \\
\hline 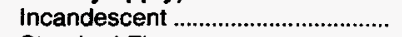 & 354 & 2,201 & 17.3 & 8.4 & 9.0 & 16.9 & 24.4 & 170.1 & 1.34 & .08 \\
\hline Standard Fluorescent ........................ & 763 & 2,478 & 17.8 & 8.0 & 9.4 & 15.9 & 22.9 & 187.1 & 1.35 & .08 \\
\hline Compact Fluorescent ........................ & 296 & 4,118 & 17.1 & 8.1 & 9.4 & 17.5 & 23.9 & 344.1 & 1.43 & .08 \\
\hline High-Intensity Discharge ................ & 329 & 4,390 & 17.6 & 7.6 & 8.5 & 14.4 & 21.5 & 312.0 & 1.25 & .07 \\
\hline Electronic Ballast ............................... & 392 & 3,736 & 16.8 & 7.2 & 10.6 & 16.2 & 23.4 & 291.0 & 1.31 & .08 \\
\hline \multicolumn{11}{|l|}{$\begin{array}{l}\text { Water-Heating Equipment (more } \\
\text { than one may apply) }\end{array}$} \\
\hline 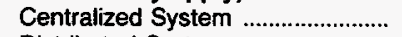 & 507 & 2,262 & 16.3 & 7.6 & 9.1 & 15.9 & 22.8 & 178.1 & 1.28 & .08 \\
\hline $\begin{array}{l}\text { Distributed System ............................ } \\
\text { Don't Know/ }\end{array}$ & 240 & 2,994 & 22.3 & 9.7 & 9.9 & 15.2 & 24.4 & 217.4 & 1.62 & .07 \\
\hline 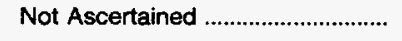 & 32 & 3,182 & 27.3 & 11.2 & 16.6 & 22.2 & 32.3 & 216.0 & 1.85 & .07 \\
\hline \multicolumn{11}{|l|}{$\begin{array}{l}\text { Commercial Refrigeration } \\
\text { Equipment (more than one may } \\
\text { apply) }\end{array}$} \\
\hline Any Equipment & 346 & 5,494 & 18.4 & 7.9 & 10.5 & 16.2 & 21.8 & 381.7 & 1.28 & .07 \\
\hline 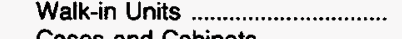 & 233 & 5,289 & 18.6 & 8.2 & 12.4 & 17.2 & 24.2 & 367.5 & 1.29 & .07 \\
\hline Cases and Cabinets ....................... & 248 & 4,967 & 16.6 & 7.1 & 12.0 & 16.2 & 22.0 & 385.0 & 1.29 & .08 \\
\hline None & 458 & 1,747 & 17.9 & 8.5 & 9.2 & 15.9 & 23.4 & 145.0 & 1.49 & .08 \\
\hline \multicolumn{11}{|l|}{$\begin{array}{l}\text { Retrofit or Purchase of any } \\
\text { Equipment Within Last Ten } \\
\text { Years (more than one may } \\
\text { apply) }\end{array}$} \\
\hline Retrofit and/or Purchase ................ & 611 & 2,881 & 17.7 & 7.6 & 9.9 & 16.1 & 22.4 & 229.7 & 1.41 & .08 \\
\hline 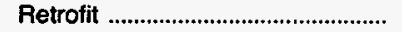 & 346 & 3,356 & 15.3 & 6.3 & 9.7 & 14.4 & 20.9 & 267.9 & 1.22 & .08 \\
\hline Purchase & 415 & 2,824 & 21.2 & 9.3 & 11.4 & 16.5 & 23.4 & 224.1 & 1.68 & .08 \\
\hline No Retrofit or Purchase .................... & 193 & 1,710 & 19.8 & 11.1 & 8.3 & 15.9 & 24.4 & 117.9 & 1.36 & .07 \\
\hline \multicolumn{11}{|l|}{$\begin{array}{l}\text { HVAC Conservation Features } \\
\text { (more than one may apply) }\end{array}$} \\
\hline VAV System & 292 & 3,900 & 18.8 & 8.6 & 12.6 & 17.3 & 24.4 & 296.9 & 1.43 & .08 \\
\hline Economizer Cycle .................................. & 431 & 3,078 & 16.3 & 7.2 & 10.4 & 16.1 & 23.9 & 241.0 & 1.28 & .08 \\
\hline 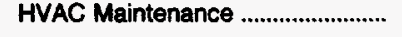 & 787 & 2,581 & 18.3 & 8.2 & 9.7 & 16.3 & 23.4 & 198.9 & 1.41 & .08 \\
\hline \multicolumn{11}{|l|}{$\begin{array}{l}\text { Lighting Conservation Features } \\
\text { (more than one may apply) }\end{array}$} \\
\hline $\begin{array}{l}\text { Specular Reflectors .......................... } \\
\text { Natural Lighting Control }\end{array}$ & 332 & 2,697 & 15.2 & 6.6 & 10.6 & 16.2 & 23.0 & 210.3 & 1.19 & .08 \\
\hline 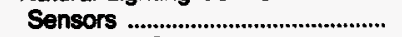 & 186 & 3,213 & 13.5 & 5.4 & 9.8 & 15.6 & 19.8 & 268.1 & 1.13 & .08 \\
\hline Occupancy Sensors .......................... & 446 & 4,092 & 17.7 & 7.6 & 11.1 & 17.8 & 24.0 & 316.8 & 1.37 & .08 \\
\hline 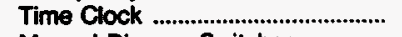 & 141 & 3,433 & 13.4 & 5.8 & 8.6 & 14.4 & 17.8 & 261.0 & 1.02 & .08 \\
\hline Manual Dimmer Switches ............... & 269 & 3,443 & 18.6 & 8.7 & 12.0 & 18.1 & 25.0 & 264.1 & 1.43 & .08 \\
\hline
\end{tabular}

See footnotes at end of table. 
Table 3.48. Electricity Consumption and Expenditure Intensities in FBSS Buildings in Federal Region 9, 1993 (Continued)

\begin{tabular}{|c|c|c|c|c|c|c|c|c|c|c|}
\hline \multirow{3}{*}{$\begin{array}{c}\text { Building } \\
\text { Characteristics }\end{array}$} & \multicolumn{7}{|c|}{ Electricity Consumption } & \multicolumn{3}{|c|}{ Electricity Expenditures } \\
\hline & \multirow{2}{*}{$\begin{array}{l}\text { Total } \\
\text { (million } \\
\text { kWh) }\end{array}$} & \multirow{2}{*}{$\begin{array}{c}\text { per } \\
\text { Building } \\
\text { (thousand } \\
\text { kWh) }\end{array}$} & \multirow{2}{*}{$\begin{array}{c}\text { per } \\
\text { Square } \\
\text { Foot } \\
\text { (kWh) }\end{array}$} & \multirow{2}{*}{$\begin{array}{c}\text { per } \\
\text { Worker } \\
\text { (thousand } \\
\text { kWh) }\end{array}$} & \multicolumn{3}{|c|}{$\begin{array}{l}\text { Distribution of } \\
\text { Building-Level Intensities } \\
\text { (kWh/square foot) }\end{array}$} & \multirow{2}{*}{$\begin{array}{c}\text { per } \\
\text { Building } \\
\text { (thousand } \\
\text { dollars) }\end{array}$} & \multirow{2}{*}{$\begin{array}{c}\text { per } \\
\text { Square } \\
\text { Foot } \\
\text { (dollars) }\end{array}$} & \multirow[b]{2}{*}{$\begin{array}{l}\text { per kWh } \\
\text { (dollars) }\end{array}$} \\
\hline & & & & & Percentile & Median & $\begin{array}{l}\text { 75th } \\
\text { Percentile }\end{array}$ & & & \\
\hline \multicolumn{11}{|l|}{$\begin{array}{l}\text { Energy Management Practices } \\
\text { (more than one may apply) } \\
\text { Energy Management and Control }\end{array}$} \\
\hline System & 372 & 5,232 & 20.4 & 8.9 & 12.6 & 19.6 & 29.9 & 359.1 & 1.40 & 0.07 \\
\hline 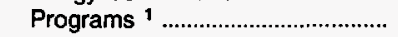 & 448 & 3,643 & 18.2 & 8.1 & 10.4 & 16.2 & 23.9 & 272.1 & 1.36 & .07 \\
\hline HVAC Maintenance Staff ${ }^{2}$........... & 244 & 4,685 & 16.7 & 6.9 & 9.8 & 17.7 & 23.3 & 348.6 & 1.24 & .07 \\
\hline \multicolumn{11}{|l|}{$\begin{array}{l}\text { Off-Hours Reduction in } \\
\text { Equipment (more than one may } \\
\text { apply) }\end{array}$} \\
\hline 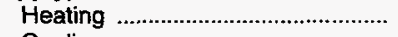 & 270 & 1,785 & 13.7 & 6.5 & 8.2 & 13.8 & 20.9 & 148.3 & 1.14 & .08 \\
\hline Cooling & 262 & 1,668 & 13.6 & 6.3 & 8.3 & 14.4 & 20.8 & 137.6 & 1.12 & .08 \\
\hline 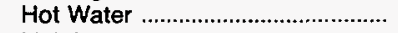 & 174 & 2,564 & 12.8 & 5.6 & 7.4 & 13.3 & 24.1 & 223.9 & 1.12 & .09 \\
\hline 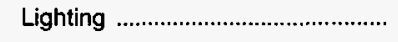 & 293 & 1,706 & 13.9 & 6.5 & 8.2 & 14.4 & 22.5 & 141.8 & 1.15 & .08 \\
\hline \multicolumn{11}{|l|}{ Building Generates Electricity } \\
\hline 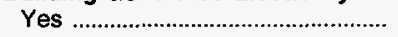 & 351 & 6,745 & 24.6 & 12.7 & 13.4 & 20.4 & 39.8 & 511.7 & 1.86 & .08 \\
\hline No & 453 & 1,660 & 15.1 & 6.5 & 9.0 & 15.3 & 22.1 & 129.7 & 1.18 & .08 \\
\hline
\end{tabular}

1 Building participates in any programs sponsored by the Federal Energy Management Program, in-house, utility, or third party.

2 HVAC maintenance staff means at least one person spends at least half their working hours maintaining the heating/cooling equipment.

$\left({ }^{*}\right)=$ Value rounds to zero in the units displayed.

NC $=$ No cases in responding sample.

Notes: - Total workers are the number of workers during the main shift. - See Glossary for explanation of abbreviations and definitions of terms used in this report. - These data are from 881 federally owned buildings having the following criteria: (1) located in Federal Regions 3 , 6 , or 9 ; (2) larger than 10,000 square feet; and (3) used for a commercial purpose, other than warehouse and storage. In addition, 9 out of 10 selected buildings were from agencies other than the Department of Defense. - Statistics for the "energy end uses" represent consumption in buildings that have end use, not consumption for a particular fuel for a particular end use. $\bullet A / C=$ Air Conditioning. $\bullet$ FBSS $=$ Federal Buildings Supplemental Survey.

- HVAC = Heating, Ventilation, and Air Conditioning. - VAV = Variable-Air Volume. $\bullet$ KWH = Kilowatthour. $\bullet$ Data are for Fiscal Year 1993

(October 1, 1992 through September 30, 1993). - Because of rounding, data may not sum to totals.

Source: Energy Information Administration, Office of Energy Markets and End Use, 1993 Federal Buildings Supplemental Survey. 
Table 3.49. Natural Gas Consumption and Expenditure Intensities in FBSS Buildings in Federal Region 3, 1993

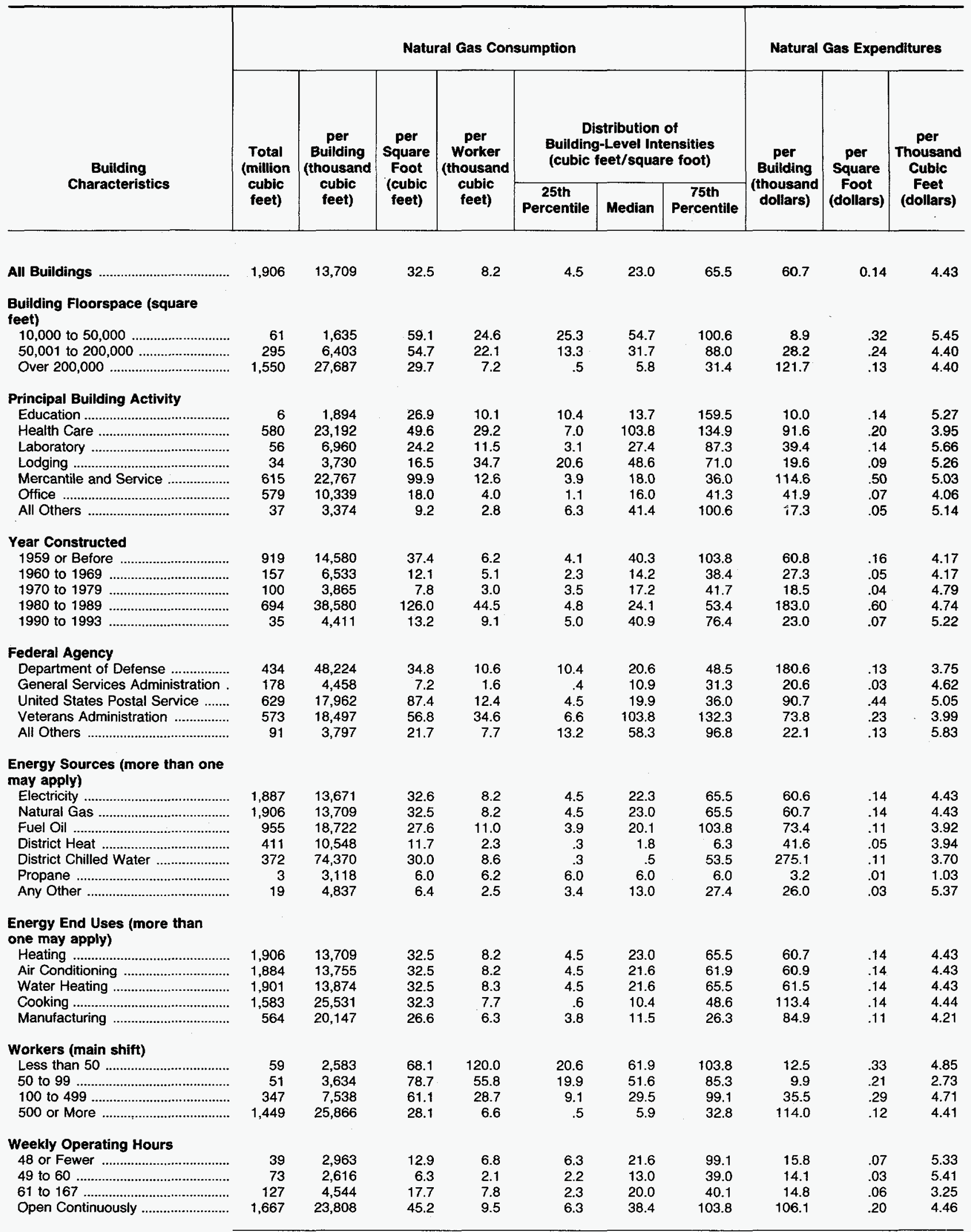

See footnotes at end of table. 


\begin{tabular}{|c|c|c|c|c|c|c|c|c|c|c|}
\hline \multirow{3}{*}{$\begin{array}{c}\text { Building } \\
\text { Characteristics }\end{array}$} & \multicolumn{7}{|c|}{ Natural Gas Consumption } & \multicolumn{3}{|c|}{ Natural Gas Expenditures } \\
\hline & \multirow{2}{*}{$\begin{array}{l}\text { Total } \\
\text { (milion } \\
\text { cubic } \\
\text { feet) }\end{array}$} & \multirow{2}{*}{$\begin{array}{c}\text { per } \\
\text { Building } \\
\text { (thousand } \\
\text { cubic } \\
\text { feet) }\end{array}$} & \multirow{2}{*}{$\begin{array}{c}\text { per } \\
\text { Square } \\
\text { Foot } \\
\text { (cubic } \\
\text { feet) }\end{array}$} & \multirow{2}{*}{$\begin{array}{c}\text { per } \\
\text { Worker } \\
\text { (thousand } \\
\text { cubic } \\
\text { feet) }\end{array}$} & \multicolumn{3}{|c|}{$\begin{array}{l}\text { Distribution of } \\
\text { Building-Level Intensities } \\
\text { (cubic feet/square foot) }\end{array}$} & \multirow{2}{*}{\begin{tabular}{|c|} 
per \\
Building \\
(thousand \\
dollars)
\end{tabular}} & \multirow{2}{*}{$\begin{array}{c}\text { per } \\
\text { Square } \\
\text { Foot } \\
\text { (dollars) }\end{array}$} & \multirow{2}{*}{\begin{tabular}{|c} 
per \\
Thousand \\
Cubic \\
Feet \\
(dollars)
\end{tabular}} \\
\hline & & & & & $\begin{array}{c}\text { 25th } \\
\text { Percentile }\end{array}$ & Median & $\begin{array}{c}\text { 75th } \\
\text { Percentile }\end{array}$ & & & \\
\hline \multicolumn{11}{|l|}{ Multibuilding Facility } \\
\hline 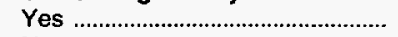 & 761 & 10,431 & 31.5 & 9.0 & 10.4 & 41.7 & 103.8 & 46.4 & 0.14 & 4.45 \\
\hline No & 1,144 & 17,335 & 33.1 & 7.8 & .9 & 13.0 & 36.0 & 76.6 & .15 & 4.42 \\
\hline \multicolumn{11}{|l|}{ Space-Heating Energy Source } \\
\hline 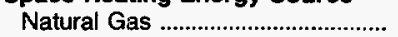 & 1,480 & 15,421 & 66.9 & 29.6 & 15.5 & 40.8 & 103.1 & 70.4 & .31 & 4.57 \\
\hline 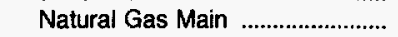 & 1,456 & 16,172 & 72.4 & 32.5 & 16.9 & 41.0 & 103.8 & 73.7 & .33 & 4.56 \\
\hline Natural Gas Secondary ............... & 25 & 4,154 & 12.3 & 4.7 & 6.3 & 23.8 & 41.7 & 21.0 & .06 & 5.05 \\
\hline Other Excluding Natural Gas ........ & 425 & 9,887 & 11.6 & 2.3 & .3 & 1.8 & 7.1 & 39.1 & .05 & 3.96 \\
\hline Building Not Heated ............................ & & & & & & & NC & NC & & NC \\
\hline \multicolumn{11}{|l|}{ Cooling Energy Source } \\
\hline 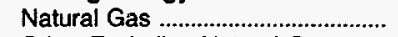 & 1 & 563 & 25.3 & 6.3 & 25.3 & 25.3 & 25.3 & 4.4 & .20 & 7.76 \\
\hline Other Excluding Natural Gas ........ & 1,884 & 13,852 & 32.5 & 8.2 & 4.2 & 21.3 & 64.6 & 61.3 & .14 & 4.43 \\
\hline A/C Not Performed ........................... & 21 & 10,538 & 28.2 & 11.7 & 26.4 & 48.7 & 71.0 & 48.6 & .13 & 4.61 \\
\hline \multicolumn{11}{|l|}{ Water-Heating Energy Source } \\
\hline 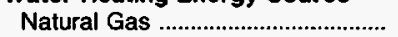 & 1,428 & 18,310 & 78.9 & 29.2 & 20.0 & 44.1 & 103.8 & 82.7 & .36 & 4.51 \\
\hline Other Excluding Natural Gas ........ & 472 & 8,008 & 11.7 & 2.6 & .5 & 5.3 & 19.2 & 33.4 & .05 & 4.17 \\
\hline Water Heating Not Performed ..... & 5 & 2,434 & 42.9 & 3.7 & 40.3 & 51.1 & 61.9 & 12.2 & .21 & 5.01 \\
\hline \multicolumn{11}{|l|}{ Cooking Energy Source } \\
\hline 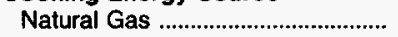 & 1,393 & 30,286 & 35.6 & 7.7 & .6 & 6.8 & 53.5 & 138.2 & .16 & 4.56 \\
\hline Other Excluding Natural Gas ........ & 190 & 11,860 & 19.0 & 7.4 & .5 & 22.3 & 46.4 & 42.0 & .07 & 3.54 \\
\hline Cooking Not Performed .................... & 323 & 4,190 & 33.6 & 12.7 & 10.4 & 35.1 & 81.2 & 18.4 & .15 & 4.38 \\
\hline \multicolumn{11}{|l|}{ Manufacturing Energy Source } \\
\hline 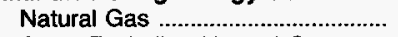 & 47 & 9,346 & 8.8 & 3.6 & 3.8 & 12.7 & 12.8 & 51.6 & .05 & 5.52 \\
\hline Other Excluding Natural Gas ........ & 517 & 22,495 & 32.5 & 6.8 & 3.9 & 10.3 & 26.4 & 92.1 & .13 & 4.09 \\
\hline Manufacturing Not Performed ..... & 1,341 & 12,085 & 35.8 & 9.4 & 4.8 & 33.0 & 81.2 & 54.7 & .16 & 4.52 \\
\hline \multicolumn{11}{|l|}{ Percent of Floorspace Heated } \\
\hline 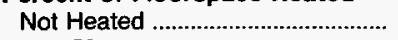 & NC & $\mathrm{NC}$ & NC & NC & NC & NC & NC & NC & NC & NC \\
\hline 1 to 50 & 16 & 3,292 & 3.7 & 2.5 & .5 & 31.4 & 41.4 & 16.4 & .02 & 4.98 \\
\hline 51 to 100 & 1,889 & 14,098 & 34.8 & 8.4 & 4.5 & 22.3 & 65.5 & 62.4 & .15 & 4.43 \\
\hline \multicolumn{11}{|l|}{$\begin{array}{l}\text { Heating Equipment (more than } \\
\text { one may apply) }\end{array}$} \\
\hline 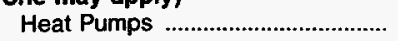 & 33 & 2,722 & 6.1 & 2.2 & 1.6 & 17.8 & 46.8 & 14.6 & .03 & 5.36 \\
\hline Furnaces & 33 & 4,681 & 92.7 & 56.0 & 16.8 & 39.7 & 100.6 & 6.6 & .13 & 1.41 \\
\hline Individual Space Heaters ............... & 1,127 & 32,207 & 54.0 & 18.1 & 3.9 & 26.4 & 88.0 & 145.2 & .24 & 4.51 \\
\hline 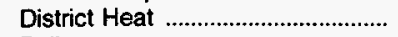 & 725 & 12,952 & 18.5 & 4.0 & .7 & 6.8 & 86.3 & 52.3 & .07 & 4.04 \\
\hline 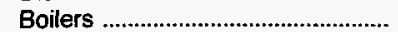 & 1,076 & 15,587 & 60.0 & 24.0 & 12.7 & 26.4 & 57.9 & 73.7 & .28 & 4.73 \\
\hline Packaged-Heating Units ................ & 638 & 39,857 & 158.8 & 50.0 & 2.5 & 19.9 & 78.1 & 205.3 & .82 & 5.15 \\
\hline \multicolumn{11}{|l|}{$\begin{array}{l}\text { Retrofit or Purchase of any } \\
\text { Equipment Within Last Ten } \\
\text { Years (more than one may } \\
\text { apply) }\end{array}$} \\
\hline Retrofit and/or Purchase .................. & 1,784 & 16,076 & 34,4 & 8.3 & 3.5 & 20.1 & 61.9 & 70.8 & .15 & 4.40 \\
\hline 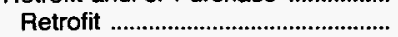 & 695 & 15,794 & 27.1 & 9.0 & 2.2 & 13.5 & 42.0 & 65.6 & .11 & 4.15 \\
\hline 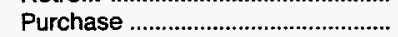 & 1,667 & 18,732 & 40.8 & 9.1 & 4.8 & 26.0 & 77.3 & 82.4 & .18 & 4.40 \\
\hline No Retrofit or Purchase ............. & 121 & 4,327 & 17.8 & 7.8 & 11.0 & 35.6 & 95.0 & 21.1 & .09 & 4.87 \\
\hline $\begin{array}{l}\text { HVAC Conservation Features } \\
\text { (more than one may apply) }\end{array}$ & & & & & & & & & & \\
\hline 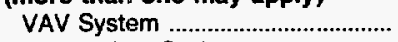 & 1,094 & 22,331 & 48.0 & 10.7 & 3.2 & 12.7 & 42.0 & 105.8 & .23 & 4.74 \\
\hline 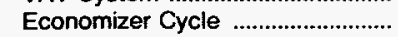 & 1,531 & 19,634 & 38.3 & 8.2 & 3.4 & 14.2 & 47.0 & 88.1 & .17 & 4.49 \\
\hline 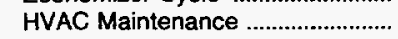 & 1,888 & 13,988 & 32.3 & 8.2 & 4.5 & 23.0 & 61.9 & 62.0 & .14 & 4.43 \\
\hline
\end{tabular}

See footnotes at end of table. 
Table 3.49. Natural Gas Consumption and Expenditure Intensities in FBSS Buildings in Federal Region 3, 1993 (Continued)

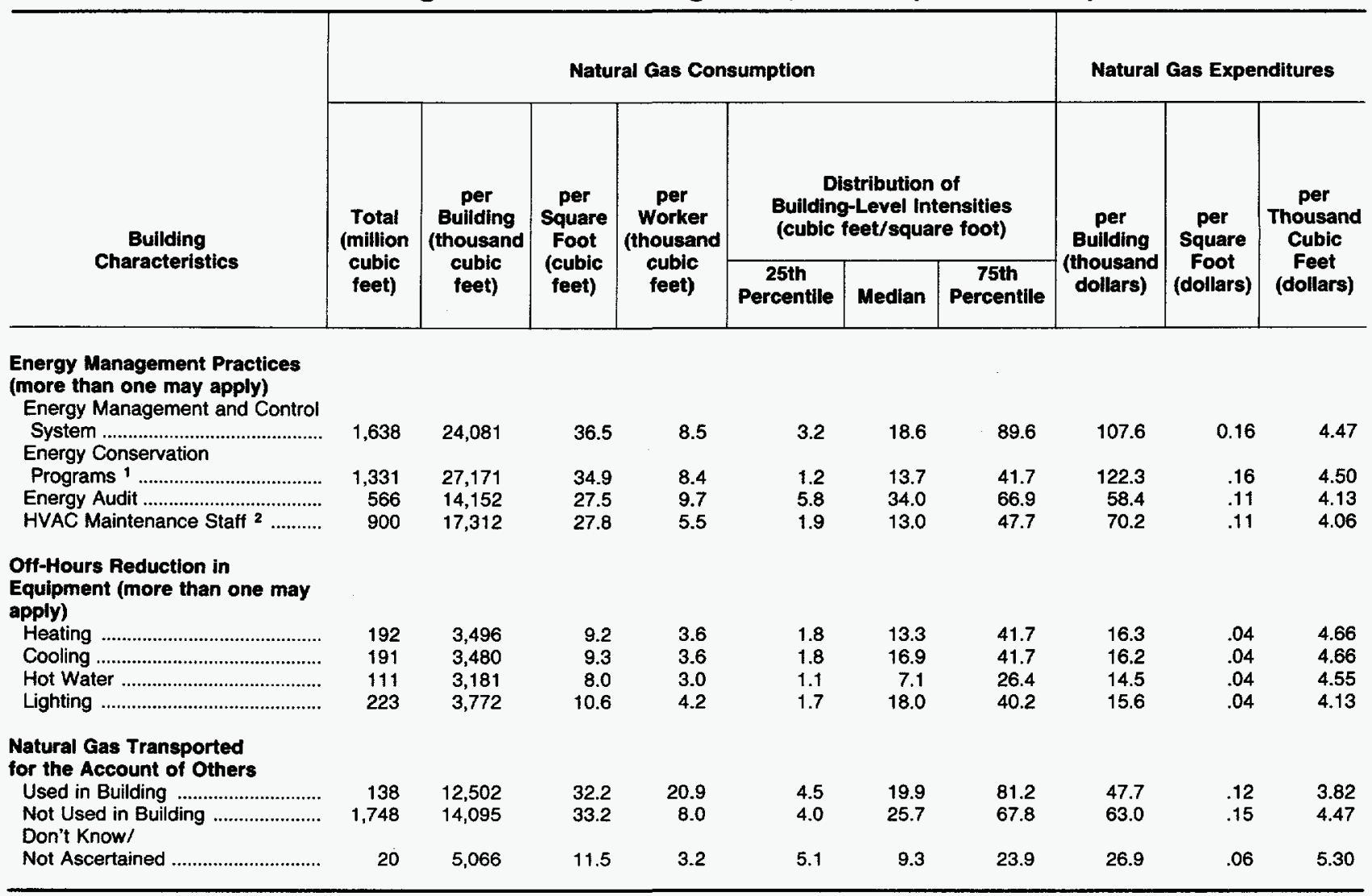

1 Building participates in any programs sponsored by the Federal Energy Management Program, in-house, utility, or third party.

2 HVAC maintenance staff means at least one person spends at least half their working hours maintaining the heating/cooling equipment.

$\mathrm{NC}=$ No cases in responding sample.

Notes: - Total workers are the number of workers during the main shift. - See Glossary for explanation of abbreviations and definitions of terms used in this report. - These data are from 881 federally owned buildings having the following criteria: (1) located in Federal Regions 3 , 6 , or 9 ; (2) larger than 10,000 square feet; and (3) used for a commercial purpose, other than warehouse and storage. In addition, 9 out of 10 selected buildings were from agencies other than the Department of Defense. - Statistics for the "energy end uses" represent consumption in buildings that have end use, not consumption for a particular fuel for a particular end use. $\bullet A / C=$ Air Conditioning. $\bullet$ FSS = Federal Buildings Supplemental Survey.

- HVAC = Heating, Ventilation, and Air Conditioning. - VAV = Variable-Air Volume. - Data are for Fiscal Year 1993 (October 1, 1992 through September 30,1993$)$. Because of rounding, data may not sum to totals.

Source: Energy Information Administration, Office of Energy Markets and End Use, 1993 Federal Buildings Supplemental Survey. 
Table 3.50. Natural Gas Consumption and Expenditure Intensities in FBSS Buildings in Federal Region 6, 1993

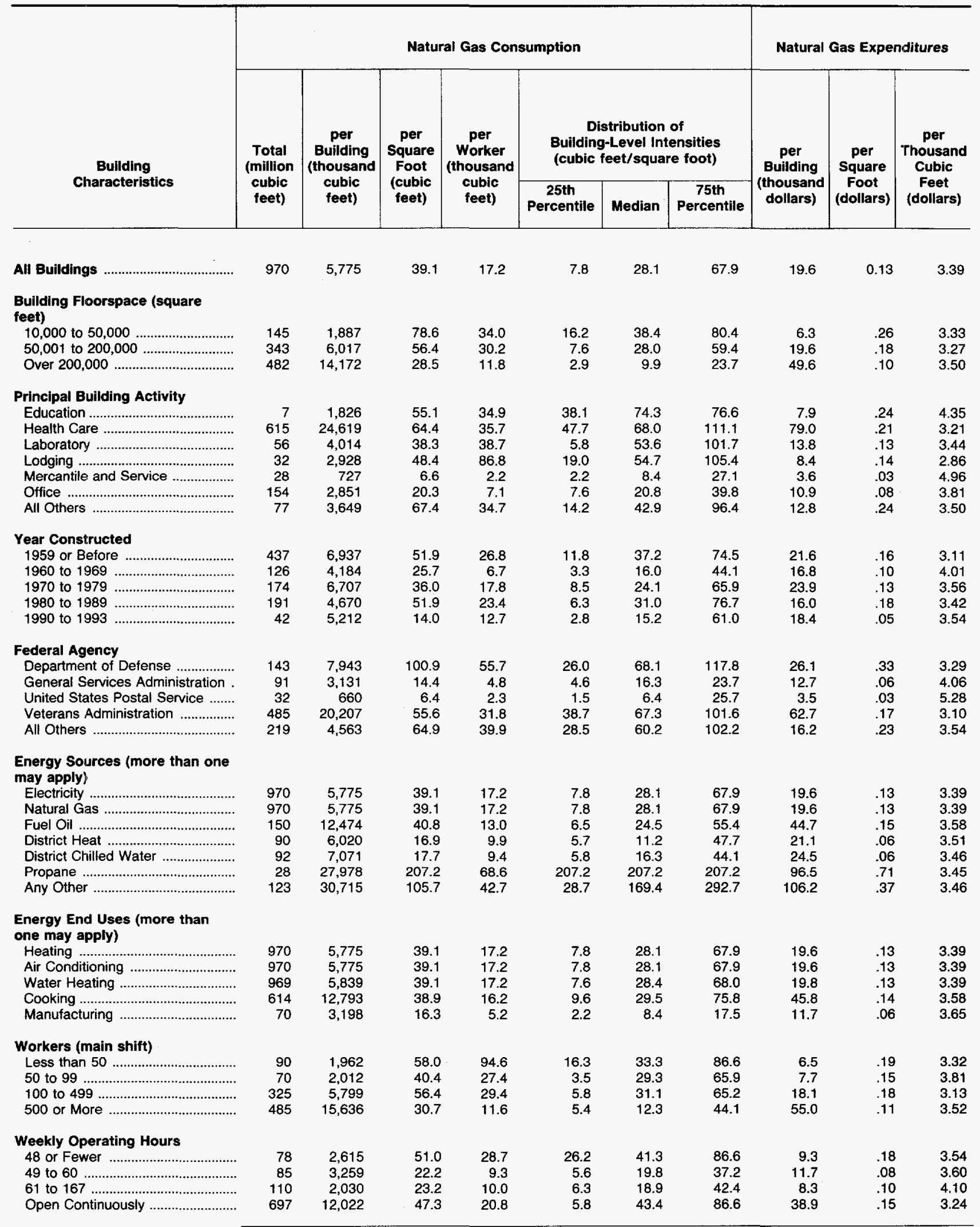

See footnotes at end of table. 
Table 3.50. Natural Gas Consumption and Expenditure Intensities in FBSS Buildings in Federal Region 6, 1993 (Continued)

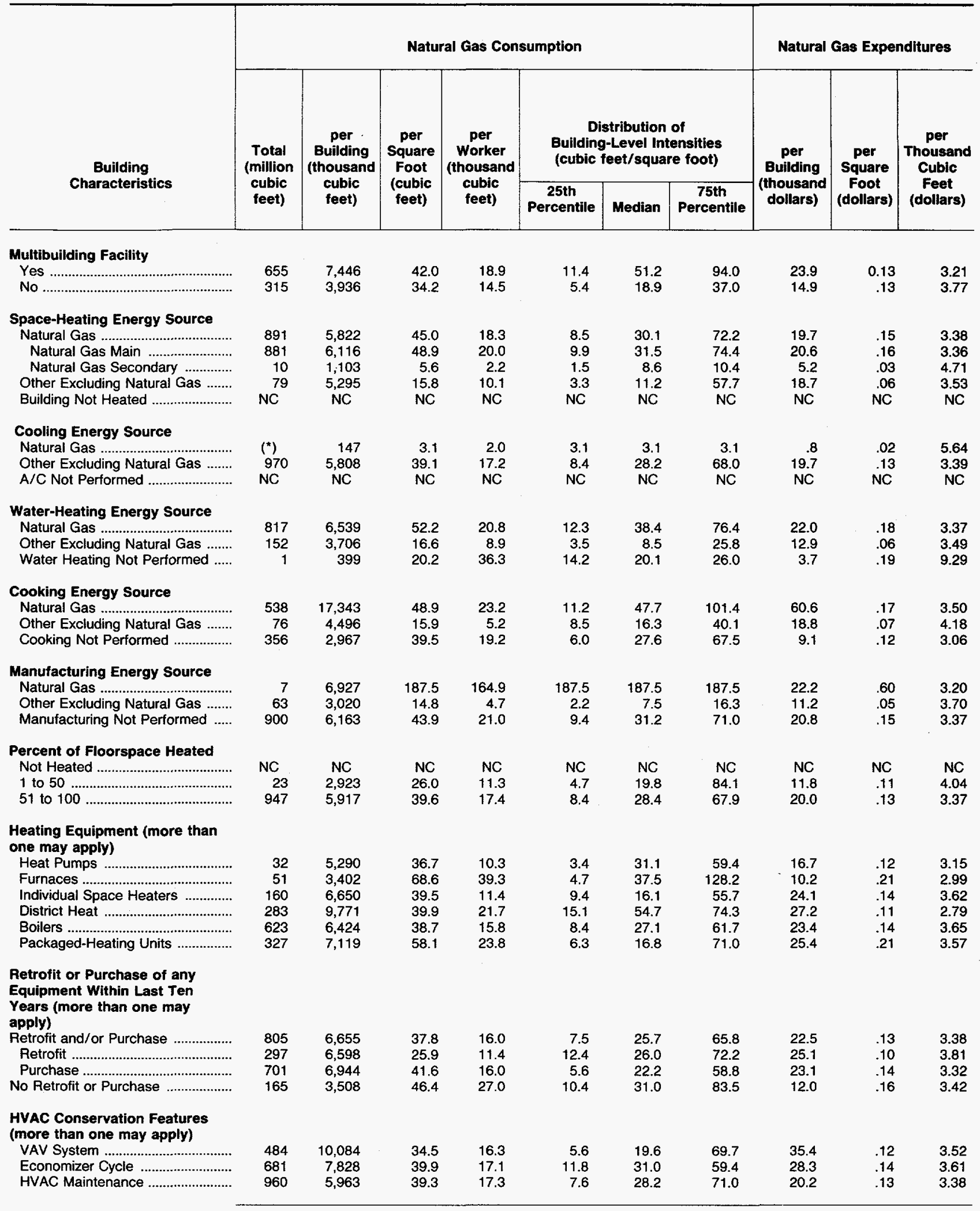

See footnotes at end of table. 
Table 3.50. Natural Gas Consumption and Expenditure Intensities in FBSS Buildings in Federal Region 6, 1993 (Continued)

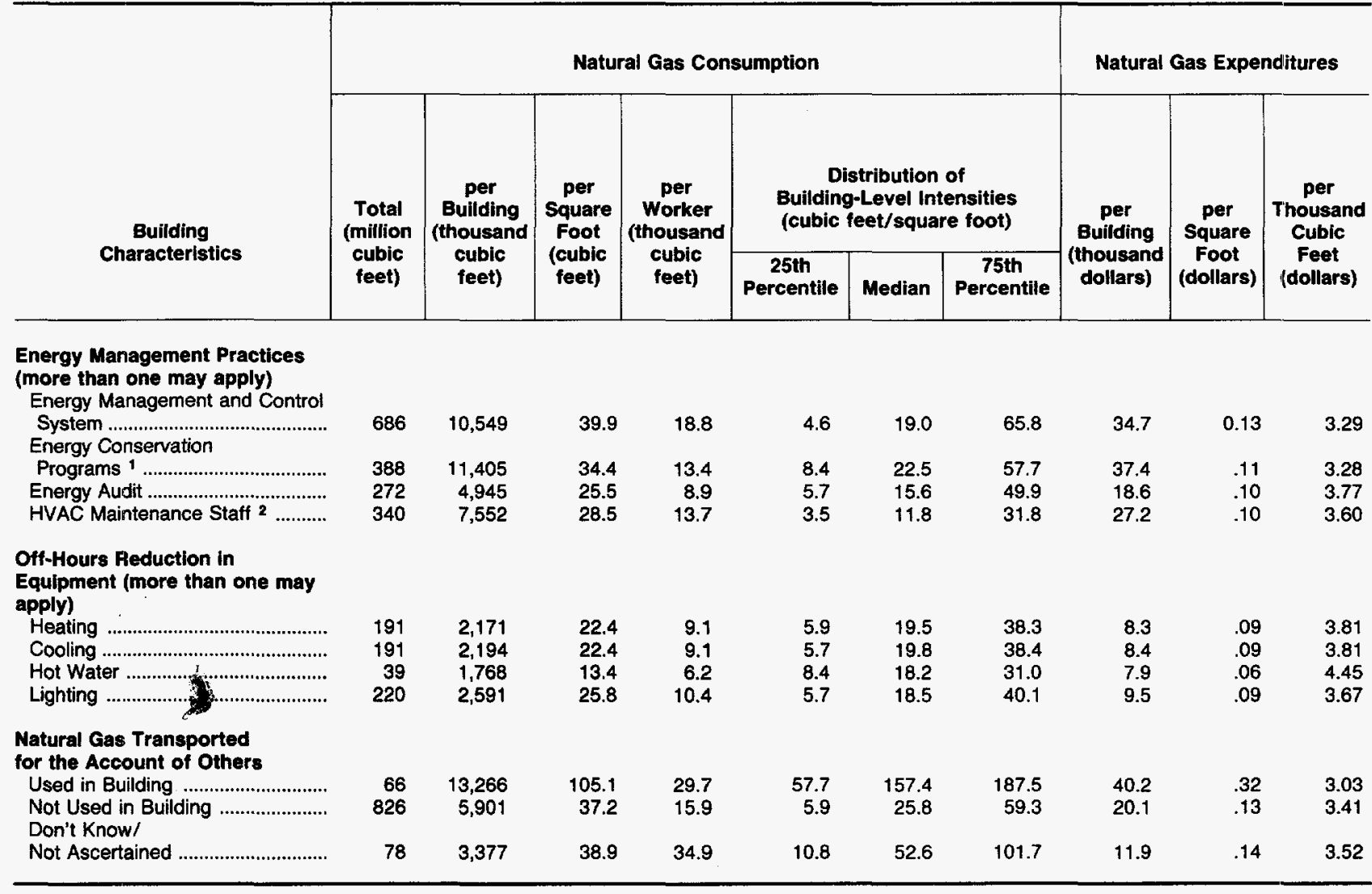

1 Building participates in any programs sponsored by the Federal Energy Management Program, in-house, utility, or third party.

2 HVAC maintenance staff means at least one person spends at least half their working hours maintaining the heating/cooling equipment.

$\left.{ }^{*}\right)$ Value rounds to zero in the units displayed.

$\mathrm{NC}=$ No cases in responding sample.

Notes: - Total workers are the number of workers during the main shift. - See Glossary for explanation of abbreviations and definitions of terms used in this report. - These data are from 881 federally owned buildings having the following criteria: (1) located in Federal Regions 3,6 , or $9 ;$ (2) larger than 10,000 square feet; and (3) used for a commercial purpose, other than warehouse and storage. In addition, 9 out of 10 selected buildings were from agencies other than the Department of Defense. - Statistics for the "energy end uses" represent consumption in buildings that have end use, not consumption for a particular fuel for a particular end use. - A/C = Air Conditioning. - FBSS = Federal Buildings Supplemental Survey.

- HVAC = Heating, Ventilation, and Air Conditioning. - VAV = Variable-Air Volume. - Data are for Fiscal Year 1993 (October 1, 1992 through September 30, 1993). Because of rounding, data may not sum to totals.

Source: Energy Information Administration, Office of Energy Markets and End Use, 1993 Federal Buildings Supplemental Survey. 
Table 3.51. Natural Gas Consumption and Expenditure Intensities in FBSS Buildings in Federal Region 9, 1993

\begin{tabular}{|c|c|c|c|c|c|c|c|c|c|c|}
\hline \multirow{2}{*}{$\begin{array}{c}\text { Building } \\
\text { Characteristics }\end{array}$} & \multicolumn{7}{|c|}{ Natural Gas Consumption } & \multicolumn{3}{|c|}{ Natural Gas Expenditures } \\
\hline & $\begin{array}{l}\text { Total } \\
\text { (million } \\
\text { cublc } \\
\text { feet) }\end{array}$ & $\begin{array}{c}\text { per } \\
\text { Building } \\
\text { (thousand } \\
\text { cublic } \\
\text { feet) }\end{array}$ & $\begin{array}{c}\text { per } \\
\text { Square } \\
\text { Foot } \\
\text { (cublc } \\
\text { feet) }\end{array}$ & $\begin{array}{c}\text { per } \\
\text { Worker } \\
\text { (thousand } \\
\text { cubic } \\
\text { feet) }\end{array}$ & \multicolumn{3}{|c|}{$\begin{array}{l}\text { Distribution of } \\
\text { Bullding-Level Intensities } \\
\text { (cubic feet/square foot) }\end{array}$} & $\begin{array}{c}\text { per } \\
\text { Bullding } \\
\text { (thousand } \\
\text { dollars) }\end{array}$ & $\begin{array}{l}\text { per } \\
\text { Square } \\
\text { Foot } \\
\text { (dollars) }\end{array}$ & $\begin{array}{c}\text { per } \\
\text { Thousano } \\
\text { Cublc } \\
\text { Feet } \\
\text { (dollars) }\end{array}$ \\
\hline All Bulldings & 1,047 & 4,655 & 30.2 & 14.0 & 11.4 & 26.6 & 57.2 & 20.6 & 0.13 & 4.42 \\
\hline \multicolumn{11}{|l|}{$\begin{array}{l}\text { Bullding Floorspace (square } \\
\text { feet) }\end{array}$} \\
\hline 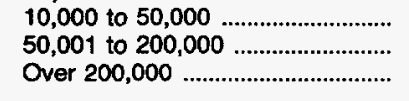 & $\begin{array}{l}109 \\
409 \\
529\end{array}$ & $\begin{array}{r}1,177 \\
4,705 \\
11,747\end{array}$ & $\begin{array}{l}47.5 \\
44.4 \\
22.8\end{array}$ & $\begin{array}{l}18.4 \\
21.3 \\
10.6\end{array}$ & $\begin{array}{r}12.5 \\
16.7 \\
5.9\end{array}$ & $\begin{array}{l}31.6 \\
30.4 \\
12.0\end{array}$ & $\begin{array}{l}82.9 \\
51.8 \\
24.4\end{array}$ & $\begin{array}{r}5.9 \\
21.8 \\
48.6\end{array}$ & $\begin{array}{l}.24 \\
.21 \\
.09\end{array}$ & $\begin{array}{l}4.97 \\
4.64 \\
4.14\end{array}$ \\
\hline 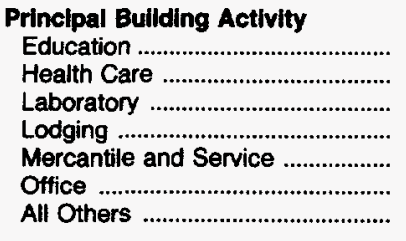 & $\begin{array}{r}18 \\
399 \\
96 \\
23 \\
54 \\
298 \\
159\end{array}$ & $\begin{array}{r}2,573 \\
15,353 \\
3,306 \\
2,519 \\
1,156 \\
4,197 \\
4,425\end{array}$ & $\begin{array}{r}66.3 \\
58.4 \\
37.6 \\
43.6 \\
8.2 \\
20.4 \\
48.0\end{array}$ & $\begin{array}{r}67.0 \\
26.9 \\
25.3 \\
87.2 \\
3.3 \\
8.8 \\
29.3\end{array}$ & $\begin{array}{r}30.4 \\
19.8 \\
29.2 \\
24.4 \\
6.0 \\
7.1 \\
20.7\end{array}$ & $\begin{array}{l}52.3 \\
57.2 \\
42.4 \\
54.5 \\
11.2 \\
20.4 \\
48.7\end{array}$ & $\begin{array}{r}63.1 \\
111.6 \\
67.9 \\
69.6 \\
18.0 \\
39.5 \\
96.5\end{array}$ & $\begin{array}{r}8.3 \\
65.6 \\
16.4 \\
12.0 \\
6.9 \\
17.5 \\
19.9\end{array}$ & $\begin{array}{l}.21 \\
.25 \\
.19 \\
.21 \\
.05 \\
.09 \\
.22\end{array}$ & $\begin{array}{l}3.22 \\
4.27 \\
4.95 \\
4.77 \\
5.94 \\
4.17 \\
4.50\end{array}$ \\
\hline 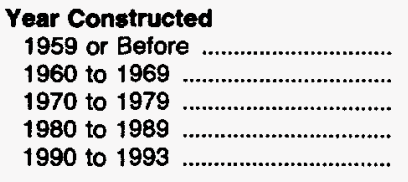 & $\begin{array}{r}382 \\
272 \\
217 \\
155 \\
22\end{array}$ & $\begin{array}{l}4,897 \\
5,672 \\
4,812 \\
3,693 \\
1,796\end{array}$ & $\begin{array}{l}41.7 \\
33.2 \\
22.0 \\
26.0 \\
14.1\end{array}$ & $\begin{array}{r}24.0 \\
14.1 \\
9.9 \\
10.7 \\
6.9\end{array}$ & $\begin{array}{r}20.0 \\
13.0 \\
7.5 \\
8.6 \\
4.5\end{array}$ & $\begin{array}{l}38.4 \\
28.9 \\
16.1 \\
21.4 \\
10.9\end{array}$ & $\begin{array}{l}60.8 \\
53.9 \\
30.0 \\
69.9 \\
43.3\end{array}$ & $\begin{array}{l}18.3 \\
25.3 \\
25.7 \\
16.0 \\
13.2\end{array}$ & $\begin{array}{l}.16 \\
.15 \\
.12 \\
.11 \\
.10\end{array}$ & $\begin{array}{l}3.74 \\
4.46 \\
5.34 \\
4.34 \\
7.35\end{array}$ \\
\hline 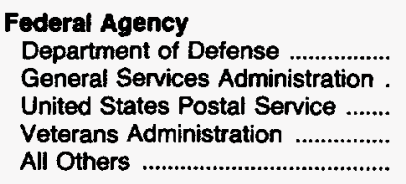 & $\begin{array}{r}162 \\
224 \\
58 \\
344 \\
260\end{array}$ & $\begin{array}{r}4,371 \\
5,884 \\
1,088 \\
11,861 \\
3,830\end{array}$ & $\begin{array}{r}34.0 \\
18.3 \\
8.2 \\
64.0 \\
49.5\end{array}$ & $\begin{array}{r}20.4 \\
8.0 \\
3.3 \\
28.8 \\
26.9\end{array}$ & $\begin{array}{r}18.5 \\
6.6 \\
5.2 \\
19.1 \\
26.7\end{array}$ & $\begin{array}{r}40.2 \\
16.6 \\
9.2 \\
57.2 \\
45.2\end{array}$ & $\begin{array}{r}62.0 \\
26.5 \\
16.1 \\
111.6 \\
80.4\end{array}$ & $\begin{array}{r}18.6 \\
23.1 \\
6.5 \\
50.2 \\
18.6\end{array}$ & $\begin{array}{l}.14 \\
.07 \\
.05 \\
.27 \\
.24\end{array}$ & $\begin{array}{l}4.25 \\
3.93 \\
5.98 \\
4.23 \\
4.86\end{array}$ \\
\hline 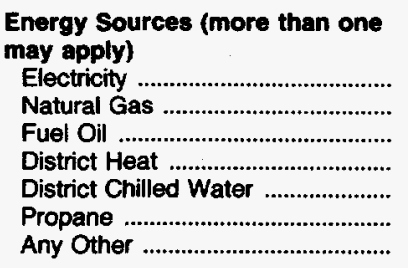 & $\begin{array}{r}1,047 \\
1,047 \\
496 \\
238 \\
146 \\
72 \\
72\end{array}$ & $\begin{array}{r}4,655 \\
4,655 \\
12,409 \\
8,814 \\
18,262 \\
71,563 \\
24,129\end{array}$ & $\begin{array}{l}30.2 \\
30.2 \\
47.1 \\
35.7 \\
38.2 \\
95.2 \\
42.7\end{array}$ & $\begin{array}{l}14.0 \\
14.0 \\
23.1 \\
18.3 \\
20.3 \\
47.7 \\
21.3\end{array}$ & $\begin{array}{r}11.4 \\
11.4 \\
24.0 \\
9.7 \\
12.9 \\
95.2 \\
11.4\end{array}$ & $\begin{array}{r}26.6 \\
26.6 \\
43.8 \\
22.8 \\
41.8 \\
95.2 \\
121.6\end{array}$ & $\begin{array}{r}57.2 \\
57.2 \\
98.3 \\
83.2 \\
157.0 \\
95.2 \\
161.0\end{array}$ & $\begin{array}{r}20.6 \\
20.6 \\
54.3 \\
37.8 \\
70.9 \\
392.3 \\
83.8\end{array}$ & $\begin{array}{l}.13 \\
.13 \\
.21 \\
.15 \\
.15 \\
.52 \\
.15\end{array}$ & $\begin{array}{l}4.42 \\
4.42 \\
4.37 \\
4.29 \\
3.88 \\
5.48 \\
3.47\end{array}$ \\
\hline 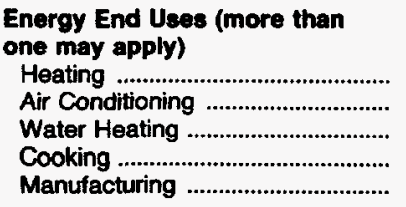 & $\begin{array}{r}1,044 \\
1,012 \\
1,044 \\
465 \\
95\end{array}$ & $\begin{array}{l}4,683 \\
4,844 \\
4,683 \\
9,126 \\
4,531\end{array}$ & $\begin{array}{l}30.7 \\
30.2 \\
30.4 \\
29.4 \\
17.5\end{array}$ & $\begin{array}{r}14.4 \\
13.8 \\
14.0 \\
12.5 \\
7.9\end{array}$ & $\begin{array}{r}11.6 \\
11.2 \\
11.4 \\
11.4 \\
7.6\end{array}$ & $\begin{array}{r}26.7 \\
26.3 \\
26.7 \\
29.6 \\
9.7\end{array}$ & $\begin{array}{l}57.3 \\
54.2 \\
57.3 \\
83.2 \\
22.6\end{array}$ & $\begin{array}{l}20.7 \\
21.1 \\
20.6 \\
42.5 \\
18.5\end{array}$ & $\begin{array}{l}.14 \\
.13 \\
.13 \\
.14 \\
.07\end{array}$ & $\begin{array}{l}4.42 \\
4.36 \\
4.40 \\
4.66 \\
4.08\end{array}$ \\
\hline 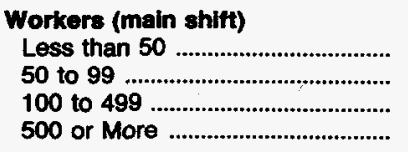 & $\begin{array}{r}77 \\
72 \\
436 \\
462\end{array}$ & $\begin{array}{r}1,408 \\
1,997 \\
4,637 \\
11,556\end{array}$ & $\begin{array}{l}38.2 \\
45.7 \\
39.9 \\
22.9\end{array}$ & $\begin{array}{r}69.8 \\
26.4 \\
23.0 \\
8.9\end{array}$ & $\begin{array}{r}14.3 \\
13.3 \\
12.5 \\
4.8\end{array}$ & $\begin{array}{l}48.2 \\
27.9 \\
26.6 \\
14.1\end{array}$ & $\begin{array}{l}83.6 \\
42.2 \\
51.8 \\
31.0\end{array}$ & $\begin{array}{r}7.7 \\
7.9 \\
21.6 \\
47.3\end{array}$ & $\begin{array}{l}.21 \\
.18 \\
.19 \\
.09\end{array}$ & $\begin{array}{l}5.45 \\
3.98 \\
4.66 \\
4.09\end{array}$ \\
\hline 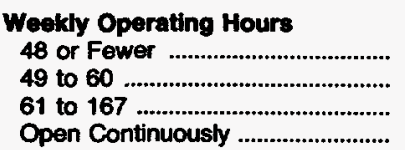 & $\begin{array}{l}149 \\
260 \\
149 \\
490\end{array}$ & $\begin{array}{l}3,465 \\
4,640 \\
2,608 \\
7,100\end{array}$ & $\begin{array}{l}49.6 \\
20.5 \\
27.9 \\
35.8\end{array}$ & $\begin{array}{r}34.9 \\
8.6 \\
15.3 \\
16.0\end{array}$ & $\begin{array}{r}20.0 \\
7.2 \\
9.3 \\
17.7\end{array}$ & $\begin{array}{l}32.8 \\
17.3 \\
18.5 \\
39.0\end{array}$ & $\begin{array}{l}82.9 \\
38.3 \\
42.4 \\
83.6\end{array}$ & $\begin{array}{l}14.1 \\
18.3 \\
12.4 \\
33.2\end{array}$ & $\begin{array}{l}.20 \\
.08 \\
.13 \\
.17\end{array}$ & $\begin{array}{l}4.07 \\
3.95 \\
4.76 \\
4.67\end{array}$ \\
\hline
\end{tabular}

See footnotes at end of table. 

FBSS Buildings in Federal Region 9, 1993 (Continued)

\begin{tabular}{|c|c|c|c|c|c|c|c|c|c|c|}
\hline \multirow{3}{*}{$\begin{array}{c}\text { Bullding } \\
\text { Characteristics }\end{array}$} & \multicolumn{7}{|c|}{ Natural Gas Consumption } & \multicolumn{3}{|c|}{ Natural Gas Expendltures } \\
\hline & \multirow{2}{*}{$\begin{array}{c}\text { Total } \\
\text { (million } \\
\text { cubic } \\
\text { feet) }\end{array}$} & \multirow{2}{*}{$\begin{array}{c}\text { per } \\
\text { Bullding } \\
\text { (thousand } \\
\text { cublc } \\
\text { feet) }\end{array}$} & \multirow{2}{*}{$\begin{array}{c}\text { per } \\
\text { Square } \\
\text { Foot } \\
\text { (cubic } \\
\text { feet) }\end{array}$} & \multirow{2}{*}{$\begin{array}{c}\text { per } \\
\text { Worker } \\
\text { (thousand } \\
\text { cubic } \\
\text { feet) }\end{array}$} & \multicolumn{3}{|c|}{$\begin{array}{l}\text { Distribution of } \\
\text { Bullding-Level Intensities } \\
\text { (cubic feet/square foot) }\end{array}$} & \multirow{2}{*}{$\begin{array}{c}\text { per } \\
\text { Bullding } \\
\text { (thousand } \\
\text { dollars) }\end{array}$} & \multirow{2}{*}{$\begin{array}{c}\text { per } \\
\text { Square } \\
\text { Foot } \\
\text { (dollars) }\end{array}$} & \multirow{2}{*}{$\begin{array}{l}\text { per } \\
\text { Thousand } \\
\text { Cublc } \\
\text { Feet } \\
\text { (dollars) }\end{array}$} \\
\hline & & & & & $\begin{array}{c}\text { 25th } \\
\text { Percentile }\end{array}$ & Medlan & $\begin{array}{c}\text { 75th } \\
\text { Percentlle }\end{array}$ & & & \\
\hline \multicolumn{11}{|l|}{ Multibullding Facility } \\
\hline 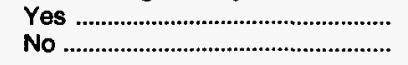 & $\begin{array}{l}720 \\
327\end{array}$ & $\begin{array}{l}6,053 \\
3,086\end{array}$ & $\begin{array}{l}38.1 \\
20.7\end{array}$ & $\begin{array}{r}17.6 \\
9.7\end{array}$ & $\begin{array}{r}19.8 \\
7.1\end{array}$ & $\begin{array}{l}40.9 \\
16.8\end{array}$ & $\begin{array}{l}69.6 \\
30.0\end{array}$ & $\begin{array}{l}27.4 \\
13.0\end{array}$ & $\begin{array}{r}0.17 \\
.09\end{array}$ & $\begin{array}{l}4.52 \\
4.20\end{array}$ \\
\hline \multicolumn{11}{|l|}{ Space-Heating Energy Source } \\
\hline 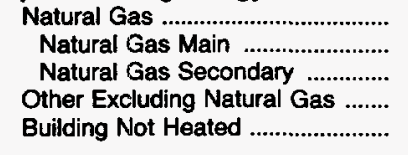 & $\begin{array}{r}904 \\
836 \\
67 \\
141 \\
3\end{array}$ & $\begin{array}{l}4,611 \\
4,521 \\
6,131 \\
5,205 \\
1,558\end{array}$ & $\begin{array}{r}31.3 \\
30.8 \\
40.6 \\
26.9 \\
4.7\end{array}$ & $\begin{array}{r}14.4 \\
14.5 \\
14.0 \\
14.0 \\
1.5\end{array}$ & $\begin{array}{r}13.2 \\
14.3 \\
5.2 \\
7.2 \\
2.0\end{array}$ & $\begin{array}{r}28.9 \\
28.6 \\
31.0 \\
12.4 \\
4.6\end{array}$ & $\begin{array}{r}60.4 \\
59.3 \\
60.8 \\
32.8 \\
7.1\end{array}$ & $\begin{array}{r}20.4 \\
20.1 \\
25.3 \\
22.6 \\
9.1\end{array}$ & $\begin{array}{l}.14 \\
.14 \\
.17 \\
.12 \\
.03\end{array}$ & $\begin{array}{l}4.43 \\
4.45 \\
4.13 \\
4.34 \\
5.87\end{array}$ \\
\hline $\begin{array}{l}\text { Cooling Energy Source } \\
\text { Natural Gas .................................... } \\
\text { Other Excluding Natural Gas ....... } \\
\text { A/C Not Performed ........................ }\end{array}$ & $\begin{array}{r}2 \\
1,011 \\
35\end{array}$ & $\begin{array}{r}309 \\
4,978 \\
2,189\end{array}$ & $\begin{array}{r}9.6 \\
30.3 \\
30.7\end{array}$ & $\begin{array}{r}3.2 \\
13.9 \\
20.4\end{array}$ & $\begin{array}{r}5.2 \\
11.4 \\
21.4\end{array}$ & $\begin{array}{l}11.2 \\
26.6 \\
53.4\end{array}$ & $\begin{array}{l}15.7 \\
57.2 \\
61.8\end{array}$ & $\begin{array}{r}2.3 \\
21.7 \\
13.3\end{array}$ & $\begin{array}{l}.07 \\
.13 \\
.19\end{array}$ & $\begin{array}{l}7.51 \\
4.36 \\
6.07\end{array}$ \\
\hline $\begin{array}{l}\text { Water-Heating Energy Source } \\
\text { Natural Gas ...................................... } \\
\text { Other Excluding Natural Gas ....... } \\
\text { Water Heating Not Performed ..... }\end{array}$ & $\begin{array}{r}813 \\
231 \\
3\end{array}$ & $\begin{array}{l}4,566 \\
5,144 \\
1,572\end{array}$ & $\begin{array}{r}30.0 \\
31.7 \\
9.7\end{array}$ & $\begin{array}{r}13.9 \\
14.5 \\
9.1\end{array}$ & $\begin{array}{r}15.7 \\
6.9 \\
9.0\end{array}$ & $\begin{array}{l}30.0 \\
12.9 \\
14.6\end{array}$ & $\begin{array}{l}60.8 \\
39.5 \\
20.2\end{array}$ & $\begin{array}{l}20.5 \\
21.2 \\
15.6\end{array}$ & $\begin{array}{l}.13 \\
.13 \\
.10\end{array}$ & $\begin{array}{l}4.48 \\
4.13 \\
9.94\end{array}$ \\
\hline $\begin{array}{l}\text { Cooking Energy Source } \\
\text { Natural Gas ................................. } \\
\text { Other Excluding Natural Gas ........ } \\
\text { Cooking Not Performed ................. }\end{array}$ & $\begin{array}{l}329 \\
136 \\
582\end{array}$ & $\begin{array}{l}9,690 \\
7,997 \\
3,345\end{array}$ & $\begin{array}{l}33.4 \\
22.8 \\
30.8\end{array}$ & $\begin{array}{l}13.0 \\
11.4 \\
15.5\end{array}$ & $\begin{array}{r}7.1 \\
12.2 \\
11.4\end{array}$ & $\begin{array}{l}26.3 \\
30.8 \\
26.5\end{array}$ & $\begin{array}{l}67.8 \\
83.2 \\
52.3\end{array}$ & $\begin{array}{l}45.8 \\
35.8 \\
14.1\end{array}$ & $\begin{array}{l}.16 \\
.10 \\
.13\end{array}$ & $\begin{array}{l}4.73 \\
4.48 \\
4.23\end{array}$ \\
\hline $\begin{array}{l}\text { Manufacturing Energy Source } \\
\text { Natural Gas .................................. } \\
\text { Other Excluding Natural Gas ....... } \\
\text { Manufacturing Not Performed ..... }\end{array}$ & $\begin{array}{r}27 \\
68 \\
952\end{array}$ & $\begin{array}{l}3,355 \\
5,256 \\
4,668\end{array}$ & $\begin{array}{l}14.6 \\
19.0 \\
32.5\end{array}$ & $\begin{array}{r}7.0 \\
8.4 \\
15.2\end{array}$ & $\begin{array}{r}8.0 \\
7.1 \\
12.5\end{array}$ & $\begin{array}{r}10.8 \\
9.3 \\
28.4\end{array}$ & $\begin{array}{l}20.4 \\
46.7 \\
57.6\end{array}$ & $\begin{array}{l}16.1 \\
20.0 \\
20.8\end{array}$ & $\begin{array}{l}.07 \\
.07 \\
.14\end{array}$ & $\begin{array}{l}4.81 \\
3.80 \\
4.45\end{array}$ \\
\hline $\begin{array}{l}\text { Percent of Floorspace Heated } \\
\text { Not Heated } \\
1 \text { to } 50 \\
51 \text { to } 100\end{array}$ & $\begin{array}{r}3 \\
76 \\
968\end{array}$ & $\begin{array}{l}1,558 \\
6,321 \\
4,590\end{array}$ & $\begin{array}{r}4.7 \\
46.1 \\
29.9\end{array}$ & $\begin{array}{r}1.5 \\
73.9 \\
13.5\end{array}$ & $\begin{array}{r}2.0 \\
5.7 \\
11.6\end{array}$ & $\begin{array}{r}4.6 \\
35.3 \\
26.6\end{array}$ & $\begin{array}{r}7.1 \\
57.9 \\
57.3\end{array}$ & $\begin{array}{r}9.1 \\
25.3 \\
20.4\end{array}$ & $\begin{array}{l}.03 \\
.18 \\
.13\end{array}$ & $\begin{array}{l}5.87 \\
4.00 \\
4.45\end{array}$ \\
\hline \multicolumn{11}{|l|}{$\begin{array}{l}\text { Heating Equipment (more than } \\
\text { one may apply) }\end{array}$} \\
\hline 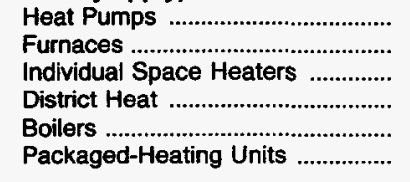 & $\begin{array}{r}76 \\
22 \\
132 \\
257 \\
617 \\
264\end{array}$ & $\begin{array}{l}2,613 \\
1,018 \\
5,753 \\
7,550 \\
4,639 \\
4,549\end{array}$ & $\begin{array}{l}29.6 \\
19.3 \\
28.9 \\
34.7 \\
26.5 \\
47.6\end{array}$ & $\begin{array}{r}12.4 \\
8.4 \\
12.7 \\
18.5 \\
11.6 \\
22.5\end{array}$ & $\begin{array}{r}7.2 \\
8.6 \\
9.2 \\
12.0 \\
14.1 \\
12.9\end{array}$ & $\begin{array}{l}36.2 \\
18.2 \\
30.4 \\
31.2 \\
27.0 \\
26.6\end{array}$ & $\begin{array}{r}49.1 \\
49.1 \\
67.8 \\
101.3 \\
51.8 \\
63.1\end{array}$ & $\begin{array}{r}10.8 \\
6.0 \\
24.7 \\
32.4 \\
22.6 \\
16.3\end{array}$ & $\begin{array}{l}.12 \\
.11 \\
.12 \\
.15 \\
.13 \\
.17\end{array}$ & $\begin{array}{l}4.12 \\
5.94 \\
4.30 \\
4.29 \\
4.87 \\
3.58\end{array}$ \\
\hline \multicolumn{11}{|l|}{$\begin{array}{l}\text { Retrofit or Purchase of any } \\
\text { Equipment Within Last Ten } \\
\text { Years (more than one may } \\
\text { apply) }\end{array}$} \\
\hline 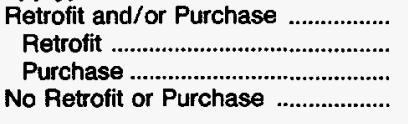 & $\begin{array}{l}876 \\
643 \\
495 \\
171\end{array}$ & $\begin{array}{l}5,409 \\
8,464 \\
4,340 \\
2,717\end{array}$ & $\begin{array}{l}29.4 \\
33.2 \\
29.9 \\
34.8\end{array}$ & $\begin{array}{l}13.4 \\
14.5 \\
13.6 \\
18.4\end{array}$ & $\begin{array}{r}11.2 \\
15.9 \\
9.6 \\
12.0\end{array}$ & $\begin{array}{l}27.6 \\
31.5 \\
26.4 \\
26.5\end{array}$ & $\begin{array}{l}57.2 \\
70.0 \\
54.2 \\
60.8\end{array}$ & $\begin{array}{l}24.2 \\
35.5 \\
21.4 \\
11.3\end{array}$ & $\begin{array}{l}.13 \\
.14 \\
.15 \\
.14\end{array}$ & $\begin{array}{l}4.47 \\
4.19 \\
4.94 \\
4.16\end{array}$ \\
\hline \multicolumn{11}{|l|}{$\begin{array}{l}\text { HVAC Conservation Features } \\
\text { (more than one may apply) }\end{array}$} \\
\hline $\begin{array}{l}\text { VAV System ................................ } \\
\text { Economizer Cycle ........................ } \\
\text { HVAC Maintenance ........................ }\end{array}$ & $\begin{array}{r}390 \\
674 \\
1,028\end{array}$ & $\begin{array}{l}6,614 \\
5,911 \\
4,780\end{array}$ & $\begin{array}{l}29.8 \\
29.6 \\
30.1\end{array}$ & $\begin{array}{l}13.9 \\
13.5 \\
13.9\end{array}$ & $\begin{array}{r}11.2 \\
9.7 \\
11.2\end{array}$ & $\begin{array}{l}22.5 \\
22.0 \\
26.5\end{array}$ & $\begin{array}{l}50.9 \\
51.8 \\
57.1\end{array}$ & $\begin{array}{l}29.8 \\
24.9 \\
21.1\end{array}$ & $\begin{array}{l}.13 \\
.12 \\
.13\end{array}$ & $\begin{array}{l}4.51 \\
4.21 \\
4.40\end{array}$ \\
\hline
\end{tabular}

See footnotes at end of table. 
Table 3.51. Natural Gas Consumption and Expenditure Intensities in FBSS Buildings in Federal Region 9, 1993 (Continued)

\begin{tabular}{|c|c|c|c|c|c|c|c|c|c|c|}
\hline \multirow{3}{*}{$\begin{array}{c}\text { Building } \\
\text { Characteristics }\end{array}$} & \multicolumn{7}{|c|}{ Natural Gas Consumption } & \multicolumn{3}{|c|}{ Natural Gas Expenditures } \\
\hline & \multirow{2}{*}{$\begin{array}{l}\text { Total } \\
\text { (million } \\
\text { cuble } \\
\text { feet) }\end{array}$} & \multirow{2}{*}{$\begin{array}{c}\text { per } \\
\text { Bullding } \\
\text { (thousand } \\
\text { cublc } \\
\text { feet) }\end{array}$} & \multirow{2}{*}{$\begin{array}{c}\text { per } \\
\text { Square } \\
\text { Foot } \\
\text { (cublc } \\
\text { feet) }\end{array}$} & \multirow{2}{*}{$\begin{array}{c}\text { per } \\
\text { Worker } \\
\text { (thousand } \\
\text { cubic } \\
\text { feet) }\end{array}$} & \multicolumn{3}{|c|}{$\begin{array}{l}\text { Distribution of } \\
\text { Bullding-Level Intensitles } \\
\text { (cubic feet/square foot) }\end{array}$} & \multirow{2}{*}{$\begin{array}{c}\text { per } \\
\text { Bullding } \\
\text { (thousand } \\
\text { dollars) }\end{array}$} & \multirow{2}{*}{$\begin{array}{c}\text { per } \\
\text { Square } \\
\text { Foot } \\
\text { (dollars) }\end{array}$} & \multirow{2}{*}{$\begin{array}{l}\text { per } \\
\text { Thousend } \\
\text { Cublc } \\
\text { Feet } \\
\text { (dollars) }\end{array}$} \\
\hline & & & & & $\begin{array}{c}\text { 25th } \\
\text { Percentlle }\end{array}$ & Median & $\begin{array}{c}\text { 75th } \\
\text { Percentlle }\end{array}$ & & & \\
\hline \multicolumn{11}{|l|}{$\begin{array}{l}\text { Energy Management Practlces } \\
\text { (more than one may apply) } \\
\text { Energy Management and Control }\end{array}$} \\
\hline $\begin{array}{l}\text { System } \\
\text { Energy Conservation }\end{array}$ & 360 & 6,319 & 24.9 & 10.9 & 8.2 & 24.4 & 59.3 & 29.1 & 0.11 & 4.61 \\
\hline 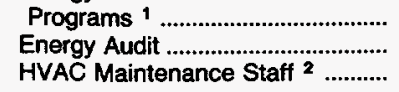 & $\begin{array}{l}655 \\
344 \\
371\end{array}$ & $\begin{array}{l}6,684 \\
5,139 \\
9,509\end{array}$ & $\begin{array}{l}29.9 \\
25.8 \\
29.5\end{array}$ & $\begin{array}{l}13.3 \\
11.0 \\
12.3\end{array}$ & $\begin{array}{r}11.6 \\
9.7 \\
6.5\end{array}$ & $\begin{array}{l}26.6 \\
22.6 \\
21.5\end{array}$ & $\begin{array}{l}57.3 \\
58.2 \\
57.3\end{array}$ & $\begin{array}{l}28.2 \\
23.9 \\
41.1\end{array}$ & $\begin{array}{l}.13 \\
.12 \\
.13\end{array}$ & $\begin{array}{l}4.22 \\
4.65 \\
4.33\end{array}$ \\
\hline \multicolumn{11}{|l|}{$\begin{array}{l}\text { Otf-Hours Reduction in } \\
\text { Equipment (more than one may } \\
\text { apply) }\end{array}$} \\
\hline 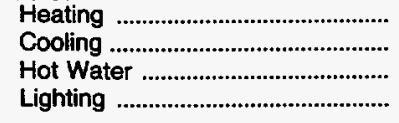 & $\begin{array}{l}453 \\
432 \\
270 \\
511\end{array}$ & $\begin{array}{l}3,571 \\
3,515 \\
4,580 \\
3,902\end{array}$ & $\begin{array}{l}24.6 \\
24.6 \\
20.7 \\
26.8\end{array}$ & $\begin{array}{r}11.5 \\
11.5 \\
9.2 \\
12.5\end{array}$ & $\begin{array}{r}9.6 \\
9.2 \\
9.6 \\
10.5\end{array}$ & $\begin{array}{l}20.9 \\
20.0 \\
20.9 \\
22.5\end{array}$ & $\begin{array}{l}39.7 \\
39.3 \\
30.8 \\
47.6\end{array}$ & $\begin{array}{l}15.0 \\
14.5 \\
17.9 \\
16.1\end{array}$ & $\begin{array}{l}.10 \\
.10 \\
.08 \\
.11\end{array}$ & $\begin{array}{l}4.19 \\
4.11 \\
3.91 \\
4.14\end{array}$ \\
\hline \multicolumn{11}{|l|}{$\begin{array}{l}\text { Natural Gas Transported } \\
\text { for the Account of Others }\end{array}$} \\
\hline 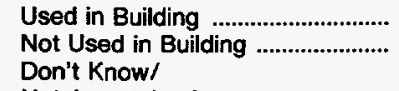 & $\begin{array}{l}223 \\
736\end{array}$ & $\begin{array}{l}9,286 \\
4,231\end{array}$ & $\begin{array}{l}42.8 \\
27.5\end{array}$ & $\begin{array}{l}17.4 \\
13.2\end{array}$ & $\begin{array}{l}12.9 \\
10.6\end{array}$ & $\begin{array}{l}42.8 \\
22.9\end{array}$ & $\begin{array}{l}57.3 \\
51.8\end{array}$ & $\begin{array}{l}39.1 \\
18.7\end{array}$ & $\begin{array}{l}.18 \\
.12\end{array}$ & $\begin{array}{l}4.21 \\
4.43\end{array}$ \\
\hline 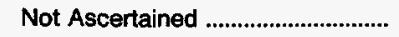 & 85 & 3,397 & 32.3 & 13.7 & 20.2 & 37.6 & 69.9 & 16.4 & .16 & 4.82 \\
\hline
\end{tabular}

1 Building particjpates in any programs sponsored by the Federal Energy Management Program, in-house, utility, or third party.

2 HVAC maintenance staff means at least one person spends at least half their working hours maintaining the heating/cooling equipment.

NC $=$ No cases in responding sample.

Notes: - Total workers are the number of workers during the main shift. - See Glossary for explanation of abbreviations and definitions of terms used in this report. - These data are from 881 federally owned buildings having the following criteria: (1) located in Federal Regions 3 , 6 , or 9 ; (2) larger than 10,000 square feet; and (3) used for a commercial purpose, other than warehouse and storage. In addition, 9 out of 10 selected buildings were from agencies other than the Department of Defense. - Statistics for the "energy end uses" represent consumption in buildings that have end use, not consumption for a particular fuel for a particular end use. $\bullet$ A/C = Air Conditioning. $\bullet$ FBSS = Federal Buildings Supplemental Survey.

- HVAC = Heating, Ventilation, and Air Conditioning. • VAV = Variable-Air Volume. - Data are for Fiscal Year 1993 (October 1, 1992 through September 30,1993$)$. Because of rounding, data may not sum to totals.

Source: Energy Information Administration, Office of Energy Markets and End Use, 1993 Federal Buildings Supplemental Survey. 
Table 3.52. District Heat Consumption and Expenditure Intensities in FBSS Buildings in Federal Region 3, 1993

\begin{tabular}{|c|c|c|c|c|c|c|c|}
\hline \multirow[b]{2}{*}{$\begin{array}{l}\text { Bullding } \\
\text { Characteristics }\end{array}$} & \multicolumn{4}{|c|}{ District Heat Consumption } & \multicolumn{3}{|c|}{ District Heat Expenditures } \\
\hline & $\begin{array}{l}\text { Total } \\
\text { (million } \\
\text { pounds) }\end{array}$ & $\begin{array}{l}\text { per } \\
\text { Bullding } \\
\text { (thousand } \\
\text { pounds) }\end{array}$ & $\begin{array}{l}\text { per } \\
\text { Square } \\
\text { foot } \\
\text { (pounds) }\end{array}$ & $\begin{array}{l}\text { per Worker } \\
\text { (thousand } \\
\text { pounds) }\end{array}$ & $\begin{array}{c}\text { per } \\
\text { Bullding } \\
\text { (thousand } \\
\text { dollars) }\end{array}$ & $\begin{array}{l}\text { per } \\
\text { Square } \\
\text { Foot } \\
\text { (dollars) }\end{array}$ & $\begin{array}{l}\text { per } \\
\text { Thousano } \\
\text { Pound } \\
\text { (dollars) }\end{array}$ \\
\hline 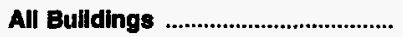 & 3,015 & 19,577 & 47.11 & 12.5 & 241.6 & 0.58 & 12.34 \\
\hline \multicolumn{8}{|l|}{$\begin{array}{l}\text { Bullding Floorspace (square } \\
\text { feet) }\end{array}$} \\
\hline 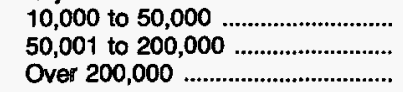 & $\begin{array}{r}187 \\
805 \\
2,022\end{array}$ & $\begin{array}{r}6,034 \\
12,786 \\
33,707\end{array}$ & $\begin{array}{r}195.10 \\
115.92 \\
36.06\end{array}$ & $\begin{array}{r}119.6 \\
60.9 \\
8.9\end{array}$ & $\begin{array}{r}56.2 \\
138.1 \\
446.2\end{array}$ & $\begin{array}{r}1.82 \\
1.25 \\
.48\end{array}$ & $\begin{array}{r}9.32 \\
10.80 \\
13.24\end{array}$ \\
\hline \multicolumn{8}{|l|}{ Principal Building Activity } \\
\hline 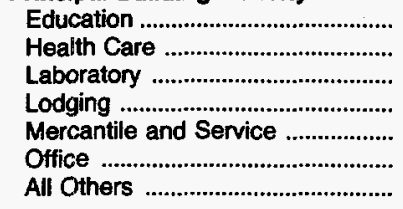 & $\begin{array}{r}19 \\
422 \\
765 \\
37 \\
69 \\
1,372 \\
331\end{array}$ & $\begin{array}{r}4,754 \\
20,091 \\
26,372 \\
5,326 \\
23,105 \\
19,883 \\
15,747\end{array}$ & $\begin{array}{r}72.05 \\
50.04 \\
217.10 \\
76.24 \\
33.16 \\
31.08 \\
65.43\end{array}$ & $\begin{array}{r}48.5 \\
31.7 \\
130.6 \\
83.8 \\
2.1 \\
7.9 \\
23.4\end{array}$ & $\begin{array}{r}55.7 \\
208.0 \\
292.3 \\
64.3 \\
283.6 \\
275.0 \\
184.2\end{array}$ & $\begin{array}{r}.84 \\
.52 \\
2.41 \\
.92 \\
.41 \\
.43 \\
.77\end{array}$ & $\begin{array}{l}11.72 \\
10.35 \\
11.08 \\
12.07 \\
12.27 \\
13.83 \\
11.70\end{array}$ \\
\hline 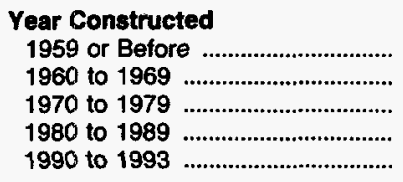 & $\begin{array}{r}1,500 \\
954 \\
244 \\
197 \\
120\end{array}$ & $\begin{array}{c}18,992 \\
19,865 \\
22,214 \\
19,678 \\
19,987\end{array}$ & $\begin{array}{l}43.45 \\
63.78 \\
27.09 \\
78.56 \\
40.21\end{array}$ & $\begin{array}{r}8.6 \\
26.5 \\
10.2 \\
44.4 \\
32.3\end{array}$ & $\begin{array}{l}228.9 \\
250.6 \\
355.4 \\
170.4 \\
247.6\end{array}$ & $\begin{array}{l}.52 \\
.80 \\
.43 \\
.68 \\
.50\end{array}$ & $\begin{array}{r}12.05 \\
12.62 \\
16.00 \\
8.66 \\
12.39\end{array}$ \\
\hline $\begin{array}{l}\text { Federal Agency } \\
\text { Department of Defense ................ } \\
\text { General Services Administration . } \\
\text { United States Postal Service ........ } \\
\text { Veterans Administration ................. } \\
\text { All Others }\end{array}$ & $\begin{array}{r}462 \\
1,009 \\
42 \\
407 \\
1,095\end{array}$ & $\begin{array}{l}35,532 \\
21,476 \\
20,837 \\
14,520 \\
17,115\end{array}$ & $\begin{array}{r}39.22 \\
29.05 \\
32.06 \\
68.33 \\
107.26\end{array}$ & $\begin{array}{r}11.3 \\
7.1 \\
1.4 \\
49.6 \\
53.3\end{array}$ & $\begin{array}{l}558.3 \\
294.9 \\
261.9 \\
146.5 \\
179.2\end{array}$ & $\begin{array}{r}.62 \\
.40 \\
.40 \\
.69 \\
1.12\end{array}$ & $\begin{array}{l}15.71 \\
13.73 \\
12.57 \\
10.09 \\
10.47\end{array}$ \\
\hline 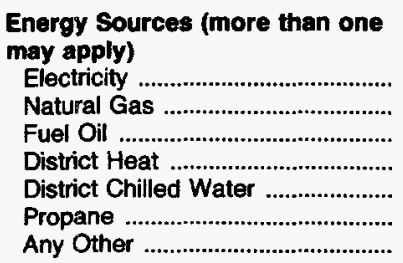 & $\begin{array}{r}3,015 \\
1,337 \\
749 \\
3,015 \\
1,273 \\
40 \\
70\end{array}$ & $\begin{array}{l}19,577 \\
34,290 \\
39,431 \\
19,577 \\
22,731 \\
19,769 \\
23,281\end{array}$ & $\begin{array}{l}47.11 \\
37.97 \\
32.81 \\
47.11 \\
55.62 \\
59.28 \\
22.00\end{array}$ & $\begin{array}{r}12.5 \\
7.6 \\
11.7 \\
12.5 \\
19.8 \\
52.7 \\
8.1\end{array}$ & $\begin{array}{l}241.6 \\
476.7 \\
599.0 \\
241.6 \\
289.3 \\
173.6 \\
174.9\end{array}$ & $\begin{array}{l}.58 \\
.53 \\
.50 \\
.58 \\
.71 \\
.52 \\
.17\end{array}$ & $\begin{array}{r}12.34 \\
13.90 \\
15.19 \\
12.34 \\
12.73 \\
8.78 \\
7.51\end{array}$ \\
\hline \multicolumn{8}{|l|}{$\begin{array}{l}\text { Energy End Uses (more than } \\
\text { one may apply) }\end{array}$} \\
\hline 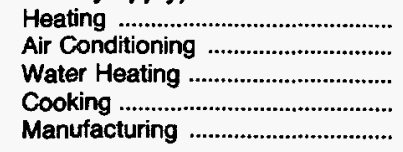 & $\begin{array}{r}3,015 \\
3,011 \\
3,001 \\
1,834 \\
845\end{array}$ & $\begin{array}{l}19,577 \\
19,683 \\
19,742 \\
32,757 \\
42,260\end{array}$ & $\begin{array}{l}47.11 \\
47.08 \\
47.33 \\
36.26 \\
43.59\end{array}$ & $\begin{array}{r}12.5 \\
12.5 \\
12.5 \\
8.7 \\
10.5\end{array}$ & $\begin{array}{l}241.6 \\
242.9 \\
243.5 \\
431.1 \\
536.9\end{array}$ & $\begin{array}{l}.58 \\
.58 \\
.58 \\
.48 \\
.55\end{array}$ & $\begin{array}{l}12.34 \\
12.34 \\
12.33 \\
13.16 \\
12.70\end{array}$ \\
\hline \multicolumn{8}{|l|}{ Workers (main shift) } \\
\hline $\begin{array}{l}\text { Less than } 50 \\
50 \text { to } 99 \\
100 \text { to } 499 \\
500 \text { or More }\end{array}$ & $\begin{array}{r}190 \\
130 \\
791 \\
1,904\end{array}$ & $\begin{array}{r}6,118 \\
7,240 \\
15,818 \\
34,620\end{array}$ & $\begin{array}{r}97.49 \\
138.75 \\
118.23 \\
34.99\end{array}$ & $\begin{array}{r}242.8 \\
106.3 \\
66.5 \\
8.4\end{array}$ & $\begin{array}{r}58.6 \\
70.1 \\
176.2 \\
460.4\end{array}$ & $\begin{array}{r}.93 \\
1.34 \\
1.32 \\
.47\end{array}$ & $\begin{array}{r}9.57 \\
9.68 \\
11.14 \\
13.30\end{array}$ \\
\hline 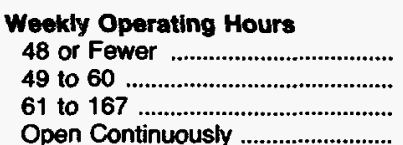 & $\begin{array}{r}354 \\
754 \\
249 \\
1,658\end{array}$ & $\begin{array}{l}11,798 \\
15,718 \\
13,816 \\
28,583\end{array}$ & $\begin{array}{l}51.57 \\
35.71 \\
38.52 \\
56.12\end{array}$ & $\begin{array}{l}22.1 \\
13.5 \\
16.6 \\
10.7\end{array}$ & $\begin{array}{l}123.3 \\
224.1 \\
146.3 \\
347.0\end{array}$ & $\begin{array}{l}.54 \\
.51 \\
.41 \\
.68\end{array}$ & $\begin{array}{l}10.45 \\
14.26 \\
10.59 \\
12.14\end{array}$ \\
\hline
\end{tabular}

See footnotes at end of table. 
Table 3.52. District Heat Consumption and Expenditure Intensities in FBSS Buildings in Federal Region 3, 1993 (Continued)

\begin{tabular}{|c|c|c|c|c|c|c|c|}
\hline \multirow[b]{2}{*}{$\begin{array}{l}\text { Bullding } \\
\text { Characteristics }\end{array}$} & \multicolumn{4}{|c|}{ District Heat Consumption } & \multicolumn{3}{|c|}{ District Heat Expenditures } \\
\hline & $\begin{array}{l}\text { Total } \\
\text { (million } \\
\text { pounds) }\end{array}$ & $\begin{array}{l}\text { per } \\
\text { Bullding } \\
\text { (thousand } \\
\text { pounds) }\end{array}$ & $\begin{array}{l}\text { per } \\
\text { Square } \\
\text { foot } \\
\text { (pounds) }\end{array}$ & $\begin{array}{l}\text { per Worker } \\
\text { (thousand } \\
\text { pounds) }\end{array}$ & $\begin{array}{l}\text { per } \\
\text { Building } \\
\text { (thousand } \\
\text { dollars) }\end{array}$ & $\begin{array}{l}\text { per } \\
\text { Square } \\
\text { Foot } \\
\text { (dollars) }\end{array}$ & $\begin{array}{l}\text { per } \\
\text { Thousand } \\
\text { Pound } \\
\text { (dollars) }\end{array}$ \\
\hline \multicolumn{8}{|l|}{$\begin{array}{l}\text { HVAC Conservation Features } \\
\text { (more than one may apply) }\end{array}$} \\
\hline 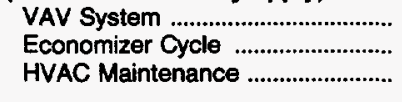 & $\begin{array}{r}814 \\
1,819 \\
2,983\end{array}$ & $\begin{array}{l}21,434 \\
23,326 \\
19,755\end{array}$ & $\begin{array}{l}38.85 \\
43.17 \\
47.09\end{array}$ & $\begin{array}{r}8.8 \\
9.5 \\
12.4\end{array}$ & $\begin{array}{l}277.7 \\
300.8 \\
244.1\end{array}$ & $\begin{array}{r}0.50 \\
.56 \\
.58\end{array}$ & $\begin{array}{l}12.96 \\
12.89 \\
12.35\end{array}$ \\
\hline \multicolumn{8}{|l|}{$\begin{array}{l}\text { Energy Management Practlces } \\
\text { (more than one may apply) } \\
\text { Energy Management and Control }\end{array}$} \\
\hline $\begin{array}{l}\text { System .................................... } \\
\text { Energy Conservation }\end{array}$ & 1,583 & 22,609 & 38.59 & 8.5 & 284.6 & .49 & 12.59 \\
\hline 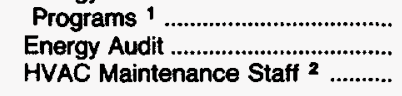 & $\begin{array}{l}1,533 \\
1,071 \\
1,491\end{array}$ & $\begin{array}{l}26,898 \\
19,836 \\
27,101\end{array}$ & $\begin{array}{l}32.90 \\
41.50 \\
40.07\end{array}$ & $\begin{array}{r}8.5 \\
15.3 \\
8.0\end{array}$ & $\begin{array}{l}366.2 \\
257.4 \\
383.7\end{array}$ & $\begin{array}{l}.45 \\
.54 \\
.57\end{array}$ & $\begin{array}{l}13.62 \\
12.98 \\
14.16\end{array}$ \\
\hline \multicolumn{8}{|l|}{$\begin{array}{l}\text { Off-Hours Reduction in } \\
\text { Equipment (more than one may } \\
\text { apply) }\end{array}$} \\
\hline 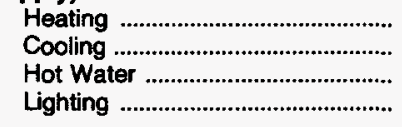 & $\begin{array}{r}1,160 \\
1,162 \\
718 \\
1,235\end{array}$ & $\begin{array}{l}15,468 \\
15,284 \\
17,090 \\
15,063\end{array}$ & $\begin{array}{l}35.82 \\
35.84 \\
39.04 \\
37.98\end{array}$ & $\begin{array}{l}13.8 \\
13.8 \\
15.6 \\
15.6\end{array}$ & $\begin{array}{l}198.6 \\
196.1 \\
215.0 \\
191.6\end{array}$ & $\begin{array}{l}.46 \\
.46 \\
.49 \\
.48\end{array}$ & $\begin{array}{l}12.84 \\
12.83 \\
12.58 \\
12.72\end{array}$ \\
\hline \multicolumn{8}{|l|}{ Building Generates Electricity } \\
\hline 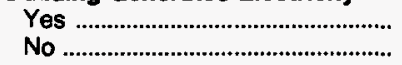 & $\begin{array}{l}1,017 \\
1,998\end{array}$ & $\begin{array}{l}29,044 \\
16,793\end{array}$ & $\begin{array}{l}41.37 \\
50.70\end{array}$ & $\begin{array}{r}9.1 \\
15.4\end{array}$ & $\begin{array}{l}364.2 \\
205.6\end{array}$ & $\begin{array}{l}.52 \\
.62\end{array}$ & $\begin{array}{l}12.54 \\
12.24\end{array}$ \\
\hline
\end{tabular}

1 Building participates in any programs sponsored by the Federal Energy Management Program, in-house, utility, or third party.

2 HVAC maintenance staff means at least one person spends at least half their working hours maintaining the heating/cooling equipment.

Notes: - Total workers are the number of workers during the main shift. - See Glossary for explanation of abbreviations and definitions of terms used in this report. - These data are from $\mathbf{8 8 1}$ federally owned buildings having the following criteria: (1) lacated in Federal Regions 3 , 6 , or 9; (2) larger than 10,000 square feet; and (3) used for a commercial purpose, other than warehouse and storage. In addition, 9 out of 10 selected buildings were from agencies other than the Department of Defense. - Statistics for the "energy end uses" represent consumption in buildings that have end use, not consumption for a particular fuel for a particular end use. - FBSS = Federal Buildings Supplemental Survey. - HVAC = Heating, Ventilation, and Air Conditioning. - VAV = Variable-Air Volume. - Data are for Fiscal Year 1993 (October 1, 1992 through September 30,1993 ). Because of rounding, data may not sum to totals.

Source: Energy Information Administration, Office of Energy Markets and End Use, 1993 Federal Buildings Supplemental Survey. 
Table 3.53. District Heat Consumption and Expenditure Intensities in FBSS Buildings in Federal Region 6, 1993

\begin{tabular}{|c|c|c|c|c|c|c|c|}
\hline \multirow[b]{2}{*}{$\begin{array}{c}\text { Bullding } \\
\text { Characteristics }\end{array}$} & \multicolumn{4}{|c|}{ District Heat Consumption } & \multicolumn{3}{|c|}{ District Heat Expenditures; } \\
\hline & $\begin{array}{l}\text { Total } \\
\text { (million } \\
\text { pounds) }\end{array}$ & $\begin{array}{l}\text { per } \\
\text { Bullding } \\
\text { (thousand } \\
\text { pounds) }\end{array}$ & $\begin{array}{l}\text { per } \\
\text { Square } \\
\text { foot } \\
\text { (pounds) }\end{array}$ & $\begin{array}{l}\text { per Worker } \\
\text { (thousand } \\
\text { pounds) }\end{array}$ & $\begin{array}{l}\text { per } \\
\text { Bullding } \\
\text { (thousand } \\
\text { dollars) }\end{array}$ & $\begin{array}{l}\text { per } \\
\text { Square } \\
\text { Foot } \\
\text { (dollars) }\end{array}$ & $\begin{array}{l}\text { per } \\
\text { Thousand } \\
\text { Pound } \\
\text { (dollars) }\end{array}$ \\
\hline 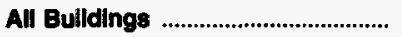 & 982 & 14,439 & 73.07 & 40.0 & 156.2 & 0.79 & 10.82 \\
\hline \multicolumn{8}{|l|}{$\begin{array}{l}\text { Bullding Floorspace (square } \\
\text { feet) }\end{array}$} \\
\hline $\begin{array}{l}10,000 \text { to } 50,000 \\
50,001 \text { to } 200,000 \\
\text { Over } 200,000\end{array}$ & $\begin{array}{r}95 \\
392 \\
495\end{array}$ & $\begin{array}{r}4,525 \\
12,639 \\
30,938\end{array}$ & $\begin{array}{r}164.13 \\
105.24 \\
54.19\end{array}$ & $\begin{array}{r}117.6 \\
50.4 \\
31.0\end{array}$ & $\begin{array}{r}46.9 \\
138.9 \\
333.4\end{array}$ & $\begin{array}{r}1.70 \\
1.16 \\
.58\end{array}$ & $\begin{array}{l}10.36 \\
10.99 \\
10.78\end{array}$ \\
\hline \multicolumn{8}{|l|}{ Principal Bullding Activity } \\
\hline 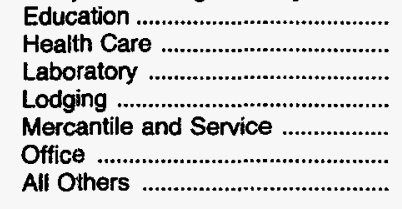 & $\begin{array}{r}5 \\
271 \\
463 \\
24 \\
\left({ }^{*}\right) \\
151 \\
67\end{array}$ & $\begin{array}{r}2,676 \\
18,077 \\
27,242 \\
4,892 \\
2 \\
8,362 \\
6,723\end{array}$ & $\begin{array}{r}152.90 \\
40.07 \\
194.80 \\
85.13 \\
.01 \\
55.54 \\
62.15\end{array}$ & $\begin{array}{r}118.9 \\
24.9 \\
140.6 \\
95.9 \\
\left({ }^{*}\right) \\
17.5 \\
70.5\end{array}$ & $\begin{array}{c}18.8 \\
175.7 \\
318.7 \\
58.4 \\
(*) \\
92.3 \\
57.9\end{array}$ & $\begin{array}{r}1.08 \\
.39 \\
2.28 \\
1.02 \\
\left({ }^{*}\right) \\
.61 \\
.54\end{array}$ & $\begin{array}{r}7.04 \\
9.72 \\
11.70 \\
11.94 \\
12.50 \\
11.04 \\
8.61\end{array}$ \\
\hline 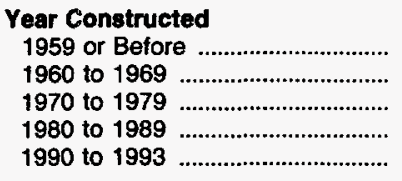 & $\begin{array}{r}570 \\
105 \\
56 \\
191 \\
60\end{array}$ & $\begin{array}{l}12,950 \\
15,026 \\
14,012 \\
17,362 \\
29,909\end{array}$ & $\begin{array}{r}98.54 \\
102.66 \\
57.43 \\
65.95 \\
21.69\end{array}$ & $\begin{array}{l}50.2 \\
36.4 \\
15.8 \\
45.9 \\
23.1\end{array}$ & $\begin{array}{l}140.9 \\
169.0 \\
116.6 \\
188.1 \\
352.5\end{array}$ & $\begin{array}{r}1.07 \\
1.15 \\
.48 \\
.71 \\
.26\end{array}$ & $\begin{array}{r}10.88 \\
11.25 \\
8.32 \\
10.83 \\
11.78\end{array}$ \\
\hline 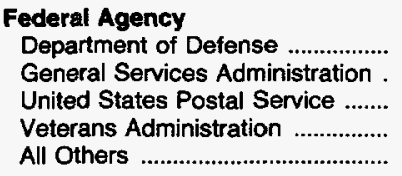 & $\begin{array}{l}14 \\
44 \\
\left({ }^{*}\right) \\
286 \\
638\end{array}$ & $\begin{array}{r}4,687 \\
8,724 \\
2 \\
15,053 \\
15,953\end{array}$ & $\begin{array}{r}57.04 \\
38.67 \\
.01 \\
41.14 \\
129.38\end{array}$ & $\begin{array}{l}27.6 \\
14.3 \\
\left({ }^{*}\right) \\
25.9 \\
67.6\end{array}$ & $\begin{array}{l}28.4 \\
103.6 \\
\left({ }^{*}\right) \\
146.1 \\
181.1\end{array}$ & $\begin{array}{r}.35 \\
.46 \\
.40 \\
1.47\end{array}$ & $\begin{array}{r}6.05 \\
11.87 \\
12.50 \\
9.71 \\
11.35\end{array}$ \\
\hline \multicolumn{8}{|l|}{$\begin{array}{l}\text { Energy Sources (more than one } \\
\text { may apply) }\end{array}$} \\
\hline 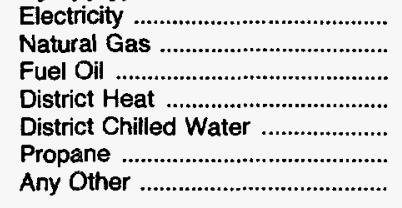 & $\begin{array}{r}982 \\
222 \\
63 \\
982 \\
343 \\
7 \\
58\end{array}$ & $\begin{array}{r}14,439 \\
14,786 \\
8,971 \\
14,439 \\
12,688 \\
3,659 \\
19,307\end{array}$ & $\begin{array}{r}73.07 \\
41.55 \\
59.38 \\
73.07 \\
46.70 \\
308.31 \\
47.21\end{array}$ & $\begin{array}{r}40.0 \\
24.4 \\
24.8 \\
40.0 \\
21.9 \\
122.0 \\
31.3\end{array}$ & $\begin{array}{r}156.2 \\
142.6 \\
88.7 \\
156.2 \\
133.2 \\
13.9 \\
181.1\end{array}$ & $\begin{array}{r}.79 \\
.40 \\
.59 \\
.79 \\
.49 \\
1.17 \\
.44\end{array}$ & $\begin{array}{r}10.82 \\
9.64 \\
9.89 \\
10.82 \\
10.50 \\
3.81 \\
9.38\end{array}$ \\
\hline \multicolumn{8}{|l|}{$\begin{array}{l}\text { Energy End Uses (more than } \\
\text { one may apply) }\end{array}$} \\
\hline $\begin{array}{l}\text { Heating } \\
\text { Air Conditioning } \\
\text { Water Heating } \\
\text { Cooking } \\
\text { Manufacturing }\end{array}$ & $\begin{array}{r}982 \\
982 \\
982 \\
264 \\
13\end{array}$ & $\begin{array}{r}14,439 \\
14,439 \\
14,439 \\
15,551 \\
3,210\end{array}$ & $\begin{array}{l}73.07 \\
73.07 \\
73.07 \\
39.46 \\
53.79\end{array}$ & $\begin{array}{l}40.0 \\
40.0 \\
40.0 \\
23.2 \\
40.8\end{array}$ & $\begin{array}{r}156.2 \\
156.2 \\
156.2 \\
145.9 \\
21.5\end{array}$ & $\begin{array}{l}.79 \\
.79 \\
.79 \\
.37 \\
.36\end{array}$ & $\begin{array}{r}10.82 \\
10.82 \\
10.82 \\
9.38 \\
6.71\end{array}$ \\
\hline 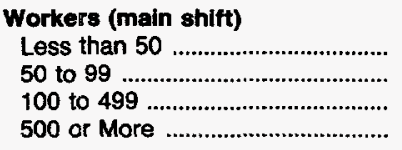 & $\begin{array}{r}97 \\
27 \\
494 \\
363\end{array}$ & $\begin{array}{r}4,633 \\
5,471 \\
18,310 \\
24,188\end{array}$ & $\begin{array}{r}91.20 \\
146.86 \\
121.45 \\
44.72\end{array}$ & $\begin{array}{r}204.8 \\
70.0 \\
75.8 \\
21.2\end{array}$ & $\begin{array}{r}50.3 \\
65.3 \\
202.2 \\
252.1\end{array}$ & $\begin{array}{r}.99 \\
1.75 \\
1.34 \\
.47\end{array}$ & $\begin{array}{l}10.86 \\
11.93 \\
11.04 \\
10.42\end{array}$ \\
\hline 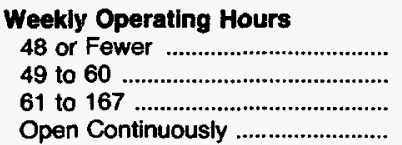 & $\begin{array}{l}106 \\
232 \\
325 \\
319\end{array}$ & $\begin{array}{l}10,583 \\
11,579 \\
17,103 \\
16,815\end{array}$ & $\begin{array}{r}103.08 \\
82.25 \\
104.44 \\
49.28\end{array}$ & $\begin{array}{r}132.1 \\
41.6 \\
47.6 \\
28.2\end{array}$ & $\begin{array}{l}117.2 \\
113.1 \\
202.2 \\
176.2\end{array}$ & $\begin{array}{r}1.14 \\
.80 \\
1.23 \\
.52\end{array}$ & $\begin{array}{r}11.08 \\
9.77 \\
11.82 \\
10.48\end{array}$ \\
\hline
\end{tabular}

See footnotes at end of table. 
Table 3.53. District Heat Consumption and Expenditure Intensities in FBSS Buildings in Federal Region 6, 1993 (Continued)

\begin{tabular}{|c|c|c|c|c|c|c|c|}
\hline \multirow[b]{2}{*}{$\begin{array}{c}\text { Bullding } \\
\text { Characteristics }\end{array}$} & \multicolumn{4}{|c|}{ District Heat Consumption } & \multicolumn{3}{|c|}{ Dlstrict Heat Expenditures } \\
\hline & $\begin{array}{l}\text { Total } \\
\text { (million } \\
\text { pounds) }\end{array}$ & $\begin{array}{l}\text { per } \\
\text { Bullding } \\
\text { (thousand } \\
\text { pounds) }\end{array}$ & $\begin{array}{l}\text { per } \\
\text { Square } \\
\text { foot } \\
\text { (pounds) }\end{array}$ & $\begin{array}{l}\text { per Worker } \\
\text { (thousand } \\
\text { pounds) }\end{array}$ & $\begin{array}{l}\text { per } \\
\text { Building } \\
\text { (thousand } \\
\text { dollars) }\end{array}$ & $\begin{array}{l}\text { per } \\
\text { Square } \\
\text { Foot } \\
\text { (dollars) }\end{array}$ & $\begin{array}{l}\text { per } \\
\text { Thousand } \\
\text { Pound } \\
\text { (dollars) }\end{array}$ \\
\hline \multicolumn{8}{|l|}{$\begin{array}{l}\text { HVAC Conservation Features } \\
\text { (more than one may apply) }\end{array}$} \\
\hline 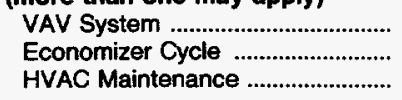 & $\begin{array}{l}393 \\
555 \\
979\end{array}$ & $\begin{array}{l}16,388 \\
15,409 \\
14,612\end{array}$ & $\begin{array}{l}47.64 \\
58.61 \\
72.98\end{array}$ & $\begin{array}{l}28.4 \\
34.1 \\
39.9\end{array}$ & $\begin{array}{l}167.9 \\
157.7 \\
158.0\end{array}$ & $\begin{array}{r}0.49 \\
.60 \\
.79\end{array}$ & $\begin{array}{l}10.24 \\
10.23 \\
10.82\end{array}$ \\
\hline \multicolumn{8}{|l|}{$\begin{array}{l}\text { Energy Management Practices } \\
\text { (more than one may apply) } \\
\text { Energy Management and Control }\end{array}$} \\
\hline 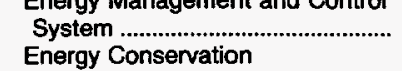 & 610 & 16,955 & 64.26 & 35.6 & 176.4 & .67 & 10.40 \\
\hline 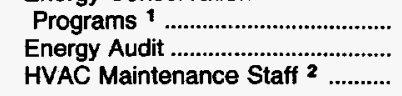 & $\begin{array}{r}194 \\
69 \\
195\end{array}$ & $\begin{array}{r}17,672 \\
13,806 \\
16,235\end{array}$ & $\begin{array}{l}38.63 \\
50.17 \\
33.38\end{array}$ & $\begin{array}{l}18.9 \\
16.7 \\
19.8\end{array}$ & $\begin{array}{l}168.7 \\
125.7 \\
171.2\end{array}$ & $\begin{array}{l}.37 \\
.46 \\
.35\end{array}$ & $\begin{array}{r}9.55 \\
9.10 \\
10.54\end{array}$ \\
\hline \multicolumn{8}{|l|}{$\begin{array}{l}\text { Off-Hours Reduction in } \\
\text { Equlpment (more than one may } \\
\text { apply) }\end{array}$} \\
\hline 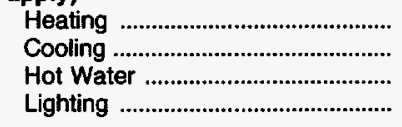 & $\begin{array}{r}387 \\
408 \\
77 \\
363\end{array}$ & $\begin{array}{r}13,361 \\
13,613 \\
8,526 \\
11,706\end{array}$ & $\begin{array}{l}88.47 \\
85.40 \\
52.16 \\
93.25\end{array}$ & $\begin{array}{l}46.6 \\
43.1 \\
17.6 \\
42.4\end{array}$ & $\begin{array}{r}140.6 \\
144.2 \\
84.5 \\
134.0\end{array}$ & $\begin{array}{r}.93 \\
.90 \\
.52 \\
1.07\end{array}$ & $\begin{array}{r}10.52 \\
10.59 \\
9.91 \\
11.45\end{array}$ \\
\hline \multicolumn{8}{|l|}{ Bullding Generates Electriclty } \\
\hline 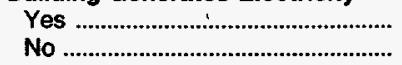 & $\begin{array}{l}154 \\
828\end{array}$ & $\begin{array}{l}19,291 \\
13,792\end{array}$ & $\begin{array}{l}32.86 \\
94.68\end{array}$ & $\begin{array}{l}25.4 \\
44.8\end{array}$ & $\begin{array}{l}199.8 \\
150.4\end{array}$ & $\begin{array}{r}.34 \\
1.03\end{array}$ & $\begin{array}{l}10.36 \\
10.91\end{array}$ \\
\hline
\end{tabular}

1 Building participates in any programs sponsored by the Federal Energy Management Program, in-house, utility, or third party.

2 HVAC maintenance staff means at least one person spends at least half their working hours maintaining the heating/cooling equipment.

(*) = Value rounds to zero in the units displayed.

Notes: - Total workers are the number of workers during the main shift. - See Glossary for explanation of abbreviations and definitions of terms used in this report. - These data are from 881 federally owned buildings having the following criteria: (1) located in Federal Regions 3 , 6 , or 9 ; (2) larger than 10,000 square feet; and (3) used for a commercial purpose, other than warehouse and storage. In addition, 9 out of 10 selected buildings were from agencies other than the Department of Defense. - Statistics for the "energy end uses" represent consumption in buildings that have end use, not consumption for a particular fuel for a particular end use. - FBSS = Federal Buildings Supplemental Survey. - HVAC = Heating, Ventilation, and Air Conditioning. - VAV = Variable-Air Volume. • Data are for Fiscal Year 1993 (October 1, 1992 through September 30, 1993). - Because of rounding, data may not sum to totals.

Source: Energy Information Administration, Office of Energy Markets and End Use, 1993 Federal Buildings Supplemental Survey. 
Table 3.54. District Heat Consumption and Expenditure Intensities in FBSS Buildings in Federal Region 9, 1993

\begin{tabular}{|c|c|c|c|c|c|c|c|}
\hline \multirow[b]{2}{*}{$\begin{array}{c}\text { Building } \\
\text { Characteristics }\end{array}$} & \multicolumn{4}{|c|}{ District Heat Consumption } & \multicolumn{3}{|c|}{ District Heat Expenditures } \\
\hline & $\begin{array}{l}\text { Total } \\
\text { (million } \\
\text { pounds) }\end{array}$ & $\begin{array}{l}\text { per } \\
\text { Building } \\
\text { (thousand } \\
\text { pounds) }\end{array}$ & $\begin{array}{l}\text { per } \\
\text { Square } \\
\text { foot } \\
\text { (pounds) }\end{array}$ & $\begin{array}{l}\text { per Worker } \\
\text { (thousand } \\
\text { pounds) }\end{array}$ & $\begin{array}{l}\text { per } \\
\text { Bullding } \\
\text { (thousand } \\
\text { dollars) }\end{array}$ & $\begin{array}{l}\text { per } \\
\text { square } \\
\text { Foot } \\
\text { (dollars) }\end{array}$ & $\begin{array}{l}\text { per } \\
\text { Theusand } \\
\text { Pound } \\
\text { (dollars) }\end{array}$ \\
\hline 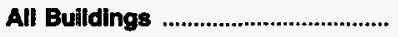 & 834 & 11,269 & 70.93 & 36.0 & 122.5 & 0.77 & 10.87 \\
\hline \multicolumn{8}{|l|}{$\begin{array}{l}\text { Bullding Floorspace (square } \\
\text { feet) }\end{array}$} \\
\hline 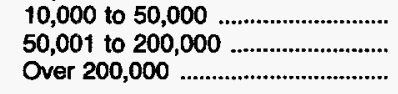 & $\begin{array}{r}88 \\
353 \\
393\end{array}$ & $\begin{array}{r}3,376 \\
10,092 \\
30,226\end{array}$ & $\begin{array}{r}109.56 \\
96.22 \\
53.94\end{array}$ & $\begin{array}{l}49.5 \\
49.2 \\
27.6\end{array}$ & $\begin{array}{r}38.6 \\
113.6 \\
314.2\end{array}$ & $\begin{array}{r}1.25 \\
1.08 \\
.56\end{array}$ & $\begin{array}{l}11.43 \\
11.25 \\
10.40\end{array}$ \\
\hline \multicolumn{8}{|l|}{ Principal Building Activity } \\
\hline 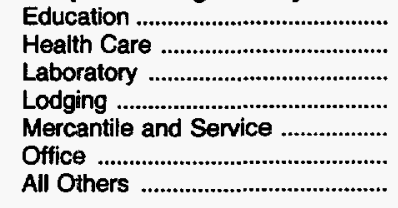 & $\begin{array}{r}14 \\
503 \\
97 \\
43 \\
5 \\
52 \\
120\end{array}$ & $\begin{array}{r}7,224 \\
13,963 \\
24,275 \\
3,587 \\
2,455 \\
6,499 \\
11,979\end{array}$ & $\begin{array}{r}85.32 \\
64.92 \\
253.76 \\
63.25 \\
84.17 \\
26.83 \\
152.30\end{array}$ & $\begin{array}{r}85.0 \\
30.7 \\
178.2 \\
120.6 \\
25.8 \\
11.6 \\
109.7\end{array}$ & $\begin{array}{r}70.3 \\
138.9 \\
286.9 \\
46.9 \\
29.5 \\
80.6 \\
151.0\end{array}$ & $\begin{array}{r}.83 \\
.65 \\
3.00 \\
.83 \\
1.01 \\
.33 \\
1.92\end{array}$ & $\begin{array}{r}9.73 \\
9.95 \\
11.82 \\
13.07 \\
12.01 \\
12.40 \\
12.61\end{array}$ \\
\hline 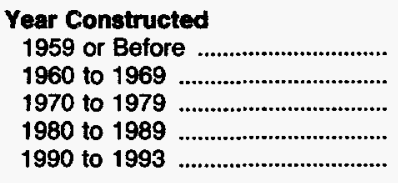 & $\begin{array}{l}230 \\
138 \\
323 \\
144 \\
\text { NC }\end{array}$ & $\begin{array}{r}6,377 \\
12,511 \\
17,955 \\
15,950 \\
\text { NC }\end{array}$ & $\begin{array}{c}76.08 \\
85.08 \\
66.77 \\
62.91 \\
\text { NC }\end{array}$ & $\begin{array}{c}38.7 \\
48.5 \\
28.5 \\
46.9 \\
\text { NC }\end{array}$ & $\begin{array}{r}76.9 \\
127.1 \\
200.2 \\
143.8 \\
\text { NC }\end{array}$ & $\begin{array}{l}.92 \\
.86 \\
.74 \\
.57 \\
\text { NC }\end{array}$ & $\begin{array}{c}12.05 \\
10.16 \\
11.15 \\
9.02 \\
\text { NC }\end{array}$ \\
\hline 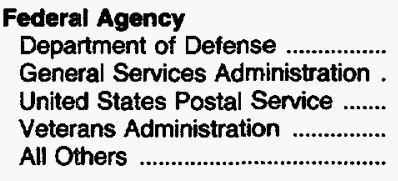 & $\begin{array}{c}169 \\
34 \\
N C \\
526 \\
105\end{array}$ & $\begin{array}{c}7,327 \\
17,050 \\
\text { NC } \\
11,692 \\
26,299\end{array}$ & $\begin{array}{c}51.06 \\
29.46 \\
\text { NC } \\
76.78 \\
235.43\end{array}$ & $\begin{array}{c}40.4 \\
11.7 \\
N C \\
34.0 \\
171.1\end{array}$ & $\begin{array}{c}93.6 \\
201.4 \\
N C \\
117.0 \\
310.3\end{array}$ & $\begin{array}{l}.65 \\
.35 \\
\text { NC } \\
.77 \\
2.78\end{array}$ & $\begin{array}{c}12.78 \\
11.81 \\
N C \\
10.01 \\
11.80\end{array}$ \\
\hline \multicolumn{8}{|l|}{$\begin{array}{l}\text { Energy Sources (more than one } \\
\text { may apply) }\end{array}$} \\
\hline 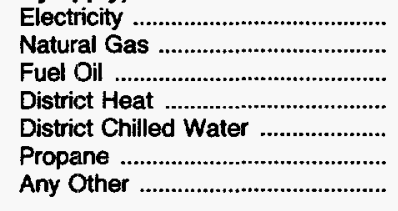 & $\begin{array}{l}828 \\
509 \\
163 \\
834 \\
349 \\
\mathrm{NC} \\
78\end{array}$ & $\begin{array}{l}11,346 \\
18,859 \\
18,081 \\
11,269 \\
13,941 \\
\text { NC } \\
39,199\end{array}$ & $\begin{array}{c}70.57 \\
76.28 \\
54.54 \\
70.93 \\
61.01 \\
\text { NC } \\
60.17\end{array}$ & $\begin{array}{l}35.8 \\
39.1 \\
27.4 \\
36.0 \\
35.5 \\
\text { NC } \\
33.5\end{array}$ & $\begin{array}{l}123.0 \\
204.6 \\
188.3 \\
122.5 \\
137.2 \\
\text { NG } \\
461.9\end{array}$ & $\begin{array}{l}.77 \\
.83 \\
.57 \\
.77 \\
.60 \\
\text { NC } \\
.71\end{array}$ & $\begin{array}{r}10.84 \\
10.85 \\
10.41 \\
10.87 \\
9.84 \\
\text { NC } \\
11.78\end{array}$ \\
\hline \multicolumn{8}{|l|}{$\begin{array}{l}\text { Energy End Uses (more than } \\
\text { one may apply) }\end{array}$} \\
\hline 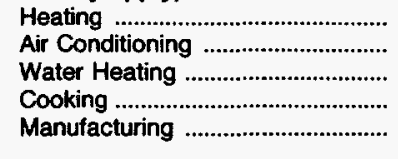 & $\begin{array}{l}834 \\
751 \\
786 \\
462 \\
161\end{array}$ & $\begin{array}{l}11,582 \\
11,919 \\
10,919 \\
23,080 \\
20,132\end{array}$ & $\begin{array}{r}71.26 \\
70.01 \\
68.76 \\
64.64 \\
105.63\end{array}$ & $\begin{array}{l}36.0 \\
35.7 \\
34.4 \\
32.5 \\
52.0\end{array}$ & $\begin{array}{l}125.9 \\
125.8 \\
116.3 \\
238.7 \\
203.9\end{array}$ & $\begin{array}{r}.77 \\
.74 \\
.73 \\
.67 \\
1.07\end{array}$ & $\begin{array}{l}10.87 \\
10.55 \\
10.65 \\
10.34 \\
10.13\end{array}$ \\
\hline $\begin{array}{l}\text { Workers (main shift) } \\
\text { Less than } 50 \\
50 \text { to } 99 \\
100 \text { to } 499 \\
500 \text { or More }\end{array}$ & $\begin{array}{r}61 \\
22 \\
445 \\
306\end{array}$ & $\begin{array}{r}3,412 \\
3,583 \\
11,416 \\
27,799\end{array}$ & $\begin{array}{r}70.49 \\
57.02 \\
104.29 \\
49.01\end{array}$ & $\begin{array}{r}404.0 \\
46.0 \\
52.6 \\
21.7\end{array}$ & $\begin{array}{r}41.9 \\
42.9 \\
127.7 \\
279.3\end{array}$ & $\begin{array}{r}.87 \\
.68 \\
1.17 \\
.49\end{array}$ & $\begin{array}{l}12.28 \\
11.98 \\
11.18 \\
10.05\end{array}$ \\
\hline 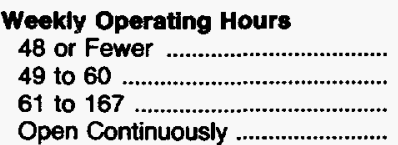 & $\begin{array}{r}75 \\
177 \\
39 \\
543\end{array}$ & $\begin{array}{r}3,566 \\
13,594 \\
39,376 \\
13,922\end{array}$ & $\begin{array}{r}60.41 \\
80.07 \\
129.21 \\
67.82\end{array}$ & $\begin{array}{r}33.8 \\
36.4 \\
116.5 \\
34.4\end{array}$ & $\begin{array}{r}38.4 \\
161.0 \\
590.6 \\
142.9\end{array}$ & $\begin{array}{r}.65 \\
.95 \\
1.94 \\
.70\end{array}$ & $\begin{array}{l}10.77 \\
11.84 \\
15.00 \\
10.27\end{array}$ \\
\hline
\end{tabular}

See footnotes at end of table. 
Table 3.54. District Heat Consumption and Expenditure Intensities in FBSS Buildings in Federal Region 9, 1993 (Continued)

\begin{tabular}{|c|c|c|c|c|c|c|c|}
\hline \multirow[b]{2}{*}{$\begin{array}{c}\text { Building } \\
\text { Characteristics }\end{array}$} & \multicolumn{4}{|c|}{ District Heat Consumption } & \multicolumn{3}{|c|}{ District Heat Expenditures } \\
\hline & $\begin{array}{l}\text { Total } \\
\text { (million } \\
\text { pounds) }\end{array}$ & $\begin{array}{l}\text { per } \\
\text { Bullding } \\
\text { (thousand } \\
\text { pounds) }\end{array}$ & $\begin{array}{l}\text { per } \\
\text { Square } \\
\text { foot } \\
\text { (pounds) }\end{array}$ & $\begin{array}{l}\text { per Worker } \\
\text { (thousand } \\
\text { pounds) }\end{array}$ & $\begin{array}{l}\text { per } \\
\text { Building } \\
\text { (thouseand } \\
\text { dollars) }\end{array}$ & $\begin{array}{l}\text { per } \\
\text { Square } \\
\text { Foot } \\
\text { (dollars) }\end{array}$ & $\begin{array}{l}\text { per } \\
\text { Thousand } \\
\text { Pound } \\
\text { (dollars) }\end{array}$ \\
\hline \multicolumn{8}{|l|}{$\begin{array}{l}\text { HVAC Conservation Features } \\
\text { (more than one may apply) }\end{array}$} \\
\hline 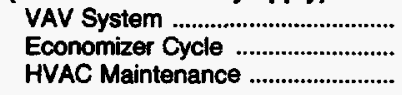 & $\begin{array}{l}274 \\
472 \\
832\end{array}$ & $\begin{array}{l}21,070 \\
16,281 \\
11,721\end{array}$ & $\begin{array}{l}56.85 \\
82.92 \\
71.28\end{array}$ & $\begin{array}{l}30.5 \\
42.0 \\
36.0\end{array}$ & $\begin{array}{l}196.6 \\
172.5 \\
127.4\end{array}$ & $\begin{array}{r}0.53 \\
.88 \\
.77\end{array}$ & $\begin{array}{r}9.33 \\
10.59 \\
10.87\end{array}$ \\
\hline \multicolumn{8}{|l|}{$\begin{array}{l}\text { Energy Management Practices } \\
\text { (more than one may apply) } \\
\text { Energy Management and Control }\end{array}$} \\
\hline System ............................................. & 298 & 22,919 & 54.91 & 30.0 & 245.9 & .59 & 10.73 \\
\hline 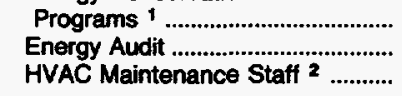 & $\begin{array}{l}402 \\
248 \\
255\end{array}$ & $\begin{array}{l}19,142 \\
10,343 \\
17,031\end{array}$ & $\begin{array}{l}69.66 \\
61.46 \\
60.77\end{array}$ & $\begin{array}{l}31.9 \\
31.5 \\
33.6\end{array}$ & $\begin{array}{l}209.1 \\
110.1 \\
180.0\end{array}$ & $\begin{array}{l}.76 \\
.65 \\
.64\end{array}$ & $\begin{array}{l}10.92 \\
10.64 \\
10.57\end{array}$ \\
\hline \multicolumn{8}{|l|}{$\begin{array}{l}\text { Off-Hours Reduction in } \\
\text { Equipment (more than one may } \\
\text { apply) }\end{array}$} \\
\hline 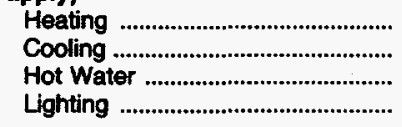 & $\begin{array}{r}153 \\
139 \\
48 \\
160\end{array}$ & $\begin{array}{r}10,204 \\
7,709 \\
7,956 \\
6,953\end{array}$ & $\begin{array}{l}70.48 \\
60.63 \\
34.19 \\
57.61\end{array}$ & $\begin{array}{l}35.2 \\
27.6 \\
14.5 \\
27.8\end{array}$ & $\begin{array}{r}117.7 \\
86.6 \\
94.3 \\
81.4\end{array}$ & $\begin{array}{l}.81 \\
.68 \\
.41 \\
.67\end{array}$ & $\begin{array}{l}11.53 \\
11.24 \\
11.85 \\
11.71\end{array}$ \\
\hline \multicolumn{8}{|l|}{ Bullding Generates Electricity } \\
\hline Yes & $\begin{array}{l}242 \\
592\end{array}$ & $\begin{array}{r}24,229 \\
9,244\end{array}$ & $\begin{array}{l}65.80 \\
73.27\end{array}$ & $\begin{array}{l}35.6 \\
36.1\end{array}$ & $\begin{array}{r}285.6 \\
97.0\end{array}$ & $\begin{array}{l}.78 \\
.77\end{array}$ & $\begin{array}{l}11.79 \\
10.49\end{array}$ \\
\hline
\end{tabular}

1 Building participates in any programs sponsored by the Federal Energy Management Program, in-house, utility, or third party.

2 HVAC maintenance staff means at least one person spends at least half their working hours maintaining the heating/cooling equipment.

NC $=$ No cases in responding sample.

Notes: - Total workers are the number of workers during the main shift. - See Glossary for explanation of abbreviations and definitions of terms used in this report. - These data are from 881 federally owned buildings having the following criteria: (1) located in Federal Regions 3 , 6. or 9; (2) larger than 10,000 square feet; and (3) used for a commercial purpose, other than warehouse and storage. In addition, 9 out of 10 selected buildings were from agencies other than the Department of Defense. - Statistics for the "energy end uses" represent consumption in buildings that have end use, not consumption for a particular fuel for a particular end use. - FBSS = Federal Buildings Supplemental Survey. - HVAC = Heating, Ventilation, and Air Conditioning. - VAV = Variable-Air Volume. - Data are for Fiscal Year 1993 (October 1, 1992 through September 30, 1993). - Because of rounding, data may not sum to totals.

Source: Energy Information Administration, Office of Energy Markets and End Use, 1993 Federal Buildings Supplemental Survey. 

Appendix A

How the Survey Was Conducted 



\title{
Appendix A
}

\section{How the Survey Was Conducted}

\author{
Introduction
}

The Federal Buildings Supplemental Survey (FBSS) conducted by the Energy Information Administration (EIA) of the U.S. Department of Energy (DOE), in conjunction with the Office of Federal Energy Management Programs (OFEMP) of DOE was a supplement to the Commercial Buildings Energy Consumption Survey (CBECS). The FBSS was conducted to assist in the implementation of the Energy Policy Act of 1992 (EPACT) by focusing a modified version of the CBECS exclusively on Federally owned and operated buildings. The CBECS, a triennial commercial buildings survey conducted by EIA, is the only source of national-level data on both commercial buildings' characteristics and related energy consumption and expenditures. Federally owned commercial buildings are one of three types of government-owned commercial buildings that are included in the CBECS sample. However, because the CBECS sample size is relatively small (6,500 sample commercial buildings of an estimated 4.8 million commercial buildings) and yields an even smaller number of federally owned government buildings, in-depth examination of energy use and characteristics of these buildings was not possible using the CBECS data. To obtain energy-related building characteristics and consumption and expenditures data for Federal commercial buildings, a sample survey of approximately 900 Federally owned and operated buildings was conducted in Federal Regions 3, 6, and 9 (See Appendix $\mathrm{C}$ for the Federal regions map). The FBSS sample selection procedures are described in the "Sample Design" section of this appendix. As with the CBECS, the "building" was the basic unit for the FBSS since the building is the energyconsuming unit. FBSS data are at the building level and data presented in this report represent the 881 responding buildings and cannot be used to generalize about Federal buildings in each region.

EIA used a computer-assisted telephone interview (CATI) to conduct voluntary interviews with the Federal building energy manager or designated person. CATI was used as a test as opposed to the usual in-person data collection method to see if building owners/managers could provide the technical information over the telephone. Under EIA's direction, a survey research firm conducted the FBSS CATI at their telephone center.

\section{At a Glance - Differences Between 1992 CBECS and 1993 FBSS}

The CBECS consists of two major data collection stages--a building characteristics survey, which is an in-person interview with the building respondent, and an energy suppliers survey which is mailed to the energy suppliers. The FBSS survey design included only one major data collection stage -- collecting both building characteristics and consumption data from the building respondent, because the Federal building respondent was thought to have consumption data available to them. Respondents were asked to provide consumption and expenditures data for electricity, natural gas, fuel oil, and district sources (steam, hot water, and chilled water). For these major fuels, the following data were requested: (1) quantity consumed or delivered; (2) cost; and (3) unit of measure. The units of measure were as follows: electricity--kilowatt $(\mathrm{kW})$ demand; natural gas including transportation gas--therms, cubic feet or 1,000 cubic feet; fuel oil--fuel-tank data; and district heating and cooling--the entire district or system. Respondents could Fax completed worksheets that were mailed to them in advance. If building respondents could not obtain these data, they were asked to provide the name, address, telephone and Fax number of the person who would most likely be able to provide the data. The data were requested for the Federal Fiscal Year 1993; that is, from October 1992 to September 1993. 
These data were collected on the Building Questionnaire, a modified version of the CBECS form tailored to include specific questions relating to the Federal Energy Management Program (FEMP); such as, questions on motors, retrofitting equipment, energy conservation program sponsorship, and availability of energy audits. Additionally, wording and structural changes to the 1992 CBECS questionnaire were incorporated into the 1993 FBSS questionnaire to facilitate the CATI mode of data collection.

\section{Questions Asked in 1993 FBSS and Not 1992 CBECS}

- Equipment Age for: heating (each type present), central chillers, refrigeration, water heating, motors 10 or more horsepower (each type present)

- Sponsorship of Retrofit/Purchase and Type of Assistance (included FEMP and Federal Energy Efficiency Fund (FEEF)) for: special energy technologies, heating equipment (each type present), central chillers, refrigeration, water-heating equipment, lighting

- FBSS asked if Electronic Ballasts were present in the building

- Motors - 10 or more horsepower: number, age, approximate number of energy-efficient motors, approximate number of motors rewound, age when rewound, general behavior when motor fails -- rewind or replace. Specifically asked for the following equipment:

$\begin{array}{ll}\text { Chillers } & \text { Heat Pumps } \\ \text { Fans } & \text { Air Compressors } \\ \text { Water Pumps } & \text { Elevators } \\ \text { Escalators } & \text { Refrigeration }\end{array}$

- Questions asked of the interviewer to assess the respondents ability to answer the motor questions and the questionnaire in general

\section{CBECS Questions Not Asked in 1993 FBSS}

- Physical Characteristics: Number of below-ground-level floors, building shape, ground-level length/width of square and rectangular buildings, attachment to other structures, renovations and demolitions

- Ownership and Occupancy Characteristics: Building owner and occupant of building, number businesses and organizations that occupy buildings, percent vacant three consecutive months, additional operating hours when equipment in use

- Conservation and Energy Management: Special space functions, opening and closing windows

This appendix has three sections: "Sample Design," "Survey of Building Characteristics and Consumption Data," and "Public-Use Data Preparation." These sections focus on components of the sample, the procedures for data collection and processing, data difficulties encountered, and procedures for handling unit and item nonresponse.

\section{Target Population}

The OFEMP requested that the FBSS provide building-level energy-related characteristics for a special sample of commercial buildings owned by the Federal Government. To meet OFEMP's requests, the FBSS target population consisted of federally owned commercial buildings: (1) operated by either GSA, some agency other than GSA, or a contract facility; and (2) in Federal Regions 3, 6, or 9 that: 
- Met the CBECS definition of a building--a structure intended for human access and totally enclosed by walls extending from the foundation to the roof

- Were primarily used for some commercial purpose--more than 50 percent of the floorspace devoted to activities that are neither residential, industrial, agricultural nor warehouse/storage

- Measured 10,000 square feet or larger -- this was increased from the CBECS 1,001 square feet size criterion because smaller buildings form a large, inherently ill-defined, group of marginal structures.

All agencies in Federal Regions 3, 6, and 9 were in the FBSS target population. ${ }^{3}$

\section{Determining Building Eligibility}

During the development of the facility and building sample lists for the FBSS frame, somewhat looser criteria were applied to prevent inaccurate exclusion of eligible buildings based on inaccurate list information. During the interview with the building owner or manager, building eligibility was determined according to the criteria listed above to allow a knowledgeable respondent to ultimately screen eligible buildings. Once the interview began, initial screening questions instructed the interviewer to terminate the interview if the respondent indicated that the building size was less than 10,000 square feet or if 50 percent or more of the square footage was used for residential, industrial, agricultural, or warehouse/storage purposes.

\section{Sample Design}

Although a comprehensive list of all Federal buildings in the target population (3 Federal regions) does not exist, there is a list of most Federal facilities. Under the direction of the OFEMP, a data base was developed, which contained energy management information on all Federally owned facilities in the United States. As of June 1994, 19,237 Federal facilities were represented in the data base. Therefore, the FBSS sample design was based upon sampling from a national list of Federal facilities created from the data base.

\section{Two-Stage Systematic Probability-Proportional-to-Size (PPS) Sample}

The sample design of the FBSS was a two-stage systematic probability-proportional-to-size (PPS) design and the twostages of the design were: (1) Selecting Facilities and (2) Selecting Buildings. Facility selection was conducted using the FEMP data base. For each facility selected in stage one, a list of buildings on the selected facility was obtained from the facility energy manager. For stage two, buildings were selected. PPS sampling is commonly used to take advantage of existing knowledge about the sample units to improve the reliability of survey estimates. For quantities roughly proportional to certain measures of size (MOS's), estimates based on PPS sampling have lower variances than estimates based on equal-probability sampling. The total square footage of a facility or building was used as a MOS since building size is well correlated with commercial activity and energy consumption, which indicates size is a good choice not only for PPS sampling, but also for ordering in the systematic selection.

\footnotetext{
${ }^{3}$ For a detailed discussion of CBECS criteria, see Appendix A, "How the Survey was Conducted" in the Commercial Buildings Energy Consumption and Expenditures 1992, DOE/EIA-0318(92), Energy Information Administration (Washington, D.C., Government Printing Office, April 1995).
} 


\section{First Stage - Selecting Facilities}

To prepare for the first-stage of the PPS sample, all Federal facilities listed in Regions 3, 6, and 9 were divided into Department of Defense (DOD) and non-DOD facilities based on agency designation, to ensure appropriate selection of DOD facilities. Next, within the DOD facilities, eight strata were formed by grouping adjacent States. Adjacent States were assumed to be similar in average temperatures and main fuel used; and, therefore, similar in energy-related characteristics. Similarly, the non-DOD facilities were grouped into eight strata by the same grouping of adjacent States. From the 15 States in Regions 3, 6, and 9 the States were grouped as follows: (1) West Virginia and Virginia, (2) Delaware and Pennsylvania, (3) Maryland and District of Columbia, (4) Oklahoma and Texas, (5) New Mexico, (6) Louisiana and Arkansas, (7) Hawaii and California, and (8) Arizona and Nevada. These groupings resulted in the listed Federal facilities being divided in a total of 16 strata--8 DOD strata and 8 non-DOD strata. From each of the 16 strata an independent systematic PPS sample of facilities was conducted; such that $N_{i}$ buildings were selected (with replacement). $\mathrm{N}_{\mathrm{i}}$ represents the number of buildings assigned to stratum I based on the ratio of the square feet of stratum I to the square feet of all facilities. A modified proportional allocation of the sample to stratum was used; 10 percent from the 8 DOD strata, 90 percent from the 8 non-DOD strata, resulting in separate proportional allocation within each of these two sets of strata. Table A1 below lists the adjacent States contained in each stratum along with square footage and sample sizes for each stratum.

Table A1. Stratum Sample Size

\begin{tabular}{|c|c|c|c|c|c|c|c|c|}
\hline Strata \# & $\begin{array}{c}\text { Strata } \\
\text { Type }\end{array}$ & $\begin{array}{l}\text { Federal } \\
\text { Region }\end{array}$ & States & Square Feet & $\begin{array}{c}\text { Number of } \\
\text { Facilities }\end{array}$ & $\begin{array}{l}\text { Number of } \\
\text { Buildings }\end{array}$ & $\mathbf{N}_{\mathbf{l}}$ & $\mathbf{R}_{\mathbf{i}}$ \\
\hline 1 & DOD & 3 & WV, VA & $62,361,720$ & 85 & 7,070 & 28 & $2,227,204$ \\
\hline 2 & DOD & 3 & $\mathrm{DE}, \mathrm{PA}$ & $29,160,956$ & 109 & 2,702 & 13 & $2,243,150$ \\
\hline 3 & DOD & 3 & $\mathrm{MD}, \mathrm{DC}$ & $53,219,624$ & 53 & 5,164 & 24 & $2,217,484$ \\
\hline 7 & DOD & 6 & OK, TX & $85,299,041$ & 138 & 10,876 & 38 & $2,244,712$ \\
\hline 8 & DOD & 6 & NM & $12,952,851$ & 12 & 2,165 & 6 & $2,158,809$ \\
\hline 9 & DOD & 6 & LA, AR & $18,895,368$ & 49 & 3,408 & 9 & $2,099,485$ \\
\hline 13 & DOD & 9 & $\mathrm{HI}, \mathrm{CA}$ & $164,757,998$ & 186 & 19,659 & 74 & $2,226,459$ \\
\hline 14 & DOD & 9 & $A Z, N V$ & $18,348,119$ & 29 & 3,230 & 8 & $2,293,515$ \\
\hline 4 & Non-DOD & 3 & W, VA & $31,238,568$ & 172 & 1,681 & 218 & 143,296 \\
\hline 5 & Non-DOD & 3 & DE, PA & $23,271,681$ & 199 & 1,099 & 162 & 143,653 \\
\hline 6 & Non-DOD & 3 & $M D, D C$ & $74,381,176$ & 167 & 1,759 & 518 & 143,593 \\
\hline 10 & Non-DOD & 6 & OK, TX & $35,509,436$ & 460 & 1,815 & 247 & 143,763 \\
\hline 11 & Non-DOD & 6 & NM & $14,218,734$ & 64 & 1,646 & 99 & 143,624 \\
\hline 12 & Non-DOD & 6 & $L A, A R$ & $13,621,885$ & 134 & 463 & 95 & 143,388 \\
\hline 15 & non-DOD & 9 & $\mathrm{HI}, \mathrm{CA}$ & $56,847,625$ & 429 & 4,368 & 396 & 143,555 \\
\hline 16 & non-DOD & 9 & $A Z, N V$ & $9,348,882$ & 134 & 959 & 65 & 143,829 \\
\hline
\end{tabular}

$N_{i}=$ Stratum sample size or the number of buildings assigned to stratum l.

$R_{i}=$ Ratio of stratum square feet to stratum sample size.

Source: Energy Information Administration, Office of Energy Markets and End Use, 1993 Federal Buildings Supplemental Survey. 


\section{Second Stage - Selecting Buildings}

In the second stage, the selected multibuilding facilities were screened for eligible buildings. Each facility was sampled independently. Once the frame of buildings for each facility was verified for accuracy, it provided the correct MOS for each eligible building and, consequently, an update for the facility MOS. The frame of buildings for each facility was then ordered by building square footage and sampled systematically with PPS.

\section{Projected Sampling Results}

The core sample size was 1,000 buildings, since 90 percent of 1,000 would provide the targeted sample size of 900 buildings. An additional sample of 1,000 buildings was selected for reserve use for ineligible buildings, nonresponse, and any replication that may have been needed. The 2,000 buildings were randomly divided into the core and reserve samples, Panel I and Panel II, respectively.

\section{Actual Sample Selected}

To achieve the FBSS sampling goal, the actual sample selected included the core (Panel I) and reserve (Panel II) buildings of which 963 buildings were from Region 3, 494 buildings were from Region 6, and 543 were from Region 9.

\section{Actual Sampling Results}

These procedures resulted in 881 completed interviews, only 19 short of the targeted goal of 900 . Of the 881 completed interviews; 310 (35.2 percent) were from Region 3; 245 (27.8 percent) were from Region 6; and 326 (37.0 percent) were from Region 9 (See Table A2). The overall response rate for this survey was 75 percent. The regional response rates are provided below.

Table A2. Regional Response Rates in Panel I and Panel II

\begin{tabular}{l|c|c|c|c|c|c|c|c|c}
\hline & \multicolumn{3}{|c|}{ Panel I Selection } & \multicolumn{3}{c|}{ Panel II Selection } & \multicolumn{4}{c}{ Total } \\
\cline { 2 - 11 } & Eligible & $\begin{array}{l}\text { Completed } \\
\text { Interviews }\end{array}$ & Percent & Eligible & $\begin{array}{l}\text { Completed } \\
\text { Interviews }\end{array}$ & Percent & Eligible & $\begin{array}{l}\text { Completed } \\
\text { Interviews }\end{array}$ & Percent \\
\hline Region 3 ... & 383 & 283 & 73.89 & 40 & 27 & 67.50 & 423 & 310 & 73.29 \\
Region 6 ... & 228 & 207 & 90.79 & 43 & 38 & 88.37 & 271 & 245 & 90.41 \\
Region 9 ... & 288 & 210 & 72.92 & 195 & 116 & 59.49 & 483 & 326 & 67.49 \\
Total ..... & 899 & 700 & 77.86 & 278 & 181 & 65.11 & 1,177 & 881 & 74.85 \\
\hline
\end{tabular}

Source: Energy Information Administration, Office of Energy Markets and End Use, 1993 Federal Buildings Supplemental Survey. 


\section{Survey of Building Characteristics and Consumption Data}

\section{Data Collection}

FBSS data collection involved many phases and began with the redesign of the 1992 CBECS Building Questionnaire to not only assist in the implementation of the 1992 EPACT but also to accommodate the CATI system. After the questionnaire redesign, the data collection phases continued with minimizing nonresponse to ensure quality data, training supervisors and interviewers, interviewing building respondents, and concluded with processing the FBSS data. Survey interviewing began July 1994 (pretests were conducted) and ended December 31, 1994. A survey contractor performed the data collection under the direction of EIA. The data were collected by the survey contractor's telephone center staff.

\section{Minimizing Nonresponse}

Prior to and throughout data collection, EIA worked closely with the three Federal regions employing several approaches to increase cooperation and participation and to ensure that respondent burden and nonresponse was minimized. There were telephone callbacks; establishment of an 800 number to address respondents' concerris or questions; and direct EIA response to customer concerns. Respondents were encouraged to call either EIA or the 800 number if they had any questions. Additionally, letters of notification and/or FBSS materials were sent to the following:

- Federal Interagency Management Task Force

- General Services Administration (GSA) and Department of Energy (DOE) regional offices

- Facility managers of facilities selected to participate in FBSS

- Building managers of the buildings selected to participate in the FBSS.

For more discussion on the efforts taken to minimize nonresponse and respondent burden as well as examples of letters sent and addressees receiving those respective letters, see Appendix E, "Outreach Efforts."

\section{Training Supervisors and Interviewers}

Because the 1993 FBSS was a CATI-administered, shortened version of the 1992 CBECS Building Questionnaire, only a shortened version of training was needed. The survey contractor conducted both the half-day supervisor training session and the one-day interviewer training session at the survey contractor's telephone center. The supervisor training session covered the in-depth FBSS subject-matter information, which included a component on monitoring the interview and providing feedback. The interviewer training session conducted on August 11, 1994, included FBSS background, key concepts related to energy use, several hours of interviewer self-study, several hours of practice interview, and administrative information. EIA personnel observed the interviewer training session and were available for assistance. From August 12 through August 15, 1994, interviewers received intense monitoring. On August 16, an interviewer debriefing was held. Monitoring also continued throughout the data collection process.

\section{Interviewing the Building Respondent}

Each interview began with a series of screening questions designed to verify the building's address and eligibility for the survey. The completed building interview lasted an average of 39 minutes. This included the time for the interviewer to ask all questions on energy-related building characteristics as well as the consumption and expenditure data. 


\section{Data Preparation for Report}

EIA data analysts reviewed and processed the data for the final data tape. Crosstabulations were run to check for internal consistency of the data. Because commercial building consumption and expenditure data are complex and interrelated, the EIA review was extensive. EIA performed data imputations and in July 1995, prepared a final data tape. Statistical tables of aggregated data were then produced and analyzed. The report text was based on these tables, which are presented both in the text and in Chapter 3, of this report.

\section{Processing the FBSS Data}

Because FBSS used CATI as the mode of data collection, most data editing occurred during the interview. The CATI system checked for completeness, inconsistencies or ambiguities in the data, accuracy of questionnaire skip patterns, and checked that only allowable values or codes were entered. After the interviews, data editing occurred during review of data frequencies and crosstabulations. These were reviewed to search for outlying values and inconsistencies that the CATI edits may not have identified. When CATI edits failed to resolve data problems, especially when the energy sources or heating and cooling equipment were involved, EIA personnel provided technical guidance, and when necessary, the survey contractor contacted the respondent by telephone for clarification. Telephone data retrieval was conducted for edit failures involving nonprogrammed-CATI edits. After having inconsistencies corrected by the contractor, EIA began the data preparation for the report. Any changes made to any questionnaire response as a result of data editing were documented.

\section{Data Editing/Data Adjustments}

Adjustments for unit nonresponse were performed. Cases missing all or part of calendar year 1993 consumption or expenditures were considered as particular kinds of item nonresponse. Adjustments for these cases were made as described under "Annual Consumption and Expenditures" in the "Nonsampling and Sampling Errors" section. For cases where the consumption data covered more than the one sampled building, the EIA implemented a special adjustment procedure--disaggregation to compute building-specific annualized consumption and expenditures.

Disaggregation. Disaggregation was generally necessary when either the building respondent reported that the energy bill for a source included more than the sampled building. In a limited number of cases, the preliminary data reviewer designated a case for disaggregation, even if the building respondent had not. A disaggregation "factor" was calculated based on the square footage of the buildings involved.

\section{Imputations}

Nonresponse to several items in otherwise completed questionnaires was treated by a technique known as hot-deck imputation. In hot-decking, when a certain response is missing for a given building, another building, called a "donor" is randomly chosen to furnish its reported value for that missing item. That value is then assigned to the building with item nonresponse (the nonrespondent, or "receiver"). To serve as a donor, a building had to be similar to the nonrespondent in characteristics correlated with the missing item. This procedure was used to reduce the bias caused by different nonresponse rates for a particular item among different types of buildings. The characteristics used to define "similar" depended on the nature of the item to be imputed. The most frequently used characteristics were: principal building activity, floorspace category, year constructed category, and Federal region. To hot-deck values for a particular item, all buildings were first grouped according to the values of the matching characteristics specified for that item. Within each group defined by the matching variables, donor buildings were assigned randomly to receiver buildings. For the FBSS, only data items considered critical for predicting energy consumption were imputed. These data items were: square footage, year constructed, principal building activity, energy sources used, end uses performed, major and minor fuel end uses, percent of floorspace heated and cooled, presence of refrigeration equipment, months the building was in use, operating hours, and number of workers. 
The general approach taken for imputing annual consumption or expenditures for a particular fuel was to use respondent cases to develop multiple linear regression equations, and then use these equations to provide imputed values for cases in which the data were missing.

\section{Public-Use Data Preparation}

In addition to the publication of this 1993 FBSS service report, the basic survey data at the microlevel were provided to the public on public-use data diskettes. These public-use diskettes are available to the public through the National Technical Information Service (NTIS) and the Office of Scientific and Technical Information (OSTI). (See Appendix $F$ for ordering information.) 
Appendix B

Types of Buildings 


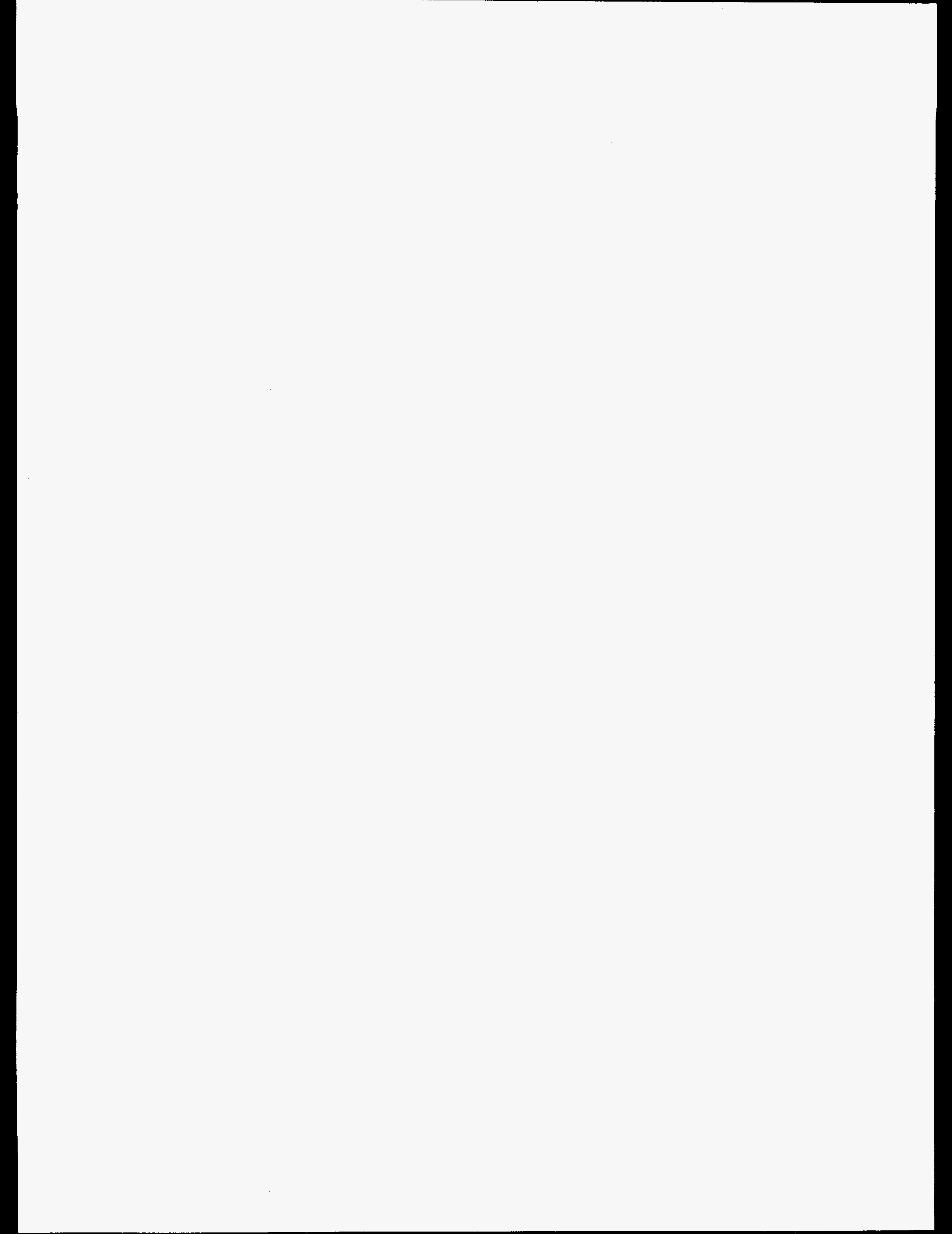




\section{Appendix B}

\section{Types of Buildings}

\section{EXAMPLES OF ACTIVITIES THAT MAY TAKE PLACE WITHIN A BUILDING}

a. Vacant. Refers to unused or unoccupied space.

b. Office/Professional. Refers to activities requiring general office space, professional offices, and administrative offices.

\author{
Data processing: \\ Computer center \\ Data entry, Keypunch \\ Financial \\ Bank \\ Brokerage Firm \\ Insurance \\ Real Estate \\ Securities \\ Professional: \\ Administration of an institution \\ Consulting \\ Corporate \\ Engineering \\ Law \\ Management \\ Medical \\ Mixed professional
}

c. Shopping Center/Mall/Retail/Service. Involves sales and displays of goods or services (excluding food).

Automotive sales and service:

Automobile dealers

Gasoline stations

Motor vehicle repair/service

Retail Sales:

Apparel stores

Building materials, Garden supply stores, Hardware

Department stores

Drugstores

Fumiture, Home equipment stores and Home furnishings

Liquor stores

Services (except food):

Dry cleaner/Car wash/Laundry

Multiservice establishments

Personal service

Post office

Shopping mall/Covered shopping center

Strip shopping center

Wholesale goods (except food) d. Laboratory. Activities utilize equipment for experimental testing or for analysis

\section{Agricultural laboratory \\ Electrical/Mechanical laboratory \\ Dental/Medical laboratory}

e. Nonrefrigerated Warehouse or Storage. Activities include the storage of goods, merchandise, raw materials or manufactured products. Does not include space in the building that is used for storage associated with another main activity in the building, such as retail or office activity.

f. Food Sales. Involves retail or wholesale sale of food

Convenience store or market

Farmer's market, Fruit/Vegetable market

Grocery store/Supermarket

Meat'Seafood store

Retail bakery

Specialty food store

g. Public Order and Safety. Activities related to the preservation of law and order or in public safety.
Courthouse
Fire station
Jail
Penitentiary/Prison
Police station
Reformatory
Sheriff's office

h. Outpatient Health Services/Clinic.

Services may be medical, dental, psychiatric or veterinary.

Dental clinic

Medical clinic

Abortion/Birth control

Ear, Eye, Nose and Throat

Emergency walk-in clinics

General

Mental health/Psychiatric clinic

Veterinary clinic 


\section{EXAMPLES OF ACTIVITIES THAT MAY TAKE PLACE WITHIN A BUILDING}

i. Industrial Processing and Manufacturing. Activities involve the processing or procurement of goods, merchandise, raw materials or food.

Assembly factory:

Apparel and other goods made from purchased material

Electrical or electronic instruments and fabricated metal tools

Leather goods

Machinery and other heavy equipment

Measuring devices and light equipment

Transportation vehicles

Construction/Natural resource procurement:

Construction site building

Mining

Food processing:

Bakery

Beverage

Cannery

Confectionery

Dairy

Grain mill

Meat-packing plant

Poultry-dressing plant

Foundry:

Glassworks

Metalworks

Rolling or Finishing mill

Steel works

Manufacturing plants:

Buildings for smelting, refining, drawing, rolling, or extruding of nonferrous metals, stone, clay, glass and concrete products

Mills:

Leather

Textile

Paper, chemical, rubber or petroleum processing

Printing, publishing

Utility or sanitary services:

Generation of electricity

Collection or disposal of refuse

Irrigation

Pumping stations

Storage, transmission or distribution

Steam supply

Sewage disposal/Treatment

Water supply/treatment j. Agricultural purposes.

Greenhouse

Livestock housing

Nursery

Produce storage

k. Refrigerated warehouse or storage. Activities include the storage of goods, merchandise, raw materials or manufactured products in buildings (or portions of buildings) specifically designed to store perishable goods or merchandise under refrigeration. Includes "cold storage" facilities, which store products at temperatures between $0^{\circ} \mathrm{F}$ and $50^{\circ} \mathrm{F}$ and "freezer facilities" which store products at between $0^{\circ} \mathrm{F}$ and $-20^{\circ} \mathrm{F}$. The refrigeration equipment consists of either a central station or individual wall hung or rooftop units. A storage space with individual freezers or refrigerators in it is not considered refrigerated warehouse or storage. Do not include space in the building that is used for storage associated with another main activity in the building, such as retail or office activity.

Cheese warehouse

Cold storage

Fur storage

1. Religious Worship.

Church

Synagogue

Mosque

m. Public Assembly. Refers to the gathering of people for social, recreational, or religious activities whether in private or nonprivate meeting halls.

Entertainment building

Artgallery/Archive/Exhibithall/Library/Museum

Concert hall

Coliseum/Arena (enclosed)

Nightclub

Observatory/Planetarium

Radio/TV studio or station

Theater/Movie/Cinema

Recreational facility:

Amusement arcade

Athletic facility/Gymnasium/Health club

or spa/ndoor racket sports/Recreation center/YMCA or YWCA

Bowling alley

Indoor pool

Poolroom

Skating rink 


\section{EXAMPLES OF ACTIVITIES THAT MAY TAKE PLACE WITHIN A BUILDING}

m. Public Assembly (cont'd)

Religious assembly:

Chapel

Church

Mosque

Synagogue

Social/Public/Civic assembly:

Assembly ball

Auditorium

Convention hall

Funeral home

Lecture hall

Lodge hall

Meeting hall

Student union

Town hall

Other enclosed assembly building:

Armory

Passenger terminal

Stadium

n. Education. Refers to where academic or technical classroom instruction is provided.

College or university classrooms/Laboratories

Elementary school

Junior high school

Preschool

Senior high school

Vocational school

Other activities that occur on school campuses should be reported separately:

Administration (see Office/Professional)

Auditorium (see Public Assembly)

Dormitory (see Hotel/Motel/Dorm)

Gymnasium (see Public Assembly)

Infirmary (see Hospital/npatient Health Services)

Library (see Public Assembly)

School for mentally retarded (see Hospital/Inpatient Health Services)

Student Union (see Public Assembly) o. Food Services. Activities involve preparation and sale of food and beverages for consumption.

Prepared meal services:

Cafeteria

Canry-out service:

Caterer

Fast food establishment

Pizza parlor

Sandwich shop

Full-service restaurant:

Bar

Bar and grill

Coffee shop

Diner

Full menu

p. Hospital/nnpatient Services. Refers to services involving overnight care.

Medical care hospital:

Chronic disease

Ear, Eye, Nose and Throat, etc.

General medical and surgical

Maternity

Medical infirmary (connected with institution)

Orthopedic

Tuberculosis/Other respiratory disease

Mental Facility:

Mental retardation/Schools for mentally retarded

Psychiatric

Rehabilitation:

Alcoholism

Substance abuse/Narcotic/Drug addiction

Physical therapy

Veterinary:

Hospital for animals

Kennel 
q. Skilled Nursing/Other Residential Care. Refers to

24-hour nursing care for extended periods of time.

Homes for the aged

Nursing homes

r. Hotel/Motel/Dorm. Refers to short- or long-term accommodations for several persons.

Short-term residence:

Convention hotel

Hotel

Inn

Motel

Shelter home

Tourist home

Long-term residence:

Boarding house

Convent Monastery

Dormitory/Sorority/Fraternity

Orphanage

s. Residential Living Quarters. Activities involve living quarters that have individual kitchen facilities.

Multi-Family:

High-rise apartments

Low-rise apartments

Single-Family:

Detached

Duplex

Townhouse/Rowhouse

Triplex

Quadriplex

t. Indoor/Enclosed Parking Garage

u. Other activities are those that do not fit into any of the previous categories. Included are:

Crematorium

Hangar

Public restrooms/Showers

Telephone exchange 
Appendix C

Federal Regions Map 



\section{Appendix C}

\section{Federal Regions Map}

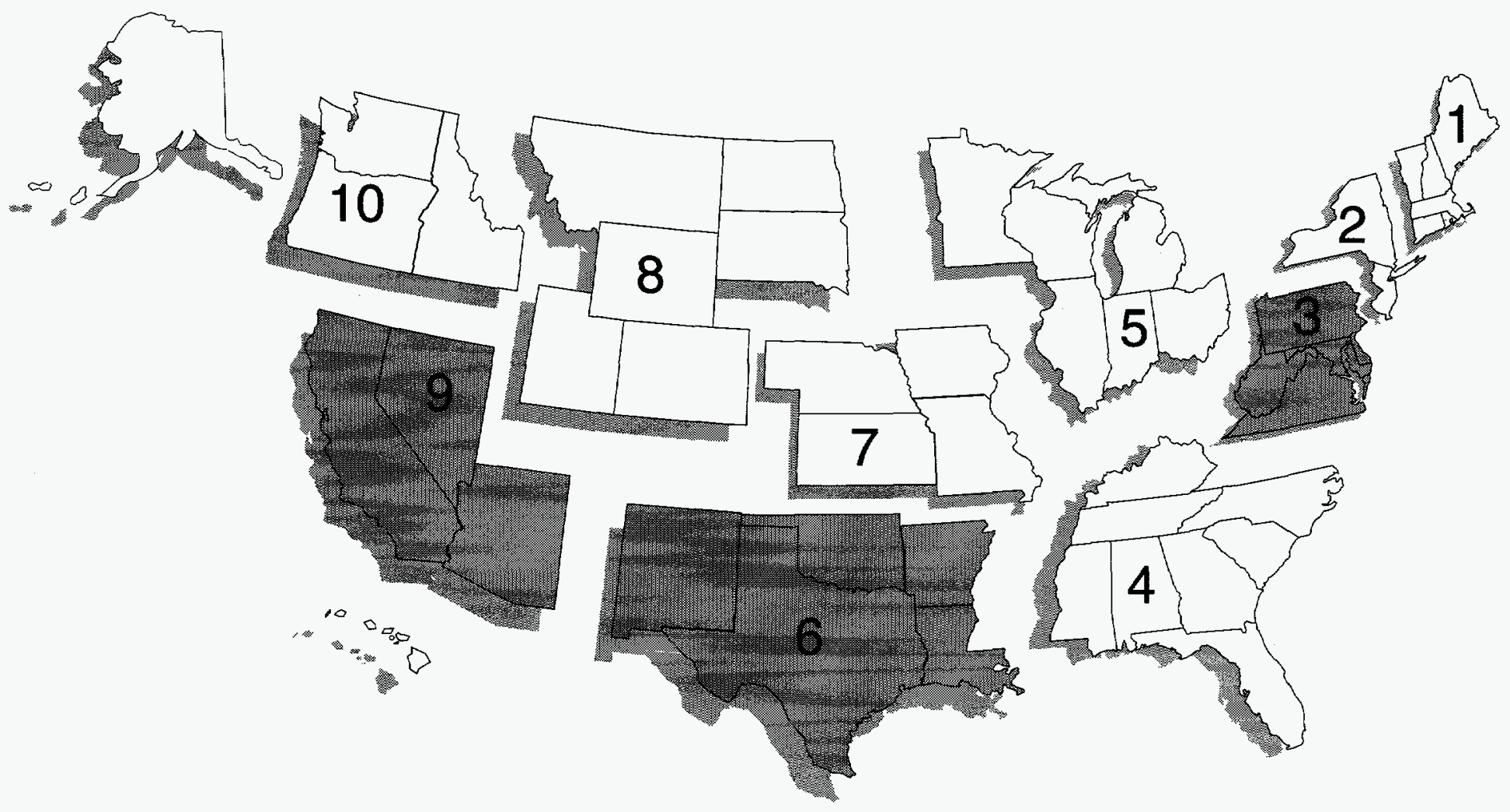

Region 3

Mid Atlantic

Delaware (DE)

District of Columbia (DC)

Maryland (MD)

Pennsylvania (PA)

Virginia (VA)

West Virginia (WV)
Region 6 Southwest

Arkansas (AR)

Louisiana (LA)

New Mexico (NM)

Oklahoma (OK)

Texas (TX)
Region 9

West

Arizona (AZ)

California (CA)

Hawaii $(\mathrm{HI})$

Nevada (NV) 
Appendix D

Metric Conversion Factors 



\section{Appendix D}

\section{Metric Conversion Factors}

Data in the Energy Information Administration publications are expressed in units, such as British thermal units, barrels, cubic feet, and short tons, that historically have been used in the United States. However, because U.S. activities involve foreign nations, most of which use metric units of measure, the United States is committed to making the transition to the metric system. The metric conversion factors presented in Table D1 can be used to calculate the metric-unit equivalents of values expressed in U.S. units. For example, 500 short tons are the equivalent of 453.6 metric tons ( 500 short tons $\times 0.9071847$ metric tons/short tons $=453.6$ metric tons).

Table D1. Metric Conversion Factors

\begin{tabular}{|c|c|c|c|c|}
\hline Type of Unit & U.S. Unit & & Conversion Factor & Metric Unit \\
\hline Mass & $\begin{array}{l}\text { Short Tons } \\
\text { Short Tons Uranium Oxide }\left(\mathrm{U}_{3} \mathrm{O}_{8}\right) \\
\text { Short Tons Uranium Fluoride }\left(\mathrm{UF}_{6}\right) \\
\text { Long Tons } \\
\text { Pounds }(\mathrm{lb}) \\
\text { Pounds Uranium Oxide }\left(\mathrm{lb} \mathrm{U}_{3} \mathrm{O}_{8}\right) \\
\text { Ounces, Avoirdupois }(\mathrm{Oz})\end{array}$ & $\begin{array}{l}x \\
x \\
x \\
x \\
x \\
x \\
x\end{array}$ & $\begin{array}{l}0.9071847 \\
0.769 \\
0.613 \\
1.016 \\
0.45359237^{a} \\
0.384645^{b} \\
28.34952\end{array}$ & $\begin{array}{l}=\text { Metric Tons }(\mathrm{t}) \\
=\text { Metric Tons Uranium }(\mathrm{U}) \\
=\text { Metric Tons Uranium }(\mathrm{U}) \\
=\text { Metric Tons }(\mathrm{t}) \\
=\text { Kilograms }(\mathrm{kg}) \\
=\text { Kilograms }(\mathrm{Kg}) \\
=\text { Grams }(\mathrm{g})\end{array}$ \\
\hline Volume & 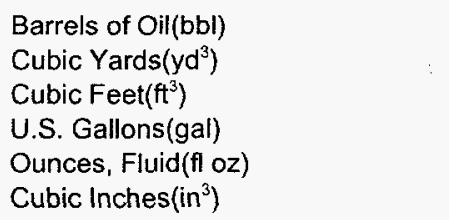 & $\begin{array}{l}x \\
x \\
x \\
x \\
x \\
x\end{array}$ & $\begin{array}{l}0.1589873 \\
0.765555 \\
0.02831685 \\
3.785412 \\
29.57353 \\
16.38706\end{array}$ & $\begin{array}{l}=\text { Cubic Meters }\left(\mathrm{m}^{3}\right) \\
=\text { Cubic Meters }\left(\mathrm{m}^{3}\right) \\
=\text { Cubic Meters }\left(\mathrm{m}^{3}\right) \\
=\text { Liter }(\mathrm{L}) \\
=\text { Milliliters }(\mathrm{ml}) \\
=\text { Milliliters }(\mathrm{ml})\end{array}$ \\
\hline Length & $\begin{array}{l}\text { Miles (mi) } \\
\text { Yards (yd) } \\
\text { Feet (ft) } \\
\text { Inches (in) }\end{array}$ & $\begin{array}{l}x \\
x \\
x \\
x\end{array}$ & $\begin{array}{l}1,609344^{a} \\
0.9144^{a} \\
0.3048^{a} \\
2.54^{a}\end{array}$ & $\begin{array}{l}=\text { Kilometers }(\mathrm{km}) \\
=\text { Meters }(\mathrm{m}) \\
=\text { Meters }(\mathrm{m}) \\
=\text { Centimeters }(\mathrm{cm})\end{array}$ \\
\hline Area & $\begin{array}{l}\text { Acres } \\
\text { Square Miles }\left(\mathrm{mi}^{2}\right) \\
\text { Square Yards }\left(\mathrm{yd}^{2}\right) \\
\text { Square Feet }\left(\mathrm{ft}^{2}\right) \\
\text { Square Inches }\left(\mathrm{in}^{2}\right)\end{array}$ & $\begin{array}{l}x \\
x \\
x \\
x \\
x\end{array}$ & $\begin{array}{l}0.40469 \\
2,589988 \\
0.8361274 \\
0.09290304^{a} \\
6.45616^{a}\end{array}$ & $\begin{array}{l}=\text { Hectares }(\mathrm{ha}) \\
=\text { Square Kilometers }\left(\mathrm{km}^{2}\right) \\
=\text { Square Meters }\left(\mathrm{m}^{2}\right) \\
=\text { Square Meters }\left(\mathrm{m}^{2}\right) \\
=\text { Square Centimeters }\left(\mathrm{cm}^{2}\right)\end{array}$ \\
\hline Temperature & Degrees Fahrenheit ${ }^{\mathrm{c}}\left({ }^{\circ} \mathrm{F}\right)$ & $x$ & 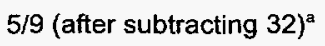 & $=$ Degrees Celsius $\left({ }^{\circ} \mathrm{C}\right)$ \\
\hline Energy & $\begin{array}{l}\text { British thermal units (Btu) } \\
\text { Calories (cal) } \\
\text { Kilowatthours (kWh) }\end{array}$ & $\begin{array}{l}x \\
x \\
x\end{array}$ & $\begin{array}{l}1,055.056 \\
4.1868 \\
3.6\end{array}$ & $\begin{array}{l}=\text { Joules }(\mathrm{J}) \\
=\text { Joules }(\mathrm{J}) \\
=\text { Megajoules }(\mathrm{MJ})\end{array}$ \\
\hline
\end{tabular}

${ }^{a}$ Exact Conversion.

${ }^{b}$ Calculated by the Energy Information Administration.

${ }^{6}$ To convert degrees Celsius $\left({ }^{\circ} \mathrm{C}\right)$ to degrees Fahrenheit $\left({ }^{\circ} \mathrm{F}\right)$ multiply by $9 / 5$, then add 32.

Sources: -General Services Administration, Federal Standard 376B, Preferred Metric Units for General Use by the Federal Government (Washington, DC, January 27, 1993), pp. 9-11, 13, and 16. -National Institute of Standards and Technology, Special Publications 330, 811, and 814. -American National Standards Institute/lnstitute of Electrical and Electronic Engineers, ANS/EEE Std.268-1982, pp 28 and 29. - Energy Information Administration, Monthly Energy Review August 1993, Appendix B, p 161. 

Appendix E

Outreach Efforts 



\section{Appendix E}

\section{Outreach Efforts}

This appendix contains examples of the letters of notification and FBSS materials that were sent prior to and throughout the FBSS data collection process to minimize nonresponse and respondent burden. This appendix also contains the names and addresses of the Federal Interagency Management Task Force and GSA and DOE regional offices contacts to whom their respective letters were sent. Also included is the thank you letter sent to FBSS respondents (Letter 5). The FBSS letters of notification and other FBSS materials were sent to the following:

Letter 1. Federal Interagency Management Task Force:

Some agencies (those without Cabinet appointees) are not represented in the Federal Interagency Management Task Force. The following nonmember agencies did not receive an advance FBSS notification letter:

- Federal Energy Management Agency

- Government Printing Office

- Interstate Commerce Commission

- National Science Foundation

Although members of the Federal Interagency Management Task Force, some agencies did not have facilities chosen to participate in the FBSS. The following member agencies did not receive an advance FBSS notification letter:

- Department of Housing and Urban Development

- General Accounting Office

- Office of Management and Budget

- Department of State

Letter 2. General Services Administration and DOE regional offices and enclosures

Letter 3. Facility Managers of facilities selected to participate in FBSS and enclosures

Letter 4. Building Managers of the buildings selected to participate in the FBSS and enclosures. 


\section{Department of Energy \\ Washington, DC 20585}

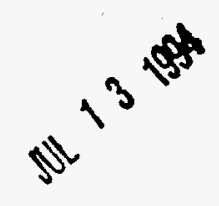

Letter 1

Mr. Victor P. Petrolati

Chief, In-House Energy Management Branch

U.S. Department of Energy

Forrestal Building, Room GF-209

1000 Independence Avenue, S.W.

Washington, D.C. 20585

Dear Mr. Petrolati:

Per our agreement at the June Interagency Federal Management Task Force meeting, enclosed is the list of your agency's facilities included as possible respondents for the Federal Buildings Supplemental Survey (FBSS). During the survey, a sample of buildings will be selected from this and similar listings for other Federal agencies.

The Energy Information Administration (EIA) of the U.S. Department of Energy (DOE, in conjunction with the Office of Federal Energy Management Programs (FEMP) of DOE, will conduct the FBSS in Federal Regions 3 (Philadelphia, PA), 6 (Dallas, TX), and 9 (San Francisco, CA). A total of 900 Federal buildings (across the 3 regions and all agencies) will be selected for the short telephone survey. Starting in late July and continuing through August, 1994, the energy managers for the selected buildings will be asked to provide energy consumption information and energyrelated building characteristics. The telephone calls will be made by Response analysis Corporation, an EIA survey contractor.

To minimize additional contacts with the buildings, calls will be made to the survey buildings prior to the actual telephone survey in order to obtain building identification information and the names and addresses of the building energy manager or other respondent. FEMP's updated building contacts information will be used, wherever available. EIA will then send advance letters to the energy managers concerning the FBSS and notifying them of the information that will be requested during the telephone survey.

Your support of the FBSS and your assistance in notifying your regional offices about the FBSS is appreciated. EIA would like to work closely with the Federal Regions to ensure that respondent burden is minimized during the FBSS. We will keep you informed of the progress of the survey. 
If you have any questions about the Federal Buildings Supplemental Survey (FBSS), please contact Rick Klimkos of FEMP at (202) 586-8287 or me at (202) 586-5744.

Sincerely,

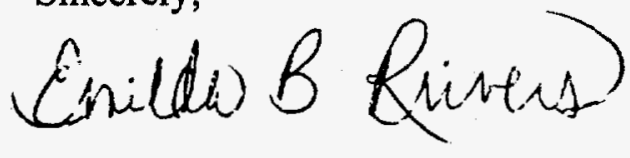

\section{Emilda B. Rivers}

Federal Buildings Supplemental Survey Project Manager Energy Information Administration 


\section{ADDRESS LIST FOR LETTER 1}

Mr. Walter D. Aughenbaugh, P.E.

Chief, Engineering Branch

Facilities Management Division

Office of Operations, Room S-313

U.S. Department of Agriculture

14th and Independence Avenue, SW

Washington, DC 20250

Mr. Ron Cyr, Energy Conservation Officer

Office of Federal Property Programs

U.S. Department of Commerce

Herbert C. Hoover Building, Room 139

14th and Constitution Avenue, NW

Washington, DC 20230

Mr. Millard Carr, Acting Director

Energy Management Policy Office

ODUSD ES

U.S. Department of Defense

400 Army Navy Drive, Room 206

Arlington, VA 22204-2884

Mr. Victor P. Petrolati

Chief, In-House Energy Management Branch

U.S. Department of Energy

Forrestal Building, Room GF-209

1000 Independence Avenue, SW

Washington, DC 20585

Mr. Phillip Wirdzek, Program Analyst

Environmental Protection Agency

PM-215, Room NEB 022

$401 \mathrm{M}$ Street, SW

Washington, DC 20460

Mr. Eric Dunham, Chief

Energy Branch, General Services Administration

Room 4327, PMFE

18 th and $F$ Streets, NW

Washington, DC 20405

Ms. Elaine Robinson, Deputy, Director

Facilities Operations Division

Department of Housing and Urban Development

Room 5180

451 7th Street, SW

Washington, DC 20410-3000
Mr. Paul Denett

Director, Office of Acquisition and Property Management

U.S. Department of the Interior

Main Interior Building, Room 5512

1849 C Street, NW

Washington, DC 20240

Mr. Charles B. Pittinger, Jr.

Head, Maintenance Management Branch

Operations and Maintenance Office

National Aeronautics and Space Administration

Mail Stop JXG, Room 3S13

300 E Street, SW

Washington, DC 20024-3210

Mr. Paul Fennewald

Maintenance Policies and Programs

U.S. Postal Service

Room 6631, 475 L'Enfant Plaza, SW

Washington, DC 20260-7311

Ms. Janet Judd, Chief

Safety, Health and Environment Division (M-46)

U.S. Department of Transportation

Room 2310, 400 7th Street, SW

Washington, DC 20590

Mr. Rajinder P. Garg, Chief

Energy Management Division (138C1)

U.S. Department of Veterans Affairs

Room 436-LAF, 810 Vermont Avenue, NW

Washington, DC 20420

Mr. Charles B. Hessler, Senior Evaluator

Energy Issues

U.S. General Accounting Office

111 Massachusetts Avenue, NW, Suite 201

Washington, DC 20001

Mr. Randy Steer, Budget Examiner

Office of Management and Budget

Energy Branch

New Executive Office Building, Room 8013

725 17th Street, NW

Washington, D.C. 20503 
Mr. Victor Ayala

Director of Facilities Management

Department of Education

Room 165

400 Maryland Avenue, SW

Washington, DC 20202

Mr. Scott Waldman

Department Wide Energy Manager

U.S. Department of Health and Human Services

HHS Cohen Building, Room 4700

330 Independence Avenue, SW

Washington, DC 20201

Mr. Jim Shivar

Federal Energy Program Manager

U.S. Department of Justice

Aerial Rios Building, Room 2211

12th and Pennsylvania Avenue, NW

Washington, DC 20530

Ms. Maggie Carson, Director

Office of Facility Management

U.S. Department of Labor

Room S-1521

200 Constitution Avenue, NW

Washington, DC 20210

Mr. Richard Iselin

Director of Facilities Management and

Support Services

Department of State

FMSS/B, Room 1480

2201 C Street, NW

Washington, DC 20520

Mr. Joseph Maty

Assistant Director of Energy Programs

Office of Management Support Systems

U.S. Department of the Treasury

Main Treasury Annex, Room 6140

1500 Pennsylvania Avenue, NW

Washington, DC 20220 


\section{Department of Energy \\ Washington, DC 20585}

$$
\text { JUL } 261994 \quad \text { Letter } 2
$$

Mr. Don Stitler

Wanamaker Building

3PMFM, Room 3319

100 E. Penn Square

Philadelphia, PA 19107

Dear Mr. Stitler:

This letter is to inform you that the Energy Information Administration (EIA) of the Department of Energy (DOE), in conjunction with the Office of Federal Energy Management Programs (FEMP) of DOE, is conducting the Federal Buildings Supplemental Survey (FBSS) and that some of the sampled buildings may be in your region. Starting in late July and continuing through mid-September, 1994, the energy managers for the selected Federally-owned buildings will be asked to provide energy consumption information and energy-related building characteristics.

The major purpose of the FBSS is to assist in the implementation of the Energy Policy Act of 1992 which requires extensive energy-related information on Federal buildings. The FBSS will be conducted in Federal Regions 3 (Philadelphia, PA), 6 (Dallas, TX), and 9 (San Francisco, CA). The Federal regions differ from the General Services Administration (GSA) regions as follows: Federal region 3 excludes all of New Jersey and includes GSA's Region NCR - Washington, DC; and Federal region 6 is GSA region 7. A total of 900 Federal buildings (across the 3 regions and several government agencies) have been selected for a telephone survey.

Information about the building size, building use, energy sources, energy end uses, conservation features and practices, and types of heating, cooling and lighting equipment is being collected through voluntary telephone interviews with the energy manager, or another individual designated by the energy manager. To minimize additional contacts with the buildings, calls will be made to the survey buildings prior to the actual telephone survey to obtain building identification information and the names and addresses of the building energy manager or other respondent. EIA will then send advance letters to the energy mangers concerning the FBSS and notifying them of the information that will be requested during the telephone survey. Enclosed is a copy of the advance letter and worksheet.

We ask that you encourage your facilities to respond, if they are contacted for this survey.

Please see the reverse side of the enclosed example letter to the building managers for the authorizing legislation and public reporting burden. 
For more information concerning this survey, please contact Emilda B. Rivers, EIA's Federal Buildings Supplemental Survey Manager at (202) 586-5744, FAX (202) 586-0018, or Internet Address ERIVERS@EIA.DOE.GOV.

Your cooperation in this important Federal buildings survey is appreciated.

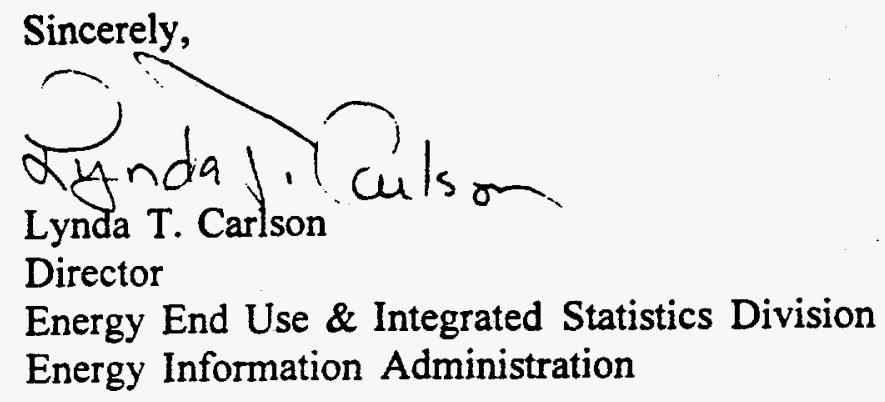

Enclosures 


\title{
EXAMPLE OF ADVANCE LETTER TO BULLDING MANAGER
}

\author{
Dear Building Manager:
}

The Energy Information Administration (EIA) of the U.S. Department of Energy (DOE), in conjunction with the Office of Federal Energy Management Programs (FEMP) of DOE, is conducting a telephone survey of 900 Federal Buildings in Federal Regions 3

(Philadelphia, PA), 6 (Dallas, TX), and 9 (San Francisco, CA). This building has been statistically selected to participate in the Federal Buildings Supplemental Survey (FBSS).

The major purpose of the FBSS is to obtain more information on energy usage and characteristics of Federal buildings, to assist in the implementation of the Energy Policy Act of 1992. Starting in late July and throughout August 1994, the building energy managers for the 900 sampled Federal buildings will be contacted by telephone. The data collection will be conducted by Response Analysis Corporation under contract to EIA.

For each of the Federal regions, a survey of the sampled buildings in that region will provide basic data on the Federally-owned buildings. Two types of data will be collected: (1) building characteristics data, which includes information on how energy is managed in Federal buildings; and (2) energy consumption data. Data collected will represent other Federal commercial buildings within those three Federal regions.

Enclosed is a FBSS worksheet with several questions that may require research prior to the telephone interview. You, or another individual designated by you to answer the FBSS worksheet, may need to contact several persons to gather the required information. For instance, the building engineer may need to provide you with information on the costs and amounts of energy used. Having the FBSS worksheet completed and available when you are contacted by telephone will expedite the interview with minimum additional burden to you.

We realize that the building interviews may be time consuming for you and we would like to thank you in advance for your participation in this important survey. Without your cooperation, we could not obtain information about how energy is used in Federal buildings. We will be happy to provide you with a profile of your building's characteristics and energy usage after all the survey data are analyzed.

Please see the reverse side of this letter for the authorizing legislation and public reporting burden.

If you have any general questions about the purpose of the Federal Buildings Supplemental Survey, please contact Emilda Rivers of EIA at (202) 586-5744.

Thank you for your time and effort.

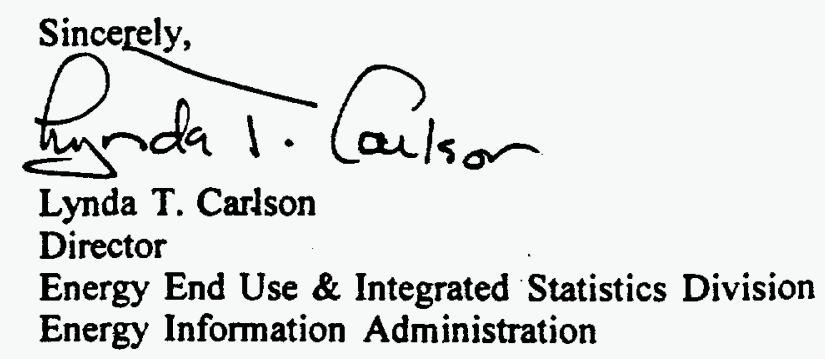

Enclosures 


\section{Federal Buildings Supplemental Survey Worksheet}

Please have the information available for the telephone survey.

Thank you for your help!

A. Building Square Footage?

B. Year major portion of construction completed?

C. Number of workers (all shifts)?

D. Energy Consumption

For fiscal year 1993 (October 1, 1992 through September 30, 1993), provide the amount of usage, units, and costs for each fuel used in the building.

Amount

Electricity

Natural Gas

Fuel Oil/ Kerosene/Diesel

District Steam

District Hot Water

District Chilled Water
Units

(circle one)

Btu's or Kwh

Btu's or $\mathrm{Cu} \mathrm{ft}$.

Btu's or Gallons

Btu's

Btu's

Ton-hours sq ft. year workers

E. Motors

1. Number and age of Motors (10 horsepower or more) that are in each of the following equipment:

Chillers
Heat Pumps
Fans
Compressors
Elevators
Escalators
Refrigeration
Water Pumps

Number Less

Number of Motors

than 10 years old

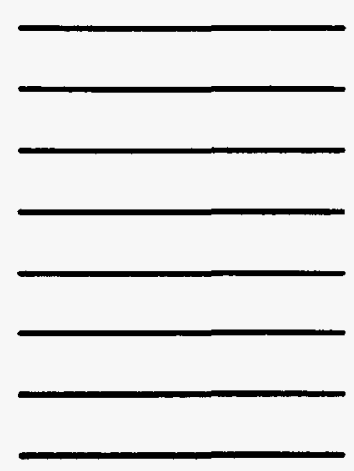

Number 10 years or older

2. Number of energy-efficient motors ( 10 horsepower or more) less than 10 years old.

3. Number of motors ( 10 horsepower or more) less than 10 years old that have been rewound:

3a. Average age of these motors that were rewound? 
General Services Administration

Mr. Steve Williford

General Services Administration

WPMOE, Room 7709

7th and D Street, S.W.

Washington, D.C. 20407

Mr. Mark Trimarchi

General Services Administration

7PMR, Room 12 A26

819 Taylor Street

Fort Worth, TX 76102

Mr. Peter Gaddy

General Services Administration

9PMFM, 30th Floor

525 Market Street

San Francisco, CA 94105

\section{Department of Energy Offices}

Curtis Carlson Jr.,

Dallas Support Office

1420 W. Mockingbird Lane, Suite 400

Dallas, TX 75247

Christopher G. McGowan

Philadelphia Support Office

1880 John F. Kennedy Blvd.

Suite 501

Philadelphia, PA 19103-7483

Martha D. Dixon

San Francisco Support Office

1301 Clay Street

Room 1060 North

Oakland, CA 94612-5219 


\section{Department of Energy}

Washington, DC 20585

Letter 3

Dear Facility Manager:

\section{NUL 011994}

To assist in the implementation of the Energy Policy Act of 1992 which requires extensive information on Federal buildings, a joint effort is being undertaken by the Energy Information Administration (EIA) and the Office of Federal Energy Management Programs (FEMP) of the Department of Energy (DOE). EIA will conduct a Federal Buildings Supplemental Survey (FBSS) in August 1994, to collect building characteristics and energy consumption information.

This survey will be conducted in Region 3 (Philadelphia, PA), Region 6 (Dallas, TX), and Region 9 (San Francisco, CA). Across these regions, a total of 900 Federal buildings have been selected for a brief telephone survey. Using scientific sampling methods, your facility was selected randomly.

The Response Analysis Corporation (RAC), under contract to EIA, will collect this information. In the next few days, someone from RAC will contact you to:

(1) verify the eligibility of the one or more buildings selected from this facility,

(2) obtain an accurate mailing address and the name of the building manager, or another individual designated by you to answer the FBSS, and

(3) schedule an appointment with the designated individual.

You are encouraged to respond. Participation in the survey is voluntary, however, your cooperation will contribute greatly to its success. The sampled buildings represent similar Federal buildings across your region. You will be asked to provide information about one or more buildings on the facility. The enclosed excerpt from the Commencial Buildings Characteristics 1992 report provides a profile of government-owned buildings as of 1992 . The RAC caller will be pleased to provide you with more information about FBSS.

For more information concerning this survey, please contact Emilda B. Rivers, ElA's Federal Buildings Supplemental Survey Manager at (202) 586-5744, FAX (202) 586-0018, or Internet Address ERIVERS@EIA.DOE.GOV.

Please see the reverse side of this letter for the authorizing legislation and public reporting burden. Your cooperation in this important Federal building survey is appreciated.

Sincerely,

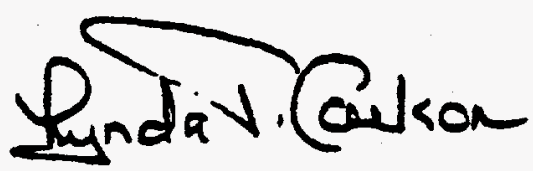

Lynda T. Carlson, Director

Energy End Use \& Integrated Statistics Division

Energy Information Administration 


\section{Authorizing Legislation:}

Authorization for collecting energy consumption data in the Commercial sector is set forth in the Federal Energy Administration Act of 1974; Public Law 93-275, as amended. The Commercial Buildings Energy Consumption Survey, Buildings Questionnaire (Federal Supplement) conducted by the Energy Information Administration collects information about energy usage in U.S. buildings.

\section{Public Reporting Burden:}

Public reporting burden for this collection of information via telephone is estimated to average 30 minutes.

Send comments regarding public reporting burden or any other aspect of this collection of information, including suggestions for reducing this burden, to:

Energy Information Administration

Office of Statistical Standards

EI-73

1000 Independence Avenue, S.W.

Washington, DC 20585

and

Office of Information and Regulatory Affairs

Office of Management and Budget

Washington, DC 20503 
Letter 3 Enclosures

DOE/EIA-0246(92)

DistributionCategoryUC-950

\title{
Commercial Buildings Characteristics 1992
}

\author{
April 1994
}

Energy Information Administration

Office of Energy Markets and End Use

U.S. Department of Energy

Washington, DC 20585 


\section{Conservation in Office Buildings and Government-Owned Buildings}

\section{Profile of Office Buildings}

The characteristics of office buildings are of increasing interest to energy analysts since office buildings consume slightly over one-fifth of all energy used in commercial buildings. An oversample of office buildings was included in the 1992 CBECS to better understand the potential for energy savings in these buildings. In 1992, office buildings comprised 16 percent ( 749 thousand buildings) of the building stock and 18 percent of the commercial floorspace (12,319 million square feet). Of these, 57 thousand ( 7.6 percent) were buildings constructed after 1986 . Below is a profile of selected energy-related characteristics for office buildings in the 1992 commercial building stock.

\section{Box 3. Conservation in Office Buildings}

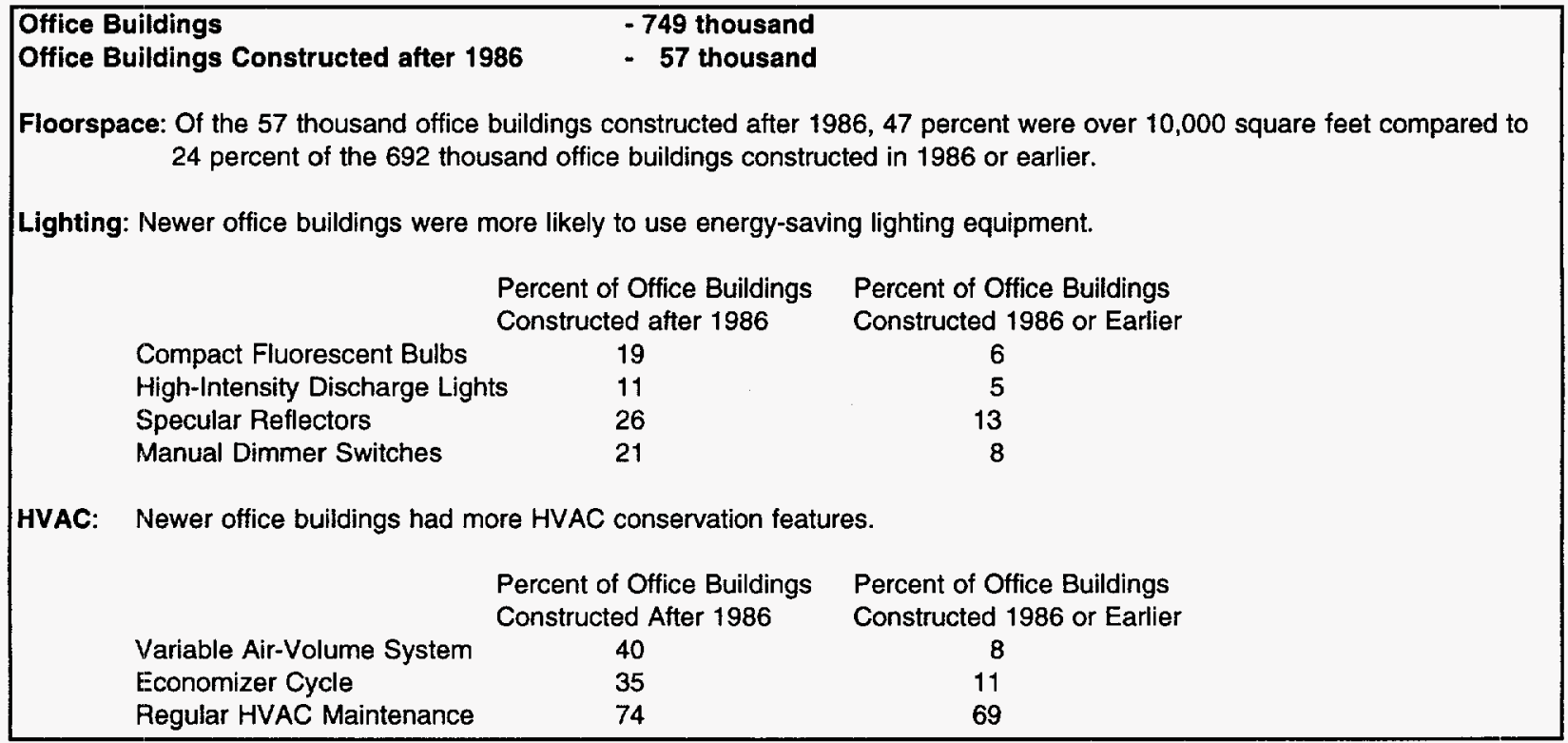

\section{Profile of Government-Owned Buildings}

With the signing of the Energy Policy Act of 1992 (EPACT), ${ }^{10}$ the CBECS is uniquely placed to determine and illustrate this legislation's impact on the commercial buildings market. This section highlights some findings for government-owned (Federal, State, and local) commercial buildings (Tables A21, A22 and A23).

\section{Box 4. Effects of EPACT on Government-Owned Buildings}

EPACT affects federally owned commercial buildings:

- By mandating a 20 percent reduction, by the year 2000 , of energy consumption per square foot in federal buildings;

- By requiring that by the year 2005 federal buildings install energy conservation features that will pay for themselves from energy savings within 10 years (i.e., a 10-year payback); and,

- By introducing financial incentives to federal buildings for energy improvement programs.

EPACT also creates, for local-owned and State-owned commercial buildings, an Energy Incentive Fund which provides up to $\$ 1$ million to States that demonstrate a commitment to improving the energy-efficiency of buildings.

${ }^{10}$ The Energy Policy Act of 1992, Title I - Energy Efficiency, Subtitle E - State and Local Assistance, Section 141 and Subtitle F - Federal Agency Energy Management, Section 152. 
Government-owned buildings represented approximately 22 percent of the commercial floorspace in 1992 , or 15.1 billion square feet in approximately 0.6 million government-owned buildings. Of these government-owned buildings, 8 percent of the floorspace was in buildings owned by the Federal government, 25 percent was in State-owned buildings, and 67 percent was in buildings owned by local governments (Figure 10). Education was the primary activity of government-owned buildings, representing 46 percent of all government buildings.
Figure 10. Ownership of Commercial Buildings, 1992

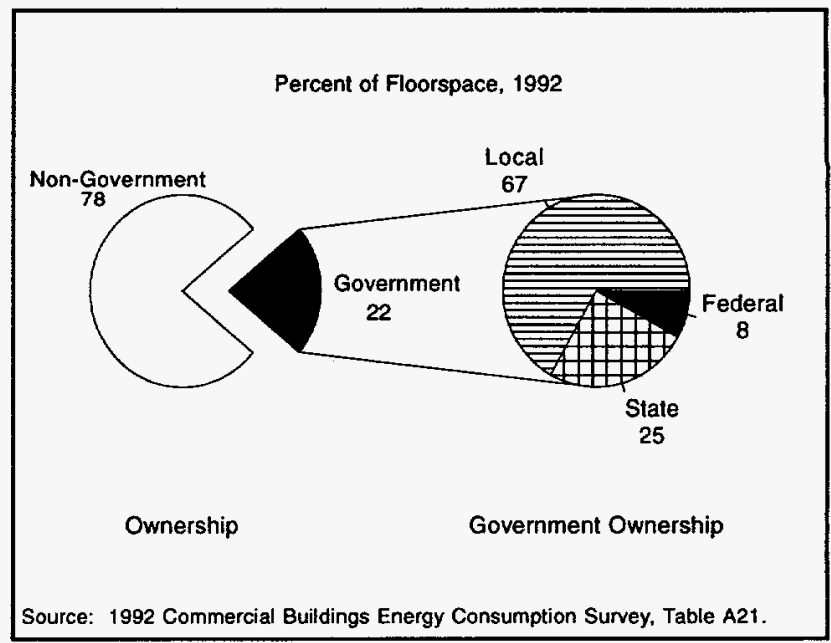

\section{Government-Owned Buildings Take Advantage of Energy-Efficient Technologies and Energy Management Practices}

Commercial buildings consume large amounts of energy for HVAC purposes. ${ }^{11}$ In 1992, as measured in floorspace, approximately 88 percent of all government buildings contained HVAC conservation features compared to 70 percent of non government buildings (Figure 11).

In 1992, government-owned buildings indicated a higher usage of energy management practices than nongovernment buildings. The 1992 CBECS included four energy management practices: EMCS, DSM, energy audits, and building energy managers. According to the CBECS, in 59 percent of government-owned floorspace energy management practices were used, compared to 36 percent of non government-owned floorspace. $^{12}$ Of the 15.1 billion square feet of government-owned floorspace: EMCS controlled 32 percent; 28 percent of the floorspace showed DSM activity; and 30 percent indicated the performance of energy audits. Although 5 percent of government-owned floorspace had a building energy manager, there was not a statistically significant difference between government-owned and non government-owned buildings.

\footnotetext{
${ }^{11}$ End-use consumption estimates are not currently available. End-use intensities (EUI) will be published at a later date.

${ }^{12}$ Estimates of any energy management practices were calculated from public use diskettes of Commercial Buildings Energy Consumption Survey.
} 
Table A21. Occupancy of Government-Owned Buildings, Number of Buildings and Floorspace, 1992

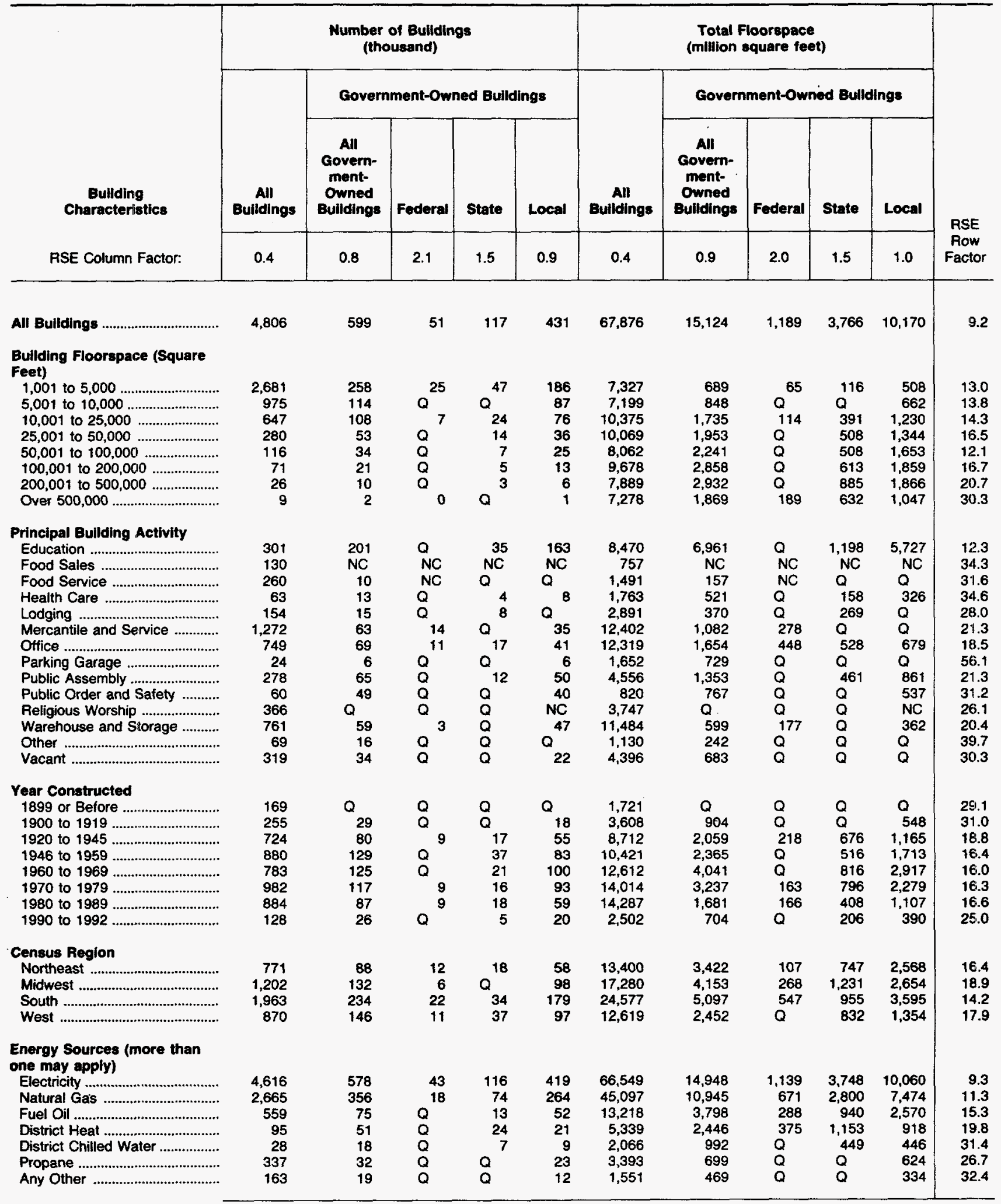

See footnotes at end of table. 
Table A21. Occupancy of Government-Owned Buildings, Number of Buildings and Floorspace, 1992 (Continued)

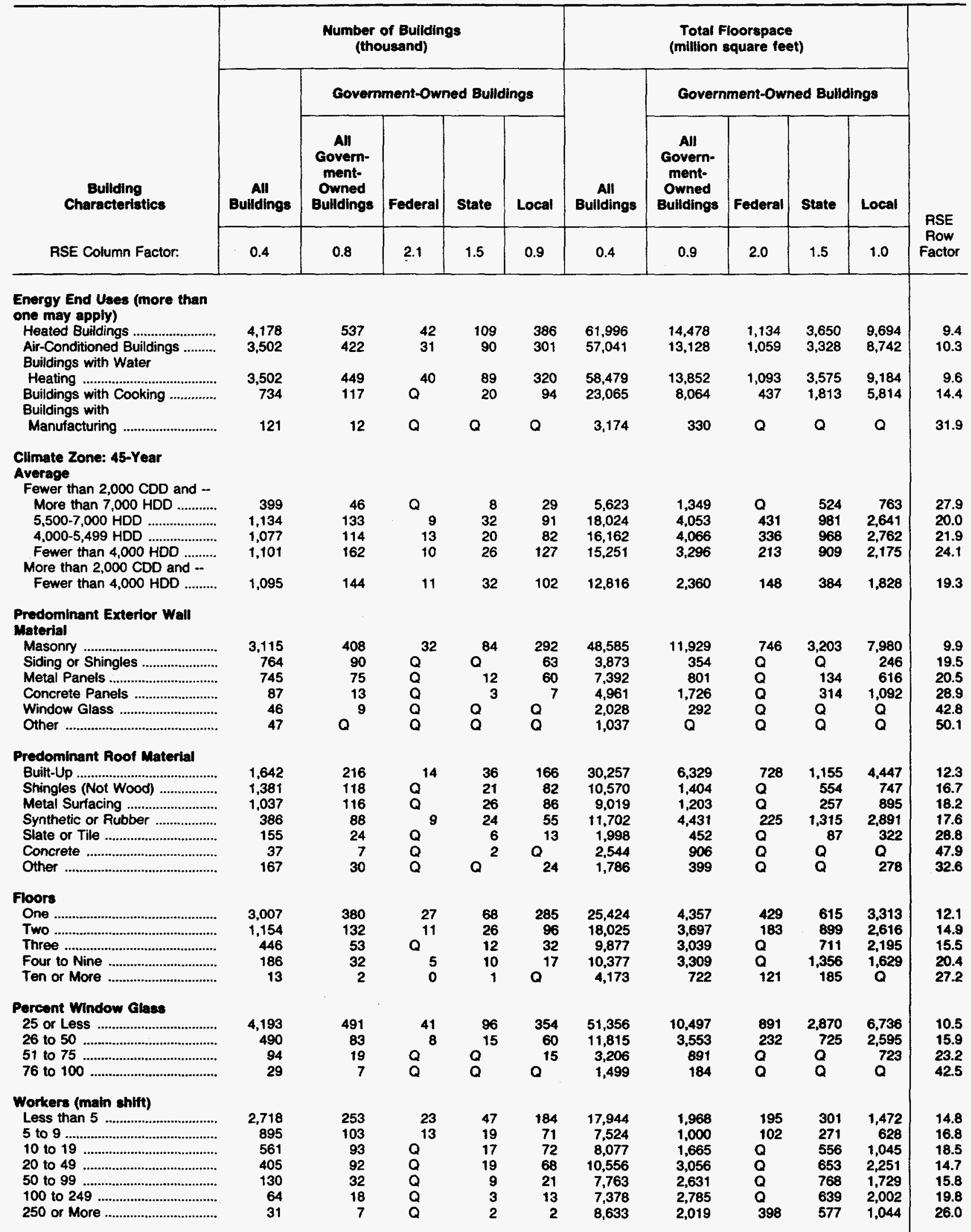

See lootnotes at end of table. 
Table A21. Occupancy of Government-Owned Buildings, Number of Buildings and Floorspace, 1992 (Continued)

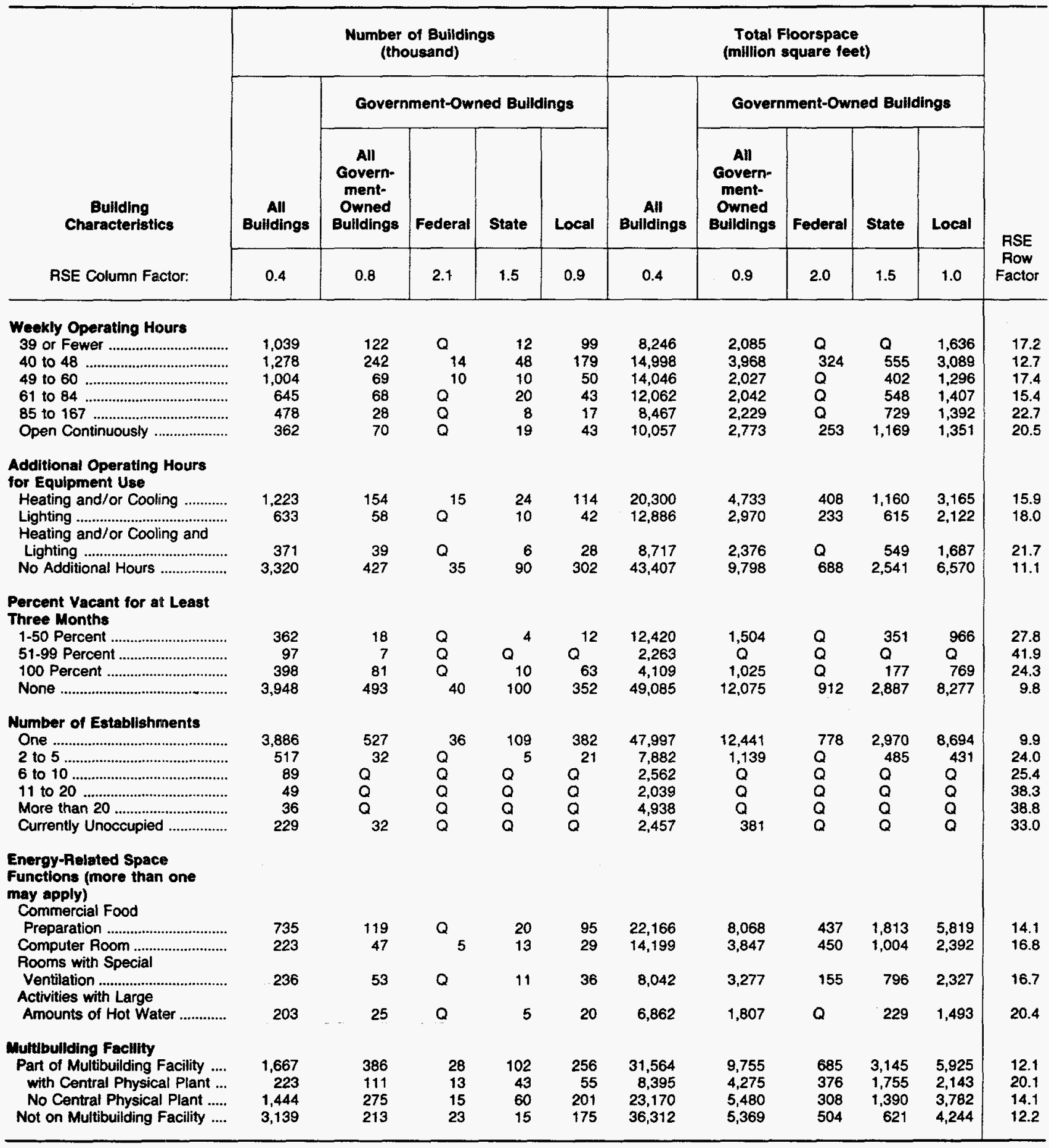

See footnotes at end of table. 

and Floorspace, 1992 (Continued)

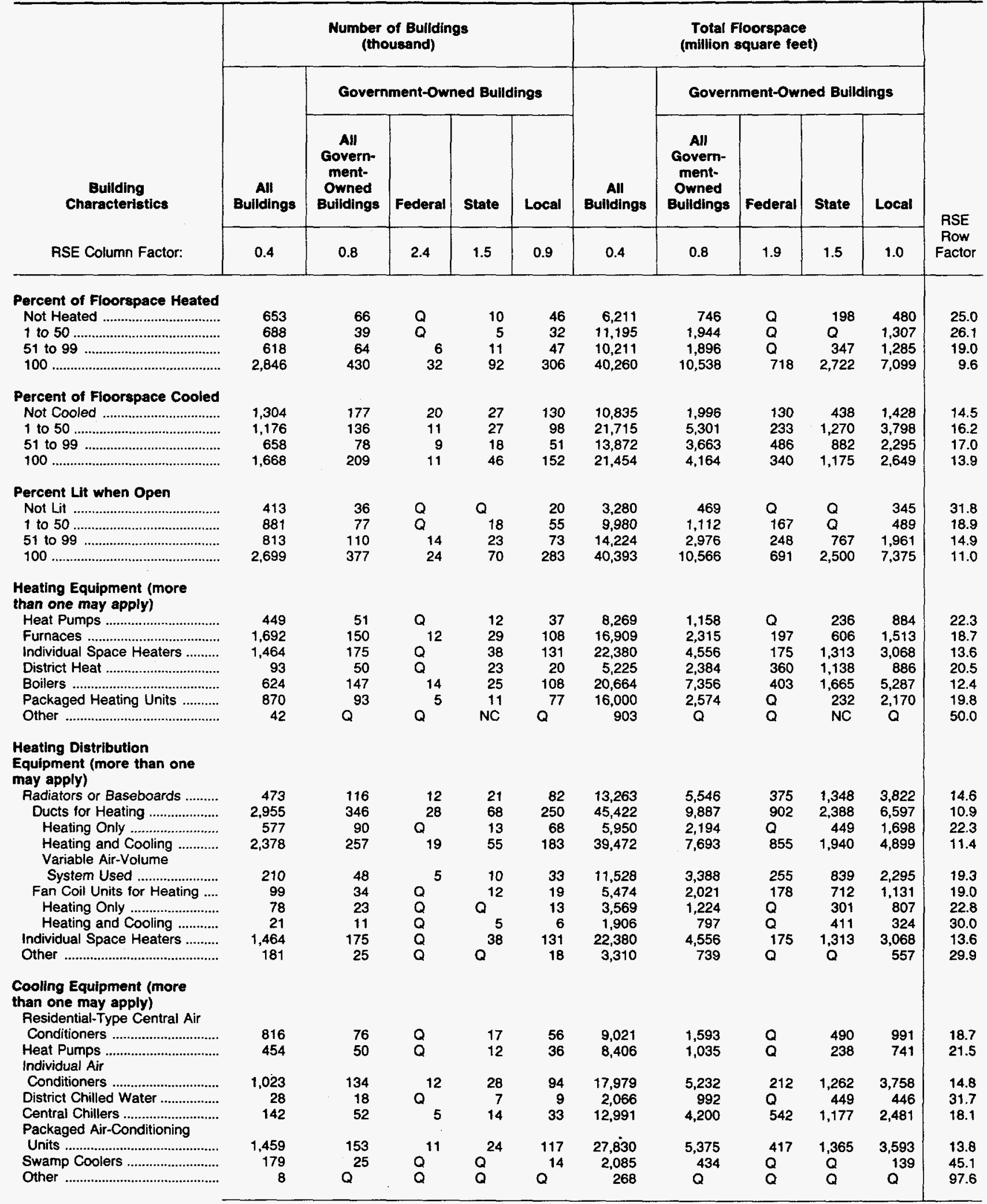

See footnotes at end of table. 
Table A21. Occupancy of Government-Owned Buildings, Number of Buildings and Floorspace, 1992 (Continued)

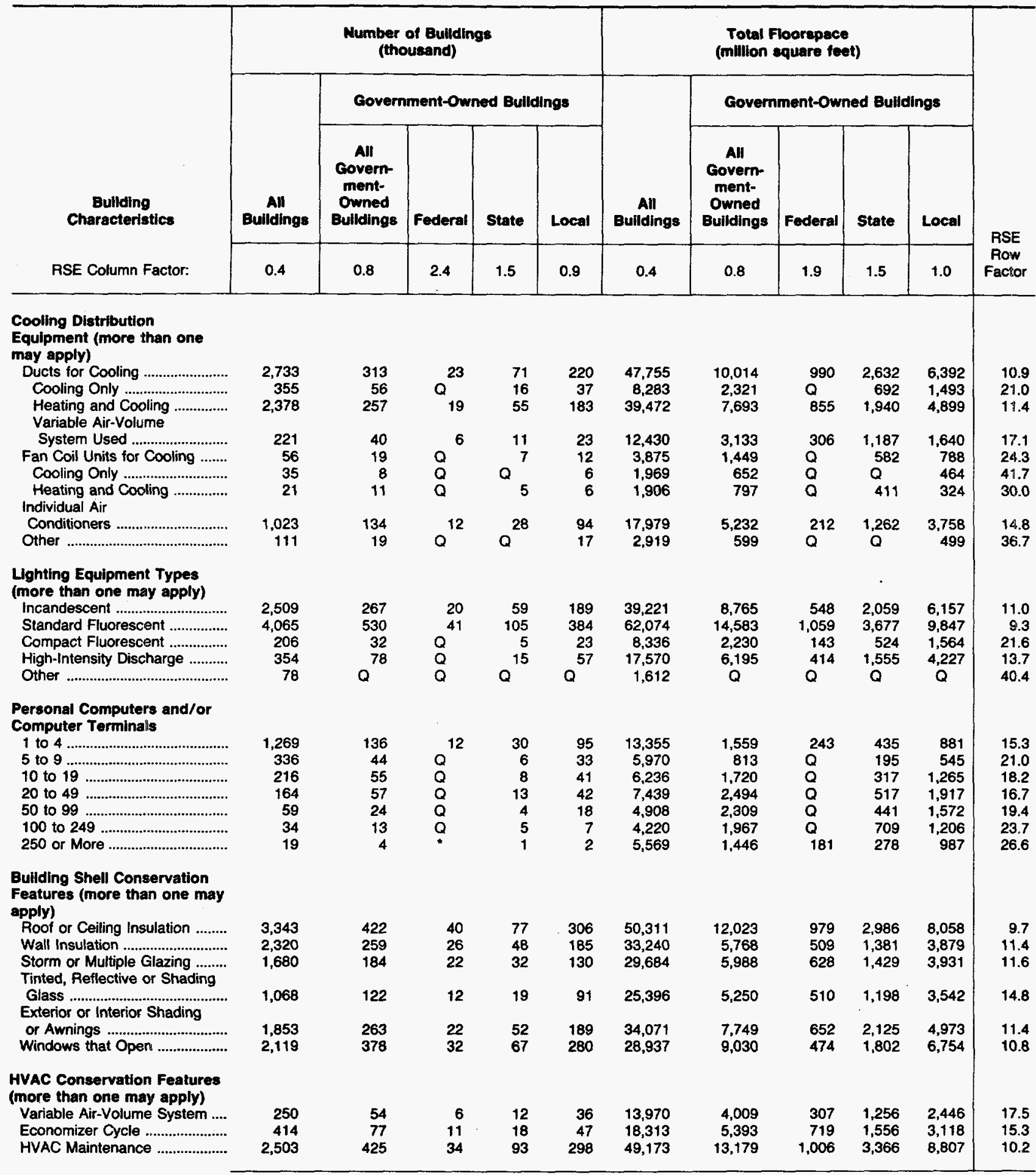

See footnotes at end of table. 
Table A21. Occupancy of Government-Owned Buildings, Number of Buildings and Floorspace, 1992 (Continued)

\begin{tabular}{|c|c|c|c|c|c|c|c|c|c|c|c|}
\hline \multirow[b]{3}{*}{$\begin{array}{l}\text { Building } \\
\text { Characteristics }\end{array}$} & \multicolumn{5}{|c|}{$\begin{array}{l}\text { Number of Bulldings } \\
\text { (thousand) }\end{array}$} & \multicolumn{5}{|c|}{$\begin{array}{l}\text { Total Floorspace } \\
\text { (million square feet) }\end{array}$} & \multirow{4}{*}{$\begin{array}{l}\text { RSE } \\
\text { Row } \\
\text { Factor }\end{array}$} \\
\hline & \multirow[b]{2}{*}{$\begin{array}{c}\text { All } \\
\text { Bulldings }\end{array}$} & \multicolumn{4}{|c|}{ Government-Owned Buildings } & \multirow[b]{2}{*}{$\begin{array}{c}\text { All } \\
\text { Buildings }\end{array}$} & \multicolumn{4}{|c|}{ Government-Owned Buildings } & \\
\hline & & $\begin{array}{l}\text { All } \\
\text { Govern- } \\
\text { ment- } \\
\text { Owned } \\
\text { Bulldings }\end{array}$ & Federal & State & Local & & $\begin{array}{l}\text { All } \\
\text { Govern- } \\
\text { ment- } \\
\text { Owned } \\
\text { Buildings }\end{array}$ & Federal & State & Local & \\
\hline RSE Column Factor: & 0.4 & 0.8 & 2.4 & 1.5 & 0.9 & 0.4 & 0.8 & 1.9 & 1.5 & 1.0 & \\
\hline \multicolumn{12}{|l|}{$\begin{array}{l}\text { Lighting Conservation } \\
\text { Features (more than one may } \\
\text { apply) }\end{array}$} \\
\hline 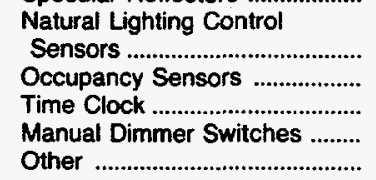 & $\begin{array}{r}74 \\
59 \\
339 \\
413 \\
78\end{array}$ & $\begin{array}{l}11 \\
14 \\
37 \\
33 \\
24\end{array}$ & $\begin{array}{l}Q \\
Q \\
Q \\
Q \\
Q\end{array}$ & $\begin{array}{r}1 \\
4 \\
6 \\
0\end{array}$ & $\begin{array}{r}9 \\
8 \\
28 \\
21 \\
15\end{array}$ & $\begin{array}{r}3,072 \\
3,629 \\
12,104 \\
12,329 \\
2,596\end{array}$ & $\begin{array}{r}681 \\
1,076 \\
1,915 \\
2,605 \\
989\end{array}$ & $\begin{array}{l}Q \\
350 \\
143 \\
Q \\
Q\end{array}$ & $\begin{array}{l}Q \\
Q \\
355 \\
756 \\
Q\end{array}$ & $\begin{array}{r}290 \\
370 \\
1,418 \\
1,737 \\
532\end{array}$ & $\begin{array}{l}31.1 \\
27.8 \\
24.8 \\
21.8 \\
29.6\end{array}$ \\
\hline \multicolumn{12}{|l|}{$\begin{array}{l}\text { Energy Conservation } \\
\text { Features (more than one may } \\
\text { apply) }\end{array}$} \\
\hline 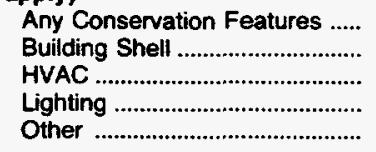 & $\begin{array}{r}4,357 \\
4,223 \\
2,604 \\
1,178 \\
264\end{array}$ & $\begin{array}{r}565 \\
539 \\
432 \\
156 \\
44\end{array}$ & $\begin{array}{r}49 \\
48 \\
34 \\
Q^{18}\end{array}$ & $\begin{array}{r}113 \\
102 \\
94 \\
33 \\
7\end{array}$ & $\begin{array}{r}404 \\
389 \\
304 \\
105 \\
33\end{array}$ & $\begin{array}{r}64,403 \\
62,056 \\
50,281 \\
29,453 \\
5,952\end{array}$ & $\begin{array}{r}14,773 \\
14,168 \\
13,276 \\
6,593 \\
1,554\end{array}$ & $\begin{array}{r}1,106 \\
1,083 \\
1,015 \\
701 \\
225\end{array}$ & $\begin{array}{r}3,731 \\
3,390 \\
3,376 \\
1,836 \\
284\end{array}$ & $\begin{array}{l}9,936 \\
9,695 \\
8,885 \\
4,057 \\
1,046\end{array}$ & $\begin{array}{r}9.3 \\
9.3 \\
10.1 \\
13.0 \\
16.4\end{array}$ \\
\hline \multicolumn{12}{|l|}{$\begin{array}{l}\text { Off-Hour Equipment } \\
\text { Reduction (more than one } \\
\text { may apply) }\end{array}$} \\
\hline $\begin{array}{l}\text { Heating } \\
\text { Cooling } \\
\text { Hot Water } \\
\text { Lighting } \\
\text { Other }\end{array}$ & $\begin{array}{r}3,400 \\
2,872 \\
578 \\
4,089 \\
547\end{array}$ & $\begin{array}{r}408 \\
321 \\
73 \\
494 \\
52\end{array}$ & $0^{29} \begin{array}{r}5 \\
33\end{array}$ & $\begin{array}{l}74 \\
58 \\
10 \\
95 \\
10\end{array}$ & $\begin{array}{r}305 \\
239 \\
58 \\
366 \\
36\end{array}$ & $\begin{array}{r}46,248 \\
42,768 \\
9,966 \\
54,944 \\
7,996\end{array}$ & $\begin{array}{r}10,572 \\
9,727 \\
2,914 \\
11,908 \\
1,767\end{array}$ & $\begin{array}{l}765 \\
758 \\
180 \\
870 \\
Q\end{array}$ & $\begin{array}{l}2,218 \\
2,083 \\
429 \\
2,546 \\
0\end{array}$ & $\begin{array}{l}7,589 \\
6,886 \\
2,306 \\
8,492 \\
1,092\end{array}$ & $\begin{array}{r}9.8 \\
10.8 \\
15.7 \\
9.4 \\
19.9\end{array}$ \\
\hline \multicolumn{12}{|l|}{$\begin{array}{l}\text { Energy Management } \\
\text { Practices (more than one may } \\
\text { apply) } \\
\text { Energy Management } \\
\text { and Control }\end{array}$} \\
\hline $\begin{array}{l}\text { System } \\
\text { Demand-Side Management }\end{array}$ & 236 & 75 & 7 & 14 & 54 & 14,320 & 4,873 & 505 & 1,413 & 2,955 & 16.5 \\
\hline 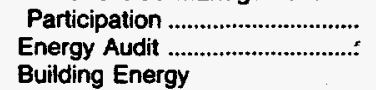 & $\begin{array}{l}315 \\
521\end{array}$ & $\begin{array}{r}86 \\
122\end{array}$ & $0^{11}$ & $\begin{array}{l}16 \\
30\end{array}$ & $\begin{array}{l}59 \\
80\end{array}$ & $\begin{array}{l}11,310 \\
14,779\end{array}$ & $\begin{array}{l}4,279 \\
4,505\end{array}$ & $\begin{array}{l}407 \\
423\end{array}$ & $\begin{array}{r}923 \\
1,328\end{array}$ & $\begin{array}{l}2,949 \\
2,755\end{array}$ & $\begin{array}{l}17.2 \\
13.9\end{array}$ \\
\hline 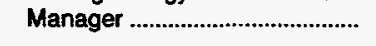 & 49 & 18 & $\mathbf{Q}$ & $Q$ & 13 & 2,311 & 777 & $\mathbf{Q}$ & 0 & 561 & 36.5 \\
\hline \multicolumn{12}{|l|}{$\begin{array}{l}\text { Demand-Side Management } \\
\text { Programs (more than one } \\
\text { may apply) }\end{array}$} \\
\hline 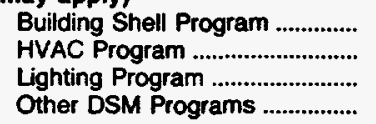 & $\begin{array}{r}36 \\
154 \\
228 \\
110\end{array}$ & $\begin{array}{l}13 \\
49 \\
62 \\
38\end{array}$ & $\begin{array}{l}Q \\
Q \\
Q \\
0\end{array}$ & $\begin{array}{r}Q \\
6 \\
14 \\
6\end{array}$ & $\begin{array}{l}\text { Q } \\
39 \\
39 \\
23\end{array}$ & $\begin{array}{l}1,079 \\
6,370 \\
8,805 \\
6,176\end{array}$ & $\begin{array}{r}367 \\
2,250 \\
3,313 \\
2,494\end{array}$ & $\begin{array}{l}Q \\
Q \\
376 \\
245\end{array}$ & $\begin{array}{l}Q \\
570 \\
730 \\
551\end{array}$ & $\begin{array}{c}Q \\
1,495 \\
2,207 \\
1,698\end{array}$ & $\begin{array}{l}37.7 \\
21.8 \\
20.6 \\
24.0\end{array}$ \\
\hline
\end{tabular}

NC $=$ No cases in sample.

$\mathrm{O}=$ Data withheld because the Relative Standard Error (RSE) was greater than 50 percent, or fewer than 20 buildings were sampled.

Notes: - To obtain a RSE percentage for any table cell, multiply the cell's corresponding RSE column and RSE row factors. - See Glossary for explanation of abbreviations and definitions of terms used in this report.

Source: Energy Information Administration, Office of Energy Markets and End Use, Form EIA-871A, "Building Questionnaire," 1992 Commercial Buildings Energy Consumption Survey. 
Dear Building Manager:

The Energy Information Administration (ElA) of the U.S. Department of Energy (DOE), in conjunction with the Office of Federal Energy Management Programs (FEMP) of DOE, is conducting a telephone survey of 900 Federal Buildings in Federal Regions 3 (Philadelphia, PA), 6 (Dallas, TX), and 9 (San Francisco, CA). This building has been statistically selected to participate in the Federal Buildings Supplemental Survey (FBSS).

The major purpose of the FBSS is to obtain more information on energy usage and characteristics of Federal buildings, to assist in the implementation of the Energy Policy Act of 1992. Starting in late July and throughout August 1994, the building energy managers for the 900 sampled Federal buildings will be contacted by telephone. The data collection will be conducted by Response Analysis Corporation under contract to EIA.

For each of the Federal regions, a survey of the sampled buildings in that region will provide basic data on the Federally-owned buildings. Two types of data will be collected: (1) building characteristics data, which includes information on how energy is managed in Federal buildings; and (2) energy consumption data. Data collected will represent other Federal commercial buildings within those three Federal regions.

Enclosed is a FBSS worksheet with several questions that may require research prior to the telephone interview: You, or another individual designated by you to answer the FBSS worksheet, may need to contact several persons to gather the required information. For instance, the building engineer may need to provide you with information on the costs and amounts of energy used. Having the FBSS worksheet completed and available when you are contacted by telephone will expedite the interview with minimum additional burden to you. Also enclosed is an exhibit booklet. You will be asked to refer to it throughout the interview; it lists response categories and items for several of the interview questions.

We realize that the building interviews may be time consuming for you and we would like to thank you in advance for you participation in this important survey. Without your cooperation, we could not obtain information about how energy is used in Federal buildings. We will be happy to provide you with a profile of your building's characteristics and energy usage after all the survey data are analyzed.

If you have any general questions about the purpose of the Federal Buildings Supplemental Survey, please contact Emilda Rivers of EIA at (202) 586-5744.

Please see the reverse side of this letter for the authorizing legislation and public reporting burden. Thank you for your time and effort.

Sincerely,

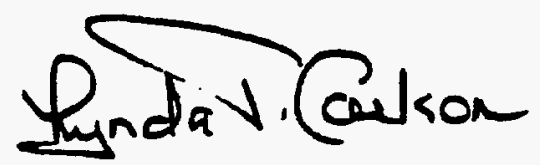

Lynda T. Carlson, Director

Energy End Use \& Integrated Statistics Division

Energy Information Administration

Enclosures 


\section{Authorizing Legislation:}

Authorization for collecting energy consumption data in the Commercial sector is set forth in the Federal Energy Administration Act of 1974; Public Law 93-275, as amended. The Commercial Buildings Energy Consumption Survey, Buildings Questionnaire (Federal Supplement) conducted by the Energy Information Administration collects information about energy usage in U.S. buildings.

\section{Public Reporting Burden:}

Public reporting burden for this collection of information via telephone is estimated to average 30 minutes.

Send comments regarding public reporting burden or any other aspect of this collection of information, including suggestions for reducing this burden, to:

Energy Information Administration

Office of Statistical Standards

EI-73

1000 Independence Avenue, S.W.

Washington, DC 20585

and

Office of Information and Regulatory Affairs

Office of Management and Budget

Washington, DC 20503 


\section{Letter 4 Enclosures}

\section{FEDERAL BUILDINGS SUPPLEMENTAL SURVEY WORKSHEET}

Please have the following information available for the telephone survey. Thank you for your help!
A. Building square footage?
B. Year major portion of construction completed?
C. Number of workers (all shifts)?
sq. ft.
year
workers

D. Energy Consumption

For fiscal year 1993 (October 1, 1992 through September 30, 1993), provide the amount of usage, units, and costs for each fuel used in the building. Complete last column only for those energy sources, if any, where your building shares a meter with other buildings.

\begin{tabular}{|c|c|c|c|c|}
\hline & Cost & Amount & $\begin{array}{c}\text { Units } \\
\text { (circle one } \\
\text { in each row) }\end{array}$ & $\begin{array}{c}\text { Total } \\
\text { square } \mathrm{ft} . \\
\text { of all buildings } \\
\text { sharing } \\
\text { same meter }\end{array}$ \\
\hline Electricity & $\$$ & & Btu's or Kwh & \\
\hline Natural Gas & $\$$ & & Btu's or $\mathrm{Cu} \mathrm{ft}$. & \\
\hline $\begin{array}{l}\text { Fuel Oil/ } \\
\text { Kerosene/Diesel }\end{array}$ & $\$$ & & Btu's or Gallons & \\
\hline District Steam & $\$$ & & Btu's & \\
\hline District Hot Water & $\Phi$ & & Btu's & \\
\hline District Chilled Water & $\$$ & 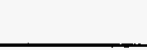 & Ton-hours & \\
\hline
\end{tabular}

"If your building shares a meter with other buildings, please try to obtain the total square footage, usage, units, and cost information (section D) for all the buildings served by the shared meter.

\section{E. Motors}

1. Number and age of motors ( 10 horsepower or more) that are in each of the following equipment:

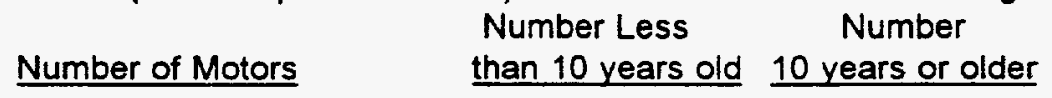

\begin{abstract}
than 10 years old 10 years or older
\end{abstract}
Chillers

Heat Pumps

Fans

Compressors

Elevators

Escalators

Refrigeration

Water Pumps

2. Number of energy-efficient motors ( 10 horsepower or more) less than 10 years old.

3. Number of motors ( 10 horsepower or more) less than 10 years old that have been rewound.

3a. (IF NON-ZERO NUMBER IN QUESTION 3): Average age of these motors that were rewound? 


\section{Department of Energy}

Washington, DC 20585

August 26, 1994

\section{Dear FBSS Respondent:}

Thank you for participating in the Federal Buildings Supplemental Survey (FBSS) that was conducted by the Energy Information Administration (EIA) of the U.S. Department of Energy (DOE), in conjunction with the Office of Federal Energy Management Programs (FEMP) of DOE. The information that you supplied will provide a baseline in Federal regions 3, 6, and 9 to measure future energy use in Federal buildings in those regions. Response Analysis Corporation, under contract to EIA, collected the information for DOE.

We really appreciate your time taken to help us in the Federal buildings survey.

Sincerely,

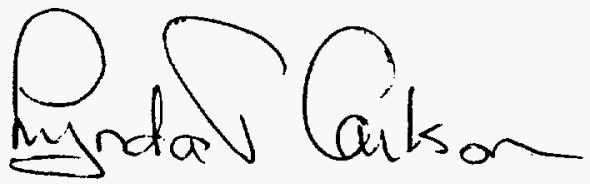

Lynda T. Carlson, Director

Energy End Use and Integrated

Statistics Division

Energy Information Administration 

Appendix F

Related EIA Energy

Consumption Publications 



\section{Appendix F}

\section{Related EIA Energy Consumption Publications}

For information about how to obtain these publications, see the inside cover of this report. Please note that the prices quoted here are subject to change.

In addition to the reports listed below, public use data tapes and data diskettes for the residential, residential transportation, and commercial sectors are available from the National Technical Information Service (NTIS). To obtain information on how to order the tapes/diskettes, you may call NTIS at 703-487-4807, FAX number 703-321-8547. Data diskettes can also be obtained from the Office of Scientific and Technical Information (OSTI). For OSTI ordering information, call 615-576-8401.

\section{Commercial Sector}

Note: The name of the Nonresidential Buildings Energy Consumption Survey was changed to the Commercial Buildings Energy Consumption Survey, beginning with the 1989 survey. The survey name was also dropped from the report title at that time and subsequently.

\section{Characteristics of Buildings}

Commercial Buildings Characteristics 1992; April 1994, DOE/EIA-0246(92), GPO Stock No. 061-003-00850-0, $\$ 28.00$.

"Commercial Buildings Characteristics 1992," Monthly Energy Review, January 1994, DOE/EIA-0035(94/01).

Commercial Buildings Characteristics 1989; June 1991, DOE/EIA-0246(89), GPO Stock No. 061-003-00699-0, \$18.00.

Nonresidential Buildings Energy Consumption Survey: Characteristics of Commercial Buildings, 1986; September 1988, DOE/EIA-0246(86), GPO Stock No. 061-003-00580-2, \$16.00.

Nonresidential Buildings Energy Consumption Survey: Characteristics of Commercial Buildings, 1983; A Supplemental Reference, DOE/EIA-M008, \$22.95. Available from the NTIS, Order No. DE-85015581.

Nonresidential Buildings Energy Consumption Survey: Characteristics of Commercial Buildings, 1983; July 1985, DOE/EIA-0246(83), GPO Stock No. 061-003-00439-3, \$7.50.

Nonresidential Buildings Energy Consumption Survey: Fuel Characteristics and Conservation Practices; June 1981, DOE/EIA-0278, GPO Stock No. 061-00300200-5, \$9.00.

Nonresidential Buildings Energy Consumption Survey: Building Characteristics; March 1981, DOE/EIA-0246, GPO Stock No. 061-003-00171-8, \$6.50.

\section{Consumption and Expenditures}

Commercial Buildings Consumption and Expenditures 1992; April 1995, DOE/EIA-0318(92), GPO Stock No. 061-00300904-2, \$31.00. 
Commercial Buildings Consumption and Expenditures 1989; April 1992, DOE/EIA-0318(89), GPO Stock No. 061-00300753-8, \$25.00.

Nonresidential Buildings Energy Consumption Survey: Commercial Buildings Consumption and Expenditures 1986; May 1989, DOE/EIA-0318(86), GPO Stock No. 061-003-00613-2, \$19.00.

Nonresidential Buildings Energy Consumption Survey: Commercial Buildings, Consumption and Expenditures 1983; September 1986, DOE/EIA-0318(83), GPO Stock No. 061-003-00496-2, \$13.00.

Nonresidential Buildings Energy Consumption Survey: 1979 Consumption and Expenditures, Part 1: Natural Gas and Electricity; March 1983, DOE/EIA-0318/1, GPO Stock No. 061-003-00298-6, \$9.50.

Nonresidential Buildings Energy Consumption Survey: 1979 Consumption and Expenditures, Part 2: Steam, Coal, Fuel Oil, LPG, and Total Fuels; December 1983, DOE/EIA-0318(79)/2, GPO Stock No. 061-003-00366-4, \$6.00.

\section{Other Publications on the Commercial Sector}

Energy Consumption Series-- Energy End-Use Intensities in Commercial Buildings, September 1994, DOE/EIA0555(94)/2, GPO Stock No. 061-003-0087-9, 9.00.

"Assessment of Energy Use in Multibuilding Facilities," Monthly Energy Review, December 1993, DOE/EIA0035(93/12).

Energy Consumption Series--Assessment of Energy Use in Multibuilding Facilities, August 1993, DOE/EIA-0555(93)/1, GPO Stock No. 061-003-00817-8, \$7.50.

Energy Consumption Series--User-Needs Study for the 1992 Commercial Buildings Energy Consumption Survey, September 1992, DOE/EIA-0555(92)/4, GPO Stock No. 061-003-00770-8, $\$ 8.50$.

Energy Consumption Series--Lighting in Commercial Buildings; March 1992, DOE/EIA-0555(92)/1, GPO Stock No. 061-003-00749-0, \$6.50.

\section{Industrial Sector}

Changes in Energy Intensity in the Manufacturing Sector 1985-1991, October 1995, DOE/EIA-0552(85-91), GPO Stock No, 061-003-00925-5, $\$ 9.00$

Manufacturing Consumption of Energy 1991, December 1994, DOE/EIA-0512(91), GPO Stock No. 061-003-008709, $\$ 34.00$.

"Energy Preview: Manufacturing Energy Consumption Survey Preliminary Estimates, 1991," Monthly Energy Review, September 1993, DOE/EIA-0035(93/01).

"Energy Efficiency in the Manufacturing Sector," Monthly Energy Review (Article), p.1, December 1992.

Manufacturing Energy Consumption Survey: Changes in Energy Intensity in the Manufacturing Sector 1980-1988, December 1991, DOE/EIA-0552(80-88), GPO Stock No. 061-003-00734-1, \$4.75.

Manufacturing Energy Consumption Survey: Manufacturing Fuel-Switching Capability 1988; September 1991, DOE/EIA-0515(88), GPO Stock No. 061-003-00720-1, \$9.00.

Manufacturing Energy Consumption Survey: Consumption of Energy, 1988; May 1991, DOE/EIA- 0512(88), GPO Stock No. 061-003-00703-8, \$11.00. 
Manufacturing Energy Consumption Survey: Energy Efficiency in Manufacturing, 1985; January 1990, DOE/EIA0516(85), GPO Stock No. 061-003-00650-7, \$4.25.

Manufacturing Energy Consumption Survey: Fuel-Switching Capability, 1985; December 1988, DOE/EIA-0515(85), GPO Stock No. 061-003-00601-9, \$3.50.

Manufacturing Energy Consumption Survey: Methodological Report, 1985; November 1988, DOE/EIA0514(85), GPO Stock No. 061-003-00595-1, \$6.00.

Manufacturing Energy Consumption Survey: Consumption of Energy, 1985; November 1988, DOE/EIA-0512(85), GPO Stock No. 061-003-00594-2, \$6.00.

"Manufacturing Sector Energy Consumption 1985 Provisional Estimates," Monthly Energy Review (Article), pp. vii-x, January 1987, DOE/EIA-0035(87/01).

Report on the 1980 Manufacturing Industries' Energy Consumption Study and Survey of Large Combustors; February 1983, DOE/EIA-0358, GPO Stock No. 061-003-00293-5, \$5.00.

Industrial Energy Consumption, Survey of Large Combustors: Report on Alternate Fuel-Burning Capabilities of Large Boilers in 1979; February 1982, DOE/EIA-0304, GPO Stock No. 061-003-0233-1, \$2.50.

Methodological Report of the 1980 Manufacturing Industries Survey of Large Combustors (EIA-463); March 1982, DOE/EIA-0306 (no GPO Stock No.).

\section{Other Publications on the Industrial Sector}

Energy Consumption Series--Derived Annual Estimates of Manufacturing Energy Consumption 1974-1988, August 1992, DOE/EIA-0555(92)/3, GPO Stock No. 061-003-00766-0, \$7.00.

Energy Consumption Series--Development of the 1991 Manufacturing Energy Consumption Survey, May 1992, DOE/EIA-0555(92)/2, GPO Stock No. 061-003-00757-1, \$5.50.

\section{Residential Sector}

\section{Housing Characteristics}

Note: The survey name was dropped from the beginning of the report title starting with the 1987 data reports.

Housing Characteristics 1993; June 1995, DOE/EIA-0314(93), GPO Stock No. 061-003-00912-3, \$23.00.

Housing Characteristics 1990; May 1992, DOE/EIA-0314(90), GPO Stock No. 061-003-00754-6, \$23.00.

Housing Characteristics 1987; May 1989, DOE/EIA-0314(87), GPO Stock No. 061-003-00619-1, \$13.00.

Residential Energy Consumption Survey: Housing Characteristics 1984; October 1986, DOE/EIA-0314(84), GPO Stock No. 061-003-00499-7, \$12.00.

Residential Energy Consumption Survey: Housing Characteristics, 1982; August 1984, DOE/EIA-0314(82), GPO Stock No. 061-003-00393-1, \$7.00. 
Residential Energy Consumption Survey Housing Characteristics, 1981; August 1983, DOE/EIA-0314(81), GPO Stock No. 061-003-00330-3, \$6.50.

Residential Energy Consumption Survey: Housing Characteristics, 1980; June 1982, DOE/EIA-0314, GPO Stock No. 061-003-00256-1, \$11.00.

Residential Energy Consumption Survey: Characteristics of the Housing Stock and Households, 1978; February 1980, DOE/EIA-0207/2, GPO Stock No. 061-003-00093-2, \$4.25.

Residential Energy Consumption Survey: Conservation; February 1980, DOE/EIA-0207/3, GPO Stock No. 061-003-00087-8, \$6.00.

Preliminary Conservation Tables from the National Interim Energy Consumption Survey; August 1979, DOE/EIA-0193/P (no GPO Stock No.).

Characteristics of the Housing Stock and Households: Preliminary Findings from the National Interim Energy Consumption Survey; October 1979, DOE/EIA-0199/P (no GPO Stock No. available).

\section{Consumption and Expenditures}

Note: The survey name was dropped from the beginning of the report title starting with the 1987 data reports. The titles were changed to Household Energy Consumption and Expenditures 1987, Part 1: National and Part 2: Regional.

Household Energy Consumption and Expenditures 1993, October 1995, DOE/EIA-0321(93), GPO Stock No. 061-003$00932-8, \$ 21.00$.

"Household Energy Consumption and Expenditures 1990," Monthly Energy Review, August 1993, DOE/EIA0035(93/08).

Household Energy Consumption and Expenditures 1990; February 1993, DOE/EIA-0321/1(90), GPO Stock No. 061003-00795-3, \$22.00.

Household Energy Consumption and Expenditures 19901S; February 1993, DOE/EIA-0321/2(90), GPO Stock No. 061003-00796-1, \$21.00.

Household Energy Consumption and Expenditures 1987, Part 1: National Data; October 1989, DOE/EIA-0321/1(87), GPO Stock No. 061-003-00635-3, \$15.00. Note: Energy end-use data are included in this report.

Household Energy Consumption and Expenditures 1987, Part 2: Regional Data; DOE/EIA-0321/2(87) (no GPO Stock No. available), $\$ 16.00$.

Residential Energy Consumption Survey: Consumption and Expenditures, April 1984 Through March 1985, Part 1: National Data; March 1987, DOE/EIA-0321/1(84), GPO Stock No. 061-003-00519-5, \$9.50.

Residential Energy Consumption Survey: Consumption and Expenditures, April 1984 Through March 1985, Part 2: Regional Data; May 1987, DOE/EIA-0321/2(84), GPO Stock No. 061-003-00528-4, \$17.00. Note: Energy end-use data are included in this report.

Residential Energy Consumption Survey: Consumption and Expenditures, April 1982 Through March 1983, Part 1: National Data; November 1984, DOE/EIA-0321/1(82), GPO Stock No. 061-003-00411-3, \$7.00.

Residential Energy Consumption Survey: Consumption and Expenditures, April 1982 Through March 1983, Part 2: Regional Data; December 1984, DOE/EIA-0321/2(82), GPO Stock No. 061-003-00414-8, \$9.50. 
Residential Energy Consumption Survey: Consumption and Expenditures, April 1981 Through March 1982, Part 1: National Data; September 1983, DOE/EIA-0321/1(81), GPO Stock No. 061-003-00340-1, \$6.00.

Residential Energy Consumption Survey: Consumption and Expenditures, April 1981 Through March 1982, Part 2: Regional Data; October 1983, DOE/EIA-0321/2(81), GPO Stock No. 061-003-00357-5, \$8.00.

Residential Energy Consumption Survey: Consumption and Expenditures, April 1980 Through March 1981, Part 1: National Data; September 1982, DOE/EIA-0321/1(80), GPO Stock No. 061-003-00278-1, \$7.50.

Residential Energy Consumption Survey: Consumption and Expenditures, April 1980 Through March 1981, Part 2: Regional Data; June 1983, DOE/EIA-0321/2(80), GPO Stock No. 061-003-00319-2, \$7.00.

Residential Energy Consumption Survey: 1979-1980 Consumption and Expenditures, Part 1: National Data (Including Conservation); April 1981, DOE/EIA-0262/1, GPO Stock No. 061-003-00191-2, \$6.50.

Residential Energy Consumption Survey: 1979-1980 Consumption and Expenditures, Part II: Regional Data; May 1981, DOE/EIA-0262/2, GPO Stock No. 061-003-00189-1, \$8.50.

Residential Energy Consumption Survey: Consumption and Expenditures, April 1978 Through March 1979; July 1980, DOE/EIA-0207/5, GPO Stock No. 061-003-00131-9, \$7.50.

Single-Family Households: Fuel Oil Inventories and Expenditures: National Interim Energy Consumption Survey; December 1979, DOE/EIA-0207/1, GPO Stock No. 061-003-00075-4, \$3.50.

\section{Other Publications on the Residential Sector}

Energy Consumption Series--Sample Design for the Residential Energy Consumption Survey, August 1994, DOE/EIA0555(94)/1, GPO Stock No. 061-003-00865-8, \$6.50.

Energy Consumption Series--User-Needs Study of the 1993 Residential Energy Consumption Survey, September 1993, DOE/EIA-0555(93)/2, GPO Stock No. 061-003-00819-4, \$13.00.

"End-Use Consumption of Residential Energy," Monthly Energy Review (Article), pp. vii-xiv, July 1987, DOE/EIA-0035(87/07).

Residential Energy Consumption Survey: Trends in Consumption and Expenditures 1978-1984 June 1987, DOE/EIA-0482, GPO Stock No. 061-003-00535-7, \$12.00.

Residential Conservation Measures; July 1986, SR/EEUD/86/01 (no GPO Stock No.).

An Economic Evaluation of Energy Conservation and Renewable Energy Tax Credits; October 1985, Service Report (no GPO Stock No.).

Residential Energy Consumption and Expenditures by End Use for 1978, 1980, and 1981; December 1984, DOE/EIA-0458, GPO Stock No. 061-003-00415-6, \$4.50.

Weatherization Program Evaluation, SR-EEUD- 84-1; August 1984 (available from the Office of the Assistant Secretary for Conservation and Renewable Energy, Department of Energy). 
Residential Energy Consumption Survey: Regression Analysis of Energy Consumption by End Use; October 1983, DOE/EIA-0431, GPO Stock No. 061-00300-347-8, \$5.00.

National Interim Energy Consumption Survey: Exploring the Variability In Energy Consumption; July 1981, DOE/EIA-0272, GPO Stock No. 061-003-00205-6, \$5.00.

National Interim Energy Consumption Survey: Exploring the Variability in Energy Consumption--A Supplement; October 1981, DOE/EIA-0272/S, GPO Stock No. 061-003-00217-0, \$4.50.

Energy Use by U.S. Households; November 1980, DOE/EIA-0248 (brochure, no GPO Stock No.).

\section{Residential Transportation Sector}

Note: The survey name was dropped from the beginning of the report title starting with the 1988 data report, and the report title was changed to Household Vehicles Energy Consumption 1988.

Household Vehicles Energy Consumption 1991; December 1993, DOE/EIA-0464(91), GPO Stock No. 061-003-00652$3, \$ 14.00$.

"Energy Preview: Residential Transportation Energy Consumption Survey Preliminary Estimates, 1991," Monthly Energy Review, January 1993, DOE/EIA-0035(93/01).

Household Vehicles Energy Consumption 1988; February 1990, DOE/EIA-0464(88), GPO Stock No. 061-003-00652-3, $\$ 11.00$.

Residential Transportation Energy Consumption Survey: Consumption Patterns of Household Vehicles 1985; April 1987, DOE/EIA-0464(85), GPO Stock No. 061-003-00521-7, \$8.50.

Residential Transportation Energy Consumption Survey: Consumption Patterns of Household Vehicles, 1983; January 1985, DOE/EIA-0464(83), GPO Stock No. 061-003-00420-2, \$4.50.

Residential Energy Consumption Survey: Consumption Patterns of Household Vehicles, Supplement:

January 1981 to September 1981; February 1983, DOE/EIA-0328, GPO Stock No. 061-003-00297-8, \$4.75.

Residential Energy Consumption Survey: Consumption Patterns of Household Vehicles, June 1979 to December 1980; April 1982, DOE/EIA-0319 (no GPO Stock No.).

\section{Cross-Sector}

Energy Consumption Series-Measuring Energy Efficiency in the United States' Economy: A Beginning; October 1995, DOE/EIA-0555(95)/2, GPO Stock No. 061-003-00935-2, \$6.50.

Energy Consumption Series--Buildings and Energy In the 1980's; June 1995, DOE/EIA-0555(95)/1, GPO Stock No. 061-003-00914-0, \$6.50.

Energy Consumption by End-Use Sector: A Comparison of Measures by Consumption and Supply Surveys; April 6, 1990, DOE/EIA-0533 (no GPO Stock No. available), \$2.50.

Natural Gas: Use and Expenditures; April 1983, DOE/EIA-0382, GPO Stock No. 061-003-00307-9, \$5.50. 


\section{Public Use Tapes}

Note: All tapes are available through the NTIS.

\section{Residential and Residential Transportation Sectors}

Residential Energy Consumption Survey: 1987 and Residential Transportation Energy Consumption Survey, 1988, Order No. PB90-501461, \$220.

Residential Energy Consumption Survey: 1984 and Residential Transportation Energy Consumption Survey, 1985; Order No. PB87-186540, \$220.

Residential Energy Consumption Survey: 1982 and Residential Transportation Energy Consumption Survey, 1983; Order No. PB85-221760, $\$ 220$.

Residential Energy Consumption Survey: Consumption and Expenditures, 1980-1981; Monthly Billing Data; Order No. PB84-166230, \$220.

Residential Energy Consumption Survey: Housing Characteristics, 1981; Consumption and Expenditures, 1981-1982; Monthly Billing Data; Order No. PB84-120476, \$220.

Residential Energy Consumption Survey: Housing Characteristics, Annualized Consumption and Expenditures, 1980-1981; Order No. PB83-199554, \$220.

Residential Energy Consumption Survey: Household Transportation Panel Monthly Gas Purchases and Vehicle and Household Characteristics, 6/79-9/81; Order No. PB84-162452, \$220.

Residential Energy Consumption Survey: Household Screener Survey, 1979-1980; Order No. PB82-114877, \$220.

Residential Energy Consumption Survey: Household Monthly Energy Consumption and Expenditures, 1978-1979; Order No. PB82-114901, \$220.

National Interim Energy Consumption Survey (Residential), 1978; Order No. PB81-108714, \$220.

\section{Commercial Sector}

Nonresidential Buildings Energy Consumption Survey: 1986 Data; Order No. PB90-500034, \$220.

Nonresidential Buildings Energy Consumption Survey: 1979 and 1983 Data; Order No. PB88-245162, \$220.

\section{Public Use Diskettes}

Note: Diskettes are available through the Office of Scientific and Technical Information (OSTI) and NTIS.

Commercial Buildings Energy Consumption and Expenditures 1992, OSTI - ASCII or dBase format, order by title, $\$ 10$ per diskette, $\$ 40 /$ set of four. NTIS - ASCII or dBase format, order by title, call for prices. 
Commercial Buildings Characteristics 1992 , OSTI - ASCII or dBase format, order by title, $\$ 10$ per diskette, $\$ 40 /$ set of four. NTIS - ASCII or dBase format: order No. PB-94-504305, call for prices.

Residential Energy Consumption Survey 1993 Data, OSTI - ASCII or dBase format, Order by title; $\$ 10$ per diskette. NTIS - ASCII format: No. Stock No. Assigned yet, Order by title.

Residential Energy Consumption Survey 1990 Data, OSTI - ASCII (3 diskettes) or dBASE (2 diskettes) format, order by title, $\$ 10$ per diskette. NTIS - ASCII format: Order No. PB-93-506103 and dBASE format: Order No. PB-93-506095.

Residential Energy Consumption Survey 1987 Data, OSTI - ASCII or dBASE format, order by title, $\$ 45$ for each set. NTIS - ASCII format: Order No. PB-91-505115, \$130, and DBASE format: Order No. PB-91-505107, \$130.

Commercial Buildings Energy Consumption Survey 1992 Data, OSTI - ASCII or dBASE format, order by title, $\$ 10$ per diskette, $\$ 40$ set of four. NTIS - ASCII or dBASE format, order by title, call for prices.

Commercial Buildings Energy Consumption Survey 1989 Data, OSTI - ASCII format, order by title, $\$ 10$ per diskette, $\$ 40$ set of four. NTIS - ASCII or dBASE format: Order No. PB92-504232, \$140.

Nonresidential Buildings Energy Consumption Survey 1986 Data, NTIS - ASCII format: Order No. PB91-506808, $\$ 130$.

Residential Transportation Energy Consumption Survey 1991 Data, OSTI - ASCII or dBASE format, order by title, call for prices. NTIS - ASCII format: Order No. PB94-500824. dBASE format: Order No. PB94-500816, call for prices.

Residential Transportation Energy Consumption Survey 1988 Data, GPO - ASCII or dBASE format, order by title, $\$ 15$ for each set. NTIS - ASCII format: Order No. PB91-507269, dBASE format: Order No. PB91-507277, \$50 each.

\section{Planned Publications}

EPACT Section 407 Data System: Results from Atlanta Clean City Fleet Vehicle Survey, planned for November 1995.

EPACT Section 407 Data Program: The Vehicle Stock and New Survey Findings, planned for December 1995.

Note: The Energy Information Administration also publishes annually the State Energy Data Report, Consumption Estimates, DOE/EIA-0214, the State Energy Price and Expenditures Report, DOE/EIA-0376; and the Monthly Energy Review, DOE/EIA-0035. These reports contain annual and monthly consumption information derived from EIA supply surveys. 


\section{Glossary}

Air-Conditioning: See Cooling.

Air-Handling Units: A method for channeling warm or cool air to different parts of a building. The process of moving the conditioned air often involves drawing air over heating or cooling coils and forcing it from a central location through ducts or air-handling units. Air-handling units are hidden in the walls or ceilings, where they use steam or hot water to heat or chilled water to cool the air inside the duct work. In the "Detailed Tables," air-handling units are included in "Ducts for Heating" in the "Heating Distribution Equipment" stub and in "Ducts for Cooling" in the "Cooling Distribution Equipment" stub. (See Cooling, Duct, and Space Heating.)

Alternative Rate Program Assistance: A type of assistance that offers special rate structures or discounts on the consumer's monthly electric bill in exchange for participation in programs aimed at cutting peak demands or changing load shape. These rates are intended to reduce consumer bills and shift hours of operation of equipment from on-peak to off-peak periods through the application of time-differentiated rates. For example, utilities often pay consumers several dollars a month (refund on their monthly electric bill) for participation in a load control program. Large commercial and industrial consumers sometimes obtain interruptible rates, which provide a discount in return for the consumer"s agreement to cut electrical loads upon request from the utility (usually during critical periods, such as summer afternoons when the system demand approaches the utility"s generating capability). (See Energy Conservation Program Assistance, Energy Conservation Programs, and Retrofit or Purchase of Any Equipment.)

Asphalt or Fiberglass Shingles: See Shingles.

Barrel: A volumetric unit of measure for crude oil and petroleum products equivalent to 42 U.S. gallons. (See Gallon.)

Baseboard: As a type of heating distribution equipment, a system in which either electric resistance coils or finned tubes carrying steam or hot water are mounted behind shallow panels along baseboards. Baseboards rely on passive convection to distribute heated air in the space. Electric baseboards are an example of an Individual Space Heater. (See Electric Baseboard and Individual Space Heater.)

Boiler: A type of space-heating equipment consisting of a vessel or tank where heat produced from the combustion of fuels such as natural gas, fuel oil, or coal is used to generate hot water or steam. Many buildings have their own boilers, while other buildings have steam or hot water piped in from a central plant. For this survey, only boilers inside the building (or serving only that particular building) are counted as part of the building"s heating system. Steam or hot water piped into a building from a central plant is considered district heat. (See Furnace, Heating, Ventilation, and Air-Conditioning (HVAC), and District Heat.)

Bottled Gas: See Liquefied Petroleum Gas (LPG) and Propane.

British Thermal Unit: See Btu (British Thermal Unit).

Btu (British Thermal Unit): A unit of energy consumed by or delivered to a building. A Btu is defined as the amount of energy required to increase the temperature of 1 pound of water by 1 degree Fahrenheit, at normal atmospheric pressure. Energy consumption is expressed in Btu in this report to allow for consumption comparisons among fuels that are measured in different units. (See Btu Conversion Factors and Metric Conversion Factors.) 
Btu Conversion Factors: The Btu conversion factors used for this survey are as follows:

\begin{tabular}{lcl}
\hline & Btu Equivalent & Unit \\
\hline Electricity & 3,412 & Kilowatthour \\
Natural Gas & 1,028 & cubic foot \\
Distillate Fuel Oils (Nos. 1,2, and 4) & 5.825 million & per Barrel \\
Residual Fuel Oils (Nos. 5 and 6) & 6.287 million & per Barrel \\
Kerosene & 5.670 million & per Barrel \\
District Heat (Steam and Hot Water) & 1,000 & pound \\
\hline Note: Btu of district hot water have been converted into equivalent pounds of steam using the conversion 1,000 Btu hot water = 1 pound steam. \\
Sources: Energy Information Administration, Monthly Energy Review (August 1995), pp. 145, 147, 149 for electricity, natural gas, distillate, \\
residual, and kerosene; and Methodological Issues In the Nonresidential Buildings Energy Consumption Survey (September 1983), pp. 173-175 \\
for district steam.
\end{tabular}

Building: In this survey, a structure totally enclosed by walls extending from the foundation to the roof, containing over 10,000 square feet of floorspace, and intended for human occupancy. Structures that were included in the survey as a specific exception were parking garages not totally enclosed by walls and a roof, as well as structures erected on pillars to elevate the first fully enclosed level, but leaving the sides at ground level open.

Excluded from the survey as nonbuildings were the following: structures (other than the exceptions just noted) that were not totally enclosed by walls and a roof (such as oil refineries, steel mills, and water towers); street lights, pumps, billboards, bridges, swimming pools, and construction sites; mobile homes and trailers, even if they housed commercial activity; and oil storage tanks. (See Commercial Building and Nonresidential Building.)

Building Envelope or Shell Energy Conservation Program: An energy conservation program that promotes reduction of energy consumption through improvements to the building envelope. Includes installation of insulation, weatherstripping, caulking, window film, and window replacement. (See Building Shell (Envelope).)

\section{Building Floorspace: See Floorspace.}

Building Shell Conservation Features: Building features designed to reduce the energy loss or gain through the shell or envelope of the building. In the "Detailed Tables," this category includes roof, ceiling or wall insulation; storm windows or double- or triple-paned glass (multiple glazing); tinted or reflective glass or shading films; and exterior or interior shadings or awnings. This category does not include participation in a building envelope or shell energy conservation program. (See Roof or Ceiling Insulation, Wall Insulation, Storm Windows, Storm Doors, Storm or Multiple Glazing, Tinted or Reflective Glass or Shading Film, and Exterior or Interior Shadings or Awnings.)

Building Shell (Envelope): The thermal envelope of the building, that is, the roof, exterior walls, and bottom floors that enclose conditioned space through which thermal energy may be transferred to or from the exterior.

Built-Up Roof: A roof covering consisting of several successive layers (each of which is called a ply), usually of roofing felt, with mopping of hot asphalt between layers and topped by a mineral-surfaced layer or by gravel embedded in a heavy coat of asphalt.

\section{Campus or Complex: See Multibuilding Facility.}

Cases or Cabinets: Refrigeration in cabinets (units) without covers or with flexible covers made of plastic or some other material, hung in strips or curtains (fringed material, usually plastic, that push aside like a bead curtain). Flexible covers stop the flow of warm air into the refrigerated space. (See Commercial Refrigeration/Freezer Equipment.)

CATI: See Computer-Assisted Telephone Interviewing (CATI). 
Central Chiller: Any centrally located air-conditioning system that produces chilled water in order to cool air. The chilled water or cold air is then distributed throughout the building using pipes or air ducts, or both. These systems are also commonly known as "chillers," "centrifugal chillers," "reciprocating chillers" or "absorption chillers." Chillers are generally located in, or just outside, the building they serve. Chillers located at central plants are included under district chilled water. (See Cooling, District Chilled Water, Central Physical Plant, and Heating, Ventilation, and AirConditioning (HVAC).)

Central Cooling: Cooling of an entire building with a refrigeration unit to condition the air. Typically, central chillers and ductwork are present in a centrally cooled building. (See Cooling.)

Central Physical Plant: A plant that is owned by, and on the grounds of, a multibuilding facility that provides district heating, district cooling, or electricity to one or more buildings on the same facility. The central physical plant may be by itself in a separate building or may be located in a building where other activities occur. (See Multibuilding Facility, District Heat, or District Chilled Water.)

Centralized Water-Heating System: Equipment to heat and store water for purposes other than space heating,which provides hot water from a single location for distribution throughout a building. A residential-type tank water heater is a good example of a centralized water heater. (See Water-Heating Equipment and Distributed/Point-of- Use Water-Heating System.)

\section{Chiller: See Central Chiller.}

Coal: A black or brownish-black solid, combustible substance formed by the partial decomposition of vegetable matter without access to air. In this report, the term includes anthracite, bituminous and subbituminous coal, as well as the derivative of coal (formed by destructive distillation or imperfect combustion) known as coke. This survey determined if coal was used in the commercial building but did not collect consumption and expenditure data on the use of coal as an energy source. In this report, coal is included in the "Any Other" category for the energy sources, main space-heating energy sources, and space-heating energy sources categories. (See Energy Source.)

Commercial Building: A building with more than 50 percent of its floorspace used for commercial activities. Commercial buildings include, but are not limited to, stores, offices, schools, churches, gymnasiums, libraries, museums, hospitals, clinics, warehouses, and jails. Agricultural buildings, residences, and manufacturing buildings were excluded from the survey. For a more complete list of buildings in the survey, see Appendix B, "Types of Buildings."

Commercial Refrigeration/Freezer Equipment: These include: commercial refrigeration/freezer units for the sale or storage of perishable materials; residential-type refrigerators/freezers; water coolers; or any other refrigeration equipment, excluding air conditioning. Freezers are designed to keep their contents below the freezing point ( 32 degrees Fahrenheit) and refrigeration equipment is designed to maintain the stored items below room temperature, but above the freezing point. In this report, data are collected on refrigeration/freezer equipment inside and/or adjacent to the building. (See Cases and Cabinets and Walk-in Refrigeration Units.)

Compact Fluorescent Light Bulbs: Designed to replace screw-in incandescent light bulbs, they are often found in table lamps, wall sconces and hall and ceiling fixtures of commercial buildings with residential type lights. They combine the efficiency of fluorescent lighting with the convenience of standard incandescent bulbs. Light is produced the same way as other fluorescent lamps. Compact fluorescent bulbs have either electronic or magnetic ballasts. (See Light Bulbs and Fluorescent Light Bulbs).

Computer-Assisted Telephone Interviewing (CATI): A computer-assisted survey process that uses the telephone for voice communications between the interviewer and the respondent. This mode of data collection was used for the 1993 Federal Buildings Supplemental Survey (FBSS).

Concrete Panel: A wall construction panel made of concrete, which is either prefabricated in a factory or poured at the site and then hoisted onto the structure. (See Precast Concrete Panel.) 
Concrete Roof: A poured concrete roof, often intended to bear the load of a parking garage that occupies the roof area of a building.

Conditional Energy Intensity: Total consumption of a particular energy source(s) or fuel(s) divided by the total floorspace of buildings that use the energy source(s) or fuel(s), i.e., the ratio of consumption to energy source-specific floorspace. This measure is used in the fuel-specific tables in the "Detailed Tables." (See Energy Source-Specific Floorspace.)

Confidence Interval: A range that is estimated to include the population value at a given confidence level, usually 95 percent. The range is calculated from the sample data. The confidence level is the expected fraction of such confidence intervals that actually do include the corresponding, unknown population value.

Conservation Features: A feature in the building designed to reduce the usage of energy. (See Building Shell Conservation Features, HVAC Conservation Features, and Lighting Conservation Features.)

Consumption: The amount of energy used in, or delivered to, a building during a given period of time. For this report, unless otherwise noted, all consumption statistics are site energy consumption, which includes electric utility sales to commercial buildings but excludes electrical system and district heat energy losses. Statistics for this report are presented on an annual basis for the 365-day period of fiscal year 1993 (October 1, 1992 through September 30, 1993). Site consumption is the amount of energy delivered to the site (building); no adjustment is made for the fuels consumed to produce electricity or district sources. Site consumption is also referred to as net energy. However, primary consumption is the amount of site consumption plus losses that occur in the electricity generation process.

Data on energy consumption were not collected by end uses separately. For example, although it might be known that electricity was used in some buildings for heating, the consumption of electricity reported for those buildings would typically include other uses of electricity as well (such as lighting and water heating). (See Btu, Conversion Losses, and Expenditures.)

Consumption per Square Foot: The aggregate ratio of total consumption for a particular set of buildings to the total floorspace of those buildings. (See Consumption, Energy Intensity, and Floorspace.)

Consumption per Worker: The aggregate ratio of total consumption to total number of workers. (See Consumption and Workers.)

Continuous-Delivery Energy Sources: Those energy sources provided continuously to a building. In this report, continuous delivery energy sources are electricity, natural gas, and district heating and cooling. (See Energy Source and Discrete-Delivery Energy Sources.)

Conversion Factors: See Btu, Btu Conversion Factors, and Metric Conversion Factors.

Conversion Losses: The amount of energy lost during generation, transmission, and distribution of energy sources particularly electricity, including plant and unaccounted-for uses. (See Consumption, Site Electricity, and Primary Electricity.)

Cooking: In this report, the use of energy for commercial or institutional food preparation. This survey asked specifically about "commercial or institutional cooking," which was intended to include any kitchen facility that was not part of a residence. This is one of six energy end uses specifically asked for in this survey. (See Energy End Use.)

Cooling: Conditioning of room air for human comfort by a refrigeration unit (such as an air conditioner or heat pump) or by a central cooling or district cooling system that circulates chilled water. Use of fans or blowers by themselves, without chilled air or water, is not included in this definition of cooling. This is one of six end uses specifically asked for in this survey. (See Energy End Use, Central Cooling, Central Chiller, Heat Pump, Heating, Ventilation, and Air-Conditioning (HVAC), and Residential-Type Central Air Conditioner.) 
Cooling Distribution Equipment: The part of a cooling system that distributes conditioned water and/or air by means of pipes, ducts, or fans. Often the distribution serves both heating and cooling. (See Duct, Individual Room AirConditioners in Wall or Windows, and Fan-Coil Unit.)

Cooling Equipment: The equipment used for cooling room air in the building for human comfort. (See Cooling Distribution Equipment and also descriptions of specific response categories collected in the FBSS: Residential-Type Central Air-Conditioner, Heat Pump, Individual Room Air-Conditioners in Walls or Windows, Central Chillers, and Packaged Units.)

Cubic Foot (cf): As a natural gas measure, the volume of gas contained in a cube with an edge that is 1 foot long at standard temperature and pressure (60 degrees Fahrenheit and 14.73 pounds standard per square inch.) The thermal content varies by the composition of the gas. (See Natural Gas and Btu Conversion Factors.)

\section{Daylighting Controls: See Natural Lighting Control Sensors.}

Decorative or Construction Glass: An exterior building wall material of glass decorative coverings such as glass blocks or spandrels, that are not window or vision (see-through) glass. Structural glass or glass curtain walls used on the outside of buildings are also included in this category. In the "Detailed Tables," decorative or construction glass is included in "Other" in the "Predominant Wall Materials" stub. (See Window or Vision Glass.)

Direct Electricity Load Control Program: A conservation program in which the utility system operator has direct control of the power supply to individual equipment on consumer premises and is able to interrupt consumer load at the time of peak load.

Discrete-Delivery Energy Sources: Energy sources that arrive at a building (site) in units or containers of a fixed size, rather than being available on a continuous basis. In this report, fuel oil is the only discrete delivery energy source. (See Energy Source and Continuous-Delivery Energy Sources.)

Distributed/Point-of-Use Water-Heating System: A system for heating hot water, for other than space-heating purposes, which is located at more than one place within a building. A point-of-use water heater is located at the faucet and heats water only as required for immediate use. Because water is not heated until it is required, this equipment is more energy efficient. (See Water-Heating Equipment and Centralized Water Heating System.)

District Chilled Water: Chilled water from an outside source used as an energy source for cooling in a building. The water is chilled in a central district system and piped into the building. Chilled water may be purchased from a utility or provided by a central physical plant in a separate building that is part of the same multibuilding facility (for example, a hospital complex or university). (See Energy Source, Central Physical Plant, and Multibuilding Facility.)

District Heat: Steam or hot water from an outside source as an energy source for space heating or another end use in a building. The steam or hot water is produced in a central plant and piped into the building. The district heat may be purchased from a utility or provided by a central physical plant in a separate building that is part of the same multibuilding facility (for example, a hospital complex or university.) For this report, district steam and district hot water are reported together as district heat in most places. (See Energy Source, Central Physical Plant, and Multibuilding Facility.)

District Hot Water: District heat in the form of hot water. (See District Heat.)

District Steam: District heat in the form of steam. (See District Heat.)

Duct: A passageway made of sheet metal or other suitable material to convey air from the heating, ventilating, and cooling systems to and from the point of utilization. (See Air-Handling Units.) 
Economizer Cycle: An HVAC conservation feature, a method of operating a ventilation system to reduce the airconditioning load. Wherever the temperature and humidity of the outdoor air are more favorable (lower heat content) than the temperature and humidity of the return air, more outdoor air is brought into the building. An economizer consists of indoor and outdoor temperature and humidity sensors, dampers, motors, and motor controls. (See HVAC Conservation Features.)

Electric Baseboard: An individual space heater with electric resistance coils mounted behind shallow panels along baseboards. Electric baseboards rely on passive convection to distribute heated air to the space. (See Individual Space Heater and Baseboard.)

Electric Utility Energy Conservation Program Sponsor: An energy conservation program sponsored by an electric utility that suggests ways to increase the energy efficiency of buildings, to reduce energy costs, to change usage patterns, or to promote the use of a different energy source. (See Energy Conservation Program Sponsor, Utility-Sponsored Energy Conservation Program, and Retrofit or Purchase of Any Equipment.)

Electricity: As an energy source for this report, electric energy supplied to a building by a central utility via power lines or from a central physical plant in a separate building that is part of the same multibuilding facility. Electric power generated within a building for exclusive use in that building is specifically excluded from the definition of electricity as an energy source. (See Energy Source, Central Physical Plant, Multibuilding Facility, Primary Electricity, and Site Electricity.)

Electricity Generation: The onsite production of electricity using electricity generators on either a regular or emergency basis. This is one of the end uses of energy specifically asked for in this survey. Not included in this survey were electricity-generating plants belonging to utility companies that produce electric power for sale to other buildings but are not part of the same multibuilding facility. (See Energy End Use, Electricity, and Multibuilding Facility.)

EMCS: See Energy Management and Control System (EMCS).

Energy Audit: In this report, an evaluation to provide information on the physical and operating characteristics of a building and its energy uses and processes that is collected at the premise or facility by trained auditors. Audit services vary from simple walk-throughs to building management training programs and site-specific process and efficiency evaluations. Audits can be initiated or sponsored and performed by a local utility, a Federal, State or local government, a building owner, or an energy service contractor. (See Energy Management Practices.)

Energy Conservation Features: In the "Detailed Tables," this includes building shell conservation features, HVAC conservation features, lighting conservation features, and other conservation features incorporated by the building. However, this category does not include participation in energy conservation programs. (See Building Shell Conservation Features, HVAC Conservation Features, and Lighting Conservation Features.)

Energy Conservation Programs: In this report, this is the planning and implementation of strategies designed to encourage consumers to improve energy efficiency, reduce energy costs, change the time of usage, or promote the use of a different energy source. This covers the complete range of load-shape objectives, including strategic conservation and load management, as well as strategic load growth.

The FBSS collected information on a variety of conservation strategies. This information included whether the building's electric or natural gas utility had sponsored any programs; whether the building had participated in, or planned to participate in, any programs sponsored by FEMP, in-house, by a utility, or by a third-party; which specific program areas the building had participated in, such as: the building envelope or direct electricity load control; identification of specific program sponsors; and what type of assistance was received through the program, such as: the Federal Energy Efficiency Fund (FEEF), general information, incentives, or alternative rates. (See Energy Management Practices.) 
Energy Conservation Program Assistance: In this report, energy conservation program assistance consists of: the Federal Energy Efficiency Fund (FEEF), general information, site-specific information, incentives, alternative-rate programs, fuel-switching programs, and other programs. This assistance can be provided by FEMP, utilities, an inhouse group, or third parties, such as an energy service company or contractor. Assistance for energy conservation programs may be monetary or nonmonetary awards to encourage consumers to buy energy-efficient equipment and to participate in programs designed to reduce energy usage. Examples of incentives are zero or low-interest loans, rebates, and direct installation of low-cost measures, such as water-heater wraps or compact fluorescent bulbs.

Energy Conservation Program Sponsor: An energy conservation program can be sponsored by FEMP, an electric or natural gas utility, in-house, or a third party, such as an energy service company or contractor. A sponsor suggests ways to increase the energy efficiency of buildings, to reduce energy costs, to change the usage patterns, or to promote the use of a different energy source through energy conservation programs.

Energy-Efficient Motors: These are also known as "high-efficiency motors" and "premium motors." They are virtually interchangeable with standard motors, but differences in construction make them more energy efficient.

Energy End Use: A use for which energy is consumed in a building. Information on six specific end uses was collected in this survey. (See Cooking, Cooling, Space Heating, Electricity Generation, Manufacturing, and Water Heating.)

Energy Intensity: The ratio of consumption to unit of measurement (floorspace, number of workers, etc.) In this report, energy intensity is usually given on an aggregate basis, as the ratio of the total consumption for a set of buildings to the total floorspace in those buildings. The energy intensity can also be computed for individual buildings. (See Consumption, Conditional Energy Intensity, and Floorspace.)

Energy Management and Control System (EMCS): An energy conservation feature that uses mini/microcomputers, instrumentation, control equipment, and software to manage a building's use of energy for heating, ventilation, airconditioning, lighting, and/or business-related processes. These systems can also manage fire control, safety, and security. Not included as EMCS are time-clock thermostats. (See Energy Management Practices.)

Energy Management Practices: In this report, involvement, as a part of the building's normal operations, in energy efficiency programs that are designed to reduce the energy used by specific end-use systems. In the "Detailed Tables," this includes the following: Energy Management and Control System, Energy Conservation Programs, Energy Audit, and HVAC Maintenance Staff. (See Energy Management and Control System (EMCS), Energy Conservation Program, Energy Audit, and HVAC Maintenance Staff.)

Energy Source: A type of energy or fuel consumed in the building. For this report, the major energy sources identified are electricity, natural gas, fuel oil, district heat, and district chilled water. In this survey, information about the use of propane, wood, coal, photovoltaic cells and solar thermal panels in commercial buildings was obtained from the building respondent. (See Electricity, Natural Gas, Fuel Oil, District Heat, District Chilled Water, Liquefied Petroleum Gas (LPG), Propane, Wood, Coal, Photovoltaic Cells (PVC"s) and Solar Thermal Panels.)

Energy Source-Specific Floorspace: Total floorspace of those buildings that use a particular fuel. (See Conditional Energy Intensity.)

Envelope: See Building Shell (Envelope).

Establishment: As defined by the Standard Industrial Classification manual developed by the Office of Management and Budget, "an economic unit, generally, at a single physical location where business is conducted or where services or industrial operations are performed." However, "establishment" is not synonymous with "building."

Evaporative Cooler (Swamp Cooler): An air-cooling unit that turns air into moist, cool air by saturating the air with water vapor. It does not cool air by use of a refrigeration unit. This type of equipment is commonly found in warm, dry climates. (See Cooling.) 
Expenditures: Funds spent for the energy consumed in, or delivered to, a building during a given period of time. For this report, all expenditure statistics are presented on an annual basis, for fiscal year 1993. The total dollar amount includes State and local taxes, fuel adjustment charges, system charges, and demand charges. The total dollar amount excludes merchandise, repair charges, and service charges. Data on energy expenditures were not collected by end uses separately. For example, although it might be known that electricity was used in some buildings for heating, the expenditures for electricity reported for those buildings would typically include other uses of electricity as well (such as lighting and water heating). (See Consumption.)

Expenditures per Million Btu: The aggregate ratio of a group of buildings' total expenditures for a given fuel to the total consumption of that fuel. (See Expenditures and Consumption.)

Expenditures per Square Foot: The aggregate ratio of a group of buildings' total expenditures for a given fuel to the total floorspace in those buildings. (See Expenditures, Floorspace, and Square Footage.)

Exterior or Interior Shadings or Awnings: A building shell conservation feature designed to reduce the flux of light into a building. Exterior shadings or awnings include any type of shading (including architectural) or awning on the outside of the building designed to limit solar penetration. Interior shadings are drapes, horizontal or vertical shades, mini blinds, or any other means of covering a window from the inside to limit the amount of solar or thermal penetration. (See Building Shell Conservation Features.)

Facility: At the sampling stage, an establishment that encompasses more than one building at a single location. Examples include college campuses and large hospital complexes. The building represents the interviewed sampling unit for this survey. Listings for the area sample ordinarily identified each building individually. For all sample buildings, a survey question determined whether the building was part of a multibuilding facility. In many cases, a building was reported during the interview to be part of a multibuilding facility even though the building had not been identified as part of a facility at the sampling stage. More rarely, a building identified as part of a facility during sampling was reported not to be part of a multibuilding facility during the interview. (See Building and Multibuilding Facility.)

Fan-Coil Unit: A type of heating or cooling distribution equipment that circulates hot or chilled water with fans but without ducts. Fan-coil units have thermostatically controlled built-in fans that draw air from the room and then across finned tubes containing hot water, steam, or chilled water. The hot water, steam or chilled water can be produced by equipment within the building or be piped into the building as part of a district heating or cooling system. (See Space Heating and Cooling.)

Federal Energy Efficiency Fund (FEEF): A fund established by the Energy Policy Act of 1992 to improve energy efficiency in Federal facilities by providing grants to Federal agencies to implement energy efficiency and water conservation projects. (See Energy Conservation Program Assistance and Retrofit or Purchase of Any Equipment.)

Federal Energy Management Program (FEMP): A Department of Energy program aiming to reduce the cost of government and make it work better through energy efficiency, use of renewable energy, and water conservation. (See Energy Conservation Program Assistance and Retrofit or Purchase of Any Equipment.)

Floors: The number of levels in the tallest section of a building that are actually considered a part of the building, including parking areas, basements, or other floors below ground level.

Floorspace: All the area enclosed by the exterior walls of a building, including indoor parking facilities, basements, hallways, lobbies, stairways, and elevator shafts. For aggregate floorspace statistics, floorspace was summed or aggregated over all buildings in a category (such as all office buildings in Federal Region 3). (See Square Footage.)

Fluorescent Light Bulbs: These are usually long, narrow, white tubes made of glass coated on the inside with fluorescent material that are connected to a fixture at both ends of the light bulb; the tubes may also be circular or $U$ shaped. The light bulb produces light by passing electricity through mercury vapor, causing the fluorescent coating to glow or fluoresce. Excluded are compact fluorescent light bulbs, which are a separate category. In the "Detailed Tables," these bulbs are included in the "Standard Fluorescent" category in the "Lighting Equipment" stub. (See Light Bulbs, and Compact Fluorescent Light Bulbs.) 
Forced Air Through Vents or Air-Handling Units: See Air-Handling Units.

Fuel: See Energy Source.

Fuel Oil: A liquid petroleum product less volatile than gasoline, used as an energy source. In this report, fuel oil includes distillate fuel oil (No. 1, 2, and 4), residual fuel oil (No. 5 and 6), and kerosene. Number 1 distillate fuel oil is used mostly as a blending stock to assure that heavier grades of fuel flow under severe cold weather conditions. Number 2 fuel oil is the most common form of heating oil. Number 2 distillate collectively refers to Number 2 heating oil and Number 2 diesel fuel. Although these products are not precisely identical, they are essentially interchangeable in most applications. Number 4 distillate is a blend of Numbers 2, 5 or Number 6 residual fuel oil, used in large stationary diesel engines and boilers equipped with fuel preheating equipment. (See Energy Source.)

Fuel-Switching: See Replacement Energy Source for Main Heating.

Fuel-Switching Program Assistance: A type of assistance where the sponsor encourages consumers to change from one fuel to another for a particular end-use service. For example, utilities might encourage consumers to replace electric water heaters with gas units or encourage industrial consumers to use electric microwave heaters instead of natural gasheaters. (See Energy Conservation Program Assistance, Energy Conservation Program, and Retrofit or Purchase of Any Equipment.)

Furnace: An enclosed chamber where fuel is burned or electrical resistance is used to heat air directly, without using steam or hot water. The warm air for heating is distributed throughout the building, typically by air ducts. (See Boiler; Duct; Space Heating; and Heating, Ventilation, Air Conditioning, and Heating (HVAC).)

Gallon: A volumetric measure equal to 4 quarts (231 cubic inches) used to measure fuel oil. One barrel equals 42 gallons. (See Barrel.)

General Information Energy Conservation Program Assistance: This type of program assistance refers to efforts of an energy conservation sponsor to inform consumers about program options through such mechanisms as brochures, bill stuffers, and workshops. (See Energy Conservation Program Assistance and Energy Conservation Programs.)

Heating: See Space Heating or Water Heating.

Heating Distribution Equipment: The part of a heating system that distributes conditioned water and/or air throughout a building by means of pipes, ducts, or fans. Often the distribution equipment serves both heating and cooling. (See Radiators, Baseboard, Duct, Individual Space Heater, and Fan-Coil Unit.)

Heating Equipment: The equipment used for heating ambient air in the building such as a heat pump, furnace, individual space heater, district steam or hot water piped in from outside the building, boiler and packaged-heating units. (See Heating Distribution Equipment, Boiler, Furnace, Heat Pump, Individual Space Heater, and Packaged Units.)

Heating, Ventilation, and Air-Conditioning (HVAC): The system or systems that condition air in a building.

Heat Pump: Heating and/or cooling equipment that draws heat into a building from outside and, during the cooling season, ejects heat from the building to the outside. Heat pumps are vapor-compression refrigeration systems whose indoor/outdoor coils are used reversibly as condensers or evaporators, depending on the need for heating or cooling. (See Cooling, Space Heating, Central Cooling, and Heating, Ventilation, and Air-Conditioning (HVAC).)

HID: See High-Intensity Discharge (HID) Light Bulbs. 
High-Intensity Discharge (HID) Light Bulbs: A lamp that produces light by passing electricity through gas, which causes the gas to glow. Examples of HID lamps are mercury vapor lamps, metal halide lamps, and high- and lowpressure sodium lamps. HID lamps have an extremely long life and emit far more lumens per fixture than do fluorescent lights. (See Light Bulbs.)

Hot-Deck Imputation: An imputation procedure for deriving a probable response to a questionnaire item concerning the commercial building using random resampling from nonmissing cases to fill in values for missing cases. (See Imputation.)

HVAC: See Heating, Ventilation, and Air-Conditioning (HVAC).

HVAC Conservation Features: A building feature designed to reduce the amount of energy consumed by the heating, cooling, and ventilating equipment. In the "Detailed Tables," this category includes the presence of variable air-volume (VAV) systems, an economizer cycle, and preventive maintenance programs for the heating and cooling equipment. (See Variable Air-Volume (VAV) System, Economizer Cycle, and Preventive Maintenance Program for the Heating and/or Cooling Equipment.)

HVAC Maintenance: See Preventive Maintenance Program for Heating and/or Cooling Equipment.

HVAC Maintenance Staff: The building employs at least one person who spends at least half of their working hours maintaining the heating and/or cooling equipment. (See Energy Management Practices.)

Imputation: A statistical method used to fill in values for missing items, designed to minimize the bias of estimates based on the filled-in data set. (See Hot-Deck Imputation, Regression)

Incandescent Light Bulbs: A light bulb that produces a soft warm light by electrically heating a tungsten filament so that it glows. Because so much of the energy is lost as heat, these are highly inefficient sources of light. The halogen light bulb is a type of incandescent light bulb, made more efficient by the addition of a halogen gas. Included in this category are the familiar type of light bulbs which screw into sockets, as well as energy-efficient incandescent bulbs such as Tungsten Halogen (spotlights), Reflector or R-Lamps (accent and task lighting), Parabolic Aluminized Reflector (PAR) lamps (flood and spot lighting), and Ellipsoidal Reflector (ER) lamps (recessed lighting). (See Light Bulbs.)

Incentives: A type of program assistance that provides cash or non-cash awards to customers to encourage the purchase or sales of conservation programs. Appliance rebate programs are the most common example on incentive programs. Incentives can also include zero or low-interest loans. (See Energy Conservation Program Assistance, Energy Conservation Programs, and Retrofit or Purchase of Any Equipment.)

Individual A/C: See Individual Room Air-Conditioners in Walls or Windows.

Individual Room Air-Conditioners in Walls or Windows: Self-contained air-conditioning units installed in either walls or windows (with heat-radiating condensers exposed to the outdoor air). These units are characterized by a lack of pipes or duct work for distributing the cool air; the units condition air only in the room or areas where they are located. In the "Detailed Tables," these are labeled as "Individual A/C." (See Cooling.)

Individual Space Heater: A free-standing or self-contained unit that generates and delivers heat to a local zone within the building. The heater may be permanently mounted in a wall or floor, or may be portable. Examples of individual space heaters include electric baseboards, electric radiant or quartz heaters, heating panels, gas- or kerosene-fired unit heaters, wood stoves, and infrared radiant heaters. These heaters are characterized by a lack of pipes or duct work for distributing hot water, steam, or warm air through the building. (See Baseboard and Electric Baseboard.)

Industrial: See Manufacturing/Industrial. 
In Scope: Meeting the requirements for eligibility in the FBSS, and, therefore, included in the population covered by the survey. These eligibility requirements were (a) that the structure be a Federal building in Region 3,6, or 9; (b) that the building be larger than 10,000 square feet; and (c) that more than 50 percent of the floorspace be used for commercial activities excluding buildings used for warehouse and storage. (See Building, Commercial, and Floorspace.)

Insulation: A building shell conservation feature consisting of material placed between the interior of a building and the outdoor environment to reduce the rate of heat loss to the environment or heat gain from the environment. Examples include glass-wool fill and foam board. (See Roof or Ceiling Insulation, Wall Insulation, and Building Shell Conservation Features.)

Intensity: The amount of a quantity per unit of measurement (floorspace, number of workers, etc.) This is a method of adjusting either the amount of energy consumed or expenditures spent, for the effects of various building characteristics such as size of the building, number of workers, or number of operating hours, to facilitate comparisons of energy across time, fuels, and buildings. (See Conditional Energy Intensity, Energy Intensity, and Expenditures per Square Foot.)

Kerosene: A petroleum distillate with properties similar to No. 1 fuel oil, used primarily in space heaters, cooking stoves, and water heaters. In this report, no distinction is made between kerosene and fuel oil. (See Fuel Oil.)

Kilowatthour (kWh): A unit of work or energy, measured as 1 kilowatt (1,000 watts) of power expended for 1 hour. One Kwh is equivalent to 3,412 Btu. (See Btu and Electricity.)

\section{Lamp: See Light Bulbs.}

Light Bulbs: A term generally used to describe a manmade source of light. The term is often used when referring to a "bulb" or "tube." The FBSS collected data only about light bulbs using electricity. (See Incandescent Light Bulbs, Fluorescent Light Bulbs, Compact Fluorescent Light Bulbs and High-Intensity Discharge (HID) Light Bulbs.)

Lighting Conservation Features: A building feature or practice designed to reduce the amount of energy consumed by the lighting system. In the "Detailed Tables," Lighting Conservation Features include natural lighting control sensors, manual dimmer switches, occupancy sensors, specular reflectors, and time clocks or timed switches. (See Natural Lighting Control Sensors, Manual Dimmer Switches, Occupancy Sensors, Specular Reflectors, and Time Clocks or Timed Switches.)

Lighting Equipment: These are light bulbs used to light the building's interior, such as incandescent light bulbs, fluorescent light bulbs, compact fluorescent light bulbs, and high-intensity discharge (HID) lights. (See Incandescent Light Bulbs, Fluorescent Light Bulbs, Compact Fluorescent Light Bulbs, High-Intensity Discharge (HID) Light Bulbs, and Electronic Ballasts.)

Liquefied Petroleum Gas (LPG): Gas fuel in liquid form supplied to a building as an energy source. The fuel is usually delivered by tank trucks and stored near the building in a tank or cylinder until used. LPG contains mostly propane, but can contain such gases as butane, propylene, butylene, or ethane. For this report, any LPG reported was assumed to be propane. (See Energy Source, Propane, and Natural Gas.)

\section{LPG: See Liquefied Petroleum Gas (LPG).}

Main Space-Heating Energy Source: The energy source used to heat most of the square footage in the building most of the time.

Major Energy Sources: The energy sources or fuels for which consumption and expenditures data were collected in the FBSS. These fuels or energy sources are: electricity, fuel oil, natural gas, district steam, district hot water, and district chilled water. District chilled water is not included in any totals for the sum of major energy sources or fuels; all other major fuels are included in these totals. (See Energy Sources.) 
Major Fuels: See Major Energy Sources.

Manual Dimmer Switches: A lighting conservation feature that changes the level of light in the building. These are like residential-style dimmer switches, which are not commonly used with fluorescent or HID lamps. (See Lighting Conservation Features.)

Manufacturing: As an energy end use, any of the energy-using operations required for manufacturing/industrial processes. Manufacturing is one of the six end uses of energy specifically requested in this survey. (See Energy End Use and Manufacturing/Industrial.)

Manufacturing/Industrial: As a building activity in this survey, activities involving the processing or procurement of goods, merchandise, raw materials, or food. These activities include: food processing; leather/textile mills; light assembly factories, such as those for apparel and electronic instruments; heavy assembly factories, such as those for machinery and other heavy equipment; paper processing; chemical or petroleum processing, metalwork, glasswork, and other similar manufacturing plants; printing and publishing; generation, transmission, or distribution of electricity, natural gas, steam, or other utility or sanitary service; and construction and natural resource procurement.

Commercial buildings (such as offices) that were associated with a manufacturing establishment were included, but the manufacturing and industrial buildings were excluded from the population covered. Such buildings could be included in the sample during the listing stage. However, buildings that had 50 percent or more of their square footage devoted to manufacturing or industrial activities were dropped from the sample during the interview stage. (See Principal Building Activity and Appendix B, "Types of Buildings.")

Masonry: A general term covering wall construction using masonry materials such as brick, concrete block, stone, and tile that are set in mortar; also included is stucco. This category does not include concrete panels since concrete panels represent a different method of constructing buildings. Concrete panels are included in the "Other" category. (See Precast Concrete Panel.)

Mean: The simple arithmetic average for a population is the sum of all the values in a population divided by the size of the population. For this report, population means are estimated by computing the weighted sum of the sample values, then dividing by the sum of the sample weights. (See Median and Weight.)

Mean Operating Hours: The arithmetic average number of operating hours per building is the weighted sum of the number of operating hours divided by the weighted sum of the number of buildings.

Mean Square Feet per Building: The arithmetic average square feet per building is the weighted sum of the total square feet divided by the weighted sum of the number of buildings.

Mean Square Feet per Worker: The arithmetic average square feet per worker is the weighted sum of the total square feet divided by the weighted sum of the total number of main shift workers.

Median: The middle value in the population; half the population has a value above the median and half has a value below. The median is different from the mean in that its estimate in not influenced much by extremes in the sample. For example, an estimate of the mean square feet per building would be affected by the inclusion of some very large buildings and would not express square footage for a "typical" building. In contrast, the median square feet would not be so affected. (See Mean.)

Metal Panels: An exterior wall construction material made of aluminum or galvanized steel panels fabricated in factories and fastened to the frame of the building to form outside walls. Pre-engineered metal buildings are also included in this category. These are included in the "Other" category in the "Predominant Exterior Wall Material" stub.

Metal Surfacing: Light-gauge metal sheets used for roofing. These are included in the "Other" category in the "Predominant Roof Material" stub. 
Metric Conversion Factors: In this report, estimates are presented in customary U.S. units. Floorspace estimates may be converted to metric units by using the relationship: 1 square foot is approximately equal to .0929 square meters. Energy estimates may be converted to metric units by using the relationship, $1 \mathrm{Btu}$ is approximately equal to 1,055 joules. One kilowatthour is exactly equal to $3,600,000$ joules. One gigajoule $\left(10_{9}\right.$ joules $)$ is approximately 278 kilowatthours (kWh).) (For additional metric conversions, see Appendix D, "Metric Conversion Factors.")

More than One May Apply: In the "Detailed Tables," a row stub accompanied by this phrase indicates overlapping categories, so that a particular building may be represented in more than one line under this stub. In general, row stubs without this designation are exclusive, that is, they divide the population of buildings into distinct groups, so that a particular building is represented in no more than one line under this stub.

Multibuilding Facility: A group of two or more buildings on the same site owned or operated by a single organization, business, or individual. Examples include university campuses and hospital complexes. (See Building and Facility.)

Natural Gas: Hydrocarbon gas (mostly methane) supplied as an energy source to individual buildings by pipelines from a central utility company. Natural gas does not refer to liquefied petroleum gas (LPG) or to privately owned gas wells operated by a building owner. (See Energy Source, Liquefied Petroleum Gas (LPG), and Propane.)

Natural Gas Transported for the Account of Others: Natural gas physically delivered to a building by a local utility, but not bought from that utility. A separate transaction is made to purchase the volume of gas and the utility is paid for the use of its pipeline to deliver the gas. Included are quantities covered by long-term contracts and quantities involved in short-term or spot-market sales. Also called "Direct-Purchase Gas," "Spot- Market Gas," "Spot Gas," "Transported Gas," and "Self-Help Gas."

Natural Gas Utility Energy Conservation Program Sponsor: An energy conservation program sponsored by a natural gas utility that suggests ways to increase the energy efficiency of buildings, to reduce energy costs, to change the usage patterns, or to promote the use of a different energy source. (See Energy Conservation Program Sponsor and Utility-Sponsored Energy Conservation Program).

Natural Lighting Control Sensors: A lighting conservation feature that takes advantage of sunlight to cut the amount of electric lighting used in a building; a control system that varies the light output of an electric lighting system in response to variations in available daylight. It is sometimes referred to as "daylighting controls" or "photocells." (See Lighting Conservation Features.)

Nonresidential Building: A building used for some purpose other than residential. Nonresidential buildings comprise three groups: commercial, manufacturing/industrial, and agricultural. Commercial buildings are the focus of this report. Additionally, the FBSS excluded buildings that were used for warehouse and storage. (See Commercial Building, Manufacturing/Industrial, Building, Residential, Principal Building Activity, Out of Scope, and Appendix B, "Types of Buildings.")

Occupancy Sensors: A lighting conservation feature that uses motion or sound to switch lights on or off; also known as "ultrasonic switching." When movement is detected, the lights turn on and remain on as long as there is movement in the room. Occupancy sensors that detect sound work like ultrasonic switching; when sound is detected, the lights turn on. In this report, occupancy sensors refer to detecting movement, not sound. (See Lighting Conservation Features.)

Off-Hour Equipment Reduction: A method of conserving energy by changing the temperature setting or reducing the use of heating, cooling, domestic hot water heating, lighting or any other equipment either manually or automatically when the building is closed. (See Conservation Features.)

Operating Hours: See Weekly Operating Hours.

Out of Scope: Violating one or more of the requirements for eligibility in the survey, therefore not included in the population covered by the FBSS. (See In Scope.) 
Packaged A/C Units: See Packaged Units.

Packaged Heating Units: See Packaged Units.

Packaged Units: Units built and assembled at a factory and installed as a self-contained unit to heat or cool all or portions of a building. Packaged units are in contrast to engineer-specified units built up from individual components for use in a given building. "Packaged Units" is a term that can apply to heating equipment, cooling equipment, or combined heating and cooling equipment. Some types of electric packaged units are also called "Direct Expansion" or DX units. (See Cooling; Heating, Ventilation, and Air-Conditioning (HVAC); and Space Heating.)

Percent Lit When Closed: The percentage of the building's square footage that is lit electrically during all hours other than the usual operating hours. (See Percent Lit When Open, Square Footage, and Weekly Operating Hours.)

Percent Lit When Open: The percentage of the building's square footage that is lit electrically during usual operating hours. (See Percent Lit When Closed, Square Footage and Weekly Operating Hours.)

Percent of Floorspace Cooled: The percentage of the building's square footage that is cooled to meet the comfort requirements of the occupants. (See Square Footage and Cooling.)

Percent of Floorspace Heated: The percentage of the building's square footage designed to be heated to at least 50 degrees Fahrenheit. (See Square Footage and Space Heating.)

Percent Window Glass: The percentage of the building's exterior wall construction material made of glass that can be seen through from the inside of the building. This percentage excludes glass covered or constructed of glass material that cannot be seen through. (See Decorative or Construction Glass and Window or Vision Glass.)

Photovoltaic Cells (PVC's): A device that produces electrical current by converting light or similar radiation. In the "Detailed Tables," PVC's are included in the "Any Other" category in the "Energy Sources," "Space-Heating Energy Sources," and "Main Space-Heating Energy Sources" stubs.

Plastic, Rubber, or Synthetic Roofing: A layer of heavy gauge plastic or rubber used for roofing. In the "Detailed Tables," plastic, rubber or synthetic roofing are included in the "Synthetic or Rubber" category in the "Predominant Roof Material" stub.

Point-of-Use Water-Heating System: See Distributed/Point-of-Use Water-Heating System.

Pounds (District Heat): A weight quantity of steam, also used in this report to denote a quantity of energy in the form of steam. The amount of usable energy obtained from a pound of steam depends on its temperature and pressure at the point of consumption and on the drop in pressure after consumption. A conversion factor of 1,000 Btu per pound was used for steam. Hot water, always reported in Btu, was converted to equivalent pounds of steam using the same factor of 1,000 Btu per pound. (See Btu, District Steam, and District Heat.)

Precast Concrete Panel: Refers to concrete panels usually made in factories and delivered to the construction site where they are hoisted onto the structure. Sometimes concrete panels are poured at the site and then hoisted on the structure. The panels are either solid or insulated. They can have plain, colored or textured finishing. In the "Detailed Tables," pre-cast concrete panels are included in the "Other" category in the "Predominant Wall Material" stub. (See Concrete Panel.)

Preventive Maintenance Program for Heating and/or Cooling Equipment: As used in this report, an HVAC conservation feature consisting of a program of routine inspection and service for the heating and/or cooling equipment. The inspection is performed on a regular basis, even if there are no apparent problems. (See HVAC Conservation Features.)

Primary Electricity: The amount of electricity delivered to commercial buildings adjusted to account for the fuels used to produce the electricity. That is, site electricity plus the conversion losses in the generation process at the utility plant. (See Consumption, Conversion Losses, Electricity and Site Electricity.) 
Primary Energy Consumption: See Consumption.

Principal Building Activity: The activity or function occupying the most floorspace in the building. The categories were designed to group buildings that have similar patterns of energy consumption. Examples of various types of principal activity include office, health care, lodging, and mercantile and service. (See Appendix B, "Types of Buildings.")

Principal Facility Activity: The main purpose for the activities across all buildings in a facility; for example, the principal building activity for a library on a school campus is "public assembly;" however, the principal facility activity is "school."

Process Heating or Cooling Energy Conservation Program: An energy conservation program designed to promote increased electric energy efficiency applications in industrial process heating or cooling. (See Energy Conservation Programs and Waste-Heat Recovery.)

Propane: A gaseous petroleum product that liquefies under pressure; propane is a major component in liquefied petroleum gas, or LPG. Any LPG reported in the CBECS was assumed to be propane. (See Liquefied Petroleum Gas (LPG).)

Quad: Quadrillion $\left(10^{15}\right)$ Btu. (See Btu.)

Radiator: A heating unit usually visibly exposed within the room or space to be heated; it transfers heat from steam or hot water by radiation to objects within visible range and by conduction to the surrounding air, which in turn is circulated by natural convection. Typically, a radiator is a freestanding, cast-iron fixture. (See Space Heating.)

Reflectors: See Specular Reflectors.

Regression: A statistical procedure used in this report to estimate consumption of, or expenditures for, energy when data were unavailable. The procedure takes into account many characteristics of buildings (such as size, age, principal activity, heating fuels). (See Imputation.)

Regular HVAC Maintenance: See Preventive Maintenance Program for Heating and/or Cooling Equipment.

Reheating Coils: A part of some air-conditioning systems, these are electric coils in air ducts used primarily to raise the temperature of circulated air after it was over cooled to remove moisture. Some buildings report reheating coils as their sole heating source. (See Air-Handling Units, Cooling, and Space Heating.)

\section{Relative Standard Error: See RSE or Relative Standard Error.}

Replacement Energy Source for Main Heating: In this report, the heating energy source to which the building could switch within one week without major modifications to the main heating equipment, without substantially reducing the area heated, and without substantially reducing the temperature maintained in the heated area.

Residential: As a building activity in this survey, activities related to use as a dwelling for one or more households. Residential buildings that contained commercial activities were included in the sample during the listing stage. However, buildings that had 50 percent or more of their square footage devoted to residential activities were considered out of scope and dropped from the sample during the interview stage. (See Principal Building Activity, In Scope, and Commercial Building.)

Residential-Type Central Air-Conditioner: There are four basic parts to a residential central air-conditioning system: (1) a condensing unit, (2) a cooling coil, (3) ductwork, and (4) a control mechanism such as a thermostat. There are two basic configurations of residential central systems: (1) a "split system" where the condensing unit is located outside and the other components are inside, and (2) a packaged-terminal air-conditioning (PTAC) unit that both heats and cools or cools only. This system contains all four components encased in one unit and is usually found in a "utility closet." If the residential type is a "PTAC," it is considered a "Packaged air-conditioning unit." 
Retrofit or Purchase of Any Equipment: The FBSS determined whether the building had retrofitted or purchased any of the heating equipment, central chillers, water heating equipment, lighting equipment, or refrigeration equipment in the past ten years. The survey also collected the sponsor and type of assistance received for these retrofits or purchases. The sponsors included an electric utility, in-house, a third party, and FEMP. The type of assistance included the Federal Energy Efficiency Fund, incentives, alternative rates, and fuel switching.

Roof or Ceiling Insulation: A building shell conservation feature consisting of insulation placed in the roof (below the waterproofing layer) or in the ceiling of the top floor in the building. (See Insulation and Building Shell Conservation Features.)

RSE or Relative Standard Error: A measure of the reliability or precision of a survey statistic. Variability occurs in survey statistics because the different samples that could be drawn would each produce different values for the survey statistics. The RSE is defined as the standard error (the square root of the variance,) of a survey estimate, divided by the survey estimate and multiplied by 100 . For example, an RSE of 10 percent means that the standard error is one-tenth as large as the survey estimate.

Sampling: The procedure used to select cases (in this survey, buildings) for interview from the population (Federal buildings in Regions 3, 6, or 9). (See Appendix A, "How the Survey Was Conducted.")

Secondary Heating Fuel: Fuels used in secondary space-heating equipment. When no secondary space-heating equipment is used, a secondary space-heating fuel that is used in the main space-heating equipment is not included in the tabulations. This occurs when, for example, wood and coal are both used in a furnace but wood is named the main space-heating fuel. Coal, in this case, is not tabulated.

Shadings or Awnings: See Exterior or Interior Shadings or Awnings.

Shakes: Flat pieces of weatherproof material laid with others in a series of overlapping rows as covering for roofs and sometimes the sides of buildings. Shakes are similar to wood shingles, but instead of having a cut and smoothly planed surface, shakes have textured grooves and a rough or "split" appearance to give a rustic feeling. These are included in the "Other" category in the "Predominant Wall Material" and "Predominant Roof Material" stubs (See Shingles, Siding, and Wooden Materials.)

Sheet Metal Panels: Includes metal panels made in factories and shipped to the building site where they are fastened to the building frame. They are usually aluminum or galvanized steel. These are included in the "Other" category in the "Predominant Wall Material" stub. (See Metal Panels.)

Shingles: Flat pieces of weatherproof material laid with others in a series of overlapping rows as covering for roofs and sometimes the walls of buildings. Shingles are manufactured in a variety of materials including fiberglass, wood, plastic, baked clay, tile, asbestos, asphalt, and aluminum. All types of shingles are included in the "Other" category in the "Predominant Roof Material" and "Predominant Wall Material" stubs. (See Siding, Shakes, Slate or Tile Shingles, and Wooden Materials.)

Siding: An exterior wall covering material made of wood, plastic (including vinyl), or metal. The structural walls may be masonry or wood. Siding is generally produced in the shape of boards applied to the outside of a building in overlapping rows. This is included in the "Other" category in the "Predominant Wall Material" stub. (See Wooden Materials.)

Site Electricity: The amount of electricity delivered to commercial buildings. (See Consumption, Conversion Losses, Electricity and Primary Electricity.)

Site-Specific Energy Conservation Program Assistance: A type of assistance that provides guidance on energy efficiency and load-management options tailored to a particular customer's facility; it often involves an on-site inspection of the customer facility to identify cost-effective energy conservation actions that could be taken. They include audits, engineering design calculations on information provided about the building, and technical assistance to architects and engineers who design new facilities. (See Energy Conservation Program Assistance and Energy Conservation Programs.) 
Slate or Tile Shingles: A type of roofing material. Tile refers to any thin, square, or rectangular piece of baked clay, stone, or concrete used as a roofing material. Slate refers to a particular stone used for roofing. These are included in the "Other" category in the "Predominant Roof Material" stub. (See Shingles.)

Solar Thermal Panels: These are thermal panels that use sunlight to heat fluids, a system that actively concentrates thermal energy from the sun by means of solar collector panels. The panels typically consist of flat, sun-oriented boxes with transparent covers, containing water tubes or air baffles under a blackened heat-absorbent panel. The energy is usually used for space heating, for water heating and/or for heating swimming pools. This is included in "Any Other" in the "Energy Sources" stub. (See Energy Sources.)

Space Heating: The use of mechanical equipment (including wood stoves and active solar heating devices) to heat all, or part, of a building to at least 50 degrees Fahrenheit. This is one of the six end uses of energy specifically asked for in this survey. (See Energy End Use.)

Specular Reflectors: A lighting conservation feature, this is the mirror-like backing of a florescent lighting fixture specifically designed to reflect light into the room. The materials and shape of the reflector are designed to reduce absorption of light within the fixture, while delivering light in the desired angular pattern. The most common materials used are silver (highest reflectivity) and aluminum (lowest cost). (See Lighting Conservation Features.)

Square Feet per Worker: The ratio of the total square footage in each category to the total number of workers in the category.

Square Footage: Floorspace, in units of square feet. One square foot is approximately equal to 0.0929 square meters. (See Floorspace and Metric Conversion Factors.)

Standard Error: A measure of the precision of an estimate, equal to the square root of the variance. (See Variance and RSE or Relative Standard Error.)

\section{Standard Fluorescent: See Fluorescent Light Bulbs.}

Standby Electricity Generation: Involves use of generators during times of high demand on utilities to avoid extra "peak-demand" charges.

Standby Electricity Generation Energy Conservation Program: An energy conservation program that encourages consumers to use generators during times of high electricity demand to avoid "peak-demand" charges. In the "Detailed Tables," this is included in "Energy Conservation Program" category in the "Energy Management Practices" stub. (See Energy Conservation Programs.)

\section{Steam: See District Steam.}

Steam or Hot Water Radiators or Baseboards: See Baseboard and Radiator.

Storm Doors: A building shell conservation feature consisting of a second door installed outside or inside a prime door creating an insulating air space. Included are sliding glass doors made of double glass or of insulating glass such as thermopane, double- or triple-pane glass as well as sliding glass doors with glass or plexiglass installed outside or inside of the door. Plastic materials covering doors or doors with storm window covering on just the glass portion of the door are counted only if they can be used year after year. (See Storm or Multiple Glazing.)

Storm or Multiple Glazing: A building shell conservation feature consisting of storm windows, storm doors, or double- or triple-pane glass that are placed on the exterior of the building to reduce the rate of heat loss. (See Building Shell Conservation Features.) 
Storm Windows: A building shell conservation feature consisting of a window or glazing material placed outside or inside a window creating an insulating air space. Windows with double glass or thermopanes are considered storm windows as well as windows with glass or plexiglass placed on the outside or inside of the window. Plastic material over windows is counted as a storm window if the same plastic material can be used year after year. (See Storm or Multiple Glazing.)

\section{Swamp Coolers: See Evaporative Cooler (Swamp Cooler) and Cooling.}

Synthetic or Rubber Roofing: See Plastic, Rubber or Synthetic Roofing.

Thermal Energy Storage (TES) or Pump Storage: The temporary storage of energy for later use. Examples of thermal storage are the storage of solar energy for night heating, the storage of summer heat for winter use, the storage of winter ice for space cooling in the summer, and the storage of heat or coolness generated electrically during time when electricity is cheaper (off-peak hours) for later use when electricity rates are higher. There are four basic types TES systems: ice storage, water storage, storage in a thermal mass such as soil, rock or other solids, and storage in other material such as glycol. The most commonly installed types of thermal energy storage systems in commercial buildings are those using ice or chilled water for cooling the building.

Thermostat: A device that adjusts the amount of heating and cooling produced and/or distributed by automatically responding to the temperature in the environment.

Third-Party Energy Conservation Program Sponsor: An energy service company (ESCO), which promotes a program sponsored by a manufacturer or distributor of energy products such as lighting or refrigeration whose goal is to encourage consumers to improve energy efficiency, reduce energy costs, change the time of usage, or promote the use of a different energy source. (See Energy Conservation Programs and Energy Conservation Program Sponsor and Retrofit or Purchase of Any Equipment.)

Time Clocks or Timed Switches: Time clocks are automatic controls that turn lights off and on at predetermined times. (See Lighting Conservation Features.)

Tinted or Reflective Glass or Shading Film: A building shell energy conservation feature consisting of tinted or reflective glass or shading films installed on the exterior glazing of a building to reduce the rate of solar penetration into the building. (See Building Shell Conservation Features.)

Transported Gas: See Natural Gas Transported for the Account of Others.

Trillion Btu: Equivalent to 1,000,000,000,000 (10 $\left.0^{12}\right)$ Btu. (See Btu.)

Utility-Sponsored Energy Conservation Program: In this report, this is any energy conservation program sponsored by an electric and/or natural gas utility to review equipment and construction features in buildings and advise on ways, among other things, to increase the energy efficiency of buildings; such as, programs to encourage the use of more energy-efficient equipment. Also, included in this survey were programs to improve the energy efficiency in the lighting system or building equipment, or the thermal efficiency of the building shell. (See Energy Conservation Program Sponsor.)

Vacant: As a principal building activity, the designation for a building in which most of the floorspace was not occupied by any tenant or establishment. A vacant building may contain occupants who are using up to 50 percent of the floorspace. The FBSS also measures vacancy in terms of the fraction of space vacant within an individual building and the fraction of time the building was in use. For all buildings, data were collected on the percent of floorspace vacant for three or more consecutive months, and/or the number of months the building was in use. (See Principal Building Activity, and Appendix B, "Types of Buildings.")

Variable Air-Volume (VAV) System: An HVAC system that supplies varying quantities of conditioned (heated or cooled) air to different parts of the building according to the heating and cooling needs of those specific areas. This is an HVAC conservation feature and is usually referred to as "VAV." (See HVAC Conservation Features.) 
Variance: A measure of the variability of a set of observations that are subject to some chance variation, equal to the expected squared difference between a single observation and the average of all possible observations obtained in the same manner. The variance is the square of the standard error of estimates. For statistics presented in this report, the variance indicates the likely difference between the value computed from the FBSS sample and the average of the values that could have been computed from all possible samples that might have been obtained by the same sample selection process. (See Standard Error.)

\section{VAV: See Variable Air-Volume (VAV) System.}

Walk-In Refrigeration Units: Refrigeration/freezer units within a building that are large enough to walk into. They may be portable or permanent, such as a meat storage locker in a butcher store. Walk-in units may or may not have a door, plastic strips, or other flexible covers. (See Commercial Refrigeration/Freezer Equipment.)

Wall Insulation: A building shell conservation feature consisting of insulation placed between the exterior and interior walls of a building. (See Insulation and Building Shell Conservation Features.)

\section{Warm-Air Furnace: See Furnace.}

Waste-Heat Recovery: Any conservation system whereby some space heating or water heating is done by actively capturing byproduct heat that would otherwise be ejected into the environment. In commercial buildings, sources of waste-heat recovery include refrigeration/air-conditioner compressors, manufacturing or other processes, data processing centers, lighting fixtures, ventilation exhaust air, and the occupants themselves. Not to be considered is the passive use of radiant heat from lighting, workers, motors, ovens, etc., when there are no special systems for collecting and redistributing heat. (See Process Heating or Cooling Energy Conservation Program.)

Water Heating: The use of energy to heat water for purposes other than space heating. This is one of the six end uses of energy specifically asked for in this survey. (See Energy End Use .)

Water-Heating Equipment: Automatically controlled, thermal insulated equipment designed for heating water at temperatures less than 180 degrees Fahrenheit for other than space heating purposes. This survey collected data to distinguish between two types of water heating equipment: centralized and distributed/point-of-use. (See Centralized Water-Heating System and Distributed/Point-of-Use Water-Heating System.)

Weekly Operating Hours: The number of hours per week that a building is used, excluding hours when the building is occupied only by maintenance, security, or other support personnel. For buildings with a schedule that varied during the year, "weekly operating hours" refers to the total weekly hours for the schedule most often followed. If operating hours varied throughout a building, the usual operating hours of the largest business in the building (based on square footage) determined the operating hours for the building.

Weight: The number of buildings in a certain Federal region that a particular sample building represents. To estimate the total value of an attribute (such as square footage) in the entire Federal region, each sample building's value is multiplied by the building's weight. Summing (aggregating) the weighted sample values provides an estimate of the Federal region total.

Window or Vision Glass: An exterior wall construction material made of glass that can be seen through from the inside of the building--the glass especially found in windows. Walls that are glass covered or constructed of glass material, but cannot be seen through, are excluded from this category. In the "Detailed Tables," window or vision glass is included in "Other" in the "Predominant Wall Materials" stub. (See Decorative or Construction Glass.)

Wood: As an energy source, wood logs, chips, or wood products that are used as fuel. In this survey, information about the use of wood as fuel in commercial buildings was obtained from the building respondent. Wood is included in the "Any Other" category in the "Energy Sources" stub. (See Energy Source.)

Wooden Materials: Wood shingles, wood shakes, or other wooden materials used as roofing materials. (The questionnaire also includes wood siding and shingles under exterior wall construction.) (See Shingles and Shakes.) 
Workers: The number of people working in a building across all shifts on a typical workday during the year. The main shift is the time when most people are in the building. Included in this definition are self-employed workers and volunteers. Excluded from this definition are customers, patients, and students, unless they are working for establishments in the building. Also excluded are employees who work out of the office, such as salespeople who report in, delivery people with routes, and messengers.

Year Constructed: The year in which the major part or the largest portion of a building was constructed. 